\title{
Data report: revised composite depth scales for Sites U1336, U1337, and U1338'
}

\author{
Roy H. Wilkens, ${ }^{2,}{ }^{3}$ Gerald R. Dickens, ${ }^{4,} 5$ Jun Tian, ${ }^{2}$ Jan Backman, ${ }^{2}$ \\ and the Expedition 320/321 Scientists ${ }^{2}$
}

\section{Chapter contents}

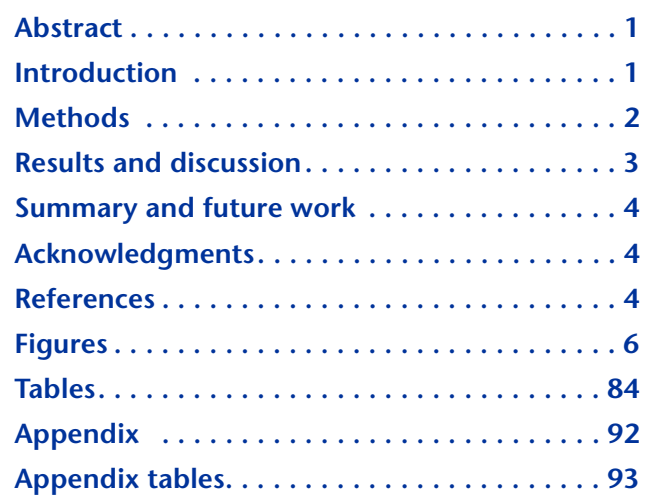

'Wilkens, R.H., Dickens, G.R., Tian, J., Backman, J., and the Expedition 320/321 Scientists, 2013. Data report: revised composite depth scales for Sites U1336, U1337, and U1338. In Pälike, H., Lyle, M., Nishi, H., Raffi, I., Gamage, K., Klaus, A., and the Expedition 320/321 Scientists, Proc. IODP, 320/ 321: Tokyo (Integrated Ocean Drilling Program Management International, Inc.). doi:10.2204/iodp.proc.320321.209.2013 'Expedition 320/321 Scientists' addresses. ${ }^{3}$ Correspondence author: rwilkens@hawaii.edu ${ }^{4}$ Department of Geological Sciences, Stockholm University, SE-10691 Stockholm, Sweden. ${ }^{5}$ Present address: Department of Earth Science, Rice University, Houston TX 77005, USA.

\section{Abstract}

The eastern equatorial Pacific (EEP) is one of the most dynamic regions of the open ocean. To fully appreciate the history of this area in the time domain, correlative and complete sedimentary records are required from multiple drill sites. One essential step for each site is the construction of an accurate composite depth scale, whereby selected intervals of successive cores from proximal holes are spliced together to render a full stratigraphic section. Here, we generate revised composite depth scales for Integrated Ocean Drilling Program (IODP) Sites U1336, U1337, and U1338, recovered during IODP Expeditions 320 and 321. Composite sections were generated using physical properties data overlain on high-resolution scanned images of adjacent core sections from all holes cored at a site. Coring disturbance, particularly deeper in the holes, prevented composite construction to total depth at each site. At Site U1336, utilizing two holes, the composite record reaches almost $135 \mathrm{~m}$ core composite depth below seafloor (CCSF). At Site U1337, with four holes, a depth of close to $450 \mathrm{~m}$ CCSF was reached with only three gaps. Using the three holes of Site U1338, a composite section of almost $400 \mathrm{~m}$ CCSF was developed with only two breaks. Composite depth records are crucial for working on these sites because sediment composition varies considerably over short $(<30 \mathrm{~cm})$ depth intervals. The composite gamma ray attenuation density records will be particularly important to a range of studies in the region because they can be coupled to those collected at earlier drill sites in the EEP.

\section{Introduction}

The eastern equatorial Pacific (EEP) delineates a region between $15^{\circ} \mathrm{N}$ and $15^{\circ} \mathrm{S}$ latitude, and between $150^{\circ} \mathrm{W}$ and the coasts of Central and South America (Fig. F1). Large zonal and meridional variations in sea surface properties (e.g., temperature, salinity, and primary productivity) characterize this area. The variance has a complex origin reflecting multiple influences; it also changes significantly on interannual timescales with El Niño Southern Oscillation (e.g., Trenberth and Caron, 2000; Pennington et al., 2006). In short, the EEP is one of the most dynamic regions of the world's oceans. 
There is considerable interest in reconstructing past sea surface properties within this region, especially during warm intervals of the late Miocene and early Pliocene (e.g., Lawrence et al., 2006; Brierley et al., 2009). However, a basic issue confronts such studies; given the extreme spatial and temporal complexities of surface water across the modern tropical Pacific, compelling arguments for regionally meaningful changes in the past require lengthy records at multiple locations that can be correlated at high temporal resolution.

Numerous sites have been drilled and cored in the EEP over the past $40 \mathrm{y}$. These include eight locations targeted by the Integrated Ocean Drilling Program (IODP) during Expeditions 320 and 321 in 2009 (see the "Expedition 320/321 summary" chapter [Pälike et al., 2010]; Fig. F1). Before the community can begin to link records between these sites and others, composite depth sections are needed. Deep-sea coring at a specific drill hole proceeds through the collection of discrete sediment cores, each retrieved after an increment of drilling. On the $\mathrm{R} / \mathrm{V}$ JOIDES Resolution, these cores, in most cases, should be 9.5 $\mathrm{m}$ in length. However, the coring process is imperfect. First, some recovered cores are less than the drilling increment, presumably because sediment has been lost. Second, the coring process typically leaves gaps (and occasionally overlaps) between successive cores. Third, there are sometimes sediment intervals disturbed by drilling. Because of these issues, coring at a single drill hole recovers a discontinuous stratigraphic record, whereas coring multiple proximal holes at a single location can lead to a continuous stratigraphic record, or "composite depth section" (e.g., Hagelberg et al., 1995).

Composite depth sections are made from spliced portions of cores from different holes. Correlative horizons are found in cores from two or more holes. By choosing such horizons that occur toward the base of a core in one hole and toward the top of a deeper core in another hole, tie points can be set so that stratigraphic records cross gaps and eliminate overlaps between successive cores in a single hole. Over the last few years, preliminary splicing has been done during the drilling expedition to evaluate stratigraphic completeness and to enhance sampling strategy. Invariably, however, some of the tie points are incorrect. Perhaps as important, intervals of core that are not part of the splice are not correlated to the composite depth section. At a drill site with three or more holes, these "off-splice" sediment intervals may comprise $60 \%-70 \%$ of the recovered sediment, but often need to be compressed or expanded, so that they can be aligned to features seen in the com- posite section. (e.g., Pälike et al., 2005; Westerhold and Röhl, 2006)

Revised composite depth sections have been presented for four of the sites drilled during Expedition 320/321: IODP Sites U1331, U1332, U1333, and U1334 (Westerhold et al., 2012). Here, we offer revised composite depth sections for IODP Sites U1336, U1337, and U1338. Age models for these sites and revised composite depth sections for other sites in the EEP await additional work.

\section{Methods}

Sediment records from different holes can be aligned at high spatial resolution using detailed physical properties measurements generated for each hole. For Sites U1336, U1337, and U1338, we used three primary sets of measurements: digital photographs, gamma ray attenuation (GRA) density, and magnetic susceptibility (MS). It is noteworthy that physical properties records for Neogene sediment at these sites are far superior to those generated in contemporaneous sediment at previous drill sites in the EEP.

Preceding Expedition 320/321, new depth scales were introduced for scientific drill holes (see "IODP Depth Scales Terminology" at www.iodp.org/program-policies/). The most fundamental depth unit is core depth below seafloor (CSF), which comes from drilling logs. This depth is the best estimate for the true depth of sediment cores and sediment samples below the seafloor. For Expedition 320/321, CSF (in meters) is the same as meters below seafloor (mbsf) used on many previous scientific drilling cruises. As emphasized above, however, successive cores in a single hole do not lead to a complete stratigraphic record.

Composite depth sections require a different depth scale. This scale is designated core composite depth below seafloor (CCSF) and typically exceeds CSF by $\sim 10 \%$. The reason for this difference lies in the methodology of splicing and core expansion during retrieval (Lisiecki and Herbert, 2007). Upon splicing, gaps between successive sediment cores are accounted for, but not the cause. The gaps arise because sediment generally expands during core recovery such that a full $9.5 \mathrm{~m}$ core represents about 9.0 at depth with some sediment lost.

Prior to constructing a spliced section the data collected by the various instruments in the core laboratory must be cleaned of "spikes" and bad intervals. Most of the spikes occur at the end of core sections where part of the measurement includes some air. Bad intervals generally are related to coring distur- 
bance or incomplete recovery. As an initial step in analysis, core section JPEG images were converted to $x-y$ data using a modification of the process described in Wilkens et al. (2009). Data were cleaned manually by overlaying data, such as GRA or MS, on top of the core images. Data that were judged to be anomalous that corresponded to either a section end or visual core disturbance were deleted (Fig. F2). In cases where GRA density is a little low but MS data or images can be correlated we did not eliminate the GRA data, as they possibly indicate a part of the core that was stretched during recovery.

To build a composite depth scale we first fix the core that best seems to capture the mudline from all of the holes cored at a site. Cores from other holes are then moved along a depth axis (CCSF) so that correlative horizons align. A tie point near the base of the first fixed core is chosen, generally at some prominent feature that is also seen near the top of a deeper (floating) core. The core gap between the top two cores in the first hole is then spanned by the floating core from the second hole. The process is continued until a depth is reached where correlative features cannot be recognized. The result of the splicing operation is a table of offsets for each individual core at a site and a second table of the locations of the tie points that define the composite section (e.g., Tables T1, T2). The results of applying the splice are illustrated with scanned core images in Figure F3 and with core data in Figure F4.

In the case where no correlation from the bottom of one core to the tops of any of the others at the site can be made, the protocol is to "append" the next deeper core of the same hole to the bottom of the composite section. This means that the offset of the upper core is carried without change to the lower core. Appends are most common in intervals of only partial core recovery. It is important to note that this procedure does not account for core expansion and gaps between cores that are seen in intervals where recovery is $100 \%$ - that is, the core liners are full. Thus, too many appends may skew calculation of overall core expansion.

In revising the composite depth scales developed during Expedition 321 coring operations, we attempted to maintain the original tie points whenever possible so that samples that were originally selected to be within the composite section will remain so, even if the depth has shifted slightly. In general, within the shallower $(<300 \mathrm{~m}$ CCSF) advanced piston corer (APC) cored intervals, changes in offsets from the original splice and/or tie points are on the order of a few tens of centimeters. Deeper at Sites U1337 and U1338 the revisions are greater because of the increased difficulty in aligning cores with partial recovery or cores that have undergone drilling disturbance during extended core barrel (XCB) coring operations. Although some intervals of deeper sections correlate well, this is not always the case. Users of these offset and splice tables should evaluate the data provided in the "Appendix" before using them as a basis for critical observations.

Once the composite section has been defined, a final stretch/compress process is required for intervals of core that are not included within the splice (offsplice). The problem and the solution process are illustrated in Figure F5. Above the interval of Core 321-U1337A-4H selected for the composite section, a boundary at $\sim 21.5 \mathrm{~m}$ CCSF corresponds to a feature at $\sim 22.0 \mathrm{~m}$ CCSF in the composite. The composite interval at these depths comes from Core 321U1337B-3H (Fig. F3). For future sampling of offsplice sediment, it is imperative that features such as this boundary be mapped to the composite so that equivalence in material can be maintained. This is accomplished by identifying tie points between offsplice intervals and the composite section and then stretching or squeezing the CCSF depth scale through interpolation to produce an adjusted CCSF scale. Tables of off-splice tie points are provided for each hole in the "Appendix." Adjusted CCSF depths are provided in the cleaned data files as well.

\section{Results and discussion}

For each site, in addition to the offset, splice, and stretch tie point tables, we have provided in the "Appendix" all of the cleaned GRA, MS, natural gamma radiation (NGR), and color reflectance data along with CSF, CCSF, and adjusted CCSF depths, where applicable.

Site U1336 consisted of only two holes. An effective splice carries to a depth of $\sim 152 \mathrm{~m} \mathrm{CCSF}$, where there is a poorly recovered interval. Below this depth there are primarily appends rather than ties between cores. Offsets and splice points are provided in Tables T1 and $\mathrm{T} 2$.

Site U1337 is more complex, with four holes and a much greater total depth than Site U1336. Below 220 m CCSF, a confused section of $\sim 50$ m CCSF was characterized by incomplete recovery in all holes. A short gap may persist at $220 \mathrm{~m} \mathrm{CCSF}$, although we chose tie points. Core $321-\mathrm{U} 1337 \mathrm{C}-9 \mathrm{H}$ has been appended to the bottom of Core $321-\mathrm{U} 1337 \mathrm{C}-8 \mathrm{H}$, suggesting another short break in recovery. At $267 \mathrm{~m}$ CCSF there is a definite break caused by poor recovery of a hard chert layer. Comparison of GRA records with downhole density logs (Malinverno, 2013) indicates that most of the section is represented in the cored material. Between 267 and 440 m CCSF continuous 
correlation can be made, although the amplitudes of the variations in properties are such that there may be some incorrect tie points. A major gap occurs at $444 \mathrm{~m}$ CCSF where Core 321-U1337A-44X is appended to Core 321-U1337A-43X. Particularly puzzling is the large gap seen in Holes U1337C and U1337D at the same level. This "void" is the result of forcing the color change at $\sim 451 \mathrm{~m}$ CCSF to the same level in all holes. It may be that this color change occurs higher in the section in the latter two holes than in Hole U1337A. This question is not resolvable using data available. Offsets and splice points are provided in Tables T3 and T4.

At Site U1338, features between all records are well aligned to $280 \mathrm{~m}$ CCSF. Ship heave of several meters during coring in Hole U1338C resulted in relatively more core disturbance in the softer sediments recovered from that hole, and the splice was subsequently made primarily from Holes U1338A and U1338B in the upper $260 \mathrm{~m}$ CCSF (to Core 321U1338A-27H). The remainder of the splice alternated between Holes U1338B and U1338C.

At the base of Core 321-U1338C-28H, overlap was lost because of poor recovery in corresponding cores from Holes U1338A and U1338B. Another gap occurred at the base of Core 321-U1338C-31H, where a chert interval was encountered. Several other ties were made on the basis of short overlaps between 280 and $360 \mathrm{~m}$ CCSF. Ties below $360 \mathrm{~m}$ CCSF were generally good to $430 \mathrm{~m}$ CCSF, whereupon poor core condition and low variability in the data used for correlation left little to do but finish out the splice with the lower four cores from Hole U1338C. Offsets and splice points are provided in Tables T5 and T6.

\section{Summary and future work}

For each site (U1336, U1337, and U1338), individual cores from adjacent drill holes can be aligned using physical properties so that composite depth sections can be constructed for long depth intervals. These enable the generation of continuous records at high temporal resolution.

Future work:

1. Place the records into the time domain with available age datums.

2. Tune these records.

3. Reevaluate composite depth intervals for other drill sites in the EEP.

4. Mesh the sites together to gain a coherent view of surface water changes in the EEP.

Tables of cleaned data, adjusted CCSF depths, and tie points used for stretching are available in the "Appendix."

\section{Acknowledgments}

Samples and data were provided by the Integrated Drilling Program (IODP). This work benefitted from reviews by Louise Anderson and Thomas Westerhold. Logan Davis assisted in data processing and his help is greatly appreciated.

\section{References}

Brierley, C.M., Fedorov, A.V., Liu, Z., Herbert, T.D., Lawrence, K.T., and LaRiviere, J.P., 2009. Greatly expanded tropical warm pool and weakened Hadley circulation in the early Pliocene. Science, 323(5922):1714-1718. doi:10.1126/science.1167625

Hagelberg, T.K., Pisias, N.G., Shackleton, N.J., Mix, A.C., and Harris, S., 1995. Refinement of a high-resolution, continuous sedimentary section for studying equatorial Pacific Ocean paleoceanography, Leg 138. In Pisias, N.G., Mayer, L.A., Janecek, T.R., Palmer-Julson, A., and van Andel, T.H. (Eds.), Proc. ODP, Sci Results, 138: College Station, TX (Ocean Drilling Program), 31-46. doi:10.2973/odp.proc.sr.138.103.1995

Lawrence, K.T., Liu, Z., and Herbert, T.D., 2006. Evolution of the eastern tropical Pacific through Plio-Pleistocene glaciation. Science, 312(5770):79-83. doi:10.1126/science. 1120395

Lisiecki, L.E., and Herbert, T.D., 2007. Automated composite depth scale construction and estimates of sediment core extension. Paleoceanography, 22(4):PA4213. doi:10.1029/2006PA001401

Malinverno, A., in press. Data report: Monte Carlo correlation of sediment records from core and downhole log measurements at Sites U1337 and U1338 (IODP Expedition 321). In Pälike, H., Lyle, M., Nishi, H., Raffi, I., Gamage, K., Klaus, A., and the Expedition 320/321 Scientists, Proc. IODP, 320/321: Tokyo (Integrated Ocean Drilling Program Management International, Inc.).

Pälike, H., Moore, T., Backman, J., Raffi, I., Lanci, L., Parés, J.M., and Janecek, T., 2005. Integrated stratigraphic correlation and improved composite depth scales for ODP Sites 1218 and 1219. In Wilson, P.A., Lyle, M., and Firth, J.V. (Eds.), Proc. ODP, Sci. Results, 199: College Station, TX (Ocean Drilling Program), 1-41. doi:10.2973/ odp.proc.sr.199.213.2005

Pälike, H., Nishi, H., Lyle, M., Raffi, I., Gamage, K., Klaus, A., and the Expedition 320/321 Scientists, 2010. Expedition 320/321 summary. In Pälike, H., Lyle, M., Nishi, H., Raffi, I., Gamage, K., Klaus, A., and the Expedition 320/ 321 Scientists, Proc. IODP, 320/321: Tokyo (Integrated Ocean Drilling Program Management International, Inc.). doi:10.2204/iodp.proc.320321.101.2010

Pennington, J.T., Mahoney, K.L., Kuwahara, V.S., Kolber, D.D., Calienes, R., and Chavez, F.P., 2006. Primary production in the eastern tropical Pacific: a review. Prog. Oceanogr., 69(2-4):285-317. doi:10.1016/ j.pocean.2006.03.012

Trenberth, K.E., and Caron, J.M., 2000. The Southern oscillation revisited: sea level pressures, surface tempera- 
tures, and precipitation. J. Clim., 13(24):4358-4365. doi:10.1175/1520-

0442(2000)013<4358:TSORSL > 2.0.CO;2

Westerhold, T., and Röhl, U., 2006. Data report: revised composite depth records for Shatsky Rise Sites 1209, 1210, and 1211. In Bralower, T.J., Premoli Silva, I., and Malone, M.J. (Eds.), Proc. ODP, Sci. Results, 198: College Station, TX (Ocean Drilling Program), 1-26. doi:10.2973/odp.proc.sr.198.122.2006

Westerhold, T., Röhl, U., Wilkens, R., Pälike, H., Lyle, M., Jones, T.D., Bown, P., Moore, T., Kamikuri, S., Acton, G., Ohneiser, C., Yamamoto, Y., Richter, C., Fitch, P., Scher, H., Liebrand, D., and the Expedition 320/321 Scientists, 2012. Revised composite depth scales and integration of IODP Sites U1331-U1334 and ODP Sites 1218-1220. In Pälike, H., Lyle, M., Nishi, H., Raffi, I., Gamage, K., Klaus, A., and the Expedition 320/321 Scientists, Proc.
IODP, 320/321: Tokyo (Integrated Ocean Drilling Program Management International, Inc.). doi:10.2204/ iodp.proc.320321.201.2012

Wilkens, R.H., Niklis, N., and Frazer, M., 2009. Data report: digital core images as data: an example from IODP Expedition 303. In Channell, J.E.T., Kanamatsu, T., Sato, T., Stein, R., Alvarez Zarikian, C.A., Malone, M.J., and the Expedition 303/306 Scientists, Proc. IODP, 303/306: College Station, TX (Integrated Ocean Drilling Program Management International, Inc.). doi:10.2204/

iodp.proc.303306.201.2009

Initial receipt: 29 June 2012

Acceptance: 12 October 2012

Publication: 19 February 2013

MS 320321-209 
Figure F1. Location map of sites drilled during Expedition 320/321.

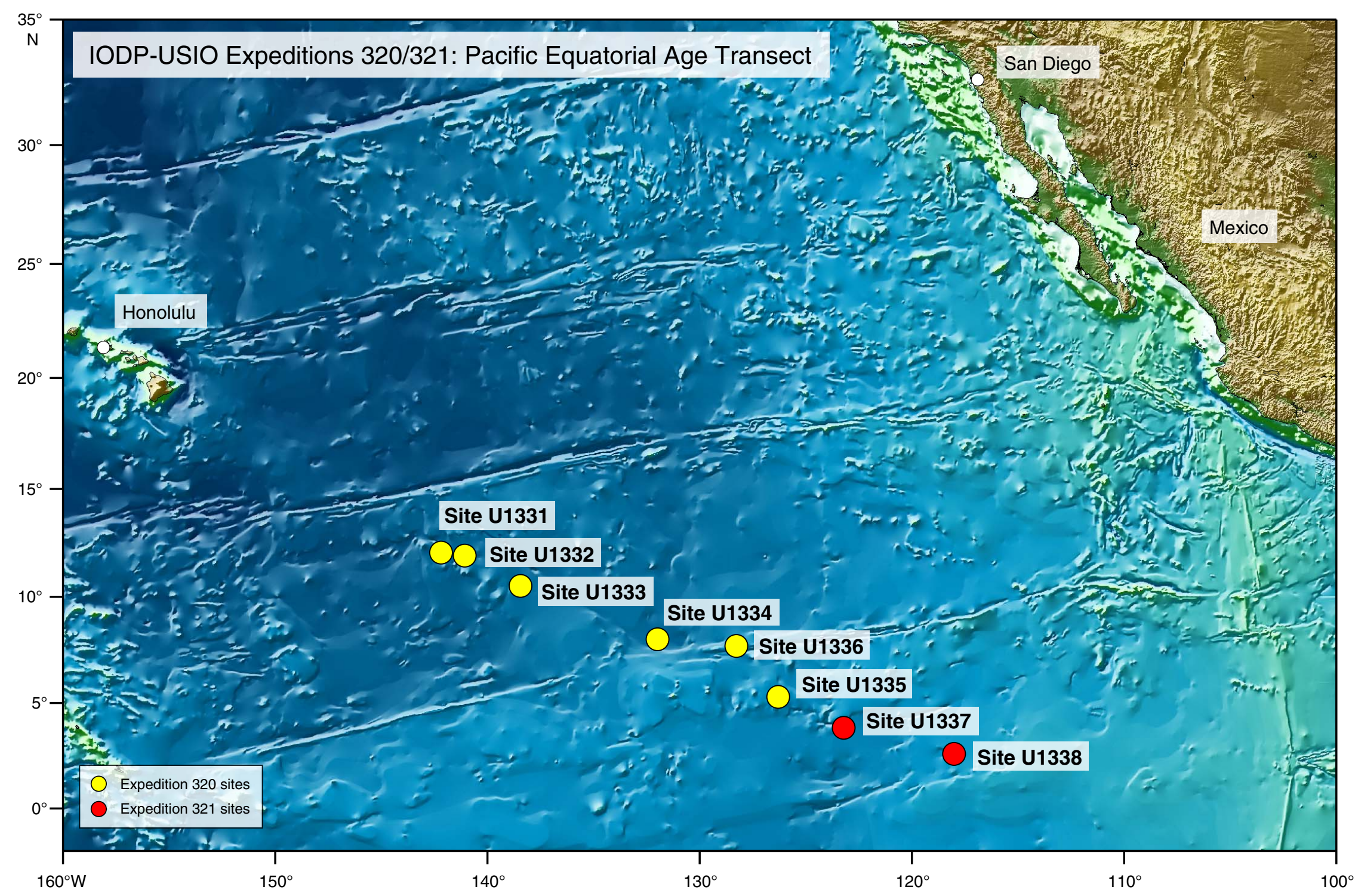


Figure F2. Example from the bottom of Core 321-U1337D-12H and the top of Core 321-U1337-13H illustrating the technique used to clean physical properties data prior to splicing. Data were plotted on top of consolidated core images (top) and against a neutral background (bottom). Sections are represented by different color symbols from Section 1 (red) to Section 7 (purple). Black symbols in the lower plot represent raw data before cleaning. Data spikes were most common at section ends.

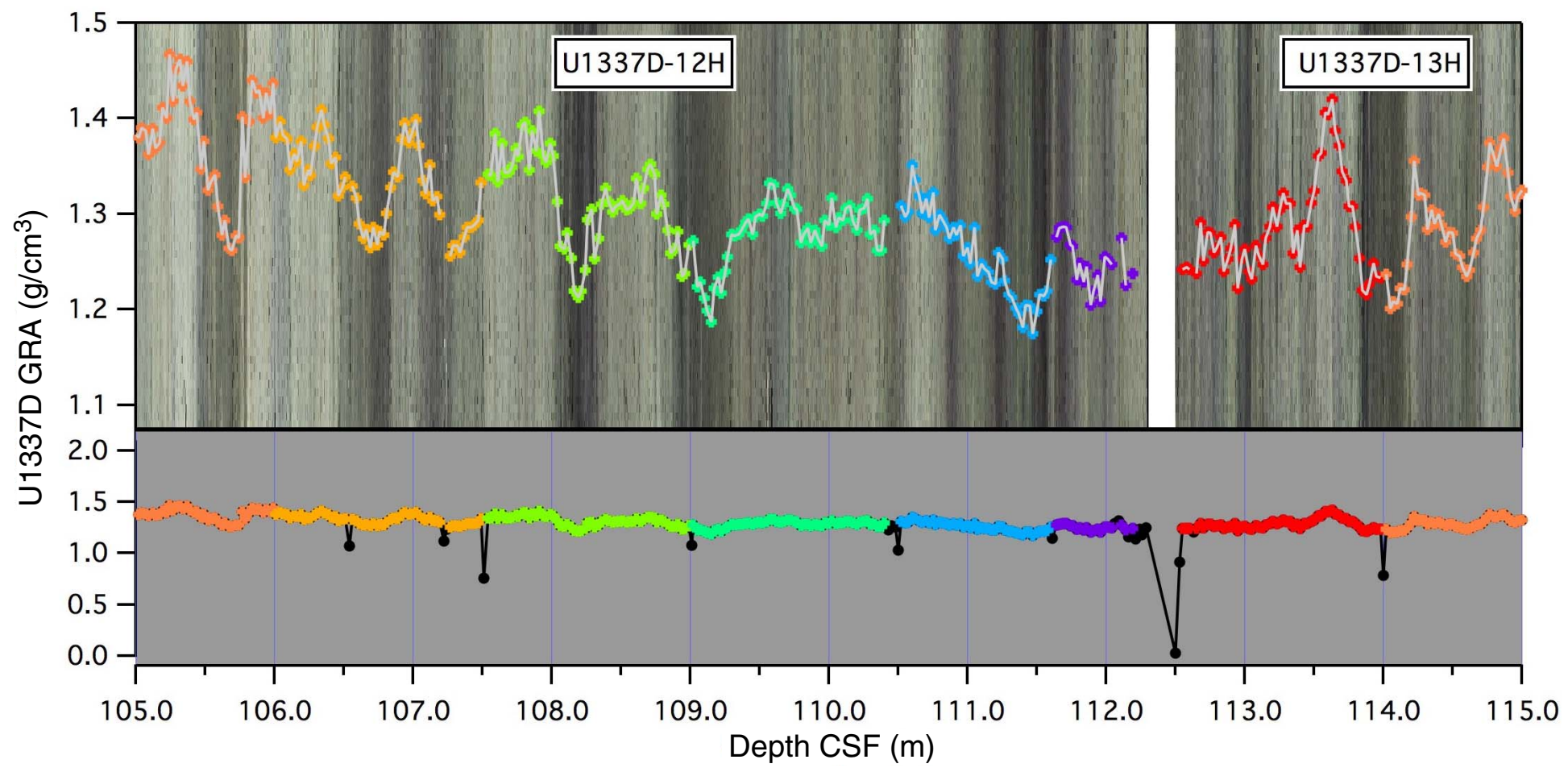


Figure F3. Spliced core images, Sites U1336-U1338. Red bars represent the top of each interval used in the splice, whereas yellow bars correspond with the bottom. Note that outside of the splice intervals within each core there is general alignment with splice features, but it is not always exact. (Continued on next 26 pages.)

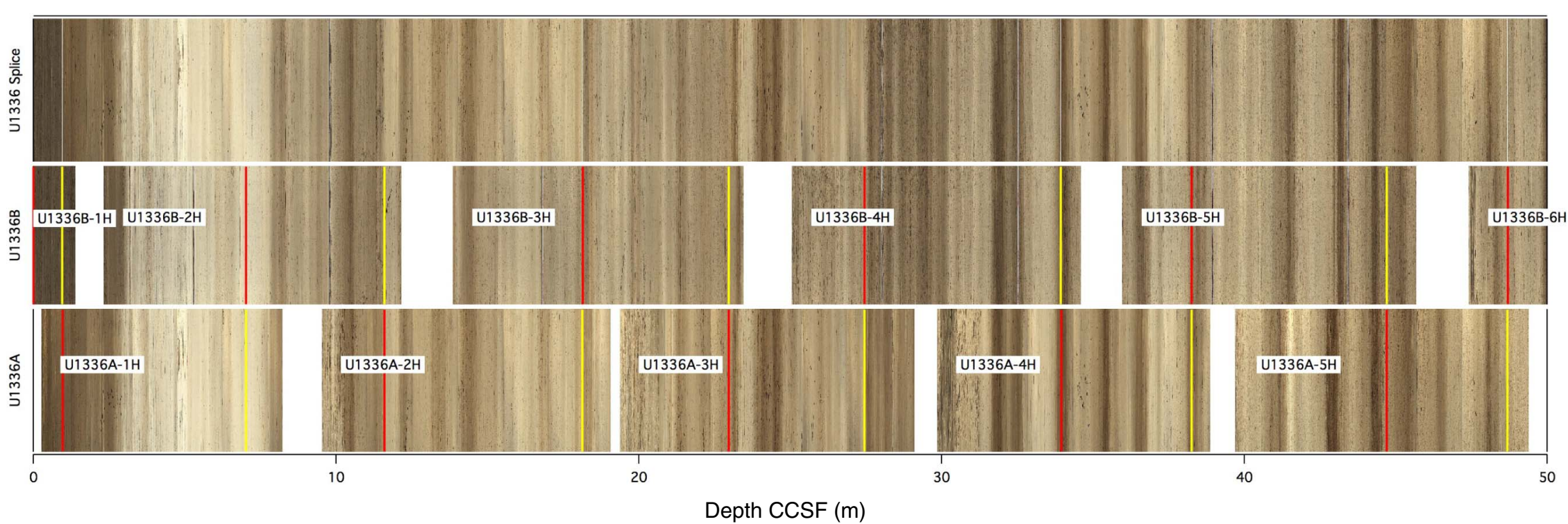


Figure F3 (continued). (Continued on next page.)

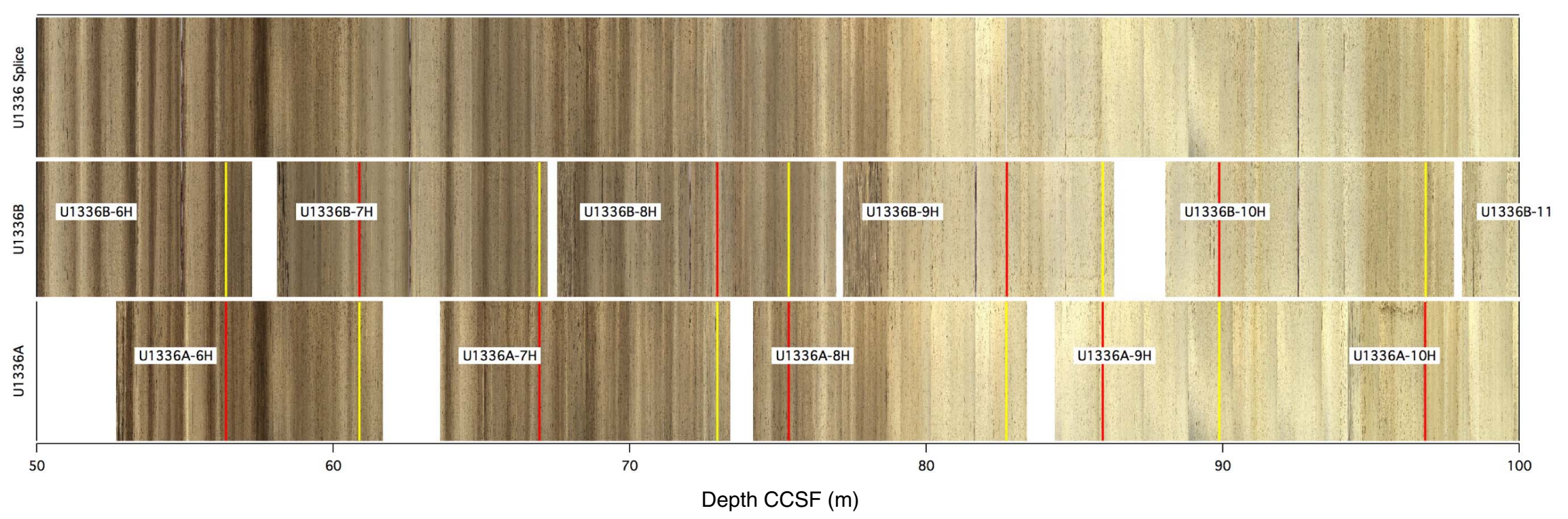


Figure F3 (continued). (Continued on next page.)

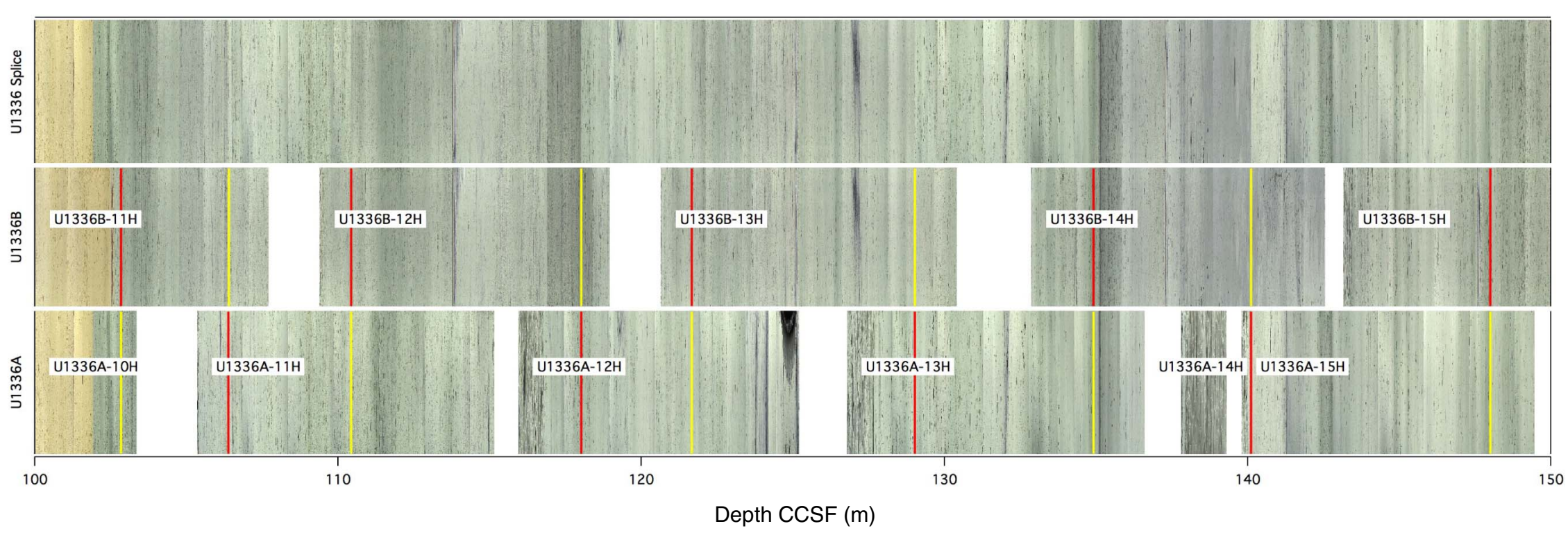




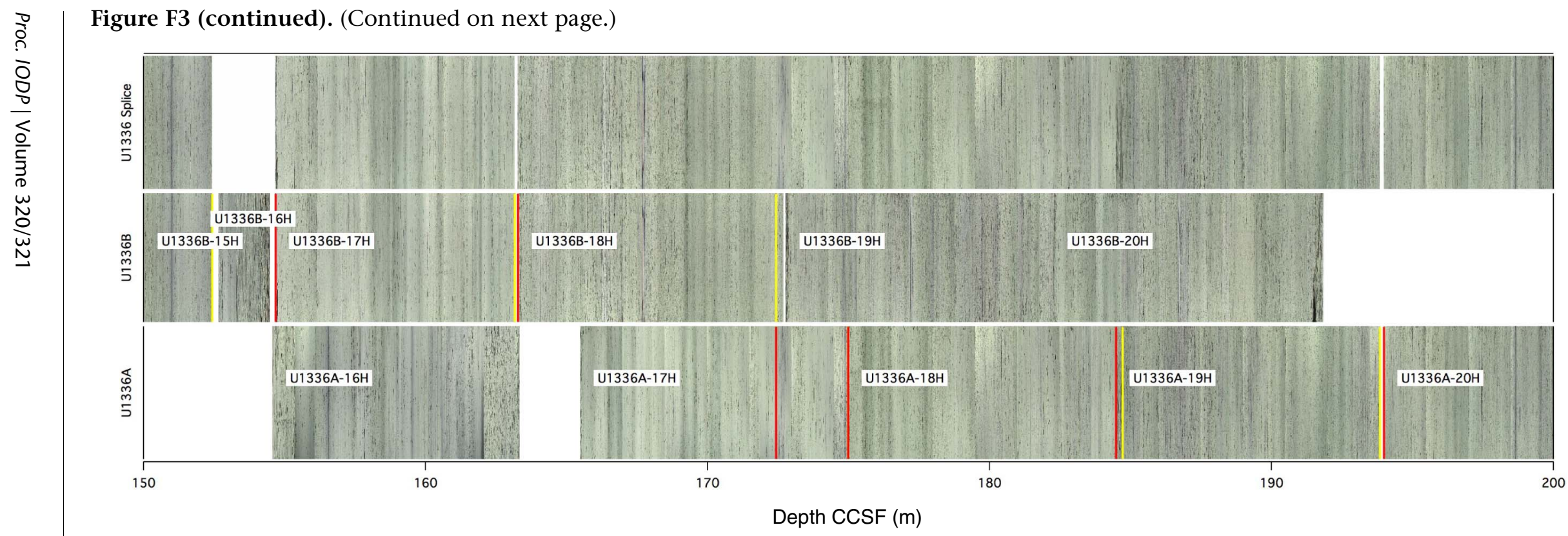




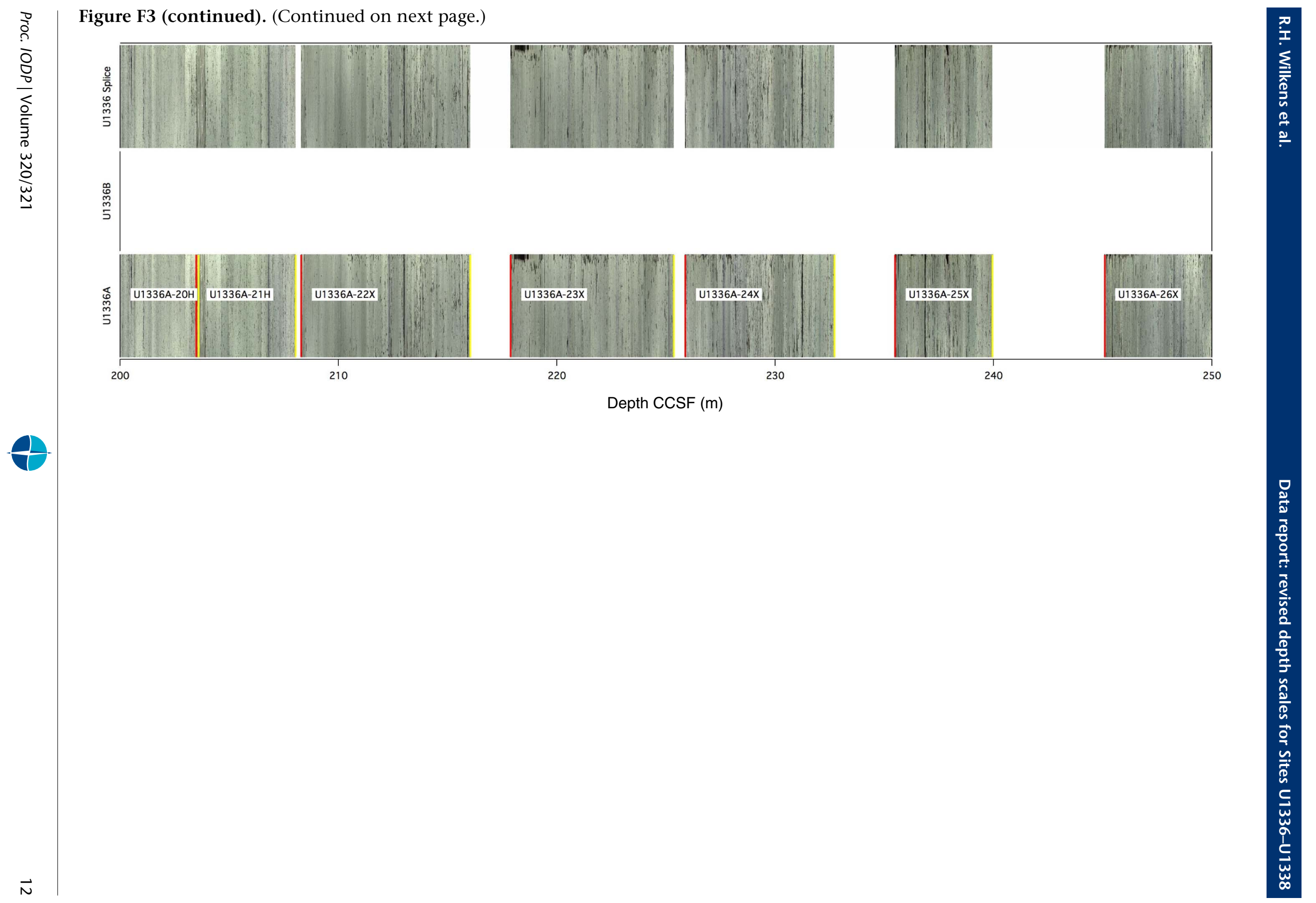




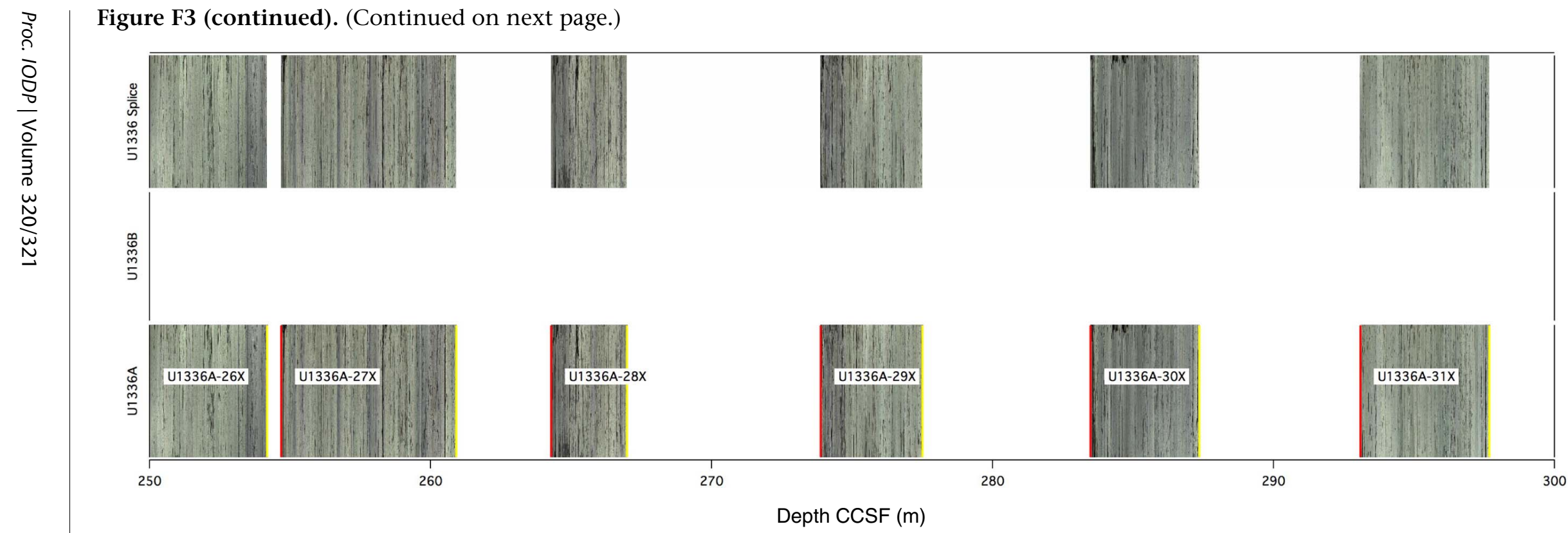




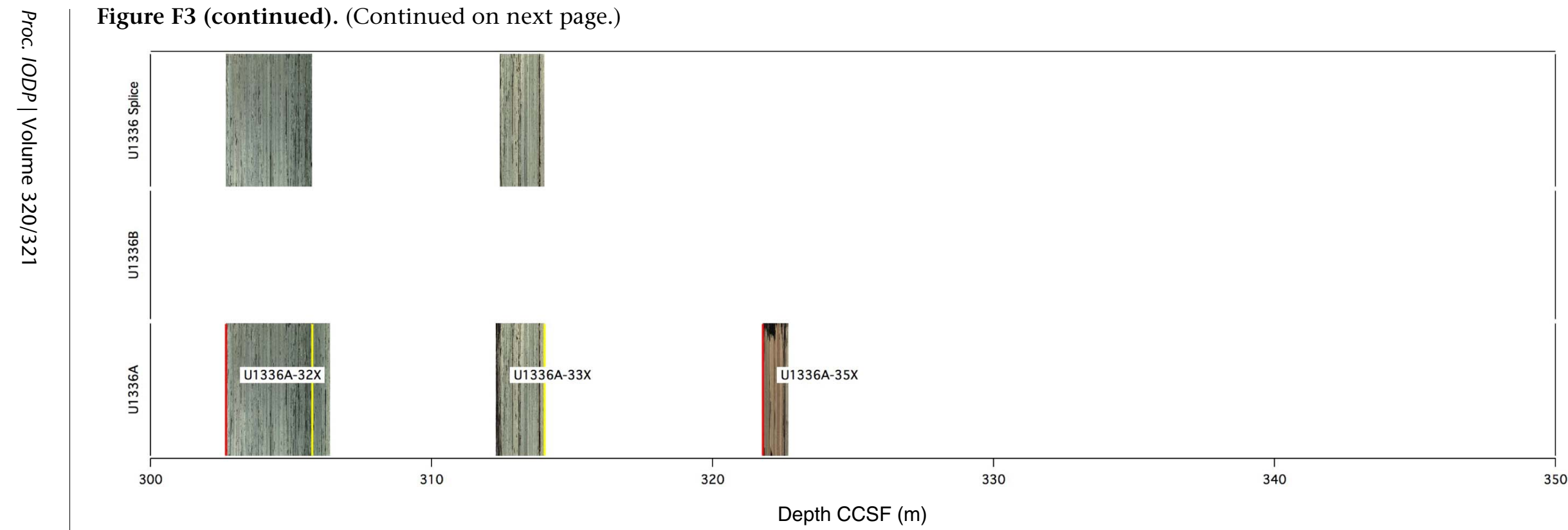




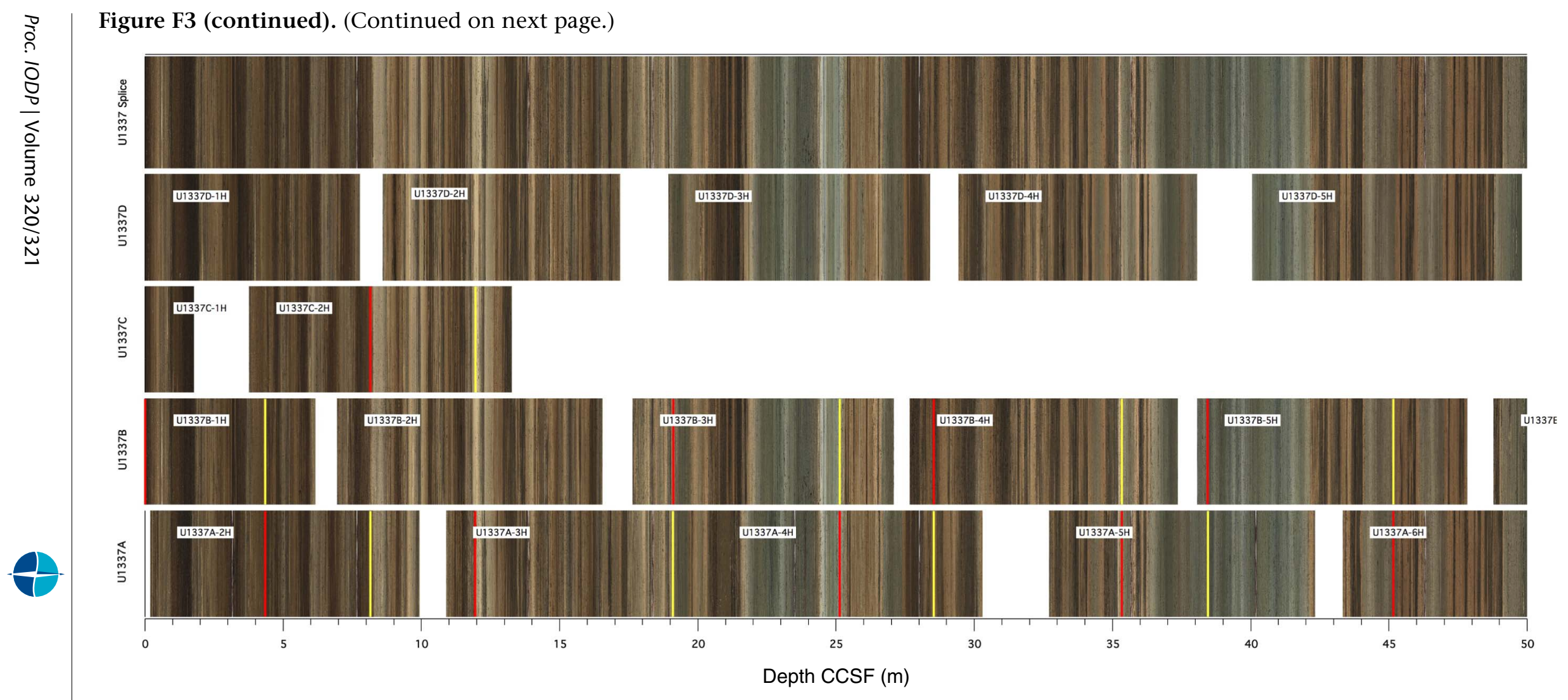




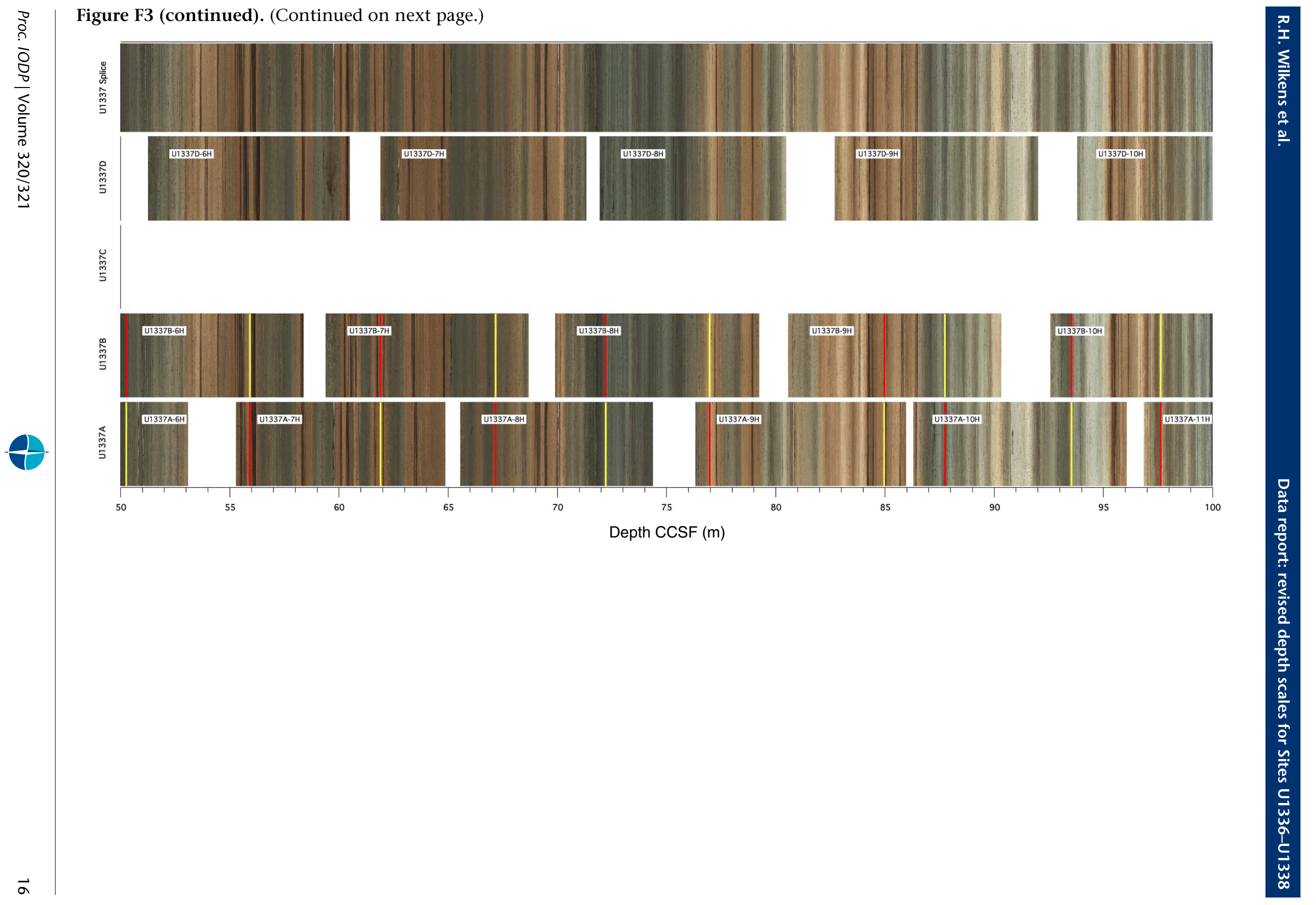




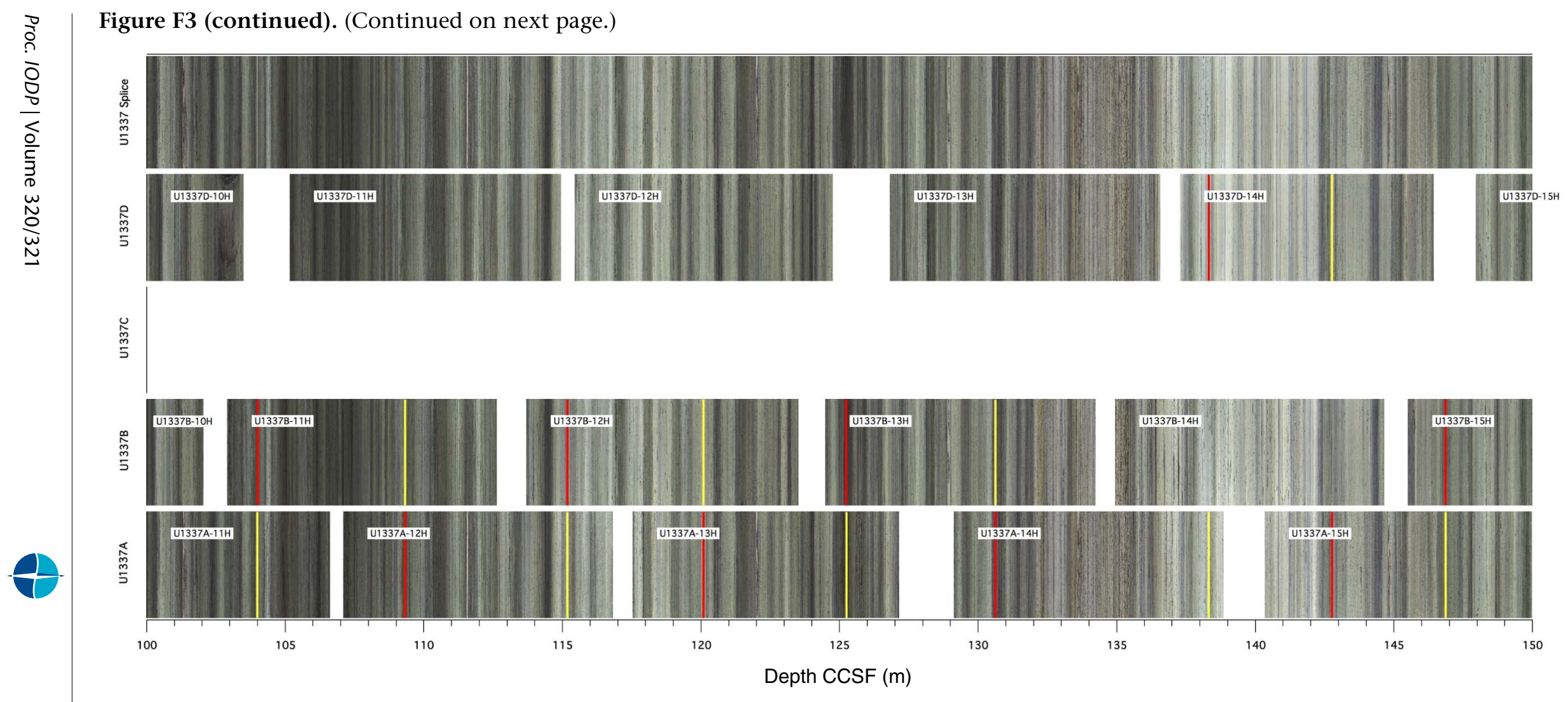




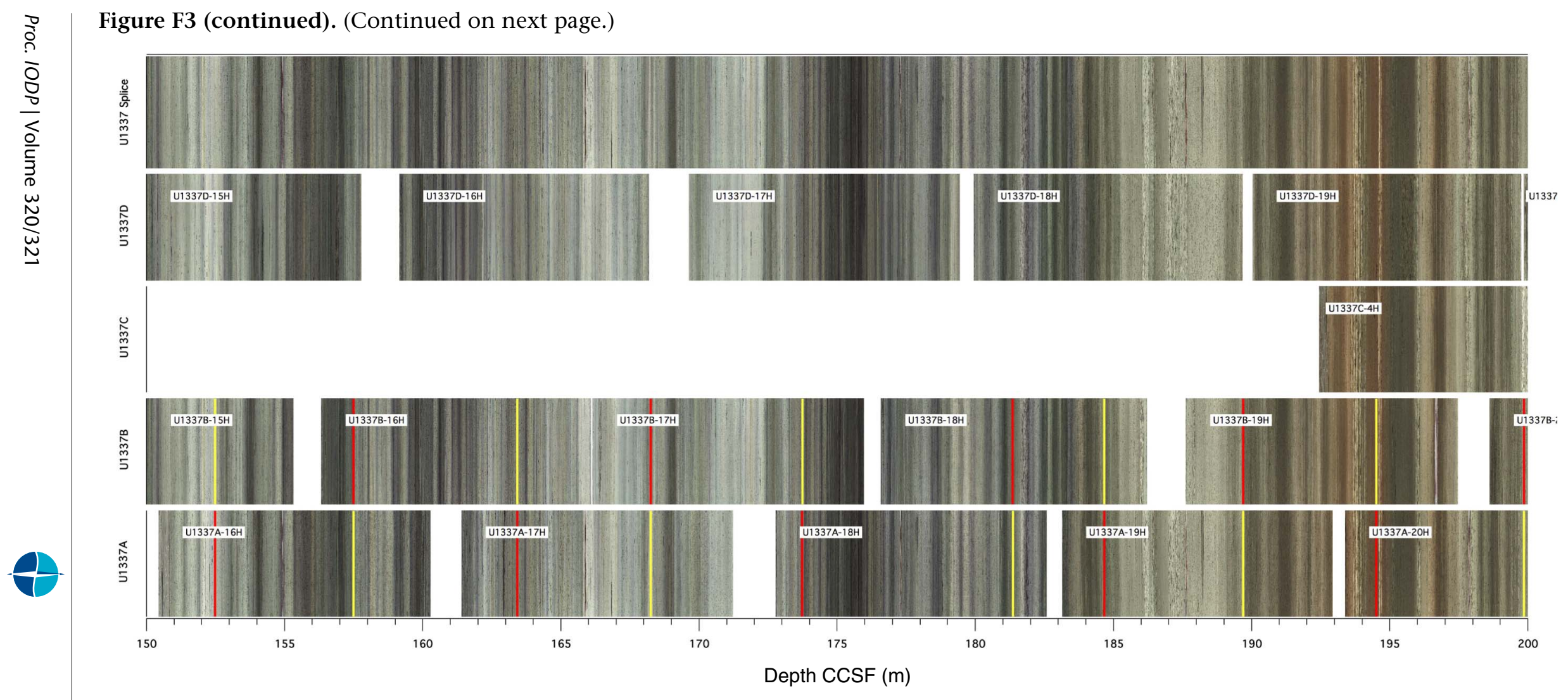




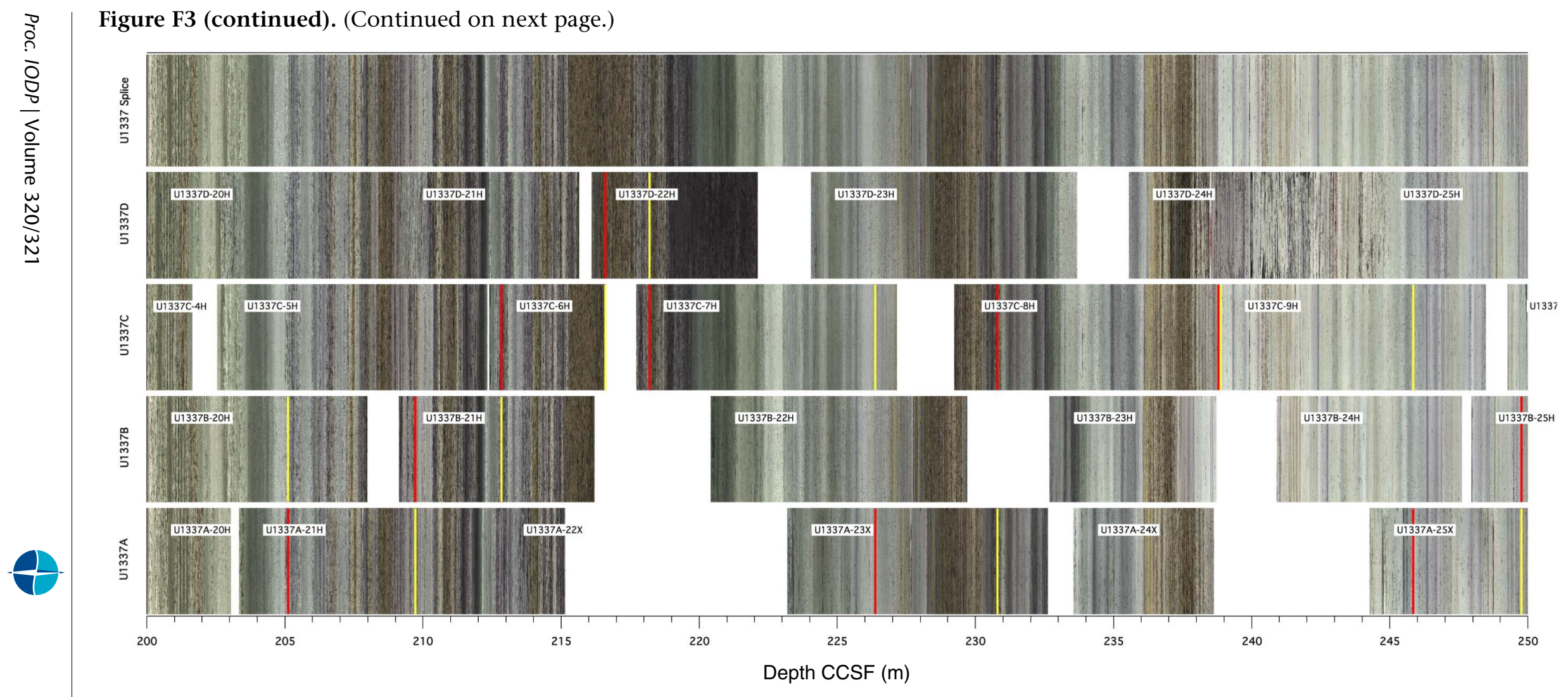



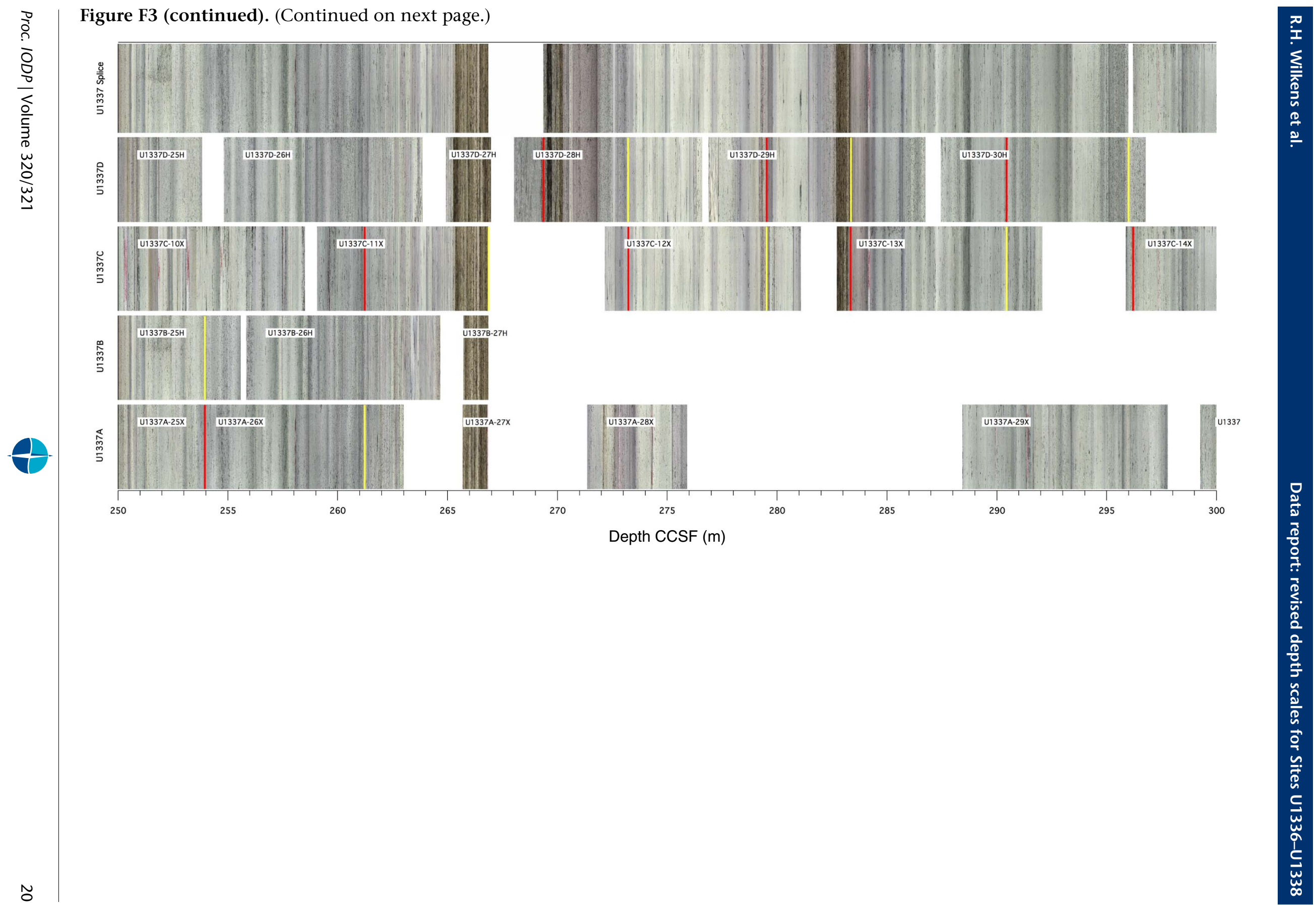


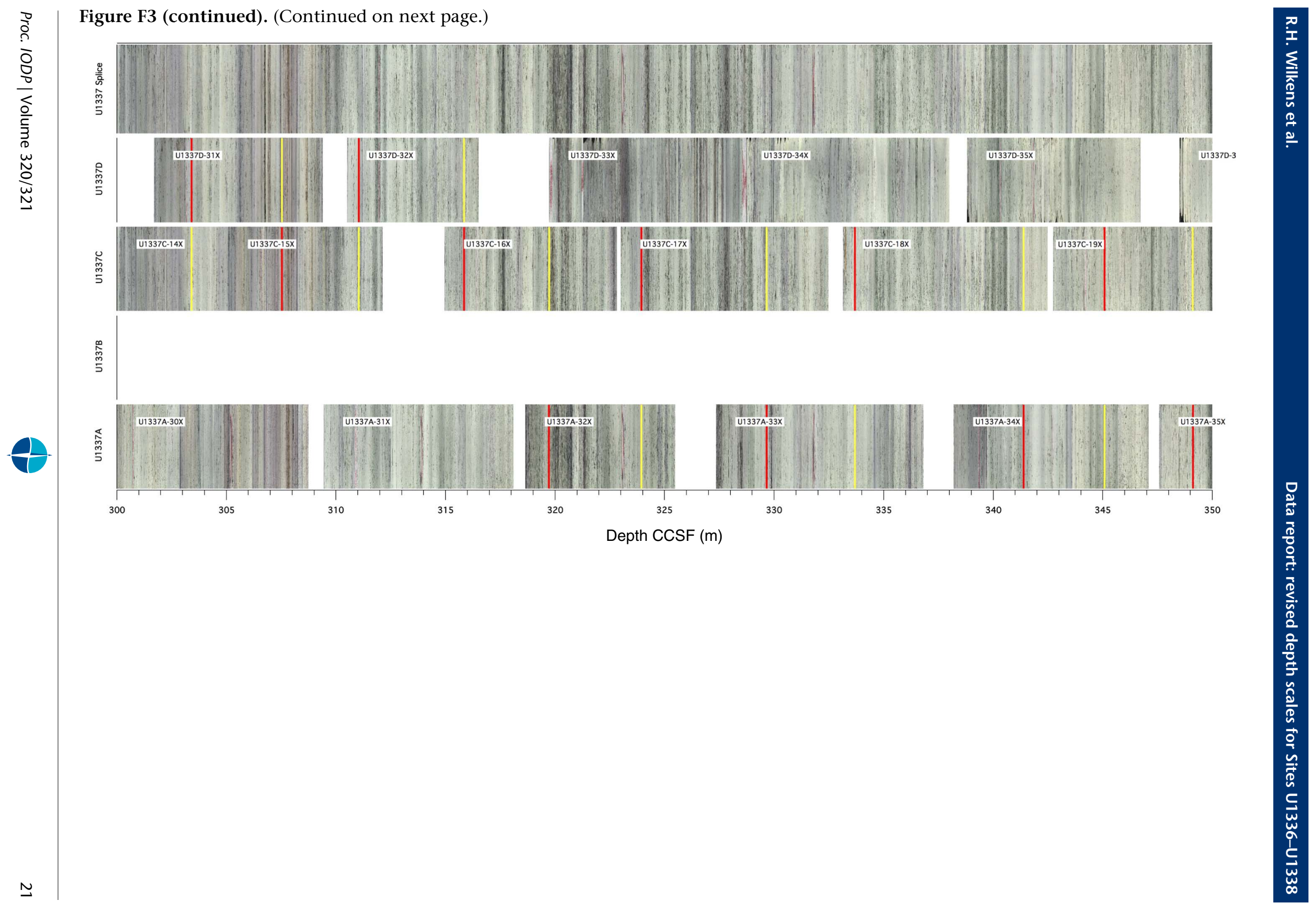


Figure F3 (continued). (Continued on next page.)
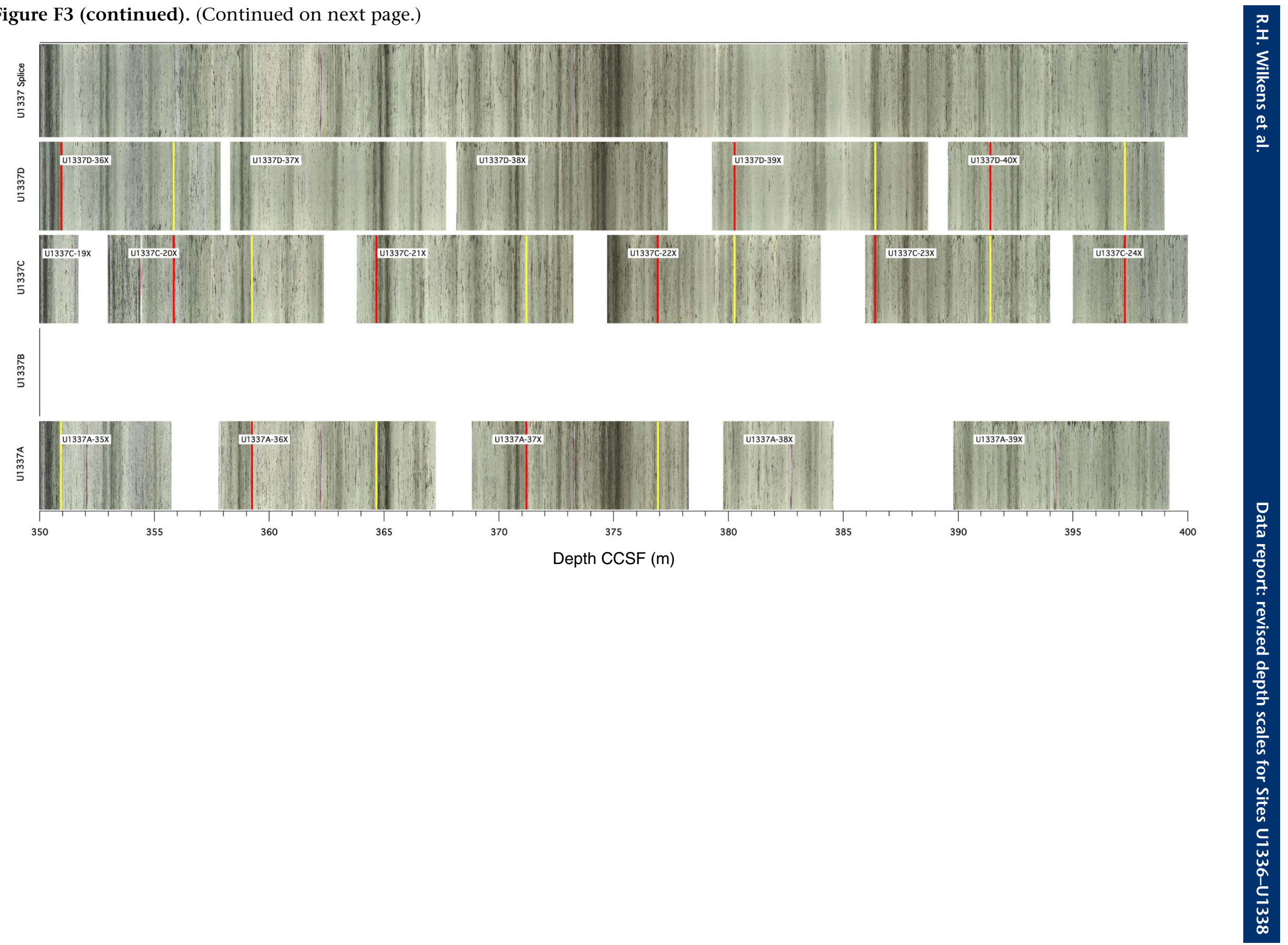
Figure F3 (continued). (Continued on next page.)

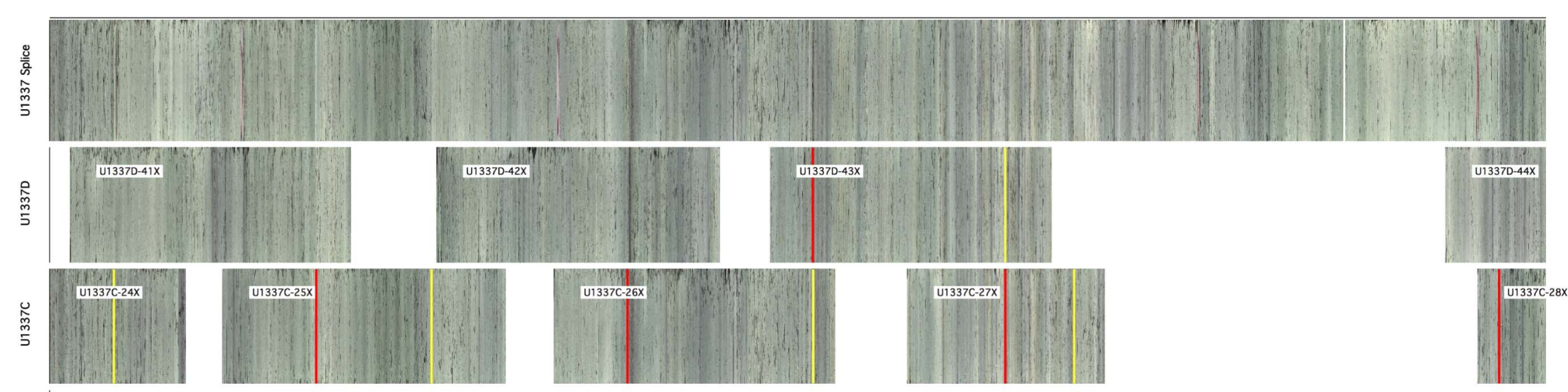

$\theta$

m
0
$m$
5
5

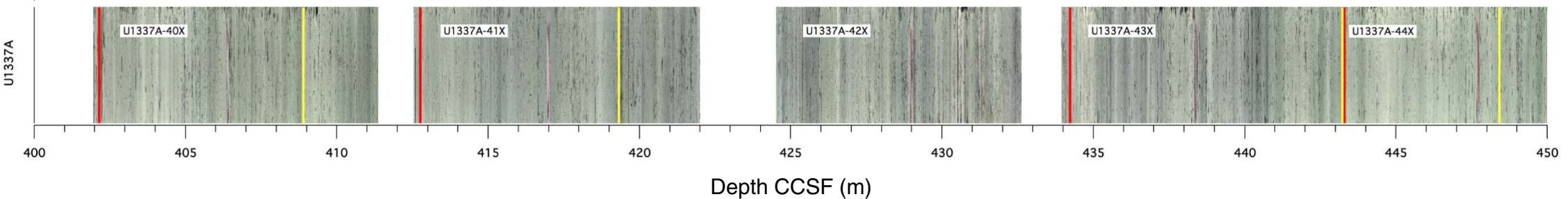



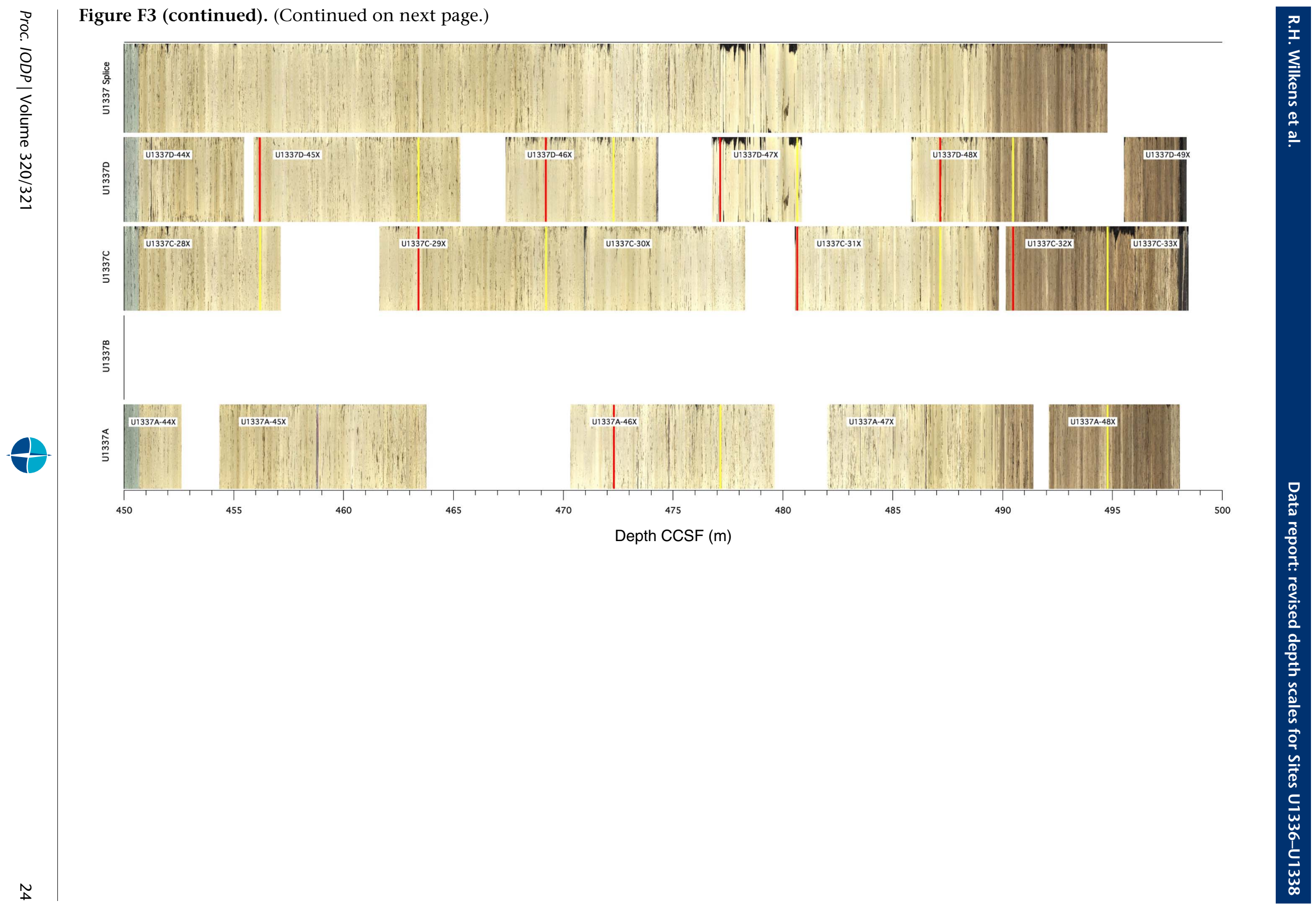
Figure F3 (continued). (Continued on next page.)
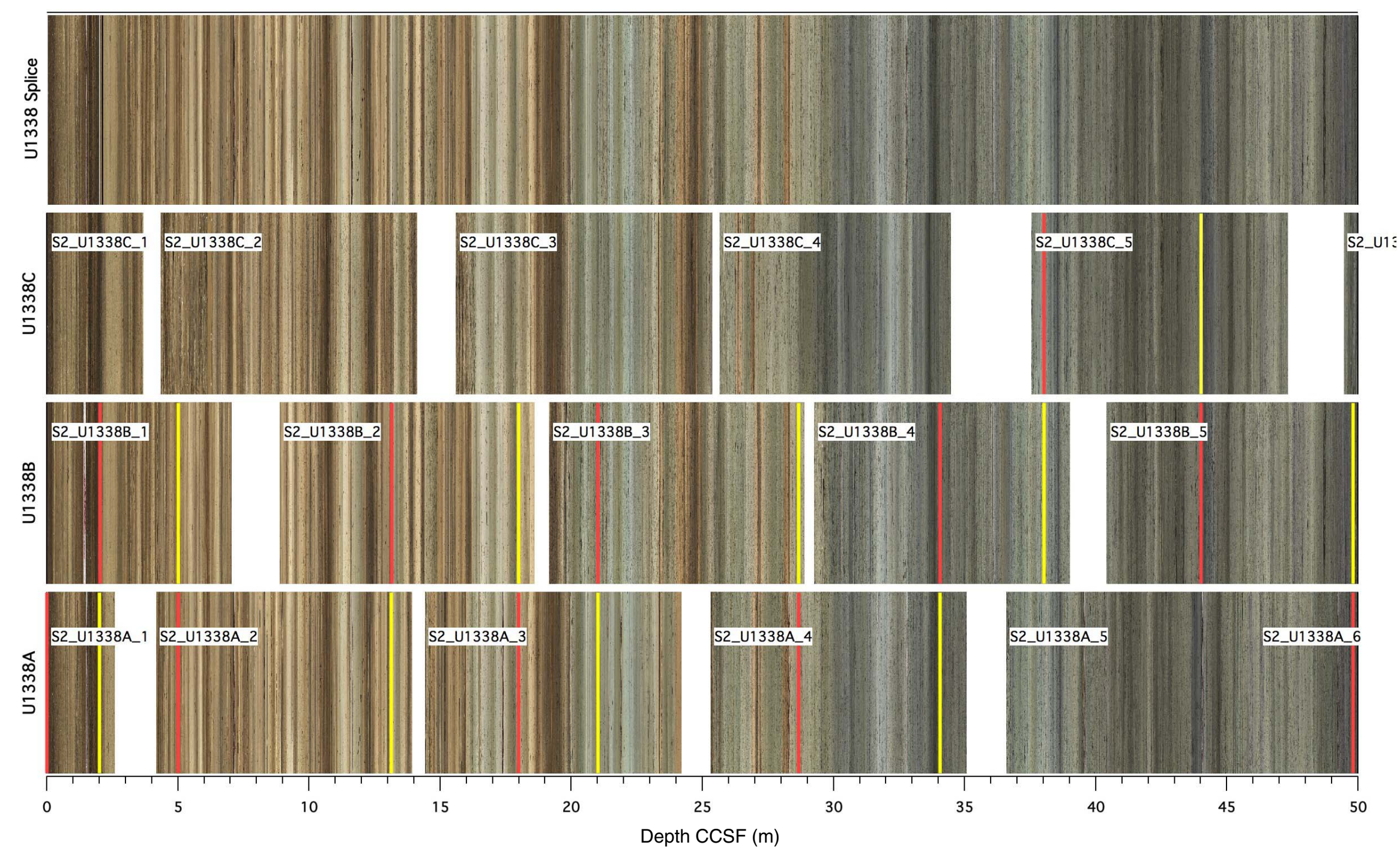
Figure F3 (continued). (Continued on next page.)
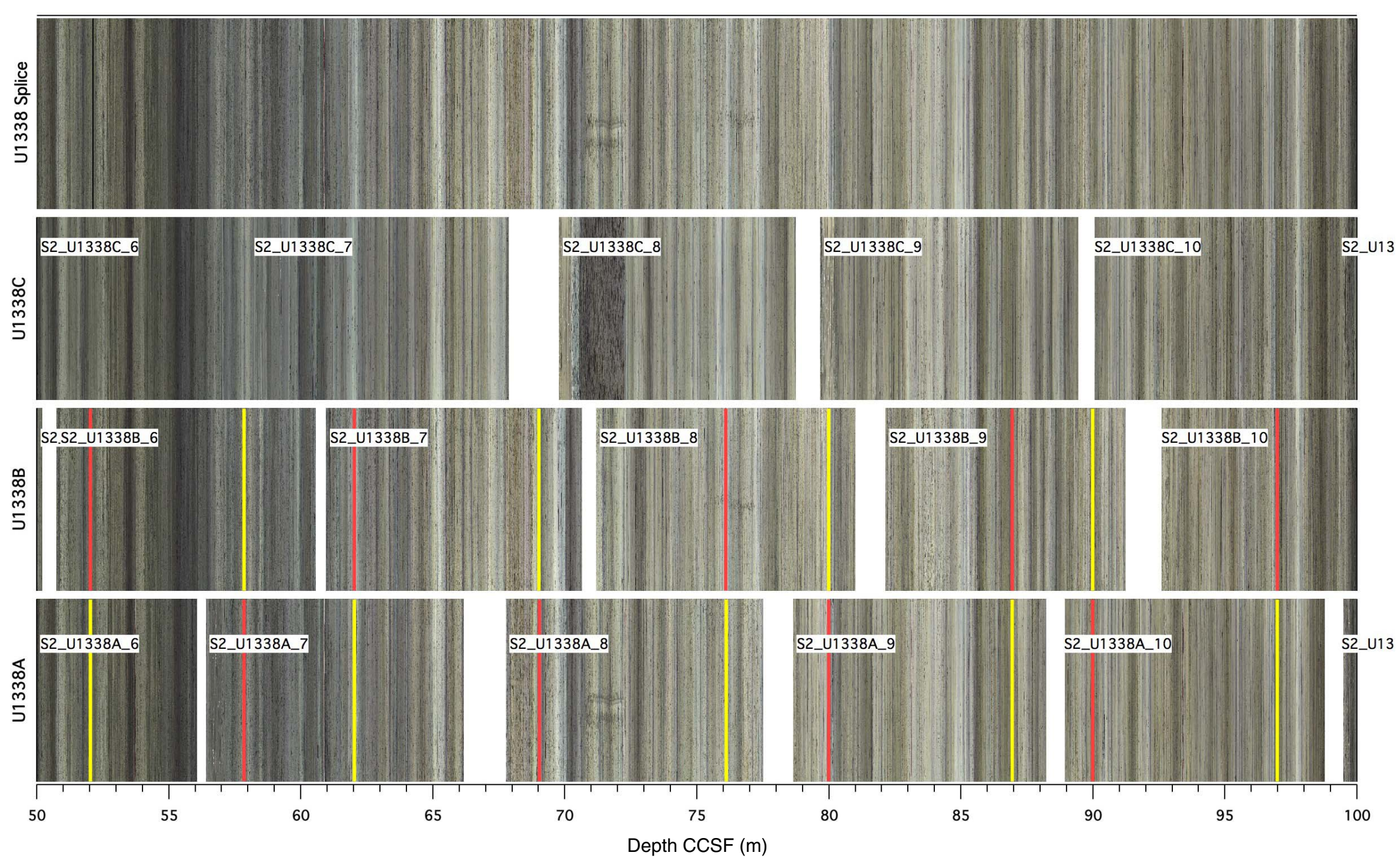
Figure F3 (continued). (Continued on next page.)
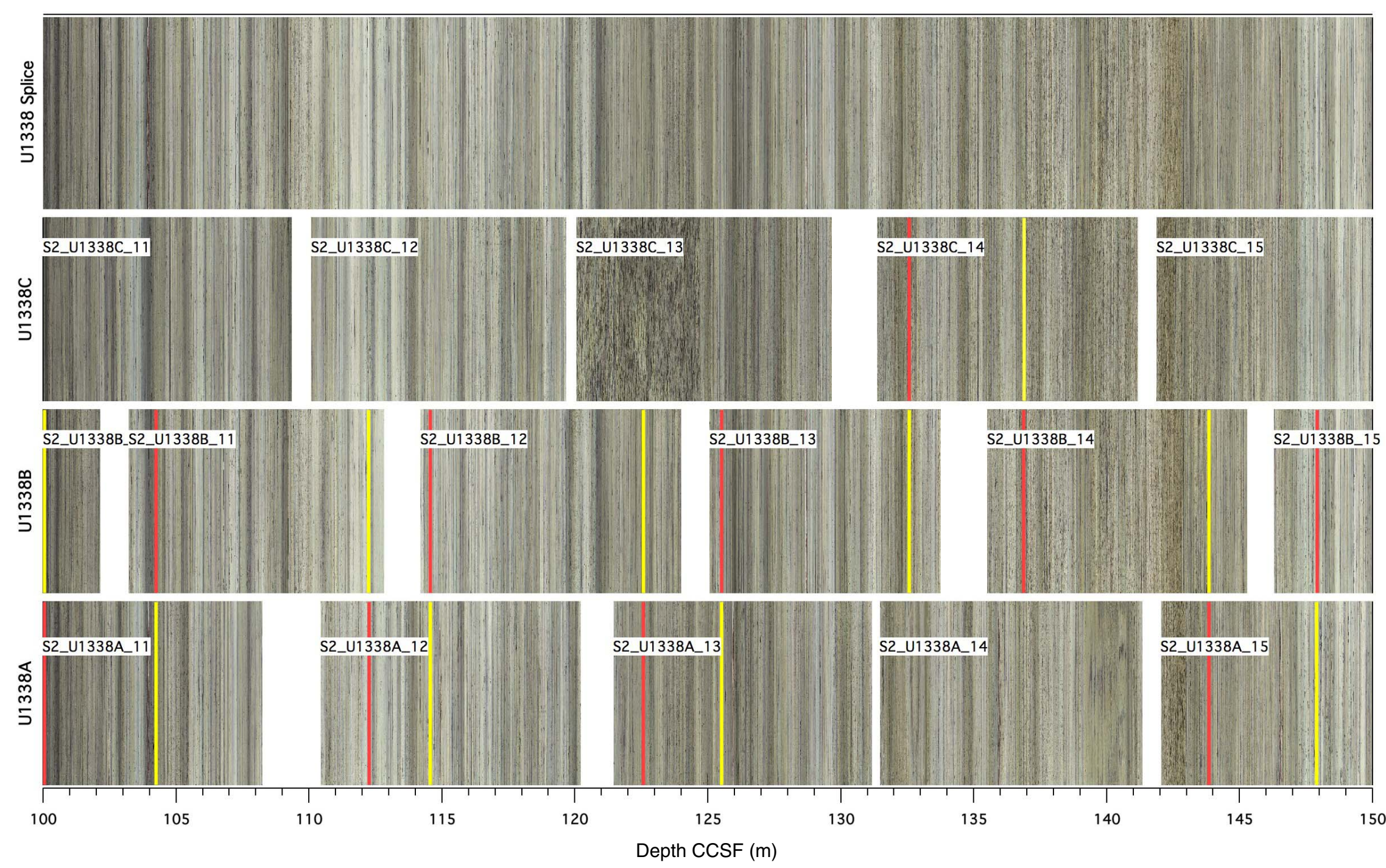
Figure F3 (continued). (Continued on next page.)
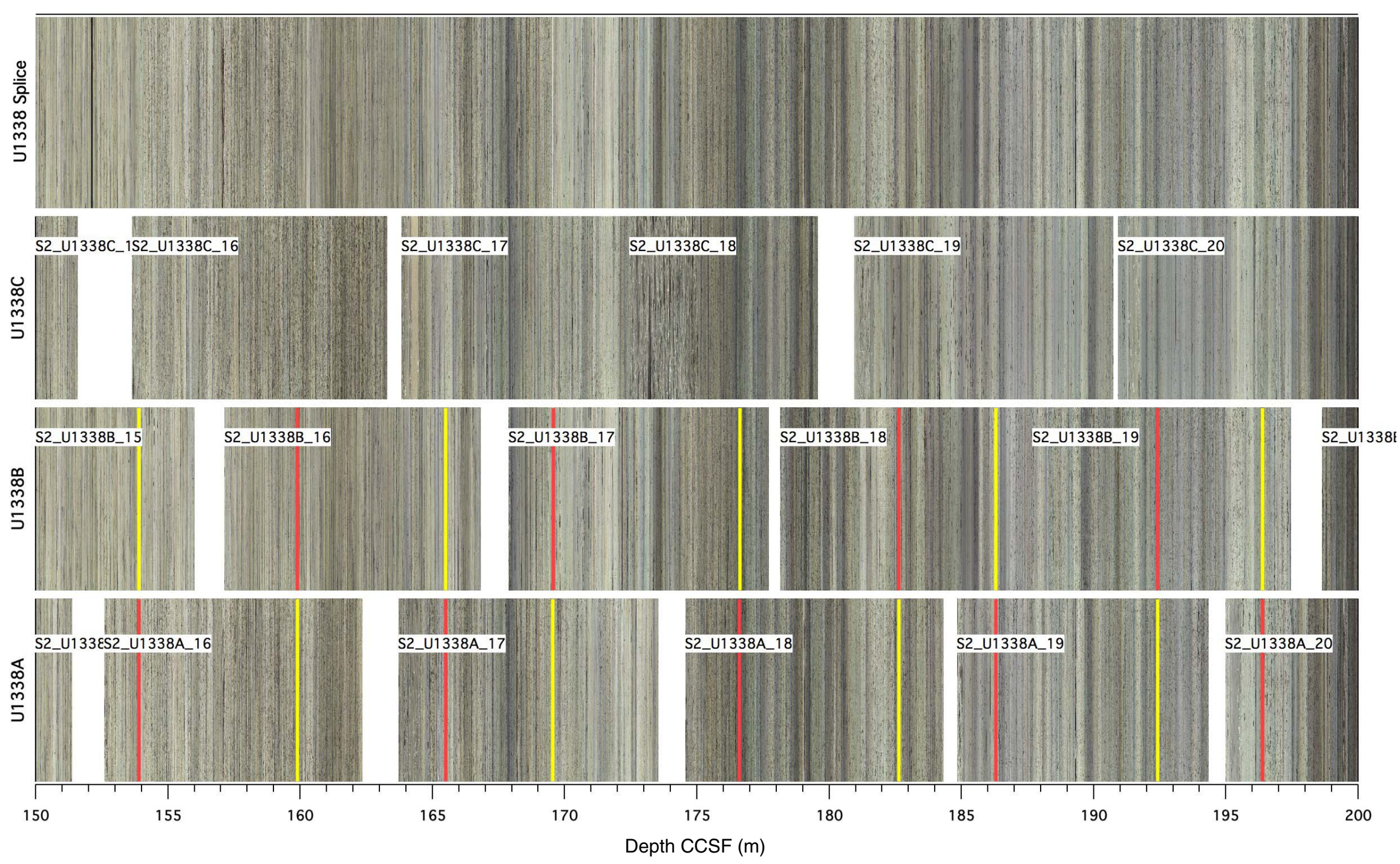
Figure F3 (continued). (Continued on next page.)
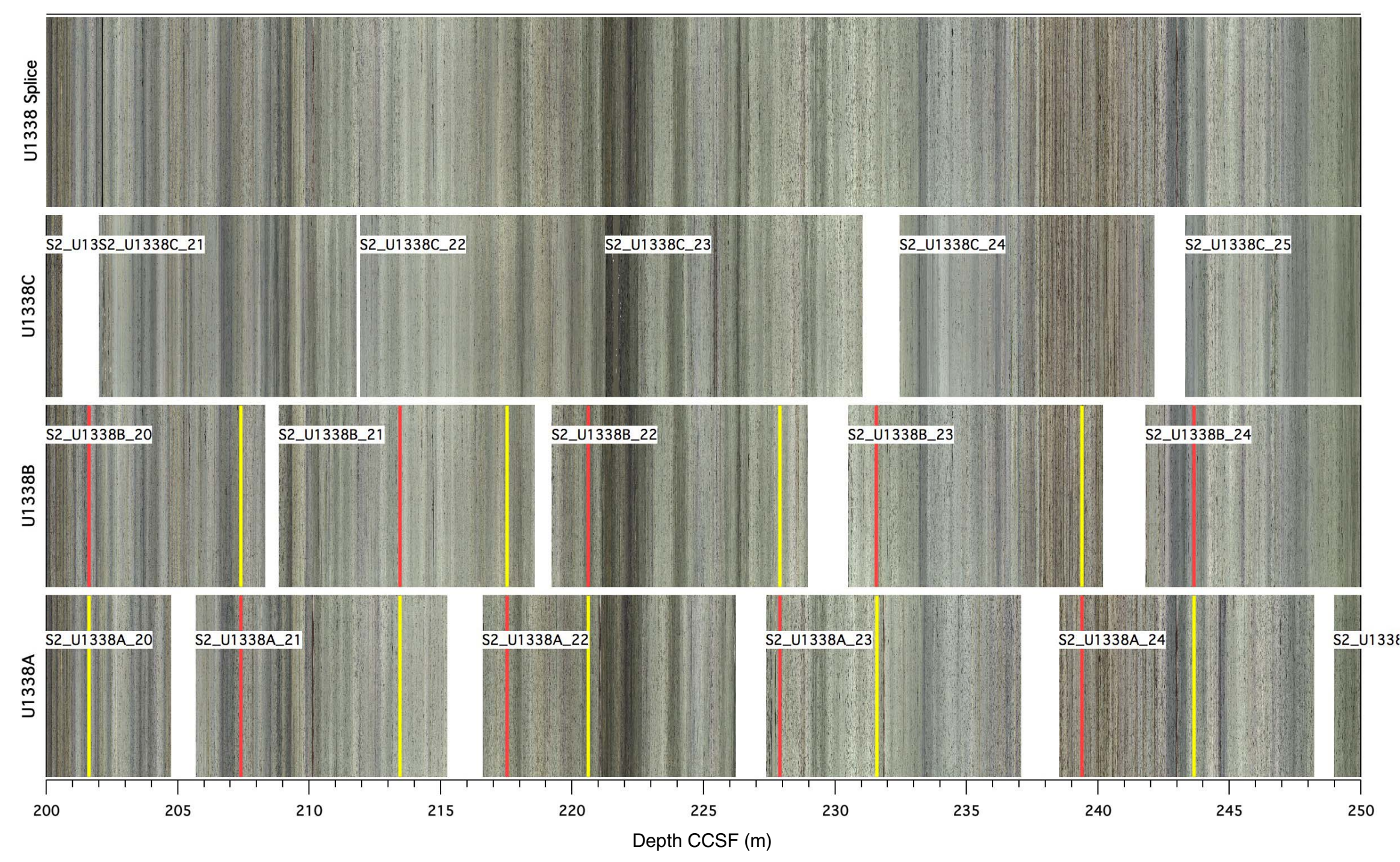


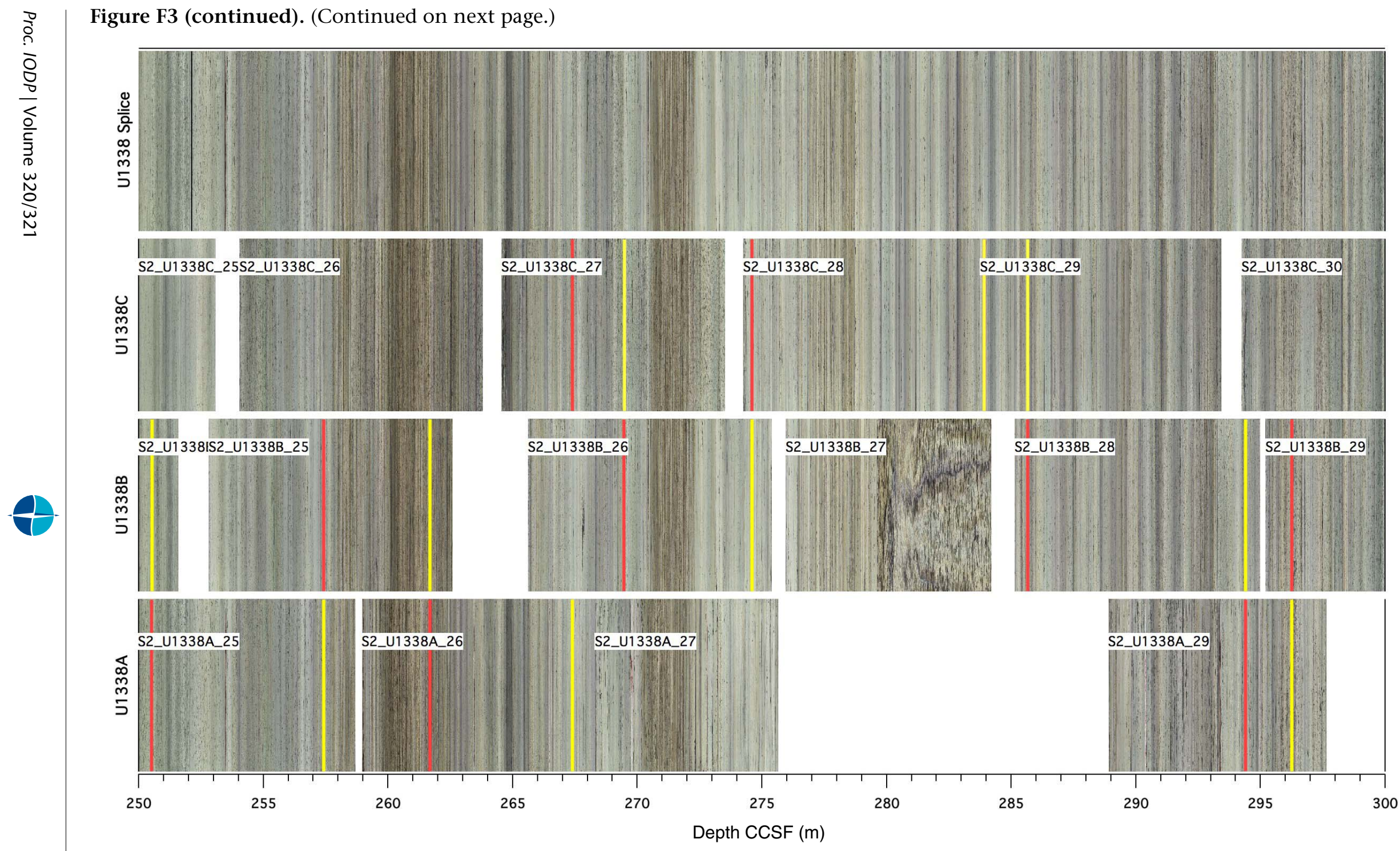


Figure F3 (continued). (Continued on next page.)
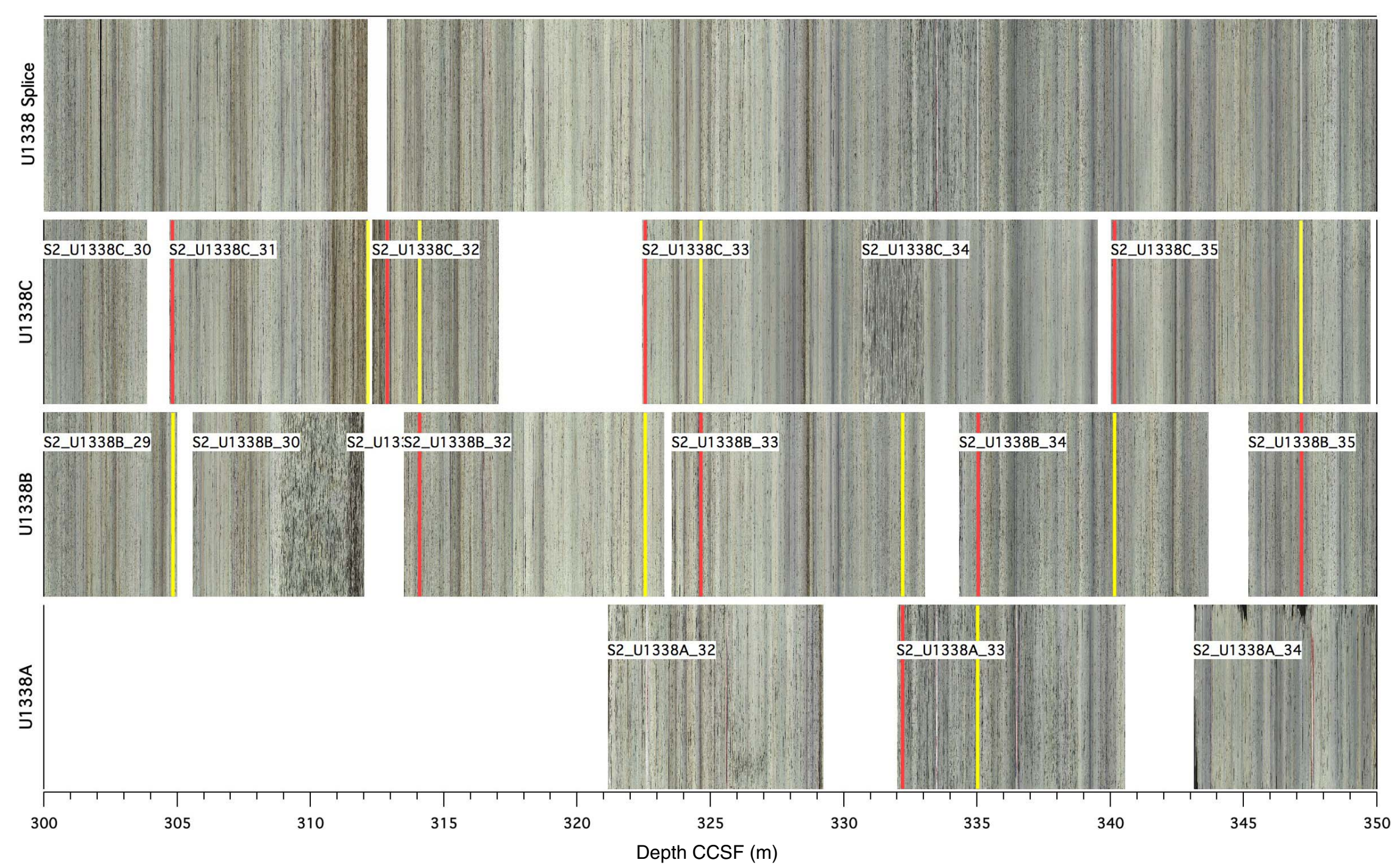
Figure F3 (continued). (Continued on next page.)
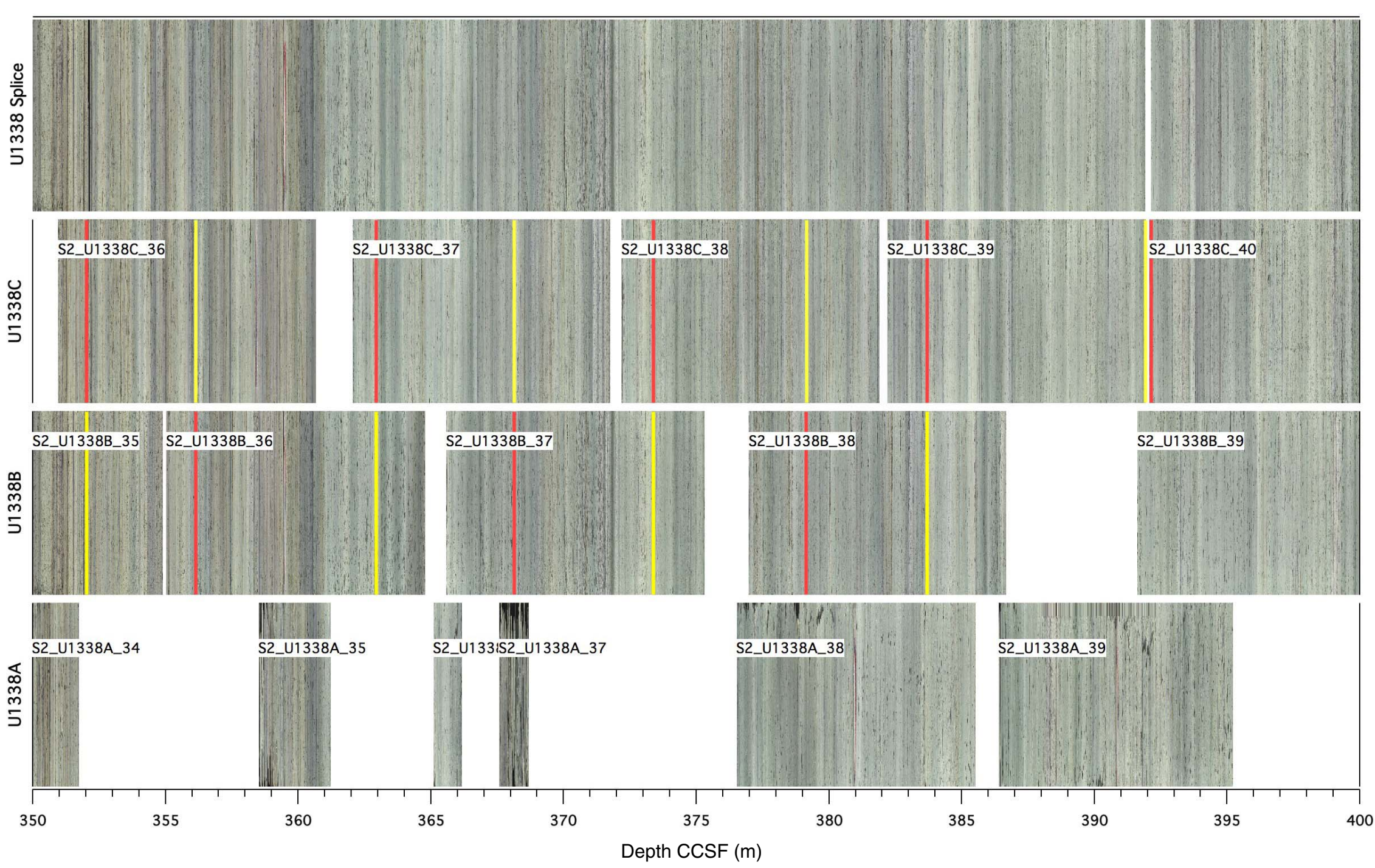
Figure F3 (continued). (Continued on next page.)
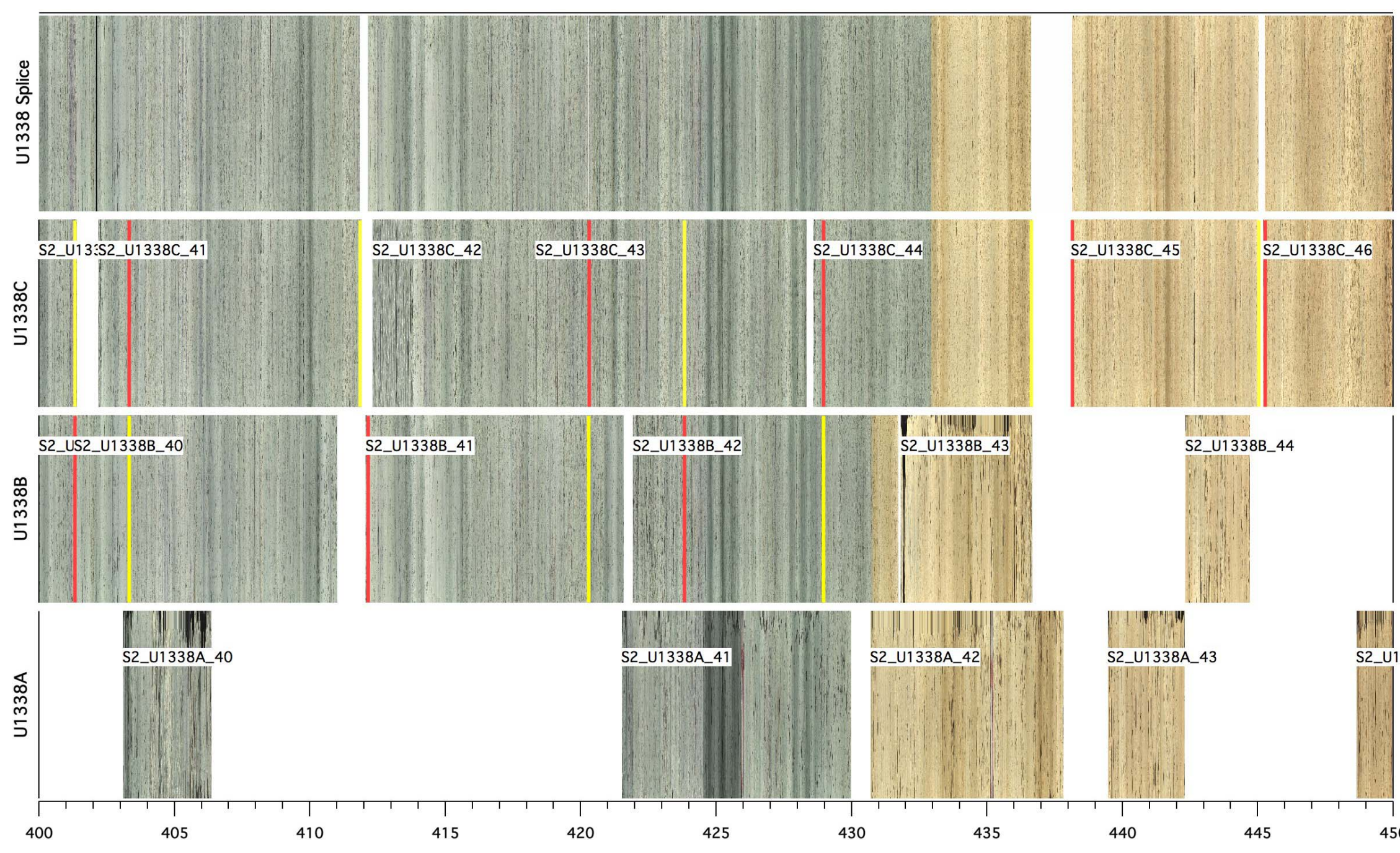

$\theta$

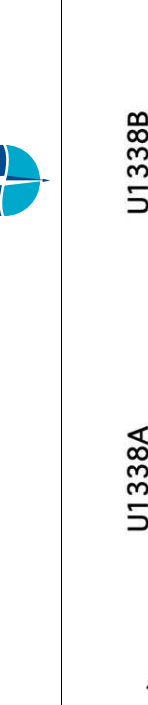

410

415
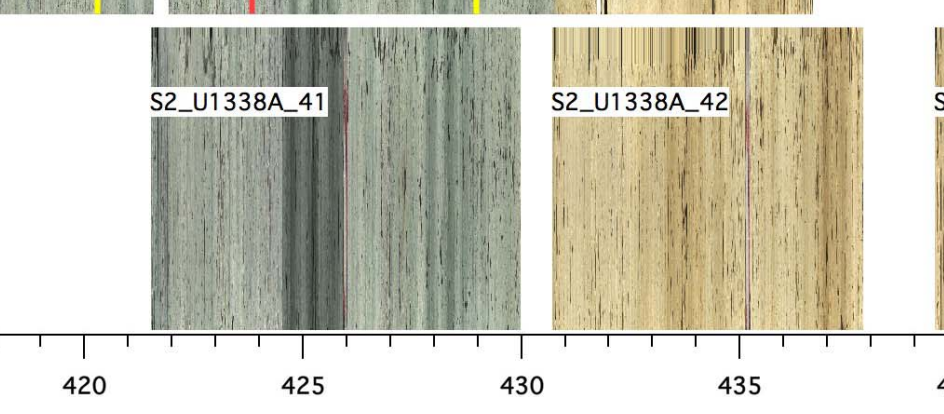

Depth CCSF (m) 


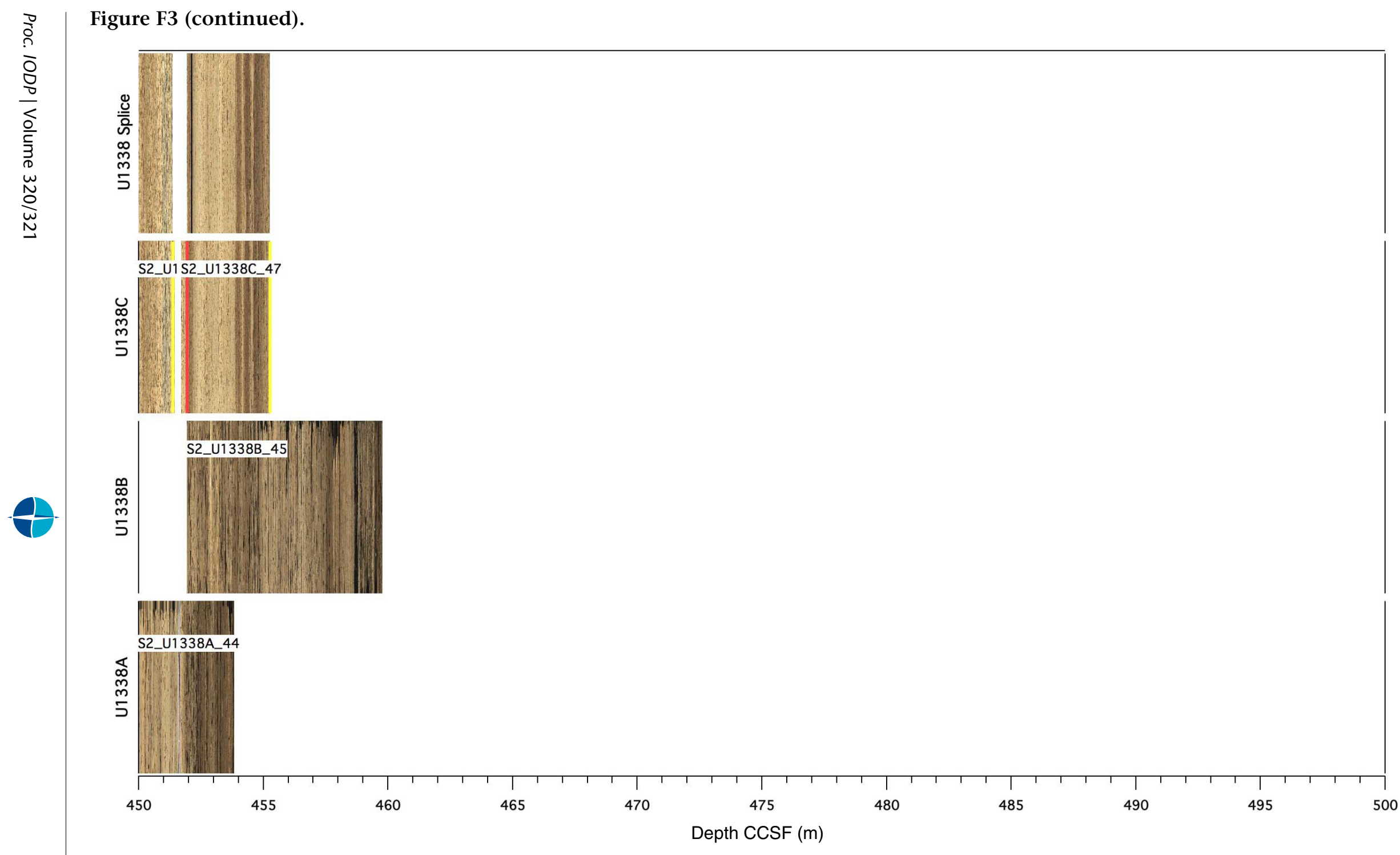


Figure F4. Spliced core data, Sites U1336-U1338. Colors of individual hole data are repeated in the spliced profile to illustrate where the splice sections come from. Note that outside of the splice intervals within each core there is general alignment with splice features, but it is not always exact. (Continued on next 47 pages.)
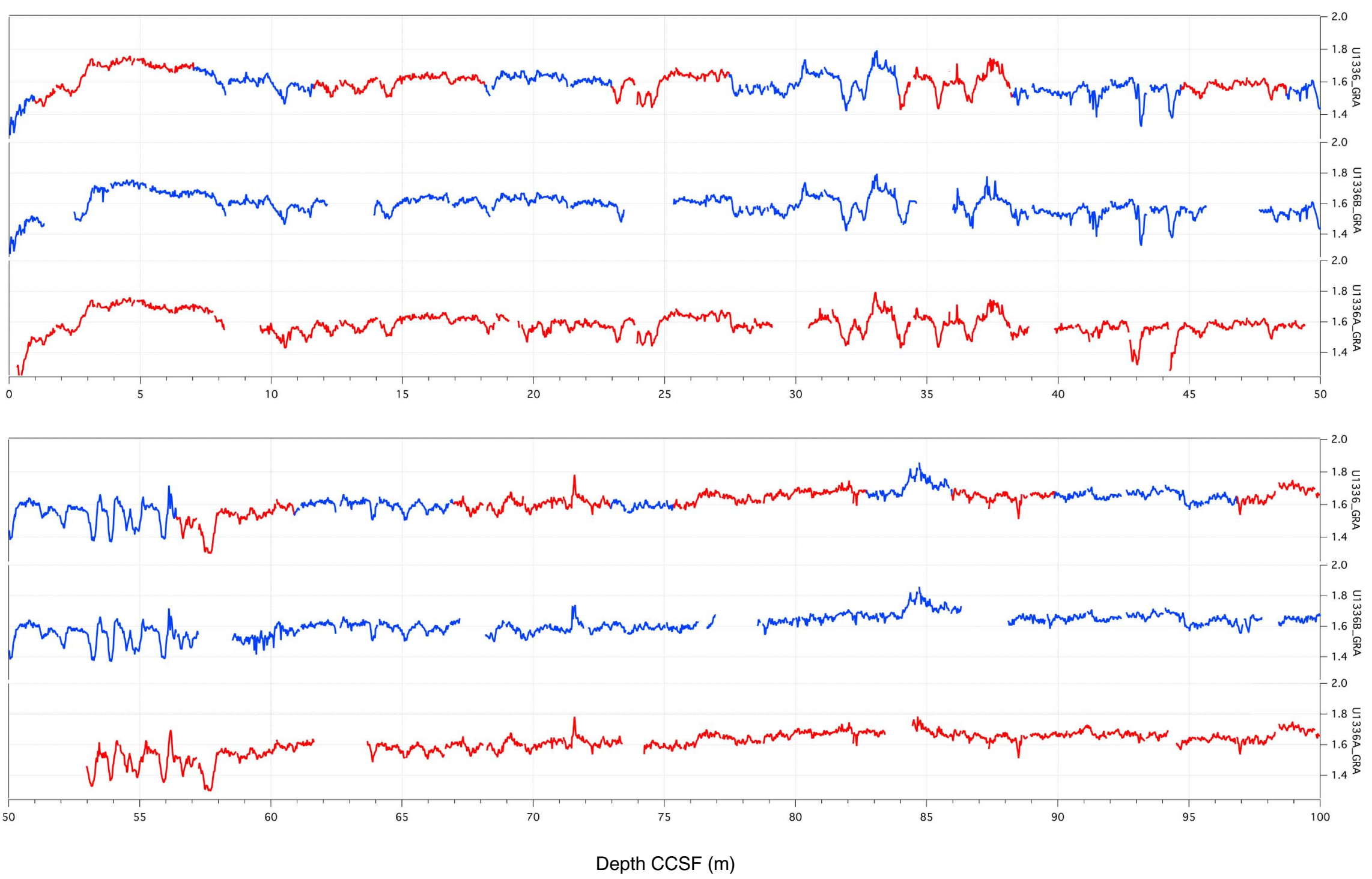
Figure F4 (continued). (Continued on next page.)
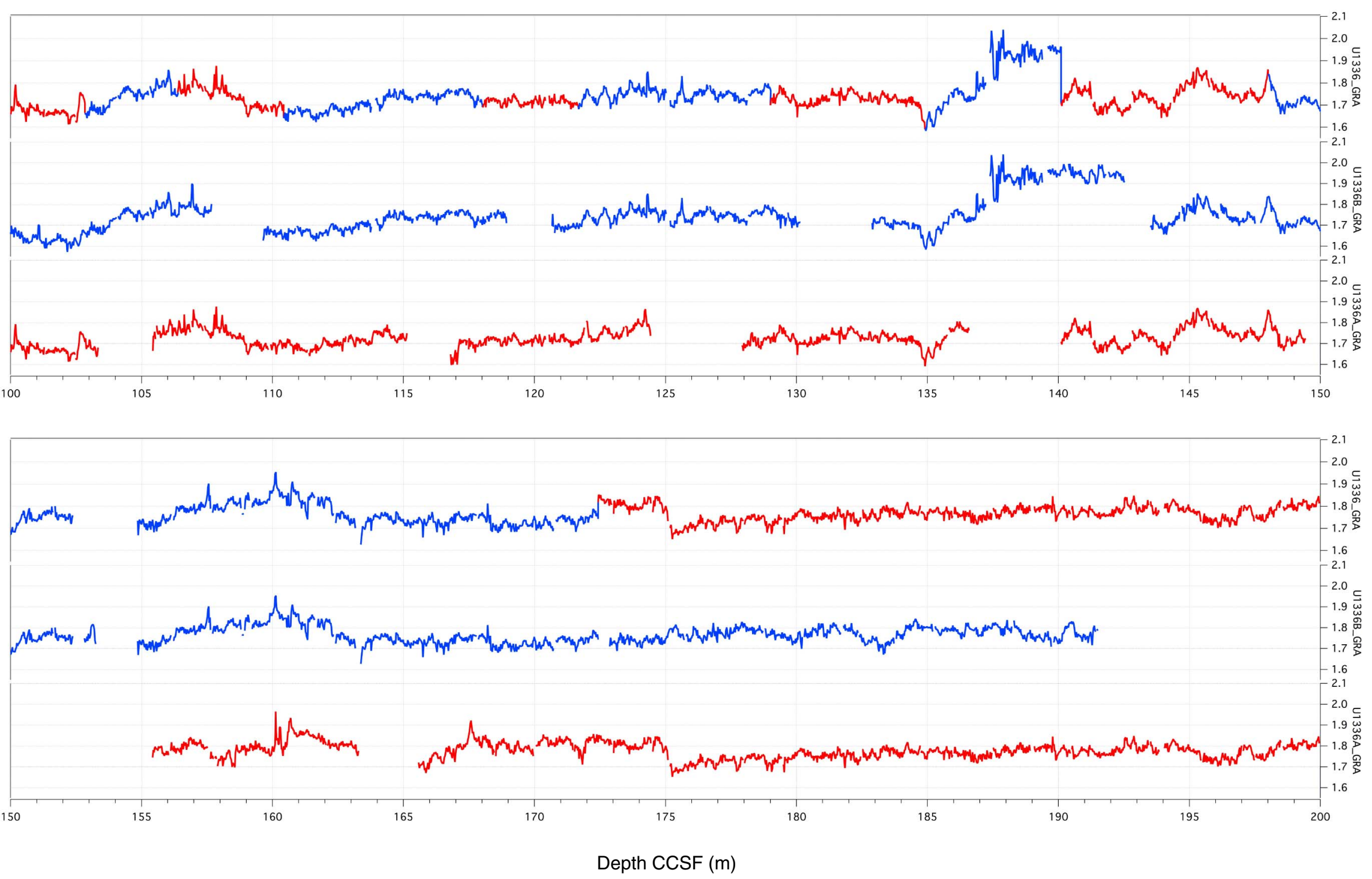
Figure F4 (continued). (Continued on next page.)

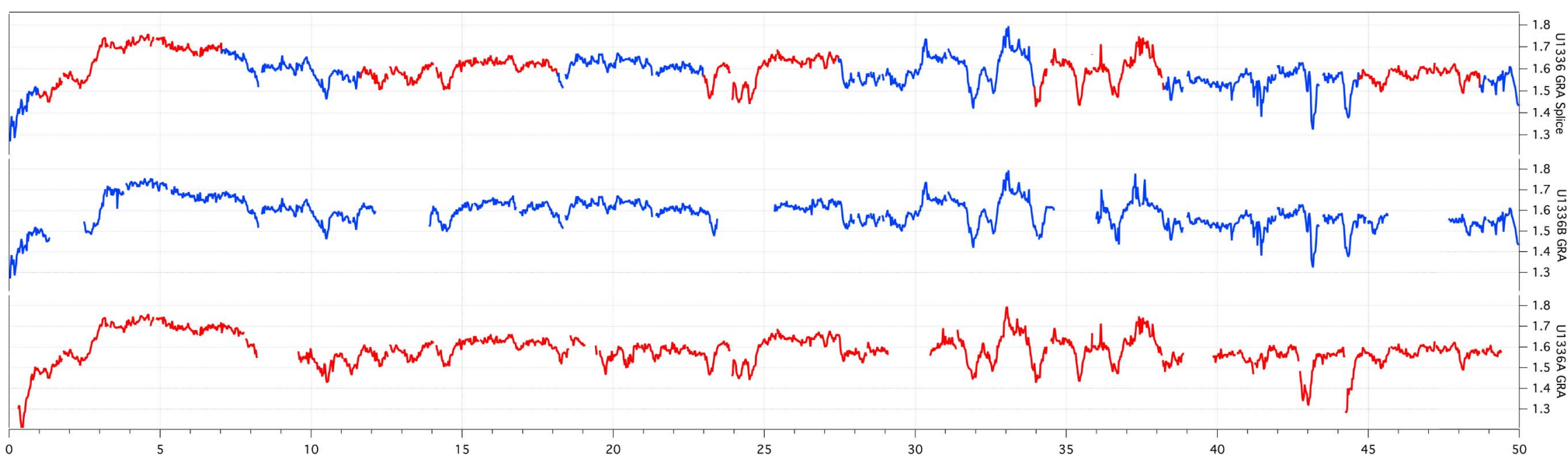

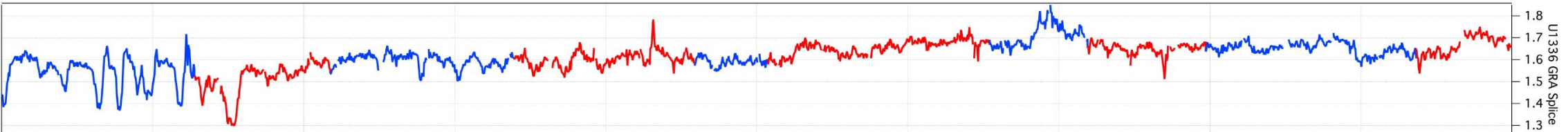

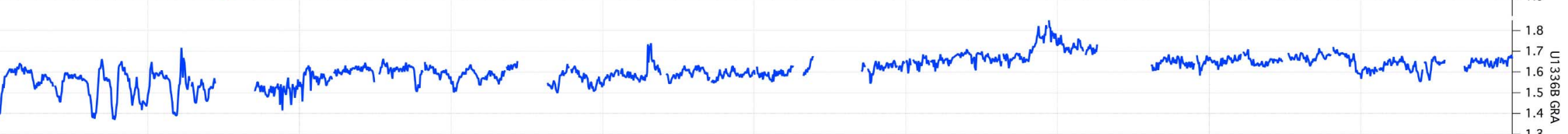

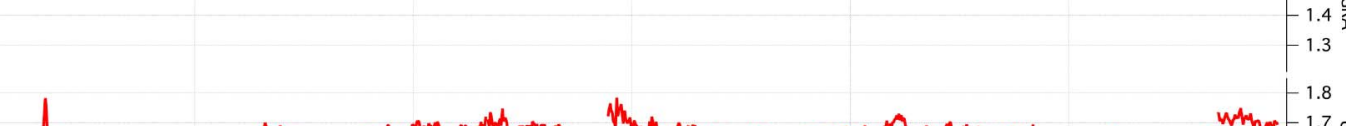

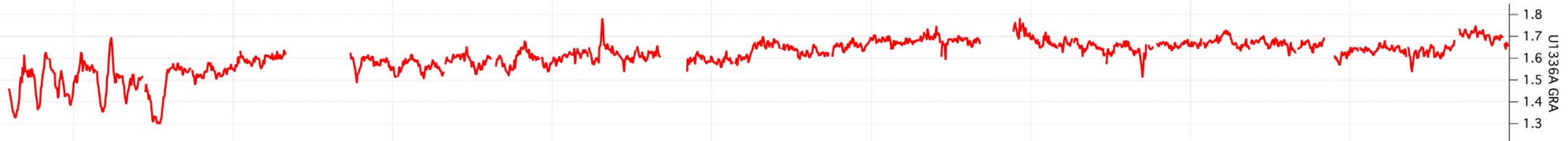
50 60

65

70

80

Depth CCSF $(m)$ 
Figure F4 (continued). (Continued on next page.)
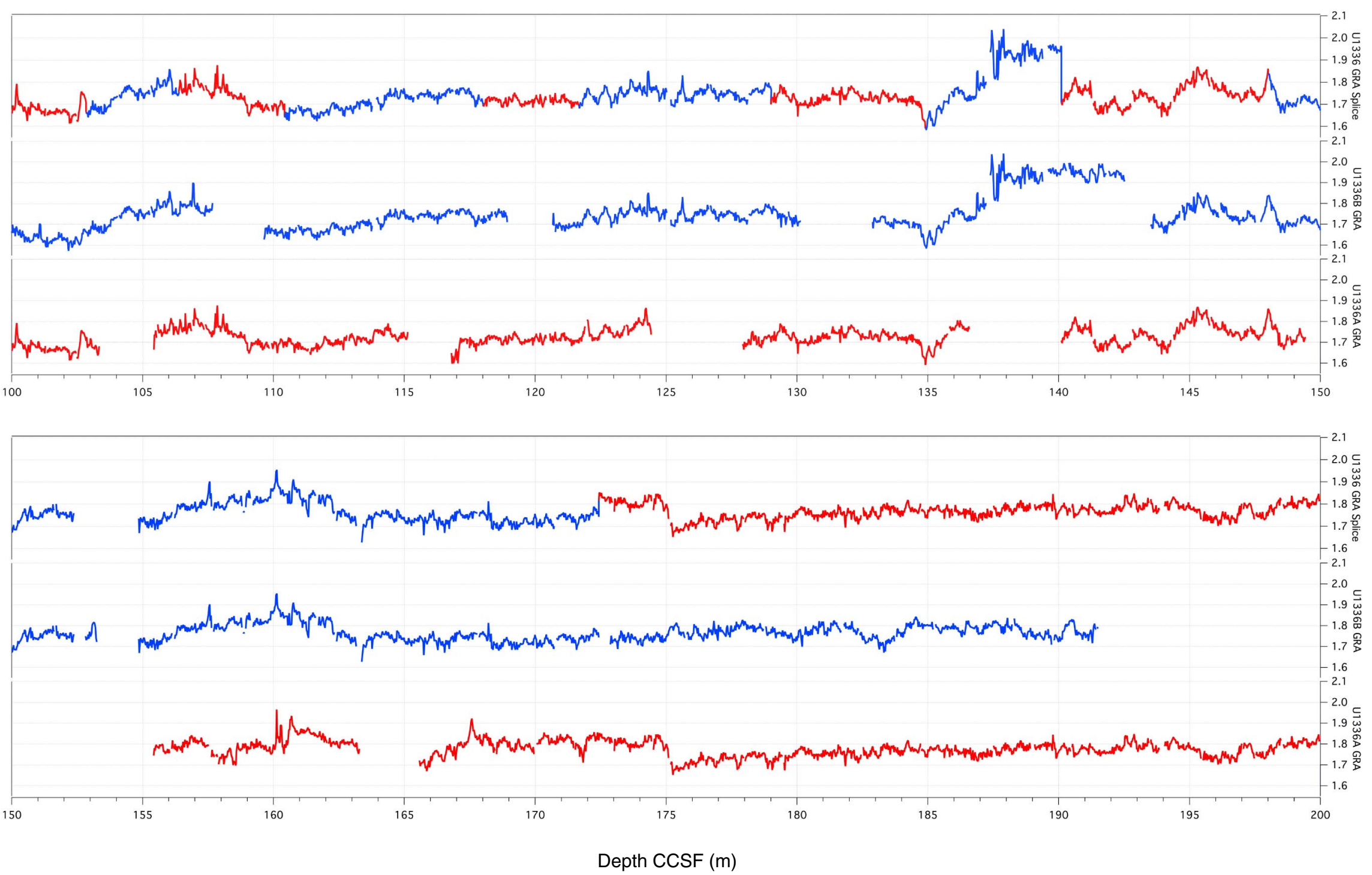


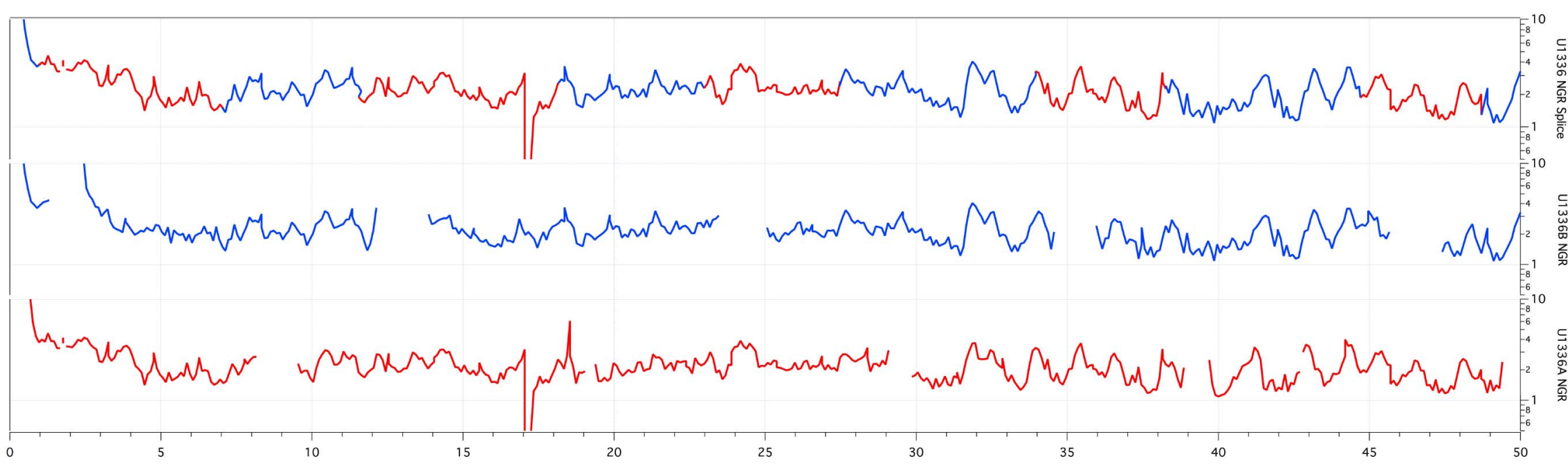

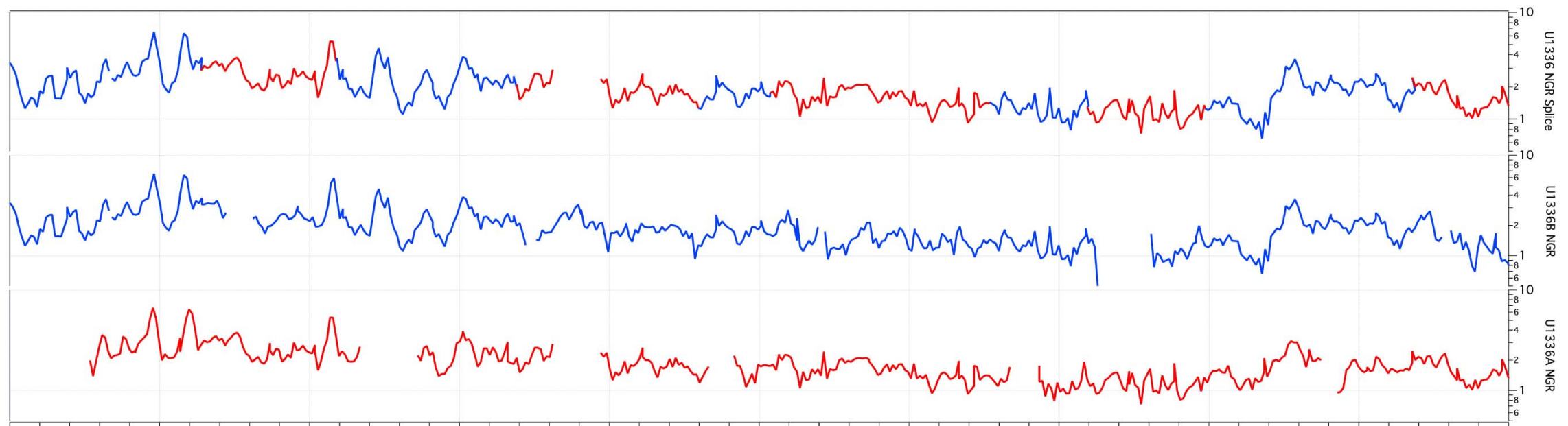




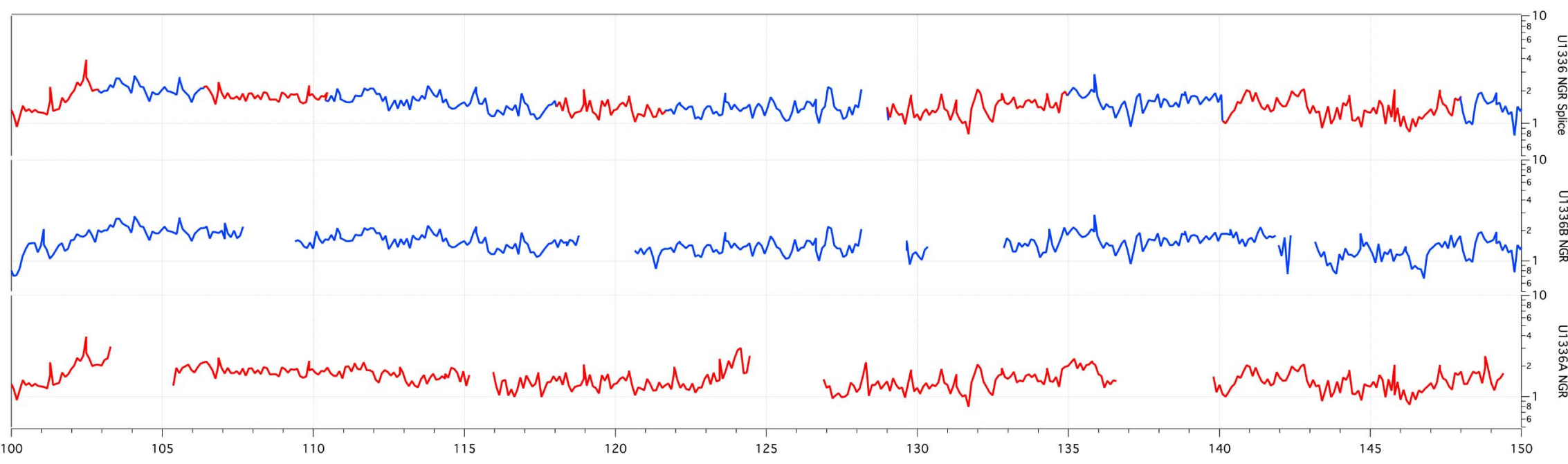

(WM


Figure F4 (continued). (Continued on next page.)

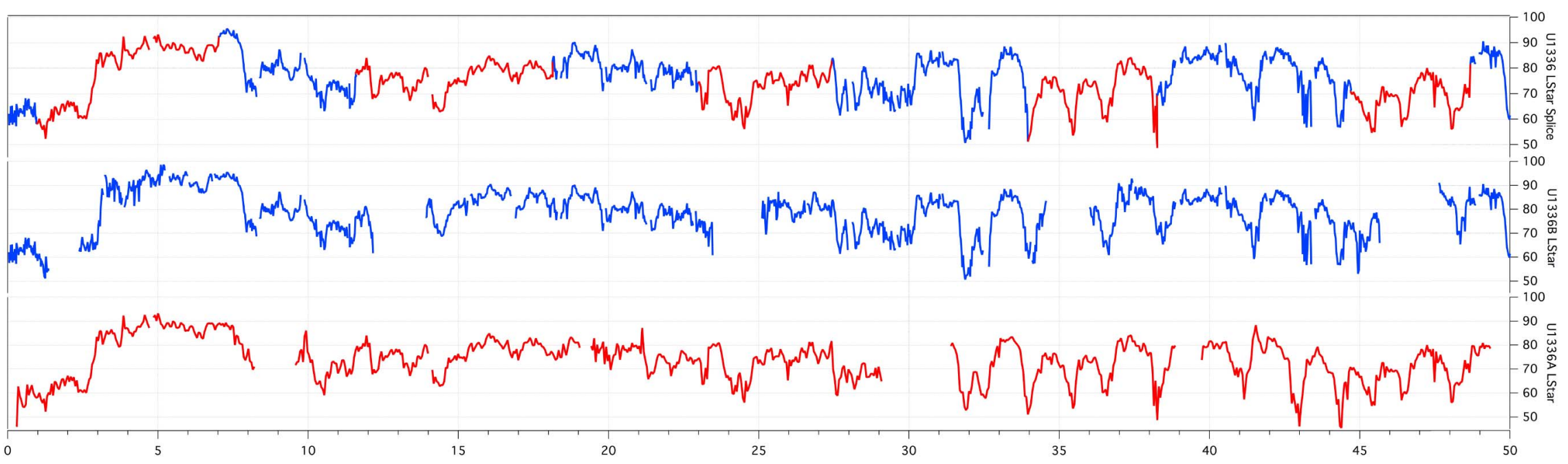

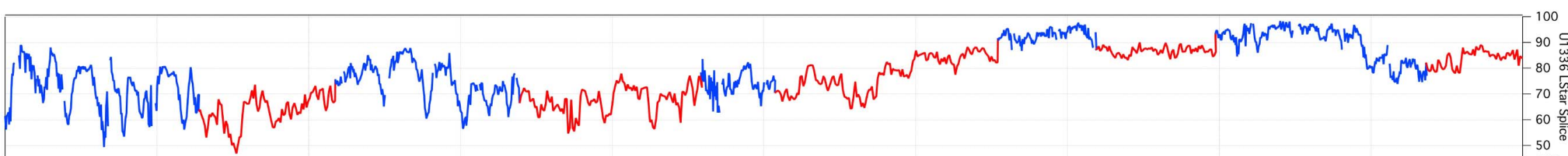

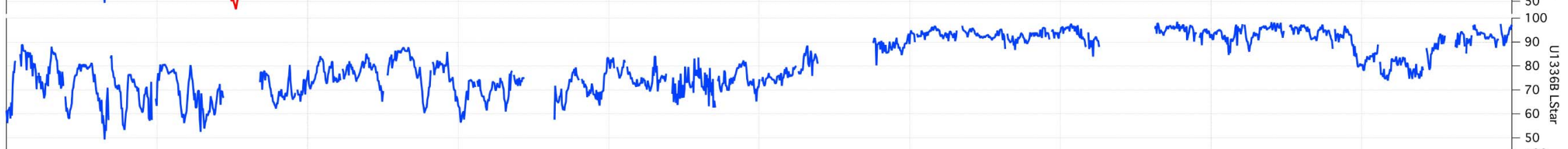

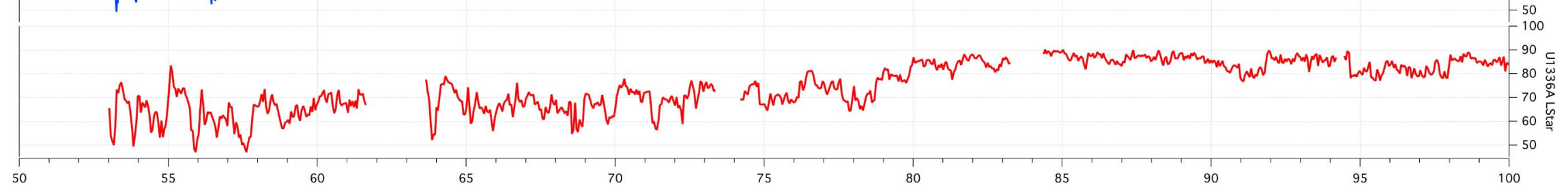


Figure F4 (continued). (Continued on next page.)

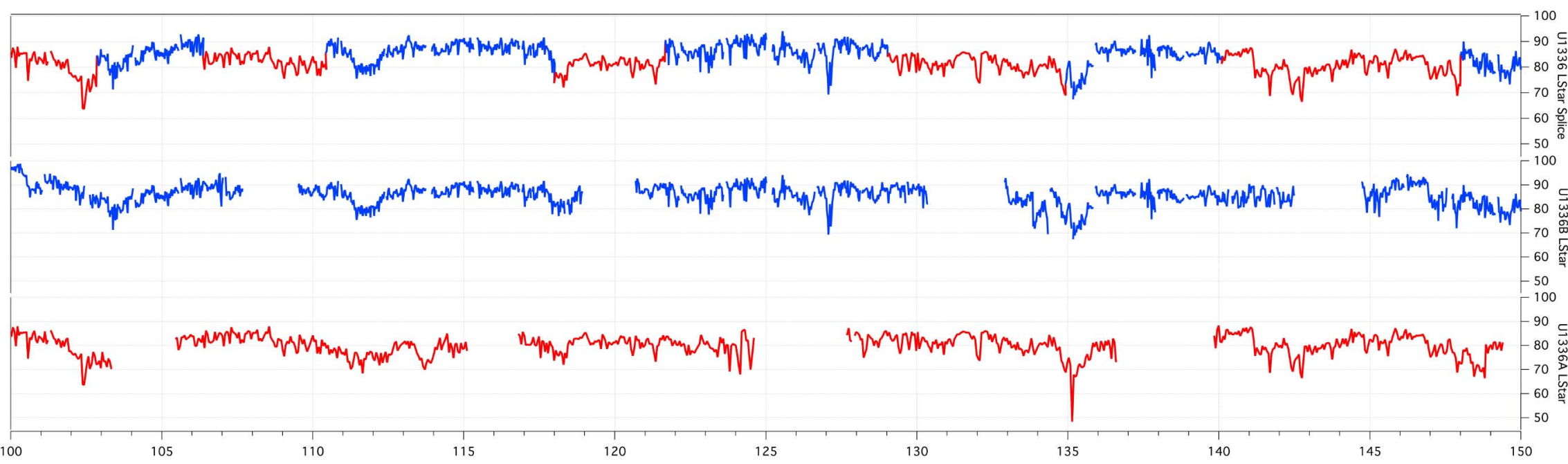

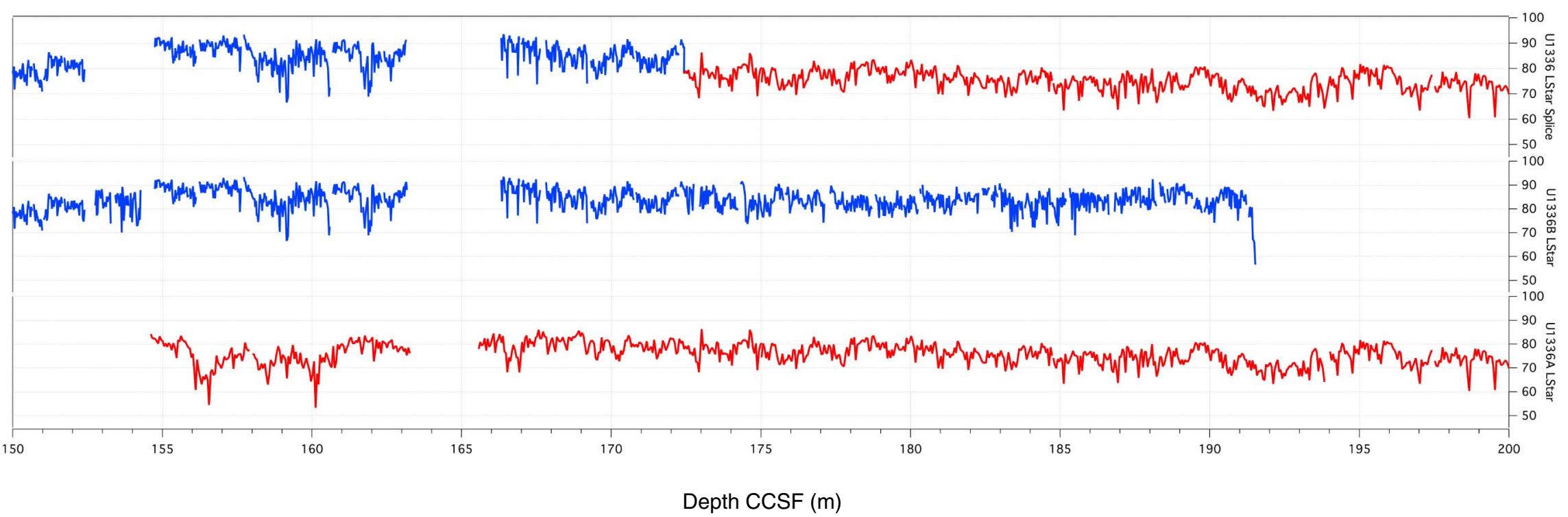


Figure F4 (continued). (Continued on next page.)

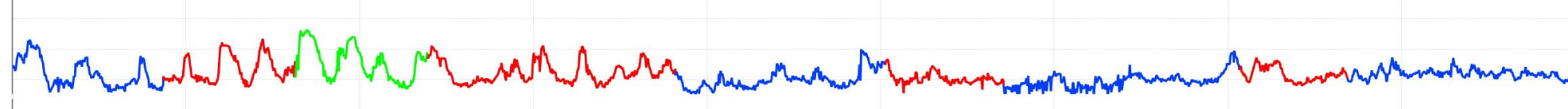

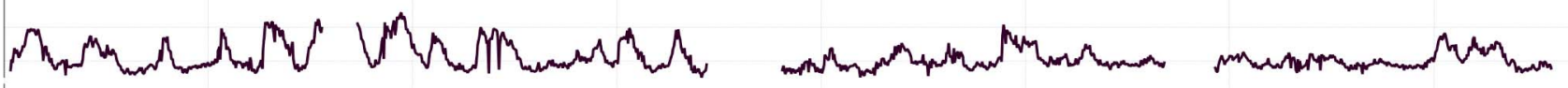
the whNomam

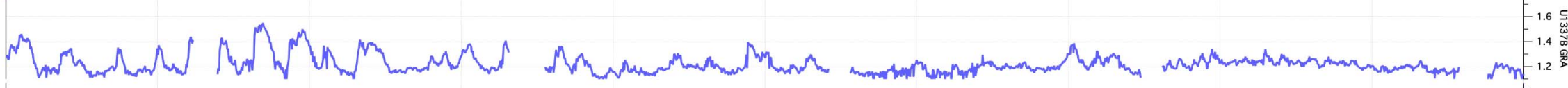
${ }_{0}^{M}$

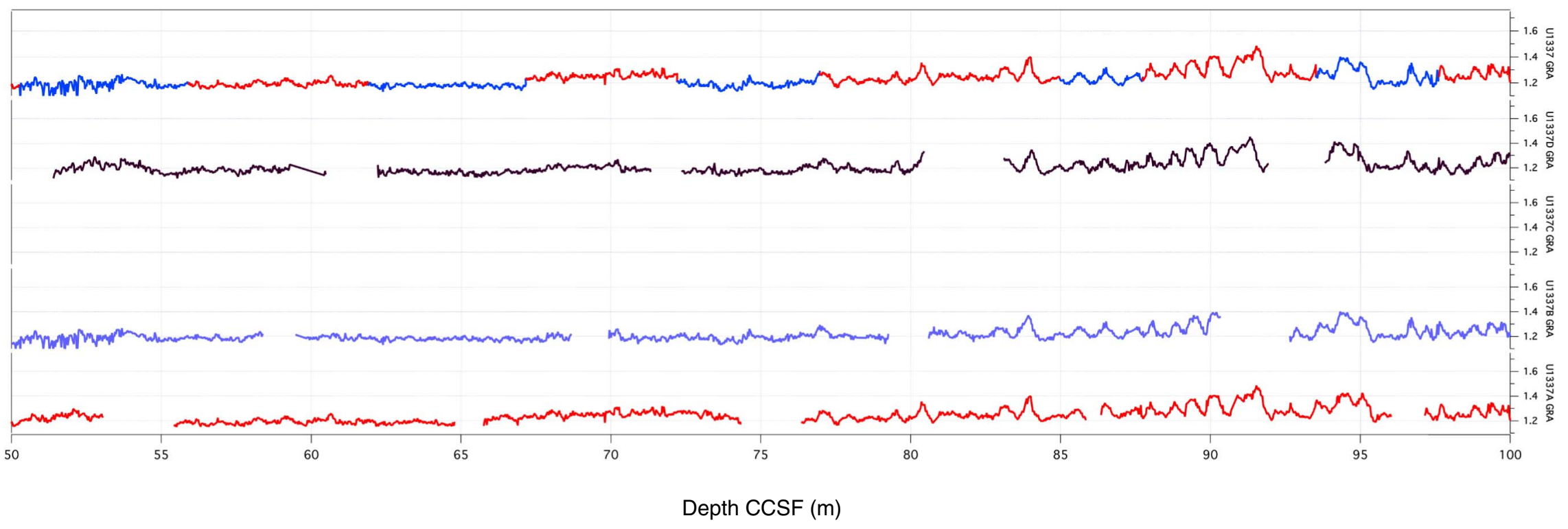


Figure F4 (continued). (Continued on next page.)
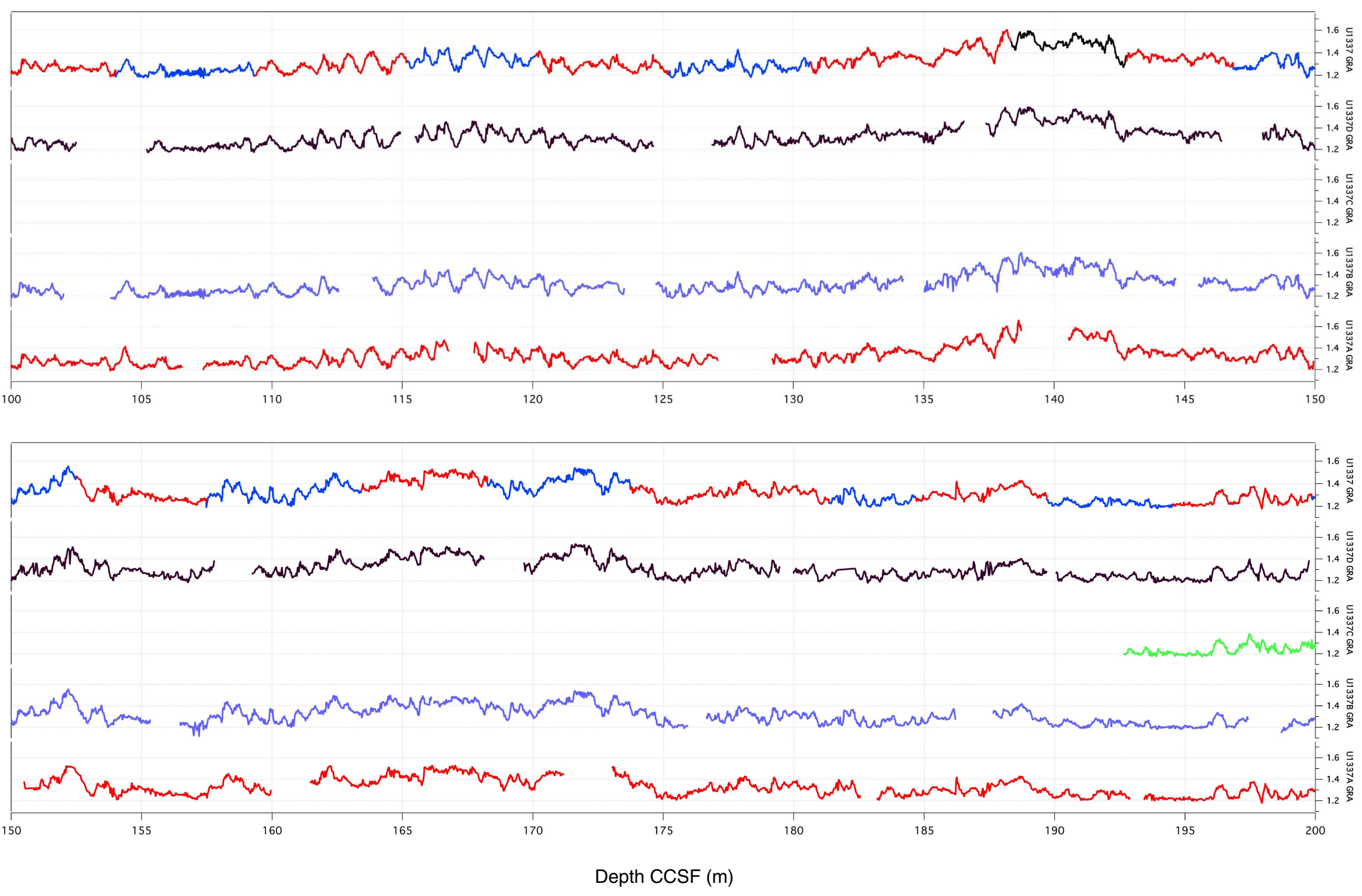

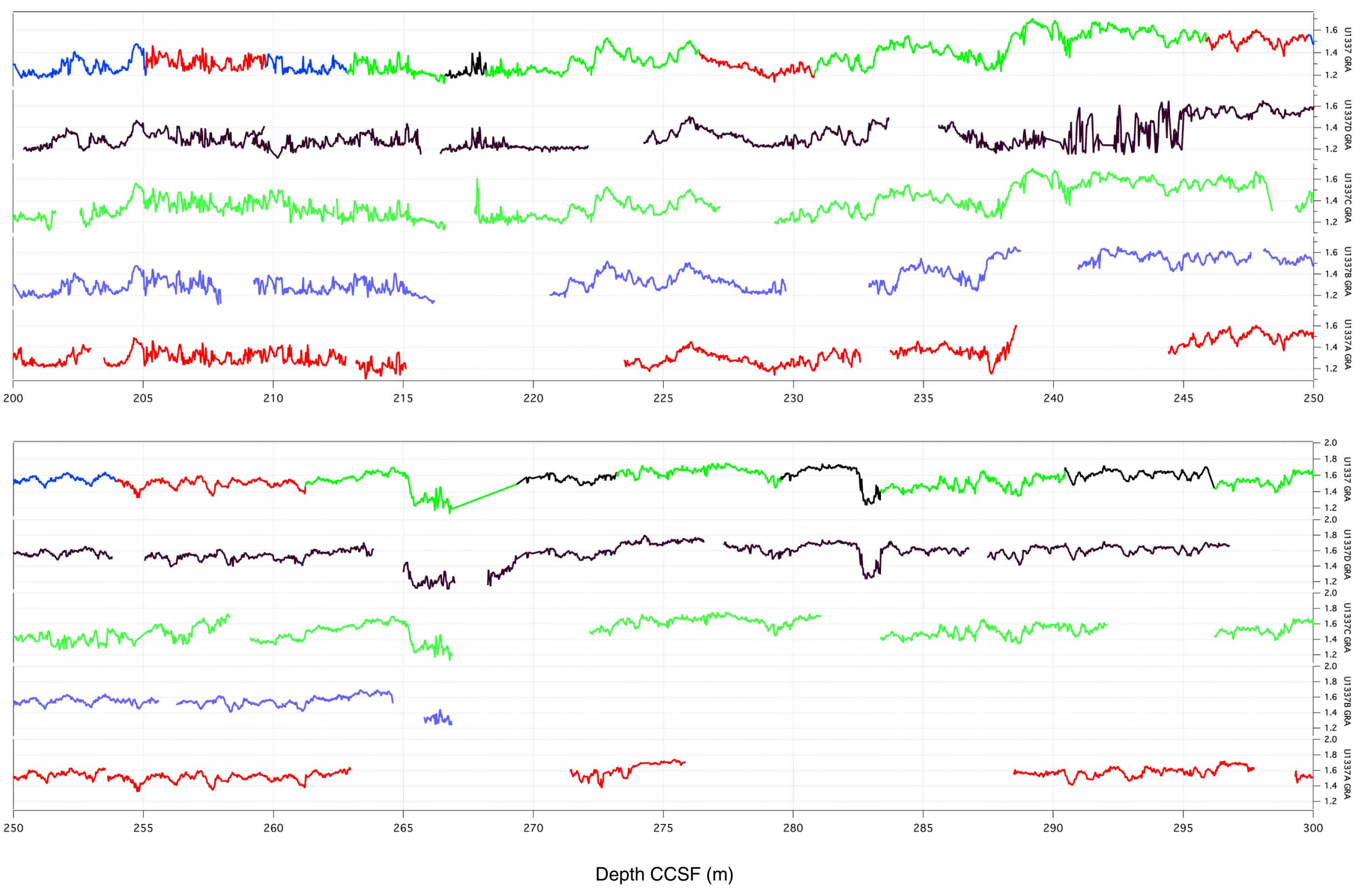
Figure F4 (continued). (Continued on next page.)
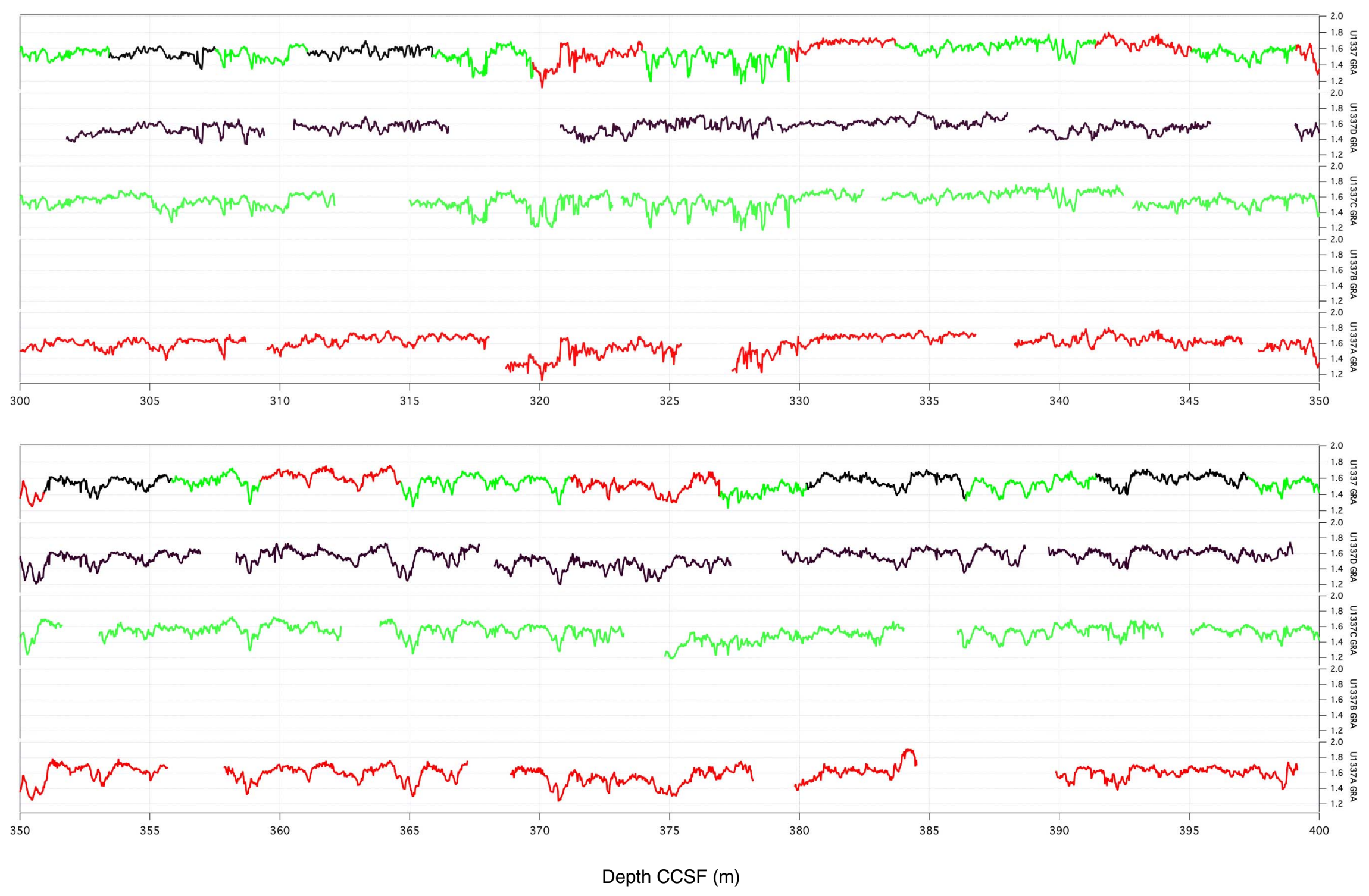


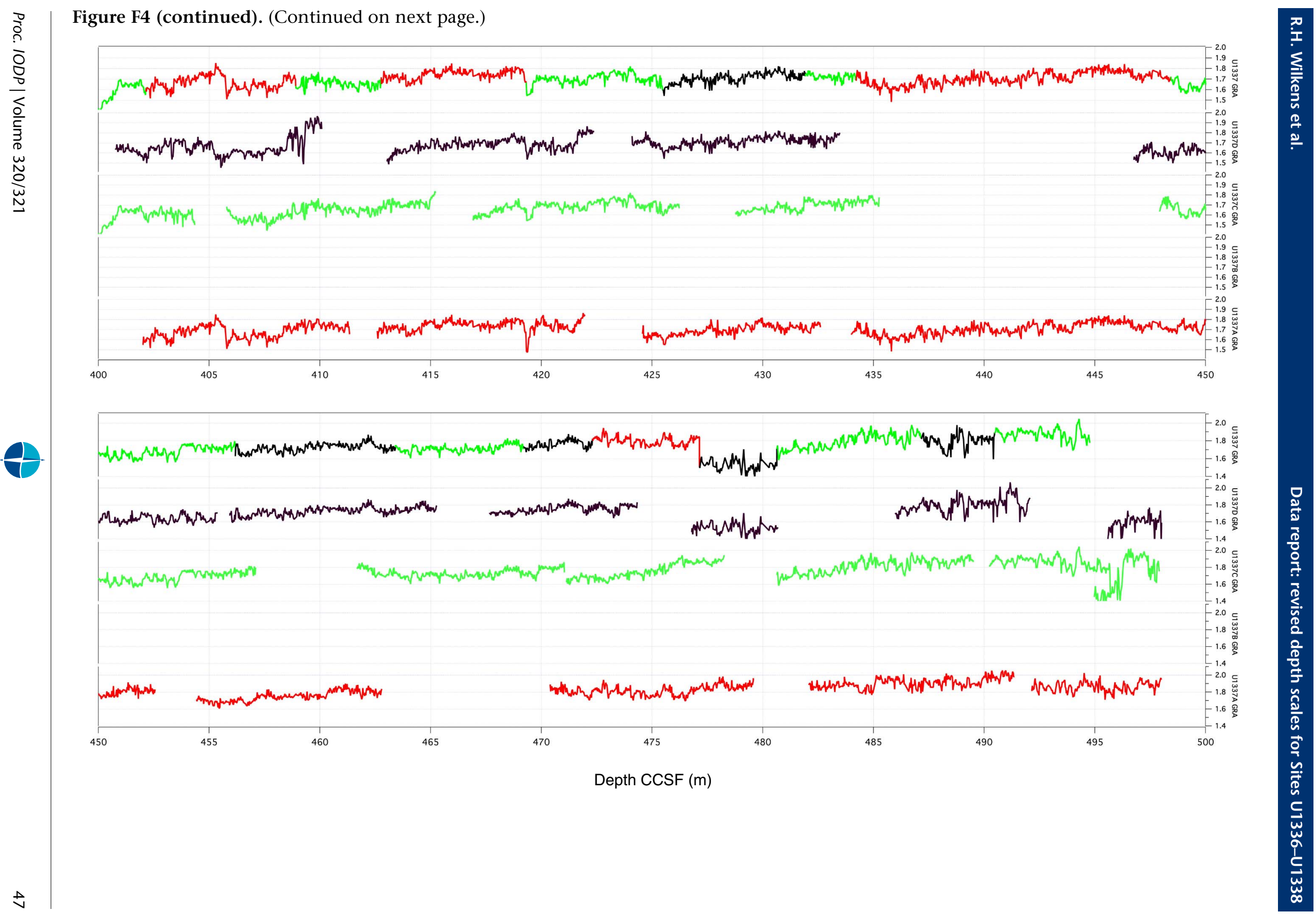




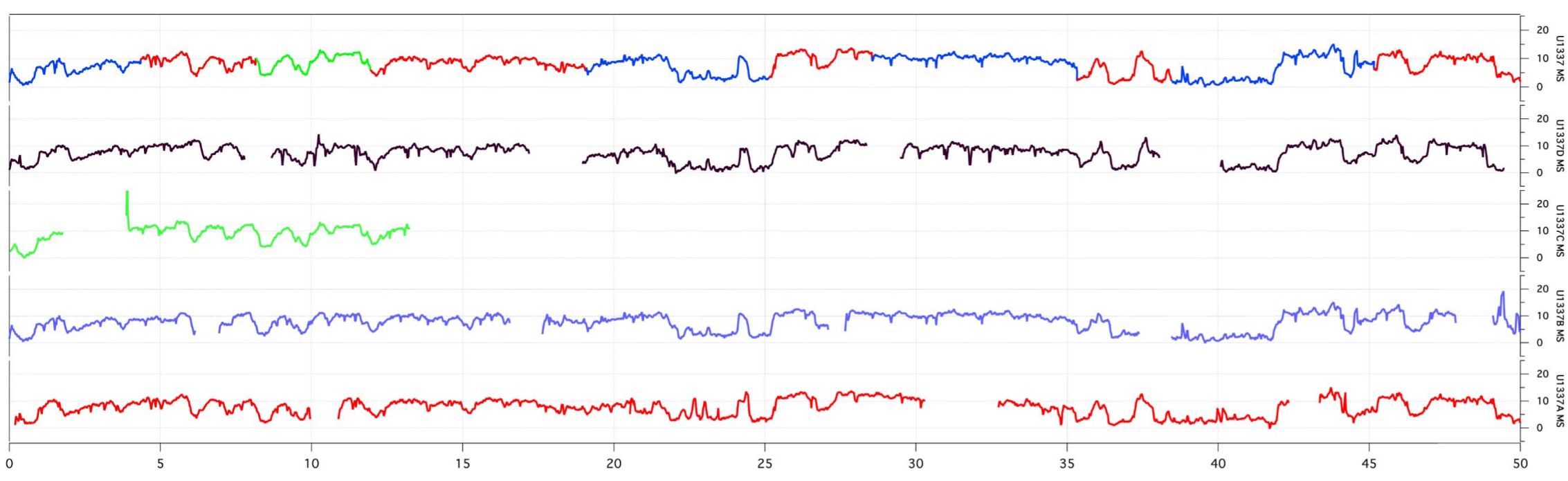

(n)


Figure F4 (continued). (Continued on next page.)
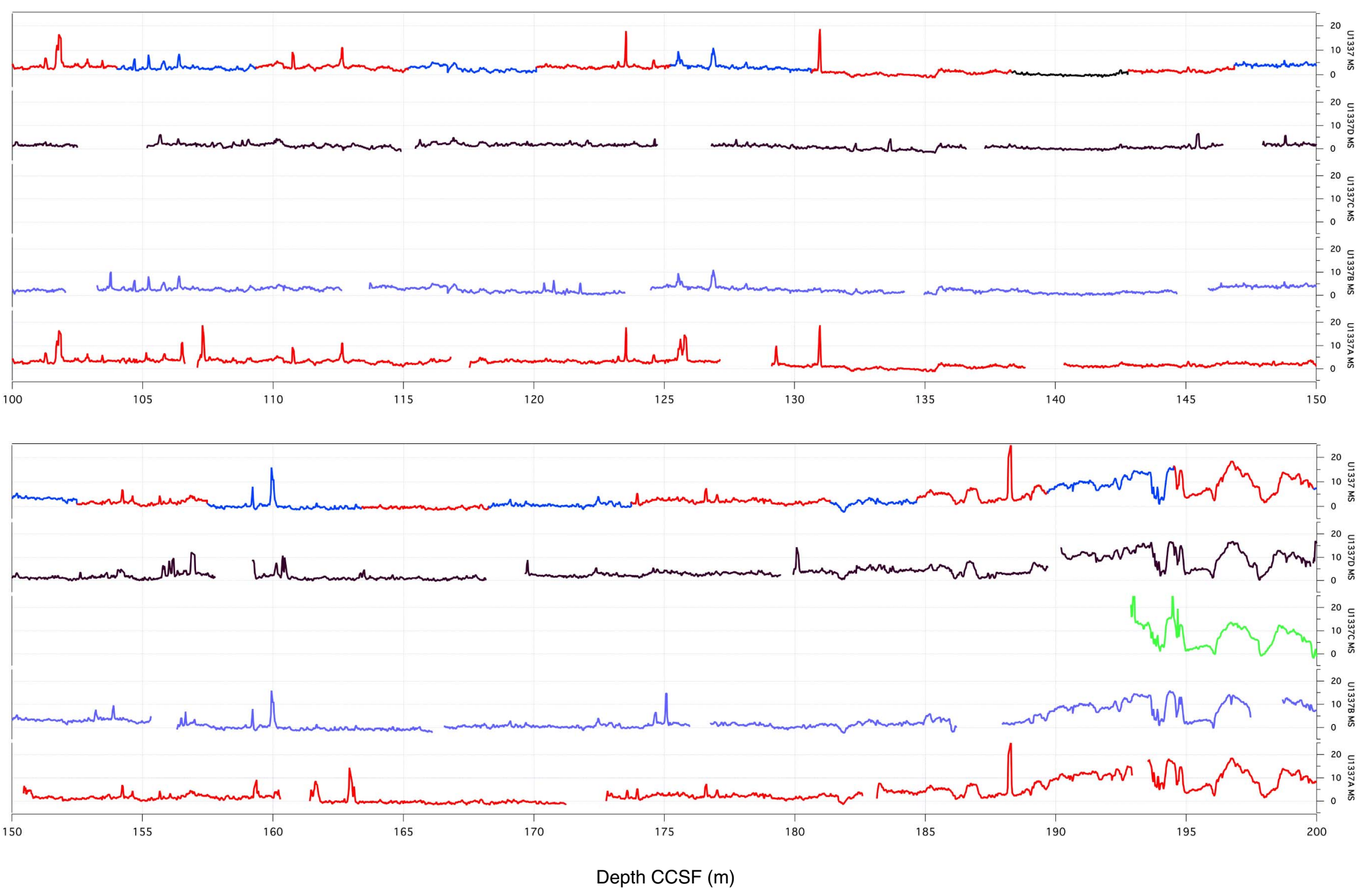
Figure F4 (continued). (Continued on next page.)
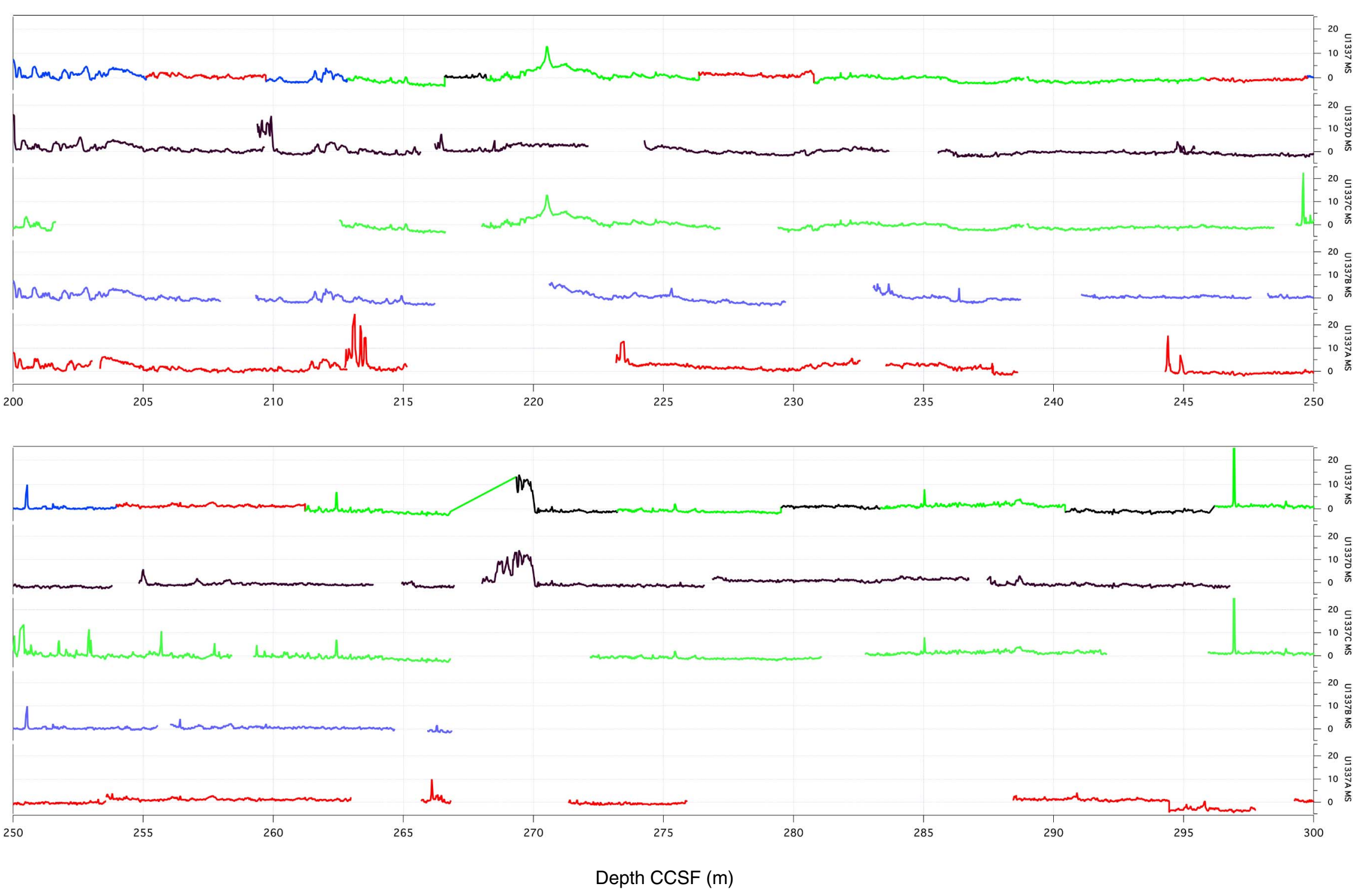
Figure F4 (continued). (Continued on next page.)
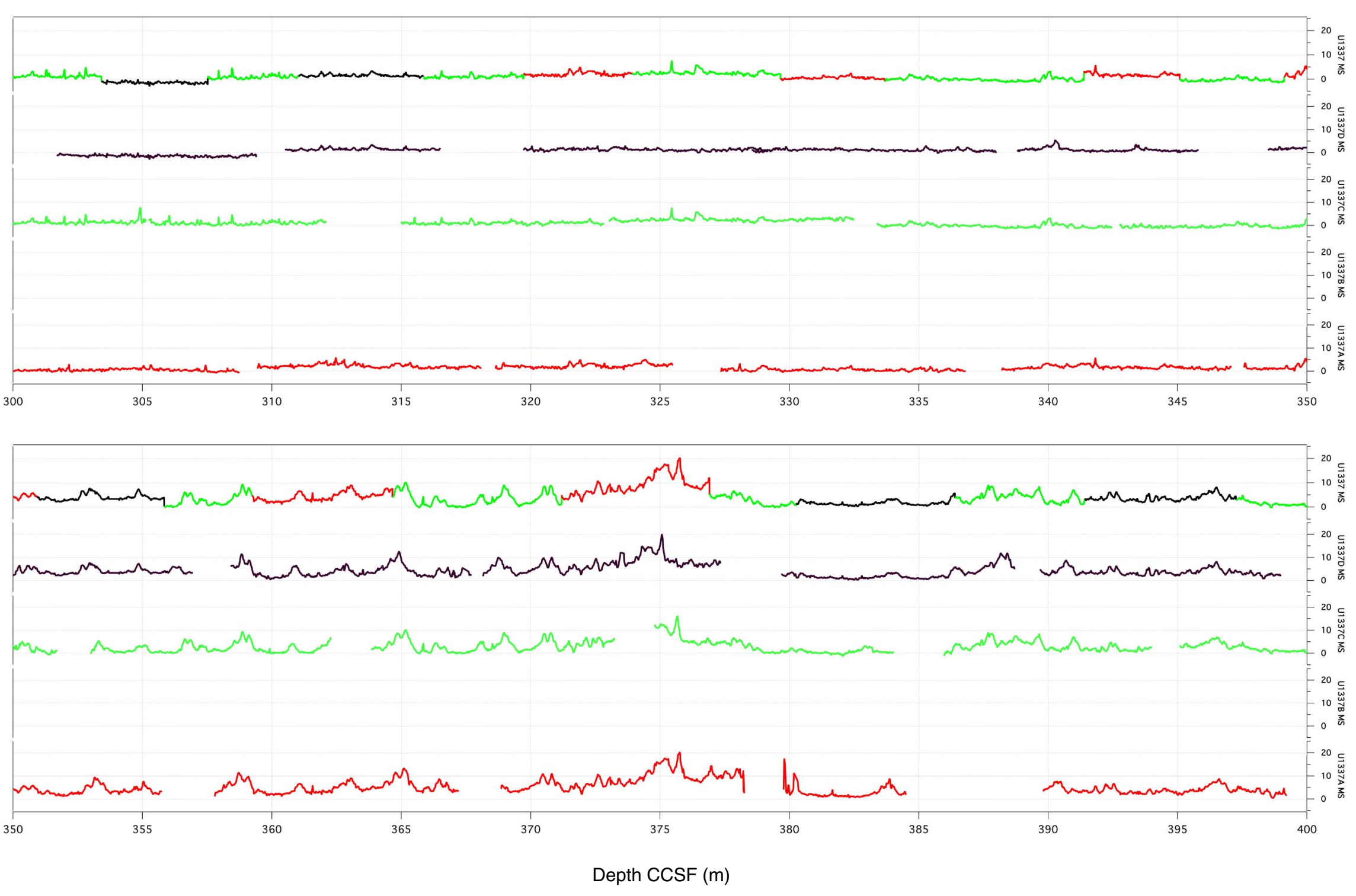


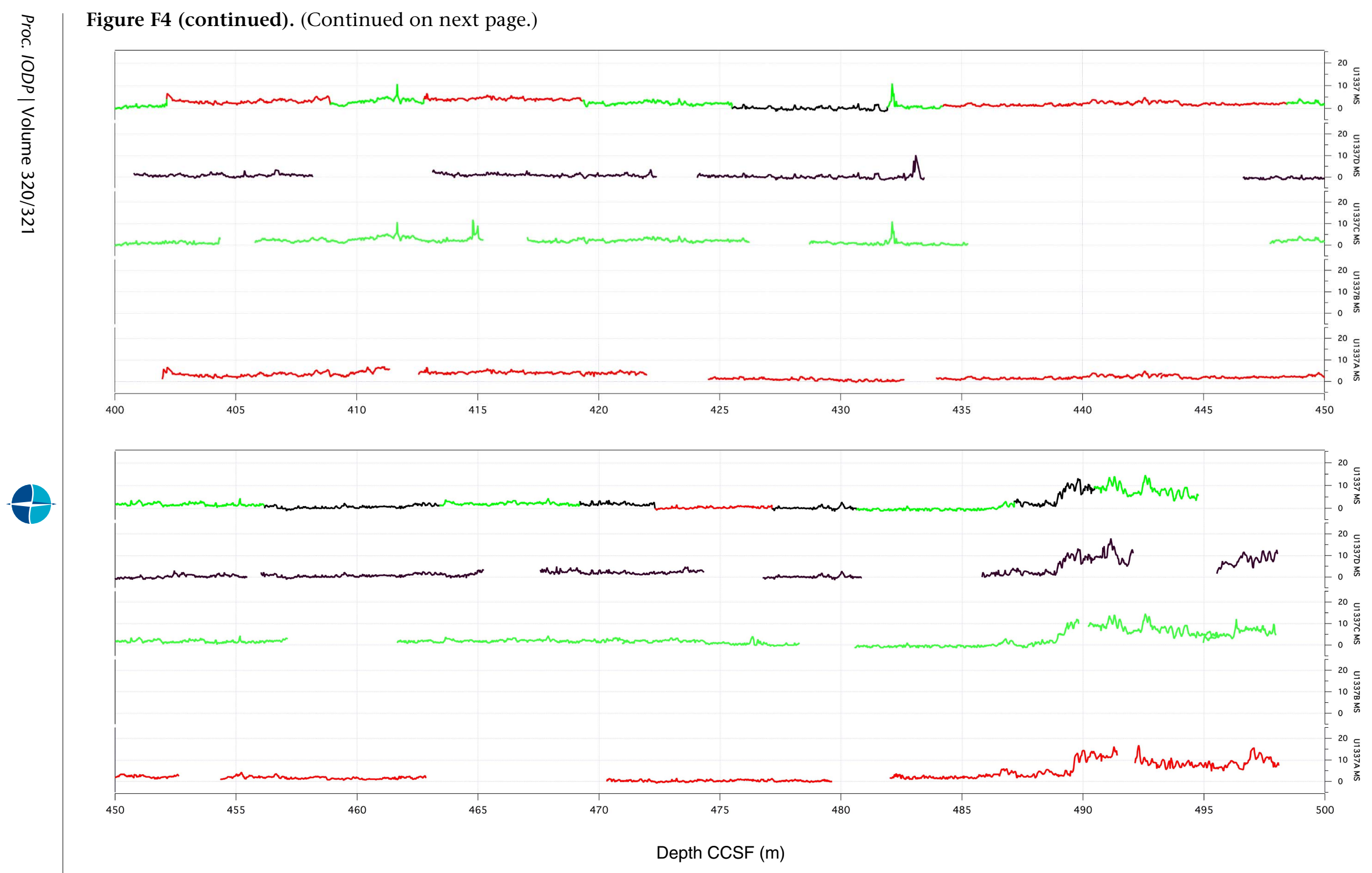




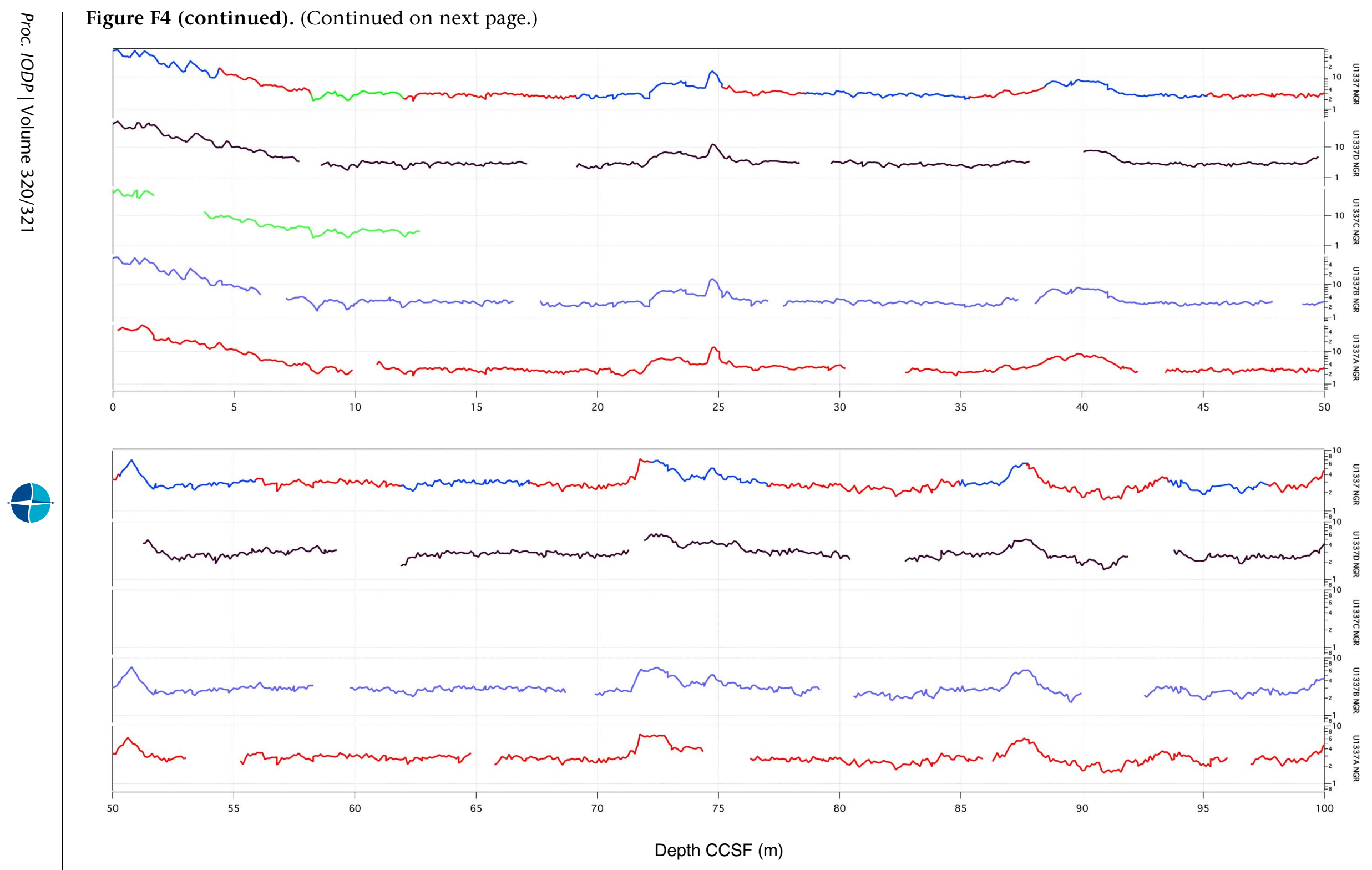


Figure F4 (continued). (Continued on next page.)
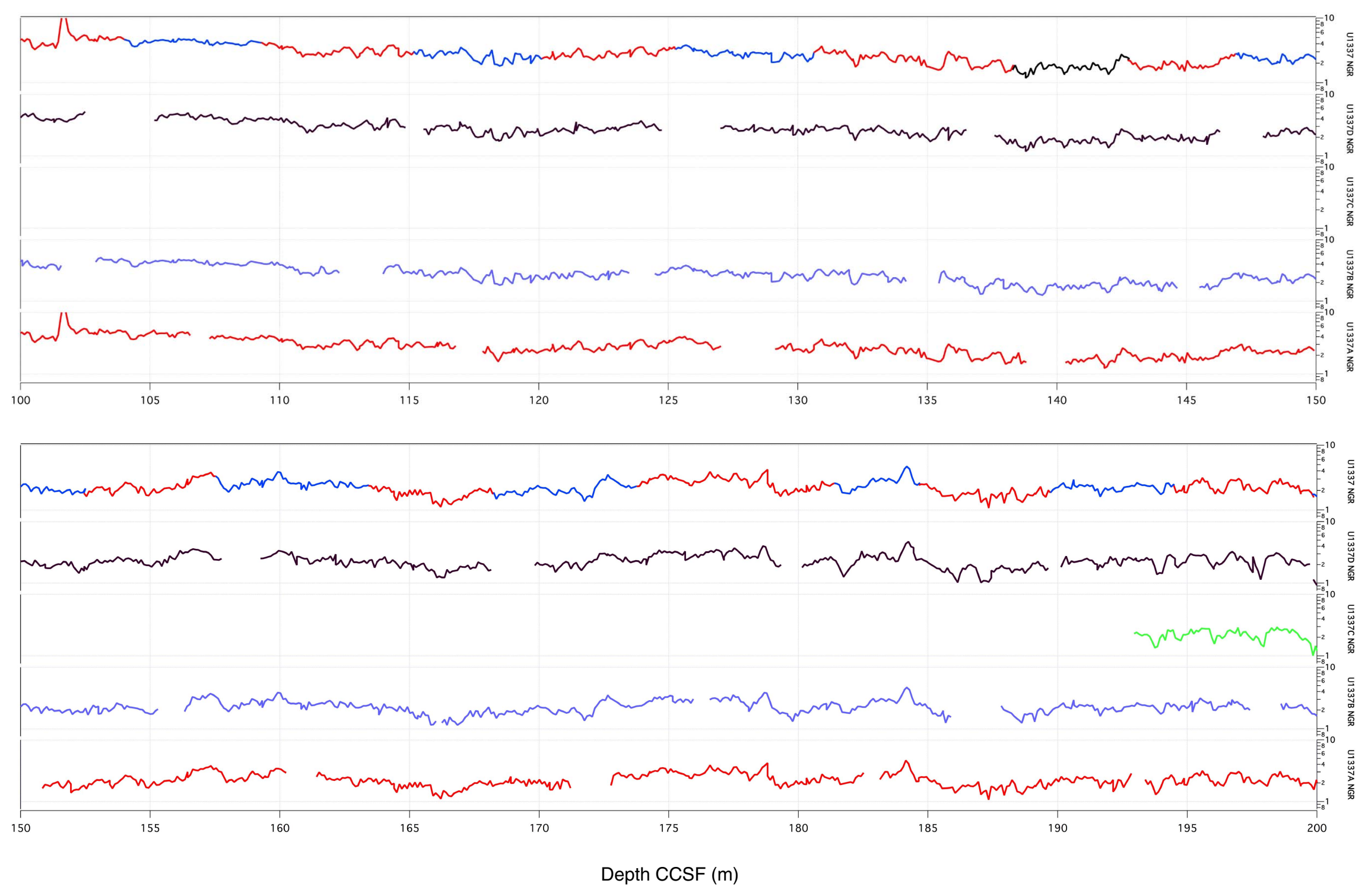

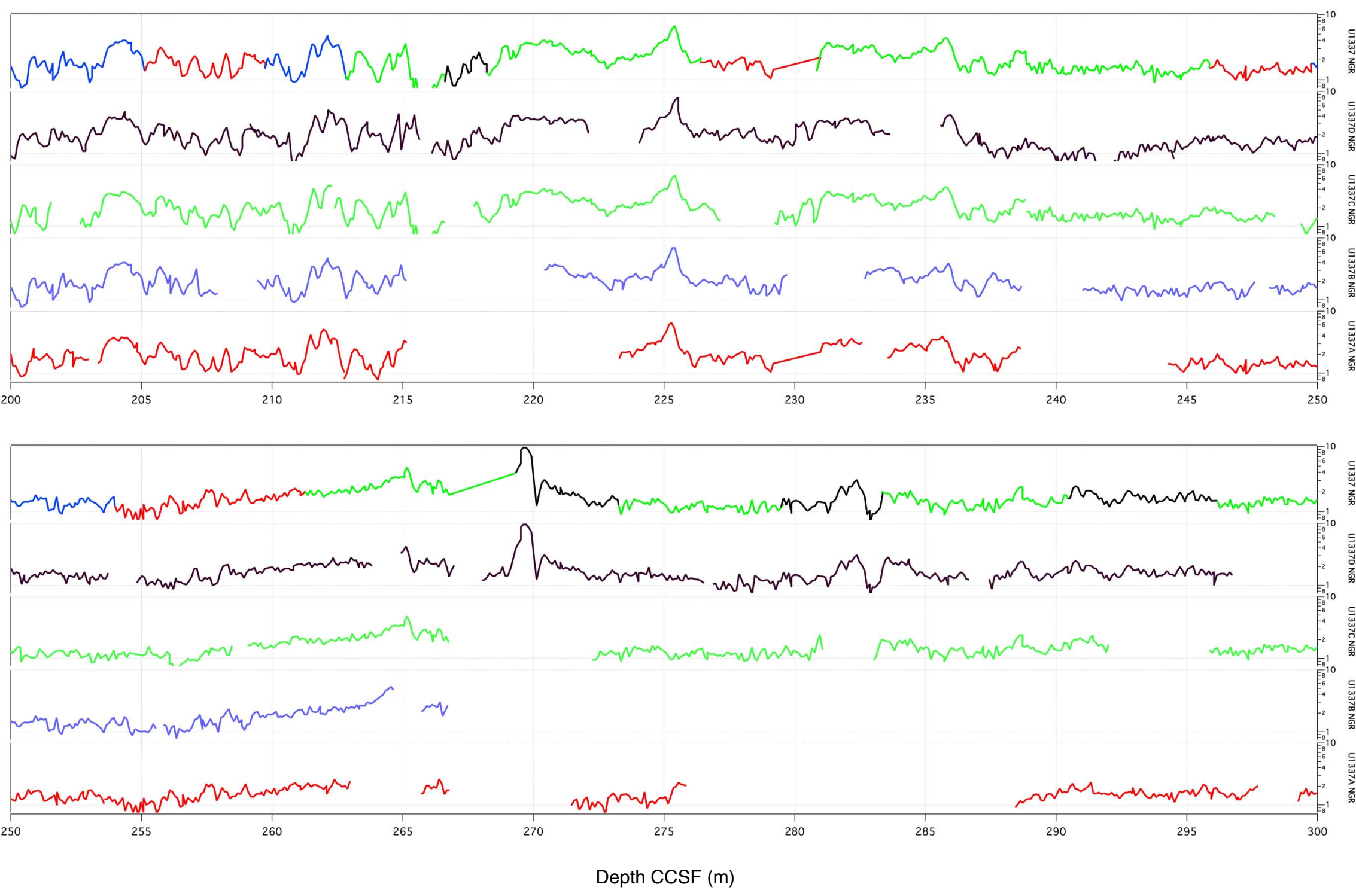
Figure F4 (continued). (Continued on next page.)
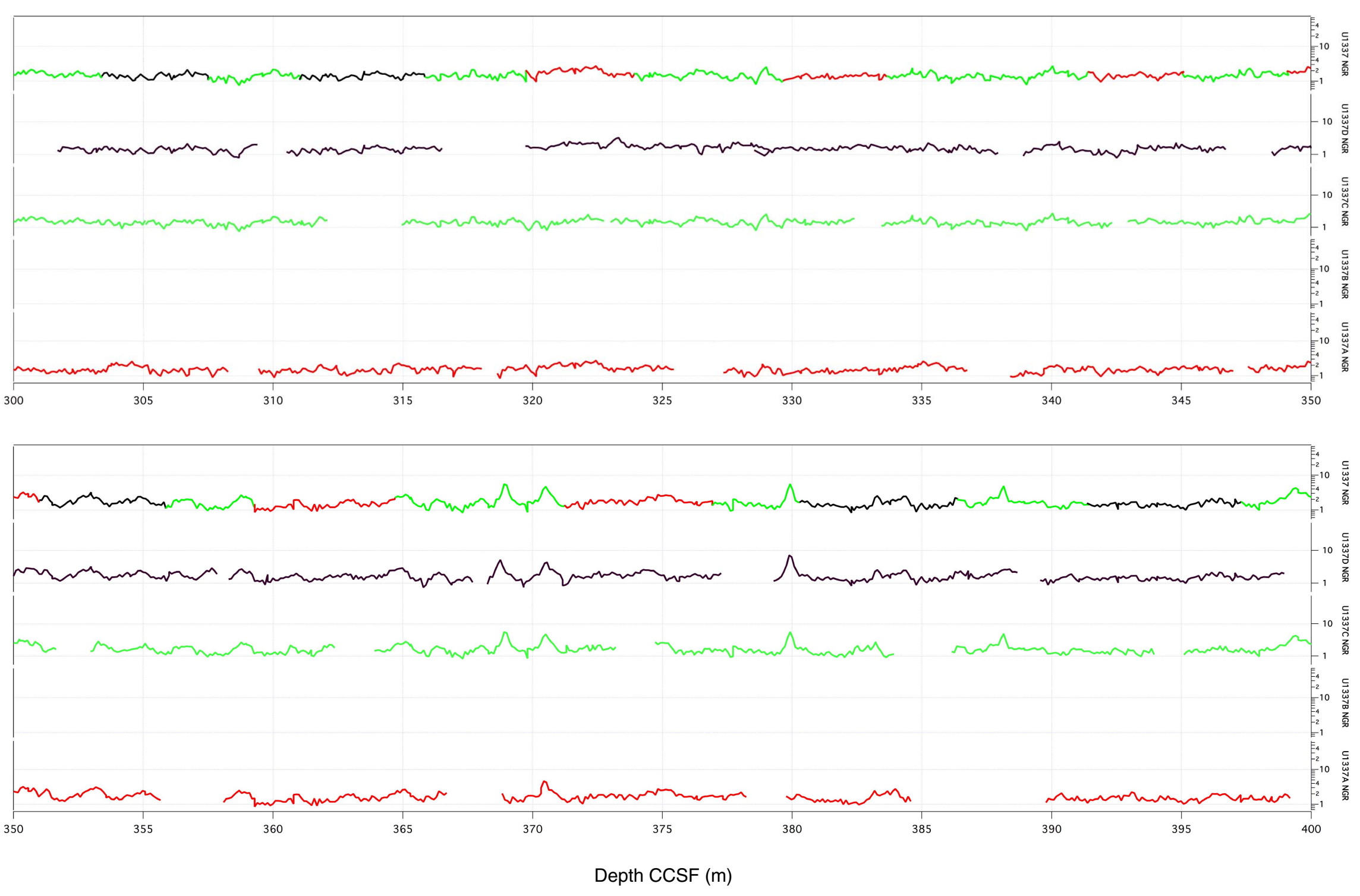
Figure F4 (continued). (Continued on next page.)
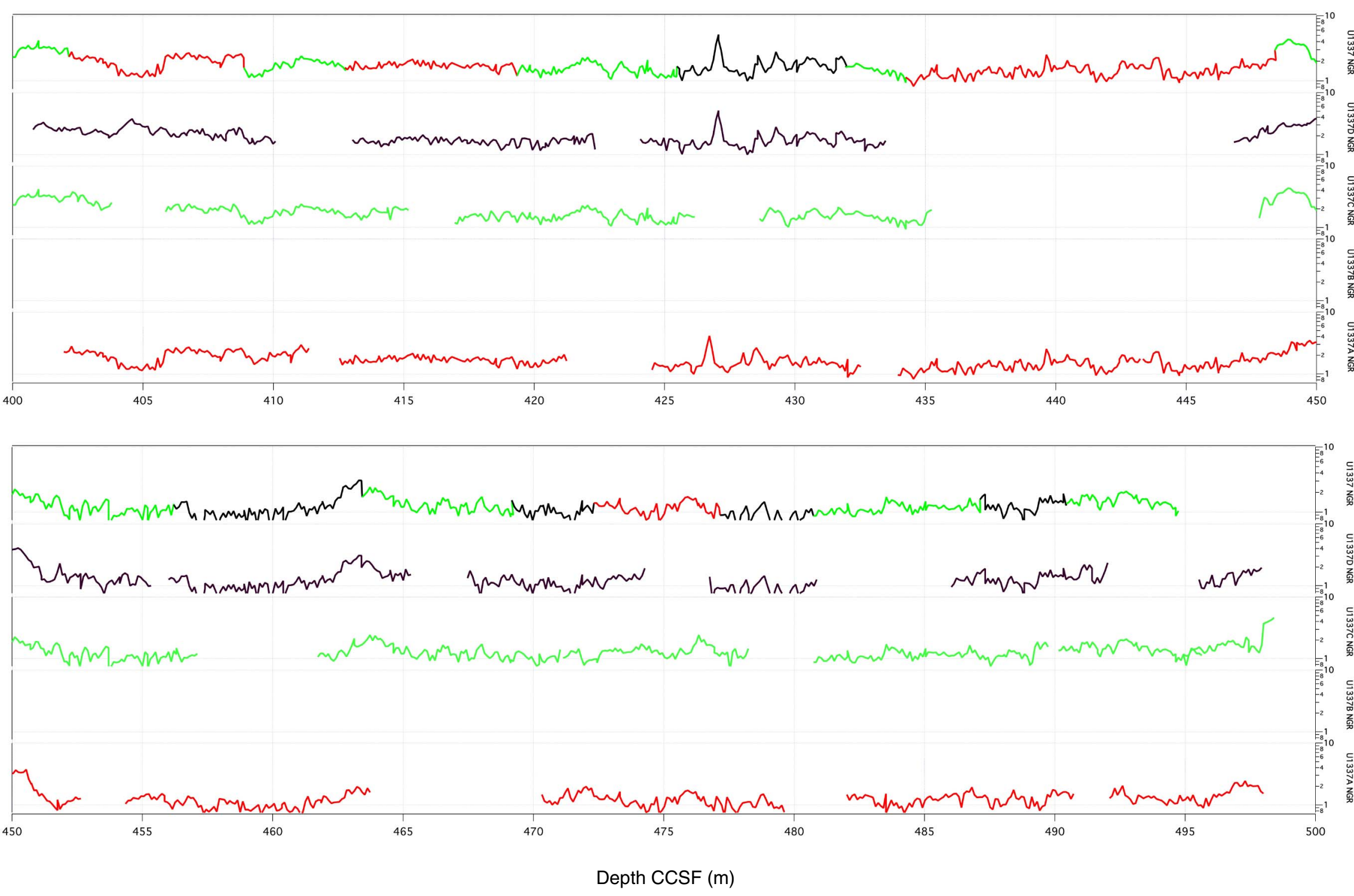


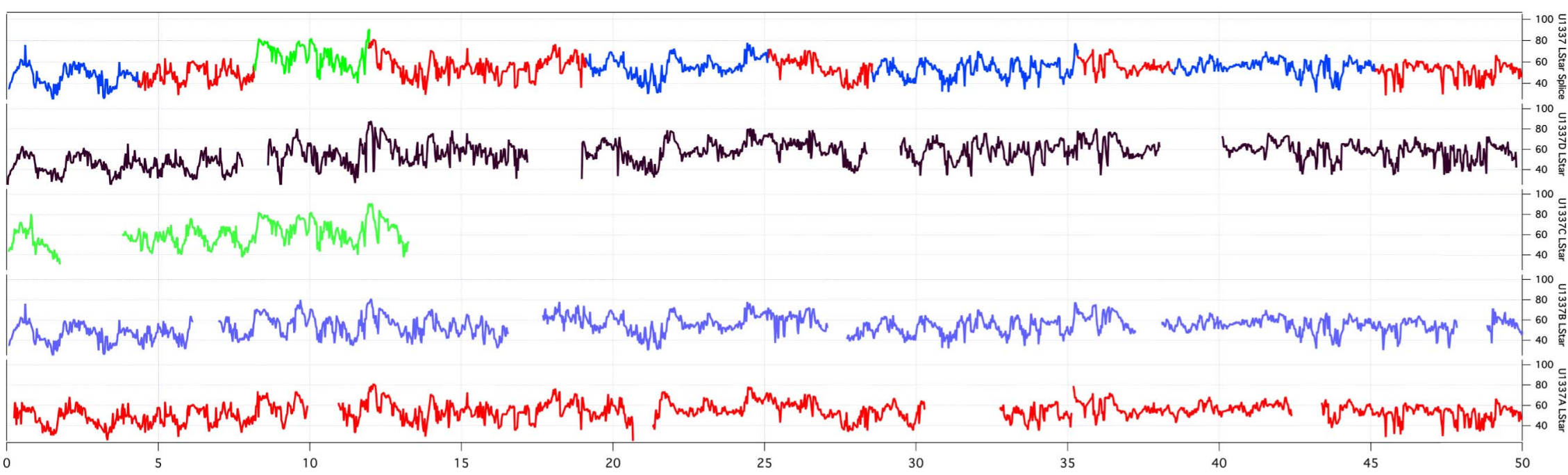

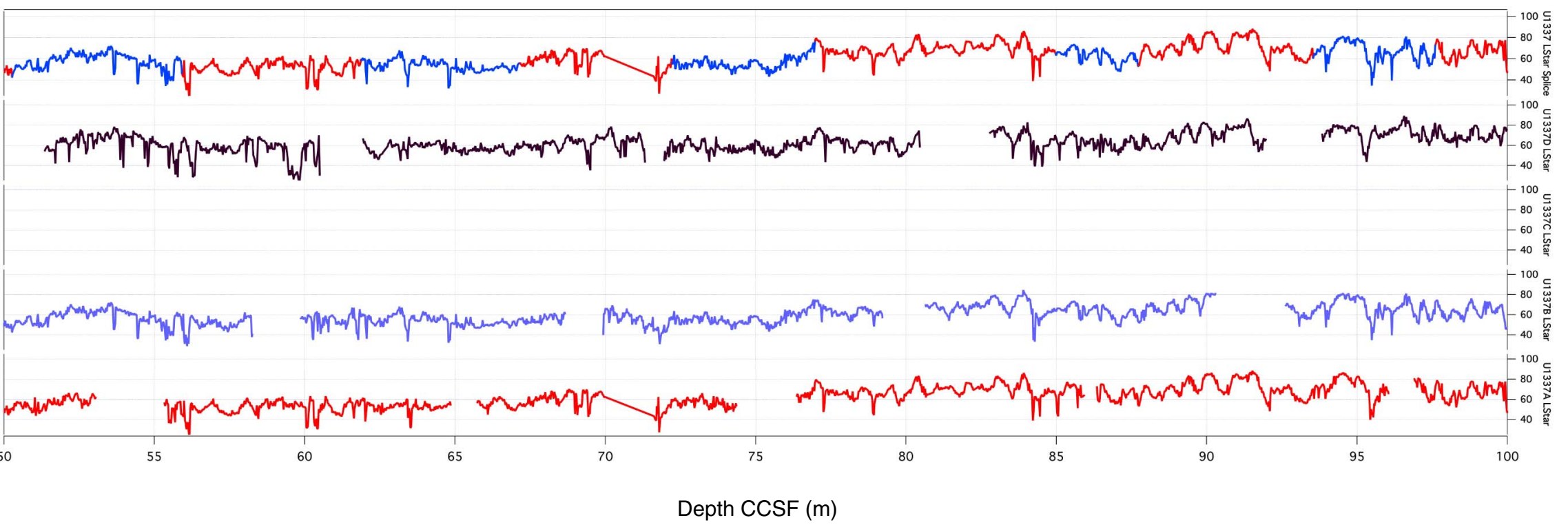




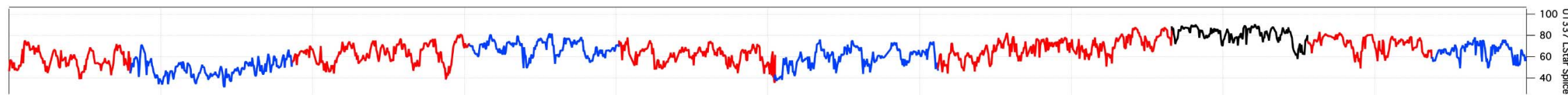

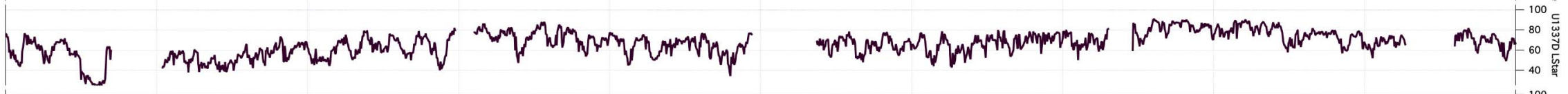

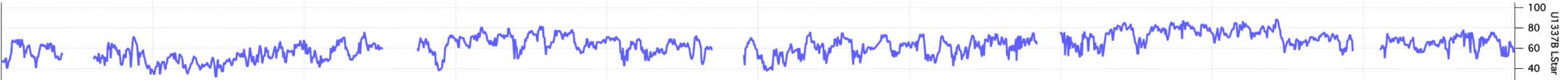

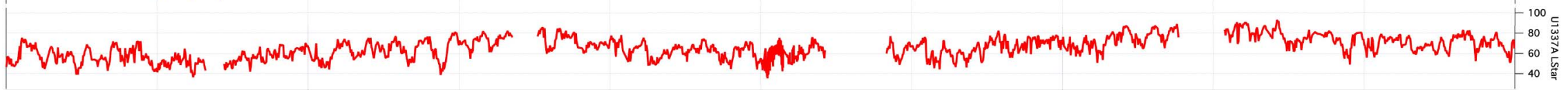
100
110
120
130

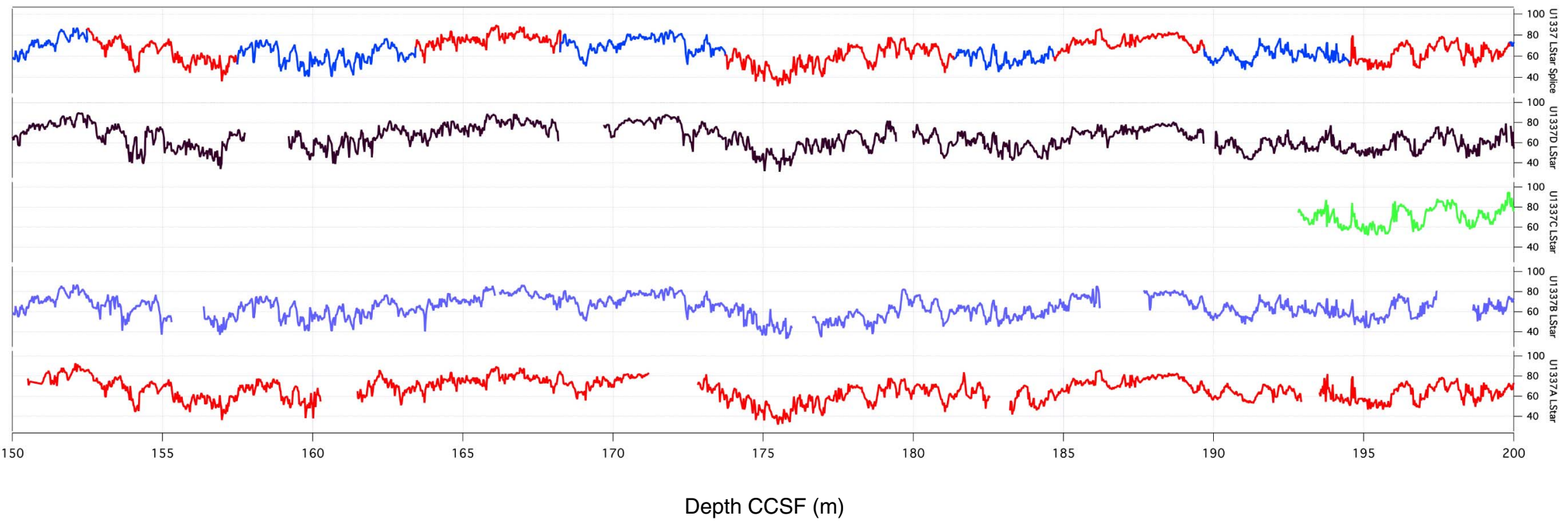


Figure F4 (continued). (Continued on next page.)
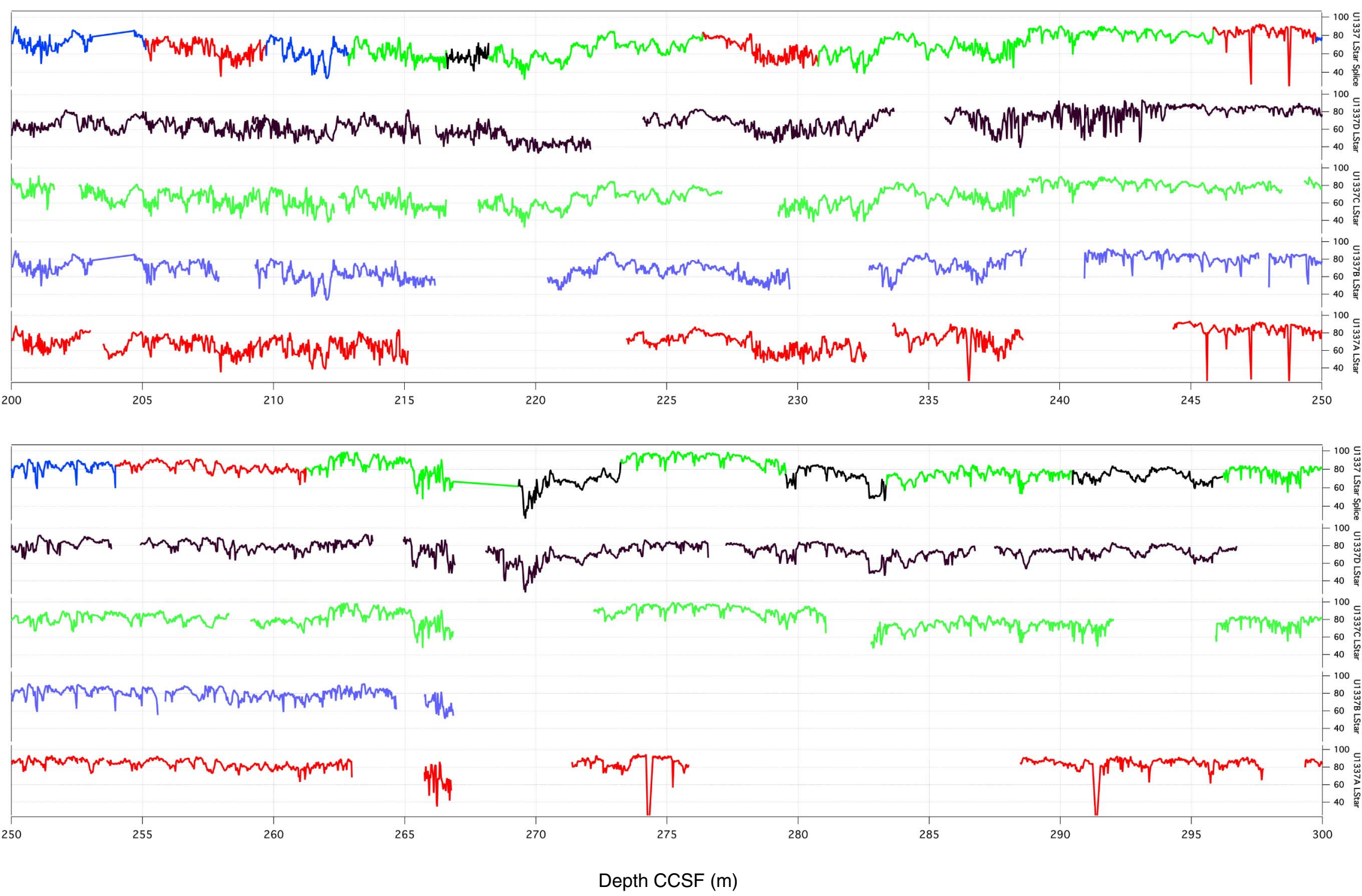
Figure F4 (continued). (Continued on next page.)
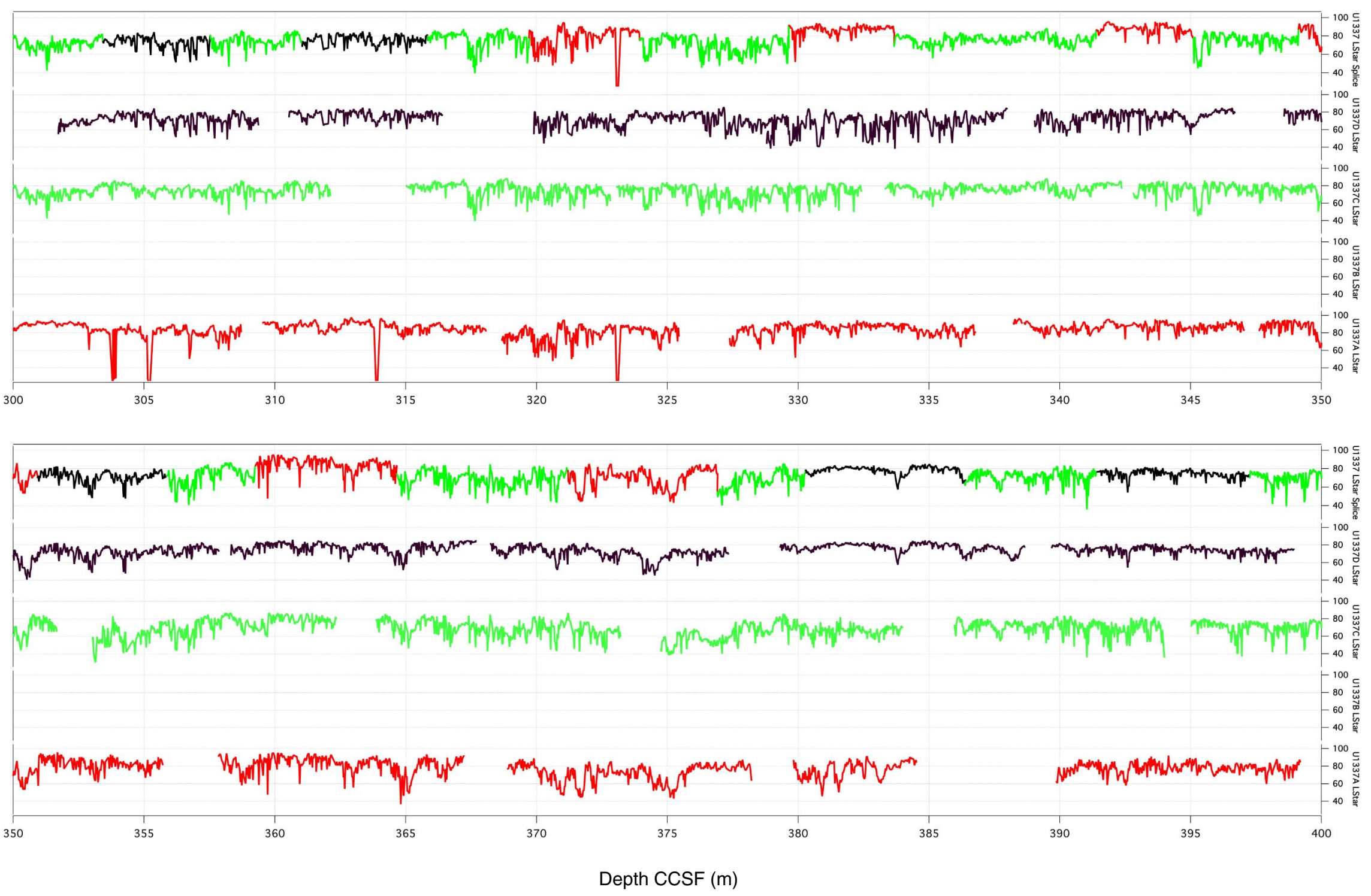
Figure F4 (continued). (Continued on next page.)

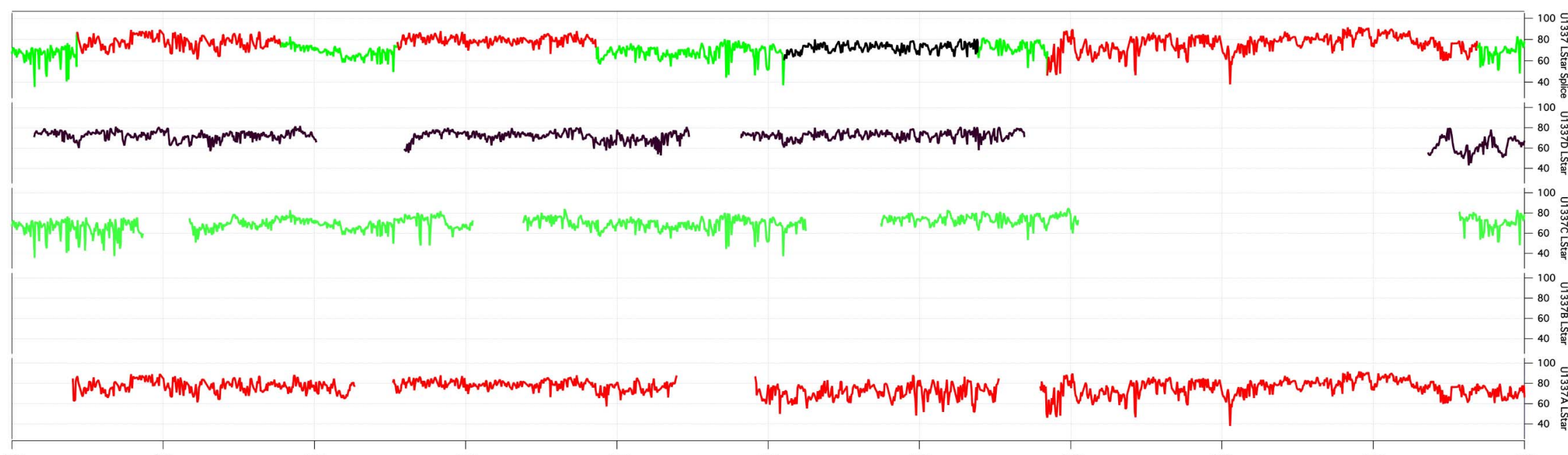

400

405

410

420

425

430

435

445

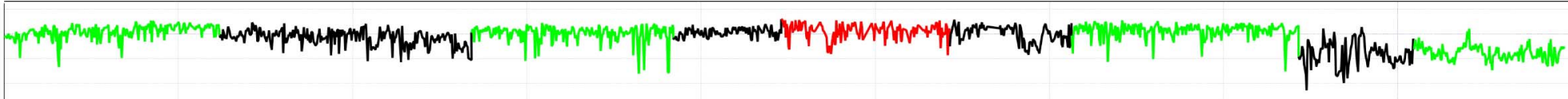

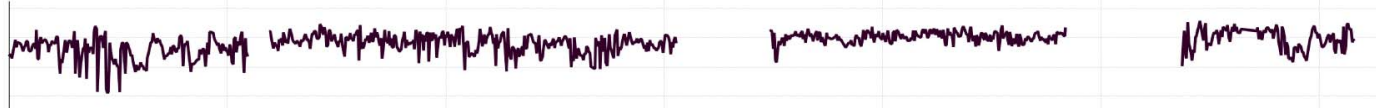

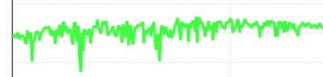

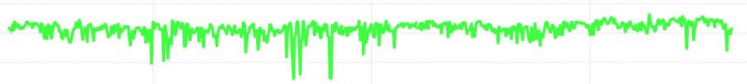

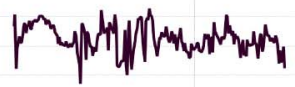

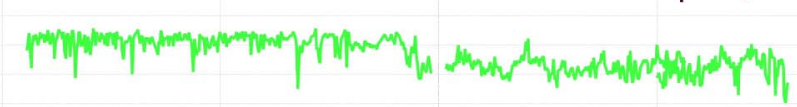

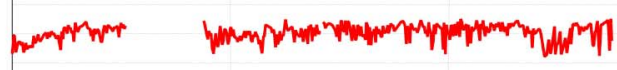

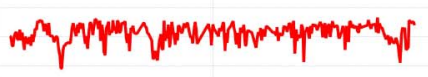

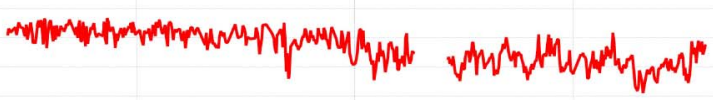

450

455

460

465

470

475

480

495

500

Depth CCSF (m) 


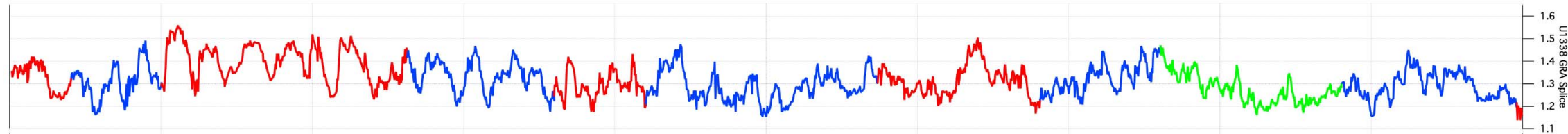

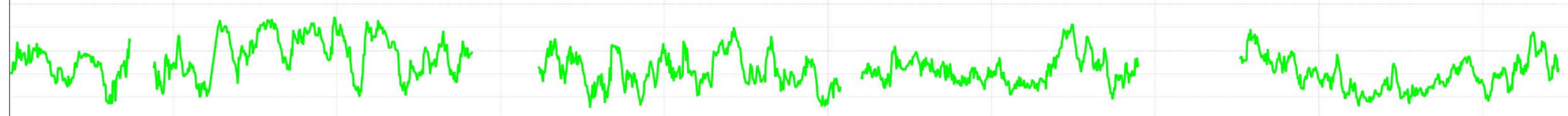

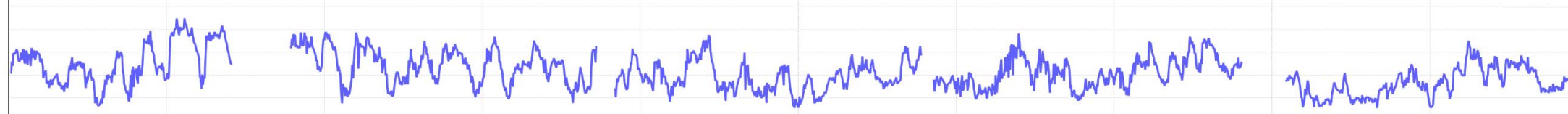

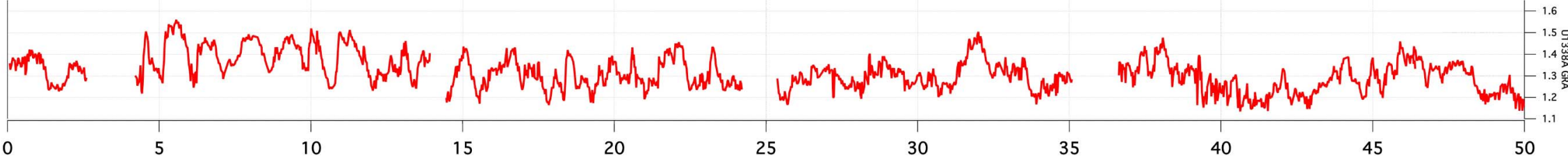

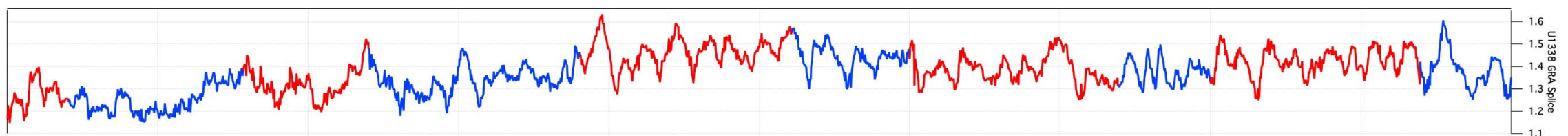

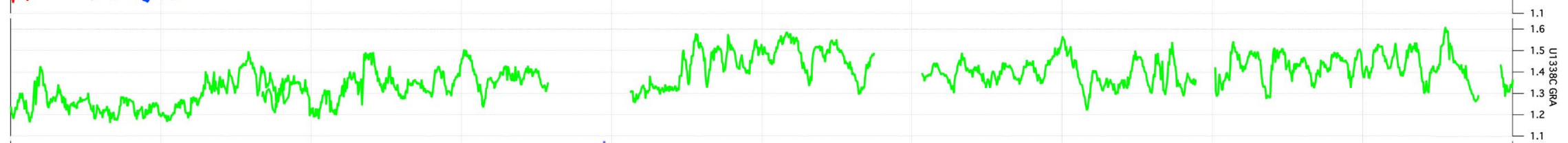

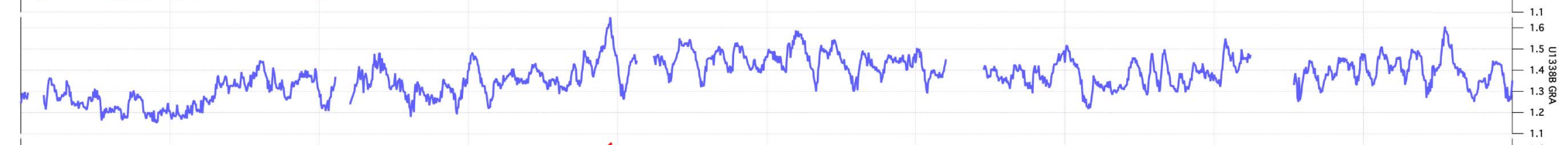

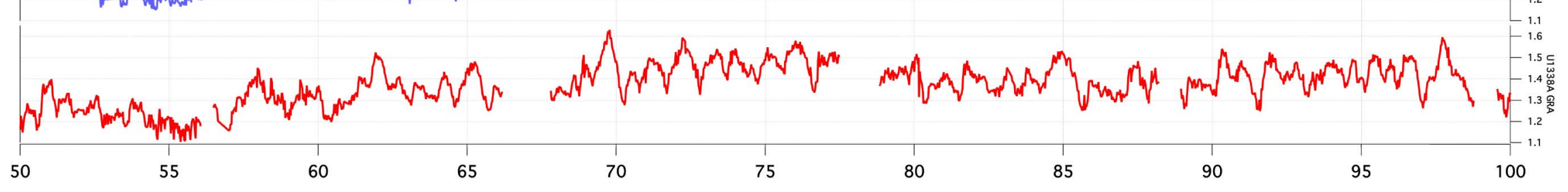



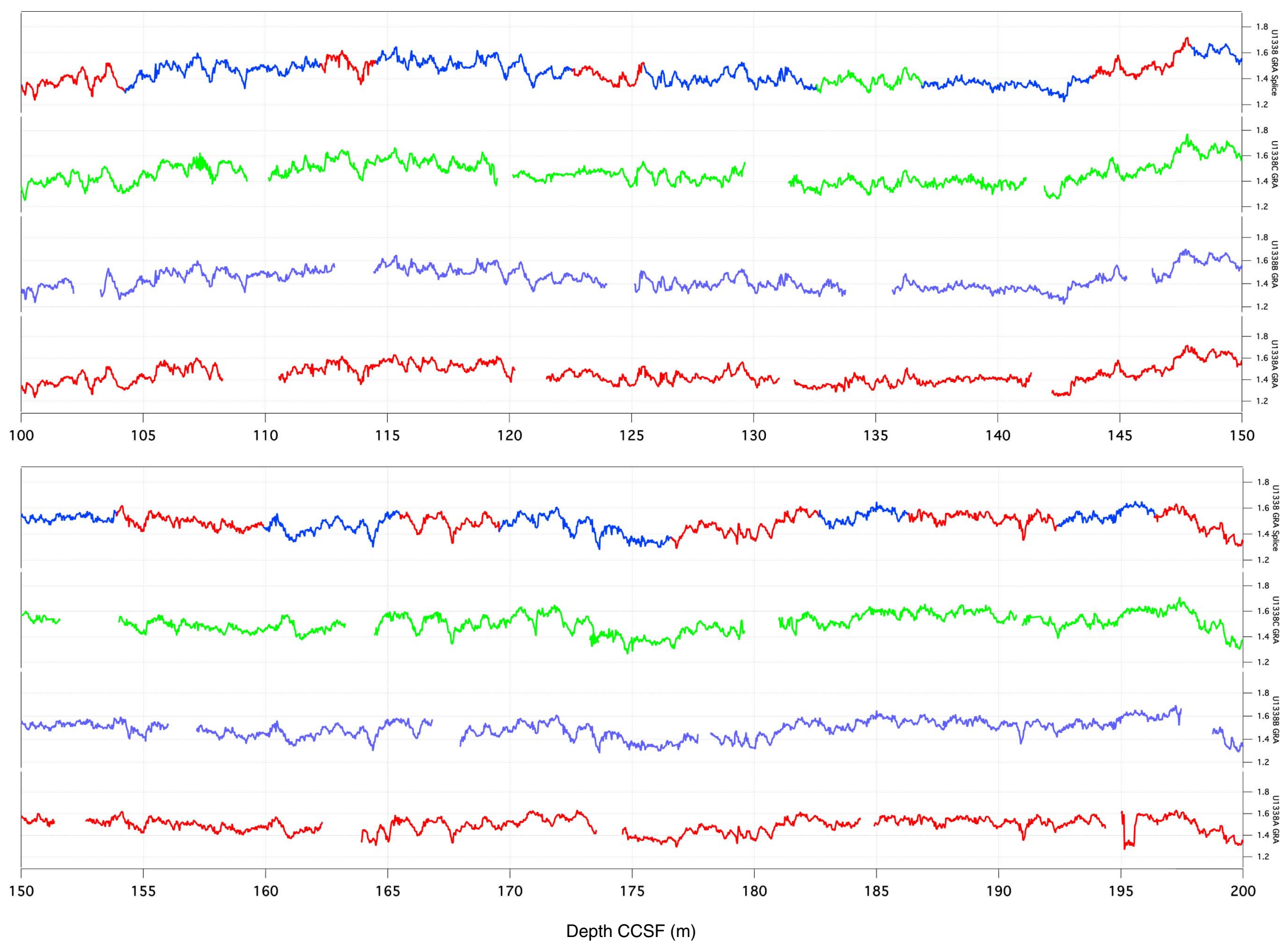


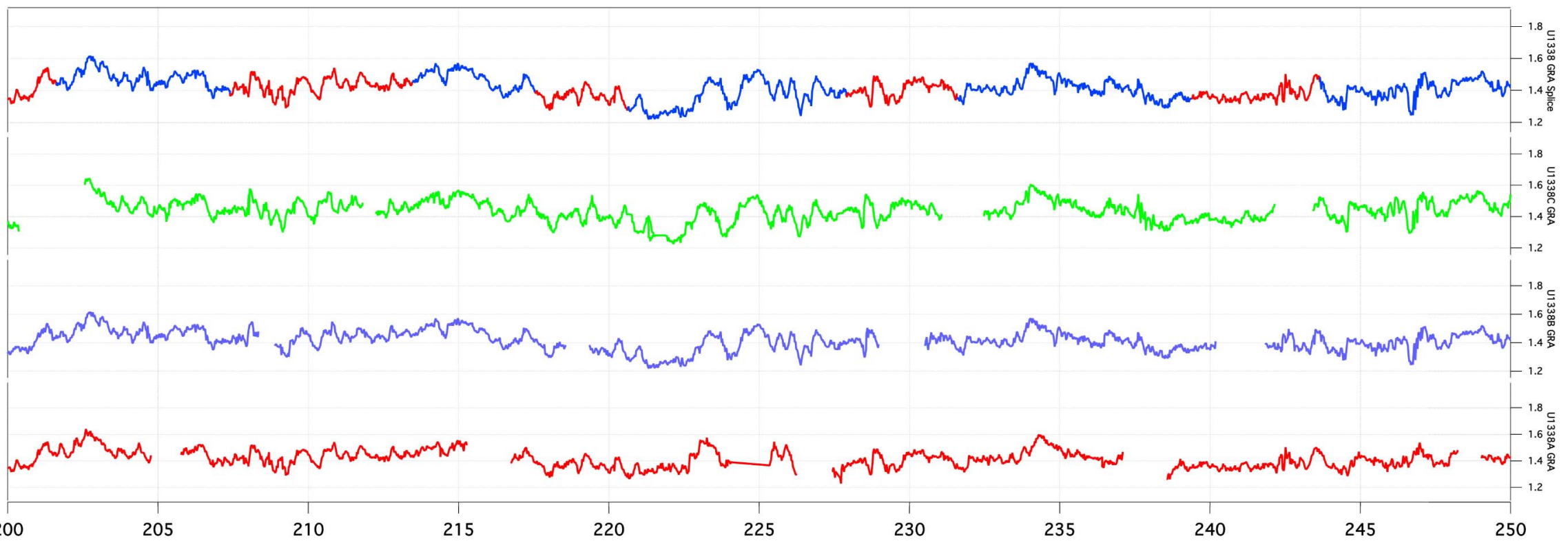

$\begin{array}{lll}28 & \\ 250 & \end{array}$


Figure F4 (continued). (Continued on next page.)

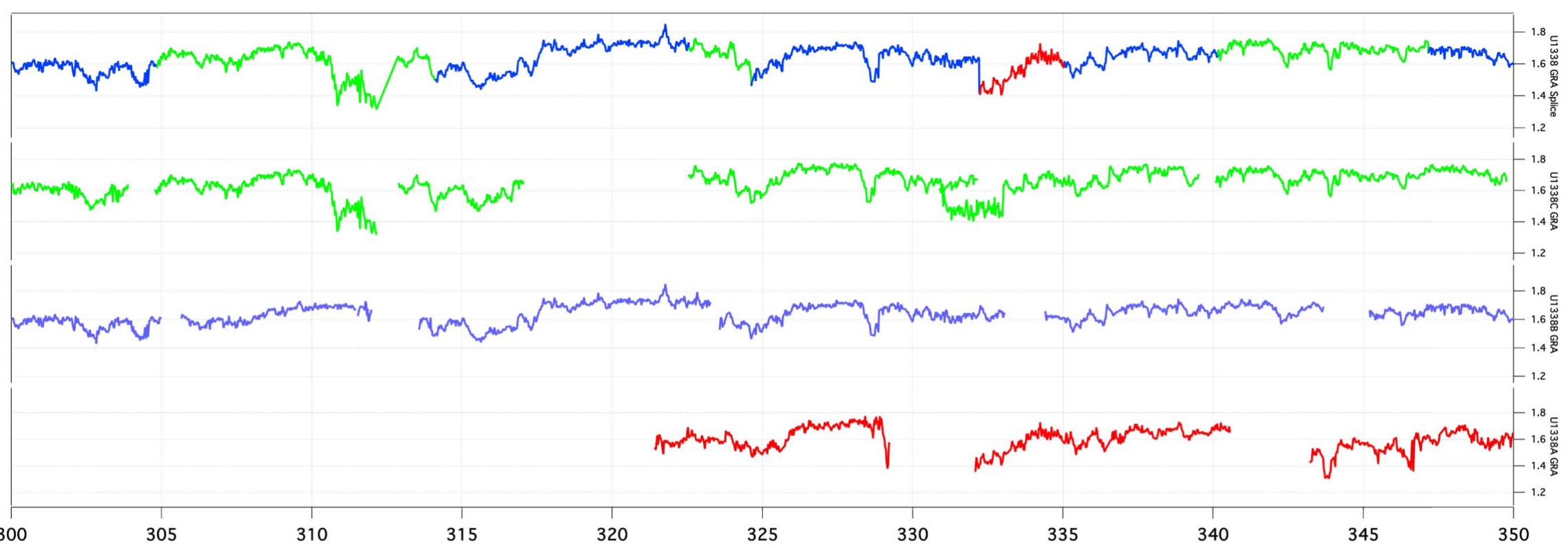

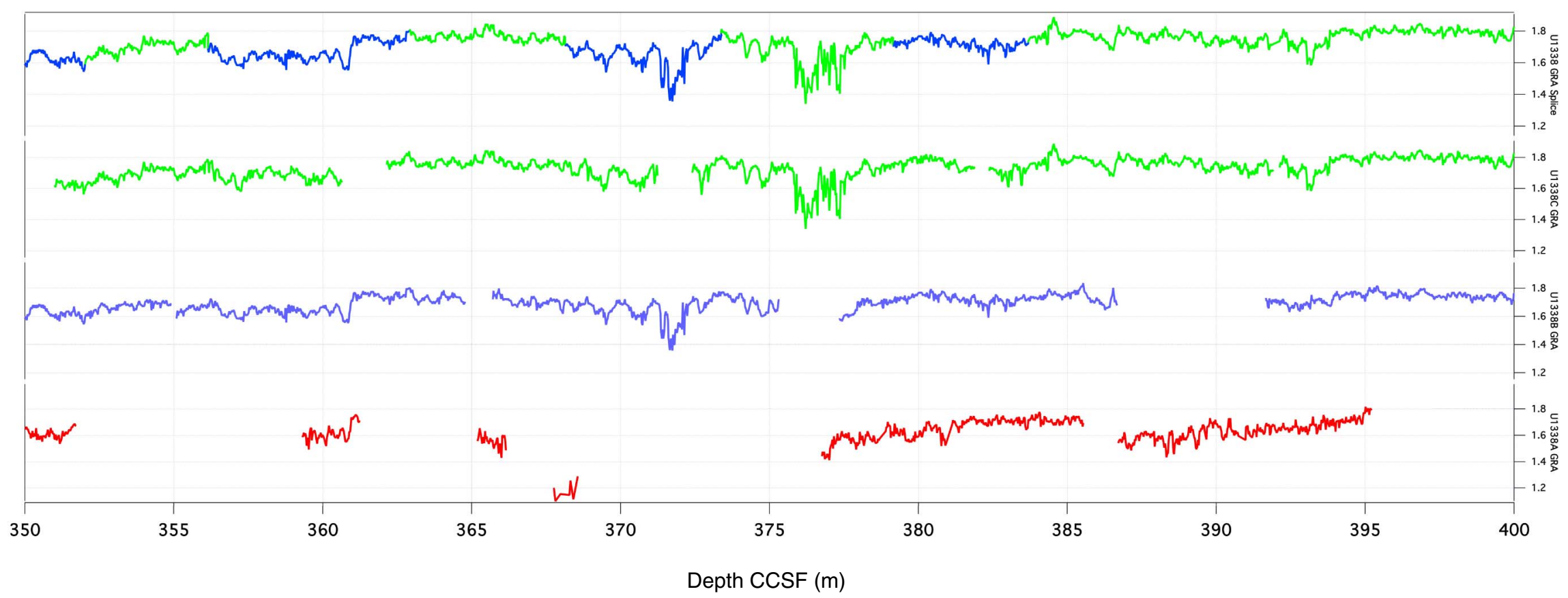




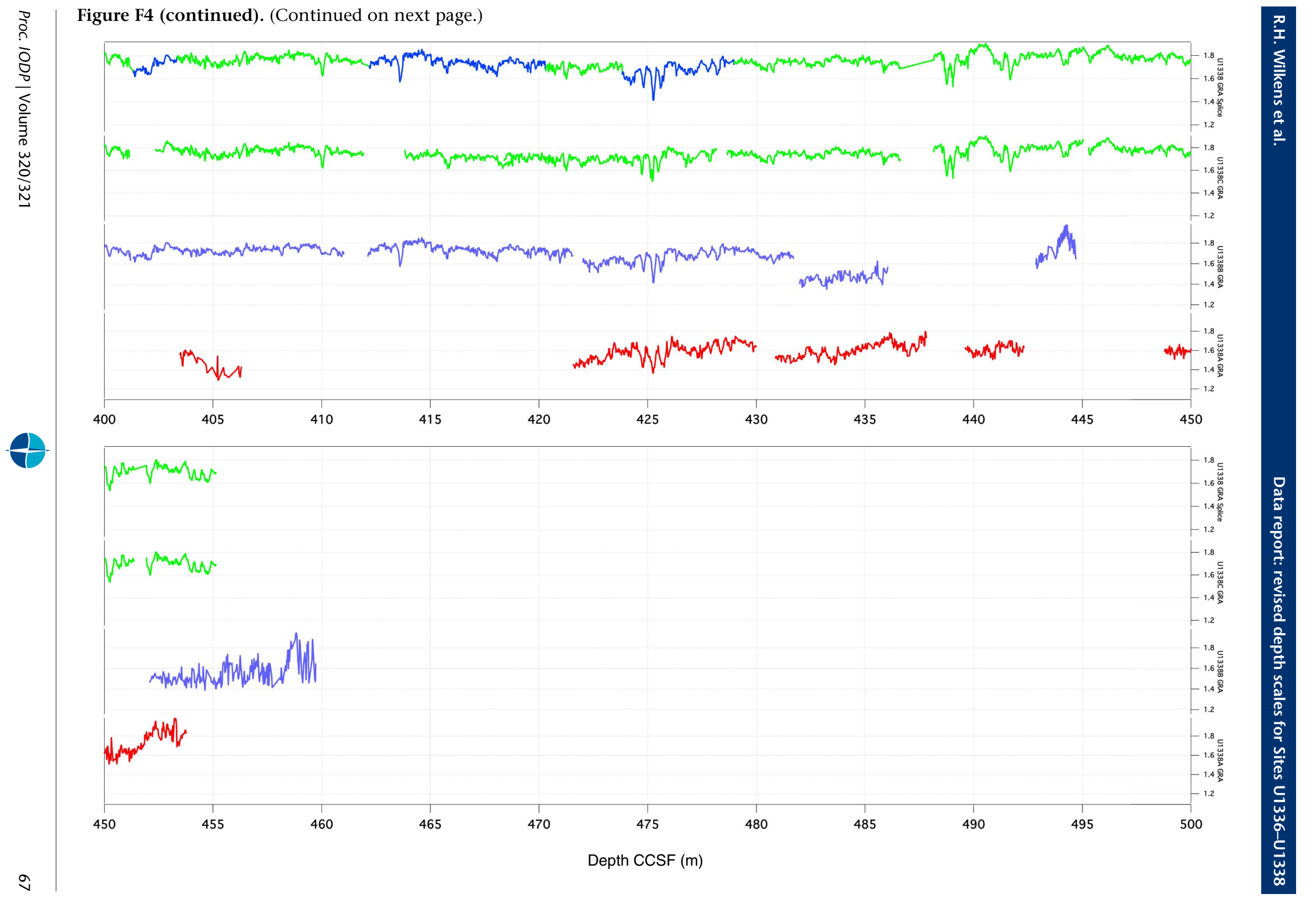



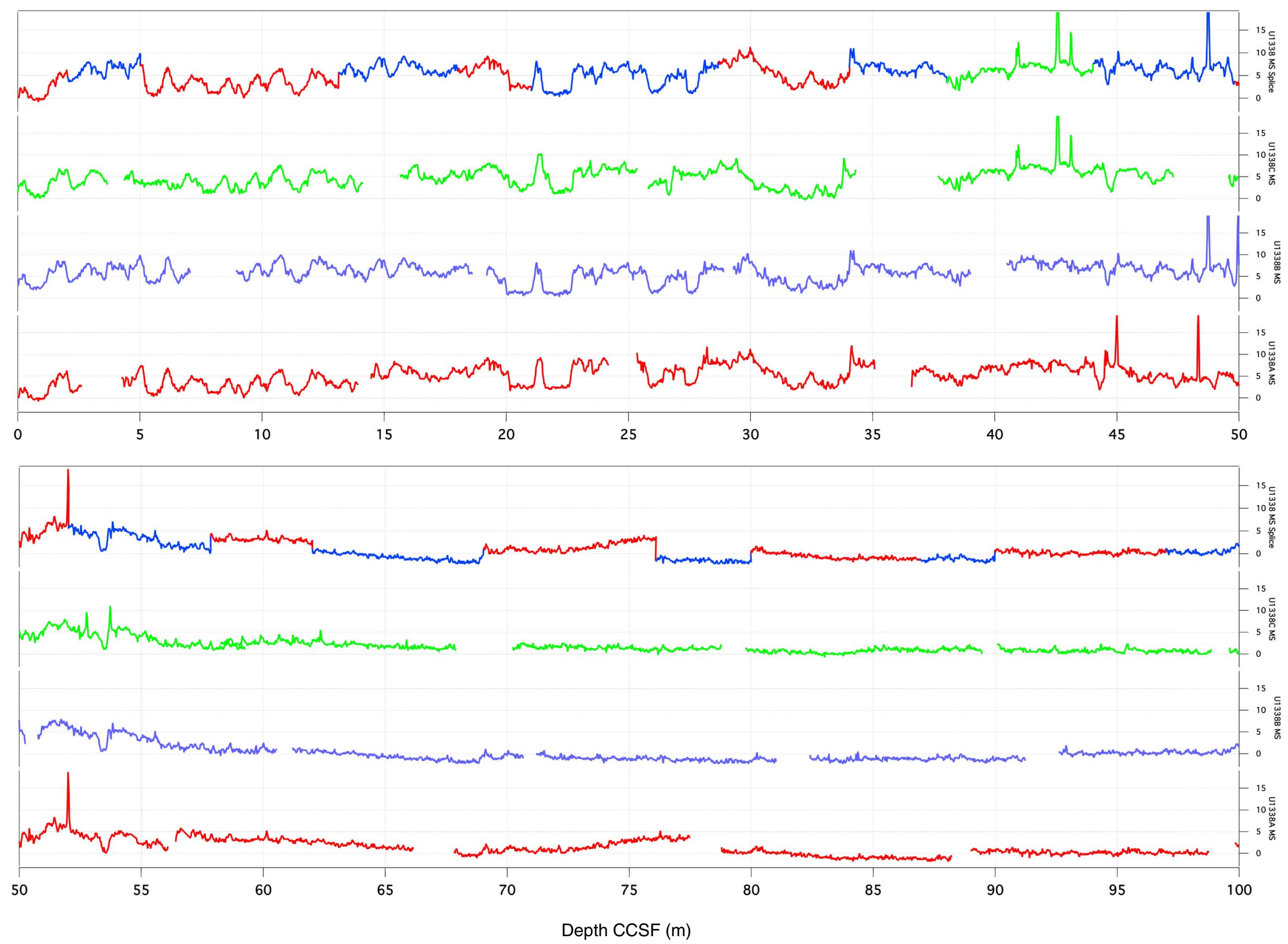
Figure F4 (continued). (Continued on next page.)

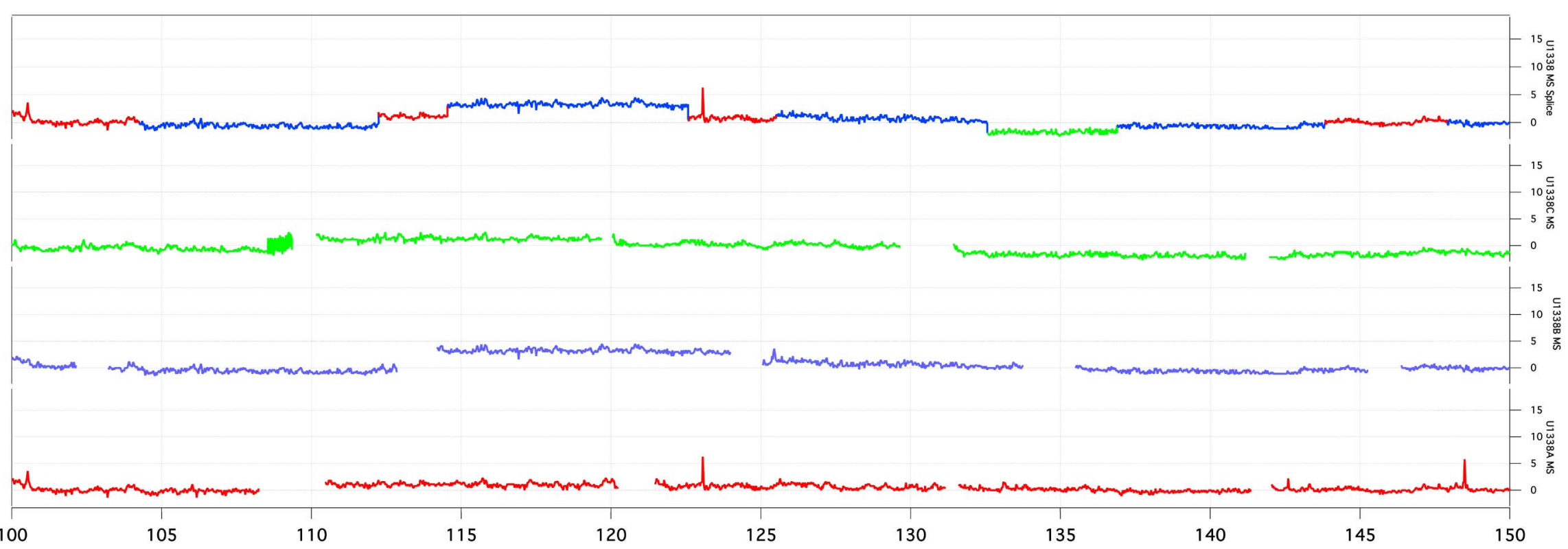

105

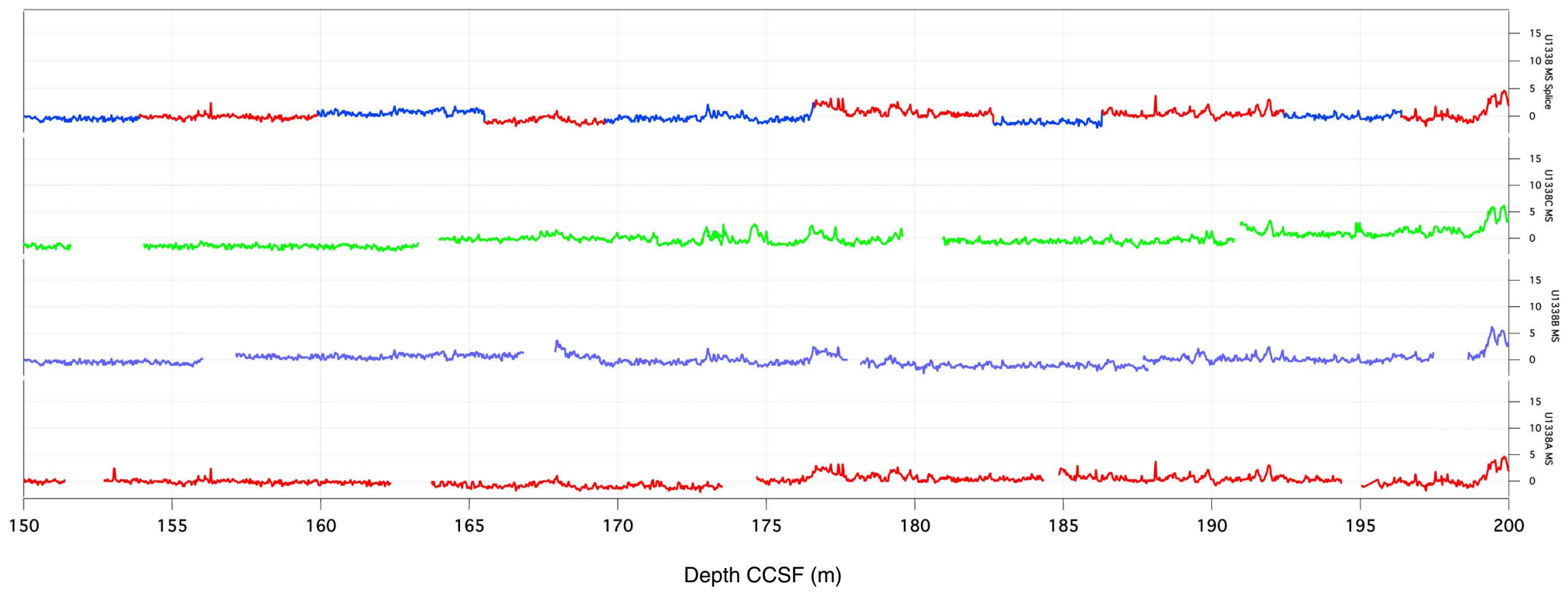


Figure F4 (continued). (Continued on next page.)
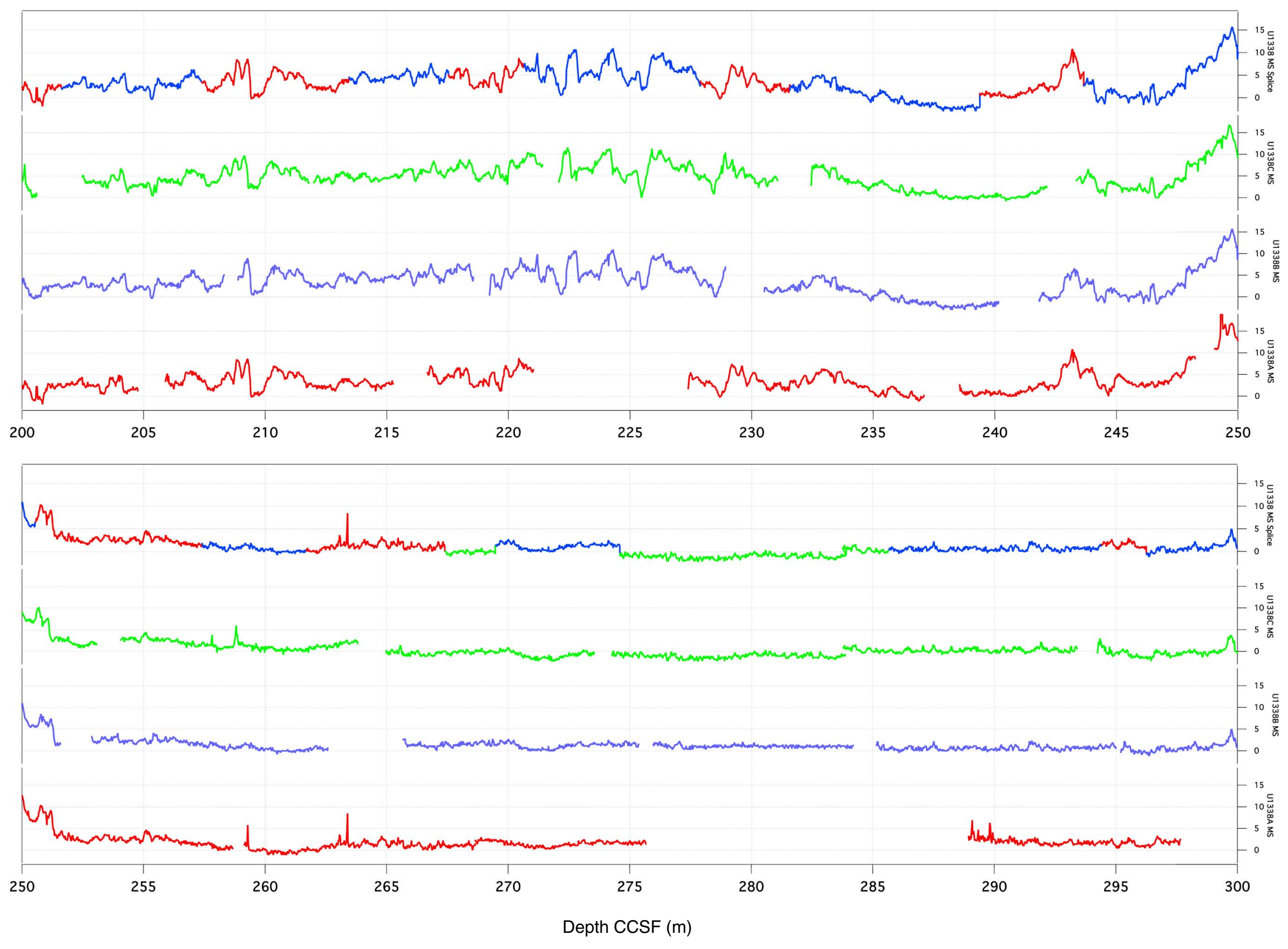
Figure F4 (continued). (Continued on next page.)

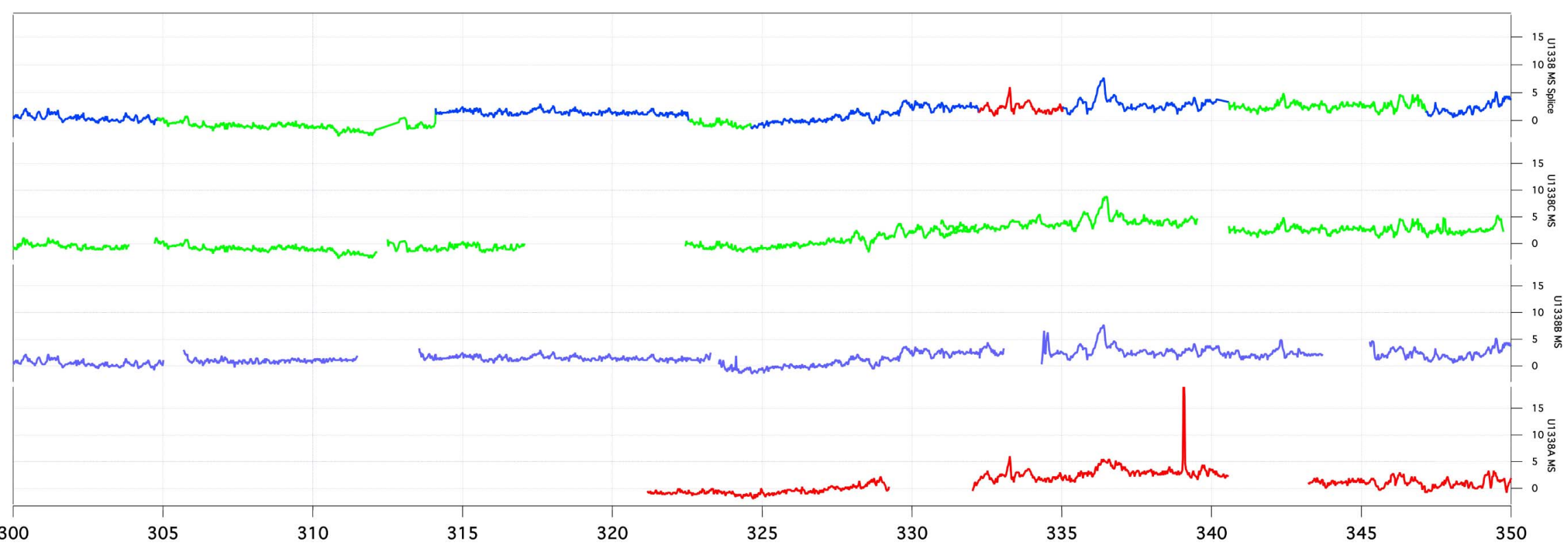

Proment

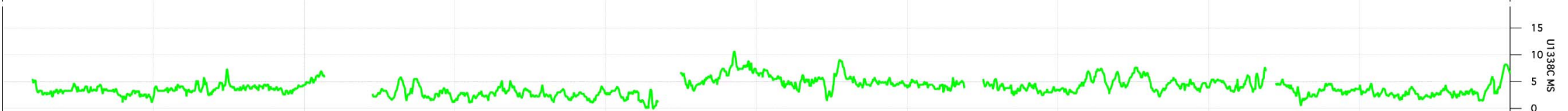

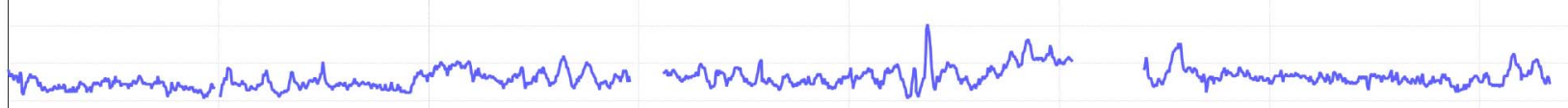

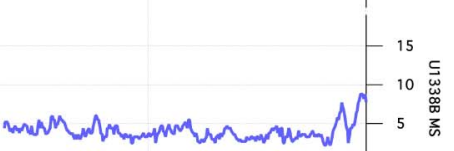

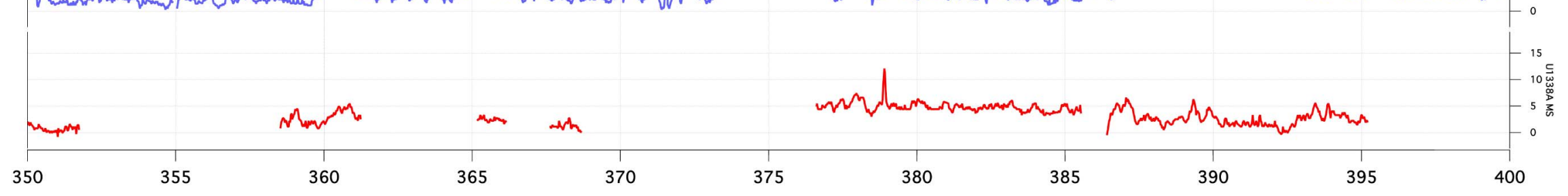


Figure F4 (continued). (Continued on next page.)
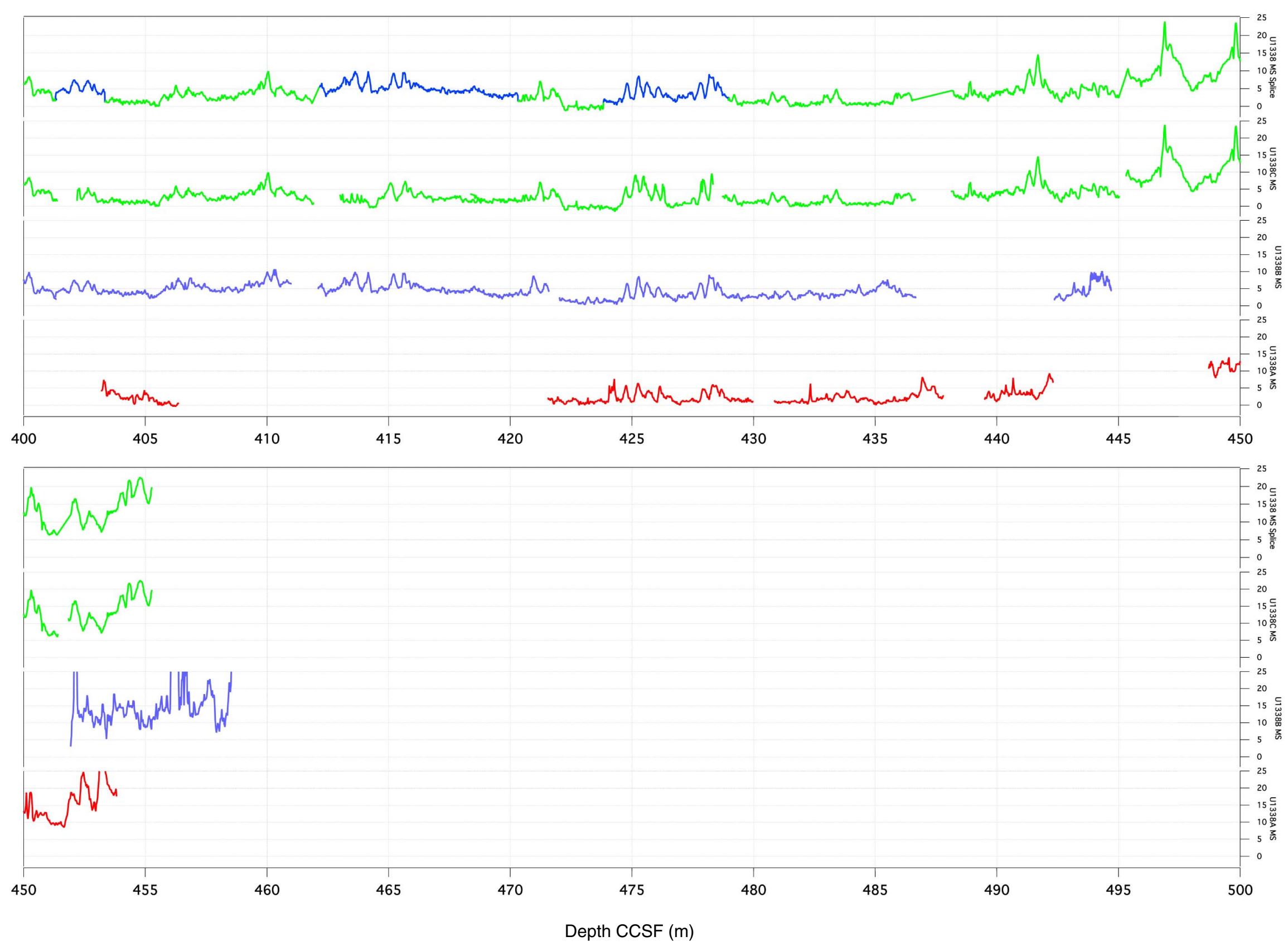
Figure F4 (continued). (Continued on next page.)

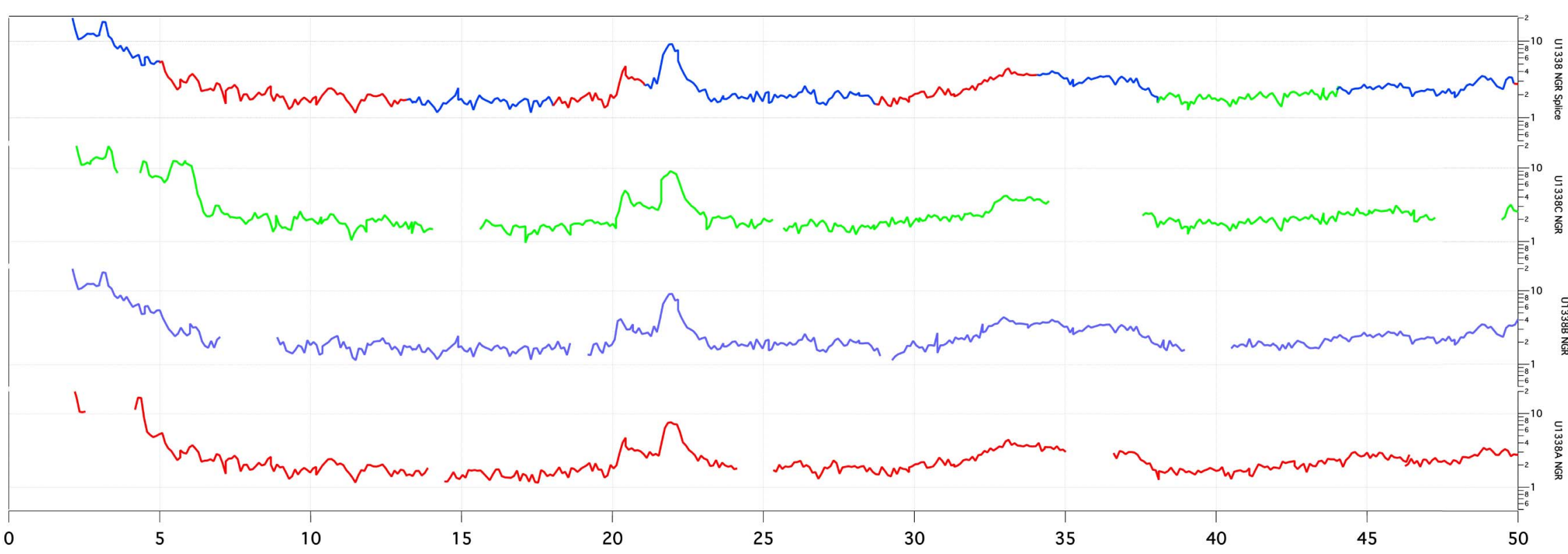

$\theta$

5
50


Figure F4 (continued). (Continued on next page.)

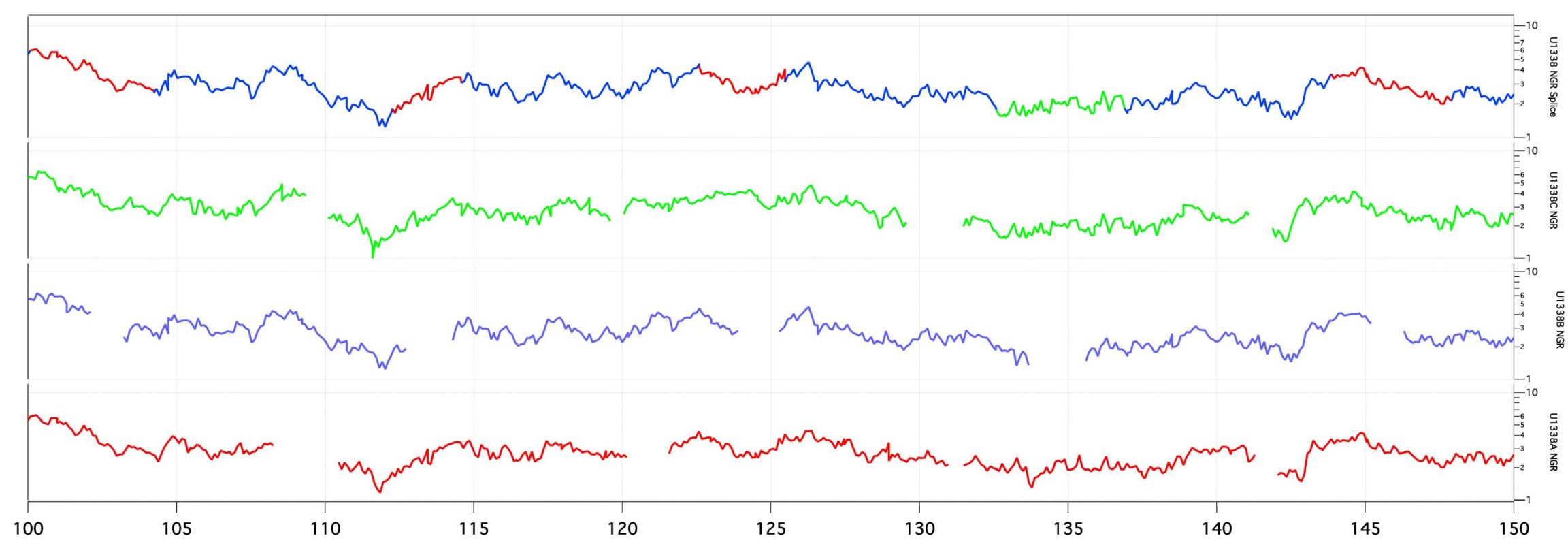

105

115

125

130

135

150

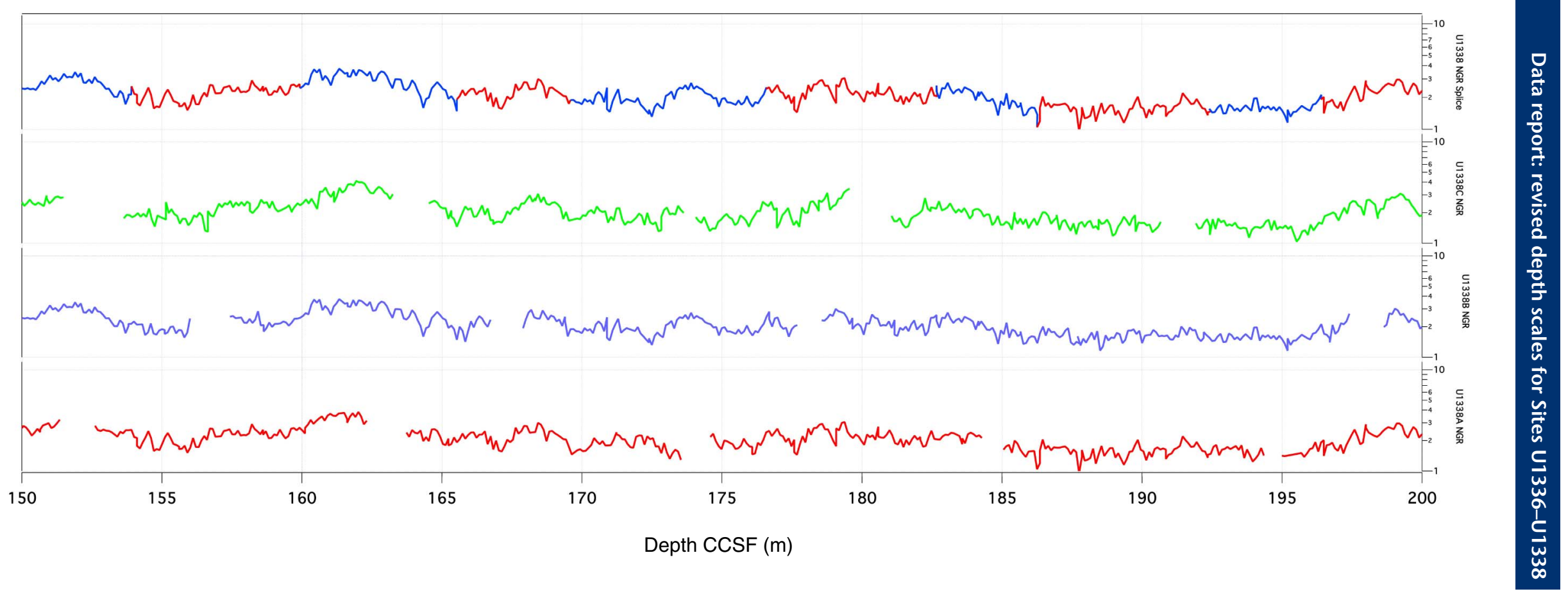



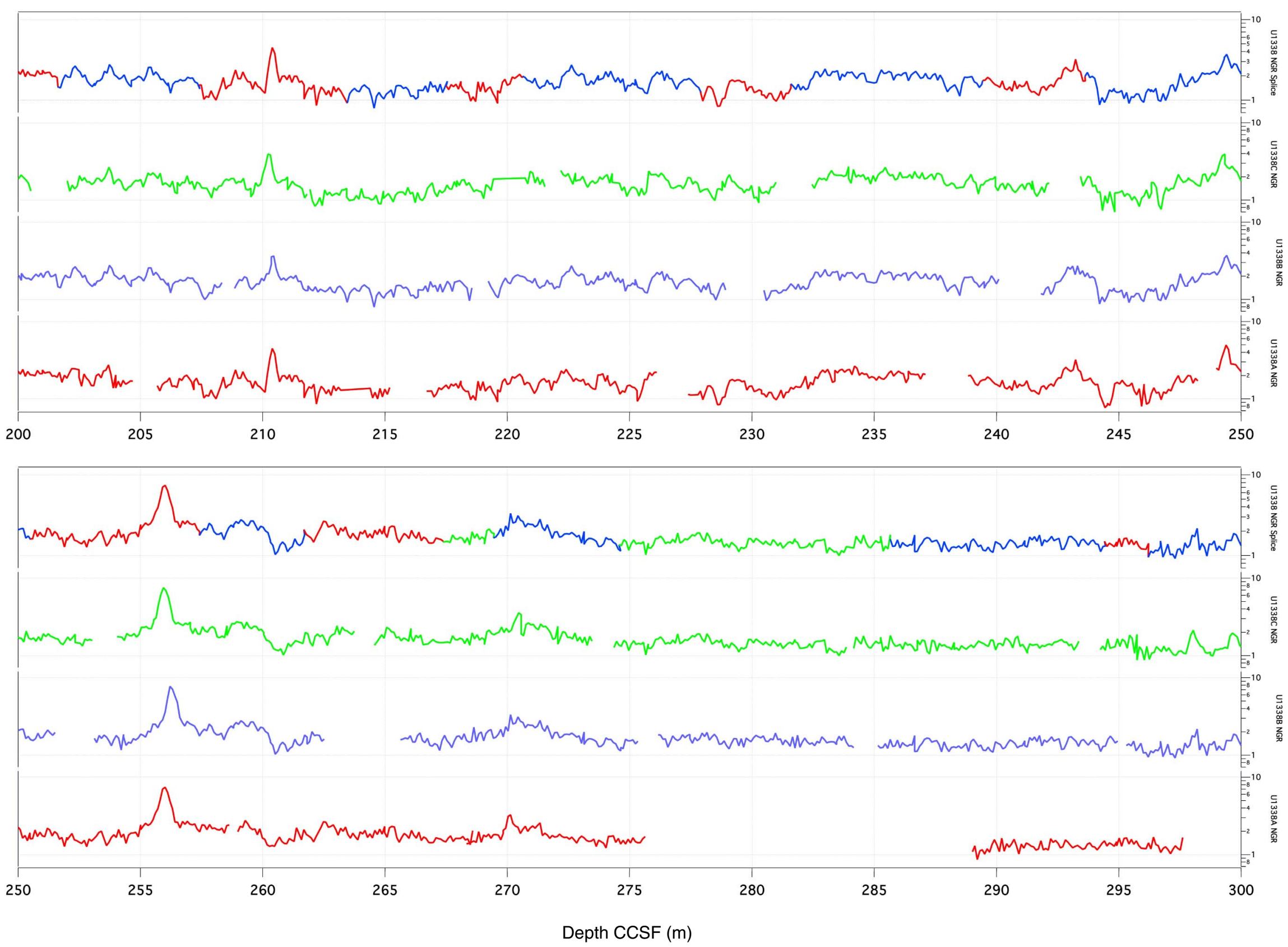
Figure F4 (continued). (Continued on next page.)

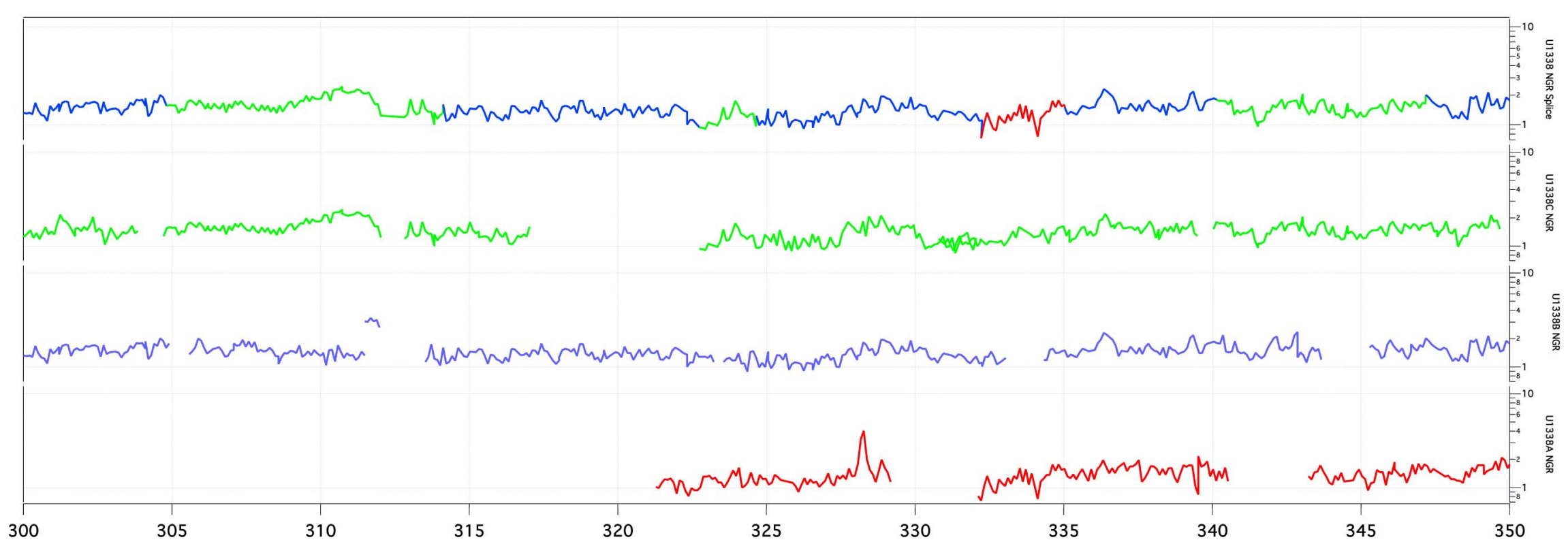

(1)




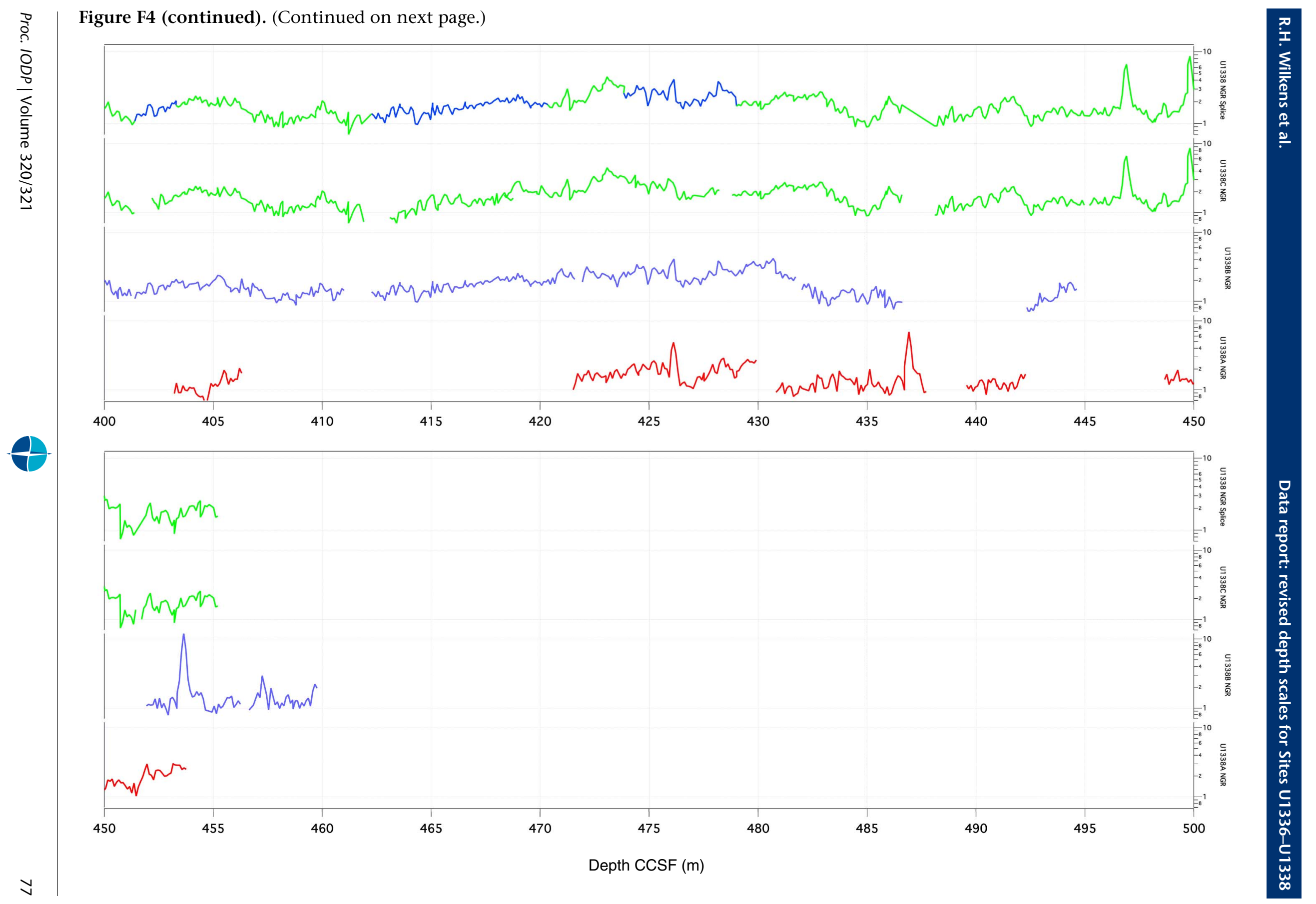




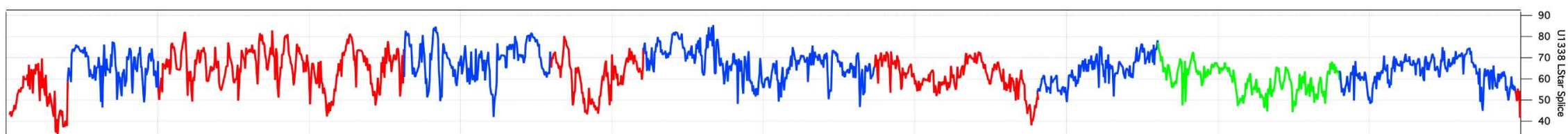

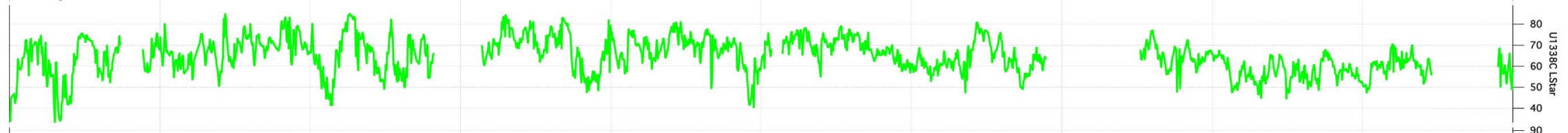

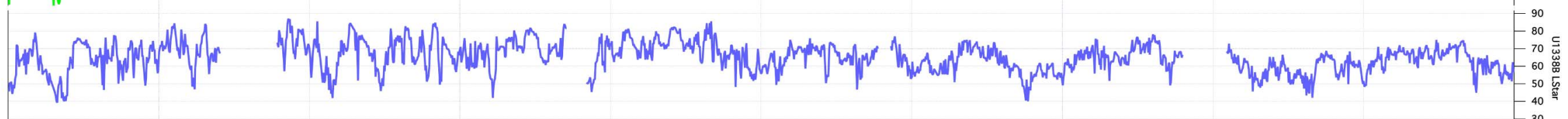

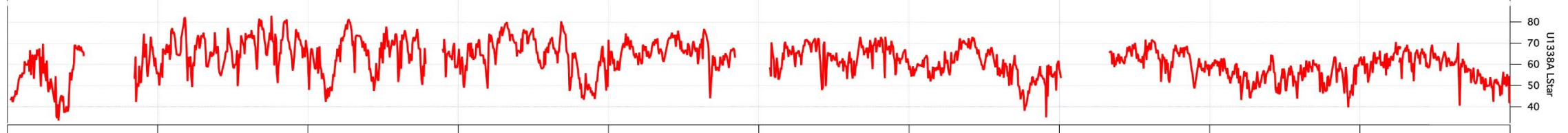

$\begin{array}{llllllllll}0 & 5 & 10 & 15 & 20 & 25 & 30 & 35 & 40 & 45\end{array}$

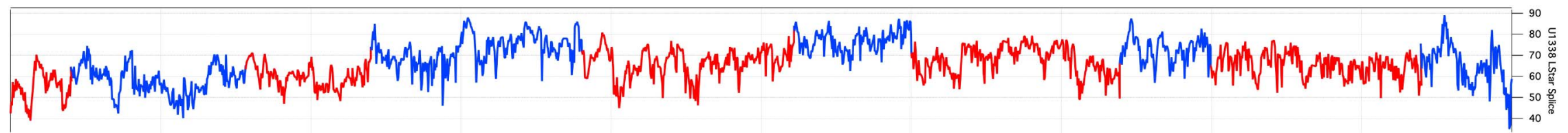

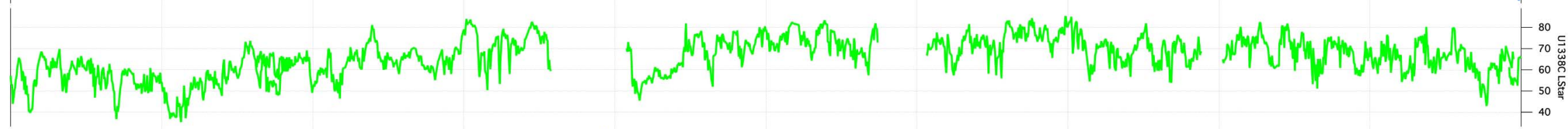

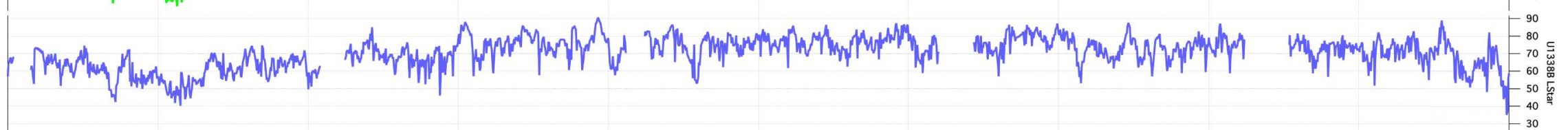

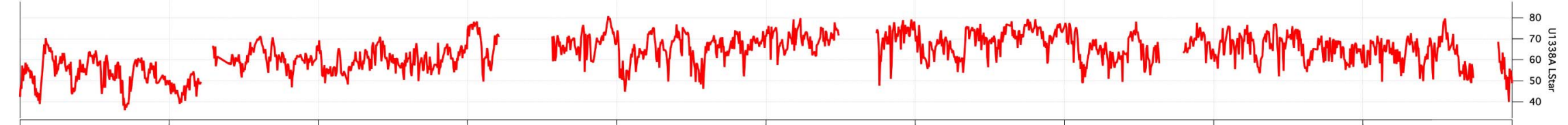

50 55

60

65 Depth CCSF (m) 


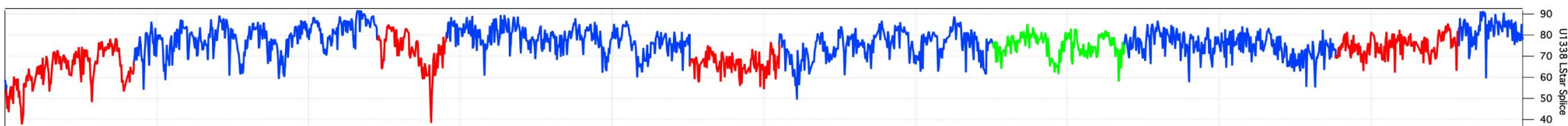

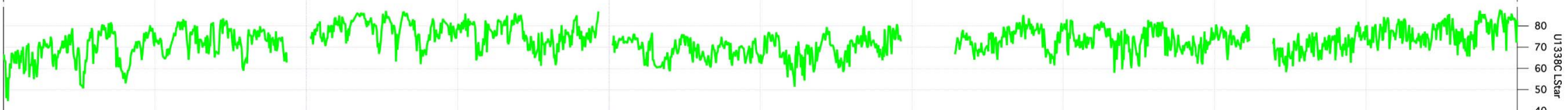

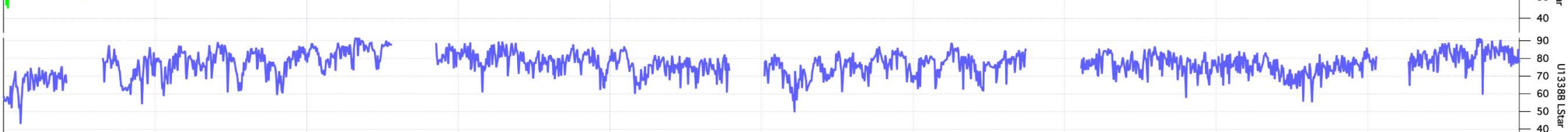

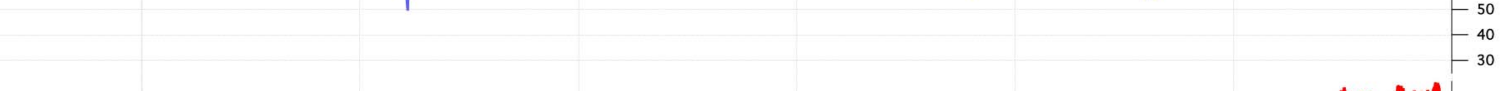

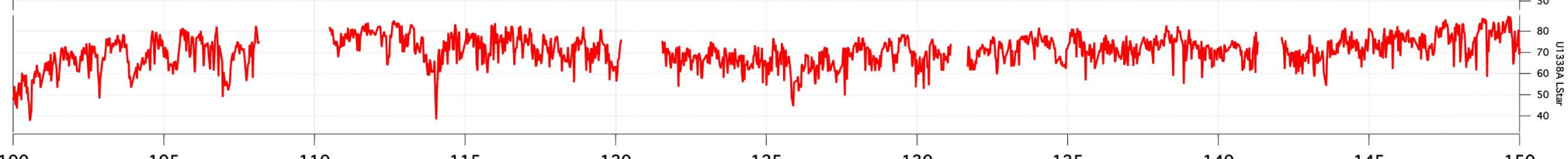

$\begin{array}{lllllllll}100 & 105 & 110 & 115 & 120 & 125 & 130 & 135 \quad 145 & 150\end{array}$

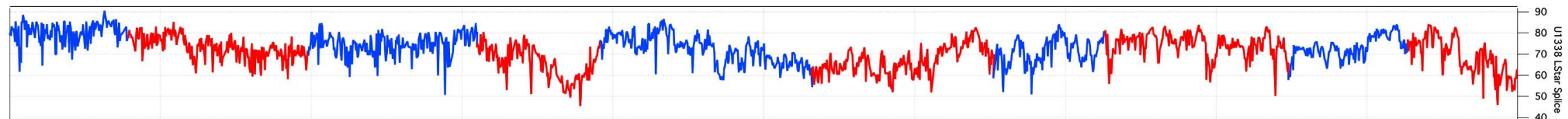

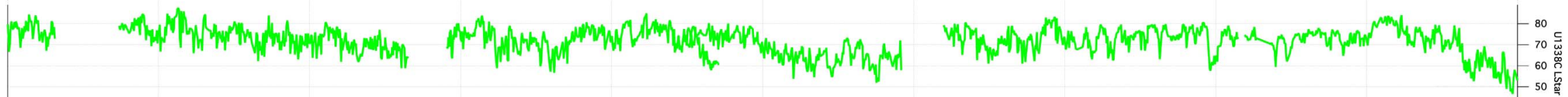

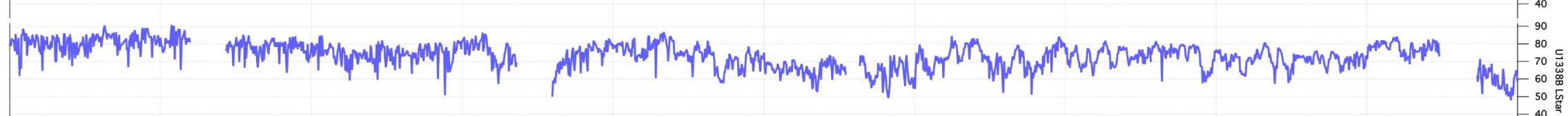

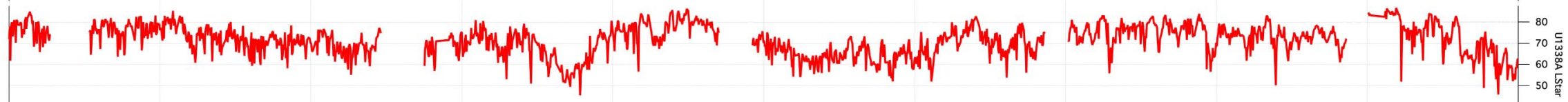

165

175

180

185

190

195

200 


W

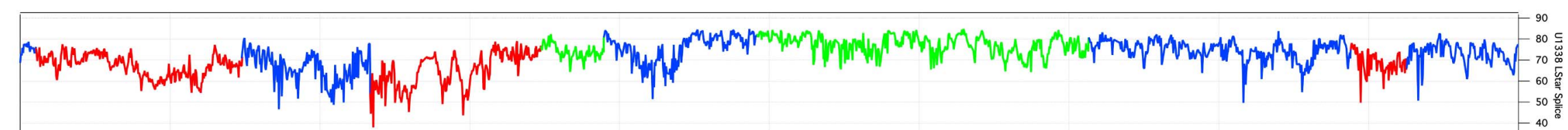

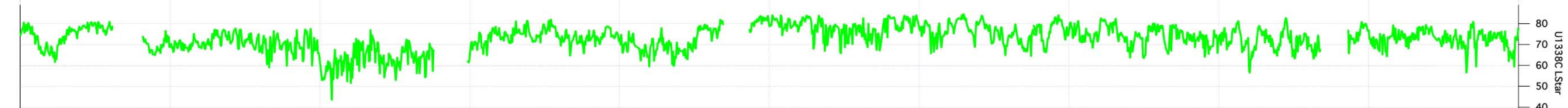

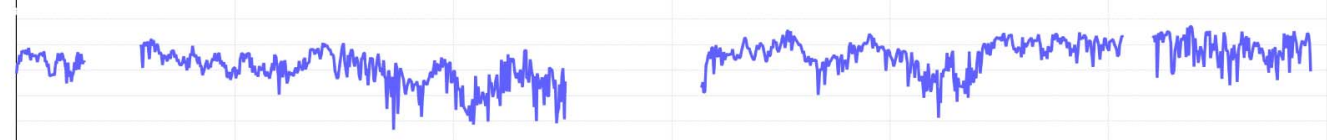

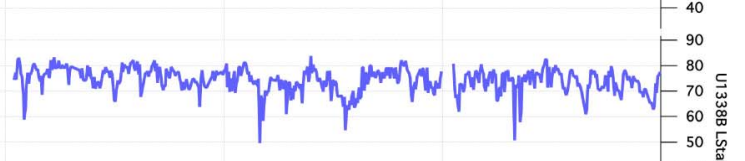

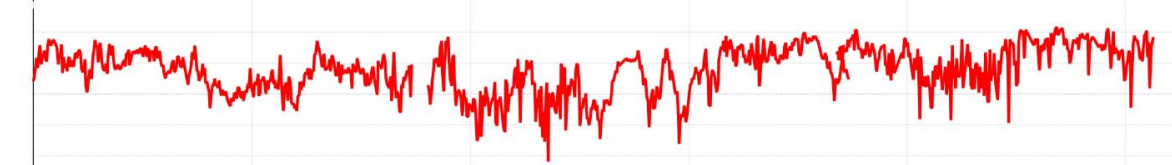

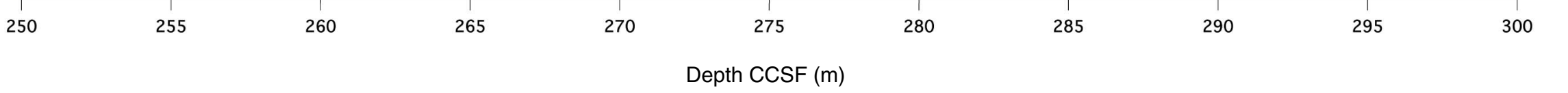




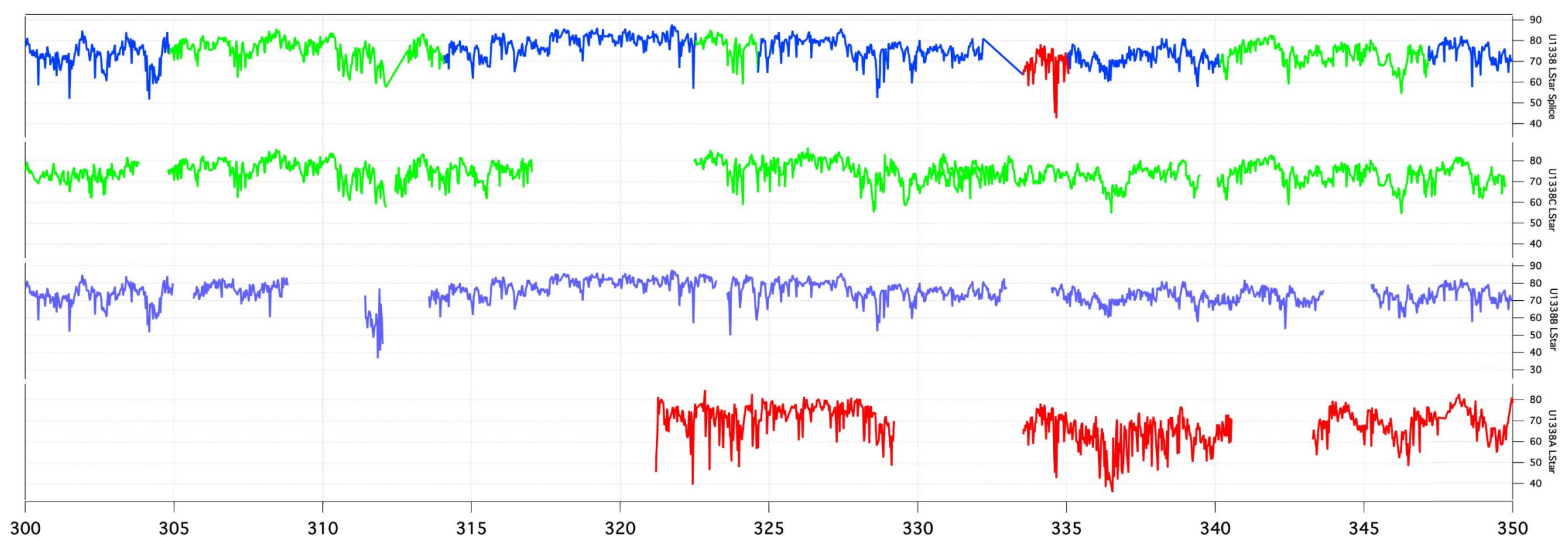

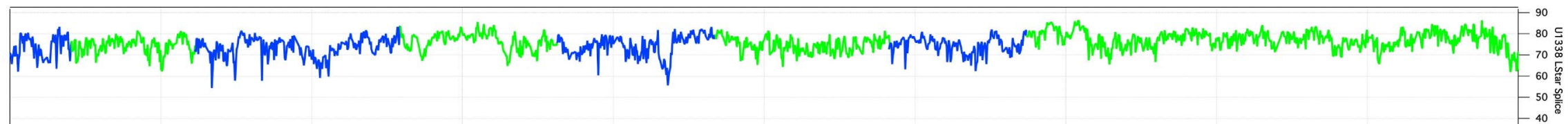

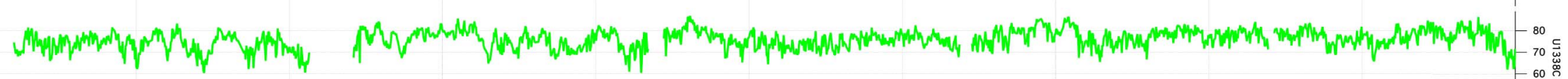

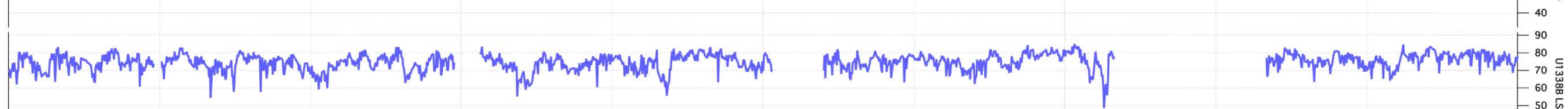

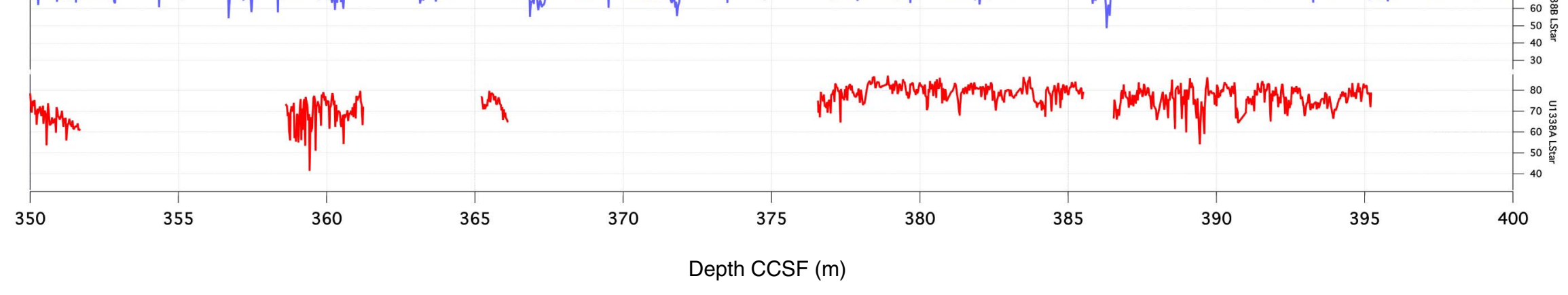




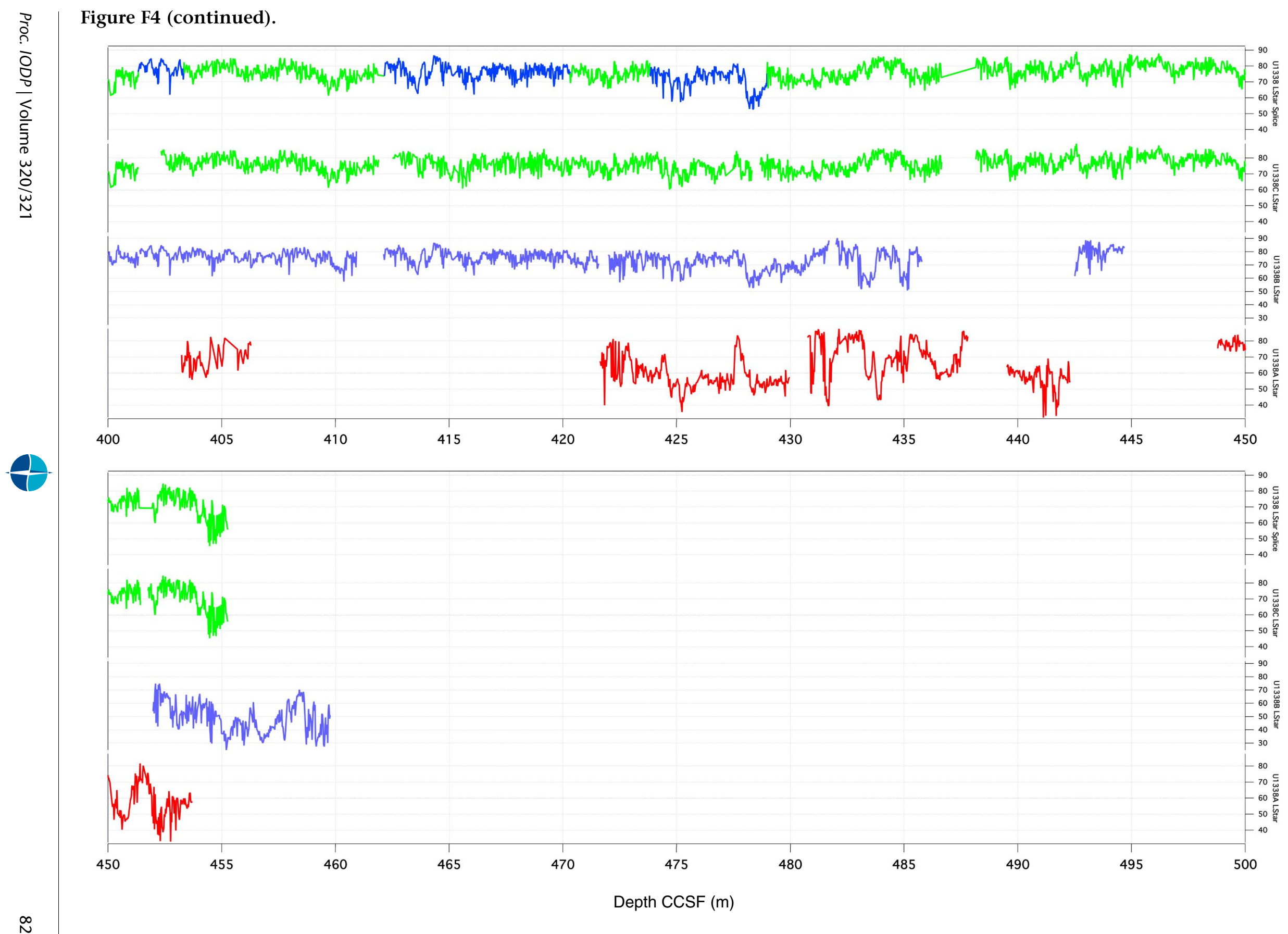


Figure F5. Example of the effects of "core stretching." Bottom: composite image of Core 321-U1337A-4H; middle: Site U1337 spliced image; top: image of Core 321-U1337A-4H plotted against adjusted (stretched) depth scale. Red symbols are plotted at tie points used to effect the stretch. Yellow vertical lines define the limits of the portion of Core 321-U1337A-4H used in the splice. Pale yellow traces are the appropriate gamma ray attenuation (GRA) data.
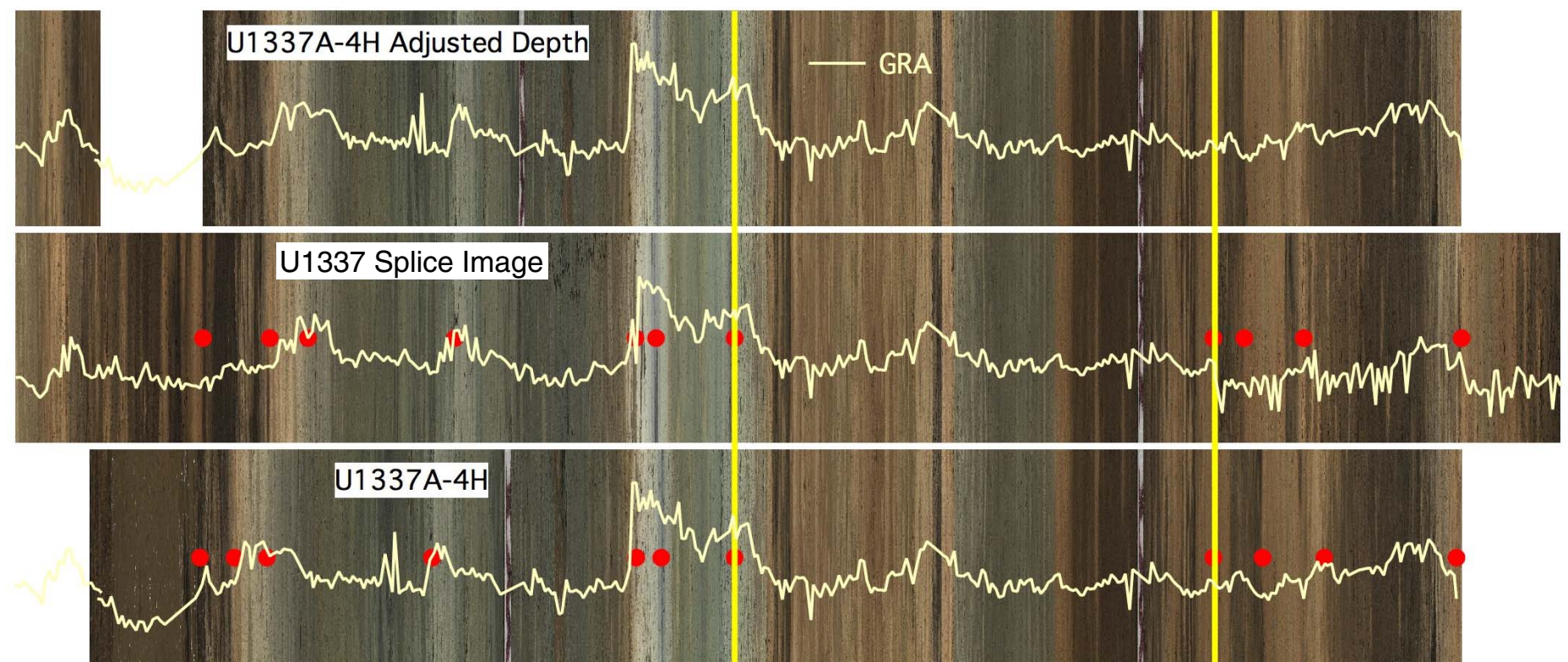

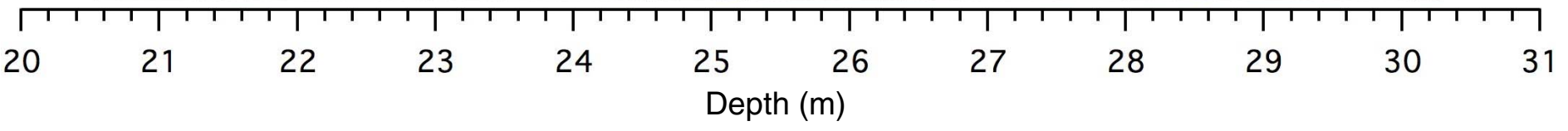


Table T1. Site U1336 splice offsets.

\begin{tabular}{|c|c|c|c|}
\hline Core & $\begin{array}{l}\text { Depth } \\
\text { CSF (m) }\end{array}$ & $\begin{array}{l}\text { Offset } \\
(\mathrm{m})\end{array}$ & $\begin{array}{c}\text { Depth } \\
\text { CCSF (m) }\end{array}$ \\
\hline \multicolumn{4}{|c|}{ 320-U1336A- } \\
\hline $1 \mathrm{H}$ & 0.0 & 0.26 & 0.26 \\
\hline $2 \mathrm{H}$ & 8.0 & 1.54 & 9.54 \\
\hline $3 \mathrm{H}$ & 17.5 & 1.88 & 19.38 \\
\hline $4 \mathrm{H}$ & 27.0 & 2.85 & 29.85 \\
\hline $5 \mathrm{H}$ & 36.5 & 3.20 & 39.70 \\
\hline $6 \mathrm{H}$ & 46.0 & 6.68 & 52.68 \\
\hline $7 \mathrm{H}$ & 55.5 & 8.11 & 63.61 \\
\hline $8 \mathrm{H}$ & 65.0 & 9.16 & 74.16 \\
\hline $9 \mathrm{H}$ & 74.5 & 9.84 & 84.34 \\
\hline $10 \mathrm{H}$ & 84.0 & 9.78 & 93.78 \\
\hline $11 \mathrm{H}$ & 93.5 & 11.86 & 105.36 \\
\hline $12 \mathrm{H}$ & 103.0 & 12.95 & 115.95 \\
\hline $13 \mathrm{H}$ & 112.5 & 14.29 & 126.79 \\
\hline $14 \mathrm{H}$ & 122.0 & 15.80 & 137.80 \\
\hline $15 \mathrm{H}$ & 123.5 & 16.30 & 139.80 \\
\hline $16 \mathrm{H}$ & 133.0 & 21.57 & 154.57 \\
\hline $17 \mathrm{H}$ & 142.0 & 23.48 & 165.48 \\
\hline $18 \mathrm{H}$ & 151.5 & 23.48 & 174.98 \\
\hline $19 \mathrm{H}$ & 161.0 & 23.48 & 184.48 \\
\hline $20 \mathrm{H}$ & 170.5 & 23.48 & 193.98 \\
\hline $21 \mathrm{H}$ & 180.0 & 23.48 & 203.48 \\
\hline $22 x$ & 184.8 & 23.48 & 208.28 \\
\hline $23 x$ & 194.4 & 23.48 & 217.88 \\
\hline $24 X$ & 202.4 & 23.48 & 225.88 \\
\hline $25 X$ & 212.0 & 23.48 & 235.48 \\
\hline $26 X$ & 221.6 & 23.48 & 245.08 \\
\hline $27 X$ & 231.2 & 23.48 & 254.68 \\
\hline $28 X$ & 240.8 & 23.48 & 264.28 \\
\hline $29 x$ & 250.4 & 23.48 & 273.88 \\
\hline $30 x$ & 260.0 & 23.48 & 283.48 \\
\hline $31 x$ & 269.6 & 23.48 & 293.08 \\
\hline $32 x$ & 279.2 & 23.48 & 302.68 \\
\hline $33 x$ & 288.8 & 23.48 & 312.28 \\
\hline $34 X$ & 293.8 & 23.48 & 317.28 \\
\hline $35 x$ & 298.3 & 23.48 & 321.78 \\
\hline \multicolumn{4}{|c|}{ 320-U1336B- } \\
\hline $1 \mathrm{H}$ & 0.0 & 0.00 & 0.00 \\
\hline $2 \mathrm{H}$ & 1.8 & 0.53 & 2.33 \\
\hline $3 \mathrm{H}$ & 11.3 & 2.56 & 13.86 \\
\hline $4 \mathrm{H}$ & 20.8 & 4.26 & 25.06 \\
\hline $5 \mathrm{H}$ & 30.3 & 5.66 & 35.96 \\
\hline $6 \mathrm{H}$ & 39.8 & 7.61 & 47.41 \\
\hline $7 \mathrm{H}$ & 49.3 & 8.81 & 58.11 \\
\hline $8 \mathrm{H}$ & 58.8 & 8.76 & 67.56 \\
\hline $9 \mathrm{H}$ & 68.3 & 8.89 & 77.19 \\
\hline $10 \mathrm{H}$ & 77.8 & 10.27 & 88.07 \\
\hline $11 \mathrm{H}$ & 87.3 & 10.77 & 98.07 \\
\hline $12 \mathrm{H}$ & 96.8 & 12.59 & 109.39 \\
\hline $13 \mathrm{H}$ & 106.3 & 14.34 & 120.64 \\
\hline $14 \mathrm{H}$ & 115.8 & 17.06 & 132.86 \\
\hline $15 \mathrm{H}$ & 125.3 & 17.87 & 143.17 \\
\hline $16 \mathrm{H}$ & 134.8 & 17.87 & 152.67 \\
\hline $17 \mathrm{H}$ & 136.8 & 17.87 & 154.67 \\
\hline $18 \mathrm{H}$ & 145.4 & 17.87 & 163.27 \\
\hline $19 \mathrm{H}$ & 154.9 & 17.87 & 172.77 \\
\hline $20 \mathrm{H}$ & 164.4 & 17.87 & 182.27 \\
\hline
\end{tabular}


Table T2. Site U1336 splice tie points.

\begin{tabular}{|c|c|c|c|c|c|c|}
\hline \multirow{2}{*}{$\begin{array}{l}\text { Hole, core, section, } \\
\text { interval }(\mathrm{cm})\end{array}$} & \multicolumn{2}{|c|}{ Depth (m) } & & \multirow{2}{*}{$\begin{array}{l}\text { Hole, core, section, } \\
\text { interval }(\mathrm{cm})\end{array}$} & \multicolumn{2}{|c|}{ Depth (m) } \\
\hline & CSF & CCSF & & & CSF & CCSF \\
\hline \multicolumn{7}{|l|}{$320-$} \\
\hline U1336B-1H-1, 95 & 0.95 & 0.95 & Tie to & U1336A-1H-1, 70 & 0.7 & 0.96 \\
\hline U1336A-1H-5, 76 & 6.76 & 7.02 & Tie to & U1336B-2H-4, 20 & 6.5 & 7.03 \\
\hline U1336B-2H-7, 26 & 11.06 & 11.59 & Tie to & U1336A-2H-2, 55 & 10.05 & 11.59 \\
\hline U1336A-2H-6, 109 & 16.59 & 18.13 & Tie to & U1336B-3H-3, 128 & 15.58 & 18.14 \\
\hline U1336B-3H-7, 10 & 20.4 & 22.96 & Tie to & U1336A-3H-3, 58 & 21.08 & 22.96 \\
\hline U1336A-3H-6, 56 & 25.56 & 27.44 & Tie to & U1336B-4H-2, 88 & 23.18 & 27.44 \\
\hline U1336B-4H-6, 137 & 29.67 & 33.93 & Tie to & U1336A-4H-3, 109 & 31.09 & 33.94 \\
\hline U1336A-4H-7, 9 & 35.4 & 38.25 & Tie to & U1336B-5H-2, 79 & 32.59 & 38.25 \\
\hline U1336B-5H-6, 124 & 39.03 & 44.69 & Tie to & U1336A-5H-4, 50 & 41.5 & 44.7 \\
\hline U1336A-5H-6, 148 & 45.48 & 48.68 & Tie to & U1336B-6H-1, 128 & 41.08 & 48.69 \\
\hline U1336B-6H-6, 147 & 48.77 & 56.38 & Tie to & U1336A-6H-3, 70 & 49.7 & 56.38 \\
\hline U1336A-6H-6, 70 & 54.2 & 60.88 & Tie to & U1336B-7H-2, 127 & 52.07 & 60.88 \\
\hline U1336B-7H-7, 13 & 58.13 & 66.94 & Tie to & U1336A-7H-3, 34 & 58.84 & 66.95 \\
\hline U1336A-7H-7, 34 & 64.84 & 72.95 & Tie to & U1336B-8H-4, 89 & 64.19 & 72.95 \\
\hline U1336B-8H-6, 30 & 66.6 & 75.36 & Tie to & U1336A-8H-1, 120 & 66.2 & 75.36 \\
\hline U1336A-8H-7, 54 & 73.54 & 82.7 & Tie to & U1336B-9H-4, 102 & 73.82 & 82.71 \\
\hline U1336B-9H-7, 6 & 77.06 & 85.95 & Tie to & U1336A-9H-2, 11 & 76.11 & 85.95 \\
\hline U1336A-9H-4, 103 & 80.03 & 89.87 & Tie to & U1336B-10H-2, 30 & 79.6 & 89.87 \\
\hline U1336B-10H-6, 127 & 86.57 & 96.84 & Tie to & U1336A-10H-3, 4 & 87.04 & 96.82 \\
\hline U1336A-10H-7, 36 & 93.06 & 102.84 & Tie to & U1336B-11H-4, 27 & 92.07 & 102.84 \\
\hline U1336B-11H-6, 82 & 95.62 & 106.39 & Tie to & U1336A-11H-1, 102 & 94.52 & 106.38 \\
\hline U1336A-11H-4, 57 & 98.57 & 110.43 & Tie to & U1336B-12H-1, 103 & 97.83 & 110.42 \\
\hline U1336B-12H-6, 112 & 105.42 & 118.01 & Tie to & U1336A-12H-2, 56 & 105.06 & 118.01 \\
\hline U1336A-12H-4, 121 & 108.71 & 121.66 & Tie to & U1336B-13H-1, 103 & 107.33 & 121.67 \\
\hline U1336B-13H-6, 88 & 114.68 & 129.02 & Tie to & U1336A-13H-2, 72 & 114.72 & 129.01 \\
\hline U1336A-13H-6, 62 & 120.62 & 134.91 & Tie to & U1336B-14H-2, 55 & 117.85 & 134.91 \\
\hline U1336B-14H-5, 124 & 123.04 & 140.1 & Append & U1336A-15H-1, 30 & 123.8 & 140.1 \\
\hline U1336A-15H-6, 70 & 131.7 & 148 & Tie to & U1336B-15H-4, 33 & 130.13 & 148 \\
\hline U1336B-15H-7, 75 & 134.55 & 152.42 & Append & U1336B-17H-1, 0 & 136.8 & 154.67 \\
\hline U1336B-17H-7, 79 & 145.29 & 163.16 & Append & U1336B-18H-1, 0 & 145.4 & 163.27 \\
\hline U1336B-18H-7, 16 & 154.56 & 172.43 & Tie to & U1336A-17H-5, 95 & 148.95 & 172.43 \\
\hline U1336A-17H-7, 50 & 151.5 & 174.98 & Append & U1336A-18H-1, 0 & 151.5 & 174.98 \\
\hline U1336A-18H-7, 74 & 161.24 & 184.72 & Append & U1336A-19H-1, 0 & 161 & 184.48 \\
\hline U1336A-19H-7, 36 & 170.36 & 193.84 & Append & U1336A-20H-1, 0 & 170.5 & 193.98 \\
\hline U1336A-20H-7, 62 & 180.12 & 203.6 & Append & U1336A-21H-1, 0 & 180 & 203.48 \\
\hline U1336A-21H-4, 56 & 184.56 & 208.04 & Append & U1336A-22X-1, 0 & 184.8 & 208.28 \\
\hline U1336A-22X-6, 45 & 192.55 & 216.03 & Append & U1336A-23X-1, 0 & 194.4 & 217.88 \\
\hline U1336A-23X-5, 147 & 201.87 & 225.35 & Append & U1336A-24X-1, 0 & 202.4 & 225.88 \\
\hline U1336A-24X-5, 84 & 209.24 & 232.72 & Append & U1336A-25X-1, 0 & 212 & 235.48 \\
\hline U1336A-25X-3, 147 & 216.47 & 239.95 & Append & U1336A-26X-1, 0 & 221.6 & 245.08 \\
\hline U1336A-26X-7, 40 & 230.7 & 254.18 & Append & U1336A-27X-1, 0 & 231.2 & 254.68 \\
\hline U1336A-27X-5, 42 & 237.42 & 260.9 & Append & U1336A-28X-1, 0 & 240.8 & 264.28 \\
\hline U1336A-28X-2, 120 & 243.5 & 266.98 & Append & U1336A-29X-1, 0 & 250.4 & 273.88 \\
\hline U1336A-29X-3, 63 & 254.03 & 277.51 & Append & U1336A-30X-1, 0 & 260 & 283.48 \\
\hline U1336A-30X-3, 87 & 263.87 & 287.35 & Append & U1336A-31X-1, 0 & 269.6 & 293.08 \\
\hline U1336A-31X-4, 40 & 274.2 & 297.68 & Append & U1336A-32X-1, 0 & 279.2 & 302.68 \\
\hline U1336A-32X-3, 7 & 282.27 & 305.75 & Append & U1336A-33X-2, 0 & 288.95 & 312.43 \\
\hline U1336A-33X-3, 58 & 290.53 & 314.01 & Append & U1336A-35X-1, 0 & 298.3 & 321.78 \\
\hline
\end{tabular}


Table T3. Site U1337 splice offsets.

\begin{tabular}{|c|c|c|c|c|c|c|c|c|c|c|c|}
\hline Core & $\begin{array}{l}\text { Depth } \\
\text { CSF }(m)\end{array}$ & $\begin{array}{l}\text { Offset } \\
(\mathrm{m})\end{array}$ & $\begin{array}{c}\text { Depth } \\
\operatorname{CCSF}(\mathrm{m})\end{array}$ & Core & $\begin{array}{l}\text { Depth } \\
\text { CSF (m) }\end{array}$ & $\begin{array}{l}\text { Offset } \\
(\mathrm{m})\end{array}$ & $\begin{array}{c}\text { Depth } \\
\text { CCSF }(\mathrm{m})\end{array}$ & Core & $\begin{array}{l}\text { Depth } \\
\text { CSF }(\mathrm{m})\end{array}$ & $\begin{array}{l}\text { Offset } \\
(\mathrm{m})\end{array}$ & $\begin{array}{c}\text { Depth } \\
\operatorname{CCSF}(m)\end{array}$ \\
\hline 321-U1337A- & & & & $5 \mathrm{H}$ & 39.0 & -0.94 & 38.06 & $31 x$ & 422.3 & 58.24 & 480.54 \\
\hline $1 \mathrm{H}$ & & 0.00 & & $6 \mathrm{H}$ & 48.5 & 0.29 & 48.79 & $32 X$ & 431.9 & 58.24 & 490.14 \\
\hline $2 \mathrm{H}$ & 5.5 & -5.31 & 0.19 & $7 \mathrm{H}$ & 58.0 & 1.39 & 59.39 & $33 x$ & 436.7 & 58.24 & 494.94 \\
\hline $3 \mathrm{H}$ & 15.0 & -4.11 & 10.89 & $8 \mathrm{H}$ & 67.5 & 2.40 & 69.90 & \multirow{2}{*}{\multicolumn{4}{|c|}{ 321-U1337D- }} \\
\hline $4 \mathrm{H}$ & 24.5 & -3.97 & 20.53 & $9 \mathrm{H}$ & 77.0 & 3.57 & 80.57 & & & & 0.00 \\
\hline $5 \mathrm{H}$ & 34.0 & -1.30 & 32.70 & $10 \mathrm{H}$ & 86.5 & 6.07 & 92.57 & $2 \mathrm{H}$ & 8.0 & 0.59 & 8.59 \\
\hline $6 \mathrm{H}$ & 43.5 & -0.17 & 43.33 & $11 \mathrm{H}$ & 96.0 & 6.90 & 102.90 & $3 \mathrm{H}$ & 17.5 & 1.44 & 18.94 \\
\hline $7 \mathrm{H}$ & 53.0 & 2.28 & 55.28 & $12 \mathrm{H}$ & 105.5 & 8.19 & 113.69 & $4 \mathrm{H}$ & 27.0 & 2.43 & 29.43 \\
\hline $8 \mathrm{H}$ & 62.5 & 3.06 & 65.56 & $13 \mathrm{H}$ & 115.0 & 9.48 & 124.48 & $5 \mathrm{H}$ & 36.5 & 3.55 & 40.05 \\
\hline $9 \mathrm{H}$ & 72.0 & 4.31 & 76.31 & $14 \mathrm{H}$ & 124.5 & 10.44 & 134.94 & $6 \mathrm{H}$ & 46.0 & 5.25 & 51.25 \\
\hline $10 \mathrm{H}$ & 81.5 & 4.80 & 86.30 & $15 \mathrm{H}$ & 134.0 & 11.50 & 145.50 & $7 \mathrm{H}$ & 55.5 & 6.39 & 61.89 \\
\hline $11 \mathrm{H}$ & 91.0 & 5.85 & 96.85 & $16 \mathrm{H}$ & 143.5 & 12.82 & 156.32 & $8 \mathrm{H}$ & 65.0 & 6.93 & 71.93 \\
\hline $12 \mathrm{H}$ & 100.5 & 6.59 & 107.09 & $17 \mathrm{H}$ & 153.0 & 13.15 & 166.15 & $9 \mathrm{H}$ & 74.5 & 8.19 & 82.69 \\
\hline $13 \mathrm{H}$ & 110.0 & 7.53 & 117.53 & $18 \mathrm{H}$ & 162.5 & 14.08 & 176.58 & $10 \mathrm{H}$ & 84.0 & 9.79 & 93.79 \\
\hline $14 \mathrm{H}$ & 119.5 & 9.62 & 129.12 & $19 \mathrm{H}$ & 172.0 & 15.62 & 187.62 & $11 \mathrm{H}$ & 93.5 & 11.66 & 105.16 \\
\hline $15 \mathrm{H}$ & 129.0 & 11.33 & 140.33 & $20 \mathrm{H}$ & 181.5 & 17.11 & 198.61 & $12 \mathrm{H}$ & 103.0 & 12.45 & 115.45 \\
\hline $16 \mathrm{H}$ & 138.5 & 11.94 & 150.44 & $21 \mathrm{H}$ & 191.0 & 18.13 & 209.13 & $13 \mathrm{H}$ & 112.5 & 14.31 & 126.81 \\
\hline $17 \mathrm{H}$ & 148.0 & 13.41 & 161.41 & $22 \mathrm{H}$ & 200.5 & 19.91 & 220.41 & $14 \mathrm{H}$ & 122.0 & 15.29 & 137.29 \\
\hline $18 \mathrm{H}$ & 157.5 & 15.27 & 172.77 & $23 \mathrm{H}$ & 210.0 & 22.68 & 232.68 & $15 \mathrm{H}$ & 131.5 & 16.45 & 147.95 \\
\hline $19 \mathrm{H}$ & 167.0 & 16.14 & 183.14 & $24 \mathrm{H}$ & 219.5 & 21.41 & 240.91 & $16 \mathrm{H}$ & 141.0 & 18.15 & 159.15 \\
\hline $20 \mathrm{H}$ & 176.5 & 16.88 & 193.38 & $25 \mathrm{H}$ & 226.4 & 21.55 & 247.95 & $17 \mathrm{H}$ & 150.5 & 19.13 & 169.63 \\
\hline $21 \mathrm{H}$ & 186.0 & 17.34 & 203.34 & $26 \mathrm{H}$ & 234.3 & 21.54 & 255.84 & $18 \mathrm{H}$ & 160.0 & 19.94 & 179.94 \\
\hline $22 X$ & 195.5 & 17.25 & 212.75 & $27 \mathrm{H}$ & 243.8 & 21.92 & 265.72 & $19 \mathrm{H}$ & 169.5 & 20.53 & 190.03 \\
\hline $23 X$ & 204.3 & 18.88 & 223.18 & \multicolumn{4}{|l|}{ 321-U1337C- } & $20 \mathrm{H}$ & 179.0 & 20.86 & 199.86 \\
\hline $24 X$ & 213.9 & 19.65 & 233.55 & $1 \mathrm{H}$ & 0.0 & 0.00 & 0.00 & $21 \mathrm{H}$ & 188.5 & 20.64 & 209.14 \\
\hline $25 X$ & 223.4 & 20.87 & 244.27 & $2 \mathrm{H}$ & 1.9 & 1.87 & 3.77 & $22 \mathrm{H}$ & 193.5 & 22.61 & 216.11 \\
\hline $26 x$ & 233.0 & 20.58 & 253.58 & $3 W$ & & & & $23 \mathrm{H}$ & 199.7 & 24.34 & 224.04 \\
\hline $27 X$ & 242.2 & 23.49 & $\begin{array}{l}265.69 \\
271.35\end{array}$ & $4 \mathrm{H}$ & 169.4 & 23.05 & 192.45 & $24 \mathrm{H}$ & 209.2 & 26.36 & 235.56 \\
\hline $28 x$ & 251.7 & 19.65 & 271.35 & $5 \mathrm{H}$ & 178.9 & 23.65 & 202.55 & $25 \mathrm{H}$ & 218.7 & 25.82 & 244.52 \\
\hline $29 X$ & 261.3 & 27.13 & 288.43 & $6 \mathrm{H}$ & 188.4 & 24.00 & 212.40 & $26 \mathrm{H}$ & 228.2 & 26.62 & 254.82 \\
\hline $30 x$ & 270.8 & 28.46 & 299.26 & $7 \mathrm{H}$ & 192.8 & 24.91 & 217.71 & $27 X$ & 237.7 & 27.22 & 264.92 \\
\hline $31 X$ & 280.5 & 28.94 & 309.44 & $8 \mathrm{H}$ & 202.3 & 26.93 & 229.23 & $28 \mathrm{H}$ & 241.4 & 26.62 & 268.02 \\
\hline $32 X$ & 290.2 & 28.44 & $\begin{array}{l}318.64 \\
327.35\end{array}$ & $9 \mathrm{H}$ & 211.8 & 26.97 & 238.77 & $29 \mathrm{H}$ & 248.0 & 28.87 & 276.87 \\
\hline $33 x$ & 299.7 & 27.65 & 327.35 & $10 x$ & 221.3 & 27.97 & 249.27 & $30 \mathrm{H}$ & 257.5 & 29.94 & 287.44 \\
\hline $34 X$ & 309.3 & 28.90 & $\begin{array}{l}338.20 \\
347.57\end{array}$ & $11 X$ & 230.9 & 28.16 & 259.06 & $31 x$ & 267.0 & 34.69 & 301.69 \\
\hline $35 X$ & 318.9 & 28.67 & 347.57 & $12 x$ & 240.5 & 31.66 & 272.16 & $32 X$ & 276.2 & 34.31 & 310.51 \\
\hline $36 X$ & 328.5 & 29.29 & $\begin{array}{l}357.79 \\
368.83\end{array}$ & $13 x$ & 250.1 & 32.61 & 282.71 & $33 x$ & 285.8 & 33.92 & 319.72 \\
\hline $37 X$ & 338.0 & 30.83 & 368.83 & $14 X$ & 259.7 & 36.17 & 295.87 & $34 X$ & 295.3 & 33.24 & 328.54 \\
\hline $38 x$ & 347.6 & 32.17 & $\begin{array}{l}379.77 \\
28078\end{array}$ & $15 X$ & 269.3 & 35.79 & 305.09 & $35 X$ & 304.9 & 33.91 & 338.81 \\
\hline $39 x$ & 357.2 & 32.58 & 389.78 & $16 X$ & 278.9 & 36.05 & 314.95 & $36 x$ & 314.5 & 33.99 & 348.49 \\
\hline $40 x$ & 366.8 & 35.16 & 401.96 & $17 X$ & 288.5 & 34.51 & 323.01 & $37 X$ & 324.1 & 34.19 & 358.29 \\
\hline $41 X$ & 376.4 & 36.14 & 412.54 & $18 x$ & 298.1 & 35.04 & 333.14 & $38 \mathrm{X}$ & 333.6 & 34.56 & 368.16 \\
\hline $42 x$ & 386.0 & 38.52 & 424.52 & $19 X$ & 307.7 & 35.04 & 342.74 & $39 x$ & 343.2 & 36.08 & 379.28 \\
\hline $43 x$ & 395.6 & 38.34 & 433.94 & $20 x$ & 317.3 & 35.67 & 352.97 & $40 x$ & 352.8 & 36.76 & 389.56 \\
\hline $44 X$ & 404.9 & 38.34 & 443.24 & $21 X$ & 326.8 & 37.00 & 363.81 & $41 x$ & 362.4 & 38.28 & 400.68 \\
\hline $45 X$ & 414.5 & 39.85 & 454.35 & $22 x$ & 336.4 & 38.32 & 374.72 & $42 X$ & 372.0 & 40.93 & 412.93 \\
\hline $46 x$ & 424.0 & 46.32 & 470.32 & $23 x$ & 346.0 & 39.95 & 385.95 & $43 x$ & 381.6 & 42.47 & 424.07 \\
\hline $47 X$ & 433.6 & 48.42 & 482.02 & $24 X$ & 355.6 & 39.40 & 395.00 & $44 X$ & 391.2 & 55.43 & 446.63 \\
\hline $48 x$ & 443.2 & 48.90 & 492.10 & $25 X$ & 365.2 & 40.57 & 405.77 & $45 X$ & 400.5 & 55.40 & 455.90 \\
\hline 321-U1337B- & & & & $26 X$ & 374.7 & 42.15 & 416.85 & $46 X$ & 410.1 & 57.27 & 467.37 \\
\hline $1 \mathrm{H}$ & 1.0 & -1.00 & 0.00 & $27 X$ & 384.3 & 44.35 & 428.65 & $47 X$ & 419.6 & 57.16 & 476.76 \\
\hline $2 \mathrm{H}$ & 10.5 & -3.56 & 6.94 & $28 x$ & 393.6 & 54.10 & 447.70 & $48 x$ & 429.1 & 56.73 & 485.83 \\
\hline $3 \mathrm{H}$ & 20.0 & -2.36 & 17.64 & $29 x$ & 403.2 & 58.43 & 461.63 & $49 x$ & 438.8 & 56.73 & 495.53 \\
\hline
\end{tabular}


Table T4. Site U1337 splice tie points. (Continued on next page.)

\begin{tabular}{|c|c|c|c|c|c|c|}
\hline \multirow{2}{*}{$\begin{array}{l}\text { Hole, core, section, } \\
\text { interval }(\mathrm{cm})\end{array}$} & \multicolumn{2}{|c|}{ Depth $(m)$} & & \multirow{2}{*}{$\begin{array}{l}\text { Hole, core, section, } \\
\text { interval }(\mathrm{cm})\end{array}$} & \multicolumn{2}{|c|}{ Depth $(m)$} \\
\hline & CSF & CCSF & & & CSF & CCSF \\
\hline \multicolumn{7}{|l|}{$321-$} \\
\hline U1337B-1H-3, 134 & 5.34 & 4.34 & Tie to & U1337A-2H-3, 115 & 9.65 & 4.34 \\
\hline U1337A-2H-6, 45 & 13.45 & 8.14 & Tie to & U1337C-2H-3, 137 & 6.27 & 8.14 \\
\hline U1337C-2H-6, 68 & 10.08 & 11.95 & Tie to & U1337A-3H-1, 105 & 16.05 & 11.94 \\
\hline U1337A-3H-6, 70 & 23.2 & 19.09 & Tie to & U1337B-3H-1, 146 & 21.46 & 19.1 \\
\hline U1337B-3H-5, 148 & 27.48 & 25.12 & Tie to & U1337A-4H-4, 9 & 29.09 & 25.12 \\
\hline U1337A-4H-6, 50 & 32.5 & 28.53 & Tie to & U1337B-4H-1, 88 & 30.38 & 28.54 \\
\hline U1337B-4H-6, 17 & 37.17 & 35.33 & Tie to & U1337A-5H-2, 113 & 36.63 & 35.33 \\
\hline U1337A-5H-4, 124 & 39.74 & 38.44 & Tie to & U1337B-5H-1, 38 & 39.38 & 38.44 \\
\hline U1337B-5H-5, 110 & 46.1 & 45.16 & Tie to & U1337A-6H-2, 32 & 45.32 & 45.15 \\
\hline U1337A-6H-5, 93 & 50.43 & 50.26 & Tie to & U1337B-6H-1, 147 & 49.97 & 50.26 \\
\hline U1337B-6H-5, 113 & 55.63 & 55.92 & Tie to & U1337A-7H-1, 64 & 53.64 & 55.92 \\
\hline U1337A-7H-5, 61 & 59.61 & 61.89 & Tie to & U1337B-7H-2, 100 & 60.5 & 61.89 \\
\hline U1337B-7H-6, 27 & 65.77 & 67.16 & Tie to & U1337A-8H-2, 9 & 64.09 & 67.15 \\
\hline U1337A-8H-5, 64 & 69.14 & 72.2 & Tie to & U1337B-8H-2, 80 & 69.8 & 72.2 \\
\hline U1337B-8H-5, 106 & 74.56 & 76.96 & Tie to & U1337A-9H-1, 65 & 72.65 & 76.96 \\
\hline U1337A-9H-6, 114 & 80.64 & 84.95 & Tie to & U1337B-9H-3, 139 & 81.39 & 84.96 \\
\hline U1337B-9H-5, 117 & 84.17 & 87.74 & Tie to & U1337A-10H-1, 143 & 82.93 & 87.73 \\
\hline U1337A-10H-5, 123 & 88.73 & 93.53 & Tie to & U1337B-10H-1, 96 & 87.46 & 93.53 \\
\hline U1337B-10H-4, 54 & 91.54 & 97.61 & Tie to & U1337A-11H-1, 76 & 91.76 & 97.61 \\
\hline U1337A-11H-5, 114 & 98.14 & 103.99 & Tie to & U1337B-11H-1, 110 & 97.1 & 104 \\
\hline U1337B-11H-5, 43 & 102.43 & 109.33 & Tie to & U1337A-12H-2, 74 & 102.74 & 109.33 \\
\hline U1337A-12H-6, 58 & 108.58 & 115.17 & Tie to & U1337B-12H-1, 148 & 106.98 & 115.17 \\
\hline U1337B-12H-5, 40 & 111.9 & 120.09 & Tie to & U1337A-13H-2, 106 & 112.56 & 120.09 \\
\hline U1337A-13H-6, 21 & 117.71 & 125.24 & Tie to & U1337B-13H-1, 75 & 115.75 & 125.23 \\
\hline U1337B-13H-5, 14 & 121.14 & 130.62 & Tie to & U1337A-14H-1, 150 & 121 & 130.62 \\
\hline U1337A-14H-7, 20 & 128.7 & 138.32 & Tie to & U1337D-14H-1, 103 & 123.03 & 138.32 \\
\hline U1337D-14H-4, 98 & 127.48 & 142.77 & Tie to & U1337A-15H-2, 94 & 131.44 & 142.77 \\
\hline U1337A-15H-5, 52 & 135.52 & 146.85 & Tie to & U1337B-15H-1, 136 & 135.36 & 146.86 \\
\hline U1337B-15H-5, 98 & 140.98 & 152.48 & Tie to & U1337A-16H-2, 54 & 140.54 & 152.48 \\
\hline U1337A-16H-5, 104 & 145.54 & 157.48 & Tie to & U1337B-16H-1, 116 & 144.66 & 157.48 \\
\hline U1337B-16H-5, 110 & 150.6 & 163.42 & Tie to & U1337A-17H-2, 50 & 150 & 163.41 \\
\hline U1337A-17H-5, 84 & 154.84 & 168.25 & Tie to & U1337B-17H-2, 59 & 155.09 & 168.24 \\
\hline U1337B-17H-6, 8 & 160.58 & 173.73 & Tie to & U1337A-18H-1, 96 & 158.46 & 173.73 \\
\hline U1337A-18H-6, 104 & 166.08 & 181.35 & Tie to & U1337B-18H-4, 27 & 167.27 & 181.35 \\
\hline U1337B-18H-6, 58 & 170.58 & 184.66 & Tie to & U1337A-19H-2, 2 & 168.52 & 184.66 \\
\hline U1337A-19H-5, 55 & 173.55 & 189.69 & Tie to & U1337B-19H-2, 57 & 174.07 & 189.69 \\
\hline U1337B-19H-5, 83 & 178.89 & 194.51 & Tie to & U1337A-20H-1, 113 & 177.63 & 194.51 \\
\hline U1337A-20H-5, 47 & 182.97 & 199.85 & Tie to & U1337B-20H-1, 124 & 182.74 & 199.85 \\
\hline U1337B-20H-5, 50 & 188 & 205.11 & Tie to & U1337A-21H-2, 27 & 187.77 & 205.11 \\
\hline U1337A-21H-5, 36 & 192.37 & 209.71 & Tie to & U1337B-21H-1, 58 & 191.58 & 209.71 \\
\hline U1337B-21H-3, 70 & 194.7 & 212.83 & Tie to & U1337C-6H-1, 43 & 188.83 & 212.83 \\
\hline U1337C-6H-3, 119 & 192.59 & 216.59 & Tie to & U1337D-22H-1, 48 & 193.98 & 216.59 \\
\hline U1337D-22H-2, 58 & 195.58 & 218.19 & Tie to & U1337C-7H-1, 48 & 193.28 & 218.19 \\
\hline U1337C-7H-6, 115 & 201.45 & 226.36 & Tie to & U1337A-23X-3, 18 & 207.48 & 226.36 \\
\hline U1337A-23X-6, 11 & 211.91 & 230.79 & Tie to & U1337C-8H-2, 5 & 203.85 & 230.78 \\
\hline U1337C-8H-7, 64 & 211.94 & 238.87 & Append & U1337C-9H-1, 2 & 211.82 & 238.79 \\
\hline U1337C-9H-5, 107 & 218.87 & 245.84 & Tie to & U1337A-25X-2, 7 & 224.96 & 245.83 \\
\hline U1337A-25X-4, 99 & 228.89 & 249.76 & Tie to & U1337B-25H-2, 31 & 228.21 & 249.76 \\
\hline U1337B-25H-4, 150 & 232.4 & 253.95 & Tie to & U1337A-26X-1, 37 & 233.37 & 253.95 \\
\hline U1337A-26X-6, 14 & 240.64 & 261.22 & Tie to & U1337C-11X-2, 66 & 233.06 & 261.22 \\
\hline U1337C-11X-6, 70 & 238.7 & 266.86 & Append & U1337D-28H-1, 133 & 242.73 & 269.35 \\
\hline U1337D-28H-4, 70 & 246.6 & 273.22 & Tie to & U1337C-12X-1, 106 & 241.56 & 273.22 \\
\hline U1337C-12X-5, 136 & 247.86 & 279.52 & Tie to & U1337D-29H-2, 115 & 250.65 & 279.52 \\
\hline U1337D-29H-5, 48 & 254.48 & 283.35 & Tie to & U1337C-13X-1, 63 & 250.73 & 283.34 \\
\hline U1337C-13X-6, 23 & 257.83 & 290.44 & Tie to & U1337D-30H-3, 1 & 260.51 & 290.45 \\
\hline U1337D-30H-6, 104 & 266.04 & 295.98 & Append & U1337C-14X-1, 33 & 260.03 & 296.2 \\
\hline U1337C-14X-6, 4 & 267.24 & 303.41 & Tie to & U1337D-31X-2, 22 & 268.72 & 303.41 \\
\hline U1337D-31X-4, 133 & 272.83 & 307.52 & Tie to & U1337C-15X-2, 93 & 271.73 & 307.52 \\
\hline U1337C-15X-4, 145 & 275.25 & 311.04 & Tie to & U1337D-32X-1, 53 & 276.73 & 311.04 \\
\hline U1337D-32X-4, 84 & 281.54 & 315.85 & Tie to & U1337C-16X-1, 90 & 279.8 & 315.85 \\
\hline U1337C-16X-4, 27 & 283.67 & 319.72 & Tie to & U1337A-32X-1, 108 & 291.28 & 319.72 \\
\hline U1337A-32X-4, 79 & 295.49 & 323.93 & Tie to & U1337C-17X-1, 93 & 289.43 & 323.94 \\
\hline U1337C-17X-5, 64 & 295.14 & 329.65 & Tie to & U1337A-33X-2, 80 & 302 & 329.65 \\
\hline U1337A-33X-5, 33 & 306.03 & 333.68 & Tie to & U1337C-18X-1, 54 & 298.64 & 333.68 \\
\hline U1337C-18X-6, 74 & 306.34 & 341.38 & Tie to & U1337A-34X-3, 18 & 312.48 & 341.38 \\
\hline U1337A-34X-5, 88 & 316.18 & 345.08 & Tie to & U1337C-19X-2, 84 & 310.04 & 345.07 \\
\hline U1337C-19X-5, 37 & 314.07 & 349.11 & Tie to & U1337A-35X-2, 4 & 320.44 & 349.11 \\
\hline
\end{tabular}


Table T4 (continued).

\begin{tabular}{|c|c|c|c|c|c|c|}
\hline \multirow{2}{*}{$\begin{array}{l}\text { Hole, core, section, } \\
\text { interval }(\mathrm{cm})\end{array}$} & \multicolumn{2}{|c|}{ Depth (m) } & & \multirow{2}{*}{$\begin{array}{l}\text { Hole, core, section, } \\
\text { interval }(\mathrm{cm})\end{array}$} & \multicolumn{2}{|c|}{ Depth (m) } \\
\hline & CSF & CCSF & & & CSF & CCSF \\
\hline U1337A-35X-3, 36 & 322.26 & 350.93 & Tie to & U1337D-36X-2, 95 & 316.95 & 350.94 \\
\hline U1337D-36X-5, 135 & 321.85 & 355.84 & Tie to & U1337C-20X-2, 137 & 320.17 & 355.84 \\
\hline U1337C-20X-5, 27 & 323.57 & 359.24 & Tie to & U1337A-36X-1, 145 & 329.95 & 359.24 \\
\hline U1337A-36X-5, 87 & 335.37 & 364.66 & Tie to & U1337C-21X-1, 85 & 327.65 & 364.65 \\
\hline U1337C-21X-5, 138 & 334.18 & 371.18 & Tie to & U1337A-37X-2, 86 & 340.36 & 371.19 \\
\hline U1337A-37X-6, 59 & 346.09 & 376.92 & Tie to & U1337C-22X-2, 69 & 338.59 & 376.91 \\
\hline U1337C-22X-4, 104 & 341.94 & 380.26 & Tie to & U1337D-39X-1, 98 & 344.18 & 380.26 \\
\hline U1337D-39X-5, 111 & 350.31 & 386.39 & Tie to & U1337C-23X-1, 44 & 346.44 & 386.39 \\
\hline U1337C-23X-4, 95 & 351.45 & 391.4 & Tie to & U1337D-40X-2, 34 & 354.64 & 391.4 \\
\hline U1337D-40X-6, 19 & 360.49 & 397.25 & Tie to & U1337C-24X-2, 76 & 357.86 & 397.26 \\
\hline U1337C-24X-5, 114 & 362.74 & 402.14 & Tie to & U1337A-40X-1, 17 & 366.97 & 402.13 \\
\hline U1337A-40X-5, 93 & 373.73 & 408.89 & Tie to & U1337C-25X-3,13 & 368.33 & 408.89 \\
\hline U1337C-25X-5, 98 & 372.18 & 412.75 & Tie to & U1337A-41X-1,22 & 376.62 & 412.76 \\
\hline U1337A-41X-5, 77 & 383.17 & 419.31 & Tie to & U1337C-26X-2,96 & 377.16 & 419.31 \\
\hline U1337C-26X-7, 15 & 383.36 & 425.51 & Tie to & U1337D-43X-1,143 & 383.03 & 425.5 \\
\hline U1337D-43X-6, 36 & 389.46 & 431.93 & Tie to & U1337C-27X-3,28 & 387.58 & 431.93 \\
\hline U1337C-27X-4, 108 & 389.88 & 434.23 & Tie to & U1337A-43X-1,28 & 395.88 & 434.22 \\
\hline U1337A-43X-7, 79 & 404.89 & 443.23 & Append & U1337A-44X-1,5 & 404.95 & 443.29 \\
\hline U1337A-44X-4, 68 & 410.08 & 448.42 & Tie to & U1337C-28X-1,72 & 394.32 & 448.42 \\
\hline U1337C-28X-6, 98 & 402.08 & 456.19 & Tie to & U1337D-45X-1,28 & 400.78 & 456.18 \\
\hline U1337D-45X-5, 150 & 408 & 463.4 & Tie to & U1337C-29X-2,27 & 404.97 & 463.4 \\
\hline U1337C-29X-6, 8 & 410.78 & 469.2 & Tie to & U1337D-46X-2,33 & 411.93 & 469.2 \\
\hline U1337D-46X-4, 42 & 415.02 & 472.29 & Tie to & U1337A-46X-2,48 & 425.98 & 472.3 \\
\hline U1337A-46X-5, 82 & 430.82 & 477.14 & Tie to & U1337D-47X-1,38 & 419.98 & 477.14 \\
\hline U1337D-47X-3, 88 & 423.48 & 480.64 & Tie to & U1337C-31X-1,10 & 422.4 & 480.64 \\
\hline U1337C-31X-5, 62 & 428.92 & 487.16 & Tie to & U1337D-48X-1,133 & 430.43 & 487.16 \\
\hline U1337D-48X-4, 14 & 433.74 & 490.47 & Tie to & U1337C-32X-1,33 & 432.23 & 490.47 \\
\hline U1337C-32X-4, 14 & 436.54 & 494.78 & Append & & & \\
\hline
\end{tabular}


Table T5. Site U1338 splice offsets.

\begin{tabular}{|c|c|c|c|c|c|c|c|}
\hline Core & $\begin{array}{l}\text { Depth } \\
\text { CSF }(m)\end{array}$ & $\begin{array}{l}\text { Offset } \\
(\mathrm{m})\end{array}$ & $\begin{array}{c}\text { Depth } \\
\text { CCSF (m) }\end{array}$ & Core & $\begin{array}{l}\text { Depth } \\
\operatorname{CSF}(\mathrm{m})\end{array}$ & $\begin{array}{l}\text { Offset } \\
(\mathrm{m})\end{array}$ & $\begin{array}{c}\text { Depth } \\
\text { CCSF }(m)\end{array}$ \\
\hline 321-U1338A- & & & & $2 \mathrm{H}$ & 7.6 & 1.29 & 8.89 \\
\hline $1 \mathrm{H}$ & 0.0 & 0.04 & 0.04 & $3 \mathrm{H}$ & 17.1 & 2.07 & 19.17 \\
\hline $2 \mathrm{H}$ & 2.7 & 1.48 & 4.18 & $4 \mathrm{H}$ & 26.6 & 2.66 & 29.26 \\
\hline $3 \mathrm{H}$ & 12.2 & 2.23 & 14.43 & $5 \mathrm{H}$ & 36.1 & 4.30 & 40.40 \\
\hline $4 \mathrm{H}$ & 21.7 & 3.62 & 25.32 & $6 \mathrm{H}$ & 45.6 & 5.14 & 50.74 \\
\hline $5 \mathrm{H}$ & 31.2 & 5.40 & 36.60 & $7 \mathrm{H}$ & 55.1 & 5.86 & 60.96 \\
\hline $6 \mathrm{H}$ & 40.7 & 5.56 & 46.26 & $8 \mathrm{H}$ & 64.6 & 6.58 & 71.18 \\
\hline $7 \mathrm{H}$ & 50.2 & 6.21 & 56.41 & $9 \mathrm{H}$ & 74.1 & 8.03 & 82.13 \\
\hline $8 \mathrm{H}$ & 59.7 & 8.08 & 67.78 & $10 \mathrm{H}$ & 83.6 & 9.00 & 92.60 \\
\hline $9 \mathrm{H}$ & 69.2 & 9.45 & 78.65 & $11 \mathrm{H}$ & 93.1 & 10.12 & 103.22 \\
\hline $10 \mathrm{H}$ & 78.7 & 10.23 & 88.93 & $12 \mathrm{H}$ & 102.6 & 11.59 & 114.19 \\
\hline $11 \mathrm{H}$ & 88.2 & 11.28 & 99.48 & $13 \mathrm{H}$ & 112.1 & 12.97 & 125.07 \\
\hline $12 \mathrm{H}$ & 97.7 & 12.75 & 110.45 & $14 \mathrm{H}$ & 121.6 & 13.90 & 135.50 \\
\hline $13 \mathrm{H}$ & 107.2 & 14.27 & 121.47 & $15 \mathrm{H}$ & 131.1 & 15.20 & 146.30 \\
\hline $14 \mathrm{H}$ & 116.7 & 14.78 & 131.48 & $16 \mathrm{H}$ & 140.6 & 16.53 & 157.13 \\
\hline $15 \mathrm{H}$ & 126.2 & 15.85 & 142.05 & $17 \mathrm{H}$ & 150.1 & 17.79 & 167.89 \\
\hline $16 \mathrm{H}$ & 135.7 & 16.91 & 152.61 & $18 \mathrm{H}$ & 159.6 & 18.56 & 178.16 \\
\hline $17 \mathrm{H}$ & 145.2 & 18.53 & 163.73 & $19 \mathrm{H}$ & 169.1 & 18.60 & 187.70 \\
\hline $18 \mathrm{H}$ & 154.7 & 19.88 & 174.58 & $20 \mathrm{H}$ & 178.6 & 20.03 & 198.63 \\
\hline $19 \mathrm{H}$ & 164.2 & 20.65 & 184.85 & $21 \mathrm{H}$ & 188.1 & 20.75 & 208.85 \\
\hline $20 \mathrm{H}$ & 173.7 & 21.29 & 194.99 & $22 \mathrm{H}$ & 197.6 & 21.62 & 219.22 \\
\hline $21 \mathrm{H}$ & 183.2 & 22.49 & 205.69 & $23 \mathrm{H}$ & 207.1 & 23.39 & 230.49 \\
\hline $22 \mathrm{H}$ & 192.7 & 23.90 & 216.60 & $24 \mathrm{H}$ & 216.6 & 25.21 & 241.81 \\
\hline $23 \mathrm{H}$ & 202.2 & 25.19 & 227.39 & $25 \mathrm{H}$ & 226.1 & 26.71 & 252.81 \\
\hline $24 \mathrm{H}$ & 211.7 & 26.83 & 238.53 & $26 \mathrm{H}$ & 238.1 & 27.53 & 265.63 \\
\hline $25 \mathrm{H}$ & 221.2 & 27.78 & 248.98 & $27 \mathrm{H}$ & 247.6 & 28.36 & 275.96 \\
\hline $26 \mathrm{H}$ & 230.7 & 28.28 & 258.98 & $28 \mathrm{H}$ & 257.1 & 28.05 & 285.15 \\
\hline $27 X$ & 240.2 & 28.13 & 268.33 & $29 \mathrm{H}$ & 266.6 & 28.60 & 295.20 \\
\hline $28 X$ & & 28.13 & 28.13 & $30 \mathrm{H}$ & 276.1 & 29.48 & 305.58 \\
\hline $29 x$ & 259.4 & 29.51 & 288.91 & $31 \mathrm{H}$ & 282.1 & 29.28 & 311.38 \\
\hline $30 x$ & & 29.34 & 29.34 & $32 \mathrm{H}$ & 282.9 & 30.62 & 313.52 \\
\hline $31 x$ & & 29.34 & 29.34 & $33 \mathrm{H}$ & 292.4 & 31.15 & 323.55 \\
\hline $32 x$ & 288.1 & 33.07 & 321.17 & $34 \mathrm{H}$ & 301.9 & 32.43 & 334.33 \\
\hline $33 x$ & 297.7 & 34.32 & 332.02 & $35 \mathrm{H}$ & 311.4 & 33.78 & 345.18 \\
\hline $34 X$ & 307.3 & 35.83 & 343.13 & $36 \mathrm{H}$ & 320.9 & 34.14 & 355.04 \\
\hline $35 x$ & 316.9 & 41.63 & 358.53 & $37 \mathrm{H}$ & 330.4 & 35.18 & 365.58 \\
\hline $36 x$ & 326.5 & 38.62 & 365.12 & $38 \mathrm{H}$ & 339.9 & 37.08 & 376.98 \\
\hline $37 X$ & 336.0 & 31.58 & 367.58 & $39 \mathrm{H}$ & 349.4 & 42.23 & 391.63 \\
\hline $38 \mathrm{X}$ & 345.5 & 31.03 & 376.53 & $40 \mathrm{H}$ & 358.9 & 42.40 & 401.30 \\
\hline $39 x$ & 354.8 & 31.61 & 386.41 & $41 \mathrm{H}$ & 368.4 & 43.67 & 412.07 \\
\hline $40 x$ & 364.4 & 38.71 & 403.11 & $42 \mathrm{H}$ & 377.9 & 44.04 & 421.94 \\
\hline $41 x$ & 374.0 & 47.52 & 421.52 & $43 x$ & 387.4 & 44.41 & 431.81 \\
\hline $42 x$ & 383.6 & 47.12 & 430.72 & $44 X$ & 397.0 & 45.33 & 442.33 \\
\hline $43 x$ & 393.2 & 46.27 & 439.47 & $45 X$ & 406.6 & 45.33 & 451.93 \\
\hline $44 X$ & 402.8 & 45.86 & 448.66 & \multicolumn{4}{|l|}{ 321-U1338C- } \\
\hline 321-U1338B- & & & & $1 \mathrm{H}$ & 0.0 & 0.00 & 0.00 \\
\hline $1 \mathrm{H}$ & 0.0 & 0.00 & 0.00 & $2 \mathrm{H}$ & 3.8 & 0.55 & 4.35 \\
\hline
\end{tabular}

\begin{tabular}{|c|c|c|c|}
\hline Core & $\begin{array}{l}\text { Depth } \\
\text { CSF }(m)\end{array}$ & $\begin{array}{l}\text { Offset } \\
(\mathrm{m})\end{array}$ & $\begin{array}{c}\text { Depth } \\
\text { CCSF }(m)\end{array}$ \\
\hline $3 \mathrm{H}$ & 13.3 & 2.31 & 15.61 \\
\hline $4 \mathrm{H}$ & 22.8 & 2.86 & 25.66 \\
\hline $5 \mathrm{H}$ & 32.3 & 5.26 & 37.56 \\
\hline $6 \mathrm{H}$ & 41.8 & 7.66 & 49.46 \\
\hline $7 \mathrm{H}$ & 51.3 & 6.80 & 58.10 \\
\hline $8 \mathrm{H}$ & 60.8 & 8.98 & 69.78 \\
\hline $9 \mathrm{H}$ & 70.3 & 9.37 & 79.67 \\
\hline $10 \mathrm{H}$ & 79.8 & 10.27 & 90.07 \\
\hline $11 \mathrm{H}$ & 89.3 & 10.25 & 99.55 \\
\hline $12 \mathrm{H}$ & 98.8 & 11.29 & 110.09 \\
\hline $13 \mathrm{H}$ & 108.3 & 11.75 & 120.05 \\
\hline $14 \mathrm{H}$ & 117.8 & 13.58 & 131.38 \\
\hline $15 \mathrm{H}$ & 127.3 & 14.58 & 141.88 \\
\hline $16 \mathrm{H}$ & 136.8 & 16.84 & 153.64 \\
\hline $17 \mathrm{H}$ & 146.3 & 17.53 & 163.83 \\
\hline $18 \mathrm{H}$ & 155.8 & 16.66 & 172.46 \\
\hline $19 \mathrm{H}$ & 161.3 & 19.65 & 180.95 \\
\hline $20 \mathrm{H}$ & 170.8 & 20.12 & 190.92 \\
\hline $21 \mathrm{H}$ & 180.3 & 21.71 & 202.01 \\
\hline $22 \mathrm{H}$ & 189.8 & 22.13 & 211.93 \\
\hline $23 \mathrm{H}$ & 199.3 & 21.98 & 221.28 \\
\hline $24 \mathrm{H}$ & 208.8 & 23.65 & 232.45 \\
\hline $25 \mathrm{H}$ & 218.3 & 25.03 & 243.33 \\
\hline $26 \mathrm{H}$ & 227.8 & 26.24 & 254.04 \\
\hline $27 \mathrm{H}$ & 237.3 & 27.26 & 264.56 \\
\hline $28 \mathrm{H}$ & 246.8 & 27.46 & 274.26 \\
\hline $29 \mathrm{H}$ & 256.3 & 27.46 & 283.76 \\
\hline $30 \mathrm{H}$ & 265.8 & 28.44 & 294.24 \\
\hline $31 \mathrm{H}$ & 275.3 & 29.42 & 304.72 \\
\hline $32 \mathrm{H}$ & 282.9 & 29.42 & 312.32 \\
\hline $33 \mathrm{H}$ & 292.4 & 30.04 & 322.44 \\
\hline $34 \mathrm{H}$ & 301.9 & 28.79 & 330.69 \\
\hline $35 \mathrm{H}$ & 306.9 & 33.13 & 340.03 \\
\hline $36 \mathrm{H}$ & 316.4 & 34.57 & 350.97 \\
\hline $37 \mathrm{H}$ & 325.9 & 36.17 & 362.07 \\
\hline $38 \mathrm{H}$ & 335.4 & 36.79 & 372.19 \\
\hline $39 \mathrm{H}$ & 344.9 & 37.30 & 382.20 \\
\hline $40 \mathrm{H}$ & 354.4 & 37.68 & 392.08 \\
\hline $41 \mathrm{H}$ & 363.9 & 38.29 & 402.19 \\
\hline $42 \mathrm{H}$ & 373.4 & 38.91 & 412.31 \\
\hline $43 \mathrm{H}$ & 377.9 & 40.45 & 418.35 \\
\hline $44 \mathrm{H}$ & 387.4 & 41.21 & 428.61 \\
\hline $45 \mathrm{H}$ & 396.9 & 41.21 & 438.11 \\
\hline $46 \mathrm{H}$ & 404.0 & 41.21 & 445.21 \\
\hline $47 \mathrm{H}$ & 410.5 & 41.21 & 451.71 \\
\hline
\end{tabular}


Table T6. Site U1338 splice tie points. (Continued on next page.)

\begin{tabular}{|c|c|c|c|c|c|c|}
\hline \multirow{2}{*}{$\begin{array}{l}\text { Hole, core, section, } \\
\text { interval }(\mathrm{cm})\end{array}$} & \multicolumn{2}{|c|}{ Depth (m) } & & \multirow{2}{*}{$\begin{array}{l}\text { Hole, core, section, } \\
\text { interval }(\mathrm{cm})\end{array}$} & \multicolumn{2}{|c|}{ Depth $(\mathrm{m})$} \\
\hline & CSF & CCSF & & & CSF & CCSF \\
\hline \multicolumn{7}{|l|}{$321-$} \\
\hline U1338A-1H-2, 47 & 1.97 & 2.01 & Tie to & U1338B-1H-2, 52 & 2.02 & 2.02 \\
\hline U1338B-1H-4, 51 & 5.01 & 5.01 & Tie to & U1338A-2H-1, 83 & 3.53 & 5.01 \\
\hline U1338A-2H-6, 145 & 11.65 & 13.13 & Tie to & U1338B-2H-3, 125 & 11.85 & 13.14 \\
\hline U1338B-2H-7, 10 & 16.7 & 17.99 & Tie to & U1338A-3H-3, 56 & 15.76 & 17.99 \\
\hline U1338A-3H-5, 58 & 18.78 & 21.01 & Tie to & U1338B-3H-2, 35 & 18.95 & 21.02 \\
\hline U1338B-3H-7, 50 & 26.6 & 28.67 & Tie to & U1338A-4H-3, 35 & 25.05 & 28.67 \\
\hline U1338A-4H-6, 124 & 30.44 & 34.06 & Tie to & U1338B-4H-4, 29 & 31.39 & 34.05 \\
\hline U1338B-4H-6, 127 & 35.37 & 38.03 & Tie to & U1338C-5H-1, 47 & 32.77 & 38.03 \\
\hline U1338C-5H-5, 46 & 38.76 & 44.02 & Tie to & U1338B-5H-3, 62 & 39.72 & 44.02 \\
\hline U1338B-5H-7, 40 & 45.5 & 49.8 & Tie to & U1338A-6H-3, 55 & 44.25 & 49.81 \\
\hline U1338A-6H-4, 127 & 46.47 & 52.03 & Tie to & U1338B-6H-1, 128 & 46.88 & 52.02 \\
\hline U1338B-6H-5, 110 & 52.7 & 57.84 & Tie to & U1338A-7H-1, 143 & 51.63 & 57.84 \\
\hline U1338A-7H-4, 110 & 55.8 & 62.01 & Tie to & U1338B-7H-1, 105 & 56.15 & 62.01 \\
\hline U1338B-7H-6, 56 & 63.16 & 69.02 & Tie to & U1338A-8H-1, 125 & 60.95 & 69.03 \\
\hline U1338A-8H-6, 82 & 68.02 & 76.1 & Tie to & U1338B-8H-4, 41 & 69.51 & 76.09 \\
\hline U1338B-8H-6, 131 & 73.41 & 79.99 & Tie to & U1338A-9H-1, 134 & 70.54 & 79.99 \\
\hline U1338A-9H-6, 78 & 77.48 & 86.93 & Tie to & U1338B-9H-4, 30 & 78.9 & 86.93 \\
\hline U1338B-9H-6, 35 & 81.95 & 89.98 & Tie to & U1338A-10H-1, 105 & 79.75 & 89.98 \\
\hline U1338A-10H-6, 55 & 86.75 & 96.98 & Tie to & U1338B-10H-3, 138 & 87.98 & 96.98 \\
\hline U1338B-10H-5, 144 & 91.04 & 100.04 & Tie to & U1338A-11H-1, 56 & 88.76 & 100.04 \\
\hline U1338A-11H-4, 27 & 92.97 & 104.25 & Tie to & U1338B-11H-1, 103 & 94.13 & 104.25 \\
\hline U1338B-11H-7, 22 & 102.12 & 112.24 & Tie to & U1338A-12H-2, 30 & 99.5 & 112.25 \\
\hline U1338A-12H-3, 110 & 101.8 & 114.55 & Tie to & U1338B-12H-1, 36 & 102.96 & 114.55 \\
\hline U1338B-12H-6, 88 & 110.98 & 122.57 & Tie to & U1338A-13H-1, 110 & 108.3 & 122.57 \\
\hline U1338A-13H-3, 104 & 111.24 & 125.51 & Tie to & U1338B-13H-1, 44 & 112.54 & 125.51 \\
\hline U1338B-13H-5, 149 & 119.59 & 132.56 & Tie to & U1338C-14H-1, 118 & 118.98 & 132.56 \\
\hline U1338C-14H-4, 101 & 123.31 & 136.89 & Tie to & U1338B-14H-1, 138 & 122.98 & 136.88 \\
\hline U1338B-14H-6, 84 & 129.94 & 143.84 & Tie to & U1338A-15H-2, 29 & 127.99 & 143.84 \\
\hline U1338A-15H-4, 135 & 132.05 & 147.9 & Tie to & U1338B-15H-2, 11 & 132.71 & 147.91 \\
\hline U1338B-15H-6, 10 & 138.7 & 153.9 & Tie to & U1338A-16H-1, 129 & 136.99 & 153.9 \\
\hline U1338A-16H-5, 128 & 142.98 & 159.89 & Tie to & U1338B-16H-2, 126 & 143.36 & 159.89 \\
\hline U1338B-16H-6, 87 & 148.97 & 165.5 & Tie to & U1338A-17H-2, 27 & 146.97 & 165.5 \\
\hline U1338A-17H-4, 132 & 151.02 & 169.55 & Tie to & U1338B-17H-2, 17 & 151.77 & 169.56 \\
\hline U1338B-17H-6, 122 & 158.82 & 176.61 & Tie to & U1338A-18H-2, 52 & 156.72 & 176.6 \\
\hline U1338A-18H-6, 56 & 162.76 & 182.64 & Tie to & U1338B-18H-3, 148 & 164.08 & 182.64 \\
\hline U1338B-18H-6, 63 & 167.73 & 186.29 & Tie to & U1338A-19H-1, 145 & 165.65 & 186.3 \\
\hline U1338A-19H-6, 6 & 171.76 & 192.41 & Tie to & U1338B-19H-4, 21 & 173.81 & 192.41 \\
\hline U1338B-19H-6, 118 & 177.78 & 196.38 & Tie to & U1338A-20H-1, 138 & 175.08 & 196.37 \\
\hline U1338A-20H-5, 63 & 180.33 & 201.62 & Tie to & U1338B-20H-2, 149 & 181.59 & 201.62 \\
\hline U1338B-20H-6, 127 & 187.37 & 207.4 & Tie to & U1338A-21H-2, 20 & 184.9 & 207.39 \\
\hline U1338A-21H-6, 26 & 190.96 & 213.45 & Tie to & U1338B-21H-4, 10 & 192.7 & 213.45 \\
\hline U1338B-21H-6, 117 & 196.77 & 217.52 & Tie to & U1338A-22H-1, 92 & 193.62 & 217.52 \\
\hline U1338A-22H-3, 101 & 196.71 & 220.61 & Tie to & U1338B-22H-1, 139 & 198.99 & 220.61 \\
\hline U1338B-22H-6, 117 & 206.27 & 227.89 & Tie to & U1338A-23H-1, 50 & 202.7 & 227.89 \\
\hline U1338A-23H-3, 119 & 206.39 & 231.58 & Tie to & U1338B-23H-1, 108 & 208.18 & 231.57 \\
\hline U1338B-23H-6, 140 & 216 & 239.39 & Tie to & U1338A-24H-1, 86 & 212.56 & 239.39 \\
\hline U1338A-24H-4, 62 & 216.82 & 243.65 & Tie to & U1338B-24H-2, 34 & 218.44 & 243.65 \\
\hline U1338B-24H-6, 122 & 225.32 & 250.53 & Tie to & U1338A-25H-2, 3 & 222.74 & 250.52 \\
\hline U1338A-25H-6, 89 & 229.63 & 257.41 & Tie to & U1338B-25H-4, 10 & 230.7 & 257.41 \\
\hline U1338B-25H-6, 137 & 234.97 & 261.68 & Tie to & U1338A-26H-2, 120 & 233.4 & 261.68 \\
\hline U1338A-26H-6, 91 & 239.11 & 267.39 & Tie to & U1338C-27H-2, 133 & 240.13 & 267.39 \\
\hline U1338C-27H-4, 41 & 242.21 & 269.47 & Tie to & U1338B-26H-3, 83 & 241.93 & 269.46 \\
\hline U1338B-26H-6, 146 & 247.06 & 274.59 & Tie to & U1338C-28H-1, 33 & 247.13 & 274.59 \\
\hline U1338C-28H-7, 64 & 256.44 & 283.9 & Append & U1338C-29H-1, 14 & 256.44 & 283.9 \\
\hline $\mathrm{U} 1338 \mathrm{C}-29 \mathrm{H}-2,39$ & 258.19 & 285.65 & Tie to & U1338B-28H-1, 50 & 257.6 & 285.65 \\
\hline U1338B-28H-7, 45 & 266.35 & 294.4 & Tie to & U1338A-29X-4, 99 & 264.89 & 294.4 \\
\hline U1338A-29X-5, 134 & 266.74 & 296.25 & Tie to & U1338B-29H-1, 105 & 267.65 & 296.25 \\
\hline U1338B-29H-7, 73 & 276.23 & 304.83 & Tie to & U1338C-31H-1, 10 & 275.4 & 304.82 \\
\hline U1338C-31H-5, 143 & 282.73 & 312.15 & Append & U1338C-32H-1, 55 & 283.45 & 312.87 \\
\hline U1338C-32H-2, 27 & 284.67 & 314.09 & Tie to & U1338B-32H-1, 57 & 283.47 & 314.09 \\
\hline U1338B-32H-7, 22 & 291.92 & 322.54 & Tie to & U1338C-33H-1, 10 & 292.5 & 322.54 \\
\hline U1338C-33H-2, 69 & 294.59 & 324.63 & Tie to & U1338B-33H-1, 108 & 293.48 & 324.63 \\
\hline U1338B-33H-6, 116 & 301.06 & 332.21 & Tie to & U1338A-33X-1, 19 & 297.89 & 332.21 \\
\hline U1338A-33X-2, 150 & 300.7 & 335.02 & Tie to & U1338B-34H-1, 70 & 302.6 & 335.03 \\
\hline U1338B-34H-4, 131 & 307.73 & 340.16 & Tie to & U1338C-35H-1, 13 & 307.03 & 340.16 \\
\hline U1338C-35H-5, 109 & 314.02 & 347.15 & Tie to & U1338B-35H-2, 48 & 313.38 & 347.16 \\
\hline U1338B-35H-5, 84 & 318.24 & 352.02 & Tie to & U1338C-36H-1, 105 & 317.45 & 352.02 \\
\hline
\end{tabular}


Table T6 (continued).

\begin{tabular}{|c|c|c|c|c|c|c|}
\hline \multirow{2}{*}{$\begin{array}{l}\text { Hole, core, section, } \\
\text { interval }(\mathrm{cm})\end{array}$} & \multicolumn{2}{|c|}{ Depth (m) } & & \multirow{2}{*}{$\begin{array}{l}\text { Hole, core, section, } \\
\text { interval }(\mathrm{cm})\end{array}$} & \multicolumn{2}{|c|}{ Depth (m) } \\
\hline & CSF & CCSF & & & CSF & CCSF \\
\hline U1338C-36H-4, 67 & 321.57 & 356.14 & Tie to & U1338B-36H-1, 110 & 322 & 356.14 \\
\hline U1338B-36H-6, 39 & 328.79 & 362.93 & Tie to & U1338C-37H-1, 86 & 326.76 & 362.93 \\
\hline U1338C-37H-5, 5 & 331.96 & 368.13 & Tie to & U1338B-37H-2, 105 & 332.95 & 368.13 \\
\hline U1338B-37H-6, 29 & 338.19 & 373.37 & Tie to & U1338C-38H-1, 118 & 336.58 & 373.37 \\
\hline U1338C-38H-5, 96 & 342.36 & 379.15 & Tie to & U1338B-38H-2, 66 & 342.06 & 379.14 \\
\hline U1338B-38H-5, 71 & 346.61 & 383.69 & Tie to & U1338C-39H-1, 149 & 346.39 & 383.69 \\
\hline U1338C-39H-7, 72 & 354.62 & 391.92 & Append & U1338C-40H-1, 5 & 354.45 & 392.13 \\
\hline U1338C-40H-7, 65 & 363.65 & 401.33 & Append & U1338B-40H-1, 3 & 358.93 & 401.33 \\
\hline U1338B-40H-2, 53 & 360.93 & 403.33 & Tie to & U1338C-41H-1, 114 & 365.04 & 403.33 \\
\hline U1338C-41H-7, 64 & 373.56 & 411.85 & Append & U1338B-41H-1, 8 & 368.48 & 412.15 \\
\hline U1338B-41H-6, 73 & 376.63 & 420.3 & Tie to & U1338C-43H-2, 46 & 379.86 & 420.31 \\
\hline U1338C-43H-4, 95 & 383.37 & 423.82 & Tie to & U1338B-42H-2, 38 & 379.78 & 423.82 \\
\hline U1338B-42H-5, 100 & 384.93 & 428.97 & Tie to & U1338C-44H-1, 36 & 387.76 & 428.97 \\
\hline U1338C-44H-6, 73 & 395.42 & 436.63 & Append & U1338C-45H-1, 5 & 396.95 & 438.16 \\
\hline U1338C-45H-5, 86 & 403.82 & 445.03 & Append & U1338C-46H-1, 5 & 404.05 & 445.26 \\
\hline U1338C-46H-5, 62 & 410.15 & 451.36 & Append & U1338C-47H-1, 23 & 410.73 & 451.94 \\
\hline U1338C-47H-3, 85 & 414.05 & 455.26 & & & & \\
\hline
\end{tabular}




\section{Appendix}

For each site, in addition to the offset, splice, and stretch tie point tables, we have provided all of the cleaned GRA, MS, NGR, and color reflectance data along with CSF, CCSF, and adjusted CCSF depths (Tables AT1-AT57). Unformatted ASCII versions of these tables are also available (see links from the table notes for each table). 
Table AT1. Gamma ray attenuation (GRA) density data, Site U1336.

\begin{tabular}{|c|c|c|c|}
\hline \multirow{2}{*}{$\begin{array}{l}\text { Core, section, } \\
\text { interval }(\mathrm{cm})\end{array}$} & \multicolumn{2}{|c|}{ Depth (m) } & \multirow{2}{*}{$\begin{array}{c}\text { GRA } \\
\left(\mathrm{g} / \mathrm{cm}^{3}\right)\end{array}$} \\
\hline & CSF & CCSF & \\
\hline \multicolumn{4}{|l|}{ 320-U1336B- } \\
\hline $1 \mathrm{H}-1,3$ & 0.03 & 0.03 & 1.27 \\
\hline $1 \mathrm{H}-1,5$ & 0.05 & 0.05 & 1.33 \\
\hline $1 \mathrm{H}-1,8$ & 0.08 & 0.08 & 1.38 \\
\hline $1 \mathrm{H}-1,10$ & 0.10 & 0.10 & 1.35 \\
\hline $1 \mathrm{H}-1,13$ & 0.13 & 0.13 & 1.34 \\
\hline $1 \mathrm{H}-1,15$ & 0.15 & 0.15 & 1.36 \\
\hline $1 \mathrm{H}-1,18$ & 0.18 & 0.18 & 1.29 \\
\hline $1 \mathrm{H}-1,20$ & 0.20 & 0.20 & 1.31 \\
\hline $1 \mathrm{H}-1,23$ & 0.23 & 0.23 & 1.34 \\
\hline $1 \mathrm{H}-1,25$ & 0.25 & 0.25 & 1.38 \\
\hline $1 \mathrm{H}-1,28$ & 0.28 & 0.28 & 1.40 \\
\hline $1 \mathrm{H}-1,30$ & 0.30 & 0.30 & 1.42 \\
\hline $1 \mathrm{H}-1,33$ & 0.33 & 0.33 & 1.40 \\
\hline $1 \mathrm{H}-1,35$ & 0.35 & 0.35 & 1.42 \\
\hline $1 \mathrm{H}-1,38$ & 0.38 & 0.38 & 1.43 \\
\hline $1 \mathrm{H}-1,40$ & 0.40 & 0.40 & 1.46 \\
\hline $1 \mathrm{H}-1,43$ & 0.43 & 0.43 & 1.45 \\
\hline $1 \mathrm{H}-1,45$ & 0.45 & 0.45 & 1.39 \\
\hline $1 \mathrm{H}-1,48$ & 0.48 & 0.48 & 1.42 \\
\hline $1 \mathrm{H}-1,50$ & 0.50 & 0.50 & 1.42 \\
\hline $1 \mathrm{H}-1,53$ & 0.53 & 0.53 & 1.43 \\
\hline $1 \mathrm{H}-1,55$ & 0.55 & 0.55 & 1.43 \\
\hline $1 \mathrm{H}-1,58$ & 0.58 & 0.58 & 1.40 \\
\hline $1 \mathrm{H}-1,60$ & 0.60 & 0.60 & 1.49 \\
\hline $1 \mathrm{H}-1,63$ & 0.63 & 0.63 & 1.45 \\
\hline $1 \mathrm{H}-1,65$ & 0.65 & 0.65 & 1.50 \\
\hline $1 \mathrm{H}-1,68$ & 0.68 & 0.68 & 1.49 \\
\hline $1 \mathrm{H}-1,70$ & 0.70 & 0.70 & 1.49 \\
\hline $1 \mathrm{H}-1,73$ & 0.73 & 0.73 & 1.50 \\
\hline $1 \mathrm{H}-1,75$ & 0.75 & 0.75 & 1.48 \\
\hline $1 \mathrm{H}-1,78$ & 0.78 & 0.78 & 1.49 \\
\hline $1 \mathrm{H}-1,80$ & 0.80 & 0.80 & 1.50 \\
\hline $1 \mathrm{H}-1,83$ & 0.83 & 0.83 & 1.50 \\
\hline $1 \mathrm{H}-1,85$ & 0.85 & 0.85 & 1.52 \\
\hline $1 \mathrm{H}-1,88$ & 0.88 & 0.88 & 1.52 \\
\hline $1 \mathrm{H}-1,90$ & 0.90 & 0.90 & 1.48 \\
\hline $1 \mathrm{H}-1,93$ & 0.93 & 0.93 & 1.51 \\
\hline $1 \mathrm{H}-1,95$ & 0.95 & 0.95 & 1.51 \\
\hline \multicolumn{4}{|l|}{ 320-U1336A- } \\
\hline $1 \mathrm{H}-1,70$ & 0.70 & 0.96 & 1.49 \\
\hline $1 \mathrm{H}-1,72$ & 0.72 & 0.98 & 1.48 \\
\hline $1 \mathrm{H}-1,74$ & 0.75 & 1.01 & 1.48 \\
\hline $1 \mathrm{H}-1,77$ & 0.77 & 1.03 & 1.47 \\
\hline $1 \mathrm{H}-1,80$ & 0.80 & 1.06 & 1.48 \\
\hline $1 \mathrm{H}-1,82$ & 0.82 & 1.08 & 1.48 \\
\hline $1 \mathrm{H}-1,84$ & 0.85 & 1.11 & 1.48 \\
\hline $1 \mathrm{H}-1,87$ & 0.87 & 1.13 & 1.48 \\
\hline $1 \mathrm{H}-1,90$ & 0.90 & 1.16 & 1.50 \\
\hline $1 \mathrm{H}-1,92$ & 0.92 & 1.18 & 1.48 \\
\hline $1 \mathrm{H}-1,95$ & 0.95 & 1.21 & 1.50 \\
\hline $1 \mathrm{H}-1,97$ & 0.97 & 1.23 & 1.48 \\
\hline $1 \mathrm{H}-1,100$ & 1.00 & 1.26 & 1.48 \\
\hline $1 \mathrm{H}-1,102$ & 1.02 & 1.28 & 1.45 \\
\hline $1 \mathrm{H}-1,105$ & 1.05 & 1.31 & 1.46 \\
\hline $1 \mathrm{H}-1,107$ & 1.07 & 1.33 & 1.45 \\
\hline $1 \mathrm{H}-1,110$ & 1.10 & 1.36 & 1.48 \\
\hline $1 \mathrm{H}-1,112$ & 1.12 & 1.38 & 1.47 \\
\hline $1 \mathrm{H}-1,115$ & 1.15 & 1.41 & 1.50 \\
\hline $1 \mathrm{H}-1,117$ & 1.17 & 1.43 & 1.51 \\
\hline $1 \mathrm{H}-1,120$ & 1.20 & 1.46 & 1.52 \\
\hline $1 \mathrm{H}-1,122$ & 1.22 & 1.48 & 1.51 \\
\hline
\end{tabular}

Only a portion of this table appears here. The complete table is available in ASCII. 
Table AT2. Magnetic susceptibility data, Site U1336.

\begin{tabular}{|c|c|c|c|}
\hline \multirow{2}{*}{$\begin{array}{l}\text { Core, section, } \\
\text { interval }(\mathrm{cm})\end{array}$} & \multicolumn{2}{|c|}{ Depth (m) } & \multirow{2}{*}{$\begin{array}{c}\text { Magnetic } \\
\text { susceptibility } \\
\text { (IU) }\end{array}$} \\
\hline & CSF & CCSF & \\
\hline \multicolumn{4}{|l|}{ 320-U1336B- } \\
\hline $1 \mathrm{H}-1,0$ & 0.00 & 0.00 & 6.30 \\
\hline $1 \mathrm{H}-1,3$ & 0.03 & 0.03 & 16.5 \\
\hline $1 \mathrm{H}-1,5$ & 0.05 & 0.05 & 13.5 \\
\hline $1 \mathrm{H}-1,8$ & 0.08 & 0.08 & 11.5 \\
\hline $1 \mathrm{H}-1,10$ & 0.10 & 0.10 & 12.1 \\
\hline $1 \mathrm{H}-1,13$ & 0.13 & 0.13 & 13.5 \\
\hline $1 \mathrm{H}-1,15$ & 0.15 & 0.15 & 16.5 \\
\hline $1 \mathrm{H}-1,18$ & 0.18 & 0.18 & 15.9 \\
\hline $1 \mathrm{H}-1,20$ & 0.20 & 0.20 & 15.7 \\
\hline $1 \mathrm{H}-1,23$ & 0.23 & 0.23 & 15.4 \\
\hline $1 \mathrm{H}-1,25$ & 0.25 & 0.25 & 15.1 \\
\hline $1 \mathrm{H}-1,28$ & 0.28 & 0.28 & 14.6 \\
\hline $1 \mathrm{H}-1,30$ & 0.30 & 0.30 & 14.3 \\
\hline $1 \mathrm{H}-1,33$ & 0.33 & 0.33 & 13.7 \\
\hline $1 \mathrm{H}-1,35$ & 0.35 & 0.35 & 13.5 \\
\hline $1 \mathrm{H}-1,38$ & 0.38 & 0.38 & 14.0 \\
\hline $1 \mathrm{H}-1,40$ & 0.40 & 0.40 & 14.3 \\
\hline $1 \mathrm{H}-1,43$ & 0.43 & 0.43 & 14.6 \\
\hline $1 \mathrm{H}-1,45$ & 0.45 & 0.45 & 15.9 \\
\hline $1 \mathrm{H}-1,48$ & 0.48 & 0.48 & 16.8 \\
\hline $1 \mathrm{H}-1,50$ & 0.50 & 0.50 & 17.6 \\
\hline $1 \mathrm{H}-1,53$ & 0.53 & 0.53 & 17.0 \\
\hline $1 \mathrm{H}-1,55$ & 0.55 & 0.55 & 16.2 \\
\hline $1 \mathrm{H}-1,58$ & 0.58 & 0.58 & 15.9 \\
\hline $1 \mathrm{H}-1,60$ & 0.60 & 0.60 & 15.4 \\
\hline $1 \mathrm{H}-1,63$ & 0.63 & 0.63 & 14.6 \\
\hline $1 \mathrm{H}-1,65$ & 0.65 & 0.65 & 14.0 \\
\hline $1 \mathrm{H}-1,68$ & 0.68 & 0.68 & 14.0 \\
\hline $1 \mathrm{H}-1,70$ & 0.70 & 0.70 & 14.0 \\
\hline $1 \mathrm{H}-1,73$ & 0.73 & 0.73 & 14.6 \\
\hline $1 \mathrm{H}-1,75$ & 0.75 & 0.75 & 15.7 \\
\hline $1 \mathrm{H}-1,78$ & 0.78 & 0.78 & 15.7 \\
\hline $1 \mathrm{H}-1,80$ & 0.80 & 0.80 & 15.9 \\
\hline $1 \mathrm{H}-1,83$ & 0.83 & 0.83 & 15.1 \\
\hline $1 \mathrm{H}-1,85$ & 0.85 & 0.85 & 14.6 \\
\hline $1 \mathrm{H}-1,88$ & 0.88 & 0.88 & 14.0 \\
\hline $1 \mathrm{H}-1,90$ & 0.90 & 0.90 & 14.8 \\
\hline $1 \mathrm{H}-1,93$ & 0.93 & 0.93 & 14.6 \\
\hline $1 \mathrm{H}-1,95$ & 0.95 & 0.95 & 14.6 \\
\hline \multicolumn{4}{|l|}{ 320-U1336A- } \\
\hline $1 \mathrm{H}-1,70$ & 0.70 & 0.96 & 15.9 \\
\hline $1 \mathrm{H}-1,72$ & 0.72 & 0.98 & 16.5 \\
\hline $1 \mathrm{H}-1,75$ & 0.75 & 1.01 & 16.5 \\
\hline $1 \mathrm{H}-1,77$ & 0.77 & 1.03 & 16.8 \\
\hline $1 \mathrm{H}-1,80$ & 0.80 & 1.06 & 17.0 \\
\hline $1 \mathrm{H}-1,82$ & 0.82 & 1.08 & 16.2 \\
\hline $1 \mathrm{H}-1,85$ & 0.85 & 1.11 & 16.5 \\
\hline $1 \mathrm{H}-1,87$ & 0.87 & 1.13 & 16.2 \\
\hline $1 \mathrm{H}-1,90$ & 0.90 & 1.16 & 16.8 \\
\hline $1 \mathrm{H}-1,92$ & 0.92 & 1.18 & 16.8 \\
\hline $1 \mathrm{H}-1,95$ & 0.95 & 1.21 & 17.0 \\
\hline $1 \mathrm{H}-1,97$ & 0.97 & 1.23 & 17.0 \\
\hline $1 \mathrm{H}-1,100$ & 1.00 & 1.26 & 18.1 \\
\hline $1 \mathrm{H}-1,102$ & 1.02 & 1.28 & 19.0 \\
\hline $1 \mathrm{H}-1,105$ & 1.05 & 1.31 & 19.2 \\
\hline $1 \mathrm{H}-1,107$ & 1.07 & 1.33 & 19.0 \\
\hline $1 \mathrm{H}-1,110$ & 1.10 & 1.36 & 18.4 \\
\hline $1 \mathrm{H}-1,112$ & 1.12 & 1.38 & 18.1 \\
\hline $1 \mathrm{H}-1,115$ & 1.15 & 1.41 & 16.8 \\
\hline
\end{tabular}

Only a portion of this table appears here. The complete table is available in ASCII. 
Table AT3. Natural gamma ray (NGR) data, Site U1336.

\begin{tabular}{|c|c|c|c|}
\hline \multirow{2}{*}{$\begin{array}{l}\text { Hole, core, section, } \\
\text { interval }(\mathrm{cm})\end{array}$} & \multicolumn{2}{|c|}{ Depth (m) } & \multirow{2}{*}{$\begin{array}{l}\text { NGR } \\
(\mathrm{cps})\end{array}$} \\
\hline & CSF & CCSF & \\
\hline \multicolumn{4}{|l|}{$320-$} \\
\hline U1336B-1H-1, 0 & 0.00 & 0.0 & 75.0 \\
\hline U1336B-1H-1, 10 & 0.10 & 0.1 & 65.0 \\
\hline U1336B-1H-1, 20 & 0.20 & 0.2 & 47.7 \\
\hline U1336B-1H-1, 30 & 0.30 & 0.3 & 26.7 \\
\hline U1336B-1H-1, 40 & 0.40 & 0.4 & 13.8 \\
\hline U1336B-1H-1, 50 & 0.50 & 0.5 & 7.9 \\
\hline U1336B-1H-1, 60 & 0.60 & 0.6 & 5.5 \\
\hline U1336B-1H-1, 70 & 0.70 & 0.7 & 4.2 \\
\hline U1336B-1H-1, 80 & 0.80 & 0.8 & 3.9 \\
\hline U1336B-1H-1, 90 & 0.90 & 0.9 & 3.6 \\
\hline U1336A-1H-1, 70 & 0.70 & 0.96 & 3.7 \\
\hline U1336A-1H-1, 70 & 0.70 & 0.96 & 3.7 \\
\hline U1336B-1H-1, 100 & 1.00 & 1.00 & 3.9 \\
\hline U1336A-1H-1, 80 & 0.80 & 1.06 & 4.0 \\
\hline U1336A-1H-1, 80 & 0.80 & 1.06 & 4.0 \\
\hline U1336A-1H-1, 90 & 0.90 & 1.16 & 3.8 \\
\hline U1336A-1H-1, 90 & 0.90 & 1.16 & 3.8 \\
\hline U1336A-1H-1, 100 & 1.00 & 1.26 & 4.6 \\
\hline U1336A-1H-1, 100 & 1.00 & 1.26 & 4.6 \\
\hline U1336A-1H-1, 110 & 1.10 & 1.36 & 3.8 \\
\hline U1336A-1H-1, 110 & 1.10 & 1.36 & 3.8 \\
\hline U1336A-1H-1, 120 & 1.20 & 1.46 & 3.8 \\
\hline U1336A-1H-1, 120 & 1.20 & 1.46 & 3.8 \\
\hline U1336A-1H-1, 130 & 1.30 & 1.56 & 3.3 \\
\hline U1336A-1H-1, 130 & 1.30 & 1.56 & 3.3 \\
\hline U1336A-1H-1, 140 & 1.40 & 1.66 & 3.3 \\
\hline U1336A-1H-1, 140 & 1.40 & 1.66 & 3.3 \\
\hline U1336A-1H-1, 150 & 1.50 & 1.76 & 4.2 \\
\hline U1336A-1H-2, 0 & 1.50 & 1.76 & 3.7 \\
\hline U1336A-1H-2, 10 & 1.60 & 1.86 & 3.4 \\
\hline U1336A-1H-2, 10 & 1.60 & 1.86 & 3.4 \\
\hline U1336A-1H-2, 20 & 1.70 & 1.96 & 3.4 \\
\hline U1336A-1H-2, 20 & 1.70 & 1.96 & 3.4 \\
\hline U1336A-1H-2, 30 & 1.80 & 2.06 & 3.3 \\
\hline U1336A-1H-2, 30 & 1.80 & 2.06 & 3.3 \\
\hline U1336A-1H-2, 40 & 1.90 & 2.16 & 3.6 \\
\hline U1336A-1H-2, 40 & 1.90 & 2.16 & 3.6 \\
\hline U1336A-1H-2, 50 & 2.00 & 2.26 & 4.0 \\
\hline U1336A-1H-2, 50 & 2.00 & 2.26 & 4.0 \\
\hline U1336A-1H-2, 60 & 2.10 & 2.36 & 3.8 \\
\hline U1336A-1H-2, 60 & 2.10 & 2.36 & 3.8 \\
\hline U1336A-1H-2, 70 & 2.20 & 2.46 & 4.2 \\
\hline U1336A-1H-2, 70 & 2.20 & 2.46 & 4.2 \\
\hline U1336A-1H-2, 80 & 2.30 & 2.56 & 4.0 \\
\hline U1336A-1H-2, 80 & 2.30 & 2.56 & 4.0 \\
\hline U1336A-1H-2, 90 & 2.40 & 2.66 & 3.6 \\
\hline U1336A-1H-2, 90 & 2.40 & 2.66 & 3.6 \\
\hline U1336A-1H-2, 100 & 2.50 & 2.76 & 3.3 \\
\hline U1336A-1H-2, 100 & 2.50 & 2.76 & 3.3 \\
\hline U1336A-1H-2, 110 & 2.60 & 2.86 & 3.2 \\
\hline U1336A-1H-2, 110 & 2.60 & 2.86 & 3.2 \\
\hline U1336A-1H-2, 120 & 2.70 & 2.96 & 2.4 \\
\hline U1336A-1H-2, 120 & 2.70 & 2.96 & 2.4 \\
\hline U1336A-1H-2, 130 & 2.80 & 3.06 & 2.4 \\
\hline U1336A-1H-2, 130 & 2.80 & 3.06 & 2.4 \\
\hline U1336A-1H-2, 140 & 2.90 & 3.16 & 2.8 \\
\hline U1336A-1H-2, 140 & 2.90 & 3.16 & 2.8 \\
\hline U1336A-1H-2, 150 & 3.00 & 3.26 & 3.8 \\
\hline U1336A-1H-3, 0 & 3.00 & 3.26 & 2.7 \\
\hline U1336A-1H-2, 150 & 3.00 & 3.26 & 3.8 \\
\hline U1336A-1H-3, 10 & 3.10 & 3.36 & 2.5 \\
\hline U1336A-1H-3, 20 & 3.20 & 3.46 & 2.6 \\
\hline
\end{tabular}

Only a portion of this table appears here. The complete table is available in ASCII. 
Table AT4. Color reflection data, Site U1336.

\begin{tabular}{|c|c|c|c|c|c|}
\hline \multirow{2}{*}{$\begin{array}{l}\text { Hole, core, section, } \\
\text { interval }(\mathrm{cm})\end{array}$} & \multicolumn{2}{|c|}{ Depth (m) } & \multirow[b]{2}{*}{$a^{*}$} & \multirow[b]{2}{*}{$b^{*}$} & \multirow[b]{2}{*}{$L^{*}$} \\
\hline & CSF & CCSF & & & \\
\hline \multicolumn{6}{|l|}{$320-$} \\
\hline U1336B-1H-1, 4 & 0.04 & 0.04 & 4.8 & 17.8 & 62.1 \\
\hline U1336B-1H-1, 5 & 0.05 & 0.05 & 5.1 & 15.0 & 57.6 \\
\hline U1336B-1H-1, 8 & 0.08 & 0.08 & 5.4 & 17.8 & 61.4 \\
\hline U1336B-1H-1, 9 & 0.09 & 0.09 & 5.2 & 16.3 & 58.6 \\
\hline U1336B-1H-1, 10 & 0.10 & 0.10 & 5.3 & 19.9 & 65.1 \\
\hline U1336B-1H-1, 13 & 0.13 & 0.13 & 5.4 & 19.2 & 61.4 \\
\hline U1336B-1H-1, 14 & 0.14 & 0.14 & 5.5 & 19.9 & 65.3 \\
\hline U1336B-1H-1, 16 & 0.16 & 0.16 & 5.4 & 17.5 & 57.8 \\
\hline U1336B-1H-1, 18 & 0.18 & 0.18 & 5.4 & 17.7 & 59.9 \\
\hline U1336B-1H-1, 19 & 0.19 & 0.19 & 5.4 & 18.6 & 62.2 \\
\hline U1336B-1H-1, 21 & 0.21 & 0.21 & 4.6 & 15.0 & 58.7 \\
\hline U1336B-1H-1, 23 & 0.23 & 0.23 & 3.9 & 12.3 & 63.4 \\
\hline U1336B-1H-1, 24 & 0.24 & 0.24 & 4.1 & 14.5 & 62.8 \\
\hline U1336B-1H-1, 26 & 0.26 & 0.26 & 4.6 & 14.9 & 61.3 \\
\hline U1336B-1H-1, 28 & 0.28 & 0.28 & 4.5 & 12.5 & 57.2 \\
\hline U1336B-1H-1, 29 & 0.29 & 0.29 & 4.8 & 16.7 & 65.9 \\
\hline U1336B-1H-1, 31 & 0.31 & 0.31 & 4.7 & 15.4 & 64.5 \\
\hline U1336B-1H-1, 33 & 0.33 & 0.33 & 4.9 & 16.2 & 63.3 \\
\hline U1336B-1H-1, 34 & 0.34 & 0.34 & 4.7 & 16.8 & 63.1 \\
\hline U1336B-1H-1, 36 & 0.36 & 0.36 & 4.9 & 15.0 & 60.9 \\
\hline U1336B-1H-1, 38 & 0.38 & 0.38 & 4.8 & 14.6 & 62.9 \\
\hline U1336B-1H-1, 39 & 0.39 & 0.39 & 4.9 & 15.8 & 62.9 \\
\hline U1336B-1H-1, 41 & 0.41 & 0.41 & 4.9 & 15.6 & 64.2 \\
\hline U1336B-1H-1, 44 & 0.44 & 0.44 & 4.9 & 16.0 & 62.4 \\
\hline U1336B-1H-1, 44 & 0.44 & 0.44 & 4.8 & 14.1 & 64.1 \\
\hline U1336B-1H-1, 46 & 0.46 & 0.46 & 4.5 & 13.9 & 62.7 \\
\hline U1336B-1H-1, 48 & 0.49 & 0.49 & 5.6 & 17.1 & 62.2 \\
\hline U1336B-1H-1, 49 & 0.49 & 0.49 & 5.6 & 17.9 & 63.1 \\
\hline U1336B-1H-1, 51 & 0.51 & 0.51 & 5.5 & 16.6 & 62.3 \\
\hline U1336B-1H-1, 54 & 0.54 & 0.54 & 5.7 & 16.3 & 65.4 \\
\hline U1336B-1H-1, 54 & 0.54 & 0.54 & 4.7 & 15.1 & 63.6 \\
\hline U1336B-1H-1, 56 & 0.56 & 0.56 & 5.6 & 16.0 & 60.9 \\
\hline U1336B-1H-1, 59 & 0.59 & 0.59 & 4.7 & 15.1 & 68.2 \\
\hline U1336B-1H-1, 59 & 0.59 & 0.59 & 5.3 & 14.6 & 64.9 \\
\hline U1336B-1H-1, 64 & 0.64 & 0.64 & 4.7 & 14.4 & 64.3 \\
\hline U1336B-1H-1, 64 & 0.64 & 0.64 & 4.5 & 14.2 & 63.4 \\
\hline U1336B-1H-1, 66 & 0.66 & 0.66 & 4.7 & 13.0 & 67.1 \\
\hline U1336B-1H-1, 69 & 0.69 & 0.69 & 5.1 & 14.8 & 67.6 \\
\hline U1336B-1H-1, 70 & 0.70 & 0.70 & 4.1 & 11.7 & 68.0 \\
\hline U1336B-1H-1, 71 & 0.71 & 0.71 & 5.6 & 16.6 & 64.0 \\
\hline U1336B-1H-1, 74 & 0.74 & 0.74 & 4.9 & 13.9 & 65.0 \\
\hline U1336B-1H-1, 74 & 0.75 & 0.75 & 4.8 & 13.4 & 64.6 \\
\hline U1336B-1H-1, 76 & 0.77 & 0.77 & 5.2 & 14.1 & 64.8 \\
\hline U1336B-1H-1, 79 & 0.79 & 0.79 & 5.4 & 15.9 & 62.0 \\
\hline U1336B-1H-1, 80 & 0.80 & 0.80 & 4.5 & 12.4 & 63.9 \\
\hline U1336B-1H-1, 82 & 0.82 & 0.82 & 5.6 & 16.3 & 62.2 \\
\hline U1336B-1H-1, 84 & 0.84 & 0.84 & 5.3 & 15.5 & 61.2 \\
\hline U1336B-1H-1, 85 & 0.85 & 0.85 & 5.4 & 15.3 & 60.8 \\
\hline U1336B-1H-1, 87 & 0.87 & 0.87 & 5.2 & 15.5 & 64.6 \\
\hline U1336B-1H-1, 89 & 0.89 & 0.89 & 5.0 & 12.7 & 65.2 \\
\hline U1336B-1H-1, 90 & 0.90 & 0.90 & 4.7 & 13.3 & 66.5 \\
\hline U1336B-1H-1, 92 & 0.92 & 0.92 & 5.9 & 16.5 & 61.8 \\
\hline U1336B-1H-1, 94 & 0.94 & 0.94 & 5.8 & 15.9 & 59.7 \\
\hline U1336A-1H-1, 69 & 0.69 & 0.95 & 5.1 & 14.1 & 58.1 \\
\hline U1336B-1H-1, 95 & 0.95 & 0.95 & 5.5 & 15.5 & 60.9 \\
\hline U1336A-1H-1, 74 & 0.74 & 1.00 & 4.9 & 13.8 & 58.4 \\
\hline U1336A-1H-1, 79 & 0.79 & 1.05 & 4.7 & 15.0 & 60.1 \\
\hline U1336A-1H-1, 84 & 0.85 & 1.11 & 5.3 & 15.9 & 57.3 \\
\hline U1336A-1H-1, 90 & 0.90 & 1.16 & 5.7 & 16.3 & 55.9 \\
\hline U1336A-1H-1, 95 & 0.95 & 1.21 & 5.4 & 14.9 & 57.6 \\
\hline U1336A-1H-1, 100 & 1.00 & 1.26 & 5.8 & 15.3 & 52.3 \\
\hline U1336A-1H-1, 105 & 1.05 & 1.31 & 5.0 & 12.2 & 58.6 \\
\hline
\end{tabular}

Only a portion of this table appears here. The complete table is available in ASCII. 
Table AT5. Gamma ray attenuation (GRA) density data, Hole U1336A.

\begin{tabular}{|c|c|c|c|c|}
\hline \multirow[b]{2}{*}{$\begin{array}{l}\text { Core, section, } \\
\text { interval }(\mathrm{cm})\end{array}$} & \multicolumn{3}{|c|}{ Depth (m) } & \multirow[b]{2}{*}{$\begin{array}{c}\text { GRA } \\
\left(\mathrm{g} / \mathrm{cm}^{3}\right)\end{array}$} \\
\hline & CSF & CCSF & $\begin{array}{l}\text { Adjusted } \\
\text { CCSF }\end{array}$ & \\
\hline \multicolumn{5}{|l|}{ 320-U1336A- } \\
\hline $1 \mathrm{H}-1,4$ & 0.04 & 0.30 & 0.07 & 1.30 \\
\hline $1 \mathrm{H}-1,7$ & 0.07 & 0.33 & 0.10 & 1.32 \\
\hline $1 \mathrm{H}-1,9$ & 0.09 & 0.35 & 0.12 & 1.29 \\
\hline $1 \mathrm{H}-1,12$ & 0.12 & 0.38 & 0.14 & 1.25 \\
\hline $1 \mathrm{H}-1,14$ & 0.14 & 0.40 & 0.16 & 1.22 \\
\hline $1 \mathrm{H}-1,17$ & 0.17 & 0.43 & 0.19 & 1.15 \\
\hline $1 \mathrm{H}-1,19$ & 0.19 & 0.45 & 0.22 & 1.20 \\
\hline $1 \mathrm{H}-1,22$ & 0.22 & 0.48 & 0.26 & 1.26 \\
\hline $1 \mathrm{H}-1,24$ & 0.24 & 0.50 & 0.29 & 1.28 \\
\hline $1 \mathrm{H}-1,27$ & 0.27 & 0.53 & 0.34 & 1.29 \\
\hline $1 \mathrm{H}-1,29$ & 0.29 & 0.55 & 0.36 & 1.34 \\
\hline $1 \mathrm{H}-1,32$ & 0.32 & 0.58 & 0.41 & 1.35 \\
\hline $1 \mathrm{H}-1,34$ & 0.35 & 0.61 & 0.45 & 1.36 \\
\hline $1 \mathrm{H}-1,37$ & 0.37 & 0.63 & 0.48 & 1.36 \\
\hline $1 \mathrm{H}-1,40$ & 0.40 & 0.66 & 0.52 & 1.39 \\
\hline $1 \mathrm{H}-1,42$ & 0.42 & 0.68 & 0.55 & 1.40 \\
\hline $1 \mathrm{H}-1,44$ & 0.45 & 0.71 & 0.60 & 1.42 \\
\hline $1 \mathrm{H}-1,47$ & 0.47 & 0.73 & 0.63 & 1.45 \\
\hline $1 \mathrm{H}-1,50$ & 0.50 & 0.76 & 0.67 & 1.47 \\
\hline $1 \mathrm{H}-1,52$ & 0.52 & 0.78 & 0.70 & 1.44 \\
\hline $1 \mathrm{H}-1,54$ & 0.55 & 0.81 & 0.74 & 1.45 \\
\hline $1 \mathrm{H}-1,57$ & 0.57 & 0.83 & 0.77 & 1.46 \\
\hline $1 \mathrm{H}-1,60$ & 0.60 & 0.86 & 0.82 & 1.50 \\
\hline $1 \mathrm{H}-1,62$ & 0.62 & 0.88 & 0.84 & 1.49 \\
\hline $1 \mathrm{H}-1,64$ & 0.65 & 0.91 & 0.89 & 1.49 \\
\hline $1 \mathrm{H}-1,67$ & 0.67 & 0.93 & 0.92 & 1.50 \\
\hline $1 \mathrm{H}-1,70$ & 0.70 & 0.96 & 0.96 & 1.49 \\
\hline $1 \mathrm{H}-1,72$ & 0.72 & 0.98 & 0.98 & 1.48 \\
\hline $1 \mathrm{H}-1,74$ & 0.75 & 1.01 & 1.01 & 1.48 \\
\hline $1 \mathrm{H}-1,77$ & 0.77 & 1.03 & 1.03 & 1.47 \\
\hline $1 \mathrm{H}-1,80$ & 0.80 & 1.06 & 1.06 & 1.48 \\
\hline $1 \mathrm{H}-1,82$ & 0.82 & 1.08 & 1.08 & 1.48 \\
\hline $1 \mathrm{H}-1,84$ & 0.85 & 1.11 & 1.11 & 1.48 \\
\hline $1 \mathrm{H}-1,87$ & 0.87 & 1.13 & 1.13 & 1.48 \\
\hline $1 \mathrm{H}-1,90$ & 0.90 & 1.16 & 1.16 & 1.50 \\
\hline $1 \mathrm{H}-1,92$ & 0.92 & 1.18 & 1.18 & 1.48 \\
\hline $1 \mathrm{H}-1,95$ & 0.95 & 1.21 & 1.21 & 1.50 \\
\hline $1 \mathrm{H}-1,97$ & 0.97 & 1.23 & 1.23 & 1.48 \\
\hline $1 \mathrm{H}-1,100$ & 1.00 & 1.26 & 1.26 & 1.48 \\
\hline $1 \mathrm{H}-1,102$ & 1.02 & 1.28 & 1.28 & 1.45 \\
\hline $1 \mathrm{H}-1,105$ & 1.05 & 1.31 & 1.31 & 1.46 \\
\hline $1 \mathrm{H}-1,107$ & 1.07 & 1.33 & 1.33 & 1.45 \\
\hline $1 \mathrm{H}-1,110$ & 1.10 & 1.36 & 1.36 & 1.48 \\
\hline $1 \mathrm{H}-1,112$ & 1.12 & 1.38 & 1.38 & 1.47 \\
\hline $1 \mathrm{H}-1,115$ & 1.15 & 1.41 & 1.41 & 1.50 \\
\hline $1 \mathrm{H}-1,117$ & 1.17 & 1.43 & 1.43 & 1.51 \\
\hline $1 \mathrm{H}-1,120$ & 1.20 & 1.46 & 1.46 & 1.52 \\
\hline $1 \mathrm{H}-1,122$ & 1.22 & 1.48 & 1.48 & 1.51 \\
\hline $1 \mathrm{H}-1,125$ & 1.25 & 1.51 & 1.51 & 1.51 \\
\hline $1 \mathrm{H}-1,127$ & 1.27 & 1.53 & 1.53 & 1.52 \\
\hline $1 \mathrm{H}-1,130$ & 1.30 & 1.56 & 1.56 & 1.51 \\
\hline $1 \mathrm{H}-1,132$ & 1.32 & 1.58 & 1.58 & 1.54 \\
\hline $1 \mathrm{H}-1,135$ & 1.35 & 1.61 & 1.61 & 1.52 \\
\hline $1 \mathrm{H}-1,137$ & 1.37 & 1.63 & 1.63 & 1.52 \\
\hline $1 \mathrm{H}-1,140$ & 1.40 & 1.66 & 1.66 & 1.53 \\
\hline $1 \mathrm{H}-1,142$ & 1.42 & 1.68 & 1.68 & 1.56 \\
\hline $1 \mathrm{H}-1,145$ & 1.45 & 1.71 & 1.71 & 1.53 \\
\hline $1 \mathrm{H}-1,147$ & 1.47 & 1.73 & 1.73 & 1.54 \\
\hline $1 \mathrm{H}-1,150$ & 1.50 & 1.76 & 1.76 & 1.54 \\
\hline
\end{tabular}

Only a portion of this table appears here. The complete table is available in ASCII. 
Table AT6. Magnetic susceptibility data, Hole U1336A.

\begin{tabular}{|c|c|c|c|c|}
\hline \multirow[b]{2}{*}{$\begin{array}{l}\text { Core, section, } \\
\text { interval }(\mathrm{cm})\end{array}$} & \multicolumn{3}{|c|}{ Depth $(m)$} & \multirow{2}{*}{$\begin{array}{l}\text { Magnetic } \\
\text { susceptibility } \\
\text { (IU) }\end{array}$} \\
\hline & CSF & CCSF & $\begin{array}{l}\text { Adjusted } \\
\text { CCSF }\end{array}$ & \\
\hline \multicolumn{5}{|l|}{ 320-U1336A- } \\
\hline $1 \mathrm{H}-1,2$ & 0.02 & 0.28 & 0.06 & 4.9 \\
\hline $1 \mathrm{H}-1,4$ & 0.04 & 0.30 & 0.07 & 10.7 \\
\hline $1 \mathrm{H}-1,7$ & 0.07 & 0.33 & 0.10 & 12.1 \\
\hline $1 \mathrm{H}-1,10$ & 0.09 & 0.35 & 0.12 & 13.2 \\
\hline $1 \mathrm{H}-1,12$ & 0.12 & 0.38 & 0.14 & 17.9 \\
\hline $1 \mathrm{H}-1,14$ & 0.14 & 0.40 & 0.16 & 17.0 \\
\hline $1 \mathrm{H}-1,17$ & 0.17 & 0.43 & 0.19 & 15.9 \\
\hline $1 \mathrm{H}-1,20$ & 0.19 & 0.45 & 0.22 & 16.5 \\
\hline $1 \mathrm{H}-1,22$ & 0.22 & 0.48 & 0.26 & 17.6 \\
\hline $1 \mathrm{H}-1,24$ & 0.24 & 0.50 & 0.29 & 17.9 \\
\hline $1 \mathrm{H}-1,27$ & 0.27 & 0.53 & 0.34 & 17.9 \\
\hline $1 \mathrm{H}-1,30$ & 0.30 & 0.56 & 0.38 & 17.9 \\
\hline $1 \mathrm{H}-1,32$ & 0.32 & 0.58 & 0.41 & 17.3 \\
\hline $1 \mathrm{H}-1,34$ & 0.35 & 0.61 & 0.45 & 16.5 \\
\hline $1 \mathrm{H}-1,37$ & 0.37 & 0.63 & 0.48 & 15.9 \\
\hline $1 \mathrm{H}-1,40$ & 0.40 & 0.66 & 0.52 & 15.4 \\
\hline $1 \mathrm{H}-1,42$ & 0.42 & 0.68 & 0.55 & 15.1 \\
\hline $1 \mathrm{H}-1,44$ & 0.45 & 0.71 & 0.60 & 15.1 \\
\hline $1 \mathrm{H}-1,47$ & 0.47 & 0.73 & 0.63 & 15.4 \\
\hline $1 \mathrm{H}-1,50$ & 0.50 & 0.76 & 0.67 & 16.2 \\
\hline $1 \mathrm{H}-1,52$ & 0.52 & 0.78 & 0.70 & 16.8 \\
\hline $1 \mathrm{H}-1,54$ & 0.55 & 0.81 & 0.74 & 16.5 \\
\hline $1 \mathrm{H}-1,57$ & 0.57 & 0.83 & 0.77 & 15.9 \\
\hline $1 \mathrm{H}-1,60$ & 0.60 & 0.86 & 0.82 & 15.4 \\
\hline $1 \mathrm{H}-1,62$ & 0.62 & 0.88 & 0.84 & 15.4 \\
\hline $1 \mathrm{H}-1,65$ & 0.65 & 0.91 & 0.89 & 15.4 \\
\hline $1 \mathrm{H}-1,67$ & 0.67 & 0.93 & 0.92 & 15.7 \\
\hline $1 \mathrm{H}-1,70$ & 0.70 & 0.96 & 0.96 & 15.9 \\
\hline $1 \mathrm{H}-1,72$ & 0.72 & 0.98 & 0.98 & 16.5 \\
\hline $1 \mathrm{H}-1,75$ & 0.75 & 1.01 & 1.01 & 16.5 \\
\hline $1 \mathrm{H}-1,77$ & 0.77 & 1.03 & 1.03 & 16.8 \\
\hline $1 \mathrm{H}-1,80$ & 0.80 & 1.06 & 1.06 & 17.0 \\
\hline $1 \mathrm{H}-1,82$ & 0.82 & 1.08 & 1.08 & 16.2 \\
\hline $1 \mathrm{H}-1,85$ & 0.85 & 1.11 & 1.11 & 16.5 \\
\hline $1 \mathrm{H}-1,87$ & 0.87 & 1.13 & 1.13 & 16.2 \\
\hline $1 \mathrm{H}-1,90$ & 0.90 & 1.16 & 1.16 & 16.8 \\
\hline $1 \mathrm{H}-1,92$ & 0.92 & 1.18 & 1.18 & 16.8 \\
\hline $1 \mathrm{H}-1,95$ & 0.95 & 1.21 & 1.21 & 17.0 \\
\hline $1 \mathrm{H}-1,97$ & 0.97 & 1.23 & 1.23 & 17.0 \\
\hline $1 \mathrm{H}-1,100$ & 1.00 & 1.26 & 1.26 & 18.1 \\
\hline $1 \mathrm{H}-1,102$ & 1.02 & 1.28 & 1.28 & 19.0 \\
\hline $1 \mathrm{H}-1,105$ & 1.05 & 1.31 & 1.31 & 19.2 \\
\hline $1 \mathrm{H}-1,107$ & 1.07 & 1.33 & 1.33 & 19.0 \\
\hline $1 \mathrm{H}-1,110$ & 1.10 & 1.36 & 1.36 & 18.4 \\
\hline $1 \mathrm{H}-1,112$ & 1.12 & 1.38 & 1.38 & 18.1 \\
\hline $1 \mathrm{H}-1,115$ & 1.15 & 1.41 & 1.41 & 16.8 \\
\hline $1 \mathrm{H}-1,117$ & 1.17 & 1.43 & 1.43 & 16.8 \\
\hline $1 \mathrm{H}-1,120$ & 1.20 & 1.46 & 1.46 & 15.9 \\
\hline $1 \mathrm{H}-1,122$ & 1.22 & 1.48 & 1.48 & 15.7 \\
\hline $1 \mathrm{H}-1,125$ & 1.25 & 1.51 & 1.51 & 15.4 \\
\hline $1 \mathrm{H}-1,127$ & 1.27 & 1.53 & 1.53 & 15.9 \\
\hline $1 \mathrm{H}-1,130$ & 1.30 & 1.56 & 1.56 & 15.7 \\
\hline $1 \mathrm{H}-1,132$ & 1.32 & 1.58 & 1.58 & 15.7 \\
\hline $1 \mathrm{H}-1,135$ & 1.35 & 1.61 & 1.61 & 15.1 \\
\hline $1 \mathrm{H}-1,137$ & 1.37 & 1.63 & 1.63 & 14.3 \\
\hline $1 \mathrm{H}-1,140$ & 1.40 & 1.66 & 1.66 & 13.7 \\
\hline $1 \mathrm{H}-1,142$ & 1.42 & 1.68 & 1.68 & 13.2 \\
\hline $1 \mathrm{H}-1,145$ & 1.45 & 1.71 & 1.71 & 13.2 \\
\hline $1 \mathrm{H}-1,147$ & 1.47 & 1.73 & 1.73 & 12.9 \\
\hline $1 \mathrm{H}-1,150$ & 1.50 & 1.76 & 1.76 & 12.1 \\
\hline
\end{tabular}

Only a portion of this table appears here. The complete table is available in ASCII. 
Table AT7. Natural gamma ray (NGR) data, Hole U1336A.

\begin{tabular}{|c|c|c|c|c|}
\hline \multirow[b]{2}{*}{$\begin{array}{l}\text { Core, section, } \\
\text { interval }(\mathrm{cm})\end{array}$} & \multicolumn{3}{|c|}{ Depth (m) } & \multirow[b]{2}{*}{$\begin{array}{l}\text { NGR } \\
\text { (cps) }\end{array}$} \\
\hline & CSF & CCSF & $\begin{array}{l}\text { Adjusted } \\
\text { CCSF }\end{array}$ & \\
\hline \multicolumn{5}{|l|}{ 320-U1336A- } \\
\hline $1 \mathrm{H}-1,0$ & 0.0 & 0.26 & 0.04 & 69.7 \\
\hline $1 \mathrm{H}-1,0$ & 0.0 & 0.26 & 0.04 & 69.7 \\
\hline $1 \mathrm{H}-1,10$ & 0.1 & 0.36 & 0.13 & 55.6 \\
\hline $1 \mathrm{H}-1,10$ & 0.1 & 0.36 & 0.13 & 55.6 \\
\hline $1 \mathrm{H}-1,20$ & 0.2 & 0.46 & 0.23 & 38.9 \\
\hline $1 \mathrm{H}-1,20$ & 0.2 & 0.46 & 0.23 & 38.9 \\
\hline $1 \mathrm{H}-1,30$ & 0.3 & 0.56 & 0.38 & 21.7 \\
\hline $1 \mathrm{H}-1,30$ & 0.3 & 0.56 & 0.38 & 21.7 \\
\hline $1 \mathrm{H}-1,40$ & 0.4 & 0.66 & 0.52 & 11.0 \\
\hline $1 \mathrm{H}-1,40$ & 0.4 & 0.66 & 0.52 & 11.0 \\
\hline $1 \mathrm{H}-1,50$ & 0.5 & 0.76 & 0.67 & 5.8 \\
\hline $1 \mathrm{H}-1,50$ & 0.5 & 0.76 & 0.67 & 5.8 \\
\hline $1 \mathrm{H}-1,60$ & 0.6 & 0.86 & 0.82 & 4.3 \\
\hline $1 \mathrm{H}-1,60$ & 0.6 & 0.86 & 0.82 & 4.3 \\
\hline $1 \mathrm{H}-1,70$ & 0.7 & 0.96 & 0.96 & 3.7 \\
\hline $1 \mathrm{H}-1,70$ & 0.7 & 0.96 & 0.96 & 3.7 \\
\hline $1 \mathrm{H}-1,80$ & 0.8 & 1.06 & 1.06 & 4.0 \\
\hline $1 \mathrm{H}-1,80$ & 0.8 & 1.06 & 1.06 & 4.0 \\
\hline $1 \mathrm{H}-1,90$ & 0.9 & 1.16 & 1.16 & 3.8 \\
\hline $1 \mathrm{H}-1,90$ & 0.9 & 1.16 & 1.16 & 3.8 \\
\hline $1 \mathrm{H}-1,100$ & 1.0 & 1.26 & 1.26 & 4.6 \\
\hline $1 \mathrm{H}-1,100$ & 1.0 & 1.26 & 1.26 & 4.6 \\
\hline $1 \mathrm{H}-1,110$ & 1.1 & 1.36 & 1.36 & 3.8 \\
\hline $1 \mathrm{H}-1,110$ & 1.1 & 1.36 & 1.36 & 3.8 \\
\hline $1 \mathrm{H}-1,120$ & 1.2 & 1.46 & 1.46 & 3.8 \\
\hline $1 \mathrm{H}-1,120$ & 1.2 & 1.46 & 1.46 & 3.8 \\
\hline $1 \mathrm{H}-1,130$ & 1.3 & 1.56 & 1.56 & 3.3 \\
\hline $1 \mathrm{H}-1,130$ & 1.3 & 1.56 & 1.56 & 3.3 \\
\hline $1 \mathrm{H}-1,140$ & 1.4 & 1.66 & 1.66 & 3.3 \\
\hline $1 \mathrm{H}-1,140$ & 1.4 & 1.66 & 1.66 & 3.3 \\
\hline $1 \mathrm{H}-1,150$ & 1.5 & 1.76 & 1.76 & 4.2 \\
\hline $1 \mathrm{H}-2,0$ & 1.5 & 1.76 & 1.76 & 3.7 \\
\hline $1 \mathrm{H}-2,10$ & 1.6 & 1.86 & 1.86 & 3.4 \\
\hline $1 \mathrm{H}-2,10$ & 1.6 & 1.86 & 1.86 & 3.4 \\
\hline $1 \mathrm{H}-2,20$ & 1.7 & 1.96 & 1.96 & 3.4 \\
\hline $1 \mathrm{H}-2,20$ & 1.7 & 1.96 & 1.96 & 3.4 \\
\hline $1 \mathrm{H}-2,30$ & 1.8 & 2.06 & 2.06 & 3.3 \\
\hline $1 \mathrm{H}-2,30$ & 1.8 & 2.06 & 2.06 & 3.3 \\
\hline $1 \mathrm{H}-2,40$ & 1.9 & 2.16 & 2.16 & 3.6 \\
\hline $1 \mathrm{H}-2,40$ & 1.9 & 2.16 & 2.16 & 3.6 \\
\hline $1 \mathrm{H}-2,50$ & 2.0 & 2.26 & 2.26 & 4.0 \\
\hline $1 \mathrm{H}-2,50$ & 2.0 & 2.26 & 2.26 & 4.0 \\
\hline $1 \mathrm{H}-2,60$ & 2.1 & 2.36 & 2.36 & 3.8 \\
\hline $1 \mathrm{H}-2,60$ & 2.1 & 2.36 & 2.36 & 3.8 \\
\hline $1 \mathrm{H}-2,70$ & 2.2 & 2.46 & 2.46 & 4.2 \\
\hline $1 \mathrm{H}-2,70$ & 2.2 & 2.46 & 2.46 & 4.2 \\
\hline $1 \mathrm{H}-2,80$ & 2.3 & 2.56 & 2.56 & 4.0 \\
\hline $1 \mathrm{H}-2,80$ & 2.3 & 2.56 & 2.56 & 4.0 \\
\hline $1 \mathrm{H}-2,90$ & 2.4 & 2.66 & 2.66 & 3.6 \\
\hline $1 \mathrm{H}-2,90$ & 2.4 & 2.66 & 2.66 & 3.6 \\
\hline $1 \mathrm{H}-2,100$ & 2.5 & 2.76 & 2.76 & 3.3 \\
\hline $1 \mathrm{H}-2,100$ & 2.5 & 2.76 & 2.76 & 3.3 \\
\hline $1 \mathrm{H}-2,110$ & 2.6 & 2.86 & 2.86 & 3.2 \\
\hline $1 \mathrm{H}-2,110$ & 2.6 & 2.86 & 2.86 & 3.2 \\
\hline $1 \mathrm{H}-2,120$ & 2.7 & 2.96 & 2.96 & 2.4 \\
\hline $1 \mathrm{H}-2,120$ & 2.7 & 2.96 & 2.96 & 2.4 \\
\hline $1 \mathrm{H}-2,130$ & 2.8 & 3.06 & 3.06 & 2.4 \\
\hline $1 \mathrm{H}-2,130$ & 2.8 & 3.06 & 3.06 & 2.4 \\
\hline $1 \mathrm{H}-2,140$ & 2.9 & 3.16 & 3.16 & 2.8 \\
\hline $1 \mathrm{H}-2,140$ & 2.9 & 3.16 & 3.16 & 2.8 \\
\hline $1 \mathrm{H}-2,150$ & 3.0 & 3.26 & 3.26 & 3.8 \\
\hline $1 \mathrm{H}-3,0$ & 3.0 & 3.26 & 3.26 & 2.7 \\
\hline
\end{tabular}

Only a portion of this table appears here. The complete table is available in ASCII. 
Table AT8. Color reflection data, Hole U1336A.

\begin{tabular}{|c|c|c|c|c|c|c|}
\hline \multirow[b]{2}{*}{$\begin{array}{l}\text { Core, section, } \\
\text { interval (cm) }\end{array}$} & \multicolumn{3}{|c|}{ Depth (m) } & \multirow[b]{2}{*}{$a^{*}$} & \multirow[b]{2}{*}{$b^{*}$} & \multirow[b]{2}{*}{$L^{*}$} \\
\hline & CSF & CCSF & $\begin{array}{l}\text { Adjusted } \\
\text { CCSF }\end{array}$ & & & \\
\hline \multicolumn{7}{|l|}{ 320-U1336A- } \\
\hline $1 \mathrm{H}-1,8.8$ & 0.09 & 0.35 & 0.12 & 4.9 & 7.7 & 93.2 \\
\hline $1 \mathrm{H}-1,13.9$ & 0.14 & 0.40 & 0.16 & 5.9 & 7.9 & 92.6 \\
\hline $1 \mathrm{H}-1,18.9$ & 0.19 & 0.45 & 0.22 & 5.7 & 8.5 & 92.5 \\
\hline $1 \mathrm{H}-1,24$ & 0.24 & 0.50 & 0.29 & 5.4 & 8.6 & 92.4 \\
\hline $1 \mathrm{H}-1,29$ & 0.29 & 0.55 & 0.36 & 5.1 & 7.9 & 91.6 \\
\hline $1 \mathrm{H}-1,34.1$ & 0.34 & 0.60 & 0.44 & 5.0 & 8.3 & 91.3 \\
\hline $1 \mathrm{H}-1,39.1$ & 0.39 & 0.65 & 0.51 & 4.6 & 8.8 & 90.6 \\
\hline $1 \mathrm{H}-1,44.2$ & 0.44 & 0.70 & 0.58 & 5.0 & 10.6 & 90.1 \\
\hline $1 \mathrm{H}-1,49.2$ & 0.49 & 0.75 & 0.66 & 5.1 & 10.1 & 90.0 \\
\hline $1 \mathrm{H}-1,54.2$ & 0.54 & 0.80 & 0.73 & 4.7 & 8.7 & 89.7 \\
\hline $1 \mathrm{H}-1,59.3$ & 0.59 & 0.85 & 0.80 & 4.9 & 9.5 & 89.3 \\
\hline $1 \mathrm{H}-1,64.3$ & 0.64 & 0.90 & 0.87 & 5.3 & 10.3 & 89.3 \\
\hline $1 \mathrm{H}-1,69.4$ & 0.69 & 0.95 & 0.95 & 5.1 & 9.6 & 89.3 \\
\hline $1 \mathrm{H}-1,74.4$ & 0.74 & 1.00 & 1.00 & 4.9 & 9.9 & 89.2 \\
\hline $1 \mathrm{H}-1,79.4$ & 0.79 & 1.05 & 1.05 & 4.7 & 9.9 & 89.2 \\
\hline $1 \mathrm{H}-1,84.5$ & 0.85 & 1.11 & 1.11 & 5.3 & 9.8 & 89.1 \\
\hline $1 \mathrm{H}-1,89.5$ & 0.90 & 1.16 & 1.16 & 5.7 & 10.3 & 89.1 \\
\hline $1 \mathrm{H}-1,94.6$ & 0.95 & 1.21 & 1.21 & 5.4 & 10.3 & 89.1 \\
\hline $1 \mathrm{H}-1,99.6$ & 1.00 & 1.26 & 1.26 & 5.8 & 10.6 & 89.0 \\
\hline $1 \mathrm{H}-1,104.7$ & 1.05 & 1.31 & 1.31 & 5.0 & 9.5 & 89.0 \\
\hline $1 \mathrm{H}-1,109.7$ & 1.10 & 1.36 & 1.36 & 4.9 & 10.1 & 88.9 \\
\hline $1 \mathrm{H}-1,114.7$ & 1.15 & 1.41 & 1.41 & 5.1 & 9.4 & 88.9 \\
\hline $1 \mathrm{H}-1,119.8$ & 1.20 & 1.46 & 1.46 & 4.9 & 10.1 & 88.8 \\
\hline $1 \mathrm{H}-1,124.8$ & 1.25 & 1.51 & 1.51 & 4.7 & 9.1 & 88.8 \\
\hline $1 \mathrm{H}-1,129.9$ & 1.30 & 1.56 & 1.56 & 4.6 & 10.3 & 88.8 \\
\hline $1 \mathrm{H}-1,134.9$ & 1.35 & 1.61 & 1.61 & 4.6 & 8.7 & 88.7 \\
\hline $1 \mathrm{H}-1,140$ & 1.40 & 1.66 & 1.66 & 5.1 & 10.4 & 88.5 \\
\hline $1 \mathrm{H}-1,145$ & 1.45 & 1.71 & 1.71 & 4.8 & 9.9 & 88.4 \\
\hline $1 \mathrm{H}-2,3.8$ & 1.54 & 1.80 & 1.80 & 4.6 & 10.5 & 88.3 \\
\hline $1 \mathrm{H}-2,8.8$ & 1.59 & 1.85 & 1.85 & 4.7 & 10.5 & 88.3 \\
\hline $1 \mathrm{H}-2,13.9$ & 1.64 & 1.90 & 1.90 & 4.9 & 10.1 & 88.2 \\
\hline $1 \mathrm{H}-2,18.9$ & 1.69 & 1.95 & 1.95 & 4.8 & 9.6 & 88.1 \\
\hline $1 \mathrm{H}-2,24$ & 1.74 & 2.00 & 2.00 & 4.3 & 10.4 & 88.0 \\
\hline $1 \mathrm{H}-2,29$ & 1.79 & 2.05 & 2.05 & 4.2 & 10.1 & 88.0 \\
\hline $1 \mathrm{H}-2,34.1$ & 1.84 & 2.10 & 2.10 & 4.1 & 10.0 & 87.9 \\
\hline $1 \mathrm{H}-2,39.1$ & 1.89 & 2.15 & 2.15 & 4.6 & 8.7 & 87.8 \\
\hline $1 \mathrm{H}-2,44.2$ & 1.94 & 2.20 & 2.20 & 4.2 & 10.1 & 87.8 \\
\hline $1 \mathrm{H}-2,49.2$ & 1.99 & 2.25 & 2.25 & 5.1 & 10.0 & 87.8 \\
\hline $1 \mathrm{H}-2,54.2$ & 2.04 & 2.30 & 2.30 & 4.3 & 9.8 & 87.7 \\
\hline $1 \mathrm{H}-2,59.3$ & 2.09 & 2.35 & 2.35 & 4.8 & 9.7 & 87.7 \\
\hline $1 \mathrm{H}-2,64.3$ & 2.14 & 2.40 & 2.40 & 4.9 & 9.0 & 87.6 \\
\hline $1 \mathrm{H}-2,69.4$ & 2.19 & 2.45 & 2.45 & 5.3 & 10.6 & 87.6 \\
\hline $1 \mathrm{H}-2,74.4$ & 2.24 & 2.50 & 2.50 & 5.1 & 8.9 & 87.6 \\
\hline $1 \mathrm{H}-2,79.4$ & 2.29 & 2.55 & 2.55 & 5.3 & 11.2 & 87.5 \\
\hline $1 \mathrm{H}-2,84.5$ & 2.35 & 2.61 & 2.61 & 5.2 & 11.0 & 87.5 \\
\hline $1 \mathrm{H}-2,89.5$ & 2.40 & 2.66 & 2.66 & 4.5 & 10.8 & 87.5 \\
\hline $1 \mathrm{H}-2,94.6$ & 2.45 & 2.71 & 2.71 & 4.8 & 10.9 & 87.5 \\
\hline $1 \mathrm{H}-2,99.6$ & 2.50 & 2.76 & 2.76 & 4.2 & 11.1 & 87.3 \\
\hline $1 \mathrm{H}-2,104.7$ & 2.55 & 2.81 & 2.81 & 3.8 & 8.6 & 87.2 \\
\hline $1 \mathrm{H}-2,109.7$ & 2.60 & 2.86 & 2.86 & 3.9 & 11.2 & 87.2 \\
\hline $1 \mathrm{H}-2,114.7$ & 2.65 & 2.91 & 2.91 & 3.4 & 8.4 & 87.1 \\
\hline $1 \mathrm{H}-2,119.8$ & 2.70 & 2.96 & 2.96 & 2.3 & 8.4 & 86.9 \\
\hline $1 \mathrm{H}-2,124.8$ & 2.75 & 3.01 & 3.01 & 2.1 & 7.7 & 86.9 \\
\hline $1 \mathrm{H}-2,129.9$ & 2.80 & 3.06 & 3.06 & 2.2 & 10.2 & 86.8 \\
\hline $1 \mathrm{H}-2,134.9$ & 2.85 & 3.11 & 3.11 & 2.3 & 9.0 & 86.5 \\
\hline $1 \mathrm{H}-2,140$ & 2.90 & 3.16 & 3.16 & 2.7 & 10.6 & 86.4 \\
\hline $1 \mathrm{H}-2,145$ & 2.95 & 3.21 & 3.21 & 2.8 & 10.9 & 86.3 \\
\hline $1 \mathrm{H}-3,3.8$ & 3.04 & 3.30 & 3.30 & 2.7 & 11.3 & 86.2 \\
\hline $1 \mathrm{H}-3,8.8$ & 3.09 & 3.35 & 3.35 & 2.5 & 11.6 & 86.0 \\
\hline $1 \mathrm{H}-3,13.9$ & 3.14 & 3.40 & 3.40 & 2.4 & 10.3 & 86.0 \\
\hline
\end{tabular}

Only a portion of this table appears here. The complete table is available in ASCII. 
Table AT9. Core adjusted data, Hole U1336A.

\begin{tabular}{|c|c|c|c|}
\hline \multirow[b]{2}{*}{$\begin{array}{l}\text { Core, section, } \\
\text { interval }(\mathrm{cm})\end{array}$} & \multicolumn{3}{|c|}{ Depth (m) } \\
\hline & $\begin{array}{l}\text { Adjusted } \\
\text { CCSF }\end{array}$ & CCSF & CSF-A \\
\hline \multicolumn{4}{|l|}{ 320-U1336A- } \\
\hline $1 \mathrm{H}-1,0$ & 0.04 & 0.26 & 0.00 \\
\hline $1 \mathrm{H}-1,17$ & 0.19 & 0.43 & 0.17 \\
\hline $1 \mathrm{H}-1,70$ & 0.96 & 0.96 & 0.70 \\
\hline $1 \mathrm{H}-5,77$ & 7.03 & 7.03 & 6.77 \\
\hline $1 \mathrm{H}-6,7$ & 7.87 & 7.83 & 7.57 \\
\hline $1 \mathrm{H}-6,33$ & 8.05 & 8.09 & 7.83 \\
\hline $1 \mathrm{H}-6,45$ & 8.21 & 8.21 & 7.95 \\
\hline $2 \mathrm{H}-1,42$ & 9.83 & 9.96 & 8.42 \\
\hline $2 \mathrm{H}-1,99$ & 10.51 & 10.53 & 8.99 \\
\hline $2 \mathrm{H}-1,148$ & 11.06 & 11.02 & 9.48 \\
\hline $2 \mathrm{H}-2,54$ & 11.59 & 11.58 & 10.04 \\
\hline $2 \mathrm{H}-6,106$ & 18.11 & 18.11 & 16.56 \\
\hline $2 \mathrm{H}-6,142$ & 18.48 & 18.46 & 16.92 \\
\hline $2 \mathrm{H}-7,14$ & 18.81 & 18.68 & 17.14 \\
\hline $2 \mathrm{H}-7,47$ & 19.13 & 19.01 & 17.47 \\
\hline $3 \mathrm{H}-1,70$ & 20.02 & 20.08 & 18.20 \\
\hline $3 \mathrm{H}-2,34$ & 21.23 & 21.22 & 19.34 \\
\hline $3 \mathrm{H}-3,60$ & 22.98 & 22.98 & 21.10 \\
\hline $3 \mathrm{H}-6,54$ & 27.41 & 27.42 & 25.54 \\
\hline $3 \mathrm{H}-6,59$ & 27.55 & 27.47 & 25.59 \\
\hline $3 \mathrm{H}-7,1$ & 28.40 & 28.39 & 26.51 \\
\hline $3 \mathrm{H}-7,28$ & 28.64 & 28.66 & 26.78 \\
\hline $3 \mathrm{H}-7,66$ & 29.01 & 29.04 & 27.16 \\
\hline $4 \mathrm{H}-1,143$ & 31.33 & 31.29 & 28.43 \\
\hline $4 \mathrm{H}-2,30$ & 31.71 & 31.65 & 28.80 \\
\hline $4 \mathrm{H}-2,131$ & 32.67 & 32.67 & 29.81 \\
\hline $4 \mathrm{H}-3,20$ & 33.04 & 33.05 & 30.20 \\
\hline $4 \mathrm{H}-3,52$ & 33.44 & 33.37 & 30.52 \\
\hline $4 \mathrm{H}-3,79$ & 33.61 & 33.65 & 30.79 \\
\hline $4 \mathrm{H}-3,90$ & 33.76 & 33.75 & 30.90 \\
\hline $4 \mathrm{H}-3,110$ & 33.95 & 33.95 & 31.10 \\
\hline $4 \mathrm{H}-7,9$ & 38.25 & 38.25 & 35.40 \\
\hline $4 \mathrm{H}-7,25$ & 38.47 & 38.41 & 35.56 \\
\hline $4 \mathrm{H}-7,59$ & 38.79 & 38.75 & 35.90 \\
\hline $5 \mathrm{H}-1,24$ & 39.83 & 39.94 & 36.74 \\
\hline $5 \mathrm{H}-1,43$ & 40.04 & 40.13 & 36.93 \\
\hline $5 \mathrm{H}-1,83$ & 40.52 & 40.54 & 37.33 \\
\hline $5 \mathrm{H}-1,147$ & 41.47 & 41.17 & 37.97 \\
\hline $5 \mathrm{H}-2,149$ & 42.93 & 42.69 & 39.49 \\
\hline $5 \mathrm{H}-3,19$ & 43.05 & 42.89 & 39.69 \\
\hline $5 \mathrm{H}-3,149$ & 44.20 & 44.19 & 40.99 \\
\hline $5 \mathrm{H}-4,34$ & 44.47 & 44.54 & 41.34 \\
\hline $5 \mathrm{H}-4,50$ & 44.70 & 44.70 & 41.50 \\
\hline $5 \mathrm{H}-6,149$ & 48.69 & 48.69 & 45.49 \\
\hline $5 \mathrm{H}-7,62$ & 49.32 & 49.32 & 46.12 \\
\hline $6 \mathrm{H}-1,108$ & 53.80 & 53.76 & 47.08 \\
\hline $6 \mathrm{H}-2,43$ & 54.65 & 54.61 & 47.93 \\
\hline $6 \mathrm{H}-3,70$ & 56.38 & 56.38 & 49.70 \\
\hline $6 \mathrm{H}-6,70$ & 60.88 & 60.88 & 54.20 \\
\hline $6 \mathrm{H}-6,85$ & 61.12 & 61.03 & 54.35 \\
\hline $6 \mathrm{H}-6,149$ & 61.61 & 61.67 & 54.99 \\
\hline 7Н-1, 18 & 63.80 & 63.79 & 55.68 \\
\hline $7 \mathrm{H}-1,125$ & 64.88 & 64.87 & 56.75 \\
\hline $7 \mathrm{H}-2,80$ & 65.95 & 65.91 & 57.80 \\
\hline $7 \mathrm{H}-3,34$ & 66.95 & 66.95 & 58.84 \\
\hline $7 \mathrm{H}-7,34$ & 72.95 & 72.95 & 64.84 \\
\hline
\end{tabular}

\begin{tabular}{|c|c|c|c|}
\hline \multirow[b]{2}{*}{$\begin{array}{l}\text { Core, section, } \\
\text { interval }(\mathrm{cm})\end{array}$} & \multicolumn{3}{|c|}{ Depth (m) } \\
\hline & $\begin{array}{l}\text { Adjusted } \\
\text { CCSF }\end{array}$ & CCSF & CSF-A \\
\hline 7H-7, 77 & 73.42 & 73.38 & 65.27 \\
\hline $8 \mathrm{H}-1,12$ & 74.14 & 74.28 & 65.12 \\
\hline $8 \mathrm{H}-1,61$ & 74.56 & 74.77 & 65.61 \\
\hline $8 \mathrm{H}-1,95$ & 74.92 & 75.11 & 65.95 \\
\hline $8 \mathrm{H}-1,120$ & 75.37 & 75.37 & 66.20 \\
\hline $8 \mathrm{H}-7,55$ & 82.71 & 82.71 & 73.55 \\
\hline $8 \mathrm{H}-7,112$ & 83.19 & 83.28 & 74.12 \\
\hline $9 \mathrm{H}-1,24$ & 84.69 & 84.58 & 74.74 \\
\hline $9 \mathrm{H}-2,11$ & 85.95 & 85.95 & 76.11 \\
\hline $9 \mathrm{H}-4,103$ & 89.87 & 89.87 & 80.03 \\
\hline $9 \mathrm{H}-5,40$ & 90.76 & 90.74 & 80.90 \\
\hline $9 \mathrm{H}-5,81$ & 91.32 & 91.15 & 81.31 \\
\hline $9 \mathrm{H}-7,26$ & 93.63 & 93.60 & 83.76 \\
\hline $10 \mathrm{H}-1,79$ & 94.65 & 94.57 & 84.79 \\
\hline $10 \mathrm{H}-2,0$ & 95.57 & 95.28 & 85.50 \\
\hline $10 \mathrm{H}-2,52$ & 95.98 & 95.80 & 86.02 \\
\hline $10 \mathrm{H}-3,4$ & 96.82 & 96.82 & 87.04 \\
\hline $10 \mathrm{H}-7,36$ & 102.84 & 102.84 & 93.06 \\
\hline $10 \mathrm{H}-7,83$ & 103.35 & 103.31 & 93.53 \\
\hline $11 \mathrm{H}-1,21$ & 105.52 & 105.57 & 93.71 \\
\hline $11 \mathrm{H}-1,47$ & 105.83 & 105.83 & 93.97 \\
\hline $11 \mathrm{H}-1,102$ & 106.38 & 106.38 & 94.52 \\
\hline $11 \mathrm{H}-4,56$ & 110.43 & 110.43 & 98.56 \\
\hline $11 \mathrm{H}-4,118$ & 111.43 & 111.04 & 99.18 \\
\hline $11 \mathrm{H}-5,58$ & 112.22 & 111.94 & 100.08 \\
\hline $11 \mathrm{H}-6,101$ & 114.24 & 113.87 & 102.01 \\
\hline $11 \mathrm{H}-7,41$ & 115.27 & 114.77 & 102.91 \\
\hline $12 \mathrm{H}-1,120$ & 117.13 & 117.15 & 104.20 \\
\hline $12 \mathrm{H}-2,56$ & 118.01 & 118.01 & 105.06 \\
\hline $12 \mathrm{H}-4,121$ & 121.66 & 121.66 & 108.71 \\
\hline $12 \mathrm{H}-5,72$ & 122.68 & 122.67 & 109.72 \\
\hline $12 \mathrm{H}-5,150$ & 123.64 & 123.45 & 110.50 \\
\hline $12 \mathrm{H}-6,78$ & 124.32 & 124.24 & 111.28 \\
\hline $13 \mathrm{H}-1,149$ & 128.14 & 128.28 & 113.99 \\
\hline $13 \mathrm{H}-2,72$ & 129.01 & 129.01 & 114.72 \\
\hline $13 \mathrm{H}-6,63$ & 134.92 & 134.92 & 120.63 \\
\hline $13 \mathrm{H}-6,149$ & 135.86 & 135.78 & 121.49 \\
\hline $13 \mathrm{H}-7,66$ & 136.47 & 136.45 & 122.16 \\
\hline $15 \mathrm{H}-1,42$ & 140.22 & 140.22 & 123.92 \\
\hline $15 \mathrm{H}-6,71$ & 148.00 & 148.01 & 131.71 \\
\hline $15 \mathrm{H}-7,40$ & 149.17 & 149.20 & 132.90 \\
\hline $16 \mathrm{H}-1,87$ & 155.40 & 155.44 & 133.87 \\
\hline $16 \mathrm{H}-2,0$ & 156.32 & 156.07 & 134.50 \\
\hline $16 \mathrm{H}-2,149$ & 157.69 & 157.56 & 135.99 \\
\hline $16 \mathrm{H}-3,94$ & 158.91 & 158.51 & 136.94 \\
\hline $16 \mathrm{H}-4,108$ & 160.13 & 160.15 & 138.58 \\
\hline 16H-5, 12 & 160.76 & 160.69 & 139.12 \\
\hline $16 \mathrm{H}-6,102$ & 163.06 & 163.09 & 141.52 \\
\hline $17 \mathrm{H}-2,150$ & 168.49 & 168.48 & 145.00 \\
\hline $17 \mathrm{H}-3,66$ & 169.51 & 169.14 & 145.66 \\
\hline $17 \mathrm{H}-3,105$ & 169.92 & 169.53 & 146.05 \\
\hline $17 \mathrm{H}-4,25$ & 170.25 & 170.23 & 146.75 \\
\hline $17 \mathrm{H}-5,95$ & 172.43 & 172.43 & 148.95 \\
\hline $17 \mathrm{H}-7,53$ & 175.01 & 175.01 & 151.53 \\
\hline $33 X-2,17$ & 312.60 & 312.60 & 289.12 \\
\hline $33 X-3,58$ & 314.01 & 314.01 & 290.53 \\
\hline
\end{tabular}

This table is also available in ASCII. 
Table AT10. Gamma ray attenuation (GRA) density data, Hole U1336B.

\begin{tabular}{|c|c|c|c|c|}
\hline \multirow[b]{2}{*}{$\begin{array}{l}\text { Core, section, } \\
\text { interval }(\mathrm{cm})\end{array}$} & \multicolumn{3}{|c|}{ Depth (m) } & \multirow[b]{2}{*}{$\begin{array}{c}\text { GRA } \\
\left(\mathrm{g} / \mathrm{cm}^{3}\right)\end{array}$} \\
\hline & CSF & CCSF & $\begin{array}{l}\text { Adjusted } \\
\text { CCSF }\end{array}$ & \\
\hline \multicolumn{5}{|l|}{ 320-U1336B- } \\
\hline $1 \mathrm{H}-1,3$ & 0.03 & 0.03 & 0.03 & 1.27 \\
\hline $1 \mathrm{H}-1,5$ & 0.05 & 0.05 & 0.05 & 1.33 \\
\hline $1 \mathrm{H}-1,8$ & 0.08 & 0.08 & 0.08 & 1.38 \\
\hline $1 \mathrm{H}-1,10$ & 0.10 & 0.10 & 0.10 & 1.35 \\
\hline $1 \mathrm{H}-1,13$ & 0.13 & 0.13 & 0.13 & 1.34 \\
\hline $1 \mathrm{H}-1,15$ & 0.15 & 0.15 & 0.15 & 1.36 \\
\hline $1 \mathrm{H}-1,18$ & 0.18 & 0.18 & 0.18 & 1.29 \\
\hline $1 \mathrm{H}-1,20$ & 0.20 & 0.20 & 0.20 & 1.31 \\
\hline $1 \mathrm{H}-1,23$ & 0.23 & 0.23 & 0.23 & 1.34 \\
\hline $1 \mathrm{H}-1,25$ & 0.25 & 0.25 & 0.25 & 1.38 \\
\hline $1 \mathrm{H}-1,28$ & 0.28 & 0.28 & 0.28 & 1.40 \\
\hline $1 \mathrm{H}-1,30$ & 0.30 & 0.30 & 0.30 & 1.42 \\
\hline $1 \mathrm{H}-1,33$ & 0.33 & 0.33 & 0.33 & 1.40 \\
\hline $1 \mathrm{H}-1,35$ & 0.35 & 0.35 & 0.35 & 1.42 \\
\hline $1 \mathrm{H}-1,38$ & 0.38 & 0.38 & 0.38 & 1.43 \\
\hline $1 \mathrm{H}-1,40$ & 0.40 & 0.40 & 0.40 & 1.46 \\
\hline $1 \mathrm{H}-1,43$ & 0.43 & 0.43 & 0.43 & 1.45 \\
\hline $1 \mathrm{H}-1,45$ & 0.45 & 0.45 & 0.45 & 1.39 \\
\hline $1 \mathrm{H}-1,48$ & 0.48 & 0.48 & 0.48 & 1.42 \\
\hline $1 \mathrm{H}-1,50$ & 0.50 & 0.50 & 0.50 & 1.42 \\
\hline $1 \mathrm{H}-1,53$ & 0.53 & 0.53 & 0.53 & 1.43 \\
\hline $1 \mathrm{H}-1,55$ & 0.55 & 0.55 & 0.55 & 1.43 \\
\hline $1 \mathrm{H}-1,58$ & 0.58 & 0.58 & 0.58 & 1.40 \\
\hline $1 \mathrm{H}-1,60$ & 0.60 & 0.60 & 0.60 & 1.49 \\
\hline $1 \mathrm{H}-1,63$ & 0.63 & 0.63 & 0.63 & 1.45 \\
\hline $1 \mathrm{H}-1,65$ & 0.65 & 0.65 & 0.65 & 1.50 \\
\hline $1 \mathrm{H}-1,68$ & 0.68 & 0.68 & 0.68 & 1.49 \\
\hline $1 \mathrm{H}-1,70$ & 0.70 & 0.70 & 0.70 & 1.49 \\
\hline $1 \mathrm{H}-1,73$ & 0.73 & 0.73 & 0.73 & 1.50 \\
\hline $1 \mathrm{H}-1,75$ & 0.75 & 0.75 & 0.75 & 1.48 \\
\hline $1 \mathrm{H}-1,78$ & 0.78 & 0.78 & 0.78 & 1.49 \\
\hline $1 \mathrm{H}-1,80$ & 0.80 & 0.80 & 0.80 & 1.50 \\
\hline $1 \mathrm{H}-1,83$ & 0.83 & 0.83 & 0.83 & 1.50 \\
\hline $1 \mathrm{H}-1,85$ & 0.85 & 0.85 & 0.85 & 1.52 \\
\hline $1 \mathrm{H}-1,88$ & 0.88 & 0.88 & 0.88 & 1.52 \\
\hline $1 \mathrm{H}-1,90$ & 0.90 & 0.90 & 0.90 & 1.48 \\
\hline $1 \mathrm{H}-1,93$ & 0.93 & 0.93 & 0.93 & 1.51 \\
\hline $1 \mathrm{H}-1,95$ & 0.95 & 0.95 & 0.95 & 1.51 \\
\hline $1 \mathrm{H}-1,98$ & 0.98 & 0.98 & 0.98 & 1.49 \\
\hline $1 \mathrm{H}-1,100$ & 1.00 & 1.00 & 1.00 & 1.50 \\
\hline $1 \mathrm{H}-1,103$ & 1.03 & 1.03 & 1.03 & 1.50 \\
\hline $1 \mathrm{H}-1,105$ & 1.05 & 1.05 & 1.05 & 1.51 \\
\hline $1 \mathrm{H}-1,108$ & 1.08 & 1.08 & 1.09 & 1.51 \\
\hline $1 \mathrm{H}-1,110$ & 1.10 & 1.10 & 1.11 & 1.50 \\
\hline $1 \mathrm{H}-1,113$ & 1.13 & 1.13 & 1.14 & 1.47 \\
\hline $1 \mathrm{H}-1,115$ & 1.15 & 1.15 & 1.16 & 1.49 \\
\hline $1 \mathrm{H}-1,118$ & 1.18 & 1.18 & 1.19 & 1.47 \\
\hline $1 \mathrm{H}-1,120$ & 1.20 & 1.20 & 1.21 & 1.48 \\
\hline $1 \mathrm{H}-1,123$ & 1.23 & 1.23 & 1.25 & 1.45 \\
\hline $1 \mathrm{H}-1,125$ & 1.25 & 1.25 & 1.27 & 1.45 \\
\hline $1 \mathrm{H}-1,128$ & 1.28 & 1.28 & 1.30 & 1.46 \\
\hline $1 \mathrm{H}-1,130$ & 1.30 & 1.30 & 1.32 & 1.45 \\
\hline $1 \mathrm{H}-1,133$ & 1.33 & 1.33 & 1.35 & 1.47 \\
\hline $1 \mathrm{H}-1,135$ & 1.35 & 1.35 & 1.36 & 1.47 \\
\hline $2 \mathrm{H}-1,15$ & 1.95 & 2.48 & 2.38 & 1.55 \\
\hline $2 \mathrm{H}-1,18$ & 1.98 & 2.51 & 2.39 & 1.52 \\
\hline $2 \mathrm{H}-1,20$ & 2.00 & 2.53 & 2.40 & 1.50 \\
\hline $2 \mathrm{H}-1,23$ & 2.03 & 2.56 & 2.42 & 1.49 \\
\hline $2 \mathrm{H}-1,25$ & 2.05 & 2.58 & 2.42 & 1.50 \\
\hline $2 \mathrm{H}-1,28$ & 2.08 & 2.61 & 2.44 & 1.50 \\
\hline $2 \mathrm{H}-1,30$ & 2.10 & 2.63 & 2.45 & 1.50 \\
\hline
\end{tabular}

Only a portion of this table appears here. The complete table is available in ASCII. 
Table AT11. Magnetic susceptibility data, Hole U1336B.

\begin{tabular}{|c|c|c|c|c|}
\hline \multirow[b]{2}{*}{$\begin{array}{l}\text { Core, section, } \\
\text { interval }(\mathrm{cm})\end{array}$} & \multicolumn{3}{|c|}{ Depth (m) } & \multirow{2}{*}{$\begin{array}{l}\text { Magnetic } \\
\text { susceptibility } \\
\text { (IU) }\end{array}$} \\
\hline & CSF & CCSF & $\begin{array}{l}\text { Adjusted } \\
\text { CCSF }\end{array}$ & \\
\hline \multicolumn{5}{|l|}{ 320-U1336B- } \\
\hline $1 \mathrm{H}-1,0$ & 0.00 & 0.00 & 0.00 & 6.3 \\
\hline $1 \mathrm{H}-1,3$ & 0.03 & 0.03 & 0.03 & 16.5 \\
\hline $1 \mathrm{H}-1,5$ & 0.05 & 0.05 & 0.05 & 13.5 \\
\hline $1 \mathrm{H}-1,8$ & 0.08 & 0.08 & 0.08 & 11.5 \\
\hline $1 \mathrm{H}-1,10$ & 0.10 & 0.10 & 0.10 & 12.1 \\
\hline $1 \mathrm{H}-1,13$ & 0.13 & 0.13 & 0.13 & 13.5 \\
\hline $1 \mathrm{H}-1,15$ & 0.15 & 0.15 & 0.15 & 16.5 \\
\hline $1 \mathrm{H}-1,18$ & 0.18 & 0.18 & 0.18 & 15.9 \\
\hline $1 \mathrm{H}-1,20$ & 0.20 & 0.20 & 0.20 & 15.7 \\
\hline $1 \mathrm{H}-1,23$ & 0.23 & 0.23 & 0.23 & 15.4 \\
\hline $1 \mathrm{H}-1,25$ & 0.25 & 0.25 & 0.25 & 15.1 \\
\hline $1 \mathrm{H}-1,28$ & 0.28 & 0.28 & 0.28 & 14.6 \\
\hline $1 \mathrm{H}-1,30$ & 0.30 & 0.30 & 0.30 & 14.3 \\
\hline $1 \mathrm{H}-1,33$ & 0.33 & 0.33 & 0.33 & 13.7 \\
\hline $1 \mathrm{H}-1,35$ & 0.35 & 0.35 & 0.35 & 13.5 \\
\hline $1 \mathrm{H}-1,38$ & 0.38 & 0.38 & 0.38 & 14.0 \\
\hline $1 \mathrm{H}-1,40$ & 0.40 & 0.40 & 0.40 & 14.3 \\
\hline $1 \mathrm{H}-1,43$ & 0.43 & 0.43 & 0.43 & 14.6 \\
\hline $1 \mathrm{H}-1,45$ & 0.45 & 0.45 & 0.45 & 15.9 \\
\hline $1 \mathrm{H}-1,48$ & 0.48 & 0.48 & 0.48 & 16.8 \\
\hline $1 \mathrm{H}-1,50$ & 0.50 & 0.50 & 0.50 & 17.6 \\
\hline $1 \mathrm{H}-1,53$ & 0.53 & 0.53 & 0.53 & 17.0 \\
\hline $1 \mathrm{H}-1,55$ & 0.55 & 0.55 & 0.55 & 16.2 \\
\hline $1 \mathrm{H}-1,58$ & 0.58 & 0.58 & 0.58 & 15.9 \\
\hline $1 \mathrm{H}-1,60$ & 0.60 & 0.60 & 0.60 & 15.4 \\
\hline $1 \mathrm{H}-1,63$ & 0.63 & 0.63 & 0.63 & 14.6 \\
\hline $1 \mathrm{H}-1,65$ & 0.65 & 0.65 & 0.65 & 14.0 \\
\hline $1 \mathrm{H}-1,68$ & 0.68 & 0.68 & 0.68 & 14.0 \\
\hline $1 \mathrm{H}-1,70$ & 0.70 & 0.70 & 0.70 & 14.0 \\
\hline $1 \mathrm{H}-1,73$ & 0.73 & 0.73 & 0.73 & 14.6 \\
\hline $1 \mathrm{H}-1,75$ & 0.75 & 0.75 & 0.75 & 15.7 \\
\hline $1 \mathrm{H}-1,78$ & 0.78 & 0.78 & 0.78 & 15.7 \\
\hline $1 \mathrm{H}-1,80$ & 0.80 & 0.80 & 0.80 & 15.9 \\
\hline $1 \mathrm{H}-1,83$ & 0.83 & 0.83 & 0.83 & 15.1 \\
\hline $1 \mathrm{H}-1,85$ & 0.85 & 0.85 & 0.85 & 14.6 \\
\hline $1 \mathrm{H}-1,88$ & 0.88 & 0.88 & 0.88 & 14.0 \\
\hline $1 \mathrm{H}-1,90$ & 0.90 & 0.90 & 0.90 & 14.8 \\
\hline $1 \mathrm{H}-1,93$ & 0.93 & 0.93 & 0.93 & 14.6 \\
\hline $1 \mathrm{H}-1,95$ & 0.95 & 0.95 & 0.95 & 14.6 \\
\hline $1 \mathrm{H}-1,98$ & 0.98 & 0.98 & 0.98 & 14.8 \\
\hline $1 \mathrm{H}-1,100$ & 1.00 & 1.00 & 1.00 & 15.4 \\
\hline $1 \mathrm{H}-1,103$ & 1.03 & 1.03 & 1.03 & 15.9 \\
\hline $1 \mathrm{H}-1,105$ & 1.05 & 1.05 & 1.05 & 15.7 \\
\hline $1 \mathrm{H}-1,108$ & 1.08 & 1.08 & 1.09 & 15.7 \\
\hline $1 \mathrm{H}-1,110$ & 1.10 & 1.10 & 1.11 & 15.7 \\
\hline $1 \mathrm{H}-1,113$ & 1.13 & 1.13 & 1.14 & 15.4 \\
\hline $1 \mathrm{H}-1,115$ & 1.15 & 1.15 & 1.16 & 15.7 \\
\hline $1 \mathrm{H}-1,118$ & 1.18 & 1.18 & 1.19 & 16.2 \\
\hline $1 \mathrm{H}-1,120$ & 1.20 & 1.20 & 1.21 & 17.0 \\
\hline $1 \mathrm{H}-1,123$ & 1.23 & 1.23 & 1.25 & 17.9 \\
\hline $1 \mathrm{H}-1,125$ & 1.25 & 1.25 & 1.27 & 18.7 \\
\hline $1 \mathrm{H}-1,128$ & 1.28 & 1.28 & 1.30 & 19.2 \\
\hline $1 \mathrm{H}-1,130$ & 1.30 & 1.30 & 1.32 & 19.0 \\
\hline $1 \mathrm{H}-1,133$ & 1.33 & 1.33 & 1.35 & 18.1 \\
\hline $1 \mathrm{H}-1,135$ & 1.35 & 1.35 & 1.36 & 17.3 \\
\hline $1 \mathrm{H}-1,138$ & 1.38 & 1.38 & 1.39 & 15.4 \\
\hline $2 \mathrm{H}-1,0$ & 1.80 & 2.33 & 2.25 & 2.2 \\
\hline $2 \mathrm{H}-1,3$ & 1.83 & 2.36 & 2.28 & 9.3 \\
\hline $2 \mathrm{H}-1,5$ & 1.85 & 2.38 & 2.30 & 14.6 \\
\hline $2 \mathrm{H}-1,8$ & 1.88 & 2.41 & 2.33 & 15.4 \\
\hline
\end{tabular}

Only a portion of this table appears here. The complete table is available in ASCII. 
Table AT12. Natural gamma ray (NGR) data, Hole U1336B.

\begin{tabular}{|c|c|c|c|c|}
\hline \multirow[b]{2}{*}{$\begin{array}{l}\text { Core, section, } \\
\text { interval }(\mathrm{cm})\end{array}$} & \multicolumn{3}{|c|}{ Depth (m) } & \multirow[b]{2}{*}{$\begin{array}{l}\text { NGR } \\
\text { (cps) }\end{array}$} \\
\hline & CSF & CCSF & $\begin{array}{l}\text { Adjusted } \\
\text { CCSF }\end{array}$ & \\
\hline \multicolumn{5}{|l|}{ 320-U1336B- } \\
\hline $1 \mathrm{H}-1,0$ & 0.0 & 0.00 & 0.00 & 75.0 \\
\hline $1 \mathrm{H}-1,10$ & 0.1 & 0.10 & 0.10 & 65.0 \\
\hline $1 \mathrm{H}-1,20$ & 0.2 & 0.20 & 0.20 & 47.7 \\
\hline $1 \mathrm{H}-1,30$ & 0.3 & 0.30 & 0.30 & 26.7 \\
\hline $1 \mathrm{H}-1,40$ & 0.4 & 0.40 & 0.40 & 13.8 \\
\hline $1 \mathrm{H}-1,50$ & 0.5 & 0.50 & 0.50 & 7.9 \\
\hline $1 \mathrm{H}-1,60$ & 0.6 & 0.60 & 0.60 & 5.5 \\
\hline $1 \mathrm{H}-1,70$ & 0.7 & 0.70 & 0.70 & 4.2 \\
\hline $1 \mathrm{H}-1,80$ & 0.8 & 0.80 & 0.80 & 3.9 \\
\hline $1 \mathrm{H}-1,90$ & 0.9 & 0.90 & 0.90 & 3.6 \\
\hline $1 \mathrm{H}-1,100$ & 1.0 & 1.00 & 1.00 & 3.9 \\
\hline $1 \mathrm{H}-1,110$ & 1.1 & 1.10 & 1.11 & 4.1 \\
\hline $1 \mathrm{H}-1,120$ & 1.2 & 1.20 & 1.21 & 4.2 \\
\hline $1 \mathrm{H}-1,130$ & 1.3 & 1.30 & 1.32 & 4.3 \\
\hline $2 \mathrm{H}-1,0$ & 1.8 & 2.33 & 2.25 & 21.7 \\
\hline $2 \mathrm{H}-1,10$ & 1.9 & 2.43 & 2.35 & 11.7 \\
\hline $2 \mathrm{H}-1,20$ & 2.0 & 2.53 & 2.40 & 5.7 \\
\hline $2 \mathrm{H}-1,30$ & 2.1 & 2.63 & 2.45 & 4.8 \\
\hline $2 \mathrm{H}-1,40$ & 2.2 & 2.73 & 2.49 & 4.4 \\
\hline $2 \mathrm{H}-1,50$ & 2.3 & 2.83 & 2.53 & 3.7 \\
\hline $2 \mathrm{H}-1,60$ & 2.4 & 2.93 & 2.60 & 3.7 \\
\hline $2 \mathrm{H}-1,70$ & 2.5 & 3.03 & 2.76 & 3.0 \\
\hline $2 \mathrm{H}-1,80$ & 2.6 & 3.13 & 2.91 & 3.3 \\
\hline $2 \mathrm{H}-1,90$ & 2.7 & 3.23 & 3.02 & 3.5 \\
\hline $2 \mathrm{H}-1,100$ & 2.8 & 3.33 & 3.13 & 2.6 \\
\hline $2 \mathrm{H}-1,110$ & 2.9 & 3.43 & 3.24 & 2.3 \\
\hline $2 \mathrm{H}-1,120$ & 3.0 & 3.53 & 3.35 & 2.2 \\
\hline $2 \mathrm{H}-1,130$ & 3.1 & 3.63 & 3.46 & 2.2 \\
\hline $2 \mathrm{H}-1,140$ & 3.2 & 3.73 & 3.57 & 2.1 \\
\hline $2 \mathrm{H}-2,0$ & 3.3 & 3.83 & 3.68 & 2.7 \\
\hline $2 \mathrm{H}-1,150$ & 3.3 & 3.83 & 3.68 & 2.9 \\
\hline $2 \mathrm{H}-2,10$ & 3.4 & 3.93 & 3.79 & 2.4 \\
\hline $2 \mathrm{H}-2,20$ & 3.5 & 4.03 & 3.89 & 2.2 \\
\hline $2 \mathrm{H}-2,30$ & 3.6 & 4.13 & 4.00 & 2.0 \\
\hline $2 \mathrm{H}-2,40$ & 3.7 & 4.23 & 4.10 & 2.0 \\
\hline $2 \mathrm{H}-2,50$ & 3.8 & 4.33 & 4.20 & 2.2 \\
\hline $2 \mathrm{H}-2,60$ & 3.9 & 4.43 & 4.31 & 2.1 \\
\hline $2 \mathrm{H}-2,70$ & 4.0 & 4.53 & 4.41 & 2.3 \\
\hline $2 \mathrm{H}-2,80$ & 4.1 & 4.63 & 4.51 & 2.2 \\
\hline $2 \mathrm{H}-2,90$ & 4.2 & 4.73 & 4.61 & 2.1 \\
\hline $2 \mathrm{H}-2,100$ & 4.3 & 4.83 & 4.72 & 2.5 \\
\hline $2 \mathrm{H}-2,110$ & 4.4 & 4.93 & 4.82 & 2.4 \\
\hline $2 \mathrm{H}-2,120$ & 4.5 & 5.03 & 4.92 & 2.1 \\
\hline $2 \mathrm{H}-2,130$ & 4.6 & 5.13 & 5.02 & 1.9 \\
\hline $2 \mathrm{H}-2,140$ & 4.7 & 5.23 & 5.11 & 2.3 \\
\hline $2 \mathrm{H}-3,0$ & 4.8 & 5.33 & 5.20 & 1.6 \\
\hline $2 \mathrm{H}-3,10$ & 4.9 & 5.43 & 5.29 & 2.2 \\
\hline $2 \mathrm{H}-3,20$ & 5.0 & 5.53 & 5.38 & 2.0 \\
\hline $2 \mathrm{H}-3,30$ & 5.1 & 5.63 & 5.47 & 2.0 \\
\hline $2 \mathrm{H}-3,40$ & 5.2 & 5.73 & 5.56 & 2.0 \\
\hline $2 \mathrm{H}-3,50$ & 5.3 & 5.83 & 5.65 & 1.7 \\
\hline $2 \mathrm{H}-3,60$ & 5.4 & 5.93 & 5.74 & 2.0 \\
\hline $2 \mathrm{H}-3,70$ & 5.5 & 6.03 & 5.84 & 1.6 \\
\hline $2 \mathrm{H}-3,80$ & 5.6 & 6.13 & 5.96 & 1.9 \\
\hline $2 \mathrm{H}-3,90$ & 5.7 & 6.23 & 6.08 & 2.0 \\
\hline $2 \mathrm{H}-3,100$ & 5.8 & 6.33 & 6.20 & 2.1 \\
\hline $2 \mathrm{H}-3,110$ & 5.9 & 6.43 & 6.32 & 2.4 \\
\hline $2 \mathrm{H}-3,120$ & 6.0 & 6.53 & 6.44 & 2.0 \\
\hline
\end{tabular}

Only a portion of this table appears here. The complete table is available in ASCII. 
Table AT13. Color reflection data, Hole U1336B.

\begin{tabular}{|c|c|c|c|c|c|c|}
\hline \multirow[b]{2}{*}{$\begin{array}{l}\text { Core, section, } \\
\text { interval }(\mathrm{cm})\end{array}$} & \multicolumn{3}{|c|}{ Depth (m) } & \multirow[b]{2}{*}{$a^{*}$} & \multirow[b]{2}{*}{$b^{*}$} & \multirow[b]{2}{*}{$L^{*}$} \\
\hline & CSF & CCSF & $\begin{array}{l}\text { Adjusted } \\
\text { CCSF }\end{array}$ & & & \\
\hline \multicolumn{7}{|l|}{ 320-U1336B- } \\
\hline 1H-1, 3.9 & 0.04 & 0.04 & 0.04 & 4.8 & 15.1 & 68.2 \\
\hline $1 \mathrm{H}-1,5.3$ & 0.05 & 0.05 & 0.05 & 5.1 & 11.7 & 68.0 \\
\hline $1 \mathrm{H}-1,7.9$ & 0.08 & 0.08 & 0.08 & 5.4 & 14.8 & 67.6 \\
\hline $1 \mathrm{H}-1,9$ & 0.09 & 0.09 & 0.09 & 5.2 & 13.0 & 67.1 \\
\hline $1 \mathrm{H}-1,10.4$ & 0.10 & 0.10 & 0.10 & 5.3 & 13.3 & 66.5 \\
\hline $1 \mathrm{H}-1,13$ & 0.13 & 0.13 & 0.13 & 5.4 & 16.7 & 65.9 \\
\hline $1 \mathrm{H}-1,14$ & 0.14 & 0.14 & 0.14 & 5.5 & 16.3 & 65.4 \\
\hline $1 \mathrm{H}-1,15.5$ & 0.16 & 0.16 & 0.16 & 5.4 & 19.9 & 65.3 \\
\hline $1 \mathrm{H}-1,18.1$ & 0.18 & 0.18 & 0.18 & 5.4 & 12.7 & 65.2 \\
\hline $1 \mathrm{H}-1,19.1$ & 0.19 & 0.19 & 0.19 & 5.4 & 19.9 & 65.1 \\
\hline $1 \mathrm{H}-1,20.6$ & 0.21 & 0.21 & 0.21 & 4.6 & 13.9 & 65.0 \\
\hline $1 \mathrm{H}-1,23.1$ & 0.23 & 0.23 & 0.23 & 3.9 & 14.6 & 64.9 \\
\hline $1 \mathrm{H}-1,24.1$ & 0.24 & 0.24 & 0.24 & 4.1 & 14.1 & 64.8 \\
\hline $1 \mathrm{H}-1,25.7$ & 0.26 & 0.26 & 0.26 & 4.6 & 15.5 & 64.6 \\
\hline $1 \mathrm{H}-1,28.2$ & 0.28 & 0.28 & 0.28 & 4.5 & 13.4 & 64.6 \\
\hline $1 \mathrm{H}-1,29.1$ & 0.29 & 0.29 & 0.29 & 4.8 & 15.4 & 64.5 \\
\hline $1 \mathrm{H}-1,30.8$ & 0.31 & 0.31 & 0.31 & 4.7 & 14.4 & 64.3 \\
\hline $1 \mathrm{H}-1,33.3$ & 0.33 & 0.33 & 0.33 & 4.9 & 15.6 & 64.2 \\
\hline $1 \mathrm{H}-1,34.2$ & 0.34 & 0.34 & 0.34 & 4.7 & 14.1 & 64.1 \\
\hline $1 \mathrm{H}-1,35.8$ & 0.36 & 0.36 & 0.36 & 4.9 & 16.6 & 64.0 \\
\hline $1 \mathrm{H}-1,38.4$ & 0.38 & 0.38 & 0.38 & 4.8 & 12.4 & 63.9 \\
\hline $1 \mathrm{H}-1,39.2$ & 0.39 & 0.39 & 0.39 & 4.9 & 15.1 & 63.6 \\
\hline $1 \mathrm{H}-1,40.9$ & 0.41 & 0.41 & 0.41 & 4.9 & 14.2 & 63.4 \\
\hline $1 \mathrm{H}-1,44.3$ & 0.44 & 0.44 & 0.44 & 4.9 & 12.3 & 63.4 \\
\hline $1 \mathrm{H}-1,43.5$ & 0.44 & 0.44 & 0.44 & 4.8 & 16.2 & 63.3 \\
\hline $1 \mathrm{H}-1,46$ & 0.46 & 0.46 & 0.46 & 4.5 & 16.8 & 63.1 \\
\hline $1 \mathrm{H}-1,49.3$ & 0.49 & 0.49 & 0.49 & 5.6 & 17.9 & 63.1 \\
\hline $1 \mathrm{H}-1,48.5$ & 0.49 & 0.49 & 0.49 & 5.6 & 14.6 & 62.9 \\
\hline $1 \mathrm{H}-1,51.1$ & 0.51 & 0.51 & 0.51 & 5.5 & 15.8 & 62.9 \\
\hline $1 \mathrm{H}-1,54.4$ & 0.54 & 0.54 & 0.54 & 4.7 & 14.5 & 62.8 \\
\hline $1 \mathrm{H}-1,53.6$ & 0.54 & 0.54 & 0.54 & 5.7 & 13.9 & 62.7 \\
\hline $1 \mathrm{H}-1,56.2$ & 0.56 & 0.56 & 0.56 & 5.6 & 16.0 & 62.4 \\
\hline $1 \mathrm{H}-1,59.4$ & 0.59 & 0.59 & 0.59 & 4.7 & 16.6 & 62.3 \\
\hline $1 \mathrm{H}-1,58.7$ & 0.59 & 0.59 & 0.59 & 5.3 & 18.6 & 62.2 \\
\hline $1 \mathrm{H}-1,63.8$ & 0.64 & 0.64 & 0.64 & 4.7 & 16.3 & 62.2 \\
\hline $1 \mathrm{H}-1,64.4$ & 0.64 & 0.64 & 0.64 & 4.5 & 17.1 & 62.2 \\
\hline $1 \mathrm{H}-1,66.3$ & 0.66 & 0.66 & 0.66 & 4.7 & 17.8 & 62.1 \\
\hline $1 \mathrm{H}-1,68.9$ & 0.69 & 0.69 & 0.69 & 5.1 & 15.9 & 62.0 \\
\hline $1 \mathrm{H}-1,69.5$ & 0.70 & 0.70 & 0.70 & 4.1 & 16.5 & 61.8 \\
\hline $1 \mathrm{H}-1,71.4$ & 0.71 & 0.71 & 0.71 & 5.6 & 17.8 & 61.4 \\
\hline $1 \mathrm{H}-1,74$ & 0.74 & 0.74 & 0.74 & 4.9 & 16.6 & 61.4 \\
\hline $1 \mathrm{H}-1,74.5$ & 0.75 & 0.75 & 0.75 & 4.8 & 17.2 & 61.4 \\
\hline $1 \mathrm{H}-1,76.5$ & 0.77 & 0.77 & 0.77 & 5.2 & 19.2 & 61.4 \\
\hline $1 \mathrm{H}-1,79.1$ & 0.79 & 0.79 & 0.79 & 5.4 & 14.5 & 61.4 \\
\hline $1 \mathrm{H}-1,79.6$ & 0.80 & 0.80 & 0.80 & 4.5 & 14.9 & 61.3 \\
\hline $1 \mathrm{H}-1,81.6$ & 0.82 & 0.82 & 0.82 & 5.6 & 15.5 & 61.2 \\
\hline $1 \mathrm{H}-1,84.1$ & 0.84 & 0.84 & 0.84 & 5.3 & 15.5 & 60.9 \\
\hline $1 \mathrm{H}-1,84.6$ & 0.85 & 0.85 & 0.85 & 5.4 & 15.0 & 60.9 \\
\hline $1 \mathrm{H}-1,86.7$ & 0.87 & 0.87 & 0.87 & 5.2 & 16.0 & 60.9 \\
\hline $1 \mathrm{H}-1,89.2$ & 0.89 & 0.89 & 0.89 & 5.0 & 15.3 & 60.8 \\
\hline $1 \mathrm{H}-1,89.7$ & 0.90 & 0.90 & 0.90 & 4.7 & 10.9 & 60.3 \\
\hline $1 \mathrm{H}-1,91.8$ & 0.92 & 0.92 & 0.92 & 5.9 & 14.7 & 60.1 \\
\hline $1 \mathrm{H}-1,94.3$ & 0.94 & 0.94 & 0.94 & 5.8 & 17.7 & 59.9 \\
\hline $1 \mathrm{H}-1,94.7$ & 0.95 & 0.95 & 0.95 & 5.5 & 15.9 & 59.7 \\
\hline $1 \mathrm{H}-1,96.9$ & 0.97 & 0.97 & 0.97 & 5.5 & 16.7 & 59.7 \\
\hline $1 \mathrm{H}-1,99.4$ & 0.99 & 0.99 & 0.99 & 5.7 & 15.8 & 59.4 \\
\hline $1 \mathrm{H}-1,99.7$ & 1.00 & 1.00 & 1.00 & 5.3 & 15.9 & 59.1 \\
\hline $1 \mathrm{H}-1,101.9$ & 1.02 & 1.02 & 1.02 & 5.9 & 15.4 & 58.7 \\
\hline $1 \mathrm{H}-1,104.5$ & 1.05 & 1.05 & 1.05 & 5.7 & 15.0 & 58.7 \\
\hline $1 \mathrm{H}-1,104.8$ & 1.05 & 1.05 & 1.05 & 5.7 & 16.3 & 58.6 \\
\hline $1 \mathrm{H}-1,107$ & 1.07 & 1.07 & 1.08 & 5.4 & 15.5 & 58.4 \\
\hline
\end{tabular}

Only a portion of this table appears here. The complete table is available in ASCII. 
Table AT14. Core adjusted data, Hole U1336B. (Continued on next page.)

\begin{tabular}{|c|c|c|c|}
\hline \multirow[b]{2}{*}{$\begin{array}{l}\text { Core, section, } \\
\text { interval }(\mathrm{cm})\end{array}$} & \multicolumn{3}{|c|}{ Depth $(\mathrm{m})$} \\
\hline & $\begin{array}{l}\text { Adjusted } \\
\text { CCSF }\end{array}$ & CCSF & CSF-A \\
\hline \multicolumn{4}{|l|}{ 320-U1336B- } \\
\hline $1 \mathrm{H}-1,0$ & 0.00 & 0.00 & 0.00 \\
\hline $1 \mathrm{H}-1,95$ & 0.95 & 0.96 & 0.95 \\
\hline $1 \mathrm{H}-1,129$ & 1.31 & 1.29 & 1.29 \\
\hline $2 \mathrm{H}-1,13$ & 2.37 & 2.46 & 1.93 \\
\hline $2 \mathrm{H}-1,58$ & 2.56 & 2.91 & 2.38 \\
\hline $2 \mathrm{H}-1,76$ & 2.87 & 3.09 & 2.56 \\
\hline $2 \mathrm{H}-2,8$ & 3.77 & 3.91 & 3.38 \\
\hline $2 \mathrm{H}-2,128$ & 5.01 & 5.11 & 4.58 \\
\hline $2 \mathrm{H}-3,67$ & 5.80 & 6.00 & 5.47 \\
\hline $2 \mathrm{H}-3,127$ & 6.53 & 6.60 & 6.07 \\
\hline $2 \mathrm{H}-4,20$ & 7.03 & 7.03 & 6.50 \\
\hline $2 \mathrm{H}-7,26$ & 11.59 & 11.59 & 11.06 \\
\hline $2 \mathrm{H}-7,70$ & 12.05 & 12.03 & 11.50 \\
\hline $3 \mathrm{H}-1,26$ & 14.16 & 14.12 & 11.56 \\
\hline $3 \mathrm{H}-3,6$ & 16.86 & 16.93 & 14.36 \\
\hline $3 \mathrm{H}-3,128$ & 18.15 & 18.14 & 15.58 \\
\hline $3 \mathrm{H}-7,14$ & 23.01 & 23.00 & 20.44 \\
\hline $3 \mathrm{H}-7,35$ & 23.08 & 23.21 & 20.65 \\
\hline $3 \mathrm{H}-7,46$ & 23.23 & 23.32 & 20.76 \\
\hline $3 \mathrm{H}-7,59$ & 23.36 & 23.45 & 20.89 \\
\hline $4 \mathrm{H}-1,24$ & 25.13 & 25.30 & 21.04 \\
\hline $4 \mathrm{H}-1,58$ & 25.54 & 25.64 & 21.38 \\
\hline $4 \mathrm{H}-1,111$ & 26.04 & 26.17 & 21.91 \\
\hline $4 \mathrm{H}-1,138$ & 26.34 & 26.44 & 22.18 \\
\hline $4 \mathrm{H}-2,56$ & 27.03 & 27.12 & 22.86 \\
\hline $4 \mathrm{H}-2,89$ & 27.45 & 27.45 & 23.19 \\
\hline $4 \mathrm{H}-6,137$ & 33.93 & 33.93 & 29.67 \\
\hline $4 \mathrm{H}-7,41$ & 34.40 & 34.47 & 30.21 \\
\hline $5 \mathrm{H}-1,6$ & 36.02 & 36.02 & 30.36 \\
\hline $5 \mathrm{H}-1,43$ & 36.40 & 36.39 & 30.73 \\
\hline $5 \mathrm{H}-1,95$ & 36.83 & 36.92 & 31.25 \\
\hline $5 \mathrm{H}-1,127$ & 37.21 & 37.23 & 31.57 \\
\hline $5 \mathrm{H}-2,69$ & 38.10 & 38.15 & 32.49 \\
\hline $5 \mathrm{H}-2,81$ & 38.27 & 38.27 & 32.61 \\
\hline $5 \mathrm{H}-6,125$ & 44.72 & 44.72 & 39.05 \\
\hline $5 \mathrm{H}-7,21$ & 45.47 & 45.17 & 39.51 \\
\hline $5 \mathrm{H}-7,48$ & 45.76 & 45.44 & 39.78 \\
\hline $6 \mathrm{H}-1,26$ & 47.55 & 47.67 & 40.06 \\
\hline $6 \mathrm{H}-1,69$ & 47.86 & 48.10 & 40.49 \\
\hline $6 \mathrm{H}-1,90$ & 48.10 & 48.31 & 40.70 \\
\hline $6 \mathrm{H}-1,128$ & 48.69 & 48.69 & 41.08 \\
\hline $6 \mathrm{H}-6,148$ & 56.39 & 56.39 & 48.78 \\
\hline $6 \mathrm{H}-7,15$ & 56.66 & 56.56 & 48.95 \\
\hline $6 \mathrm{H}-7,45$ & 56.85 & 56.86 & 49.25 \\
\hline $6 \mathrm{H}-7,60$ & 57.00 & 57.01 & 49.40 \\
\hline $6 \mathrm{H}-7,79$ & 57.18 & 57.20 & 49.59 \\
\hline 7H-1, 41 & 58.48 & 58.52 & 49.71 \\
\hline $7 \mathrm{H}-1,53$ & 58.70 & 58.64 & 49.83 \\
\hline $7 \mathrm{H}-1,89$ & 58.84 & 59.00 & 50.19 \\
\hline $7 \mathrm{H}-1,128$ & 59.31 & 59.39 & 50.58 \\
\hline $7 \mathrm{H}-2,25$ & 59.92 & 59.86 & 51.05 \\
\hline $7 \mathrm{H}-2,68$ & 60.20 & 60.29 & 51.48 \\
\hline $7 \mathrm{H}-2,89$ & 60.43 & 60.50 & 51.69 \\
\hline $7 \mathrm{H}-2,105$ & 60.63 & 60.66 & 51.85 \\
\hline $7 \mathrm{H}-2,127$ & 60.88 & 60.88 & 52.07 \\
\hline 7H-7, 15 & 66.96 & 66.96 & 58.15 \\
\hline 7H-7, 39 & 67.18 & 67.20 & 58.39 \\
\hline $8 \mathrm{H}-1,68$ & 68.50 & 68.24 & 59.48 \\
\hline $8 \mathrm{H}-1,93$ & 68.78 & 68.50 & 59.73 \\
\hline $8 \mathrm{H}-2,48$ & 69.74 & 69.54 & 60.78 \\
\hline $8 \mathrm{H}-2,65$ & 69.86 & 69.71 & 60.95 \\
\hline $8 \mathrm{H}-2,136$ & 70.49 & 70.42 & 61.66 \\
\hline $8 \mathrm{H}-3,67$ & 71.28 & 71.23 & 62.47 \\
\hline $8 \mathrm{H}-3,93$ & 71.48 & 71.49 & 62.73 \\
\hline $8 \mathrm{H}-3,111$ & 71.64 & 71.67 & 62.91 \\
\hline $8 \mathrm{H}-4,17$ & 72.24 & 72.23 & 63.47 \\
\hline $8 \mathrm{H}-4,39$ & 72.47 & 72.45 & 63.69 \\
\hline
\end{tabular}

\begin{tabular}{|c|c|c|c|}
\hline \multirow[b]{2}{*}{$\begin{array}{l}\text { Core, section, } \\
\text { interval }(\mathrm{cm})\end{array}$} & \multicolumn{3}{|c|}{ Depth $(m)$} \\
\hline & $\begin{array}{l}\text { Adjusted } \\
\text { CCSF }\end{array}$ & CCSF & CSF-A \\
\hline $8 \mathrm{H}-4,67$ & 72.72 & 72.73 & 63.97 \\
\hline $8 \mathrm{H}-4,89$ & 72.95 & 72.95 & 64.19 \\
\hline $8 \mathrm{H}-6,31$ & 75.37 & 75.37 & 66.61 \\
\hline $8 \mathrm{H}-6,71$ & 75.89 & 75.77 & 67.01 \\
\hline $8 \mathrm{H}-6,100$ & 76.13 & 76.06 & 67.30 \\
\hline $8 \mathrm{H}-7,8$ & 76.33 & 76.34 & 67.58 \\
\hline $8 \mathrm{H}-7,36$ & 76.66 & 76.62 & 67.86 \\
\hline $8 \mathrm{H}-7,65$ & 76.85 & 76.91 & 68.15 \\
\hline $9 \mathrm{H}-1,135$ & 78.55 & 78.54 & 69.65 \\
\hline $9 \mathrm{H}-3,2$ & 80.20 & 80.21 & 71.32 \\
\hline $9 \mathrm{H}-3,82$ & 80.98 & 81.01 & 72.12 \\
\hline $9 \mathrm{H}-3,138$ & 81.64 & 81.57 & 72.68 \\
\hline $9 \mathrm{H}-4,60$ & 82.33 & 82.29 & 73.40 \\
\hline $9 \mathrm{H}-4,103$ & 82.72 & 82.72 & 73.83 \\
\hline $9 \mathrm{H}-7,5$ & 85.95 & 85.95 & 77.05 \\
\hline $9 \mathrm{H}-7,34$ & 86.23 & 86.24 & 77.34 \\
\hline $10 \mathrm{H}-1,24$ & 88.15 & 88.31 & 78.04 \\
\hline $10 \mathrm{H}-1,98$ & 89.12 & 89.05 & 78.78 \\
\hline $10 \mathrm{H}-2,29$ & 89.86 & 89.86 & 79.59 \\
\hline $10 \mathrm{H}-6,123$ & 96.80 & 96.80 & 86.53 \\
\hline $10 \mathrm{H}-7,4$ & 97.56 & 97.11 & 86.84 \\
\hline $10 \mathrm{H}-7,42$ & 98.21 & 97.49 & 87.22 \\
\hline $11 \mathrm{H}-1,35$ & 98.42 & 98.42 & 87.65 \\
\hline $11 \mathrm{H}-3,1$ & 100.19 & 101.08 & 90.31 \\
\hline $11 \mathrm{H}-3,25$ & 100.60 & 101.32 & 90.55 \\
\hline $11 \mathrm{H}-4,3$ & 101.91 & 102.60 & 91.83 \\
\hline $11 \mathrm{H}-4,27$ & 102.84 & 102.84 & 92.07 \\
\hline $11 \mathrm{H}-6,82$ & 106.39 & 106.39 & 95.62 \\
\hline $11 \mathrm{H}-6,136$ & 106.97 & 106.94 & 96.16 \\
\hline $12 \mathrm{H}-1,27$ & 109.60 & 109.66 & 97.07 \\
\hline $12 \mathrm{H}-1,44$ & 109.74 & 109.83 & 97.24 \\
\hline $12 \mathrm{H}-1,93$ & 110.29 & 110.32 & 97.73 \\
\hline $12 \mathrm{H}-1,102$ & 110.41 & 110.41 & 97.82 \\
\hline $12 \mathrm{H}-6,112$ & 118.01 & 118.01 & 105.42 \\
\hline $12 \mathrm{H}-7,4$ & 118.33 & 118.43 & 105.84 \\
\hline $12 \mathrm{H}-7,41$ & 118.81 & 118.81 & 106.21 \\
\hline $12 \mathrm{H}-7,53$ & 118.93 & 118.92 & 106.33 \\
\hline $13 \mathrm{H}-1,13$ & 120.62 & 120.77 & 106.43 \\
\hline $13 \mathrm{H}-1,48$ & 120.96 & 121.12 & 106.78 \\
\hline $13 \mathrm{H}-1,103$ & 121.67 & 121.67 & 107.33 \\
\hline $13 \mathrm{H}-6,87$ & 129.01 & 129.01 & 114.67 \\
\hline $13 \mathrm{H}-6,144$ & 129.78 & 129.58 & 115.24 \\
\hline $13 \mathrm{H}-7,48$ & 130.14 & 130.12 & 115.78 \\
\hline $14 \mathrm{H}-1,7$ & 132.79 & 132.93 & 115.87 \\
\hline $14 \mathrm{H}-1,31$ & 133.13 & 133.17 & 116.11 \\
\hline $14 \mathrm{H}-1,59$ & 133.54 & 133.45 & 116.39 \\
\hline $14 \mathrm{H}-1,149$ & 134.28 & 134.35 & 117.29 \\
\hline $14 \mathrm{H}-2,57$ & 134.93 & 134.93 & 117.87 \\
\hline $14 \mathrm{H}-5,124$ & 140.10 & 140.10 & 123.04 \\
\hline $14 \mathrm{H}-6,69$ & 141.09 & 141.05 & 123.99 \\
\hline $14 \mathrm{H}-7,23$ & 142.28 & 142.09 & 125.03 \\
\hline $15 \mathrm{H}-1,47$ & 143.61 & 143.64 & 125.77 \\
\hline $15 \mathrm{H}-2,27$ & 144.92 & 144.94 & 127.07 \\
\hline $15 \mathrm{H}-2,64$ & 145.29 & 145.31 & 127.44 \\
\hline $15 \mathrm{H}-2,94$ & 145.62 & 145.61 & 127.74 \\
\hline $15 \mathrm{H}-3,78$ & 146.93 & 146.95 & 129.08 \\
\hline $15 \mathrm{H}-3,125$ & 147.46 & 147.42 & 129.55 \\
\hline $15 \mathrm{H}-4,33$ & 148.00 & 148.00 & 130.13 \\
\hline $15 \mathrm{H}-7,74$ & 152.41 & 152.41 & 134.54 \\
\hline $17 \mathrm{H}-1,9$ & 154.76 & 154.76 & 136.89 \\
\hline $17 \mathrm{H}-7,77$ & 163.14 & 163.14 & 145.27 \\
\hline $18 \mathrm{H}-1,1$ & 163.28 & 163.28 & 145.41 \\
\hline $18 \mathrm{H}-7,41$ & 172.69 & 172.68 & 154.81 \\
\hline $19 \mathrm{H}-1,93$ & 173.70 & 173.70 & 155.83 \\
\hline $19 \mathrm{H}-1,136$ & 173.98 & 174.13 & 156.26 \\
\hline $19 \mathrm{H}-2,34$ & 174.43 & 174.61 & 156.74 \\
\hline $19 \mathrm{H}-2,80$ & 175.06 & 175.07 & 157.20 \\
\hline $19 \mathrm{H}-4,4$ & 176.81 & 177.31 & 159.44 \\
\hline
\end{tabular}


Table AT14 (continued).

\begin{tabular}{lccc}
\hline & \multicolumn{3}{c}{ Depth $(\mathrm{m})$} \\
\cline { 2 - 4 } $\begin{array}{l}\text { Core, section, } \\
\text { interval }(\mathrm{cm})\end{array}$ & $\begin{array}{c}\text { Adjusted } \\
\text { CCSF }\end{array}$ & CCSF & CSF-A \\
\hline $19 \mathrm{H}-4,99$ & 178.04 & 178.26 & 160.39 \\
$19 \mathrm{H}-5,43$ & 178.88 & 179.20 & 161.33 \\
$19 \mathrm{H}-5,85$ & 179.87 & 179.62 & 161.75 \\
$19 \mathrm{H}-5,139$ & 180.27 & 180.16 & 162.29 \\
$19 \mathrm{H}-6,46$ & 181.02 & 180.73 & 162.86 \\
$19 \mathrm{H}-6,82$ & 181.68 & 181.09 & 163.22 \\
$19 \mathrm{H}-7,1$ & 182.47 & 181.78 & 163.91 \\
$19 \mathrm{H}-7,24$ & 182.60 & 182.01 & 164.14 \\
$19 \mathrm{H}-7,55$ & 182.86 & 182.32 & 164.45 \\
$20 \mathrm{H}-1,147$ & 183.59 & 183.74 & 165.87 \\
$20 \mathrm{H}-2,26$ & 184.01 & 184.03 & 166.16 \\
$20 \mathrm{H}-2,111$ & 185.07 & 184.88 & 167.01 \\
$20 \mathrm{H}-3,78$ & 185.99 & 186.05 & 168.18 \\
$20 \mathrm{H}-4,72$ & 187.50 & 187.49 & 169.62 \\
$20 \mathrm{H}-5,4$ & 189.00 & 188.31 & 170.44 \\
$20 \mathrm{H}-5,106$ & 190.28 & 189.33 & 171.46 \\
$20 \mathrm{H}-7,3$ & 191.64 & 191.30 & 173.43 \\
\hline
\end{tabular}

This table is also available in ASCII. 
Table AT15. Gamma ray attenuation (GRA) density data, Site U1337.

\begin{tabular}{|c|c|c|c|}
\hline \multirow{2}{*}{$\begin{array}{l}\text { Core, section, } \\
\text { interval }(\mathrm{cm})\end{array}$} & \multicolumn{2}{|c|}{ Depth (m) } & \multirow{2}{*}{$\begin{array}{c}\text { GRA } \\
\left(\mathrm{g} / \mathrm{cm}^{3}\right)\end{array}$} \\
\hline & CSF & CCSF & \\
\hline \multicolumn{4}{|l|}{ 321-U1337B- } \\
\hline $1 \mathrm{H}-1,2.5$ & 1.02 & 0.02 & 1.29 \\
\hline $1 \mathrm{H}-1,5$ & 1.05 & 0.05 & 1.28 \\
\hline $1 \mathrm{H}-1,7.5$ & 1.08 & 0.08 & 1.25 \\
\hline $1 \mathrm{H}-1,10$ & 1.10 & 0.10 & 1.27 \\
\hline $1 \mathrm{H}-1,12.5$ & 1.13 & 0.13 & 1.33 \\
\hline $1 \mathrm{H}-1,15$ & 1.15 & 0.15 & 1.35 \\
\hline $1 \mathrm{H}-1,17.5$ & 1.18 & 0.18 & 1.38 \\
\hline $1 \mathrm{H}-1,20$ & 1.20 & 0.20 & 1.37 \\
\hline $1 \mathrm{H}-1,22.5$ & 1.23 & 0.23 & 1.35 \\
\hline $1 \mathrm{H}-1,25$ & 1.25 & 0.25 & 1.36 \\
\hline $1 \mathrm{H}-1,27.6$ & 1.28 & 0.28 & 1.32 \\
\hline $1 \mathrm{H}-1,30.1$ & 1.30 & 0.30 & 1.33 \\
\hline $1 \mathrm{H}-1,32.6$ & 1.33 & 0.33 & 1.30 \\
\hline $1 \mathrm{H}-1,35.1$ & 1.35 & 0.35 & 1.35 \\
\hline $1 \mathrm{H}-1,37.6$ & 1.38 & 0.38 & 1.37 \\
\hline $1 \mathrm{H}-1,40.1$ & 1.40 & 0.40 & 1.34 \\
\hline $1 \mathrm{H}-1,42.6$ & 1.43 & 0.43 & 1.40 \\
\hline $1 \mathrm{H}-1,45.1$ & 1.45 & 0.45 & 1.45 \\
\hline $1 \mathrm{H}-1,47.6$ & 1.48 & 0.48 & 1.43 \\
\hline $1 \mathrm{H}-1,50.1$ & 1.50 & 0.50 & 1.46 \\
\hline $1 \mathrm{H}-1,52.6$ & 1.53 & 0.53 & 1.44 \\
\hline $1 \mathrm{H}-1,55.1$ & 1.55 & 0.55 & 1.41 \\
\hline $1 \mathrm{H}-1,57.6$ & 1.58 & 0.58 & 1.42 \\
\hline $1 \mathrm{H}-1,60.1$ & 1.60 & 0.60 & 1.42 \\
\hline $1 \mathrm{H}-1,62.6$ & 1.63 & 0.63 & 1.40 \\
\hline $1 \mathrm{H}-1,65.1$ & 1.65 & 0.65 & 1.41 \\
\hline $1 \mathrm{H}-1,67.6$ & 1.68 & 0.68 & 1.39 \\
\hline $1 \mathrm{H}-1,70.1$ & 1.70 & 0.70 & 1.43 \\
\hline $1 \mathrm{H}-1,72.6$ & 1.73 & 0.73 & 1.41 \\
\hline $1 \mathrm{H}-1,75.1$ & 1.75 & 0.75 & 1.41 \\
\hline $1 \mathrm{H}-1,77.6$ & 1.78 & 0.78 & 1.40 \\
\hline $1 \mathrm{H}-1,80.1$ & 1.80 & 0.80 & 1.34 \\
\hline $1 \mathrm{H}-1,82.6$ & 1.83 & 0.83 & 1.36 \\
\hline $1 \mathrm{H}-1,85.1$ & 1.85 & 0.85 & 1.33 \\
\hline $1 \mathrm{H}-1,87.6$ & 1.88 & 0.88 & 1.30 \\
\hline $1 \mathrm{H}-1,90.2$ & 1.90 & 0.90 & 1.27 \\
\hline $1 \mathrm{H}-1,92.7$ & 1.93 & 0.93 & 1.22 \\
\hline $1 \mathrm{H}-1,95.2$ & 1.95 & 0.95 & 1.23 \\
\hline $1 \mathrm{H}-1,97.7$ & 1.98 & 0.98 & 1.20 \\
\hline $1 \mathrm{H}-1,100.2$ & 2.00 & 1.00 & 1.18 \\
\hline $1 \mathrm{H}-1,102.7$ & 2.03 & 1.03 & 1.18 \\
\hline $1 \mathrm{H}-1,105.2$ & 2.05 & 1.05 & 1.14 \\
\hline $1 \mathrm{H}-1,107.7$ & 2.08 & 1.08 & 1.11 \\
\hline $1 \mathrm{H}-1,110.2$ & 2.10 & 1.10 & 1.12 \\
\hline $1 \mathrm{H}-1,117.7$ & 2.18 & 1.18 & 1.16 \\
\hline $1 \mathrm{H}-1,120.2$ & 2.20 & 1.20 & 1.18 \\
\hline $1 \mathrm{H}-1,122.7$ & 2.23 & 1.23 & 1.20 \\
\hline $1 \mathrm{H}-1,125.2$ & 2.25 & 1.25 & 1.18 \\
\hline $1 \mathrm{H}-1,127.7$ & 2.28 & 1.28 & 1.16 \\
\hline $1 \mathrm{H}-1,130.2$ & 2.30 & 1.30 & 1.21 \\
\hline $1 \mathrm{H}-1,132.7$ & 2.33 & 1.33 & 1.11 \\
\hline $1 \mathrm{H}-1,135.2$ & 2.35 & 1.35 & 1.16 \\
\hline $1 \mathrm{H}-1,137.7$ & 2.38 & 1.38 & 1.20 \\
\hline $1 \mathrm{H}-1,140.2$ & 2.40 & 1.40 & 1.19 \\
\hline $1 \mathrm{H}-1,142.7$ & 2.43 & 1.43 & 1.16 \\
\hline $1 \mathrm{H}-1,145.3$ & 2.45 & 1.45 & 1.18 \\
\hline $1 \mathrm{H}-1,147.8$ & 2.48 & 1.48 & 1.14 \\
\hline $1 \mathrm{H}-2,2$ & 2.52 & 1.52 & 1.19 \\
\hline $1 \mathrm{H}-2,4.5$ & 2.55 & 1.55 & 1.17 \\
\hline $1 \mathrm{H}-2,7$ & 2.57 & 1.57 & 1.19 \\
\hline $1 \mathrm{H}-2,9.5$ & 2.60 & 1.60 & 1.18 \\
\hline $1 \mathrm{H}-2,12$ & 2.62 & 1.62 & 1.16 \\
\hline
\end{tabular}

Only a portion of this table appears here. The complete table is available in ASCII. 
Table AT16. Magnetic susceptibility data, Site U1337.

\begin{tabular}{|c|c|c|c|}
\hline \multirow{2}{*}{$\begin{array}{l}\text { Core, section, } \\
\text { interval }(\mathrm{cm})\end{array}$} & \multicolumn{2}{|c|}{ Depth (m) } & \multirow{2}{*}{$\begin{array}{c}\text { Magnetic } \\
\text { susceptibility } \\
\text { (IU) }\end{array}$} \\
\hline & CSF & CCSF & \\
\hline \multicolumn{4}{|l|}{ 321-U1337B- } \\
\hline $1 \mathrm{H}-1,0.1$ & 1.00 & 0.00 & 1.6 \\
\hline $1 \mathrm{H}-1,2.6$ & 1.03 & 0.03 & 4.4 \\
\hline $1 \mathrm{H}-1,5.1$ & 1.05 & 0.05 & 4.9 \\
\hline $1 \mathrm{H}-1,7.6$ & 1.08 & 0.08 & 6.6 \\
\hline $1 \mathrm{H}-1,10.1$ & 1.10 & 0.10 & 4.9 \\
\hline $1 \mathrm{H}-1,12.6$ & 1.13 & 0.13 & 5.2 \\
\hline $1 \mathrm{H}-1,15.1$ & 1.15 & 0.15 & 4.4 \\
\hline $1 \mathrm{H}-1,17.6$ & 1.18 & 0.18 & 4.1 \\
\hline $1 \mathrm{H}-1,20.1$ & 1.20 & 0.20 & 3.3 \\
\hline $1 \mathrm{H}-1,22.6$ & 1.23 & 0.23 & 3.3 \\
\hline $1 \mathrm{H}-1,25.1$ & 1.25 & 0.25 & 3.0 \\
\hline $1 \mathrm{H}-1,27.6$ & 1.28 & 0.28 & 2.2 \\
\hline $1 \mathrm{H}-1,30.1$ & 1.30 & 0.30 & 2.7 \\
\hline $1 \mathrm{H}-1,32.6$ & 1.33 & 0.33 & 2.2 \\
\hline $1 \mathrm{H}-1,35.1$ & 1.35 & 0.35 & 1.6 \\
\hline $1 \mathrm{H}-1,37.6$ & 1.38 & 0.38 & 1.4 \\
\hline $1 \mathrm{H}-1,40.1$ & 1.40 & 0.40 & 1.6 \\
\hline $1 \mathrm{H}-1,42.6$ & 1.43 & 0.43 & 1.1 \\
\hline $1 \mathrm{H}-1,45.1$ & 1.45 & 0.45 & 0.5 \\
\hline $1 \mathrm{H}-1,47.6$ & 1.48 & 0.48 & 1.1 \\
\hline $1 \mathrm{H}-1,50.1$ & 1.50 & 0.50 & 1.1 \\
\hline $1 \mathrm{H}-1,52.6$ & 1.53 & 0.53 & 1.1 \\
\hline $1 \mathrm{H}-1,55.2$ & 1.55 & 0.55 & 1.6 \\
\hline $1 \mathrm{H}-1,57.7$ & 1.58 & 0.58 & 1.6 \\
\hline $1 \mathrm{H}-1,60.2$ & 1.60 & 0.60 & 0.8 \\
\hline $1 \mathrm{H}-1,62.7$ & 1.63 & 0.63 & 1.6 \\
\hline $1 \mathrm{H}-1,65.2$ & 1.65 & 0.65 & 1.9 \\
\hline $1 \mathrm{H}-1,67.7$ & 1.68 & 0.68 & 1.9 \\
\hline $1 \mathrm{H}-1,70.2$ & 1.70 & 0.70 & 1.6 \\
\hline $1 \mathrm{H}-1,72.7$ & 1.73 & 0.73 & 1.6 \\
\hline $1 \mathrm{H}-1,75.2$ & 1.75 & 0.75 & 2.2 \\
\hline $1 \mathrm{H}-1,77.7$ & 1.78 & 0.78 & 3.0 \\
\hline $1 \mathrm{H}-1,80.2$ & 1.80 & 0.80 & 3.0 \\
\hline $1 \mathrm{H}-1,82.7$ & 1.83 & 0.83 & 3.8 \\
\hline $1 \mathrm{H}-1,85.2$ & 1.85 & 0.85 & 5.2 \\
\hline $1 \mathrm{H}-1,87.7$ & 1.88 & 0.88 & 5.8 \\
\hline $1 \mathrm{H}-1,90.2$ & 1.90 & 0.90 & 6.9 \\
\hline $1 \mathrm{H}-1,92.7$ & 1.93 & 0.93 & 6.9 \\
\hline $1 \mathrm{H}-1,95.2$ & 1.95 & 0.95 & 7.1 \\
\hline $1 \mathrm{H}-1,97.7$ & 1.98 & 0.98 & 6.9 \\
\hline $1 \mathrm{H}-1,100.2$ & 2.00 & 1.00 & 7.1 \\
\hline $1 \mathrm{H}-1,102.7$ & 2.03 & 1.03 & 7.1 \\
\hline $1 \mathrm{H}-1,105.2$ & 2.05 & 1.05 & 6.6 \\
\hline $1 \mathrm{H}-1,107.7$ & 2.08 & 1.08 & 6.6 \\
\hline $1 \mathrm{H}-1,110.2$ & 2.10 & 1.10 & 6.6 \\
\hline $1 \mathrm{H}-1,112.8$ & 2.13 & 1.13 & 5.2 \\
\hline $1 \mathrm{H}-1,115.2$ & 2.15 & 1.15 & 4.4 \\
\hline $1 \mathrm{H}-1,117.8$ & 2.18 & 1.18 & 6.6 \\
\hline $1 \mathrm{H}-1,120.3$ & 2.20 & 1.20 & 7.1 \\
\hline $1 \mathrm{H}-1,122.8$ & 2.23 & 1.23 & 7.4 \\
\hline $1 \mathrm{H}-1,125.3$ & 2.25 & 1.25 & 7.4 \\
\hline $1 \mathrm{H}-1,127.8$ & 2.28 & 1.28 & 7.4 \\
\hline $1 \mathrm{H}-1,130.3$ & 2.30 & 1.30 & 7.7 \\
\hline $1 \mathrm{H}-1,132.8$ & 2.33 & 1.33 & 8.2 \\
\hline $1 \mathrm{H}-1,135.3$ & 2.35 & 1.35 & 8.5 \\
\hline $1 \mathrm{H}-1,137.8$ & 2.38 & 1.38 & 8.8 \\
\hline $1 \mathrm{H}-1,140.3$ & 2.40 & 1.40 & 8.8 \\
\hline $1 \mathrm{H}-1,142.8$ & 2.43 & 1.43 & 9.1 \\
\hline $1 \mathrm{H}-1,145.3$ & 2.45 & 1.45 & 9.1 \\
\hline $1 \mathrm{H}-1,147.8$ & 2.48 & 1.48 & 7.7 \\
\hline
\end{tabular}

Only a portion of this table appears here. The complete table is available in ASCII. 
Table AT17. Natural gamma ray (NGR) data, Site U1337.

\begin{tabular}{|c|c|c|c|}
\hline \multirow{2}{*}{$\begin{array}{l}\text { Core, section, } \\
\text { interval }(\mathrm{cm})\end{array}$} & \multicolumn{2}{|c|}{ Depth (m) } & \multirow{2}{*}{$\begin{array}{l}\text { NGR } \\
(\mathrm{cps})\end{array}$} \\
\hline & CSF & CCSF & \\
\hline \multicolumn{4}{|l|}{ 321-U1337B- } \\
\hline $1 \mathrm{H}-1,0$ & 1.00 & 0.00 & 65.0 \\
\hline $1 \mathrm{H}-1,10$ & 1.10 & 0.10 & 67.0 \\
\hline $1 \mathrm{H}-1,20$ & 1.20 & 0.20 & 67.8 \\
\hline $1 \mathrm{H}-1,30$ & 1.30 & 0.30 & 56.6 \\
\hline $1 \mathrm{H}-1,40$ & 1.40 & 0.40 & 43.9 \\
\hline $1 \mathrm{H}-1,50$ & 1.50 & 0.50 & 42.9 \\
\hline $1 \mathrm{H}-1,60$ & 1.60 & 0.60 & 42.8 \\
\hline $1 \mathrm{H}-1,70$ & 1.70 & 0.70 & 41.0 \\
\hline $1 \mathrm{H}-1,80$ & 1.80 & 0.80 & 50.5 \\
\hline $1 \mathrm{H}-1,90$ & 1.90 & 0.90 & 65.5 \\
\hline $1 \mathrm{H}-1,100$ & 2.00 & 1.00 & 52.7 \\
\hline $1 \mathrm{H}-1,110$ & 2.10 & 1.10 & 42.0 \\
\hline $1 \mathrm{H}-1,120$ & 2.20 & 1.20 & 51.7 \\
\hline $1 \mathrm{H}-1,130$ & 2.30 & 1.30 & 62.8 \\
\hline $1 \mathrm{H}-1,140$ & 2.40 & 1.40 & 57.6 \\
\hline $1 \mathrm{H}-1,150$ & 2.50 & 1.50 & 48.6 \\
\hline $1 \mathrm{H}-2,0$ & 2.50 & 1.50 & 46.8 \\
\hline $1 \mathrm{H}-2,10$ & 2.60 & 1.60 & 46.6 \\
\hline $1 \mathrm{H}-2,20$ & 2.70 & 1.70 & 42.9 \\
\hline $1 \mathrm{H}-2,30$ & 2.80 & 1.80 & 34.1 \\
\hline $1 \mathrm{H}-2,40$ & 2.90 & 1.90 & 26.0 \\
\hline $1 \mathrm{H}-2,50$ & 3.00 & 2.00 & 24.6 \\
\hline $1 \mathrm{H}-2,60$ & 3.10 & 2.10 & 26.1 \\
\hline $1 \mathrm{H}-2,70$ & 3.20 & 2.20 & 22.0 \\
\hline $1 \mathrm{H}-2,80$ & 3.30 & 2.30 & 19.2 \\
\hline $1 \mathrm{H}-2,90$ & 3.40 & 2.40 & 25.5 \\
\hline $1 \mathrm{H}-2,100$ & 3.50 & 2.50 & 29.4 \\
\hline $1 \mathrm{H}-2,110$ & 3.60 & 2.60 & 24.1 \\
\hline $1 \mathrm{H}-2,120$ & 3.70 & 2.70 & 20.2 \\
\hline $1 \mathrm{H}-2,130$ & 3.80 & 2.80 & 16.2 \\
\hline $1 \mathrm{H}-2,140$ & 3.90 & 2.90 & 14.3 \\
\hline $1 \mathrm{H}-2,150$ & 4.00 & 3.00 & 15.4 \\
\hline $1 \mathrm{H}-3,0$ & 4.00 & 3.00 & 18.4 \\
\hline $1 \mathrm{H}-3,10$ & 4.10 & 3.10 & 23.9 \\
\hline $1 \mathrm{H}-3,20$ & 4.20 & 3.20 & 31.1 \\
\hline $1 \mathrm{H}-3,30$ & 4.30 & 3.30 & 25.9 \\
\hline $1 \mathrm{H}-3,40$ & 4.40 & 3.40 & 23.4 \\
\hline $1 \mathrm{H}-3,50$ & 4.50 & 3.50 & 19.8 \\
\hline $1 \mathrm{H}-3,60$ & 4.60 & 3.60 & 16.7 \\
\hline $1 \mathrm{H}-3,70$ & 4.70 & 3.70 & 15.2 \\
\hline $1 \mathrm{H}-3,80$ & 4.80 & 3.80 & 16.0 \\
\hline $1 \mathrm{H}-3,90$ & 4.90 & 3.90 & 13.9 \\
\hline $1 \mathrm{H}-3,100$ & 5.00 & 4.00 & 9.9 \\
\hline $1 \mathrm{H}-3,110$ & 5.10 & 4.10 & 9.5 \\
\hline $1 \mathrm{H}-3,120$ & 5.20 & 4.20 & 9.5 \\
\hline $1 \mathrm{H}-3,130$ & 5.30 & 4.30 & 11.8 \\
\hline \multicolumn{4}{|l|}{ 321-U1337A- } \\
\hline $2 \mathrm{H}-3,120$ & 9.70 & 4.39 & 19.0 \\
\hline $2 \mathrm{H}-3,130$ & 9.80 & 4.49 & 16.3 \\
\hline $2 \mathrm{H}-3,140$ & 9.90 & 4.59 & 13.1 \\
\hline $2 \mathrm{H}-3,150$ & 10.00 & 4.69 & 11.4 \\
\hline $2 \mathrm{H}-4,0$ & 10.00 & 4.69 & 11.4 \\
\hline $2 \mathrm{H}-4,10$ & 10.10 & 4.79 & 11.7 \\
\hline $2 \mathrm{H}-4,20$ & 10.20 & 4.89 & 12.0 \\
\hline $2 \mathrm{H}-4,30$ & 10.30 & 4.99 & 11.3 \\
\hline $2 \mathrm{H}-4,40$ & 10.40 & 5.09 & 11.4 \\
\hline $2 \mathrm{H}-4,50$ & 10.50 & 5.19 & 9.9 \\
\hline $2 \mathrm{H}-4,60$ & 10.60 & 5.29 & 9.2 \\
\hline $2 \mathrm{H}-4,70$ & 10.70 & 5.39 & 8.2 \\
\hline $2 \mathrm{H}-4,80$ & 10.80 & 5.49 & 8.2 \\
\hline
\end{tabular}

Only a portion of this table appears here. The complete table is available in ASCII. 
Table AT18. Color reflection data, Site U1337.

\begin{tabular}{|c|c|c|c|c|c|}
\hline $\begin{array}{l}\text { Core, section, } \\
\text { interval }(\mathrm{cm})\end{array}$ & $\begin{array}{l}\text { Depth } \\
\operatorname{CSF}(m)\end{array}$ & $\begin{array}{c}\text { Depth } \\
\operatorname{CCSF}(\mathrm{m})\end{array}$ & $a^{*}$ & $b^{*}$ & $\mathrm{~L}^{*}$ \\
\hline \multicolumn{6}{|l|}{ 321-U1337B- } \\
\hline 1H-1, 5.2 & 1.05 & 0.05 & 6.8 & 10.3 & 35.1 \\
\hline $1 \mathrm{H}-1,7.7$ & 1.08 & 0.08 & 6.4 & 9.7 & 35.2 \\
\hline $1 \mathrm{H}-1,10.2$ & 1.10 & 0.10 & 6.0 & 10.5 & 38.9 \\
\hline $1 \mathrm{H}-1,12.8$ & 1.13 & 0.13 & 3.4 & 6.8 & 41.1 \\
\hline $1 \mathrm{H}-1,15.3$ & 1.15 & 0.15 & 6.0 & 11.9 & 41.0 \\
\hline $1 \mathrm{H}-1,17.9$ & 1.18 & 0.18 & 4.9 & 9.9 & 43.9 \\
\hline $1 \mathrm{H}-1,20.4$ & 1.20 & 0.20 & 4.3 & 9.2 & 46.2 \\
\hline $1 \mathrm{H}-1,22.9$ & 1.23 & 0.23 & 6.0 & 14.2 & 49.7 \\
\hline $1 \mathrm{H}-1,25.5$ & 1.25 & 0.25 & 5.3 & 12.2 & 50.8 \\
\hline $1 \mathrm{H}-1,28$ & 1.28 & 0.28 & 4.2 & 10.5 & 49.6 \\
\hline $1 \mathrm{H}-1,30.6$ & 1.31 & 0.31 & 5.8 & 14.6 & 53.0 \\
\hline $1 \mathrm{H}-1,33.1$ & 1.33 & 0.33 & 6.2 & 17.1 & 51.5 \\
\hline $1 \mathrm{H}-1,35.6$ & 1.36 & 0.36 & 6.1 & 19.0 & 56.1 \\
\hline $1 \mathrm{H}-1,38.2$ & 1.38 & 0.38 & 6.2 & 18.1 & 53.3 \\
\hline $1 \mathrm{H}-1,40.7$ & 1.41 & 0.41 & 5.7 & 19.1 & 55.9 \\
\hline $1 \mathrm{H}-1,43.3$ & 1.43 & 0.43 & 5.8 & 19.9 & 58.5 \\
\hline $1 \mathrm{H}-1,45.8$ & 1.46 & 0.46 & 5.3 & 19.9 & 62.9 \\
\hline $1 \mathrm{H}-1,48.4$ & 1.48 & 0.48 & 4.9 & 15.9 & 60.8 \\
\hline $1 \mathrm{H}-1,50.9$ & 1.51 & 0.51 & 4.5 & 14.6 & 61.9 \\
\hline $1 \mathrm{H}-1,53.4$ & 1.53 & 0.53 & 3.7 & 12.2 & 57.0 \\
\hline $1 \mathrm{H}-1,56$ & 1.56 & 0.56 & 2.4 & 9.1 & 58.1 \\
\hline $1 \mathrm{H}-1,58.5$ & 1.59 & 0.59 & 3.2 & 12.1 & 57.1 \\
\hline $1 \mathrm{H}-1,61.1$ & 1.61 & 0.61 & 1.4 & 13.8 & 76.1 \\
\hline $1 \mathrm{H}-1,63.6$ & 1.64 & 0.64 & 4.5 & 14.8 & 56.3 \\
\hline $1 \mathrm{H}-1,66.1$ & 1.66 & 0.66 & 3.2 & 10.6 & 55.3 \\
\hline $1 \mathrm{H}-1,68.7$ & 1.69 & 0.69 & 4.0 & 13.1 & 59.0 \\
\hline $1 \mathrm{H}-1,71.2$ & 1.71 & 0.71 & 4.5 & 15.4 & 56.0 \\
\hline $1 \mathrm{H}-1,73.8$ & 1.74 & 0.74 & 4.8 & 17.7 & 62.0 \\
\hline $1 \mathrm{H}-1,76.3$ & 1.76 & 0.76 & 4.2 & 14.1 & 58.2 \\
\hline $1 \mathrm{H}-1,78.9$ & 1.79 & 0.79 & 3.3 & 12.2 & 56.2 \\
\hline $1 \mathrm{H}-1,81.4$ & 1.81 & 0.81 & 3.5 & 12.2 & 53.7 \\
\hline $1 \mathrm{H}-1,83.9$ & 1.84 & 0.84 & 4.0 & 15.3 & 51.5 \\
\hline $1 \mathrm{H}-1,86.5$ & 1.87 & 0.87 & 2.8 & 11.5 & 48.3 \\
\hline $1 \mathrm{H}-1,89$ & 1.89 & 0.89 & 3.1 & 10.4 & 40.8 \\
\hline $1 \mathrm{H}-1,91.6$ & 1.92 & 0.92 & 3.2 & 8.8 & 36.6 \\
\hline $1 \mathrm{H}-1,94.1$ & 1.94 & 0.94 & 5.3 & 9.1 & 35.5 \\
\hline $1 \mathrm{H}-1,96.7$ & 1.97 & 0.97 & 3.5 & 6.6 & 40.8 \\
\hline $1 \mathrm{H}-1,99.2$ & 1.99 & 0.99 & 4.1 & 11.8 & 51.8 \\
\hline $1 \mathrm{H}-1,101.7$ & 2.02 & 1.02 & 7.1 & 15.1 & 42.1 \\
\hline $1 \mathrm{H}-1,104.3$ & 2.04 & 1.04 & 5.3 & 11.5 & 44.4 \\
\hline $1 \mathrm{H}-1,106.8$ & 2.07 & 1.07 & 4.8 & 11.9 & 44.8 \\
\hline $1 \mathrm{H}-1,109.4$ & 2.09 & 1.09 & 7.4 & 15.4 & 39.5 \\
\hline $1 \mathrm{H}-1,111.9$ & 2.12 & 1.12 & 6.9 & 15.7 & 41.8 \\
\hline $1 \mathrm{H}-1,114.4$ & 2.14 & 1.14 & 6.8 & 14.4 & 40.7 \\
\hline $1 \mathrm{H}-1,117$ & 2.17 & 1.17 & 7.9 & 15.5 & 40.3 \\
\hline $1 \mathrm{H}-1,119.5$ & 2.19 & 1.19 & 7.5 & 16.4 & 40.8 \\
\hline $1 \mathrm{H}-1,122.1$ & 2.22 & 1.22 & 7.6 & 14.6 & 40.7 \\
\hline $1 \mathrm{H}-1,124.6$ & 2.25 & 1.25 & 4.2 & 8.1 & 42.1 \\
\hline $1 \mathrm{H}-1,127.2$ & 2.27 & 1.27 & 4.0 & 7.7 & 40.6 \\
\hline $1 \mathrm{H}-1,129.7$ & 2.30 & 1.30 & 5.3 & 10.2 & 35.6 \\
\hline $1 \mathrm{H}-1,132.2$ & 2.32 & 1.32 & 5.8 & 10.7 & 35.3 \\
\hline $1 \mathrm{H}-1,134.8$ & 2.35 & 1.35 & 6.0 & 7.4 & 32.6 \\
\hline $1 \mathrm{H}-1,137.3$ & 2.37 & 1.37 & 3.7 & 8.1 & 40.8 \\
\hline $1 \mathrm{H}-1,139.9$ & 2.40 & 1.40 & 4.9 & 8.8 & 37.4 \\
\hline $1 \mathrm{H}-1,142.4$ & 2.42 & 1.42 & 3.4 & 5.3 & 36.7 \\
\hline $1 \mathrm{H}-1,145$ & 2.45 & 1.45 & 3.8 & 5.8 & 34.5 \\
\hline $1 \mathrm{H}-1,147.5$ & 2.48 & 1.48 & 3.1 & 2.3 & 29.0 \\
\hline $2 \mathrm{H}-1,2.6$ & 2.53 & 1.53 & 5.6 & 0.3 & 23.8 \\
\hline $2 \mathrm{H}-1,5.2$ & 2.55 & 1.55 & 3.7 & 6.7 & 39.7 \\
\hline
\end{tabular}

Only a portion of this table appears here. The complete table is available in ASCII. 
Table AT19. Gamma ray attenuation (GRA) density data, Hole U1337A.

\begin{tabular}{|c|c|c|c|c|}
\hline \multirow[b]{2}{*}{$\begin{array}{l}\text { Core, section, } \\
\text { interval }(\mathrm{cm})\end{array}$} & \multicolumn{3}{|c|}{ Depth (m) } & \multirow[b]{2}{*}{$\begin{array}{c}\text { GRA } \\
\left(\mathrm{g} / \mathrm{cm}^{3}\right)\end{array}$} \\
\hline & CSF & CCSF & $\begin{array}{l}\text { Adjusted } \\
\text { CCSF }\end{array}$ & \\
\hline \multicolumn{5}{|l|}{ 321-U1337A- } \\
\hline $2 \mathrm{H}-1,5$ & 5.55 & 0.24 & 0.12 & 1.32 \\
\hline $2 \mathrm{H}-1,7.5$ & 5.58 & 0.27 & 0.15 & 1.35 \\
\hline $2 \mathrm{H}-1,10$ & 5.60 & 0.29 & 0.17 & 1.43 \\
\hline $2 \mathrm{H}-1,12.5$ & 5.63 & 0.32 & 0.21 & 1.39 \\
\hline $2 \mathrm{H}-1,15$ & 5.65 & 0.34 & 0.23 & 1.36 \\
\hline $2 \mathrm{H}-1,17.5$ & 5.68 & 0.37 & 0.26 & 1.42 \\
\hline $2 \mathrm{H}-1,20$ & 5.70 & 0.39 & 0.29 & 1.38 \\
\hline $2 \mathrm{H}-1,22.5$ & 5.73 & 0.42 & 0.32 & 1.40 \\
\hline $2 \mathrm{H}-1,25$ & 5.75 & 0.44 & 0.34 & 1.37 \\
\hline $2 \mathrm{H}-1,27.5$ & 5.78 & 0.47 & 0.37 & 1.35 \\
\hline $2 \mathrm{H}-1,30$ & 5.80 & 0.49 & 0.40 & 1.40 \\
\hline $2 \mathrm{H}-1,32.5$ & 5.83 & 0.52 & 0.43 & 1.43 \\
\hline $2 \mathrm{H}-1,35.1$ & 5.85 & 0.54 & 0.45 & 1.44 \\
\hline $2 \mathrm{H}-1,37.6$ & 5.88 & 0.57 & 0.48 & 1.43 \\
\hline $2 \mathrm{H}-1,40.1$ & 5.90 & 0.59 & 0.51 & 1.43 \\
\hline $2 \mathrm{H}-1,42.6$ & 5.93 & 0.62 & 0.54 & 1.43 \\
\hline $2 \mathrm{H}-1,45.1$ & 5.95 & 0.64 & 0.56 & 1.44 \\
\hline $2 \mathrm{H}-1,47.6$ & 5.98 & 0.67 & 0.59 & 1.40 \\
\hline $2 \mathrm{H}-1,50.1$ & 6.00 & 0.69 & 0.62 & 1.43 \\
\hline $2 \mathrm{H}-1,52.6$ & 6.03 & 0.72 & 0.65 & 1.42 \\
\hline $2 \mathrm{H}-1,55.1$ & 6.05 & 0.74 & 0.67 & 1.43 \\
\hline $2 \mathrm{H}-1,57.6$ & 6.08 & 0.77 & 0.71 & 1.42 \\
\hline $2 \mathrm{H}-1,60.1$ & 6.10 & 0.79 & 0.73 & 1.43 \\
\hline $2 \mathrm{H}-1,62.6$ & 6.13 & 0.82 & 0.76 & 1.42 \\
\hline $2 \mathrm{H}-1,65.1$ & 6.15 & 0.84 & 0.78 & 1.42 \\
\hline $2 \mathrm{H}-1,67.6$ & 6.18 & 0.87 & 0.82 & 1.41 \\
\hline $2 \mathrm{H}-1,70.1$ & 6.20 & 0.89 & 0.84 & 1.38 \\
\hline $2 \mathrm{H}-1,72.6$ & 6.23 & 0.92 & 0.87 & 1.38 \\
\hline $2 \mathrm{H}-1,75.1$ & 6.25 & 0.94 & 0.90 & 1.32 \\
\hline $2 \mathrm{H}-1,77.6$ & 6.28 & 0.97 & 0.93 & 1.25 \\
\hline $2 \mathrm{H}-1,80.1$ & 6.30 & 0.99 & 0.96 & 1.17 \\
\hline $2 \mathrm{H}-1,82.6$ & 6.33 & 1.02 & 0.99 & 1.18 \\
\hline $2 \mathrm{H}-1,85.1$ & 6.35 & 1.04 & 1.02 & 1.17 \\
\hline $2 \mathrm{H}-1,87.6$ & 6.38 & 1.07 & 1.05 & 1.19 \\
\hline $2 \mathrm{H}-1,90.1$ & 6.40 & 1.09 & 1.08 & 1.19 \\
\hline $2 \mathrm{H}-1,92.6$ & 6.43 & 1.12 & 1.11 & 1.18 \\
\hline $2 \mathrm{H}-1,95.1$ & 6.45 & 1.14 & 1.14 & 1.18 \\
\hline $2 \mathrm{H}-1,97.6$ & 6.48 & 1.17 & 1.17 & 1.19 \\
\hline $2 \mathrm{H}-1,100.2$ & 6.50 & 1.19 & 1.20 & 1.21 \\
\hline $2 \mathrm{H}-1,102.7$ & 6.53 & 1.22 & 1.23 & 1.18 \\
\hline $2 \mathrm{H}-1,105.2$ & 6.55 & 1.24 & 1.26 & 1.20 \\
\hline $2 \mathrm{H}-1,107.7$ & 6.58 & 1.27 & 1.29 & 1.20 \\
\hline $2 \mathrm{H}-1,110.2$ & 6.60 & 1.29 & 1.32 & 1.21 \\
\hline $2 \mathrm{H}-1,112.7$ & 6.63 & 1.32 & 1.35 & 1.17 \\
\hline $2 \mathrm{H}-1,115.2$ & 6.65 & 1.34 & 1.38 & 1.20 \\
\hline $2 \mathrm{H}-1,117.7$ & 6.68 & 1.37 & 1.42 & 1.19 \\
\hline $2 \mathrm{H}-1,120.2$ & 6.70 & 1.39 & 1.44 & 1.17 \\
\hline $2 \mathrm{H}-1,122.7$ & 6.73 & 1.42 & 1.48 & 1.18 \\
\hline $2 \mathrm{H}-1,125.2$ & 6.75 & 1.44 & 1.50 & 1.18 \\
\hline $2 \mathrm{H}-1,127.7$ & 6.78 & 1.47 & 1.54 & 1.19 \\
\hline $2 \mathrm{H}-1,130.2$ & 6.80 & 1.49 & 1.56 & 1.18 \\
\hline $2 \mathrm{H}-1,132.7$ & 6.83 & 1.52 & 1.60 & 1.18 \\
\hline $2 \mathrm{H}-1,135.2$ & 6.85 & 1.54 & 1.62 & 1.16 \\
\hline $2 \mathrm{H}-1,137.7$ & 6.88 & 1.57 & 1.66 & 1.16 \\
\hline $2 \mathrm{H}-1,140.2$ & 6.90 & 1.59 & 1.68 & 1.18 \\
\hline $2 \mathrm{H}-1,142.7$ & 6.93 & 1.62 & 1.72 & 1.24 \\
\hline $2 \mathrm{H}-1,145.2$ & 6.95 & 1.64 & 1.74 & 1.23 \\
\hline $2 \mathrm{H}-2,4.8$ & 7.05 & 1.74 & 1.87 & 1.31 \\
\hline $2 \mathrm{H}-2,7.3$ & 7.07 & 1.76 & 1.89 & 1.30 \\
\hline $2 \mathrm{H}-2,9.8$ & 7.10 & 1.79 & 1.93 & 1.33 \\
\hline $2 \mathrm{H}-2,12.3$ & 7.12 & 1.81 & 1.96 & 1.29 \\
\hline $2 \mathrm{H}-2,14.8$ & 7.15 & 1.84 & 2.00 & 1.32 \\
\hline
\end{tabular}

Only a portion of this table appears here. The complete table is available in ASCII. 
Table AT20. Magnetic susceptibility data, Hole U1337A.

\begin{tabular}{|c|c|c|c|c|}
\hline \multirow[b]{2}{*}{$\begin{array}{l}\text { Core, section, } \\
\text { interval }(\mathrm{cm})\end{array}$} & \multicolumn{3}{|c|}{ Depth (m) } & \multirow{2}{*}{$\begin{array}{c}\text { Magnetic } \\
\text { susceptibility } \\
\text { (IU) }\end{array}$} \\
\hline & CSF & CCSF & $\begin{array}{l}\text { Adjusted } \\
\text { CCSF }\end{array}$ & \\
\hline \multicolumn{5}{|l|}{ 321-U1337A- } \\
\hline $2 \mathrm{H}-1,0.1$ & 5.50 & 0.19 & 0.06 & 1.1 \\
\hline $2 \mathrm{H}-1,2.5$ & 5.53 & 0.22 & 0.10 & 4.4 \\
\hline $2 \mathrm{H}-1,5.1$ & 5.55 & 0.24 & 0.12 & 3.3 \\
\hline $2 \mathrm{H}-1,7.6$ & 5.58 & 0.27 & 0.15 & 3.6 \\
\hline $2 \mathrm{H}-1,10.1$ & 5.60 & 0.29 & 0.17 & 2.5 \\
\hline $2 \mathrm{H}-1,12.6$ & 5.63 & 0.32 & 0.21 & 3.3 \\
\hline $2 \mathrm{H}-1,15.1$ & 5.65 & 0.34 & 0.23 & 5.5 \\
\hline $2 \mathrm{H}-1,17.6$ & 5.68 & 0.37 & 0.26 & 4.4 \\
\hline $2 \mathrm{H}-1,20.1$ & 5.70 & 0.39 & 0.29 & 3.0 \\
\hline $2 \mathrm{H}-1,22.6$ & 5.73 & 0.42 & 0.32 & 3.3 \\
\hline $2 \mathrm{H}-1,25.1$ & 5.75 & 0.44 & 0.34 & 3.3 \\
\hline $2 \mathrm{H}-1,27.6$ & 5.78 & 0.47 & 0.37 & 3.3 \\
\hline $2 \mathrm{H}-1,30.1$ & 5.80 & 0.49 & 0.40 & 2.7 \\
\hline $2 \mathrm{H}-1,32.6$ & 5.83 & 0.52 & 0.43 & 1.6 \\
\hline $2 \mathrm{H}-1,35.1$ & 5.85 & 0.54 & 0.45 & 1.9 \\
\hline $2 \mathrm{H}-1,37.6$ & 5.88 & 0.57 & 0.48 & 2.2 \\
\hline $2 \mathrm{H}-1,40.1$ & 5.90 & 0.59 & 0.51 & 1.6 \\
\hline $2 \mathrm{H}-1,42.6$ & 5.93 & 0.62 & 0.54 & 1.9 \\
\hline $2 \mathrm{H}-1,45.1$ & 5.95 & 0.64 & 0.56 & 1.9 \\
\hline $2 \mathrm{H}-1,47.6$ & 5.98 & 0.67 & 0.59 & 2.2 \\
\hline $2 \mathrm{H}-1,50.1$ & 6.00 & 0.69 & 0.62 & 1.6 \\
\hline $2 \mathrm{H}-1,52.6$ & 6.03 & 0.72 & 0.65 & 2.2 \\
\hline $2 \mathrm{H}-1,55.1$ & 6.05 & 0.74 & 0.67 & 1.9 \\
\hline $2 \mathrm{H}-1,57.6$ & 6.08 & 0.77 & 0.71 & 1.9 \\
\hline $2 \mathrm{H}-1,60.1$ & 6.10 & 0.79 & 0.73 & 1.9 \\
\hline $2 \mathrm{H}-1,62.6$ & 6.13 & 0.82 & 0.76 & 2.2 \\
\hline $2 \mathrm{H}-1,65.2$ & 6.15 & 0.84 & 0.78 & 2.2 \\
\hline $2 \mathrm{H}-1,67.7$ & 6.18 & 0.87 & 0.82 & 3.0 \\
\hline $2 \mathrm{H}-1,70.2$ & 6.20 & 0.89 & 0.84 & 2.7 \\
\hline $2 \mathrm{H}-1,72.7$ & 6.23 & 0.92 & 0.87 & 3.6 \\
\hline $2 \mathrm{H}-1,75.2$ & 6.25 & 0.94 & 0.90 & 4.7 \\
\hline $2 \mathrm{H}-1,77.7$ & 6.28 & 0.97 & 0.93 & 7.1 \\
\hline $2 \mathrm{H}-1,80.2$ & 6.30 & 0.99 & 0.96 & 7.1 \\
\hline $2 \mathrm{H}-1,82.7$ & 6.33 & 1.02 & 0.99 & 7.7 \\
\hline $2 \mathrm{H}-1,85.2$ & 6.35 & 1.04 & 1.02 & 7.1 \\
\hline $2 \mathrm{H}-1,87.7$ & 6.38 & 1.07 & 1.05 & 5.2 \\
\hline $2 \mathrm{H}-1,90.2$ & 6.40 & 1.09 & 1.08 & 7.7 \\
\hline $2 \mathrm{H}-1,92.7$ & 6.43 & 1.12 & 1.11 & 7.4 \\
\hline $2 \mathrm{H}-1,95.2$ & 6.45 & 1.14 & 1.14 & 8.2 \\
\hline $2 \mathrm{H}-1,97.7$ & 6.48 & 1.17 & 1.17 & 8.0 \\
\hline $2 \mathrm{H}-1,100.2$ & 6.50 & 1.19 & 1.20 & 8.2 \\
\hline $2 \mathrm{H}-1,102.7$ & 6.53 & 1.22 & 1.23 & 9.1 \\
\hline $2 \mathrm{H}-1,105.2$ & 6.55 & 1.24 & 1.26 & 8.8 \\
\hline $2 \mathrm{H}-1,107.7$ & 6.58 & 1.27 & 1.29 & 9.3 \\
\hline $2 \mathrm{H}-1,110.2$ & 6.60 & 1.29 & 1.32 & 10.2 \\
\hline $2 \mathrm{H}-1,112.7$ & 6.63 & 1.32 & 1.35 & 9.9 \\
\hline $2 \mathrm{H}-1,115.2$ & 6.65 & 1.34 & 1.38 & 9.9 \\
\hline $2 \mathrm{H}-1,117.7$ & 6.68 & 1.37 & 1.42 & 10.2 \\
\hline $2 \mathrm{H}-1,120.2$ & 6.70 & 1.39 & 1.44 & 9.9 \\
\hline $2 \mathrm{H}-1,122.8$ & 6.73 & 1.42 & 1.48 & 9.9 \\
\hline $2 \mathrm{H}-1,125.2$ & 6.75 & 1.44 & 1.50 & 9.9 \\
\hline $2 \mathrm{H}-1,127.8$ & 6.78 & 1.47 & 1.54 & 10.7 \\
\hline $2 \mathrm{H}-1,130.3$ & 6.80 & 1.49 & 1.56 & 10.2 \\
\hline $2 \mathrm{H}-1,132.8$ & 6.83 & 1.52 & 1.60 & 9.6 \\
\hline $2 \mathrm{H}-1,135.3$ & 6.85 & 1.54 & 1.62 & 10.2 \\
\hline $2 \mathrm{H}-1,137.8$ & 6.88 & 1.57 & 1.66 & 9.3 \\
\hline $2 \mathrm{H}-1,140.3$ & 6.90 & 1.59 & 1.68 & 9.1 \\
\hline $2 \mathrm{H}-1,142.8$ & 6.93 & 1.62 & 1.72 & 7.7 \\
\hline $2 \mathrm{H}-1,145.3$ & 6.95 & 1.64 & 1.74 & 9.6 \\
\hline $2 \mathrm{H}-1,147.8$ & 6.98 & 1.67 & 1.78 & 8.8 \\
\hline
\end{tabular}

Only a portion of this table appears here. The complete table is available in ASCII. 
Table AT21. Natural gamma ray (NGR) data, Hole U1337A.

\begin{tabular}{|c|c|c|c|c|}
\hline \multirow[b]{2}{*}{$\begin{array}{l}\text { Core, section, } \\
\text { interval }(\mathrm{cm})\end{array}$} & \multicolumn{3}{|c|}{ Depth $(\mathrm{m})$} & \multirow[b]{2}{*}{$\begin{array}{l}\text { NGR } \\
(\mathrm{cps})\end{array}$} \\
\hline & CSF & CCSF & $\begin{array}{l}\text { Adjusted } \\
\text { CCSF }\end{array}$ & \\
\hline \multicolumn{5}{|l|}{ 321-U1337A- } \\
\hline $2 \mathrm{H}-1,0$ & 5.5 & 0.19 & 0.06 & 44.4 \\
\hline $2 \mathrm{H}-1,10$ & 5.6 & 0.29 & 0.17 & 48.7 \\
\hline $2 \mathrm{H}-1,20$ & 5.7 & 0.39 & 0.29 & 55.4 \\
\hline $2 \mathrm{H}-1,30$ & 5.8 & 0.49 & 0.40 & 50.9 \\
\hline $2 \mathrm{H}-1,40$ & 5.9 & 0.59 & 0.51 & 48.2 \\
\hline $2 \mathrm{H}-1,50$ & 6.0 & 0.69 & 0.62 & 46.6 \\
\hline $2 \mathrm{H}-1,60$ & 6.1 & 0.79 & 0.73 & 44.1 \\
\hline $2 \mathrm{H}-1,70$ & 6.2 & 0.89 & 0.84 & 46.4 \\
\hline $2 \mathrm{H}-1,80$ & 6.3 & 0.99 & 0.96 & 47.7 \\
\hline $2 \mathrm{H}-1,90$ & 6.4 & 1.09 & 1.08 & 53.7 \\
\hline $2 \mathrm{H}-1,100$ & 6.5 & 1.19 & 1.20 & 65.6 \\
\hline $2 \mathrm{H}-1,110$ & 6.6 & 1.29 & 1.32 & 61.1 \\
\hline $2 \mathrm{H}-1,120$ & 6.7 & 1.39 & 1.44 & 55.3 \\
\hline $2 \mathrm{H}-1,130$ & 6.8 & 1.49 & 1.56 & 49.0 \\
\hline $2 \mathrm{H}-1,140$ & 6.9 & 1.59 & 1.68 & 38.9 \\
\hline $2 \mathrm{H}-1,150$ & 7.0 & 1.69 & 1.80 & 31.5 \\
\hline $2 \mathrm{H}-2,0$ & 7.0 & 1.69 & 1.80 & 24.2 \\
\hline $2 \mathrm{H}-2,10$ & 7.1 & 1.79 & 1.93 & 22.6 \\
\hline $2 \mathrm{H}-2,20$ & 7.2 & 1.89 & 2.07 & 22.5 \\
\hline $2 \mathrm{H}-2,30$ & 7.3 & 1.99 & 2.18 & 23.9 \\
\hline $2 \mathrm{H}-2,40$ & 7.4 & 2.09 & 2.24 & 23.1 \\
\hline $2 \mathrm{H}-2,50$ & 7.5 & 2.19 & 2.30 & 22.4 \\
\hline $2 \mathrm{H}-2,60$ & 7.6 & 2.29 & 2.37 & 26.6 \\
\hline $2 \mathrm{H}-2,70$ & 7.7 & 2.39 & 2.46 & 20.8 \\
\hline $2 \mathrm{H}-2,80$ & 7.8 & 2.49 & 2.56 & 18.7 \\
\hline $2 \mathrm{H}-2,90$ & 7.9 & 2.59 & 2.66 & 19.2 \\
\hline $2 \mathrm{H}-2,100$ & 8.0 & 2.69 & 2.75 & 18.0 \\
\hline $2 \mathrm{H}-2,110$ & 8.1 & 2.79 & 2.85 & 17.5 \\
\hline $2 \mathrm{H}-2,120$ & 8.2 & 2.89 & 2.94 & 20.0 \\
\hline $2 \mathrm{H}-2,130$ & 8.3 & 2.99 & 3.04 & 22.1 \\
\hline $2 \mathrm{H}-2,140$ & 8.4 & 3.09 & 3.14 & 22.2 \\
\hline $2 \mathrm{H}-3,0$ & 8.5 & 3.19 & 3.23 & 20.5 \\
\hline $2 \mathrm{H}-3,10$ & 8.6 & 3.29 & 3.34 & 20.5 \\
\hline $2 \mathrm{H}-3,20$ & 8.7 & 3.39 & 3.46 & 21.6 \\
\hline $2 \mathrm{H}-3,30$ & 8.8 & 3.49 & 3.56 & 18.7 \\
\hline $2 \mathrm{H}-3,40$ & 8.9 & 3.59 & 3.65 & 16.5 \\
\hline $2 \mathrm{H}-3,50$ & 9.0 & 3.69 & 3.75 & 17.6 \\
\hline $2 \mathrm{H}-3,60$ & 9.1 & 3.79 & 3.84 & 17.1 \\
\hline $2 \mathrm{H}-3,70$ & 9.2 & 3.89 & 3.93 & 13.5 \\
\hline $2 \mathrm{H}-3,80$ & 9.3 & 3.99 & 4.02 & 12.3 \\
\hline $2 \mathrm{H}-3,90$ & 9.4 & 4.09 & 4.11 & 13.1 \\
\hline $2 \mathrm{H}-3,100$ & 9.5 & 4.19 & 4.20 & 13.4 \\
\hline $2 \mathrm{H}-3,110$ & 9.6 & 4.29 & 4.30 & 16.7 \\
\hline $2 \mathrm{H}-3,120$ & 9.7 & 4.39 & 4.39 & 19.0 \\
\hline $2 \mathrm{H}-3,130$ & 9.8 & 4.49 & 4.49 & 16.3 \\
\hline $2 \mathrm{H}-3,140$ & 9.9 & 4.59 & 4.59 & 13.1 \\
\hline $2 \mathrm{H}-3,150$ & 10.0 & 4.69 & 4.69 & 11.4 \\
\hline $2 \mathrm{H}-4,0$ & 10.0 & 4.69 & 4.69 & 11.4 \\
\hline $2 \mathrm{H}-4,10$ & 10.1 & 4.79 & 4.79 & 11.7 \\
\hline $2 \mathrm{H}-4,20$ & 10.2 & 4.89 & 4.89 & 12.0 \\
\hline $2 \mathrm{H}-4,30$ & 10.3 & 4.99 & 4.99 & 11.3 \\
\hline $2 \mathrm{H}-4,40$ & 10.4 & 5.09 & 5.09 & 11.4 \\
\hline $2 \mathrm{H}-4,50$ & 10.5 & 5.19 & 5.19 & 9.9 \\
\hline $2 \mathrm{H}-4,60$ & 10.6 & 5.29 & 5.29 & 9.2 \\
\hline $2 \mathrm{H}-4,70$ & 10.7 & 5.39 & 5.39 & 8.2 \\
\hline $2 \mathrm{H}-4,80$ & 10.8 & 5.49 & 5.49 & 8.2 \\
\hline $2 \mathrm{H}-4,90$ & 10.9 & 5.59 & 5.59 & 9.7 \\
\hline $2 \mathrm{H}-4,100$ & 11.0 & 5.69 & 5.69 & 9.3 \\
\hline $2 \mathrm{H}-4,110$ & 11.1 & 5.79 & 5.79 & 8.4 \\
\hline $2 \mathrm{H}-4,120$ & 11.2 & 5.89 & 5.89 & 8.0 \\
\hline
\end{tabular}

Only a portion of this table appears here. The complete table is available in ASCII. 
Table AT22. Color reflection data, Hole U1337A.

\begin{tabular}{|c|c|c|c|c|c|c|}
\hline \multirow[b]{2}{*}{$\begin{array}{l}\text { Core, section, } \\
\text { interval }(\mathrm{cm})\end{array}$} & \multicolumn{3}{|c|}{ Depth (m) } & \multirow[b]{2}{*}{$a^{*}$} & \multirow[b]{2}{*}{$b^{*}$} & \multirow[b]{2}{*}{$L^{*}$} \\
\hline & CSF & CCSF & $\begin{array}{l}\text { Adjusted } \\
\text { CCSF }\end{array}$ & & & \\
\hline \multicolumn{7}{|l|}{ 321-U1337A- } \\
\hline $2 \mathrm{H}-1,5.2$ & 5.55 & 0.24 & 0.12 & 4.1 & 7.7 & 47.3 \\
\hline $2 \mathrm{H}-1,7.7$ & 5.58 & 0.27 & 0.15 & 5.6 & 17.6 & 63.2 \\
\hline $2 \mathrm{H}-1,10.3$ & 5.60 & 0.29 & 0.17 & 5.4 & 17.3 & 60.1 \\
\hline $2 \mathrm{H}-1,12.8$ & 5.63 & 0.32 & 0.21 & 6.8 & 9.6 & 40.6 \\
\hline $2 \mathrm{H}-1,15.3$ & 5.65 & 0.34 & 0.23 & 5.2 & 16.7 & 58.2 \\
\hline $2 \mathrm{H}-1,17.9$ & 5.68 & 0.37 & 0.26 & 5.8 & 11.6 & 46.7 \\
\hline $2 \mathrm{H}-1,20.4$ & 5.70 & 0.39 & 0.29 & 7.1 & 16.6 & 52.7 \\
\hline $2 \mathrm{H}-1,23$ & 5.73 & 0.42 & 0.32 & 5.4 & 13.0 & 52.9 \\
\hline $2 \mathrm{H}-1,25.5$ & 5.76 & 0.45 & 0.35 & 4.2 & 14.1 & 55.5 \\
\hline $2 \mathrm{H}-1,28$ & 5.78 & 0.47 & 0.37 & 6.5 & 9.8 & 41.3 \\
\hline $2 \mathrm{H}-1,30.6$ & 5.81 & 0.50 & 0.41 & 5.4 & 17.9 & 63.3 \\
\hline $2 \mathrm{H}-1,33.1$ & 5.83 & 0.52 & 0.43 & 4.5 & 13.5 & 59.1 \\
\hline $2 \mathrm{H}-1,35.7$ & 5.86 & 0.55 & 0.46 & 4.5 & 13.4 & 54.6 \\
\hline $2 \mathrm{H}-1,38.2$ & 5.88 & 0.57 & 0.48 & 3.6 & 10.1 & 60.0 \\
\hline $2 \mathrm{H}-1,40.7$ & 5.91 & 0.60 & 0.52 & 3.6 & 10.3 & 55.5 \\
\hline $2 \mathrm{H}-1,43.3$ & 5.93 & 0.62 & 0.54 & 4.7 & 15.0 & 53.9 \\
\hline $2 \mathrm{H}-1,45.8$ & 5.96 & 0.65 & 0.57 & 4.4 & 13.9 & 54.3 \\
\hline $2 \mathrm{H}-1,48.4$ & 5.98 & 0.67 & 0.59 & 4.1 & 15.2 & 58.1 \\
\hline $2 \mathrm{H}-1,50.9$ & 6.01 & 0.70 & 0.63 & 4.6 & 14.1 & 53.4 \\
\hline $2 \mathrm{H}-1,53.4$ & 6.03 & 0.72 & 0.65 & 4.6 & 13.4 & 57.9 \\
\hline $2 \mathrm{H}-1,56$ & 6.06 & 0.75 & 0.68 & 3.9 & 11.3 & 57.2 \\
\hline $2 \mathrm{H}-1,58.5$ & 6.09 & 0.78 & 0.72 & 4.6 & 14.3 & 54.4 \\
\hline $2 \mathrm{H}-1,61.1$ & 6.11 & 0.80 & 0.74 & 4.5 & 13.6 & 52.7 \\
\hline $2 \mathrm{H}-1,63.6$ & 6.14 & 0.83 & 0.77 & 3.7 & 10.9 & 57.3 \\
\hline $2 \mathrm{H}-1,66.2$ & 6.16 & 0.85 & 0.79 & 3.6 & 11.5 & 58.8 \\
\hline $2 \mathrm{H}-1,68.7$ & 6.19 & 0.88 & 0.83 & 4.6 & 14.0 & 55.7 \\
\hline $2 \mathrm{H}-1,71.2$ & 6.21 & 0.90 & 0.85 & 4.1 & 12.9 & 55.0 \\
\hline $2 \mathrm{H}-1,73.8$ & 6.24 & 0.93 & 0.88 & 3.5 & 9.8 & 52.5 \\
\hline $2 \mathrm{H}-1,76.3$ & 6.26 & 0.95 & 0.91 & 2.9 & 6.3 & 43.7 \\
\hline $2 \mathrm{H}-1,78.9$ & 6.29 & 0.98 & 0.94 & 7.2 & 15.2 & 43.3 \\
\hline $2 \mathrm{H}-1,81.4$ & 6.31 & 1.00 & 0.97 & 7.0 & 14.5 & 43.4 \\
\hline $2 \mathrm{H}-1,83.9$ & 6.34 & 1.03 & 1.00 & 5.7 & 9.2 & 44.7 \\
\hline $2 \mathrm{H}-1,86.5$ & 6.37 & 1.06 & 1.04 & 5.4 & 8.6 & 45.5 \\
\hline $2 \mathrm{H}-1,89$ & 6.39 & 1.08 & 1.06 & 5.1 & 8.7 & 48.4 \\
\hline $2 \mathrm{H}-1,91.6$ & 6.42 & 1.11 & 1.10 & 7.8 & 15.5 & 43.5 \\
\hline $2 \mathrm{H}-1,94.1$ & 6.44 & 1.13 & 1.13 & 5.2 & 7.3 & 42.4 \\
\hline $2 \mathrm{H}-1,96.7$ & 6.47 & 1.16 & 1.16 & 3.4 & 5.6 & 41.6 \\
\hline $2 \mathrm{H}-1,99.2$ & 6.49 & 1.18 & 1.19 & 5.8 & 5.8 & 32.6 \\
\hline $2 \mathrm{H}-1,101.7$ & 6.52 & 1.21 & 1.22 & 4.0 & 5.1 & 39.8 \\
\hline $2 \mathrm{H}-1,104.3$ & 6.54 & 1.23 & 1.25 & 3.5 & 4.4 & 39.7 \\
\hline $2 \mathrm{H}-1,106.8$ & 6.57 & 1.26 & 1.28 & 4.6 & 4.7 & 34.6 \\
\hline $2 \mathrm{H}-1,109.4$ & 6.59 & 1.28 & 1.31 & 5.1 & 4.5 & 34.5 \\
\hline $2 \mathrm{H}-1,111.9$ & 6.62 & 1.31 & 1.34 & 3.9 & 2.8 & 36.9 \\
\hline $2 \mathrm{H}-1,114.5$ & 6.65 & 1.34 & 1.38 & 4.2 & 7.1 & 44.9 \\
\hline $2 \mathrm{H}-1,117$ & 6.67 & 1.36 & 1.40 & 5.2 & 6.5 & 41.6 \\
\hline $2 \mathrm{H}-1,119.5$ & 6.70 & 1.39 & 1.44 & 3.5 & 3.0 & 35.5 \\
\hline $2 \mathrm{H}-1,122.1$ & 6.72 & 1.41 & 1.46 & 5.0 & 4.5 & 34.3 \\
\hline $2 \mathrm{H}-1,124.6$ & 6.75 & 1.44 & 1.50 & 3.9 & 4.6 & 38.7 \\
\hline $2 \mathrm{H}-1,127.2$ & 6.77 & 1.46 & 1.52 & 5.7 & 5.0 & 30.6 \\
\hline $2 \mathrm{H}-1,129.7$ & 6.80 & 1.49 & 1.56 & 3.8 & 2.6 & 33.1 \\
\hline $2 \mathrm{H}-1,132.3$ & 6.82 & 1.51 & 1.58 & 3.0 & 1.9 & 34.5 \\
\hline $2 \mathrm{H}-1,134.8$ & 6.85 & 1.54 & 1.62 & 3.8 & 1.6 & 30.5 \\
\hline $2 \mathrm{H}-1,137.3$ & 6.87 & 1.56 & 1.64 & 2.6 & 2.5 & 36.8 \\
\hline $2 \mathrm{H}-1,139.9$ & 6.90 & 1.59 & 1.68 & 3.8 & 4.7 & 32.7 \\
\hline $2 \mathrm{H}-1,142.4$ & 6.92 & 1.61 & 1.70 & 4.1 & 5.5 & 32.8 \\
\hline $2 \mathrm{H}-1,145$ & 6.95 & 1.64 & 1.74 & 3.6 & 6.2 & 35.4 \\
\hline $2 \mathrm{H}-2,5.2$ & 7.05 & 1.74 & 1.87 & 3.4 & 10.3 & 57.8 \\
\hline $2 \mathrm{H}-2,7.7$ & 7.08 & 1.77 & 1.91 & 4.9 & 13.1 & 48.7 \\
\hline $2 \mathrm{H}-2,10.3$ & 7.10 & 1.79 & 1.93 & 3.8 & 13.5 & 57.2 \\
\hline
\end{tabular}

Only a portion of this table appears here. The complete table is available in ASCII. 
Table AT23. Core adjusted data, Hole U1337A. (Continued on next three pages.)

\begin{tabular}{|c|c|c|c|}
\hline \multirow[b]{2}{*}{$\begin{array}{l}\text { Core, section, } \\
\text { interval }(\mathrm{cm})\end{array}$} & \multicolumn{3}{|c|}{ Depth $(\mathrm{m})$} \\
\hline & $\begin{array}{l}\text { Adjusted } \\
\text { CCSF }\end{array}$ & CCSF & CSF-A \\
\hline \multicolumn{4}{|l|}{ 321-U1337A- } \\
\hline $2 \mathrm{H}-1,10$ & 0.17 & 0.29 & $\begin{array}{l}5.60 \\
623\end{array}$ \\
\hline $2 \mathrm{H}-1,73$ & 0.87 & 0.92 & 6.23 \\
\hline $2 \mathrm{H}-1,150$ & 1.80 & 1.69 & 7.00 \\
\hline $2 \mathrm{H}-2,27$ & 2.16 & 1.96 & 7.27 \\
\hline $2 \mathrm{H}-2,56$ & 2.33 & 2.25 & 7.56 \\
\hline $2 \mathrm{H}-3,5$ & 3.28 & 3.24 & 8.55 \\
\hline $2 \mathrm{H}-3,25$ & 3.51 & 3.44 & 8.75 \\
\hline $2 \mathrm{H}-3,43$ & 3.68 & 3.62 & 8.93 \\
\hline $2 \mathrm{H}-3,116$ & 4.35 & 4.35 & 9.66 \\
\hline $2 \mathrm{H}-6,45$ & 8.14 & 8.14 & 13.45 \\
\hline $2 \mathrm{H}-6,87$ & 8.54 & 8.56 & 13.87 \\
\hline $2 \mathrm{H}-7,60$ & 9.87 & 9.79 & 15.10 \\
\hline $3 \mathrm{H}-1,7$ & 10.85 & 10.96 & 15.07 \\
\hline $3 \mathrm{H}-1,21$ & 10.97 & 11.10 & 15.21 \\
\hline $3 \mathrm{H}-1,58$ & 11.38 & 11.47 & 15.58 \\
\hline $3 \mathrm{H}-1,79$ & 11.59 & 11.68 & 15.79 \\
\hline $3 \mathrm{H}-1,98$ & 11.78 & 11.87 & 15.98 \\
\hline $3 \mathrm{H}-1,105$ & 11.94 & 11.94 & 16.05 \\
\hline $3 \mathrm{H}-6,70$ & 19.09 & 19.09 & 23.20 \\
\hline $3 \mathrm{H}-6,91$ & 19.33 & 19.30 & 23.41 \\
\hline $3 \mathrm{H}-6,132$ & 19.71 & 19.71 & 23.82 \\
\hline $3 \mathrm{H}-7,48$ & 20.36 & 20.37 & 24.48 \\
\hline $3 \mathrm{H}-7,74$ & 20.61 & 20.63 & 24.74 \\
\hline $4 \mathrm{H}-1,78$ & 21.33 & 21.31 & 25.28 \\
\hline $4 \mathrm{H}-1,103$ & 21.81 & 21.56 & 25.53 \\
\hline $4 \mathrm{H}-1,126$ & 22.08 & 21.79 & 25.76 \\
\hline $4 \mathrm{H}-2,93$ & 23.12 & 22.96 & 26.93 \\
\hline $4 \mathrm{H}-3,89$ & 24.41 & 24.42 & 28.39 \\
\hline $4 \mathrm{H}-3,107$ & 24.56 & 24.60 & 28.57 \\
\hline $4 \mathrm{H}-4,9$ & 25.12 & 25.12 & 29.09 \\
\hline $4 \mathrm{H}-6,50$ & 28.53 & 28.53 & 32.50 \\
\hline $4 \mathrm{H}-6,84$ & 28.75 & 28.88 & 32.84 \\
\hline $4 \mathrm{H}-6,129$ & 29.17 & 29.32 & 33.29 \\
\hline $4 \mathrm{H}-7,73$ & 30.30 & 30.26 & 34.23 \\
\hline $5 \mathrm{H}-1,8$ & 32.75 & 32.78 & 34.08 \\
\hline $5 \mathrm{H}-1,40$ & 33.11 & 33.10 & 34.40 \\
\hline $5 \mathrm{H}-1,71$ & 33.44 & 33.41 & 34.71 \\
\hline $5 \mathrm{H}-1,107$ & 33.78 & 33.77 & 35.07 \\
\hline $5 \mathrm{H}-1,138$ & 34.11 & 34.08 & 35.38 \\
\hline $5 \mathrm{H}-2,48$ & 34.75 & 34.68 & 35.98 \\
\hline $5 \mathrm{H}-2,113$ & 35.33 & 35.33 & 36.63 \\
\hline $5 \mathrm{H}-4,125$ & 38.45 & 38.45 & 39.75 \\
\hline $5 \mathrm{H}-4,141$ & 38.56 & 38.61 & 39.91 \\
\hline $5 \mathrm{H}-5,59$ & 39.27 & 39.30 & 40.59 \\
\hline $5 \mathrm{H}-5,77$ & 39.51 & 39.47 & 40.77 \\
\hline $5 \mathrm{H}-5,100$ & 39.72 & 39.70 & 41.00 \\
\hline $5 \mathrm{H}-6,73$ & 40.95 & 40.93 & 42.23 \\
\hline $5 \mathrm{H}-6,120$ & 41.41 & 41.40 & 42.70 \\
\hline $5 \mathrm{H}-7,39$ & 42.16 & 42.09 & 43.39 \\
\hline $5 \mathrm{H}-7,57$ & 42.28 & 42.28 & 43.57 \\
\hline $6 \mathrm{H}-1,12$ & 43.49 & 43.45 & 43.62 \\
\hline $6 \mathrm{H}-1,72$ & 44.12 & 44.05 & 44.22 \\
\hline $6 \mathrm{H}-2,33$ & 45.16 & 45.16 & 45.33 \\
\hline $6 \mathrm{H}-5,94$ & 50.27 & 50.27 & 50.44 \\
\hline $6 \mathrm{H}-6,19$ & 51.14 & 51.03 & 51.19 \\
\hline $6 \mathrm{H}-6,56$ & 51.39 & 51.39 & 51.56 \\
\hline $6 \mathrm{H}-6,62$ & 51.47 & 51.45 & 51.62 \\
\hline $6 \mathrm{H}-6,146$ & 52.44 & 52.29 & 52.46 \\
\hline $6 \mathrm{H}-7,28$ & 52.81 & 52.61 & 52.78 \\
\hline $6 \mathrm{H}-7,65$ & 53.01 & 52.98 & 53.15 \\
\hline 7H-1, 15 & 55.40 & 55.43 & 53.15 \\
\hline $7 \mathrm{H}-1,28$ & 55.56 & 55.56 & 53.28 \\
\hline $7 \mathrm{H}-1,55$ & 55.87 & 55.83 & 53.55 \\
\hline $7 \mathrm{H}-1,64$ & 55.92 & 55.92 & 53.64 \\
\hline $7 \mathrm{H}-5,63$ & 61.91 & 61.91 & 59.63 \\
\hline 7H-5, 102 & 62.31 & 62.31 & 60.02 \\
\hline $7 \mathrm{H}-6,23$ & 62.96 & 63.01 & 60.73 \\
\hline
\end{tabular}

\begin{tabular}{|c|c|c|c|}
\hline \multirow[b]{2}{*}{$\begin{array}{l}\text { Core, section, } \\
\text { interval }(\mathrm{cm})\end{array}$} & \multicolumn{3}{|c|}{ Depth (m) } \\
\hline & $\begin{array}{l}\text { Adjusted } \\
\text { CCSF }\end{array}$ & CCSF & CSF-A \\
\hline $7 \mathrm{H}-6,64$ & 63.31 & 63.42 & 61.14 \\
\hline $7 \mathrm{H}-7,52$ & 64.75 & 64.81 & 62.52 \\
\hline $8 \mathrm{H}-1,21$ & 65.83 & 65.77 & 62.71 \\
\hline $8 \mathrm{H}-1,53$ & 66.09 & 66.09 & 63.03 \\
\hline $8 \mathrm{H}-1,77$ & 66.49 & 66.33 & 63.27 \\
\hline $8 \mathrm{H}-1,87$ & 66.58 & 66.43 & 63.37 \\
\hline $8 \mathrm{H}-2,8$ & 67.14 & 67.14 & 64.08 \\
\hline $8 \mathrm{H}-5,66$ & 72.22 & 72.22 & 69.16 \\
\hline $8 \mathrm{H}-6,2$ & 73.24 & 73.09 & 70.02 \\
\hline $8 \mathrm{H}-6,22$ & 73.41 & 73.28 & 70.22 \\
\hline $8 \mathrm{H}-6,95$ & 74.21 & 74.01 & 70.95 \\
\hline $8 \mathrm{H}-6,118$ & 74.33 & 74.24 & 71.18 \\
\hline $9 \mathrm{H}-1,4$ & 76.37 & 76.35 & 72.04 \\
\hline $9 \mathrm{H}-1,65$ & 76.96 & 76.96 & 72.65 \\
\hline $9 \mathrm{H}-6,115$ & 84.96 & 84.96 & 80.65 \\
\hline $9 \mathrm{H}-6,123$ & 85.09 & 85.04 & 80.73 \\
\hline $9 \mathrm{H}-7,34$ & 85.74 & 85.65 & 81.34 \\
\hline $9 \mathrm{H}-7,47$ & 85.86 & 85.78 & 81.47 \\
\hline $9 \mathrm{H}-7,55$ & 85.94 & 85.86 & 81.55 \\
\hline $10 \mathrm{H}-1,6$ & 86.37 & 86.36 & 81.56 \\
\hline $10 \mathrm{H}-1,17$ & 86.51 & 86.47 & 81.67 \\
\hline $10 \mathrm{H}-1,29$ & 86.58 & 86.59 & 81.79 \\
\hline $10 \mathrm{H}-1,102$ & 87.35 & 87.32 & 82.52 \\
\hline $10 \mathrm{H}-1,144$ & 87.74 & 87.74 & 82.94 \\
\hline $10 \mathrm{H}-5,122$ & 93.52 & 93.52 & 88.72 \\
\hline $10 \mathrm{H}-5,143$ & 93.78 & 93.73 & 88.93 \\
\hline $10 \mathrm{H}-6,15$ & 93.97 & 93.95 & 89.15 \\
\hline $10 \mathrm{H}-6,138$ & 95.17 & 95.18 & 90.38 \\
\hline $10 \mathrm{H}-7,11$ & 95.39 & 95.41 & 90.61 \\
\hline $10 \mathrm{H}-7,43$ & 95.69 & 95.73 & 90.93 \\
\hline $10 \mathrm{H}-7,69$ & 95.97 & 95.99 & 91.19 \\
\hline $11 \mathrm{H}-1,33$ & 97.17 & 97.18 & 91.33 \\
\hline $11 \mathrm{H}-1,75$ & 97.60 & 97.60 & 91.75 \\
\hline $11 \mathrm{H}-5,115$ & 104.00 & 104.00 & 98.15 \\
\hline $11 \mathrm{H}-5,137$ & 104.27 & 104.22 & 98.37 \\
\hline $11 \mathrm{H}-6,15$ & 104.52 & 104.50 & 98.65 \\
\hline $11 \mathrm{H}-6,110$ & 105.44 & 105.46 & 99.60 \\
\hline $11 \mathrm{H}-7,35$ & 106.14 & 106.20 & 100.35 \\
\hline $11 \mathrm{H}-7,70$ & 106.46 & 106.55 & 100.70 \\
\hline $12 \mathrm{H}-1,35$ & 107.38 & 107.44 & 100.85 \\
\hline $12 \mathrm{H}-1,102$ & 108.14 & 108.11 & 101.52 \\
\hline $12 \mathrm{H}-1,123$ & 108.40 & 108.32 & 101.73 \\
\hline $12 \mathrm{H}-1,149$ & 108.64 & 108.58 & 101.99 \\
\hline $12 \mathrm{H}-2,28$ & 108.96 & 108.87 & 102.28 \\
\hline $12 \mathrm{H}-2,62$ & 109.28 & 109.21 & 102.62 \\
\hline $12 \mathrm{H}-2,74$ & 109.33 & 109.33 & 102.74 \\
\hline $12 \mathrm{H}-6,58$ & 115.17 & 115.17 & 108.58 \\
\hline $12 \mathrm{H}-6,119$ & 115.82 & 115.78 & 109.19 \\
\hline $12 \mathrm{H}-7,20$ & 116.37 & 116.29 & 109.70 \\
\hline $12 \mathrm{H}-7,48$ & 116.70 & 116.57 & 109.98 \\
\hline $12 \mathrm{H}-7,67$ & 116.85 & 116.76 & 110.17 \\
\hline $13 \mathrm{H}-1,32$ & 117.90 & 117.85 & 110.32 \\
\hline $13 \mathrm{H}-1,71$ & 118.26 & 118.24 & 110.71 \\
\hline $13 \mathrm{H}-1,89$ & 118.47 & 118.42 & 110.89 \\
\hline $13 \mathrm{H}-1,136$ & 118.95 & 118.89 & 111.36 \\
\hline $13 \mathrm{H}-2,22$ & 119.29 & 119.25 & 111.72 \\
\hline $13 \mathrm{H}-2,106$ & 120.09 & 120.09 & 112.56 \\
\hline $13 \mathrm{H}-6,20$ & 125.23 & 125.23 & 117.70 \\
\hline $13 \mathrm{H}-6,40$ & 125.46 & 125.43 & 117.90 \\
\hline $13 \mathrm{H}-6,52$ & 125.63 & 125.55 & 118.02 \\
\hline $13 \mathrm{H}-6,109$ & 126.10 & 126.12 & 118.59 \\
\hline $13 \mathrm{H}-7,6$ & 126.54 & 126.59 & 119.06 \\
\hline $13 \mathrm{H}-7,39$ & 126.87 & 126.92 & 119.39 \\
\hline $13 \mathrm{H}-7,56$ & 127.11 & 127.09 & 119.56 \\
\hline $14 \mathrm{H}-1,13$ & 129.20 & 129.25 & 119.63 \\
\hline $14 \mathrm{H}-1,28$ & 129.38 & 129.40 & 119.78 \\
\hline $14 \mathrm{H}-1,51$ & 129.59 & 129.63 & 120.01 \\
\hline $14 \mathrm{H}-1,75$ & 129.83 & 129.87 & 120.25 \\
\hline
\end{tabular}


Table AT23 (continued). (Continued on next page.)

\begin{tabular}{|c|c|c|c|c|c|c|c|}
\hline \multirow[b]{2}{*}{$\begin{array}{l}\text { Core, section, } \\
\text { interval (cm) }\end{array}$} & \multicolumn{3}{|c|}{ Depth $(m)$} & \multirow[b]{2}{*}{$\begin{array}{l}\text { Core, section, } \\
\text { interval }(\mathrm{cm})\end{array}$} & \multicolumn{3}{|c|}{ Depth (m) } \\
\hline & $\begin{array}{l}\text { Adjusted } \\
\text { CCSF }\end{array}$ & CCSF & CSF-A & & $\begin{array}{l}\text { Adjusted } \\
\text { CCSF }\end{array}$ & CCSF & CSF-A \\
\hline $14 \mathrm{H}-1,137$ & 130.50 & 130.49 & 120.87 & $20 \mathrm{H}-1,78$ & 194.15 & 194.16 & 177.28 \\
\hline $14 \mathrm{H}-2,5$ & 130.67 & 130.67 & 121.05 & $20 \mathrm{H}-1,113$ & 194.51 & 194.51 & 177.63 \\
\hline $14 \mathrm{H}-7,20$ & 138.32 & 138.32 & 128.70 & $20 \mathrm{H}-5,48$ & 199.86 & 199.86 & 182.98 \\
\hline $14 \mathrm{H}-7,25$ & 138.39 & 138.37 & 128.75 & $20 \mathrm{H}-5,140$ & 200.73 & 200.78 & 183.90 \\
\hline $14 \mathrm{H}-7,35$ & 138.49 & 138.47 & 128.85 & $20 \mathrm{H}-6,33$ & 201.13 & 201.21 & 184.33 \\
\hline $14 \mathrm{H}-7,60$ & 138.74 & 138.73 & 129.10 & $20 \mathrm{H}-6,84$ & 201.60 & 201.73 & 184.84 \\
\hline $15 \mathrm{H}-1,33$ & 140.65 & 140.66 & 129.33 & $20 \mathrm{H}-6,144$ & 202.10 & 202.33 & 185.44 \\
\hline $15 \mathrm{H}-1,51$ & 140.78 & 140.84 & 129.51 & $20 \mathrm{H}-7,22$ & 202.31 & 202.60 & 185.72 \\
\hline $15 \mathrm{H}-1,63$ & 140.90 & 140.96 & 129.63 & $20 \mathrm{H}-7,49$ & 202.53 & 202.87 & 185.99 \\
\hline $15 \mathrm{H}-1,88$ & 141.21 & 141.21 & 129.88 & $20 \mathrm{H}-7,61$ & 202.71 & 202.99 & 186.11 \\
\hline $15 \mathrm{H}-1,109$ & 141.37 & 141.42 & 130.09 & $21 \mathrm{H}-1,14$ & 203.66 & 203.49 & 186.14 \\
\hline $15 \mathrm{H}-2,0$ & 141.78 & 141.83 & 130.50 & $21 \mathrm{H}-1,107$ & 204.43 & 204.41 & 187.07 \\
\hline $15 \mathrm{H}-2,13$ & 141.88 & 141.96 & 130.63 & $21 \mathrm{H}-2,27$ & 205.11 & 205.11 & 187.77 \\
\hline $15 \mathrm{H}-2,47$ & 142.27 & 142.30 & 130.97 & $21 \mathrm{H}-5,36$ & 209.71 & 209.71 & 192.37 \\
\hline $15 \mathrm{H}-2,71$ & 142.51 & 142.54 & 131.21 & $21 \mathrm{H}-5,48$ & 209.77 & 209.83 & 192.49 \\
\hline $15 \mathrm{H}-2,94$ & 142.77 & 142.77 & 131.44 & $21 \mathrm{H}-5,77$ & 210.09 & 210.12 & 192.78 \\
\hline $15 \mathrm{H}-5,53$ & 146.86 & 146.86 & 135.53 & $21 \mathrm{H}-5,99$ & 210.34 & 210.34 & 193.00 \\
\hline $15 \mathrm{H}-5,98$ & 147.35 & 147.32 & 135.98 & $21 \mathrm{H}-5,136$ & 210.73 & 210.71 & 193.37 \\
\hline $15 \mathrm{H}-5,150$ & 147.82 & 147.83 & 136.50 & $21 \mathrm{H}-6,18$ & 211.11 & 211.05 & 193.71 \\
\hline $15 \mathrm{H}-6,82$ & 148.63 & 148.65 & 137.32 & $21 \mathrm{H}-6,88$ & 211.90 & 211.75 & 194.41 \\
\hline $15 \mathrm{H}-7,33$ & 149.60 & 149.66 & 138.33 & $21 \mathrm{H}-6,133$ & 212.32 & 212.20 & 194.86 \\
\hline $15 \mathrm{H}-7,60$ & 149.88 & 149.93 & 138.60 & $21 \mathrm{H}-7,27$ & 212.65 & 212.54 & 195.20 \\
\hline $16 \mathrm{H}-1,48$ & 150.91 & 150.92 & 138.98 & $21 \mathrm{H}-7,47$ & 212.87 & 212.74 & 195.40 \\
\hline $16 \mathrm{H}-1,65$ & 151.11 & 151.09 & 139.15 & $22 X-1,57$ & 213.24 & 213.32 & 196.07 \\
\hline $16 \mathrm{H}-1,86$ & 151.31 & 151.30 & 139.36 & $22 X-1,111$ & 213.99 & 213.86 & 196.61 \\
\hline $16 \mathrm{H}-1,98$ & 151.47 & 151.42 & 139.48 & $22 X-1,141$ & 214.28 & 214.16 & 196.91 \\
\hline $16 \mathrm{H}-1,146$ & 151.89 & 151.91 & 139.96 & $22 X-2,35$ & 214.65 & 214.60 & 197.35 \\
\hline $16 \mathrm{H}-2,54$ & 152.48 & 152.48 & 140.54 & $22 X-2,57$ & 214.85 & 214.82 & 197.57 \\
\hline $16 \mathrm{H}-5,103$ & 157.47 & 157.47 & 145.53 & $23 X-1,24$ & 223.94 & 223.42 & 204.54 \\
\hline $16 \mathrm{H}-6,6$ & 158.00 & 158.00 & 146.06 & $23 X-1,35$ & 224.09 & 223.54 & 204.65 \\
\hline $16 \mathrm{H}-6,42$ & 158.34 & 158.36 & 146.42 & $23 X-1,59$ & 224.34 & 223.77 & 204.89 \\
\hline $16 \mathrm{H}-6,99$ & 158.85 & 158.93 & 146.99 & $23 X-1,94$ & 224.60 & 224.12 & 205.24 \\
\hline $16 \mathrm{H}-7,9$ & 159.48 & 159.53 & 147.59 & $23 X-2,80$ & 225.50 & 225.48 & 206.60 \\
\hline $16 \mathrm{H}-7,32$ & 159.72 & 159.76 & 147.82 & $23 X-3,19$ & 226.37 & 226.37 & 207.49 \\
\hline $16 \mathrm{H}-7,52$ & 159.88 & 159.96 & 148.02 & $23 X-6,11$ & 230.79 & 230.79 & 211.91 \\
\hline $17 \mathrm{H}-1,10$ & 161.71 & 161.51 & 148.10 & $23 X-6,82$ & 231.50 & 231.50 & 212.62 \\
\hline $17 \mathrm{H}-1,43$ & 161.98 & 161.84 & 148.43 & $23 X-7,19$ & 232.03 & 231.97 & 213.09 \\
\hline $17 \mathrm{H}-1,81$ & 162.37 & 162.23 & 148.81 & $23 X-7,64$ & 232.50 & 232.42 & 213.54 \\
\hline $17 \mathrm{H}-1,102$ & 162.62 & 162.43 & 149.02 & $23 X-7,79$ & 232.62 & 232.57 & 213.69 \\
\hline $17 \mathrm{H}-1,132$ & 162.85 & 162.73 & 149.32 & $24 X-1,35$ & 234.46 & 233.90 & 214.25 \\
\hline $17 \mathrm{H}-2,50$ & 163.41 & 163.41 & 150.00 & $24 X-1,75$ & 234.94 & 234.30 & 214.65 \\
\hline $17 \mathrm{H}-5,83$ & 168.24 & 168.24 & 154.83 & $24 X-1,127$ & 235.28 & 234.82 & 215.17 \\
\hline $17 \mathrm{H}-6,18$ & 169.16 & 169.09 & 155.68 & $24 X-2,68$ & 235.87 & 235.73 & 216.08 \\
\hline $17 \mathrm{H}-6,45$ & 169.47 & 169.36 & 155.95 & $24 X-2,103$ & 236.10 & 236.08 & 216.43 \\
\hline $17 \mathrm{H}-6,141$ & 170.40 & 170.32 & 156.91 & $24 X-3,23$ & 236.74 & 236.78 & 217.13 \\
\hline $17 \mathrm{H}-7,6$ & 170.60 & 170.47 & 157.06 & $24 X-3,78$ & 237.24 & 237.33 & 217.68 \\
\hline $17 \mathrm{H}-7,63$ & 171.12 & 171.04 & 157.63 & $24 X-4,47$ & 238.09 & 238.12 & 218.47 \\
\hline $17 \mathrm{H}-7,75$ & 171.21 & 171.16 & 157.75 & $24 X-4,84$ & 238.48 & 238.49 & 218.84 \\
\hline $18 \mathrm{H}-1,32$ & 173.10 & 173.09 & 157.82 & $25 X-1,18$ & 244.45 & 244.45 & 223.58 \\
\hline $18 \mathrm{H}-1,47$ & 173.26 & 173.24 & 157.97 & $25 X-1,101$ & 245.36 & 245.28 & 224.41 \\
\hline $18 \mathrm{H}-1,96$ & 173.73 & 173.73 & 158.46 & $25 X-1,118$ & 245.53 & 245.45 & 224.58 \\
\hline $18 \mathrm{H}-6,104$ & 181.35 & 181.35 & 166.08 & $25 X-2,7$ & 245.85 & 245.84 & 224.97 \\
\hline $18 \mathrm{H}-6,135$ & 181.68 & 181.66 & 166.39 & $25 X-4,99$ & 249.76 & 249.76 & 228.89 \\
\hline $18 \mathrm{H}-7,49$ & 182.33 & 182.31 & 167.03 & $25 X-4,118$ & 249.96 & 249.96 & 229.08 \\
\hline $18 \mathrm{H}-7,72$ & 182.56 & 182.53 & 167.26 & $25 X-5,17$ & 250.50 & 250.44 & 229.57 \\
\hline $19 \mathrm{H}-1,6$ & 183.21 & 183.20 & 167.06 & $25 X-5,34$ & 250.63 & 250.61 & 229.74 \\
\hline $19 \mathrm{H}-1,17$ & 183.31 & 183.31 & 167.17 & $25 X-5,55$ & 250.84 & 250.82 & 229.95 \\
\hline $19 \mathrm{H}-1,68$ & 183.88 & 183.82 & 167.68 & $25 X-5,103$ & 251.25 & 251.30 & 230.43 \\
\hline $19 \mathrm{H}-1,108$ & 184.22 & 184.22 & 168.08 & $25 X-5,150$ & 251.66 & 251.77 & 230.90 \\
\hline $19 \mathrm{H}-2,4$ & 184.68 & 184.68 & 168.54 & $25 X-7,29$ & 253.00 & 253.06 & 232.19 \\
\hline $19 \mathrm{H}-5,55$ & 189.69 & 189.69 & 173.55 & $25 X-7,73$ & 253.41 & 253.50 & 232.63 \\
\hline $19 \mathrm{H}-6,30$ & 190.85 & 190.94 & 174.80 & $26 X-1,9$ & 253.72 & 253.67 & 233.09 \\
\hline $19 \mathrm{H}-6,138$ & 191.94 & 192.02 & 175.88 & $26 X-1,38$ & 253.96 & 253.96 & 233.38 \\
\hline $19 \mathrm{H}-7,72$ & 192.73 & 192.86 & 176.72 & $26 X-6,14$ & 261.22 & 261.22 & 240.64 \\
\hline $20 \mathrm{H}-1,8$ & 193.44 & 193.46 & 176.58 & $26 \mathrm{X}-6,28$ & 261.48 & 261.36 & 240.78 \\
\hline $20 \mathrm{H}-1,30$ & 193.68 & 193.68 & 176.80 & $26 X-6,80$ & 262.01 & 261.88 & 241.30 \\
\hline $20 \mathrm{H}-1,43$ & 193.79 & 193.81 & 176.93 & $26 \mathrm{X}-6,84$ & 262.05 & 261.92 & 241.34 \\
\hline $20 \mathrm{H}-1,61$ & 193.98 & 193.99 & 177.11 & $26 \mathrm{X}-6,92$ & 262.12 & 262.00 & 241.42 \\
\hline
\end{tabular}


Table AT23 (continued). (Continued on next page.)

\begin{tabular}{|c|c|c|c|}
\hline \multirow[b]{2}{*}{$\begin{array}{l}\text { Core, section, } \\
\text { interval }(\mathrm{cm})\end{array}$} & \multicolumn{3}{|c|}{ Depth $(\mathrm{m})$} \\
\hline & $\begin{array}{l}\text { Adjusted } \\
\text { CCSF }\end{array}$ & CCSF & CSF-A \\
\hline $26 X-7,36$ & 262.64 & 262.44 & 241.86 \\
\hline $26 X-7,40$ & 262.71 & 262.48 & 241.90 \\
\hline $26 X-7,87$ & 263.24 & 262.95 & 242.37 \\
\hline $27 X-1,0$ & 265.71 & 265.69 & 242.20 \\
\hline $27 X-1,8$ & 265.79 & 265.77 & 242.28 \\
\hline $27 X-1,12$ & 265.86 & 265.81 & 242.32 \\
\hline $27 X-1,29$ & 266.12 & 265.99 & 242.49 \\
\hline $27 X-1,48$ & 266.26 & 266.17 & 242.68 \\
\hline $27 X-1,63$ & 266.37 & 266.32 & 242.83 \\
\hline $27 X-1,74$ & 266.44 & 266.43 & 242.94 \\
\hline $27 X-1,103$ & 266.70 & 266.72 & 243.23 \\
\hline $27 X-1,110$ & 266.79 & 266.79 & 243.30 \\
\hline $28 \mathrm{X}-1,11$ & 271.46 & 271.46 & 251.81 \\
\hline $28 X-4,46$ & 275.81 & 275.81 & 256.16 \\
\hline $29 X-1,15$ & 288.88 & 288.58 & 261.45 \\
\hline $29 X-2,79$ & 290.79 & 290.72 & 263.59 \\
\hline $29 X-3,22$ & 291.52 & 291.65 & 264.52 \\
\hline $29 X-4,48$ & 293.25 & 293.41 & 266.28 \\
\hline $29 X-4,132$ & 293.94 & 294.25 & 267.12 \\
\hline $29 X-5,131$ & 295.12 & 295.74 & 268.61 \\
\hline $29 X-7,30$ & 297.32 & 297.23 & 270.10 \\
\hline $30 \times-1,13$ & 299.17 & 299.39 & 270.93 \\
\hline $30 X-5,147$ & 306.76 & 306.73 & 278.27 \\
\hline $30 X-6,111$ & 307.87 & 307.87 & 279.41 \\
\hline $30 X-6,150$ & 308.24 & 308.26 & 279.80 \\
\hline $30 X-7,38$ & 308.71 & 308.64 & 280.18 \\
\hline $31 X-1,13$ & 309.78 & 309.58 & 280.63 \\
\hline $31 X-1,130$ & 310.92 & 310.74 & 281.80 \\
\hline $31 X-2,72$ & 311.77 & 311.66 & 282.72 \\
\hline $31 x-3,5$ & 312.28 & 312.49 & 283.55 \\
\hline $31 X-4,23$ & 313.29 & 314.17 & 285.23 \\
\hline $31 X-4,89$ & 314.04 & 314.83 & 285.89 \\
\hline $31 X-5,12$ & 315.45 & 315.56 & 286.62 \\
\hline $31 X-5,48$ & 316.04 & 315.92 & 286.98 \\
\hline $31 X-6,84$ & 317.38 & 317.78 & 288.84 \\
\hline $32 X-1,31$ & 319.02 & 318.95 & 290.51 \\
\hline $32 X-1,88$ & 319.48 & 319.52 & 291.08 \\
\hline $32 X-1,108$ & 319.72 & 319.72 & 291.28 \\
\hline $32 X-4,80$ & 323.94 & 323.94 & 295.50 \\
\hline $32 X-5,13$ & 324.74 & 324.77 & 296.33 \\
\hline $32 X-5,45$ & 325.20 & 325.09 & 296.65 \\
\hline $32 X-5,78$ & 325.40 & 325.42 & 296.98 \\
\hline $33 X-1,6$ & 327.78 & 327.41 & 299.76 \\
\hline $33 X-1,29$ & 327.98 & 327.64 & 299.99 \\
\hline $33 X-1,123$ & 328.71 & 328.58 & 300.93 \\
\hline $33 X-2,39$ & 329.08 & 329.24 & 301.59 \\
\hline $33 X-2,80$ & 329.65 & 329.65 & 302.00 \\
\hline $33 X-5,34$ & 333.69 & 333.69 & 306.04 \\
\hline $33 X-5,124$ & 334.40 & 334.59 & 306.94 \\
\hline $33 X-5,149$ & 334.60 & 334.84 & 307.19 \\
\hline $33 X-6,36$ & 335.02 & 335.21 & 307.56 \\
\hline $33 X-6,48$ & 335.26 & 335.33 & 307.68 \\
\hline $33 X-6,94$ & 335.75 & 335.80 & 308.14 \\
\hline $33 X-7,72$ & 336.77 & 336.77 & 309.12 \\
\hline $34 X-1,5$ & 338.24 & 338.25 & 309.35 \\
\hline $34 \mathrm{X}-2,0$ & 339.64 & 339.70 & 310.80 \\
\hline $34 X-2,102$ & 340.88 & 340.72 & 311.82 \\
\hline $34 X-3,17$ & 341.38 & 341.38 & 312.47 \\
\hline $34 X-5,88$ & 345.08 & 345.08 & 316.18 \\
\hline $34 X-6,134$ & 347.08 & 347.04 & 318.14 \\
\hline $35 X-1,2$ & 347.59 & 347.59 & 318.92 \\
\hline $35 X-2,4$ & 349.11 & 349.11 & 320.44 \\
\hline $35 X-3,36$ & 350.93 & 350.93 & 322.26 \\
\hline $35 X-4,68$ & 352.61 & 352.75 & 324.08 \\
\hline $35 X-4,88$ & 352.76 & 352.95 & 324.28 \\
\hline $35 X-4,99$ & 352.87 & 353.06 & 324.39 \\
\hline $35 X-5,19$ & 353.46 & 353.76 & 325.09 \\
\hline $35 X-5,140$ & 354.74 & 354.97 & 326.30 \\
\hline
\end{tabular}

\begin{tabular}{|c|c|c|c|}
\hline \multirow[b]{2}{*}{$\begin{array}{l}\text { Core, section, } \\
\text { interval (cm) }\end{array}$} & \multicolumn{3}{|c|}{ Depth $(\mathrm{m})$} \\
\hline & $\begin{array}{l}\text { Adjusted } \\
\text { CCSF }\end{array}$ & CCSF & CSF-A \\
\hline $35 X-6,5$ & 354.93 & 355.12 & 326.45 \\
\hline $35 X-6,62$ & 355.69 & 355.69 & 327.02 \\
\hline $36 X-1,3$ & 357.90 & 357.82 & 328.53 \\
\hline $36 X-1,23$ & 358.13 & 358.02 & 328.73 \\
\hline $36 X-1,57$ & 358.53 & 358.37 & 329.07 \\
\hline $36 \mathrm{X}-1,92$ & 358.83 & 358.71 & 329.42 \\
\hline $36 \mathrm{X}-1,145$ & 359.24 & 359.24 & 329.95 \\
\hline $36 X-5,87$ & 364.66 & 364.66 & 335.37 \\
\hline $36 X-6,0$ & 365.29 & 365.29 & 336.00 \\
\hline $36 X-6,98$ & 366.11 & 366.27 & 336.98 \\
\hline $36 \mathrm{X}-6,122$ & 366.38 & 366.51 & 337.22 \\
\hline $36 X-6,135$ & 366.50 & 366.64 & 337.35 \\
\hline $36 X-7,15$ & 366.72 & 366.84 & 337.55 \\
\hline $36 X-7,50$ & 367.13 & 367.19 & 337.90 \\
\hline $37 X-1,10$ & 369.30 & 368.93 & 338.10 \\
\hline $37 X-2,1$ & 370.36 & 370.34 & 339.51 \\
\hline $37 X-2,22$ & 370.55 & 370.55 & 339.72 \\
\hline $37 X-2,31$ & 370.64 & 370.64 & 339.81 \\
\hline $37 X-2,54$ & 370.82 & 370.87 & 340.04 \\
\hline $37 X-2,87$ & 371.20 & 371.20 & 340.37 \\
\hline $37 X-6,58$ & 376.91 & 376.91 & 346.08 \\
\hline $37 X-6,93$ & 377.14 & 377.26 & 346.43 \\
\hline $37 X-7,13$ & 377.38 & 377.46 & 346.63 \\
\hline $37 X-7,60$ & 377.74 & 377.93 & 347.10 \\
\hline $37 X-7,75$ & 377.93 & 378.08 & 347.25 \\
\hline $37 X-7,86$ & 378.02 & 378.19 & 347.36 \\
\hline $38 X-1,2$ & 379.71 & 379.79 & 347.62 \\
\hline $38 X-1,13$ & 379.82 & 379.90 & 347.73 \\
\hline $38 \mathrm{X}-1,48$ & 380.31 & 380.25 & 348.08 \\
\hline $38 \mathrm{X}-2,126$ & 382.66 & 382.53 & 350.36 \\
\hline $38 X-3,67$ & 383.69 & 383.44 & 351.27 \\
\hline $38 X-4,15$ & 384.26 & 383.92 & 351.75 \\
\hline $39 X-1,8$ & 390.40 & 389.86 & 357.28 \\
\hline $39 X-1,51$ & 390.85 & 390.29 & 357.71 \\
\hline $39 X-1,87$ & 391.19 & 390.65 & 358.07 \\
\hline $39 X-2,55$ & 392.03 & 391.83 & 359.25 \\
\hline $39 X-2,89$ & 392.30 & 392.17 & 359.59 \\
\hline $39 X-2,135$ & 392.66 & 392.63 & 360.05 \\
\hline $39 X-3,34$ & 393.14 & 393.12 & 360.54 \\
\hline $40 X-1,18$ & 402.14 & 402.14 & 366.98 \\
\hline $40 X-5,93$ & 408.89 & 408.89 & 373.73 \\
\hline $40 X-6,1$ & 409.43 & 409.47 & 374.31 \\
\hline $40 X-6,117$ & 410.60 & 410.64 & 375.47 \\
\hline $40 X-7,9$ & 410.84 & 410.75 & 375.59 \\
\hline $40 X-7,64$ & 411.44 & 411.31 & 376.14 \\
\hline $41 X-1,22$ & 412.76 & 412.76 & 376.62 \\
\hline $41 X-5,78$ & 419.32 & 419.32 & 383.18 \\
\hline $41 X-6,53$ & 420.67 & 420.57 & 384.43 \\
\hline $41 X-6,109$ & 421.12 & 421.13 & 384.99 \\
\hline $41 X-7,50$ & 421.77 & 421.74 & 385.60 \\
\hline $42 X-1,96$ & 425.52 & 425.48 & 386.96 \\
\hline $42 X-2,82$ & 427.13 & 426.84 & 388.32 \\
\hline $42 X-3,46$ & 428.11 & 427.98 & 389.46 \\
\hline $42 X-3,130$ & 428.84 & 428.82 & 390.30 \\
\hline $42 X-4,30$ & 429.25 & 429.32 & 390.80 \\
\hline $42 X-4,69$ & 429.62 & 429.71 & 391.19 \\
\hline $42 X-4,126$ & 430.29 & 430.28 & 391.76 \\
\hline $42 X-5,45$ & 430.96 & 430.97 & 392.45 \\
\hline $42 X-5,109$ & 431.54 & 431.61 & 393.09 \\
\hline $42 X-6,43$ & 432.45 & 432.45 & 393.93 \\
\hline $43 X-1,1$ & 433.95 & 433.95 & 395.61 \\
\hline $43 X-7,77$ & 443.21 & 443.21 & 404.87 \\
\hline $44 X-1,9$ & 443.33 & 443.33 & 404.99 \\
\hline $44 X-4,70$ & 448.44 & 448.44 & 410.10 \\
\hline $44 X-5,41$ & 449.58 & 449.65 & 411.31 \\
\hline $44 X-5,73$ & 449.82 & 449.97 & 411.63 \\
\hline $44 X-5,145$ & 450.67 & 450.69 & 412.35 \\
\hline $44 X-7,83$ & 452.57 & 452.57 & 414.23 \\
\hline
\end{tabular}


Table AT23 (continued).

\begin{tabular}{lccc}
\hline & \multicolumn{3}{c}{ Depth $(\mathrm{m})$} \\
\cline { 2 - 4 } $\begin{array}{l}\text { Core, section, } \\
\text { interval }(\mathrm{cm})\end{array}$ & $\begin{array}{c}\text { Adjusted } \\
\text { CCSF }\end{array}$ & CCSF & CSF-A \\
\hline 45X-1, 22 & 454.57 & 454.57 & 414.72 \\
$45 X-2,80$ & 456.61 & 456.66 & 416.80 \\
$45 X-5,6$ & 460.39 & 460.41 & 420.56 \\
$45 X-6,97$ & 462.46 & 462.82 & 422.97 \\
$46 X-1,8$ & 470.40 & 470.40 & 424.08 \\
$46 X-2,49$ & 472.31 & 472.31 & 425.99 \\
$46 X-5,82$ & 477.14 & 477.14 & 430.82 \\
$46 X-7,69$ & 479.61 & 479.61 & 433.29 \\
$47 X-1,4$ & 482.06 & 482.06 & 433.64 \\
$47 X-4,0$ & 486.71 & 486.52 & 438.10 \\
$47 X-4,139$ & 488.20 & 487.91 & 439.49 \\
$47 X-5,118$ & 488.88 & 489.20 & 440.78 \\
$47 X-6,55$ & 489.90 & 490.07 & 441.65 \\
$47 X-6,65$ & 490.01 & 490.17 & 441.75 \\
$47 X-7,54$ & 491.05 & 491.28 & 442.86 \\
$48 X-1,3$ & 492.45 & 492.13 & 443.23 \\
$48 X-1,28$ & 492.74 & 492.38 & 443.48 \\
$48 X-1,122$ & 493.32 & 493.32 & 444.42 \\
$48 X-2,24$ & 493.84 & 493.84 & 444.94 \\
$48 X-2,82$ & 494.31 & 494.42 & 445.52 \\
$48 X-2,111$ & 494.62 & 494.71 & 445.81 \\
\hline
\end{tabular}

This table is also available in ASCII. 
Table AT24. Gamma ray attenuation (GRA) density data, Hole U1337B.

\begin{tabular}{|c|c|c|c|c|}
\hline \multirow[b]{2}{*}{$\begin{array}{l}\text { Core, section, } \\
\text { interval }(\mathrm{cm})\end{array}$} & \multicolumn{3}{|c|}{ Depth (m) } & \multirow[b]{2}{*}{$\begin{array}{c}\text { GRA } \\
\left(\mathrm{g} / \mathrm{cm}^{3}\right)\end{array}$} \\
\hline & CSF & CCSF & $\begin{array}{l}\text { Adjusted } \\
\text { CCSF }\end{array}$ & \\
\hline \multicolumn{5}{|l|}{ 321-U1337B- } \\
\hline $1 \mathrm{H}-1,2.5$ & 1.02 & 0.02 & 0.02 & 1.29 \\
\hline $1 \mathrm{H}-1,5$ & 1.05 & 0.05 & 0.05 & 1.28 \\
\hline $1 \mathrm{H}-1,7.5$ & 1.08 & 0.08 & 0.08 & 1.25 \\
\hline $1 \mathrm{H}-1,10$ & 1.10 & 0.10 & 0.10 & 1.27 \\
\hline $1 \mathrm{H}-1,12.5$ & 1.13 & 0.13 & 0.13 & 1.33 \\
\hline $1 \mathrm{H}-1,15$ & 1.15 & 0.15 & 0.15 & 1.35 \\
\hline $1 \mathrm{H}-1,17.5$ & 1.18 & 0.18 & 0.18 & 1.38 \\
\hline $1 \mathrm{H}-1,20$ & 1.20 & 0.20 & 0.20 & 1.37 \\
\hline $1 \mathrm{H}-1,22.5$ & 1.23 & 0.23 & 0.23 & 1.35 \\
\hline $1 \mathrm{H}-1,25$ & 1.25 & 0.25 & 0.25 & 1.36 \\
\hline $1 \mathrm{H}-1,27.6$ & 1.28 & 0.28 & 0.28 & 1.32 \\
\hline $1 \mathrm{H}-1,30.1$ & 1.30 & 0.30 & 0.30 & 1.33 \\
\hline $1 \mathrm{H}-1,32.6$ & 1.33 & 0.33 & 0.33 & 1.30 \\
\hline $1 \mathrm{H}-1,35.1$ & 1.35 & 0.35 & 0.35 & 1.35 \\
\hline $1 \mathrm{H}-1,37.6$ & 1.38 & 0.38 & 0.38 & 1.37 \\
\hline $1 \mathrm{H}-1,40.1$ & 1.40 & 0.40 & 0.40 & 1.34 \\
\hline $1 \mathrm{H}-1,42.6$ & 1.43 & 0.43 & 0.43 & 1.40 \\
\hline $1 \mathrm{H}-1,45.1$ & 1.45 & 0.45 & 0.45 & 1.45 \\
\hline $1 \mathrm{H}-1,47.6$ & 1.48 & 0.48 & 0.48 & 1.43 \\
\hline $1 \mathrm{H}-1,50.1$ & 1.50 & 0.50 & 0.50 & 1.46 \\
\hline $1 \mathrm{H}-1,52.6$ & 1.53 & 0.53 & 0.53 & 1.44 \\
\hline $1 \mathrm{H}-1,55.1$ & 1.55 & 0.55 & 0.55 & 1.41 \\
\hline $1 \mathrm{H}-1,57.6$ & 1.58 & 0.58 & 0.58 & 1.42 \\
\hline $1 \mathrm{H}-1,60.1$ & 1.60 & 0.60 & 0.60 & 1.42 \\
\hline $1 \mathrm{H}-1,62.6$ & 1.63 & 0.63 & 0.63 & 1.40 \\
\hline $1 \mathrm{H}-1,65.1$ & 1.65 & 0.65 & 0.65 & 1.41 \\
\hline $1 \mathrm{H}-1,67.6$ & 1.68 & 0.68 & 0.68 & 1.39 \\
\hline $1 \mathrm{H}-1,70.1$ & 1.70 & 0.70 & 0.70 & 1.43 \\
\hline $1 \mathrm{H}-1,72.6$ & 1.73 & 0.73 & 0.73 & 1.41 \\
\hline $1 \mathrm{H}-1,75.1$ & 1.75 & 0.75 & 0.75 & 1.41 \\
\hline $1 \mathrm{H}-1,77.6$ & 1.78 & 0.78 & 0.78 & 1.40 \\
\hline $1 \mathrm{H}-1,80.1$ & 1.80 & 0.80 & 0.80 & 1.34 \\
\hline $1 \mathrm{H}-1,82.6$ & 1.83 & 0.83 & 0.83 & 1.36 \\
\hline $1 \mathrm{H}-1,85.1$ & 1.85 & 0.85 & 0.85 & 1.33 \\
\hline $1 \mathrm{H}-1,87.6$ & 1.88 & 0.88 & 0.88 & 1.30 \\
\hline $1 \mathrm{H}-1,90.2$ & 1.90 & 0.90 & 0.90 & 1.27 \\
\hline $1 \mathrm{H}-1,92.7$ & 1.93 & 0.93 & 0.93 & 1.22 \\
\hline $1 \mathrm{H}-1,95.2$ & 1.95 & 0.95 & 0.95 & 1.23 \\
\hline $1 \mathrm{H}-1,97.7$ & 1.98 & 0.98 & 0.98 & 1.2 \\
\hline $1 \mathrm{H}-1,100.2$ & 2.00 & 1.00 & 1.00 & 1.18 \\
\hline $1 \mathrm{H}-1,102.7$ & 2.03 & 1.03 & 1.03 & 1.18 \\
\hline $1 \mathrm{H}-1,105.2$ & 2.05 & 1.05 & 1.05 & 1.14 \\
\hline $1 \mathrm{H}-1,107.7$ & 2.08 & 1.08 & 1.08 & 1.11 \\
\hline $1 \mathrm{H}-1,110.2$ & 2.10 & 1.10 & 1.10 & 1.12 \\
\hline $1 \mathrm{H}-1,117.7$ & 2.18 & 1.18 & 1.18 & 1.16 \\
\hline $1 \mathrm{H}-1,120.2$ & 2.20 & 1.20 & 1.20 & 1.18 \\
\hline $1 \mathrm{H}-1,122.7$ & 2.23 & 1.23 & 1.23 & 1.20 \\
\hline $1 \mathrm{H}-1,125.2$ & 2.25 & 1.25 & 1.25 & 1.18 \\
\hline $1 \mathrm{H}-1,127.7$ & 2.28 & 1.28 & 1.28 & 1.16 \\
\hline $1 \mathrm{H}-1,130.2$ & 2.30 & 1.30 & 1.30 & 1.21 \\
\hline $1 \mathrm{H}-1,132.7$ & 2.33 & 1.33 & 1.33 & 1.11 \\
\hline $1 \mathrm{H}-1,135.2$ & 2.35 & 1.35 & 1.35 & 1.16 \\
\hline $1 \mathrm{H}-1,137.7$ & 2.38 & 1.38 & 1.38 & 1.20 \\
\hline $1 \mathrm{H}-1,140.2$ & 2.40 & 1.40 & 1.40 & 1.19 \\
\hline $1 \mathrm{H}-1,142.7$ & 2.43 & 1.43 & 1.43 & 1.16 \\
\hline $1 \mathrm{H}-1,145.3$ & 2.45 & 1.45 & 1.45 & 1.18 \\
\hline $1 \mathrm{H}-1,147.8$ & 2.48 & 1.48 & 1.48 & 1.14 \\
\hline $1 \mathrm{H}-2,2$ & 2.52 & 1.52 & 1.52 & 1.19 \\
\hline $1 \mathrm{H}-2,4.5$ & 2.55 & 1.55 & 1.55 & 1.17 \\
\hline $1 \mathrm{H}-2,7$ & 2.57 & 1.57 & 1.57 & 1.19 \\
\hline
\end{tabular}

Only a portion of this table appears here. The complete table is available in ASCII. 
Table AT25. Magnetic susceptibility data, Hole U1337B.

\begin{tabular}{|c|c|c|c|c|}
\hline \multirow[b]{2}{*}{$\begin{array}{l}\text { Core, section, } \\
\text { interval }(\mathrm{cm})\end{array}$} & \multicolumn{3}{|c|}{ Depth $(m)$} & \multirow{2}{*}{$\begin{array}{l}\text { Magnetic } \\
\text { susceptibility } \\
\text { (IU) }\end{array}$} \\
\hline & CSF & CCSF & $\begin{array}{l}\text { Adjusted } \\
\text { CCSF }\end{array}$ & \\
\hline \multicolumn{5}{|l|}{ 321-U1337B- } \\
\hline $1 \mathrm{H}-1,0.1$ & 1.00 & 0.00 & 0.00 & 1.6 \\
\hline $1 \mathrm{H}-1,2.6$ & 1.03 & 0.03 & 0.03 & 4.4 \\
\hline $1 \mathrm{H}-1,5.1$ & 1.05 & 0.05 & 0.05 & 4.9 \\
\hline $1 \mathrm{H}-1,7.6$ & 1.08 & 0.08 & 0.08 & 6.6 \\
\hline $1 \mathrm{H}-1,10.1$ & 1.10 & 0.10 & 0.10 & 4.9 \\
\hline $1 \mathrm{H}-1,12.6$ & 1.13 & 0.13 & 0.13 & 5.2 \\
\hline $1 \mathrm{H}-1,15.1$ & 1.15 & 0.15 & 0.15 & 4.4 \\
\hline $1 \mathrm{H}-1,17.6$ & 1.18 & 0.18 & 0.18 & 4.1 \\
\hline $1 \mathrm{H}-1,20.1$ & 1.20 & 0.20 & 0.20 & 3.3 \\
\hline $1 \mathrm{H}-1,22.6$ & 1.23 & 0.23 & 0.23 & 3.3 \\
\hline $1 \mathrm{H}-1,25.1$ & 1.25 & 0.25 & 0.25 & 3.0 \\
\hline $1 \mathrm{H}-1,27.6$ & 1.28 & 0.28 & 0.28 & 2.2 \\
\hline $1 \mathrm{H}-1,30.1$ & 1.30 & 0.30 & 0.30 & 2.7 \\
\hline $1 \mathrm{H}-1,32.6$ & 1.33 & 0.33 & 0.33 & 2.2 \\
\hline $1 \mathrm{H}-1,35.1$ & 1.35 & 0.35 & 0.35 & 1.6 \\
\hline $1 \mathrm{H}-1,37.6$ & 1.38 & 0.38 & 0.38 & 1.4 \\
\hline $1 \mathrm{H}-1,40.1$ & 1.40 & 0.40 & 0.40 & 1.6 \\
\hline $1 \mathrm{H}-1,42.6$ & 1.43 & 0.43 & 0.43 & 1.1 \\
\hline $1 \mathrm{H}-1,45.1$ & 1.45 & 0.45 & 0.45 & 0.5 \\
\hline $1 \mathrm{H}-1,47.6$ & 1.48 & 0.48 & 0.48 & 1.1 \\
\hline $1 \mathrm{H}-1,50.1$ & 1.50 & 0.50 & 0.50 & 1.1 \\
\hline $1 \mathrm{H}-1,52.6$ & 1.53 & 0.53 & 0.53 & 1.1 \\
\hline $1 \mathrm{H}-1,55.2$ & 1.55 & 0.55 & 0.55 & 1.6 \\
\hline $1 \mathrm{H}-1,57.7$ & 1.58 & 0.58 & 0.58 & 1.6 \\
\hline $1 \mathrm{H}-1,60.2$ & 1.60 & 0.60 & 0.60 & 0.8 \\
\hline $1 \mathrm{H}-1,62.7$ & 1.63 & 0.63 & 0.63 & 1.6 \\
\hline $1 \mathrm{H}-1,65.2$ & 1.65 & 0.65 & 0.65 & 1.9 \\
\hline $1 \mathrm{H}-1,67.7$ & 1.68 & 0.68 & 0.68 & 1.9 \\
\hline $1 \mathrm{H}-1,70.2$ & 1.70 & 0.70 & 0.70 & 1.6 \\
\hline $1 \mathrm{H}-1,72.7$ & 1.73 & 0.73 & 0.73 & 1.6 \\
\hline $1 \mathrm{H}-1,75.2$ & 1.75 & 0.75 & 0.75 & 2.2 \\
\hline $1 \mathrm{H}-1,77.7$ & 1.78 & 0.78 & 0.78 & 3.0 \\
\hline $1 \mathrm{H}-1,80.2$ & 1.80 & 0.80 & 0.80 & 3.0 \\
\hline $1 \mathrm{H}-1,82.7$ & 1.83 & 0.83 & 0.83 & 3.8 \\
\hline $1 \mathrm{H}-1,85.2$ & 1.85 & 0.85 & 0.85 & 5.2 \\
\hline $1 \mathrm{H}-1,87.7$ & 1.88 & 0.88 & 0.88 & 5.8 \\
\hline $1 \mathrm{H}-1,90.2$ & 1.90 & 0.90 & 0.90 & 6.9 \\
\hline $1 \mathrm{H}-1,92.7$ & 1.93 & 0.93 & 0.93 & 6.9 \\
\hline $1 \mathrm{H}-1,95.2$ & 1.95 & 0.95 & 0.95 & 7.1 \\
\hline $1 \mathrm{H}-1,97.7$ & 1.98 & 0.98 & 0.98 & 6.9 \\
\hline $1 \mathrm{H}-1,100.2$ & 2.00 & 1.00 & 1.00 & 7.1 \\
\hline $1 \mathrm{H}-1,102.7$ & 2.03 & 1.03 & 1.03 & 7.1 \\
\hline $1 \mathrm{H}-1,105.2$ & 2.05 & 1.05 & 1.05 & 6.6 \\
\hline $1 \mathrm{H}-1,107.7$ & 2.08 & 1.08 & 1.08 & 6.6 \\
\hline $1 \mathrm{H}-1,110.2$ & 2.10 & 1.10 & 1.10 & 6.6 \\
\hline $1 \mathrm{H}-1,112.8$ & 2.13 & 1.13 & 1.13 & 5.2 \\
\hline $1 \mathrm{H}-1,115.2$ & 2.15 & 1.15 & 1.15 & 4.4 \\
\hline $1 \mathrm{H}-1,117.8$ & 2.18 & 1.18 & 1.18 & 6.6 \\
\hline $1 \mathrm{H}-1,120.3$ & 2.20 & 1.20 & 1.20 & 7.1 \\
\hline $1 \mathrm{H}-1,122.8$ & 2.23 & 1.23 & 1.23 & 7.4 \\
\hline $1 \mathrm{H}-1,125.3$ & 2.25 & 1.25 & 1.25 & 7.4 \\
\hline $1 \mathrm{H}-1,127.8$ & 2.28 & 1.28 & 1.28 & 7.4 \\
\hline $1 \mathrm{H}-1,130.3$ & 2.30 & 1.30 & 1.30 & 7.7 \\
\hline $1 \mathrm{H}-1,132.8$ & 2.33 & 1.33 & 1.33 & 8.2 \\
\hline $1 \mathrm{H}-1,135.3$ & 2.35 & 1.35 & 1.35 & 8.5 \\
\hline $1 \mathrm{H}-1,137.8$ & 2.38 & 1.38 & 1.38 & 8.8 \\
\hline $1 \mathrm{H}-1,140.3$ & 2.40 & 1.40 & 1.40 & 8.8 \\
\hline $1 \mathrm{H}-1,142.8$ & 2.43 & 1.43 & 1.43 & 9.1 \\
\hline $1 \mathrm{H}-1,145.3$ & 2.45 & 1.45 & 1.45 & 9.1 \\
\hline $1 \mathrm{H}-1,147.8$ & 2.48 & 1.48 & 1.48 & 7.7 \\
\hline $1 \mathrm{H}-1,150.3$ & 2.50 & 1.50 & 1.50 & 4.7 \\
\hline
\end{tabular}

Only a portion of this table appears here. The complete table is available in ASCII. 
Table AT26. Natural gamma ray (NGR) data, Hole U1337B.

\begin{tabular}{|c|c|c|c|c|}
\hline \multirow[b]{2}{*}{$\begin{array}{l}\text { Core, section, } \\
\text { interval }(\mathrm{cm})\end{array}$} & \multicolumn{3}{|c|}{ Depth (m) } & \multirow[b]{2}{*}{$\begin{array}{l}\text { NGR } \\
\text { (cps) }\end{array}$} \\
\hline & CSF & CCSF & $\begin{array}{l}\text { Adjusted } \\
\text { CCSF }\end{array}$ & \\
\hline \multicolumn{5}{|l|}{ 321-U1337B- } \\
\hline $1 \mathrm{H}-1,0$ & 1.00 & 0.00 & 0.00 & 65.0 \\
\hline $1 \mathrm{H}-1,10$ & 1.10 & 0.10 & 0.10 & 67.0 \\
\hline $1 \mathrm{H}-1,20$ & 1.20 & 0.20 & 0.20 & 67.8 \\
\hline $1 \mathrm{H}-1,30$ & 1.30 & 0.30 & 0.30 & 56.6 \\
\hline $1 \mathrm{H}-1,40$ & 1.40 & 0.40 & 0.40 & 43.9 \\
\hline $1 \mathrm{H}-1,50$ & 1.50 & 0.50 & 0.50 & 42.9 \\
\hline $1 \mathrm{H}-1,60$ & 1.60 & 0.60 & 0.60 & 42.8 \\
\hline $1 \mathrm{H}-1,70$ & 1.70 & 0.70 & 0.70 & 41.0 \\
\hline $1 \mathrm{H}-1,80$ & 1.80 & 0.80 & 0.80 & 50.5 \\
\hline $1 \mathrm{H}-1,90$ & 1.90 & 0.90 & 0.90 & 65.5 \\
\hline $1 \mathrm{H}-1,100$ & 2.00 & 1.00 & 1.00 & 52.7 \\
\hline $1 \mathrm{H}-1,110$ & 2.10 & 1.10 & 1.10 & 42.0 \\
\hline $1 \mathrm{H}-1,120$ & 2.20 & 1.20 & 1.20 & 51.7 \\
\hline $1 \mathrm{H}-1,130$ & 2.30 & 1.30 & 1.30 & 62.8 \\
\hline $1 \mathrm{H}-1,140$ & 2.40 & 1.40 & 1.40 & 57.6 \\
\hline $1 \mathrm{H}-1,150$ & 2.50 & 1.50 & 1.50 & 48.6 \\
\hline $1 \mathrm{H}-2,0$ & 2.50 & 1.50 & 1.50 & 46.8 \\
\hline $1 \mathrm{H}-2,10$ & 2.60 & 1.60 & 1.60 & 46.6 \\
\hline $1 \mathrm{H}-2,20$ & 2.70 & 1.70 & 1.70 & 42.9 \\
\hline $1 \mathrm{H}-2,30$ & 2.80 & 1.80 & 1.80 & 34.1 \\
\hline $1 \mathrm{H}-2,40$ & 2.90 & 1.90 & 1.90 & 26.0 \\
\hline $1 \mathrm{H}-2,50$ & 3.00 & 2.00 & 2.00 & 24.6 \\
\hline $1 \mathrm{H}-2,60$ & 3.10 & 2.10 & 2.10 & 26.1 \\
\hline $1 \mathrm{H}-2,70$ & 3.20 & 2.20 & 2.20 & 22.0 \\
\hline $1 \mathrm{H}-2,80$ & 3.30 & 2.30 & 2.30 & 19.2 \\
\hline $1 \mathrm{H}-2,90$ & 3.40 & 2.40 & 2.40 & 25.5 \\
\hline $1 \mathrm{H}-2,100$ & 3.50 & 2.50 & 2.50 & 29.4 \\
\hline $1 \mathrm{H}-2,110$ & 3.60 & 2.60 & 2.60 & 24.1 \\
\hline $1 \mathrm{H}-2,120$ & 3.70 & 2.70 & 2.70 & 20.2 \\
\hline $1 \mathrm{H}-2,130$ & 3.80 & 2.80 & 2.80 & 16.2 \\
\hline $1 \mathrm{H}-2,140$ & 3.90 & 2.90 & 2.90 & 14.3 \\
\hline $1 \mathrm{H}-2,150$ & 4.00 & 3.00 & 3.00 & 15.4 \\
\hline $1 \mathrm{H}-3,0$ & 4.00 & 3.00 & 3.00 & 18.4 \\
\hline $1 \mathrm{H}-3,10$ & 4.10 & 3.10 & 3.10 & 23.9 \\
\hline $1 \mathrm{H}-3,20$ & 4.20 & 3.20 & 3.20 & 31.1 \\
\hline $1 \mathrm{H}-3,30$ & 4.30 & 3.30 & 3.30 & 25.9 \\
\hline $1 \mathrm{H}-3,40$ & 4.40 & 3.40 & 3.40 & 23.4 \\
\hline $1 \mathrm{H}-3,50$ & 4.50 & 3.50 & 3.50 & 19.8 \\
\hline $1 \mathrm{H}-3,60$ & 4.60 & 3.60 & 3.60 & 16.7 \\
\hline $1 \mathrm{H}-3,70$ & 4.70 & 3.70 & 3.70 & 15.2 \\
\hline $1 \mathrm{H}-3,80$ & 4.80 & 3.80 & 3.80 & 16.0 \\
\hline $1 \mathrm{H}-3,90$ & 4.90 & 3.90 & 3.90 & 13.9 \\
\hline $1 \mathrm{H}-3,100$ & 5.00 & 4.00 & 4.00 & 9.9 \\
\hline $1 \mathrm{H}-3,110$ & 5.10 & 4.10 & 4.10 & 9.5 \\
\hline $1 \mathrm{H}-3,120$ & 5.20 & 4.20 & 4.21 & 9.5 \\
\hline $1 \mathrm{H}-3,130$ & 5.30 & 4.30 & 4.33 & 11.8 \\
\hline $1 \mathrm{H}-3,140$ & 5.40 & 4.40 & 4.44 & 13.4 \\
\hline $1 \mathrm{H}-3,150$ & 5.50 & 4.50 & 4.55 & 12.6 \\
\hline $1 \mathrm{H}-4,0$ & 5.50 & 4.50 & 4.55 & 9.8 \\
\hline $1 \mathrm{H}-4,10$ & 5.60 & 4.60 & 4.66 & 9.9 \\
\hline $1 \mathrm{H}-4,20$ & 5.70 & 4.70 & 4.74 & 9.7 \\
\hline $1 \mathrm{H}-4,30$ & 5.80 & 4.80 & 4.83 & 10.1 \\
\hline $1 \mathrm{H}-4,40$ & 5.90 & 4.90 & 4.91 & 9.8 \\
\hline $1 \mathrm{H}-4,50$ & 6.00 & 5.00 & 4.99 & 8.4 \\
\hline $1 \mathrm{H}-4,60$ & 6.10 & 5.10 & 5.07 & 8.9 \\
\hline $1 \mathrm{H}-4,70$ & 6.20 & 5.20 & 5.14 & 8.6 \\
\hline $1 \mathrm{H}-4,80$ & 6.30 & 5.30 & 5.24 & 7.8 \\
\hline $1 \mathrm{H}-4,90$ & 6.40 & 5.40 & 5.35 & 7.4 \\
\hline $1 \mathrm{H}-4,100$ & 6.50 & 5.50 & 5.45 & 7.3 \\
\hline $1 \mathrm{H}-5,0$ & 6.50 & 5.50 & 5.45 & 6.4 \\
\hline
\end{tabular}

Only a portion of this table appears here. The complete table is available in ASCII. 
Table AT27. Color reflection data, Hole U1337B.

\begin{tabular}{|c|c|c|c|c|c|c|}
\hline \multirow[b]{2}{*}{$\begin{array}{l}\text { Core, section, } \\
\text { interval }(\mathrm{cm})\end{array}$} & \multicolumn{3}{|c|}{ Depth (m) } & \multirow[b]{2}{*}{$a^{*}$} & \multirow[b]{2}{*}{$b^{*}$} & \multirow[b]{2}{*}{$\mathrm{L}^{*}$} \\
\hline & CSF & CCSF & $\begin{array}{c}\text { Adjusted } \\
\text { CCSF }\end{array}$ & & & \\
\hline \multicolumn{7}{|l|}{ 321-U1337B- } \\
\hline $1 \mathrm{H}-1,5.2$ & 1.05 & 0.05 & 0.05 & 6.8 & 10.3 & 35.1 \\
\hline $1 \mathrm{H}-1,7.7$ & 1.08 & 0.08 & 0.08 & 6.4 & 9.7 & 35.2 \\
\hline $1 \mathrm{H}-1,10.2$ & 1.10 & 0.10 & 0.10 & 6.0 & 10.5 & 38.9 \\
\hline $1 \mathrm{H}-1,12.8$ & 1.13 & 0.13 & 0.13 & 3.4 & 6.8 & 41.1 \\
\hline $1 \mathrm{H}-1,15.3$ & 1.15 & 0.15 & 0.15 & 6.0 & 11.9 & 41.0 \\
\hline $1 \mathrm{H}-1,17.9$ & 1.18 & 0.18 & 0.18 & 4.9 & 9.9 & 43.9 \\
\hline $1 \mathrm{H}-1,20.4$ & 1.20 & 0.20 & 0.20 & 4.3 & 9.2 & 46.2 \\
\hline $1 \mathrm{H}-1,22.9$ & 1.23 & 0.23 & 0.23 & 6.0 & 14.2 & 49.7 \\
\hline $1 \mathrm{H}-1,25.5$ & 1.25 & 0.25 & 0.25 & 5.3 & 12.2 & 50.8 \\
\hline $1 \mathrm{H}-1,28$ & 1.28 & 0.28 & 0.28 & 4.2 & 10.5 & 49.6 \\
\hline $1 \mathrm{H}-1,30.6$ & 1.31 & 0.31 & 0.31 & 5.8 & 14.6 & 53.0 \\
\hline $1 \mathrm{H}-1,33.1$ & 1.33 & 0.33 & 0.33 & 6.2 & 17.1 & 51.5 \\
\hline $1 \mathrm{H}-1,35.6$ & 1.36 & 0.36 & 0.36 & 6.1 & 19.0 & 56.1 \\
\hline $1 \mathrm{H}-1,38.2$ & 1.38 & 0.38 & 0.38 & 6.2 & 18.1 & 53.3 \\
\hline $1 \mathrm{H}-1,40.7$ & 1.41 & 0.41 & 0.41 & 5.7 & 19.1 & 55.9 \\
\hline $1 \mathrm{H}-1,43.3$ & 1.43 & 0.43 & 0.43 & 5.8 & 19.9 & 58.5 \\
\hline $1 \mathrm{H}-1,45.8$ & 1.46 & 0.46 & 0.46 & 5.3 & 19.9 & 62.9 \\
\hline $1 \mathrm{H}-1,48.4$ & 1.48 & 0.48 & 0.48 & 4.9 & 15.9 & 60.8 \\
\hline $1 \mathrm{H}-1,50.9$ & 1.51 & 0.51 & 0.51 & 4.5 & 14.6 & 61.9 \\
\hline $1 \mathrm{H}-1,53.4$ & 1.53 & 0.53 & 0.53 & 3.7 & 12.2 & 57.0 \\
\hline $1 \mathrm{H}-1,56$ & 1.56 & 0.56 & 0.56 & 2.4 & 9.1 & 58.1 \\
\hline $1 \mathrm{H}-1,58.5$ & 1.59 & 0.59 & 0.59 & 3.2 & 12.1 & 57.1 \\
\hline $1 \mathrm{H}-1,61.1$ & 1.61 & 0.61 & 0.61 & 1.4 & 13.8 & 76.1 \\
\hline $1 \mathrm{H}-1,63.6$ & 1.64 & 0.64 & 0.64 & 4.5 & 14.8 & 56.3 \\
\hline $1 \mathrm{H}-1,66.1$ & 1.66 & 0.66 & 0.66 & 3.2 & 10.6 & 55.3 \\
\hline $1 \mathrm{H}-1,68.7$ & 1.69 & 0.69 & 0.69 & 4.0 & 13.1 & 59.0 \\
\hline $1 \mathrm{H}-1,71.2$ & 1.71 & 0.71 & 0.71 & 4.5 & 15.4 & 56.0 \\
\hline $1 \mathrm{H}-1,73.8$ & 1.74 & 0.74 & 0.74 & 4.8 & 17.7 & 62.0 \\
\hline $1 \mathrm{H}-1,76.3$ & 1.76 & 0.76 & 0.76 & 4.2 & 14.1 & 58.2 \\
\hline $1 \mathrm{H}-1,78.9$ & 1.79 & 0.79 & 0.79 & 3.3 & 12.2 & 56.2 \\
\hline $1 \mathrm{H}-1,81.4$ & 1.81 & 0.81 & 0.81 & 3.5 & 12.2 & 53.7 \\
\hline $1 \mathrm{H}-1,83.9$ & 1.84 & 0.84 & 0.84 & 4.0 & 15.3 & 51.5 \\
\hline $1 \mathrm{H}-1,86.5$ & 1.87 & 0.87 & 0.87 & 2.8 & 11.5 & 48.3 \\
\hline $1 \mathrm{H}-1,89$ & 1.89 & 0.89 & 0.89 & 3.1 & 10.4 & 40.8 \\
\hline $1 \mathrm{H}-1,91.6$ & 1.92 & 0.92 & 0.92 & 3.2 & 8.8 & 36.6 \\
\hline $1 \mathrm{H}-1,94.1$ & 1.94 & 0.94 & 0.94 & 5.3 & 9.1 & 35.5 \\
\hline $1 \mathrm{H}-1,96.7$ & 1.97 & 0.97 & 0.97 & 3.5 & 6.6 & 40.8 \\
\hline $1 \mathrm{H}-1,99.2$ & 1.99 & 0.99 & 0.99 & 4.1 & 11.8 & 51.8 \\
\hline $1 \mathrm{H}-1,101.7$ & 2.02 & 1.02 & 1.02 & 7.1 & 15.1 & 42.1 \\
\hline $1 \mathrm{H}-1,104.3$ & 2.04 & 1.04 & 1.04 & 5.3 & 11.5 & 44.4 \\
\hline $1 \mathrm{H}-1,106.8$ & 2.07 & 1.07 & 1.07 & 4.8 & 11.9 & 44.8 \\
\hline $1 \mathrm{H}-1,109.4$ & 2.09 & 1.09 & 1.09 & 7.4 & 15.4 & 39.5 \\
\hline $1 \mathrm{H}-1,111.9$ & 2.12 & 1.12 & 1.12 & 6.9 & 15.7 & 41.8 \\
\hline $1 \mathrm{H}-1,114.4$ & 2.14 & 1.14 & 1.14 & 6.8 & 14.4 & 40.7 \\
\hline $1 \mathrm{H}-1,117$ & 2.17 & 1.17 & 1.17 & 7.9 & 15.5 & 40.3 \\
\hline $1 \mathrm{H}-1,119.5$ & 2.19 & 1.19 & 1.19 & 7.5 & 16.4 & 40.8 \\
\hline $1 \mathrm{H}-1,122.1$ & 2.22 & 1.22 & 1.22 & 7.6 & 14.6 & 40.7 \\
\hline $1 \mathrm{H}-1,124.6$ & 2.25 & 1.25 & 1.25 & 4.2 & 8.1 & 42.1 \\
\hline $1 \mathrm{H}-1,127.2$ & 2.27 & 1.27 & 1.27 & 4.0 & 7.7 & 40.6 \\
\hline $1 \mathrm{H}-1,129.7$ & 2.30 & 1.30 & 1.30 & 5.3 & 10.2 & 35.6 \\
\hline $1 \mathrm{H}-1,132.2$ & 2.32 & 1.32 & 1.32 & 5.8 & 10.7 & 35.3 \\
\hline $1 \mathrm{H}-1,134.8$ & 2.35 & 1.35 & 1.35 & 6.0 & 7.4 & 32.6 \\
\hline $1 \mathrm{H}-1,137.3$ & 2.37 & 1.37 & 1.37 & 3.7 & 8.1 & 40.8 \\
\hline $1 \mathrm{H}-1,139.9$ & 2.40 & 1.40 & 1.40 & 4.9 & 8.8 & 37.4 \\
\hline $1 \mathrm{H}-1,142.4$ & 2.42 & 1.42 & 1.42 & 3.4 & 5.3 & 36.7 \\
\hline $1 \mathrm{H}-1,145$ & 2.45 & 1.45 & 1.45 & 3.8 & 5.8 & 34.5 \\
\hline $1 \mathrm{H}-1,147.5$ & 2.48 & 1.48 & 1.48 & 3.1 & 2.3 & 29.0 \\
\hline $1 \mathrm{H}-2,2.6$ & 2.53 & 1.53 & 1.53 & 5.6 & 0.3 & 23.8 \\
\hline $1 \mathrm{H}-2,5.2$ & 2.55 & 1.55 & 1.55 & 3.7 & 6.7 & 39.7 \\
\hline
\end{tabular}

Only a portion of this table appears here. The complete table is available in ASCII. 
Table AT28. Core adjusted data, Hole U1337B. (Continued on next page.)

\begin{tabular}{|c|c|c|c|}
\hline \multirow[b]{2}{*}{$\begin{array}{l}\text { Core, section, } \\
\text { interval }(\mathrm{cm})\end{array}$} & \multicolumn{3}{|c|}{ Depth (m) } \\
\hline & $\begin{array}{l}\text { Adjusted } \\
\text { CCSF }\end{array}$ & CCSF & CSF-A \\
\hline \multicolumn{4}{|l|}{ 321-U1337B- } \\
\hline $1 \mathrm{H}-1,3$ & 0.03 & 0.03 & 1.03 \\
\hline $1 \mathrm{H}-3,110$ & 4.10 & 4.10 & 5.10 \\
\hline $1 \mathrm{H}-4,8$ & 4.65 & 4.58 & 5.58 \\
\hline $1 \mathrm{H}-4,45$ & 4.95 & 4.95 & 5.95 \\
\hline $1 \mathrm{H}-4,60$ & 5.07 & 5.10 & 6.10 \\
\hline $1 \mathrm{H}-4,73$ & 5.16 & 5.23 & 6.23 \\
\hline $1 \mathrm{H}-5,41$ & 5.88 & 5.91 & 6.91 \\
\hline $1 \mathrm{H}-5,57$ & 6.05 & 6.07 & 7.07 \\
\hline $2 \mathrm{H}-1,14$ & 7.21 & 7.08 & 10.64 \\
\hline $2 \mathrm{H}-1,32$ & 7.37 & 7.26 & 10.82 \\
\hline $2 \mathrm{H}-1,61$ & 7.62 & 7.55 & 11.11 \\
\hline $2 \mathrm{H}-1,87$ & 7.87 & 7.81 & 11.37 \\
\hline $2 \mathrm{H}-1,124$ & 8.29 & 8.18 & 11.74 \\
\hline $2 \mathrm{H}-2,20$ & 8.68 & 8.64 & 12.20 \\
\hline $2 \mathrm{H}-2,46$ & 9.01 & 8.90 & 12.46 \\
\hline $2 \mathrm{H}-2,86$ & 9.35 & 9.30 & 12.86 \\
\hline $2 \mathrm{H}-2,111$ & 9.59 & 9.55 & 13.11 \\
\hline $2 \mathrm{H}-2,142$ & 9.88 & 9.86 & 13.42 \\
\hline $2 \mathrm{H}-3,26$ & 10.21 & 10.20 & 13.76 \\
\hline $2 \mathrm{H}-3,56$ & 10.52 & 10.50 & 14.06 \\
\hline $2 \mathrm{H}-3,83$ & 10.83 & 10.77 & 14.33 \\
\hline $2 \mathrm{H}-4,6$ & 11.53 & 11.51 & 15.06 \\
\hline $2 \mathrm{H}-4,40$ & 11.82 & 11.84 & 15.40 \\
\hline $2 \mathrm{H}-4,101$ & 12.50 & 12.45 & 16.01 \\
\hline $2 \mathrm{H}-4,121$ & 12.72 & 12.66 & 16.21 \\
\hline $2 \mathrm{H}-5,21$ & 13.15 & 13.15 & 16.71 \\
\hline $2 \mathrm{H}-5,108$ & 14.08 & 14.02 & 17.58 \\
\hline $2 \mathrm{H}-6,7$ & 14.48 & 14.51 & 18.07 \\
\hline $2 \mathrm{H}-6,59$ & 15.01 & 15.03 & 18.59 \\
\hline $2 \mathrm{H}-6,82$ & 15.27 & 15.26 & 18.82 \\
\hline $2 \mathrm{H}-7,53$ & 16.40 & 16.47 & 20.03 \\
\hline $3 \mathrm{H}-1,48$ & 18.09 & 18.12 & 20.48 \\
\hline $3 \mathrm{H}-1,66$ & 18.32 & 18.30 & 20.66 \\
\hline $3 \mathrm{H}-1,101$ & 18.58 & 18.65 & 21.01 \\
\hline $3 \mathrm{H}-1,135$ & 18.94 & 18.99 & 21.35 \\
\hline $3 \mathrm{H}-2,12$ & 19.26 & 19.26 & 21.62 \\
\hline $3 \mathrm{H}-5,121$ & 24.83 & 24.85 & 27.21 \\
\hline $3 \mathrm{H}-6,1$ & 25.21 & 25.15 & 27.51 \\
\hline $3 \mathrm{H}-6,89$ & 26.02 & 26.04 & 28.39 \\
\hline $3 \mathrm{H}-7,4$ & 26.34 & 26.38 & 28.74 \\
\hline $3 \mathrm{H}-7,29$ & 26.69 & 26.63 & 28.99 \\
\hline $3 \mathrm{H}-7,69$ & 27.04 & 27.03 & 29.39 \\
\hline $4 \mathrm{H}-1,23$ & 27.78 & 27.89 & 29.73 \\
\hline $4 \mathrm{H}-1,86$ & 28.51 & 28.52 & 30.36 \\
\hline $4 \mathrm{H}-1,93$ & 28.60 & 28.59 & 30.43 \\
\hline $4 \mathrm{H}-6,19$ & 35.35 & 35.36 & 37.19 \\
\hline $4 \mathrm{H}-6,77$ & 35.81 & 35.93 & 37.77 \\
\hline $4 \mathrm{H}-6,124$ & 36.28 & 36.40 & 38.24 \\
\hline $4 \mathrm{H}-7,1$ & 36.50 & 36.67 & 38.51 \\
\hline $4 \mathrm{H}-7,66$ & 37.30 & 37.32 & 39.16 \\
\hline $5 \mathrm{H}-1,6$ & 38.06 & 38.12 & 39.06 \\
\hline $5 \mathrm{H}-1,28$ & 38.30 & 38.35 & 39.28 \\
\hline $5 \mathrm{H}-1,40$ & 38.46 & 38.46 & 39.40 \\
\hline $5 \mathrm{H}-5,107$ & 45.15 & 45.13 & 46.07 \\
\hline $5 \mathrm{H}-5,125$ & 45.30 & 45.32 & 46.25 \\
\hline $5 \mathrm{H}-6,55$ & 46.29 & 46.11 & 47.05 \\
\hline $5 \mathrm{H}-6,142$ & 47.10 & 46.98 & 47.92 \\
\hline $5 \mathrm{H}-7,62$ & 47.80 & 47.68 & 48.62 \\
\hline $6 \mathrm{H}-1,23$ & 49.13 & 49.02 & 48.73 \\
\hline $6 \mathrm{H}-1,108$ & 49.89 & 49.87 & 49.58 \\
\hline $6 \mathrm{H}-1,147$ & 50.26 & 50.26 & 49.97 \\
\hline $6 \mathrm{H}-5,112$ & 55.91 & 55.91 & 55.62 \\
\hline $6 \mathrm{H}-6,0$ & 56.37 & 56.29 & 56.00 \\
\hline $6 \mathrm{H}-6,53$ & 56.87 & 56.82 & 56.53 \\
\hline $6 \mathrm{H}-7,50$ & 58.02 & 57.99 & 57.70 \\
\hline $6 \mathrm{H}-7,74$ & 58.26 & 58.24 & 57.94 \\
\hline $7 \mathrm{H}-1,67$ & 59.89 & 60.07 & 58.67 \\
\hline
\end{tabular}

\begin{tabular}{llll}
\hline & \multicolumn{3}{c}{ Depth (m) } \\
\cline { 2 - 4 } $\begin{array}{c}\text { Core, section, } \\
\text { interval (cm) }\end{array}$ & $\begin{array}{c}\text { Adjusted } \\
\text { CCSF }\end{array}$ & CCSF & CSF-A \\
\hline
\end{tabular}

\begin{tabular}{llll}
\hline 7H-1, 144 & 60.68 & 60.83 & 59.44 \\
7H-2,13 & 60.90 & 61.02 & 59.63 \\
\hline $7 H$
\end{tabular}

$\begin{array}{llll}7 \mathrm{H}-2,55 & 61.38 & 61.44 & 60.05\end{array}$

$\begin{array}{llll}7 \mathrm{H}-2,103 & 61.92 & 61.92 & 60.53\end{array}$

$\begin{array}{llll}7 \mathrm{H}-6,27 & 67.16 & 67.16 & 65.77\end{array}$

$\begin{array}{llll}7 \mathrm{H}-7,2 & 67.97 & 67.91 & 66.52\end{array}$

$\begin{array}{llll}7 \mathrm{H}-7,58 & 68.43 & 68.47 & 67.08\end{array}$

$\begin{array}{llll}7 \mathrm{H}-7,78 & 68.65 & 68.68 & 67.28\end{array}$

$8 \mathrm{H}-1,50 \quad 70.40 \quad 70.40 \quad 68.00$

$\begin{array}{llll}8 \mathrm{H}-1,139 & 71.22 & 71.29 & 68.89\end{array}$

$\begin{array}{llll}8 \mathrm{H}-2,33 & 71.75 & 71.73 & 69.33\end{array}$

$\begin{array}{llll}8 \mathrm{H}-2,82 & 72.22 & 72.22 & 69.82\end{array}$

$\begin{array}{llll}8 \mathrm{H}-5,107 & 76.97 & 76.97 & 74.57\end{array}$

$\begin{array}{llll}8 \mathrm{H}-6,12 & 77.54 & 77.53 & 75.12\end{array}$

$\begin{array}{llll}8 \mathrm{H}-6,105 & 78.42 & 78.45 & 76.05\end{array}$

$8 \mathrm{H}-7,44 \quad 78.90 \quad 78.91 \quad 76.51$

$\begin{array}{llll}8 \mathrm{H}-7,66 & 79.17 & 79.13 & 76.73\end{array}$

$\begin{array}{llll}9 \mathrm{H}-1,23 & 80.95 & 80.80 & 77.23\end{array}$

$\begin{array}{llll}9 \mathrm{H}-1,126 & 82.08 & 81.83 & 78.26\end{array}$

$\begin{array}{llll}9 \mathrm{H}-2,74 & 82.98 & 82.81 & 79.24\end{array}$

$\begin{array}{llll}9 \mathrm{H}-2,117 & 83.22 & 83.24 & 79.67\end{array}$

$\begin{array}{llll}9 \mathrm{H}-2,140 & 83.61 & 83.47 & 79.90\end{array}$

$9 \mathrm{H}-3,36$

$9 \mathrm{H}-3,76$

$9 \mathrm{H}-3,140$

$9 \mathrm{H}-5,117$

$9 \mathrm{H}-6,3$

$9 \mathrm{H}-6,53$

$9 \mathrm{H}-6,77$

$9 \mathrm{H}-6,124$

$9 \mathrm{H}-7,2$

$9 \mathrm{H}-7,26$

$9 \mathrm{H}-7,48$

$10 \mathrm{H}-1,31$

$10 \mathrm{H}-1,98$

$10 \mathrm{H}-4,54$

$10 \mathrm{H}-5,20$

$10 \mathrm{H}-5,72$

$10 \mathrm{H}-5,128$

$10 \mathrm{H}-6,26$

$10 \mathrm{H}-6,70$

$10 \mathrm{H}-6,111$

$10 \mathrm{H}-7,26$

$11 \mathrm{H}-1,49$

$11 \mathrm{H}-1,60$

$11 \mathrm{H}-1,92$

$11 \mathrm{H}-1,112$

$11 \mathrm{H}-5,86$

$11 \mathrm{H}-5,117$

$11 \mathrm{H}-6,85$

$11 \mathrm{H}-6,132$

$11 \mathrm{H}-7,5$

$11 \mathrm{H}-7,31$

$12 \mathrm{H}-1,28$

$12 \mathrm{H}-1,82$

$12 \mathrm{H}-2,0$

$12 \mathrm{H}-5,38$

$12 \mathrm{H}-5,92$

$12 \mathrm{H}-5,146$

$12 \mathrm{H}-6,29$

$12 \mathrm{H}-6,149$

$12 \mathrm{H}-7,59$

$13 \mathrm{H}-1,34$

$13 \mathrm{H}-1,75$

$13 \mathrm{H}-5,19$

$13 \mathrm{H}-5,55$

$13 \mathrm{H}-5,89$

$13 \mathrm{H}-5,144$
Depth (m)

\begin{tabular}{lccc} 
Core, section, & & Adjusted & \\
interval $(\mathrm{cm})$ & CCSF & CCSF & CSF-A \\
\hline $13 \mathrm{H}-6,19$ & 132.21 & 132.18 & 122.69
\end{tabular}

$\begin{array}{llll}13 \mathrm{H}-6,95 & 132.86 & 132.93 & 123.45\end{array}$

$\begin{array}{llll}13 \mathrm{H}-6,131 & 133.32 & 133.29 & 123.81\end{array}$

$\begin{array}{llll}13 \mathrm{H}-7,15 & 133.64 & 133.63 & 124.15\end{array}$

$\begin{array}{llll}13 \mathrm{H}-7,63 & 134.03 & 134.11 & 124.63\end{array}$

$\begin{array}{llll}14 \mathrm{H}-1,44 & 135.38 & 135.38 & 124.94\end{array}$

$\begin{array}{llll}14 \mathrm{H}-1,83 & 135.77 & 135.77 & 125.33\end{array}$

$\begin{array}{llll}14 \mathrm{H}-1,133 & 136.26 & 136.28 & 125.83\end{array}$

$\begin{array}{llll}14 \mathrm{H}-2,9 & 136.65 & 136.53 & 126.09\end{array}$

$\begin{array}{llll}14 \mathrm{H}-2,73 & 137.17 & 137.17 & 126.73\end{array}$

$\begin{array}{llll}14 \mathrm{H}-2,138 & 137.70 & 137.82 & 127.38\end{array}$

$\begin{array}{llll}14 \mathrm{H}-3,11 & 138.03 & 138.06 & 127.61\end{array}$

$\begin{array}{llll}14 \mathrm{H}-3,58 & 138.50 & 138.52 & 128.08\end{array}$

$\begin{array}{llll}14 \mathrm{H}-3,79 & 138.68 & 138.73 & 128.29\end{array}$

$\begin{array}{llll}14 \mathrm{H}-3,136 & 139.22 & 139.30 & 128.86\end{array}$

$\begin{array}{llll}14 \mathrm{H}-4,22 & 139.64 & 139.66 & 129.22\end{array}$

$\begin{array}{llll}14 \mathrm{H}-4,113 & 140.51 & 140.57 & 130.13\end{array}$

$\begin{array}{llll}14 \mathrm{H}-5,4 & 140.82 & 140.98 & 130.54\end{array}$

$\begin{array}{llll}14 \mathrm{H}-5,96 & 141.94 & 141.90 & 131.46\end{array}$

$\begin{array}{llll}14 \mathrm{H}-6,18 & 142.66 & 142.62 & 132.18\end{array}$

$\begin{array}{llll}14 \mathrm{H}-6,75 & 143.28 & 143.19 & 132.75\end{array}$

$\begin{array}{llll}14 \mathrm{H}-7,30 & 144.29 & 144.25 & 133.80\end{array}$

$\begin{array}{llll}15 \mathrm{H}-1,28 & 145.51 & 145.78 & 134.28\end{array}$

$\begin{array}{llll}15 \mathrm{H}-1,65 & 146.06 & 146.15 & 134.65\end{array}$

$15 \mathrm{H}-1,140 \quad 146.90 \quad 146.90 \quad 135.40$

$\begin{array}{llll}15 \mathrm{H}-5,99 & 152.49 & 152.49 & 140.99\end{array}$

$\begin{array}{llll}15 \mathrm{H}-5,144 & 153.09 & 152.94 & 141.44\end{array}$

$\begin{array}{llll}15 \mathrm{H}-6,44 & 153.64 & 153.45 & 141.94\end{array}$

$\begin{array}{llll}15 \mathrm{H}-6,72 & 154.08 & 153.72 & 142.22\end{array}$

$\begin{array}{llll}15 \mathrm{H}-6,133 & 154.62 & 154.33 & 142.83\end{array}$

$\begin{array}{llll}16 \mathrm{H}-1,38 & 156.68 & 156.70 & 143.88\end{array}$

$\begin{array}{llll}16 \mathrm{H}-1,115 & 157.47 & 157.47 & 144.65\end{array}$

$\begin{array}{llll}16 \mathrm{H}-5,111 & 163.43 & 163.43 & 150.61\end{array}$

$\begin{array}{llll}16 \mathrm{H}-6,65 & 164.50 & 164.47 & 151.65\end{array}$

$\begin{array}{llll}16 \mathrm{H}-6,132 & 165.28 & 165.14 & 152.32\end{array}$

$\begin{array}{llll}16 \mathrm{H}-7,28 & 165.75 & 165.60 & 152.78\end{array}$

$\begin{array}{llll}16 \mathrm{H}-7,69 & 166.16 & 166.02 & 153.19\end{array}$

$\begin{array}{llll}17 \mathrm{H}-1,31 & 166.22 & 166.46 & 153.31\end{array}$

$\begin{array}{llll}17 \mathrm{H}-1,77 & 166.79 & 166.92 & 153.77\end{array}$

$\begin{array}{llll}17 \mathrm{H}-1,135 & 167.48 & 167.50 & 154.35\end{array}$

$\begin{array}{llll}17 \mathrm{H}-2,50 & 168.03 & 168.15 & 155.00\end{array}$

$\begin{array}{llll}17 \mathrm{H}-2,62 & 168.28 & 168.27 & 155.12\end{array}$

$\begin{array}{llll}17 \mathrm{H}-6,10 & 173.75 & 173.75 & 160.60\end{array}$

$\begin{array}{llll}17 \mathrm{H}-6,65 & 174.37 & 174.30 & 161.15\end{array}$

$\begin{array}{llll}17 \mathrm{H}-6,102 & 174.65 & 174.67 & 161.52\end{array}$

$\begin{array}{llll}17 \mathrm{H}-6,131 & 174.96 & 174.96 & 161.81\end{array}$

$\begin{array}{llll}17 \mathrm{H}-7,25 & 175.48 & 175.40 & 162.25\end{array}$

$\begin{array}{llll}17 \mathrm{H}-7,76 & 175.97 & 175.91 & 162.76\end{array}$

$\begin{array}{llll}18 \mathrm{H}-1,17 & 176.83 & 176.75 & 162.67\end{array}$

$\begin{array}{llll}18 \mathrm{H}-1,89 & 177.54 & 177.47 & 163.39\end{array}$

$\begin{array}{llll}18 \mathrm{H}-1,127 & 177.87 & 177.85 & 163.77\end{array}$

$\begin{array}{llll}18 \mathrm{H}-2,18 & 178.32 & 178.26 & 164.18\end{array}$

$\begin{array}{llll}18 \mathrm{H}-2,81 & 178.91 & 178.89 & 164.81\end{array}$

$\begin{array}{llll}18 \mathrm{H}-2,118 & 179.27 & 179.26 & 165.18\end{array}$

$\begin{array}{llll}18 \mathrm{H}-3,7 & 179.64 & 179.65 & 165.57\end{array}$

$\begin{array}{llll}18 \mathrm{H}-3,50 & 180.12 & 180.09 & 166.00\end{array}$

$\begin{array}{llll}18 \mathrm{H}-3,119 & 180.75 & 180.77 & 166.69\end{array}$

$\begin{array}{llll}18 \mathrm{H}-4,30 & 181.38 & 181.38 & 167.30\end{array}$

$\begin{array}{llll}18 \mathrm{H}-6,61 & 184.69 & 184.69 & 170.61\end{array}$

$\begin{array}{llll}18 \mathrm{H}-6,123 & 185.33 & 185.31 & 171.23\end{array}$

$\begin{array}{llll}18 \mathrm{H}-7,54 & 186.25 & 186.12 & 172.04\end{array}$

$\begin{array}{llll}19 \mathrm{H}-1,8 & 187.57 & 187.70 & 172.08\end{array}$

$\begin{array}{llll}19 \mathrm{H}-1,45 & 187.95 & 188.07 & 172.45\end{array}$

$\begin{array}{llll}19 \mathrm{H}-1,71 & 188.09 & 188.33 & 172.71\end{array}$

$\begin{array}{llll}19 \mathrm{H}-1,112 & 188.76 & 188.74 & 173.12\end{array}$

$\begin{array}{llll}19 \mathrm{H}-2,6 & 189.17 & 189.18 & 173.56\end{array}$

$\begin{array}{llll}19 \mathrm{H}-2,59 & 189.71 & 189.71 & 174.09\end{array}$

$\begin{array}{llll}19 \mathrm{H}-5,83 & 194.51 & 194.51 & 178.89\end{array}$ 
Table AT28 (continued).

\begin{tabular}{lccc}
\hline & \multicolumn{3}{c}{ Depth (m) } \\
\cline { 2 - 4 } $\begin{array}{l}\text { Core, section, } \\
\text { interval (cm) }\end{array}$ & $\begin{array}{c}\text { Adjusted } \\
\text { CCSF }\end{array}$ & CCSF & CSF-A \\
\hline $19 \mathrm{H}-6,68$ & 195.97 & 195.88 & 180.26 \\
$19 \mathrm{H}-6,103$ & 196.22 & 196.23 & 180.61 \\
$19 \mathrm{H}-6,124$ & 196.46 & 196.44 & 180.82 \\
$19 \mathrm{H}-6,140$ & 196.69 & 196.60 & 180.98 \\
$19 \mathrm{H}-7,29$ & 197.12 & 197.02 & 181.39 \\
$19 \mathrm{H}-7,67$ & 197.48 & 197.39 & 181.77 \\
$2 \mathrm{H}-1,13$ & 198.60 & 198.74 & 181.63 \\
$20 \mathrm{H}-1,59$ & 199.09 & 199.20 & 182.09 \\
$20 \mathrm{H}-1,129$ & 199.90 & 199.90 & 182.79 \\
$20 \mathrm{H}-5,48$ & 205.10 & 205.10 & 187.98 \\
$20 \mathrm{H}-5,75$ & 205.35 & 205.37 & 188.25 \\
$20 \mathrm{H}-5,121$ & 205.87 & 205.82 & 188.71 \\
$20 \mathrm{H}-6,9$ & 206.31 & 206.20 & 189.09 \\
$20 \mathrm{H}-6,27$ & 206.43 & 206.38 & 189.27 \\
$20 \mathrm{H}-6,109$ & 207.29 & 207.21 & 190.09 \\
$20 \mathrm{H}-7,66$ & 207.99 & 207.87 & 190.76 \\
$21 \mathrm{H}-1,14$ & 209.31 & 209.27 & 191.14 \\
$21 \mathrm{H}-1,31$ & 209.54 & 209.44 & 191.31 \\
$21 \mathrm{H}-1,58$ & 209.72 & 209.71 & 191.58 \\
$21 \mathrm{H}-3,69$ & 212.82 & 212.82 & 194.69 \\
$21 \mathrm{H}-3,101$ & 213.13 & 213.14 & 195.01 \\
$21 \mathrm{H}-3,143$ & 213.62 & 213.56 & 195.43 \\
$21 \mathrm{H}-4,74$ & 214.49 & 214.37 & 196.24 \\
$21 \mathrm{H}-4,105$ & 214.81 & 214.68 & 196.55 \\
$21 \mathrm{H}-4,149$ & 215.19 & 215.12 & 196.99 \\
$21 \mathrm{H}-5,99$ & 216.14 & 216.12 & 197.99 \\
$22 \mathrm{H}-1,17$ & 220.65 & 220.59 & 200.67 \\
$22 \mathrm{H}-1,110$ & 221.46 & 221.51 & 201.60 \\
$22 \mathrm{H}-2,18$ & 222.04 & 222.09 & 202.18 \\
$22 \mathrm{H}-2,54$ & 222.43 & 222.45 & 202.54 \\
$22 \mathrm{H}-2,94$ & 222.86 & 222.85 & 202.94 \\
$22 \mathrm{H}-3,8$ & 223.54 & 223.49 & 203.58 \\
$22 \mathrm{H}-3,61$ & 224.03 & 224.02 & 204.11 \\
$22 \mathrm{H}-3,110$ & 224.55 & 224.51 & 204.60 \\
$22 \mathrm{H}-4,34$ & 225.24 & 225.26 & 205.34 \\
$22 \mathrm{H}-4,65$ & 225.62 & 225.56 & 205.65 \\
$22 \mathrm{H}-4,109$ & 225.98 & 226.00 & 206.09 \\
$22 \mathrm{H}-5,16$ & 226.40 & 226.57 & 206.66 \\
$22 \mathrm{H}-5,129$ & 228.25 & 227.70 & 207.79 \\
$22 \mathrm{H}-6,27$ & 228.78 & 228.18 & 208.27 \\
$22 \mathrm{H}-7,37$ & 229.55 & 229.28 & 209.37 \\
$22 \mathrm{H}-7,67$ & 229.89 & 229.58 & 209.67 \\
$23 \mathrm{H}-1,25$ & 231.86 & 232.94 & 210.25 \\
$23 \mathrm{H}-1,60$ & 232.25 & 233.28 & 210.60 \\
$23 \mathrm{H}-1,98$ & 232.74 & 233.66 & 210.98 \\
$23 \mathrm{H}-1,149$ & 233.26 & 234.17 & 211.49 \\
$23 \mathrm{H}-2,36$ & 233.73 & 234.54 & 211.86 \\
& & &
\end{tabular}

\begin{tabular}{lccc}
\hline & \multicolumn{3}{c}{ Depth (m) } \\
\cline { 2 - 4 } $\begin{array}{l}\text { Core, section, } \\
\text { interval (cm) }\end{array}$ & $\begin{array}{c}\text { Adjusted } \\
\text { CCSF }\end{array}$ & CCSF & CSF-A \\
\hline $23 \mathrm{H}-2,74$ & 234.43 & 234.92 & 212.24 \\
$23 \mathrm{H}-3,28$ & 235.92 & 235.96 & 213.28 \\
$23 \mathrm{H}-3,78$ & 236.56 & 236.46 & 213.78 \\
$23 \mathrm{H}-3,142$ & 237.79 & 237.10 & 214.42 \\
$23 \mathrm{H}-4,28$ & 238.39 & 237.46 & 214.78 \\
$23 \mathrm{H}-4,70$ & 239.20 & 237.88 & 215.20 \\
$23 \mathrm{H}-5,11$ & 240.05 & 238.29 & 215.61 \\
$24 \mathrm{H}-1,10$ & 240.96 & 241.01 & 219.60 \\
$24 \mathrm{H}-1,94$ & 241.47 & 241.85 & 220.44 \\
$24 \mathrm{H}-1,148$ & 242.05 & 242.39 & 220.98 \\
$24 \mathrm{H}-2,75$ & 242.89 & 243.16 & 221.75 \\
$24 \mathrm{H}-2,131$ & 243.35 & 243.72 & 222.31 \\
$24 \mathrm{H}-3,60$ & 244.30 & 244.51 & 223.10 \\
$24 \mathrm{H}-3,122$ & 245.01 & 245.13 & 223.72 \\
$24 \mathrm{H}-4,11$ & 245.42 & 245.53 & 224.11 \\
$24 \mathrm{H}-4,58$ & 245.89 & 245.99 & 224.58 \\
$24 \mathrm{H}-4,107$ & 246.41 & 246.48 & 225.07 \\
$24 \mathrm{H}-4,148$ & 246.79 & 246.89 & 225.48 \\
$24 \mathrm{H}-5,62$ & 247.42 & 247.53 & 226.12 \\
$25 \mathrm{H}-1,18$ & 248.04 & 248.13 & 226.58 \\
$25 \mathrm{H}-1,96$ & 248.64 & 248.91 & 227.36 \\
$25 \mathrm{H}-1,103$ & 249.00 & 248.98 & 227.43 \\
$25 \mathrm{H}-1,148$ & 249.35 & 249.43 & 227.88 \\
$25 \mathrm{H}-2,31$ & 249.76 & 249.76 & 228.21 \\
$25 \mathrm{H}-5,1$ & 253.96 & 253.96 & 232.41 \\
$25 \mathrm{H}-5,86$ & 254.80 & 254.81 & 233.26 \\
$25 \mathrm{H}-6,34$ & 255.29 & 255.29 & 233.74 \\
$26 \mathrm{H}-1,137$ & 256.23 & 257.21 & 235.67 \\
$26 \mathrm{H}-2,60$ & 257.21 & 257.94 & 236.40 \\
$26 \mathrm{H}-2,101$ & 257.69 & 258.36 & 236.81 \\
$26 \mathrm{H}-2,133$ & 258.23 & 258.67 & 237.13 \\
$26 \mathrm{H}-3,58$ & 258.88 & 259.42 & 237.88 \\
$26 \mathrm{H}-3,136$ & 259.94 & 260.20 & 238.66 \\
$26 \mathrm{H}-4,79$ & 261.17 & 261.13 & 239.59 \\
$26 \mathrm{H}-4,123$ & 261.71 & 261.57 & 240.03 \\
$26 \mathrm{H}-6,0$ & 263.59 & 263.34 & 241.80 \\
$26 \mathrm{H}-6,100$ & 264.42 & 264.34 & 242.80 \\
$27 \mathrm{H}-1,14$ & 265.76 & 265.86 & 243.94 \\
$27 \mathrm{H}-1,26$ & 265.93 & 265.98 & 244.06 \\
$27 \mathrm{H}-1,33$ & 266.02 & 266.05 & 244.13 \\
$27 \mathrm{H}-1,48$ & 266.17 & 266.20 & 244.28 \\
$27 \mathrm{H}-1,55$ & 266.26 & 266.27 & 244.35 \\
$27 \mathrm{H}-1,70$ & 266.43 & 266.42 & 244.50 \\
$27 \mathrm{H}-1,85$ & 266.53 & 266.57 & 244.65 \\
$27 \mathrm{H}-1,105$ & 266.78 & 266.77 & 244.85 \\
$27 \mathrm{H}-1,110$ & 266.82 & 266.82 & 244.90 \\
\hline & & & \\
\hline & & &
\end{tabular}

This table is also available in ASCII. 
Table AT29. Gamma ray attenuation (GRA) density data, Hole U1337C.

\begin{tabular}{|c|c|c|c|c|}
\hline \multirow[b]{2}{*}{$\begin{array}{l}\text { Core, section, } \\
\text { interval }(\mathrm{cm})\end{array}$} & \multicolumn{3}{|c|}{ Depth (m) } & \multirow[b]{2}{*}{$\begin{array}{c}\text { GRA } \\
\left(\mathrm{g} / \mathrm{cm}^{3}\right)\end{array}$} \\
\hline & CSF & CCSF & $\begin{array}{l}\text { Adjusted } \\
\text { CCSF }\end{array}$ & \\
\hline \multicolumn{5}{|l|}{ 321-U1337C- } \\
\hline $1 \mathrm{H}-1,2.5$ & 0.03 & 0.03 & 0.03 & 1.03 \\
\hline $1 \mathrm{H}-1,5$ & 0.05 & 0.05 & 0.05 & 1.04 \\
\hline $1 \mathrm{H}-1,7.5$ & 0.08 & 0.08 & 0.08 & 1.08 \\
\hline $1 \mathrm{H}-1,10$ & 0.10 & 0.10 & 0.10 & 1.22 \\
\hline $1 \mathrm{H}-1,12.5$ & 0.13 & 0.13 & 0.13 & 1.21 \\
\hline $1 \mathrm{H}-1,15$ & 0.15 & 0.15 & 0.15 & 1.31 \\
\hline $1 \mathrm{H}-1,17.5$ & 0.18 & 0.18 & 0.18 & 1.34 \\
\hline $1 \mathrm{H}-1,20$ & 0.20 & 0.20 & 0.20 & 1.36 \\
\hline $1 \mathrm{H}-1,22.5$ & 0.23 & 0.23 & 0.23 & 1.35 \\
\hline $1 \mathrm{H}-1,25.1$ & 0.25 & 0.25 & 0.25 & 1.37 \\
\hline $1 \mathrm{H}-1,27.6$ & 0.28 & 0.28 & 0.28 & 1.36 \\
\hline $1 \mathrm{H}-1,30.1$ & 0.30 & 0.30 & 0.30 & 1.38 \\
\hline $1 \mathrm{H}-1,32.6$ & 0.33 & 0.33 & 0.33 & 1.34 \\
\hline $1 \mathrm{H}-1,35.1$ & 0.35 & 0.35 & 0.35 & 1.36 \\
\hline $1 \mathrm{H}-1,37.6$ & 0.38 & 0.38 & 0.38 & 1.36 \\
\hline $1 \mathrm{H}-1,40.1$ & 0.40 & 0.40 & 0.40 & 1.36 \\
\hline $1 \mathrm{H}-1,42.6$ & 0.43 & 0.43 & 0.43 & 1.38 \\
\hline $1 \mathrm{H}-1,45.1$ & 0.45 & 0.45 & 0.45 & 1.41 \\
\hline $1 \mathrm{H}-1,47.6$ & 0.48 & 0.48 & 0.48 & 1.45 \\
\hline $1 \mathrm{H}-1,50.1$ & 0.50 & 0.50 & 0.50 & 1.43 \\
\hline $1 \mathrm{H}-1,52.6$ & 0.53 & 0.53 & 0.53 & 1.48 \\
\hline $1 \mathrm{H}-1,55.1$ & 0.55 & 0.55 & 0.55 & 1.46 \\
\hline $1 \mathrm{H}-1,57.6$ & 0.58 & 0.58 & 0.58 & 1.45 \\
\hline $1 \mathrm{H}-1,60.1$ & 0.60 & 0.60 & 0.60 & 1.45 \\
\hline $1 \mathrm{H}-1,62.6$ & 0.63 & 0.63 & 0.63 & 1.44 \\
\hline $1 \mathrm{H}-1,65.1$ & 0.65 & 0.65 & 0.65 & 1.44 \\
\hline $1 \mathrm{H}-1,67.6$ & 0.68 & 0.68 & 0.68 & 1.43 \\
\hline $1 \mathrm{H}-1,70.1$ & 0.70 & 0.70 & 0.70 & 1.42 \\
\hline $1 \mathrm{H}-1,72.6$ & 0.73 & 0.73 & 0.73 & 1.41 \\
\hline $1 \mathrm{H}-1,75.1$ & 0.75 & 0.75 & 0.75 & 1.44 \\
\hline $1 \mathrm{H}-1,77.6$ & 0.78 & 0.78 & 0.78 & 1.42 \\
\hline $1 \mathrm{H}-1,80.2$ & 0.80 & 0.80 & 0.80 & 1.40 \\
\hline $1 \mathrm{H}-1,82.7$ & 0.83 & 0.83 & 0.83 & 1.39 \\
\hline $1 \mathrm{H}-1,85.2$ & 0.85 & 0.85 & 0.85 & 1.39 \\
\hline $1 \mathrm{H}-1,87.7$ & 0.88 & 0.88 & 0.88 & 1.37 \\
\hline $1 \mathrm{H}-1,90.2$ & 0.90 & 0.90 & 0.90 & 1.34 \\
\hline $1 \mathrm{H}-1,92.7$ & 0.93 & 0.93 & 0.93 & 1.32 \\
\hline $1 \mathrm{H}-1,95.2$ & 0.95 & 0.95 & 0.95 & 1.26 \\
\hline $1 \mathrm{H}-1,97.7$ & 0.98 & 0.98 & 0.98 & 1.23 \\
\hline $1 \mathrm{H}-1,100.2$ & 1.00 & 1.00 & 1.00 & 1.26 \\
\hline $1 \mathrm{H}-2,3.5$ & 1.03 & 1.03 & 1.03 & 1.21 \\
\hline $1 \mathrm{H}-2,6$ & 1.06 & 1.06 & 1.06 & 1.20 \\
\hline $1 \mathrm{H}-2,8.5$ & 1.09 & 1.09 & 1.08 & 1.20 \\
\hline $1 \mathrm{H}-2,11$ & 1.11 & 1.11 & 1.10 & 1.17 \\
\hline $1 \mathrm{H}-2,13.5$ & 1.14 & 1.14 & 1.13 & 1.17 \\
\hline $1 \mathrm{H}-2,16$ & 1.16 & 1.16 & 1.15 & 1.18 \\
\hline $1 \mathrm{H}-2,18.5$ & 1.19 & 1.19 & 1.18 & 1.19 \\
\hline $1 \mathrm{H}-2,21$ & 1.21 & 1.21 & 1.20 & 1.19 \\
\hline $1 \mathrm{H}-2,23.5$ & 1.24 & 1.24 & 1.23 & 1.18 \\
\hline $1 \mathrm{H}-2,26$ & 1.26 & 1.26 & 1.25 & 1.20 \\
\hline $1 \mathrm{H}-2,28.5$ & 1.29 & 1.29 & 1.28 & 1.21 \\
\hline $1 \mathrm{H}-2,31$ & 1.31 & 1.31 & 1.30 & 1.22 \\
\hline $1 \mathrm{H}-2,33.5$ & 1.34 & 1.34 & 1.32 & 1.21 \\
\hline $1 \mathrm{H}-2,36$ & 1.36 & 1.36 & 1.34 & 1.21 \\
\hline $1 \mathrm{H}-2,38.6$ & 1.39 & 1.39 & 1.37 & 1.19 \\
\hline $1 \mathrm{H}-2,41.1$ & 1.41 & 1.41 & 1.39 & 1.21 \\
\hline $1 \mathrm{H}-2,43.6$ & 1.44 & 1.44 & 1.42 & 1.17 \\
\hline $1 \mathrm{H}-2,46.1$ & 1.46 & 1.46 & 1.44 & 1.18 \\
\hline $1 \mathrm{H}-2,48.6$ & 1.49 & 1.49 & 1.47 & 1.15 \\
\hline
\end{tabular}

Only a portion of this table appears here. The complete table is available in ASCII. 
Table AT30. Magnetic susceptibility data, Hole U1337C.

\begin{tabular}{|c|c|c|c|c|}
\hline \multirow[b]{2}{*}{$\begin{array}{l}\text { Core, section, } \\
\text { interval (cm) }\end{array}$} & \multicolumn{3}{|c|}{ Depth $(m)$} & \multirow{2}{*}{$\begin{array}{l}\text { Magnetic } \\
\text { susceptibility } \\
\text { (IU) }\end{array}$} \\
\hline & CSF & CCSF & $\begin{array}{l}\text { Adjusted } \\
\text { CCSF }\end{array}$ & \\
\hline \multicolumn{5}{|l|}{ 321-U1337C- } \\
\hline $1 \mathrm{H}-1,2.6$ & 0.03 & 0.03 & 0.03 & 2.2 \\
\hline $1 \mathrm{H}-1,5.1$ & 0.05 & 0.05 & 0.05 & 3.0 \\
\hline $1 \mathrm{H}-1,7.6$ & 0.08 & 0.08 & 0.08 & 3.0 \\
\hline $1 \mathrm{H}-1,10.1$ & 0.10 & 0.10 & 0.10 & 3.6 \\
\hline $1 \mathrm{H}-1,12.6$ & 0.13 & 0.13 & 0.13 & 4.1 \\
\hline $1 \mathrm{H}-1,15.1$ & 0.15 & 0.15 & 0.15 & 4.4 \\
\hline $1 \mathrm{H}-1,17.6$ & 0.18 & 0.18 & 0.18 & 5.2 \\
\hline $1 \mathrm{H}-1,20.1$ & 0.20 & 0.20 & 0.20 & 4.4 \\
\hline $1 \mathrm{H}-1,22.6$ & 0.23 & 0.23 & 0.23 & 4.4 \\
\hline $1 \mathrm{H}-1,25.1$ & 0.25 & 0.25 & 0.25 & 3.3 \\
\hline $1 \mathrm{H}-1,27.6$ & 0.28 & 0.28 & 0.28 & 2.2 \\
\hline $1 \mathrm{H}-1,30.1$ & 0.30 & 0.30 & 0.30 & 2.2 \\
\hline $1 \mathrm{H}-1,32.6$ & 0.33 & 0.33 & 0.33 & 1.6 \\
\hline $1 \mathrm{H}-1,35.1$ & 0.35 & 0.35 & 0.35 & 1.6 \\
\hline $1 \mathrm{H}-1,37.6$ & 0.38 & 0.38 & 0.38 & 1.4 \\
\hline $1 \mathrm{H}-1,40.1$ & 0.40 & 0.40 & 0.40 & 1.4 \\
\hline $1 \mathrm{H}-1,42.6$ & 0.43 & 0.43 & 0.43 & 0.8 \\
\hline $1 \mathrm{H}-1,45.1$ & 0.45 & 0.45 & 0.45 & 0.5 \\
\hline $1 \mathrm{H}-1,47.7$ & 0.48 & 0.48 & 0.48 & 0.3 \\
\hline $1 \mathrm{H}-1,50.2$ & 0.50 & 0.50 & 0.50 & 0.0 \\
\hline $1 \mathrm{H}-1,52.7$ & 0.53 & 0.53 & 0.53 & 0.3 \\
\hline $1 \mathrm{H}-1,55.2$ & 0.55 & 0.55 & 0.55 & 0.8 \\
\hline $1 \mathrm{H}-1,57.7$ & 0.58 & 0.58 & 0.58 & 1.4 \\
\hline $1 \mathrm{H}-1,60.2$ & 0.60 & 0.60 & 0.60 & 1.1 \\
\hline $1 \mathrm{H}-1,62.7$ & 0.63 & 0.63 & 0.63 & 1.1 \\
\hline $1 \mathrm{H}-1,65.2$ & 0.65 & 0.65 & 0.65 & 1.1 \\
\hline $1 \mathrm{H}-1,67.7$ & 0.68 & 0.68 & 0.68 & 2.2 \\
\hline $1 \mathrm{H}-1,70.2$ & 0.70 & 0.70 & 0.70 & 1.4 \\
\hline $1 \mathrm{H}-1,72.7$ & 0.73 & 0.73 & 0.73 & 1.9 \\
\hline $1 \mathrm{H}-1,75.2$ & 0.75 & 0.75 & 0.75 & 1.9 \\
\hline $1 \mathrm{H}-1,77.7$ & 0.78 & 0.78 & 0.78 & 1.9 \\
\hline $1 \mathrm{H}-1,80.2$ & 0.80 & 0.80 & 0.80 & 1.9 \\
\hline $1 \mathrm{H}-1,82.7$ & 0.83 & 0.83 & 0.83 & 2.5 \\
\hline $1 \mathrm{H}-1,85.2$ & 0.85 & 0.85 & 0.85 & 3.0 \\
\hline $1 \mathrm{H}-1,87.7$ & 0.88 & 0.88 & 0.88 & 3.6 \\
\hline $1 \mathrm{H}-1,90.2$ & 0.90 & 0.90 & 0.90 & 4.7 \\
\hline $1 \mathrm{H}-1,92.7$ & 0.93 & 0.93 & 0.93 & 5.2 \\
\hline $1 \mathrm{H}-1,95.2$ & 0.95 & 0.95 & 0.95 & 7.4 \\
\hline $1 \mathrm{H}-1,97.7$ & 0.98 & 0.98 & 0.98 & 6.9 \\
\hline $1 \mathrm{H}-1,100.2$ & 1.00 & 1.00 & 1.00 & 6.3 \\
\hline $1 \mathrm{H}-2,3.5$ & 1.04 & 1.04 & 1.04 & 6.6 \\
\hline $1 \mathrm{H}-2,6.1$ & 1.06 & 1.06 & 1.06 & 7.1 \\
\hline $1 \mathrm{H}-2,8.6$ & 1.09 & 1.09 & 1.08 & 7.1 \\
\hline $1 \mathrm{H}-2,11.1$ & 1.11 & 1.11 & 1.10 & 7.7 \\
\hline $1 \mathrm{H}-2,13.6$ & 1.14 & 1.14 & 1.13 & 7.1 \\
\hline $1 \mathrm{H}-2,16.1$ & 1.16 & 1.16 & 1.15 & 6.6 \\
\hline $1 \mathrm{H}-2,18.6$ & 1.19 & 1.19 & 1.18 & 6.6 \\
\hline $1 \mathrm{H}-2,21.1$ & 1.21 & 1.21 & 1.20 & 6.9 \\
\hline $1 \mathrm{H}-2,23.6$ & 1.24 & 1.24 & 1.23 & 6.9 \\
\hline $1 \mathrm{H}-2,26.1$ & 1.26 & 1.26 & 1.25 & 7.1 \\
\hline $1 \mathrm{H}-2,28.6$ & 1.29 & 1.29 & 1.28 & 6.9 \\
\hline $1 \mathrm{H}-2,31.1$ & 1.31 & 1.31 & 1.30 & 6.9 \\
\hline $1 \mathrm{H}-2,33.6$ & 1.34 & 1.34 & 1.32 & 7.7 \\
\hline $1 \mathrm{H}-2,36.1$ & 1.36 & 1.36 & 1.34 & 8.2 \\
\hline $1 \mathrm{H}-2,38.6$ & 1.39 & 1.39 & 1.37 & 8.5 \\
\hline $1 \mathrm{H}-2,41.1$ & 1.41 & 1.41 & 1.39 & 8.8 \\
\hline $1 \mathrm{H}-2,43.6$ & 1.44 & 1.44 & 1.42 & 8.8 \\
\hline $1 \mathrm{H}-2,46.1$ & 1.46 & 1.46 & 1.44 & 9.6 \\
\hline $1 \mathrm{H}-2,48.6$ & 1.49 & 1.49 & 1.47 & 9.3 \\
\hline $1 \mathrm{H}-2,51.1$ & 1.51 & 1.51 & 1.49 & 9.3 \\
\hline $1 \mathrm{H}-2,53.6$ & 1.54 & 1.54 & 1.52 & 9.1 \\
\hline
\end{tabular}

Only a portion of this table appears here. The complete table is available in ASCII. 
Table AT31. Natural gamma ray (NGR) data, Hole U1337C.

\begin{tabular}{|c|c|c|c|c|}
\hline \multirow[b]{2}{*}{$\begin{array}{l}\text { Core, section, } \\
\text { interval }(\mathrm{cm})\end{array}$} & \multicolumn{3}{|c|}{ Depth (m) } & \multirow[b]{2}{*}{$\begin{array}{l}\text { NGR } \\
\text { (cps) }\end{array}$} \\
\hline & CSF & CCSF & $\begin{array}{l}\text { Adjusted } \\
\text { CCSF }\end{array}$ & \\
\hline \multicolumn{5}{|l|}{ 321-U1337C- } \\
\hline $1 \mathrm{H}-1,0$ & 0.0 & 0.00 & 0.00 & 53.8 \\
\hline $1 \mathrm{H}-1,10$ & 0.1 & 0.10 & 0.10 & 64.3 \\
\hline $1 \mathrm{H}-1,20$ & 0.2 & 0.20 & 0.20 & 73.7 \\
\hline $1 \mathrm{H}-1,30$ & 0.3 & 0.30 & 0.30 & 60.9 \\
\hline $1 \mathrm{H}-1,40$ & 0.4 & 0.40 & 0.40 & 46.4 \\
\hline $1 \mathrm{H}-1,50$ & 0.5 & 0.50 & 0.50 & 43.5 \\
\hline $1 \mathrm{H}-1,60$ & 0.6 & 0.60 & 0.60 & 49.2 \\
\hline $1 \mathrm{H}-1,70$ & 0.7 & 0.70 & 0.70 & 45.5 \\
\hline $1 \mathrm{H}-1,80$ & 0.8 & 0.80 & 0.80 & 43.8 \\
\hline $1 \mathrm{H}-1,90$ & 0.9 & 0.90 & 0.90 & 62.2 \\
\hline $1 \mathrm{H}-1,100$ & 1.0 & 1.00 & 1.00 & 68.5 \\
\hline $1 \mathrm{H}-2,0$ & 1.0 & 1.00 & 1.00 & 40.0 \\
\hline $1 \mathrm{H}-2,10$ & 1.1 & 1.10 & 1.09 & 38.0 \\
\hline $1 \mathrm{H}-2,20$ & 1.2 & 1.20 & 1.19 & 49.4 \\
\hline $1 \mathrm{H}-2,30$ & 1.3 & 1.30 & 1.29 & 63.7 \\
\hline $1 \mathrm{H}-2,40$ & 1.4 & 1.40 & 1.38 & 64.3 \\
\hline $1 \mathrm{H}-2,50$ & 1.5 & 1.50 & 1.48 & 58.9 \\
\hline $1 \mathrm{H}-2,60$ & 1.6 & 1.60 & 1.57 & 51.8 \\
\hline $1 \mathrm{H}-2,70$ & 1.7 & 1.70 & 1.67 & 46.3 \\
\hline $2 \mathrm{H}-1,0$ & 1.9 & 3.77 & 3.77 & 13.4 \\
\hline $2 \mathrm{H}-1,10$ & 2.0 & 3.87 & 3.87 & 11.5 \\
\hline $2 \mathrm{H}-1,20$ & 2.1 & 3.97 & 3.97 & 8.9 \\
\hline $2 \mathrm{H}-1,30$ & 2.2 & 4.07 & 4.07 & 8.1 \\
\hline $2 \mathrm{H}-1,40$ & 2.3 & 4.17 & 4.17 & 8.3 \\
\hline $2 \mathrm{H}-1,50$ & 2.4 & 4.27 & 4.28 & 9.3 \\
\hline $2 \mathrm{H}-1,60$ & 2.5 & 4.37 & 4.38 & 9.2 \\
\hline $2 \mathrm{H}-1,70$ & 2.6 & 4.47 & 4.48 & 10.1 \\
\hline $2 \mathrm{H}-1,80$ & 2.7 & 4.57 & 4.58 & 8.9 \\
\hline $2 \mathrm{H}-1,90$ & 2.8 & 4.67 & 4.68 & 9.4 \\
\hline $2 \mathrm{H}-1,100$ & 2.9 & 4.77 & 4.78 & 9.7 \\
\hline $2 \mathrm{H}-1,110$ & 3.0 & 4.87 & 4.89 & 8.8 \\
\hline $2 \mathrm{H}-1,120$ & 3.1 & 4.97 & 5.00 & 7.9 \\
\hline $2 \mathrm{H}-1,130$ & 3.2 & 5.07 & 5.11 & 7.5 \\
\hline $2 \mathrm{H}-1,140$ & 3.3 & 5.17 & 5.23 & 7.2 \\
\hline $2 \mathrm{H}-1,150$ & 3.4 & 5.27 & 5.33 & 7.0 \\
\hline $2 \mathrm{H}-2,0$ & 3.4 & 5.27 & 5.33 & 6.1 \\
\hline $2 \mathrm{H}-2,10$ & 3.5 & 5.37 & 5.43 & 5.9 \\
\hline $2 \mathrm{H}-2,20$ & 3.6 & 5.47 & 5.53 & 6.8 \\
\hline $2 \mathrm{H}-2,30$ & 3.7 & 5.57 & 5.63 & 7.8 \\
\hline $2 \mathrm{H}-2,40$ & 3.8 & 5.67 & 5.73 & 7.5 \\
\hline $2 \mathrm{H}-2,50$ & 3.9 & 5.77 & 5.83 & 7.0 \\
\hline $2 \mathrm{H}-2,60$ & 4.0 & 5.87 & 5.93 & 6.6 \\
\hline $2 \mathrm{H}-2,70$ & 4.1 & 5.97 & 6.03 & 5.1 \\
\hline $2 \mathrm{H}-2,80$ & 4.2 & 6.07 & 6.12 & 4.2 \\
\hline $2 \mathrm{H}-2,90$ & 4.3 & 6.17 & 6.21 & 4.2 \\
\hline $2 \mathrm{H}-2,100$ & 4.4 & 6.27 & 6.31 & 4.8 \\
\hline $2 \mathrm{H}-2,110$ & 4.5 & 6.37 & 6.40 & 5.0 \\
\hline $2 \mathrm{H}-2,120$ & 4.6 & 6.47 & 6.49 & 5.1 \\
\hline $2 \mathrm{H}-2,130$ & 4.7 & 6.57 & 6.59 & 4.8 \\
\hline $2 \mathrm{H}-2,140$ & 4.8 & 6.67 & 6.68 & 4.0 \\
\hline $2 \mathrm{H}-2,150$ & 4.9 & 6.77 & 6.77 & 4.3 \\
\hline $2 \mathrm{H}-3,0$ & 4.9 & 6.77 & 6.77 & 4.4 \\
\hline $2 \mathrm{H}-3,10$ & 5.0 & 6.87 & 6.87 & 4.2 \\
\hline $2 \mathrm{H}-3,20$ & 5.1 & 6.97 & 6.96 & 4.0 \\
\hline $2 \mathrm{H}-3,30$ & 5.2 & 7.07 & 7.05 & 4.0 \\
\hline $2 \mathrm{H}-3,40$ & 5.3 & 7.17 & 7.15 & 3.3 \\
\hline $2 \mathrm{H}-3,50$ & 5.4 & 7.27 & 7.24 & 3.4 \\
\hline $2 \mathrm{H}-3,60$ & 5.5 & 7.37 & 7.34 & 3.9 \\
\hline $2 \mathrm{H}-3,70$ & 5.6 & 7.47 & 7.43 & 3.9 \\
\hline
\end{tabular}

Only a portion of this table appears here. The complete table is available in ASCII. 
Table AT32. Color reflection data, Hole U1337C.

\begin{tabular}{|c|c|c|c|c|c|c|}
\hline \multirow[b]{2}{*}{$\begin{array}{l}\text { Core, section, } \\
\text { interval }(\mathrm{cm})\end{array}$} & \multicolumn{3}{|c|}{ Depth (m) } & \multirow[b]{2}{*}{$a^{*}$} & \multirow[b]{2}{*}{$b^{*}$} & \multirow[b]{2}{*}{$L^{*}$} \\
\hline & CSF & CCSF & $\begin{array}{l}\text { Adjusted } \\
\text { CCSF }\end{array}$ & & & \\
\hline \multicolumn{7}{|l|}{ 321-U1337C- } \\
\hline $1 \mathrm{H}-1,6$ & 0.06 & 0.06 & 0.06 & 6.6 & 8.2 & 43.3 \\
\hline $1 \mathrm{H}-1,8.5$ & 0.09 & 0.09 & 0.09 & 6.2 & 9.0 & 45.9 \\
\hline $1 \mathrm{H}-1,11.1$ & 0.11 & 0.11 & 0.11 & 6.1 & 9.9 & 46.8 \\
\hline $1 \mathrm{H}-1,13.6$ & 0.14 & 0.14 & 0.14 & 5.8 & 10.1 & 47.7 \\
\hline $1 \mathrm{H}-1,16.1$ & 0.16 & 0.16 & 0.16 & 5.9 & 7.4 & 44.8 \\
\hline $1 \mathrm{H}-1,18.7$ & 0.19 & 0.19 & 0.19 & 4.8 & 7.5 & 52.2 \\
\hline $1 \mathrm{H}-1,21.2$ & 0.21 & 0.21 & 0.21 & 6.9 & 12.9 & 51.5 \\
\hline $1 \mathrm{H}-1,23.8$ & 0.24 & 0.24 & 0.24 & 6.8 & 13.7 & 61.5 \\
\hline $1 \mathrm{H}-1,26.3$ & 0.26 & 0.26 & 0.26 & 5.4 & 10.3 & 65.9 \\
\hline $1 \mathrm{H}-1,28.8$ & 0.29 & 0.29 & 0.29 & 5.5 & 9.7 & 60.8 \\
\hline $1 \mathrm{H}-1,31.4$ & 0.31 & 0.31 & 0.31 & 5.8 & 12.0 & 65.0 \\
\hline $1 \mathrm{H}-1,33.9$ & 0.34 & 0.34 & 0.34 & 6.0 & 14.8 & 62.7 \\
\hline $1 \mathrm{H}-1,36.5$ & 0.37 & 0.37 & 0.37 & 6.8 & 18.2 & 63.9 \\
\hline $1 \mathrm{H}-1,39$ & 0.39 & 0.39 & 0.39 & 5.5 & 12.0 & 61.5 \\
\hline $1 \mathrm{H}-1,41.6$ & 0.42 & 0.42 & 0.42 & 5.3 & 16.4 & 68.3 \\
\hline $1 \mathrm{H}-1,44.1$ & 0.44 & 0.44 & 0.44 & 5.5 & 17.1 & 71.9 \\
\hline $1 \mathrm{H}-1,46.6$ & 0.47 & 0.47 & 0.47 & 3.2 & 15.1 & 72.0 \\
\hline $1 \mathrm{H}-1,49.2$ & 0.49 & 0.49 & 0.49 & 5.4 & 16.7 & 70.2 \\
\hline $1 \mathrm{H}-1,51.7$ & 0.52 & 0.52 & 0.52 & 5.0 & 14.0 & 66.0 \\
\hline $1 \mathrm{H}-1,54.3$ & 0.54 & 0.54 & 0.54 & 3.5 & 9.5 & 61.5 \\
\hline $1 \mathrm{H}-1,56.8$ & 0.57 & 0.57 & 0.57 & 4.0 & 10.4 & 62.9 \\
\hline $1 \mathrm{H}-1,59.4$ & 0.59 & 0.59 & 0.59 & 3.8 & 10.3 & 64.4 \\
\hline $1 \mathrm{H}-1,61.9$ & 0.62 & 0.62 & 0.62 & 4.4 & 14.7 & 66.6 \\
\hline $1 \mathrm{H}-1,64.4$ & 0.64 & 0.64 & 0.64 & 3.3 & 11.3 & 55.6 \\
\hline $1 \mathrm{H}-1,67$ & 0.67 & 0.67 & 0.67 & 4.0 & 11.1 & 65.0 \\
\hline $1 \mathrm{H}-1,69.5$ & 0.70 & 0.70 & 0.70 & 4.2 & 13.0 & 60.0 \\
\hline $1 \mathrm{H}-1,72.1$ & 0.72 & 0.72 & 0.72 & 4.2 & 14.2 & 64.5 \\
\hline $1 \mathrm{H}-1,74.6$ & 0.75 & 0.75 & 0.75 & 4.6 & 14.6 & 66.7 \\
\hline $1 \mathrm{H}-1,77.2$ & 0.77 & 0.77 & 0.77 & 3.9 & 10.1 & 65.8 \\
\hline $1 \mathrm{H}-1,79.7$ & 0.80 & 0.80 & 0.80 & 3.6 & 14.3 & 80.5 \\
\hline $1 \mathrm{H}-1,82.2$ & 0.82 & 0.82 & 0.82 & 4.5 & 16.5 & 75.4 \\
\hline $1 \mathrm{H}-1,84.8$ & 0.85 & 0.85 & 0.85 & 3.2 & 9.1 & 60.2 \\
\hline $1 \mathrm{H}-1,87.3$ & 0.87 & 0.87 & 0.87 & 3.4 & 9.4 & 49.1 \\
\hline $1 \mathrm{H}-1,89.9$ & 0.90 & 0.90 & 0.90 & 3.7 & 8.6 & 53.2 \\
\hline $1 \mathrm{H}-1,92.4$ & 0.92 & 0.92 & 0.92 & 2.9 & 7.6 & 52.5 \\
\hline $1 \mathrm{H}-1,95$ & 0.95 & 0.95 & 0.95 & 5.4 & 7.7 & 40.2 \\
\hline $1 \mathrm{H}-1,97.5$ & 0.98 & 0.98 & 0.98 & 4.6 & 12.2 & 49.8 \\
\hline $1 \mathrm{H}-2,3.8$ & 1.04 & 1.04 & 1.04 & 5.0 & 10.8 & 57.1 \\
\hline $1 \mathrm{H}-2,6.4$ & 1.06 & 1.06 & 1.06 & 6.9 & 13.2 & 50.7 \\
\hline $1 \mathrm{H}-2,8.9$ & 1.09 & 1.09 & 1.08 & 6.9 & 12.1 & 49.9 \\
\hline $1 \mathrm{H}-2,11.5$ & 1.12 & 1.12 & 1.11 & 6.9 & 12.0 & 50.9 \\
\hline $1 \mathrm{H}-2,14$ & 1.14 & 1.14 & 1.13 & 5.1 & 6.9 & 55.0 \\
\hline $1 \mathrm{H}-2,16.6$ & 1.17 & 1.17 & 1.16 & 5.5 & 10.4 & 55.5 \\
\hline $1 \mathrm{H}-2,19.1$ & 1.19 & 1.19 & 1.18 & 6.4 & 9.5 & 50.2 \\
\hline $1 \mathrm{H}-2,21.6$ & 1.22 & 1.22 & 1.21 & 6.4 & 9.3 & 50.5 \\
\hline $1 \mathrm{H}-2,24.2$ & 1.24 & 1.24 & 1.23 & 5.1 & 6.0 & 48.6 \\
\hline $1 \mathrm{H}-2,26.7$ & 1.27 & 1.27 & 1.26 & 4.4 & 5.5 & 48.3 \\
\hline $1 \mathrm{H}-2,29.3$ & 1.29 & 1.29 & 1.28 & 6.0 & 8.6 & 50.7 \\
\hline $1 \mathrm{H}-2,31.8$ & 1.32 & 1.32 & 1.30 & 4.1 & 5.1 & 49.6 \\
\hline $1 \mathrm{H}-2,34.4$ & 1.34 & 1.34 & 1.32 & 5.6 & 7.7 & 49.4 \\
\hline $1 \mathrm{H}-2,36.9$ & 1.37 & 1.37 & 1.35 & 3.4 & 2.3 & 44.1 \\
\hline $1 \mathrm{H}-2,39.4$ & 1.39 & 1.39 & 1.37 & 3.9 & 4.5 & 46.8 \\
\hline $1 \mathrm{H}-2,42$ & 1.42 & 1.42 & 1.40 & 3.2 & 0.5 & 42.3 \\
\hline $1 \mathrm{H}-2,44.5$ & 1.45 & 1.45 & 1.43 & 2.9 & 0.6 & 44.3 \\
\hline $1 \mathrm{H}-2,47.1$ & 1.47 & 1.47 & 1.45 & 3.2 & 1.0 & 42.0 \\
\hline $1 \mathrm{H}-2,49.6$ & 1.50 & 1.50 & 1.48 & 3.4 & 2.6 & 43.1 \\
\hline $1 \mathrm{H}-2,52.2$ & 1.52 & 1.52 & 1.50 & 4.5 & 3.3 & 36.2 \\
\hline $1 \mathrm{H}-2,54.7$ & 1.55 & 1.55 & 1.53 & 6.3 & 4.7 & 36.3 \\
\hline $1 \mathrm{H}-2,57.2$ & 1.57 & 1.57 & 1.54 & 2.5 & 1.1 & 45.1 \\
\hline $1 \mathrm{H}-2,59.8$ & 1.60 & 1.60 & 1.57 & 4.7 & 3.5 & 37.6 \\
\hline
\end{tabular}

Only a portion of this table appears here. The complete table is available in ASCII. 
Table AT33. Core adjusted data, Hole U1337C.

\begin{tabular}{|c|c|c|c|}
\hline \multirow[b]{2}{*}{$\begin{array}{l}\text { Core, section, } \\
\text { interval }(\mathrm{cm})\end{array}$} & \multicolumn{3}{|c|}{ Depth (m) } \\
\hline & $\begin{array}{l}\text { Adjusted } \\
\text { CCSF }\end{array}$ & CCSF & CSF-A \\
\hline \multicolumn{4}{|l|}{ 321-U1337C- } \\
\hline $1 \mathrm{H}-1,0$ & 0.00 & 0.00 & 0.00 \\
\hline $1 \mathrm{H}-1,96$ & 0.96 & 0.96 & 0.96 \\
\hline $2 \mathrm{H}-1,-196$ & 1.77 & 1.81 & -0.06 \\
\hline $2 \mathrm{H}-1,0$ & 3.77 & 3.77 & 1.90 \\
\hline $2 \mathrm{H}-1,114$ & 4.93 & 4.91 & 3.04 \\
\hline $2 \mathrm{H}-1,140$ & 5.23 & 5.17 & 3.30 \\
\hline $2 \mathrm{H}-2,66$ & 5.99 & 5.93 & 4.06 \\
\hline $2 \mathrm{H}-3,4$ & 6.81 & 6.81 & 4.94 \\
\hline $2 \mathrm{H}-3,103$ & 7.75 & 7.80 & 5.93 \\
\hline $2 \mathrm{H}-3,142$ & 8.19 & 8.19 & 6.32 \\
\hline $2 \mathrm{H}-6,68$ & 11.95 & 11.95 & 10.08 \\
\hline $2 \mathrm{H}-6,102$ & 12.34 & 12.29 & 10.42 \\
\hline $2 \mathrm{H}-6,135$ & 12.66 & 12.62 & 10.75 \\
\hline $2 \mathrm{H}-7,60$ & 13.27 & 13.27 & 11.40 \\
\hline $4 \mathrm{H}-1,32$ & 192.81 & 192.76 & 169.72 \\
\hline $4 \mathrm{H}-1,32$ & 192.81 & 192.76 & 169.72 \\
\hline $4 \mathrm{H}-1,99$ & 193.47 & 193.44 & 170.39 \\
\hline $4 \mathrm{H}-4,10$ & 197.09 & 197.05 & 174.00 \\
\hline $4 \mathrm{H}-4,123$ & 198.31 & 198.18 & 175.13 \\
\hline $4 \mathrm{H}-5,36$ & 198.93 & 198.81 & 175.76 \\
\hline $4 \mathrm{H}-5,99$ & 199.65 & 199.44 & 176.39 \\
\hline $4 \mathrm{H}-5,134$ & 200.08 & 199.78 & 176.74 \\
\hline $4 \mathrm{H}-6,53$ & 200.84 & 200.48 & 177.43 \\
\hline $4 \mathrm{H}-6,82$ & 201.14 & 200.77 & 177.72 \\
\hline $4 \mathrm{H}-7,0$ & 201.37 & 200.95 & 177.90 \\
\hline $4 \mathrm{H}-7,70$ & 202.02 & 201.64 & 178.60 \\
\hline $5 \mathrm{H}-1,0$ & 202.66 & 202.55 & 178.90 \\
\hline $5 \mathrm{H}-1,25$ & 202.92 & 202.80 & 179.15 \\
\hline $5 \mathrm{H}-1,105$ & 203.67 & 203.60 & 179.95 \\
\hline $5 \mathrm{H}-2,121$ & 205.26 & 205.27 & 181.61 \\
\hline $5 \mathrm{H}-4,17$ & 207.25 & 207.22 & 183.57 \\
\hline $5 \mathrm{H}-4,43$ & 207.54 & 207.48 & 183.83 \\
\hline $5 \mathrm{H}-5,62$ & 209.19 & 209.17 & 185.52 \\
\hline $5 \mathrm{H}-8,66$ & 211.88 & 211.94 & 188.29 \\
\hline $5 \mathrm{H}-8,104$ & 212.26 & 212.33 & 188.67 \\
\hline $6 \mathrm{H}-1,0$ & 212.40 & 212.40 & 188.40 \\
\hline $6 \mathrm{H}-1,43$ & 212.83 & 212.83 & 188.83 \\
\hline $6 \mathrm{H}-3,122$ & 216.59 & 216.62 & 192.62 \\
\hline $7 \mathrm{H}-1,17$ & 217.95 & 217.88 & 192.97 \\
\hline $7 \mathrm{H}-1,49$ & 218.18 & 218.20 & 193.29 \\
\hline 7H-6, 116 & 226.38 & 226.38 & 201.46 \\
\hline $7 \mathrm{H}-7,68$ & 227.35 & 227.15 & 202.23 \\
\hline $8 \mathrm{H}-1,0$ & 229.23 & 229.23 & 202.30 \\
\hline $8 \mathrm{H}-2,6$ & 230.79 & 230.79 & 203.86 \\
\hline $8 \mathrm{H}-7,54$ & 238.77 & 238.77 & 211.84 \\
\hline $8 \mathrm{H}-7,67$ & 238.90 & 238.90 & 211.97 \\
\hline $9 \mathrm{H}-5,107$ & 245.84 & 245.84 & 218.87 \\
\hline $9 \mathrm{H}-6,7$ & 246.36 & 246.33 & 219.37 \\
\hline $9 \mathrm{H}-6,70$ & 246.95 & 246.97 & 220.00 \\
\hline $9 \mathrm{H}-7,15$ & 247.98 & 247.91 & 220.95 \\
\hline $9 \mathrm{H}-7,71$ & 248.42 & 248.48 & 221.51 \\
\hline $10 X-1,1$ & 249.27 & 249.27 & 221.31 \\
\hline $10 X-2,6$ & 250.56 & 250.46 & 222.49 \\
\hline $10 \times-2,33$ & 250.85 & 250.73 & 222.76 \\
\hline $10 X-8,15$ & 257.75 & 257.72 & 229.75 \\
\hline $10 X-8,76$ & 258.40 & 258.33 & 230.36 \\
\hline $11 \mathrm{X}-1,1$ & 259.07 & 259.07 & 230.91 \\
\hline $11 X-1,105$ & 260.29 & 260.11 & 231.95 \\
\hline $11 X-2,50$ & 260.94 & 261.06 & 232.90 \\
\hline $11 X-2,67$ & 261.23 & 261.23 & 233.07 \\
\hline
\end{tabular}

\begin{tabular}{|c|c|c|c|}
\hline \multirow[b]{2}{*}{$\begin{array}{l}\text { Core, section, } \\
\text { interval }(\mathrm{cm})\end{array}$} & \multicolumn{3}{|c|}{ Depth $(\mathrm{m})$} \\
\hline & $\begin{array}{l}\text { Adjusted } \\
\text { CCSF }\end{array}$ & CCSF & CSF-A \\
\hline $11 X-6,68$ & 266.84 & 266.84 & 238.68 \\
\hline $12 X-1,1$ & 272.18 & 272.17 & 240.51 \\
\hline $12 X-1,50$ & 272.67 & 272.66 & 241.00 \\
\hline $12 X-1,109$ & 273.24 & 273.24 & 241.59 \\
\hline $12 X-5,135$ & 279.51 & 279.51 & 247.85 \\
\hline $12 X-6,21$ & 279.88 & 279.87 & 248.21 \\
\hline $12 X-6,97$ & 281.14 & 280.63 & 248.97 \\
\hline $12 X-6,108$ & 281.42 & 280.74 & 249.08 \\
\hline $12 X-6,140$ & 281.69 & 281.06 & 249.40 \\
\hline $13 X-1,0$ & 282.89 & 282.72 & 250.10 \\
\hline $13 X-1,43$ & 283.26 & 283.14 & 250.53 \\
\hline $13 X-1,63$ & 283.38 & 283.34 & 250.73 \\
\hline $13 X-6,27$ & 290.46 & 290.48 & 257.87 \\
\hline $13 X-6,79$ & 290.85 & 291.01 & 258.39 \\
\hline $13 X-6,115$ & 291.30 & 291.37 & 258.75 \\
\hline $13 X-7,32$ & 291.53 & 291.74 & 259.12 \\
\hline $13 X-7,66$ & 291.80 & 292.07 & 259.46 \\
\hline $14 \mathrm{X}-1,32$ & 296.19 & 296.19 & 260.02 \\
\hline $14 X-6,4$ & 303.42 & 303.41 & 267.24 \\
\hline $14 X-7,64$ & 305.11 & 305.11 & 268.94 \\
\hline $14 X-7,62$ & 305.29 & 305.09 & 268.92 \\
\hline $15 X-1,107$ & 306.16 & 306.16 & 270.37 \\
\hline $15 X-2,93$ & 307.52 & 307.52 & 271.73 \\
\hline $15 X-4,147$ & 311.06 & 311.06 & 275.27 \\
\hline $15 X-5,82$ & 311.93 & 311.91 & 276.12 \\
\hline $15 X-5,94$ & 312.07 & 312.03 & 276.24 \\
\hline $15 X-5,105$ & 312.24 & 312.14 & 276.35 \\
\hline $16 \mathrm{X}-1,0$ & 314.95 & 314.95 & 278.90 \\
\hline $16 X-1,91$ & 315.86 & 315.86 & 279.81 \\
\hline $16 X-4,28$ & 319.73 & 319.73 & 283.68 \\
\hline $16 X-5,53$ & 321.39 & 321.48 & 285.43 \\
\hline $16 X-6,54$ & 321.92 & 322.69 & 286.64 \\
\hline $17 X-1,0$ & 323.01 & 323.01 & 288.50 \\
\hline $17 X-1,93$ & 323.94 & 323.94 & 289.43 \\
\hline $17 X-5,67$ & 329.67 & 329.67 & 295.17 \\
\hline $17 X-7,77$ & 332.48 & 332.48 & 297.97 \\
\hline $18 \mathrm{X}-1,0$ & 333.14 & 333.14 & 298.10 \\
\hline $18 X-1,54$ & 333.68 & 333.68 & 298.64 \\
\hline $18 X-6,75$ & 341.39 & 341.39 & 306.35 \\
\hline $18 X-7,65$ & 342.49 & 342.49 & 307.45 \\
\hline $19 X-1,0$ & 342.74 & 342.74 & 307.70 \\
\hline $19 X-2,84$ & 345.08 & 345.08 & 310.04 \\
\hline $19 X-5,38$ & 349.12 & 349.12 & 314.08 \\
\hline $19 X-5,110$ & 349.78 & 349.84 & 314.80 \\
\hline $19 X-5,137$ & 350.15 & 350.10 & 315.07 \\
\hline $19 X-6,38$ & 350.99 & 350.62 & 315.58 \\
\hline $19 X-6,145$ & 351.96 & 351.69 & 316.65 \\
\hline $20 X-1,0$ & 352.66 & 352.97 & 317.30 \\
\hline $20 \mathrm{X}-1,30$ & 353.06 & 353.27 & 317.60 \\
\hline $20 X-2,138$ & 355.85 & 355.85 & 320.18 \\
\hline $20 X-5,28$ & 359.25 & 359.25 & 323.58 \\
\hline $20 X-6,36$ & 361.11 & 360.83 & 325.16 \\
\hline $20 X-7,75$ & 363.03 & 362.22 & 326.55 \\
\hline $20 X-7,91$ & 363.22 & 362.38 & 326.71 \\
\hline $21 X-1,0$ & 363.80 & 363.80 & 326.80 \\
\hline $21 X-1,86$ & 364.67 & 364.67 & 327.66 \\
\hline $21 X-5,141$ & 371.22 & 371.22 & 334.21 \\
\hline $21 X-6,32$ & 371.96 & 371.62 & 334.62 \\
\hline $21 X-6,82$ & 372.65 & 372.12 & 335.12 \\
\hline $21 X-7,31$ & 373.36 & 372.62 & 335.61 \\
\hline $21 X-7,93$ & 374.40 & 373.24 & 336.23 \\
\hline
\end{tabular}

\begin{tabular}{|c|c|c|c|}
\hline \multirow[b]{2}{*}{$\begin{array}{l}\text { Core, section, } \\
\text { interval (cm) }\end{array}$} & \multicolumn{3}{|c|}{ Depth (m) } \\
\hline & $\begin{array}{l}\text { Adjusted } \\
\text { CCSF }\end{array}$ & CCSF & CSF-A \\
\hline $22 X-1,0$ & 374.81 & 374.72 & 336.40 \\
\hline $22 X-1,50$ & 375.30 & 375.22 & 336.90 \\
\hline $22 X-2,69$ & 376.92 & 376.92 & 338.59 \\
\hline $22 X-4,105$ & 380.27 & 380.27 & 341.95 \\
\hline $22 X-6,78$ & 383.98 & 383.00 & 344.68 \\
\hline $22 X-7,4$ & 384.30 & 383.26 & 344.94 \\
\hline $22 X-7,80$ & 385.07 & 384.02 & 345.70 \\
\hline $23 X-1,0$ & 385.95 & 385.95 & 346.00 \\
\hline $23 \mathrm{X}-1,28$ & 386.16 & 386.22 & 346.28 \\
\hline $23 X-1,45$ & 386.40 & 386.40 & 346.45 \\
\hline $23 X-4,95$ & 391.40 & 391.39 & 351.45 \\
\hline $23 X-5,62$ & 392.62 & 392.56 & 352.62 \\
\hline $23 X-6,45$ & 393.77 & 393.40 & 353.45 \\
\hline $23 X-6,106$ & 394.51 & 394.00 & 354.06 \\
\hline $24 X-1,0$ & 395.17 & 395.00 & 355.60 \\
\hline $24 X-1,48$ & 395.69 & 395.48 & 356.08 \\
\hline $24 X-2,46$ & 396.84 & 396.96 & 357.56 \\
\hline $24 X-2,76$ & 397.26 & 397.26 & 357.86 \\
\hline $24 X-5,114$ & 402.14 & 402.14 & 362.74 \\
\hline $24 X-7,76$ & 404.56 & 404.56 & 365.16 \\
\hline $25 X-1,0$ & 405.77 & 405.77 & 365.20 \\
\hline $25 X-2,35$ & 407.60 & 407.62 & 367.05 \\
\hline $25 X-2,84$ & 408.27 & 408.10 & 367.54 \\
\hline $25 X-3,14$ & 408.90 & 408.90 & 368.34 \\
\hline $25 X-5,100$ & 412.77 & 412.77 & 372.20 \\
\hline $25 X-6,109$ & 414.77 & 414.36 & 373.79 \\
\hline $25 X-7,53$ & 415.41 & 414.90 & 374.34 \\
\hline $25 X-7,86$ & 415.76 & 415.24 & 374.67 \\
\hline $26 X-1,0$ & 416.85 & 416.85 & 374.70 \\
\hline $26 \mathrm{X}-2,24$ & 418.59 & 418.59 & 376.44 \\
\hline $26 \mathrm{X}-2,97$ & 419.32 & 419.32 & 377.17 \\
\hline $26 X-7,14$ & 425.50 & 425.50 & 383.35 \\
\hline $26 X-7,56$ & 425.97 & 425.92 & 383.77 \\
\hline $26 X-7,89$ & 426.24 & 426.25 & 384.10 \\
\hline $27 X-1,0$ & 428.61 & 428.65 & 384.30 \\
\hline $27 X-1,35$ & 428.88 & 429.00 & 384.65 \\
\hline $27 X-1,91$ & 429.62 & 429.55 & 385.21 \\
\hline $27 X-1,121$ & 429.94 & 429.85 & 385.51 \\
\hline $27 X-2,130$ & 431.42 & 431.45 & 387.10 \\
\hline $27 X-3,28$ & 431.93 & 431.92 & 387.58 \\
\hline $27 X-4,108$ & 434.23 & 434.23 & 389.88 \\
\hline $27 X-5,102$ & 435.26 & 435.26 & 390.92 \\
\hline $28 \mathrm{X}-1,0$ & 447.70 & 447.70 & 393.60 \\
\hline $28 X-1,73$ & 448.43 & 448.43 & 394.33 \\
\hline $28 X-6,100$ & 456.20 & 456.20 & 402.10 \\
\hline $28 X-7,43$ & 456.71 & 456.74 & 402.63 \\
\hline $28 X-7,83$ & 457.13 & 457.13 & 403.03 \\
\hline $29 X-1,0$ & 461.63 & 461.63 & 403.20 \\
\hline $29 \mathrm{X}-2,28$ & 463.40 & 463.40 & 404.98 \\
\hline $29 X-6,8$ & 469.21 & 469.21 & 410.78 \\
\hline $29 X-7,82$ & 471.05 & 471.05 & 412.62 \\
\hline $30 X-1,21$ & 471.15 & 471.15 & 412.91 \\
\hline $30 X-5,134$ & 478.28 & 478.28 & 420.04 \\
\hline $31 X-1,1$ & 480.55 & 480.55 & 422.31 \\
\hline $31 X-1,11$ & 480.65 & 480.65 & 422.41 \\
\hline $31 X-5,65$ & 487.19 & 487.19 & 428.95 \\
\hline $31 X-7,77$ & 489.81 & 489.81 & 431.57 \\
\hline $32 X-1,1$ & 490.15 & 490.15 & 431.91 \\
\hline $32 X-1,34$ & 490.48 & 490.48 & 432.24 \\
\hline $32 X-4,13$ & 494.76 & 494.77 & 436.53 \\
\hline $32 X-4,12$ & 494.76 & 494.76 & 436.52 \\
\hline
\end{tabular}

This table is also available in ASCII. 
Table AT34. Gamma ray attenuation (GRA) density data, Hole U1337D.

\begin{tabular}{|c|c|c|c|c|}
\hline \multirow[b]{2}{*}{$\begin{array}{l}\text { Core, section, } \\
\text { interval }(\mathrm{cm})\end{array}$} & \multicolumn{3}{|c|}{ Depth (m) } & \multirow[b]{2}{*}{$\begin{array}{c}\text { GRA } \\
\left(\mathrm{g} / \mathrm{cm}^{3}\right)\end{array}$} \\
\hline & CSF & CCSF & $\begin{array}{l}\text { Adjusted } \\
\text { CCSF }\end{array}$ & \\
\hline \multicolumn{5}{|l|}{ 321-U1337D- } \\
\hline $1 \mathrm{H}-1,15$ & 0.15 & 0.15 & 0.14 & 1.14 \\
\hline $1 \mathrm{H}-1,17.5$ & 0.18 & 0.18 & 0.16 & 1.21 \\
\hline $1 \mathrm{H}-1,20$ & 0.20 & 0.20 & 0.18 & 1.19 \\
\hline $1 \mathrm{H}-1,22.5$ & 0.23 & 0.23 & 0.21 & 1.25 \\
\hline $1 \mathrm{H}-1,25$ & 0.25 & 0.25 & 0.23 & 1.28 \\
\hline $1 \mathrm{H}-1,27.6$ & 0.28 & 0.28 & 0.26 & 1.24 \\
\hline $1 \mathrm{H}-1,30.1$ & 0.30 & 0.30 & 0.27 & 1.27 \\
\hline $1 \mathrm{H}-1,32.6$ & 0.33 & 0.33 & 0.30 & 1.25 \\
\hline $1 \mathrm{H}-1,35.1$ & 0.35 & 0.35 & 0.32 & 1.22 \\
\hline $1 \mathrm{H}-1,37.6$ & 0.38 & 0.38 & 0.35 & 1.23 \\
\hline $1 \mathrm{H}-1,40.1$ & 0.40 & 0.40 & 0.36 & 1.25 \\
\hline $1 \mathrm{H}-1,42.6$ & 0.43 & 0.43 & 0.39 & 1.26 \\
\hline $1 \mathrm{H}-1,45.1$ & 0.45 & 0.45 & 0.41 & 1.29 \\
\hline $1 \mathrm{H}-1,47.6$ & 0.48 & 0.48 & 0.44 & 1.28 \\
\hline $1 \mathrm{H}-1,50.1$ & 0.50 & 0.50 & 0.46 & 1.30 \\
\hline $1 \mathrm{H}-1,52.6$ & 0.53 & 0.53 & 0.48 & 1.34 \\
\hline $1 \mathrm{H}-1,55.1$ & 0.55 & 0.55 & 0.50 & 1.36 \\
\hline $1 \mathrm{H}-1,57.6$ & 0.58 & 0.58 & 0.53 & 1.39 \\
\hline $1 \mathrm{H}-1,60.1$ & 0.60 & 0.60 & 0.55 & 1.37 \\
\hline $1 \mathrm{H}-1,62.6$ & 0.63 & 0.63 & 0.57 & 1.38 \\
\hline $1 \mathrm{H}-1,65.1$ & 0.65 & 0.65 & 0.59 & 1.39 \\
\hline $1 \mathrm{H}-1,67.6$ & 0.68 & 0.68 & 0.62 & 1.38 \\
\hline $1 \mathrm{H}-1,70.1$ & 0.70 & 0.70 & 0.64 & 1.38 \\
\hline $1 \mathrm{H}-1,72.6$ & 0.73 & 0.73 & 0.67 & 1.39 \\
\hline $1 \mathrm{H}-1,75.1$ & 0.75 & 0.75 & 0.68 & 1.38 \\
\hline $1 \mathrm{H}-1,77.6$ & 0.78 & 0.78 & 0.71 & 1.35 \\
\hline $1 \mathrm{H}-1,80.1$ & 0.80 & 0.80 & 0.73 & 1.37 \\
\hline $1 \mathrm{H}-1,82.6$ & 0.83 & 0.83 & 0.76 & 1.39 \\
\hline $1 \mathrm{H}-1,85.1$ & 0.85 & 0.85 & 0.77 & 1.36 \\
\hline $1 \mathrm{H}-1,87.6$ & 0.88 & 0.88 & 0.80 & 1.33 \\
\hline $1 \mathrm{H}-1,90.1$ & 0.90 & 0.90 & 0.82 & 1.32 \\
\hline $1 \mathrm{H}-1,92.7$ & 0.93 & 0.93 & 0.85 & 1.28 \\
\hline $1 \mathrm{H}-1,95.2$ & 0.95 & 0.95 & 0.87 & 1.20 \\
\hline $1 \mathrm{H}-1,97.7$ & 0.98 & 0.98 & 0.89 & 1.27 \\
\hline $1 \mathrm{H}-1,100.2$ & 1.00 & 1.00 & 0.91 & 1.29 \\
\hline $1 \mathrm{H}-1,102.7$ & 1.03 & 1.03 & 0.94 & 1.29 \\
\hline $1 \mathrm{H}-1,105.2$ & 1.05 & 1.05 & 0.96 & 1.23 \\
\hline $1 \mathrm{H}-1,107.7$ & 1.08 & 1.08 & 0.98 & 1.25 \\
\hline $1 \mathrm{H}-1,110.2$ & 1.10 & 1.10 & 1.00 & 1.20 \\
\hline $1 \mathrm{H}-1,112.7$ & 1.13 & 1.13 & 1.03 & 1.19 \\
\hline $1 \mathrm{H}-1,115.2$ & 1.15 & 1.15 & 1.05 & 1.22 \\
\hline $1 \mathrm{H}-1,117.7$ & 1.18 & 1.18 & 1.07 & 1.17 \\
\hline $1 \mathrm{H}-1,120.2$ & 1.20 & 1.20 & 1.09 & 1.18 \\
\hline $1 \mathrm{H}-1,122.7$ & 1.23 & 1.23 & 1.12 & 1.15 \\
\hline $1 \mathrm{H}-1,125.2$ & 1.25 & 1.25 & 1.14 & 1.15 \\
\hline $1 \mathrm{H}-1,127.7$ & 1.28 & 1.28 & 1.16 & 1.16 \\
\hline $1 \mathrm{H}-1,130.2$ & 1.30 & 1.30 & 1.18 & 1.18 \\
\hline $1 \mathrm{H}-1,132.7$ & 1.33 & 1.33 & 1.21 & 1.19 \\
\hline $1 \mathrm{H}-1,135.2$ & 1.35 & 1.35 & 1.23 & 1.18 \\
\hline $1 \mathrm{H}-1,137.7$ & 1.38 & 1.38 & 1.26 & 1.19 \\
\hline $1 \mathrm{H}-1,140.2$ & 1.40 & 1.40 & 1.27 & 1.20 \\
\hline $1 \mathrm{H}-1,142.7$ & 1.43 & 1.43 & 1.30 & 1.21 \\
\hline $1 \mathrm{H}-1,145.3$ & 1.45 & 1.45 & 1.32 & 1.20 \\
\hline $1 \mathrm{H}-1,147.8$ & 1.48 & 1.48 & 1.35 & 1.18 \\
\hline $1 \mathrm{H}-1,150.3$ & 1.50 & 1.50 & 1.36 & 1.11 \\
\hline $1 \mathrm{H}-2,1.8$ & 1.52 & 1.52 & 1.38 & 1.21 \\
\hline $1 \mathrm{H}-2,4.3$ & 1.54 & 1.54 & 1.40 & 1.17 \\
\hline $1 \mathrm{H}-2,6.8$ & 1.57 & 1.57 & 1.43 & 1.16 \\
\hline
\end{tabular}

Only a portion of this table appears here. The complete table is available in ASCII. 
Table AT35. Magnetic susceptibility data, Hole U1337D.

\begin{tabular}{|c|c|c|c|c|}
\hline \multirow[b]{2}{*}{$\begin{array}{l}\text { Core, section, } \\
\text { interval }(\mathrm{cm})\end{array}$} & \multicolumn{3}{|c|}{ Depth $(m)$} & \multirow{2}{*}{$\begin{array}{l}\text { Magnetic } \\
\text { susceptibility } \\
\text { (IU) }\end{array}$} \\
\hline & CSF & CCSF & $\begin{array}{l}\text { Adjusted } \\
\text { CCSF }\end{array}$ & \\
\hline \multicolumn{5}{|l|}{ 321-U1337D- } \\
\hline $1 \mathrm{H}-1,0.1$ & 0.00 & 0.00 & 0.00 & 1.1 \\
\hline $1 \mathrm{H}-1,2.6$ & 0.03 & 0.03 & 0.03 & 2.7 \\
\hline $1 \mathrm{H}-1,5.1$ & 0.05 & 0.05 & 0.05 & 4.1 \\
\hline $1 \mathrm{H}-1,7.6$ & 0.08 & 0.08 & 0.07 & 5.2 \\
\hline $1 \mathrm{H}-1,10.1$ & 0.10 & 0.10 & 0.09 & 4.4 \\
\hline $1 \mathrm{H}-1,12.6$ & 0.13 & 0.13 & 0.12 & 4.4 \\
\hline $1 \mathrm{H}-1,15.1$ & 0.15 & 0.15 & 0.14 & 4.7 \\
\hline $1 \mathrm{H}-1,17.6$ & 0.18 & 0.18 & 0.16 & 4.7 \\
\hline $1 \mathrm{H}-1,20.1$ & 0.20 & 0.20 & 0.18 & 4.4 \\
\hline $1 \mathrm{H}-1,22.6$ & 0.23 & 0.23 & 0.21 & 4.1 \\
\hline $1 \mathrm{H}-1,25.1$ & 0.25 & 0.25 & 0.23 & 3.8 \\
\hline $1 \mathrm{H}-1,27.6$ & 0.28 & 0.28 & 0.26 & 3.8 \\
\hline $1 \mathrm{H}-1,30.1$ & 0.30 & 0.30 & 0.27 & 3.3 \\
\hline $1 \mathrm{H}-1,32.6$ & 0.33 & 0.33 & 0.30 & 5.5 \\
\hline $1 \mathrm{H}-1,35.1$ & 0.35 & 0.35 & 0.32 & 6.6 \\
\hline $1 \mathrm{H}-1,37.6$ & 0.38 & 0.38 & 0.35 & 2.5 \\
\hline $1 \mathrm{H}-1,40.1$ & 0.40 & 0.40 & 0.36 & 2.2 \\
\hline $1 \mathrm{H}-1,42.6$ & 0.43 & 0.43 & 0.39 & 2.2 \\
\hline $1 \mathrm{H}-1,45.1$ & 0.45 & 0.45 & 0.41 & 2.5 \\
\hline $1 \mathrm{H}-1,47.6$ & 0.48 & 0.48 & 0.44 & 2.2 \\
\hline $1 \mathrm{H}-1,50.1$ & 0.50 & 0.50 & 0.46 & 1.9 \\
\hline $1 \mathrm{H}-1,52.6$ & 0.53 & 0.53 & 0.48 & 1.4 \\
\hline $1 \mathrm{H}-1,55.1$ & 0.55 & 0.55 & 0.50 & 1.6 \\
\hline $1 \mathrm{H}-1,57.7$ & 0.58 & 0.58 & 0.53 & 1.4 \\
\hline $1 \mathrm{H}-1,60.2$ & 0.60 & 0.60 & 0.55 & 2.2 \\
\hline $1 \mathrm{H}-1,62.7$ & 0.63 & 0.63 & 0.57 & 1.6 \\
\hline $1 \mathrm{H}-1,65.2$ & 0.65 & 0.65 & 0.59 & 1.6 \\
\hline $1 \mathrm{H}-1,67.7$ & 0.68 & 0.68 & 0.62 & 2.5 \\
\hline $1 \mathrm{H}-1,70.2$ & 0.70 & 0.70 & 0.64 & 2.2 \\
\hline $1 \mathrm{H}-1,72.7$ & 0.73 & 0.73 & 0.67 & 2.2 \\
\hline $1 \mathrm{H}-1,75.2$ & 0.75 & 0.75 & 0.68 & 2.5 \\
\hline $1 \mathrm{H}-1,77.7$ & 0.78 & 0.78 & 0.71 & 1.9 \\
\hline $1 \mathrm{H}-1,80.2$ & 0.80 & 0.80 & 0.73 & 2.2 \\
\hline $1 \mathrm{H}-1,82.7$ & 0.83 & 0.83 & 0.76 & 2.5 \\
\hline $1 \mathrm{H}-1,85.2$ & 0.85 & 0.85 & 0.77 & 2.7 \\
\hline $1 \mathrm{H}-1,87.7$ & 0.88 & 0.88 & 0.80 & 3.0 \\
\hline $1 \mathrm{H}-1,90.2$ & 0.90 & 0.90 & 0.82 & 3.3 \\
\hline $1 \mathrm{H}-1,92.7$ & 0.93 & 0.93 & 0.85 & 3.6 \\
\hline $1 \mathrm{H}-1,95.2$ & 0.95 & 0.95 & 0.87 & 4.4 \\
\hline $1 \mathrm{H}-1,97.7$ & 0.98 & 0.98 & 0.89 & 5.8 \\
\hline $1 \mathrm{H}-1,100.2$ & 1.00 & 1.00 & 0.91 & 7.7 \\
\hline $1 \mathrm{H}-1,102.7$ & 1.03 & 1.03 & 0.94 & 7.7 \\
\hline $1 \mathrm{H}-1,105.2$ & 1.05 & 1.05 & 0.96 & 8.0 \\
\hline $1 \mathrm{H}-1,107.7$ & 1.08 & 1.08 & 0.98 & 7.7 \\
\hline $1 \mathrm{H}-1,110.2$ & 1.10 & 1.10 & 1.00 & 7.7 \\
\hline $1 \mathrm{H}-1,112.8$ & 1.13 & 1.13 & 1.03 & 7.7 \\
\hline $1 \mathrm{H}-1,115.2$ & 1.15 & 1.15 & 1.05 & 7.4 \\
\hline $1 \mathrm{H}-1,117.8$ & 1.18 & 1.18 & 1.07 & 7.7 \\
\hline $1 \mathrm{H}-1,120.3$ & 1.20 & 1.20 & 1.09 & 7.4 \\
\hline $1 \mathrm{H}-1,122.8$ & 1.23 & 1.23 & 1.12 & 6.9 \\
\hline $1 \mathrm{H}-1,125.3$ & 1.25 & 1.25 & 1.14 & 6.0 \\
\hline $1 \mathrm{H}-1,127.8$ & 1.28 & 1.28 & 1.16 & 6.3 \\
\hline $1 \mathrm{H}-1,130.3$ & 1.30 & 1.30 & 1.18 & 7.1 \\
\hline $1 \mathrm{H}-1,132.8$ & 1.33 & 1.33 & 1.21 & 7.1 \\
\hline $1 \mathrm{H}-1,135.3$ & 1.35 & 1.35 & 1.23 & 7.4 \\
\hline $1 \mathrm{H}-1,137.8$ & 1.38 & 1.38 & 1.26 & 8.0 \\
\hline $1 \mathrm{H}-1,140.3$ & 1.40 & 1.40 & 1.27 & 8.8 \\
\hline $1 \mathrm{H}-1,142.8$ & 1.43 & 1.43 & 1.30 & 8.2 \\
\hline $1 \mathrm{H}-1,145.3$ & 1.45 & 1.45 & 1.32 & 8.2 \\
\hline $1 \mathrm{H}-1,147.8$ & 1.48 & 1.48 & 1.35 & 8.5 \\
\hline $1 \mathrm{H}-1,150.3$ & 1.50 & 1.50 & 1.36 & 7.7 \\
\hline
\end{tabular}

Only a portion of this table appears here. The complete table is available in ASCII. 
Table AT36. Natural gamma ray (NGR) data, Hole U1337D.

\begin{tabular}{|c|c|c|c|c|}
\hline \multirow[b]{2}{*}{$\begin{array}{l}\text { Core, section, } \\
\text { interval }(\mathrm{cm})\end{array}$} & \multicolumn{3}{|c|}{ Depth (m) } & \multirow[b]{2}{*}{$\begin{array}{l}\text { NGR } \\
\text { (cps) }\end{array}$} \\
\hline & CSF & CCSF & $\begin{array}{l}\text { Adjusted } \\
\text { CCSF }\end{array}$ & \\
\hline \multicolumn{5}{|l|}{ 321-U1337D- } \\
\hline $1 \mathrm{H}-1,0$ & 0.0 & 0.0 & 0.00 & 56.10 \\
\hline $1 \mathrm{H}-1,10$ & 0.1 & 0.1 & 0.09 & 63.60 \\
\hline $1 \mathrm{H}-1,20$ & 0.2 & 0.2 & 0.18 & 69.00 \\
\hline $1 \mathrm{H}-1,30$ & 0.3 & 0.3 & 0.27 & 57.50 \\
\hline $1 \mathrm{H}-1,40$ & 0.4 & 0.4 & 0.36 & 46.00 \\
\hline $1 \mathrm{H}-1,50$ & 0.5 & 0.5 & 0.46 & 39.60 \\
\hline $1 \mathrm{H}-1,60$ & 0.6 & 0.6 & 0.55 & 41.50 \\
\hline $1 \mathrm{H}-1,70$ & 0.7 & 0.7 & 0.64 & 44.30 \\
\hline $1 \mathrm{H}-1,80$ & 0.8 & 0.8 & 0.73 & 39.40 \\
\hline $1 \mathrm{H}-1,90$ & 0.9 & 0.9 & 0.82 & 41.60 \\
\hline $1 \mathrm{H}-1,100$ & 1.0 & 1.0 & 0.91 & 61.80 \\
\hline $1 \mathrm{H}-1,110$ & 1.1 & 1.1 & 1.00 & 61.10 \\
\hline $1 \mathrm{H}-1,120$ & 1.2 & 1.2 & 1.09 & 43.10 \\
\hline $1 \mathrm{H}-1,130$ & 1.3 & 1.3 & 1.18 & 43.50 \\
\hline $1 \mathrm{H}-1,140$ & 1.4 & 1.4 & 1.27 & 55.10 \\
\hline $1 \mathrm{H}-1,150$ & 1.5 & 1.5 & 1.36 & 61.10 \\
\hline $1 \mathrm{H}-2,0$ & 1.5 & 1.5 & 1.36 & 53.20 \\
\hline $1 \mathrm{H}-2,10$ & 1.6 & 1.6 & 1.45 & 51.60 \\
\hline $1 \mathrm{H}-2,20$ & 1.7 & 1.7 & 1.55 & 53.50 \\
\hline $1 \mathrm{H}-2,30$ & 1.8 & 1.8 & 1.64 & 46.10 \\
\hline $1 \mathrm{H}-2,40$ & 1.9 & 1.9 & 1.73 & 36.70 \\
\hline $1 \mathrm{H}-2,50$ & 2.0 & 2.0 & 1.82 & 28.90 \\
\hline $1 \mathrm{H}-2,60$ & 2.1 & 2.1 & 1.92 & 23.20 \\
\hline $1 \mathrm{H}-2,70$ & 2.2 & 2.2 & 2.02 & 23.70 \\
\hline $1 \mathrm{H}-2,80$ & 2.3 & 2.3 & 2.12 & 24.30 \\
\hline $1 \mathrm{H}-2,90$ & 2.4 & 2.4 & 2.22 & 19.90 \\
\hline $1 \mathrm{H}-2,100$ & 2.5 & 2.5 & 2.31 & 19.20 \\
\hline $1 \mathrm{H}-2,110$ & 2.6 & 2.6 & 2.41 & 20.90 \\
\hline $1 \mathrm{H}-2,120$ & 2.7 & 2.7 & 2.51 & 20.40 \\
\hline $1 \mathrm{H}-2,130$ & 2.8 & 2.8 & 2.61 & 18.00 \\
\hline $1 \mathrm{H}-2,140$ & 2.9 & 2.9 & 2.71 & 15.80 \\
\hline $1 \mathrm{H}-2,150$ & 3.0 & 3.0 & 2.81 & 15.80 \\
\hline $1 \mathrm{H}-3,0$ & 3.0 & 3.0 & 2.81 & 14.30 \\
\hline $1 \mathrm{H}-3,10$ & 3.1 & 3.1 & 2.91 & 15.90 \\
\hline $1 \mathrm{H}-3,20$ & 3.2 & 3.2 & 3.01 & 19.50 \\
\hline $1 \mathrm{H}-3,30$ & 3.3 & 3.3 & 3.11 & 22.80 \\
\hline $1 \mathrm{H}-3,40$ & 3.4 & 3.4 & 3.21 & 28.30 \\
\hline $1 \mathrm{H}-3,50$ & 3.5 & 3.5 & 3.30 & 27.10 \\
\hline $1 \mathrm{H}-3,60$ & 3.6 & 3.6 & 3.40 & 23.30 \\
\hline $1 \mathrm{H}-3,70$ & 3.7 & 3.7 & 3.50 & 20.70 \\
\hline $1 \mathrm{H}-3,80$ & 3.8 & 3.8 & 3.60 & 18.00 \\
\hline $1 \mathrm{H}-3,90$ & 3.9 & 3.9 & 3.70 & 16.80 \\
\hline $1 \mathrm{H}-3,100$ & 4.0 & 4.0 & 3.79 & 16.70 \\
\hline $1 \mathrm{H}-3,110$ & 4.1 & 4.1 & 3.89 & 15.00 \\
\hline $1 \mathrm{H}-3,120$ & 4.2 & 4.2 & 3.98 & 10.70 \\
\hline $1 \mathrm{H}-3,130$ & 4.3 & 4.3 & 4.07 & 9.60 \\
\hline $1 \mathrm{H}-3,140$ & 4.4 & 4.4 & 4.16 & 9.80 \\
\hline $1 \mathrm{H}-4,0$ & 4.5 & 4.5 & 4.25 & 9.70 \\
\hline $1 \mathrm{H}-4,10$ & 4.6 & 4.6 & 4.35 & 12.40 \\
\hline $1 \mathrm{H}-4,20$ & 4.7 & 4.7 & 4.44 & 15.90 \\
\hline $1 \mathrm{H}-4,30$ & 4.8 & 4.8 & 4.53 & 14.00 \\
\hline $1 \mathrm{H}-4,40$ & 4.9 & 4.9 & 4.62 & 9.90 \\
\hline $1 \mathrm{H}-4,50$ & 5.0 & 5.0 & 4.72 & 9.90 \\
\hline $1 \mathrm{H}-4,60$ & 5.1 & 5.1 & 4.81 & 10.20 \\
\hline $1 \mathrm{H}-4,70$ & 5.2 & 5.2 & 4.90 & 10.10 \\
\hline $1 \mathrm{H}-4,80$ & 5.3 & 5.3 & 4.99 & 9.00 \\
\hline $1 \mathrm{H}-4,90$ & 5.4 & 5.4 & 5.09 & 9.40 \\
\hline $1 \mathrm{H}-4,100$ & 5.5 & 5.5 & 5.18 & 9.20 \\
\hline $1 \mathrm{H}-4,110$ & 5.6 & 5.6 & 5.27 & 8.00 \\
\hline
\end{tabular}

Only a portion of this table appears here. The complete table is available in ASCII. 
Table AT37. Color reflection data, Hole U1337D.

\begin{tabular}{|c|c|c|c|c|c|c|}
\hline \multirow[b]{2}{*}{$\begin{array}{l}\text { Core, section, } \\
\text { interval }(\mathrm{cm})\end{array}$} & \multicolumn{3}{|c|}{ Depth (m) } & \multirow[b]{2}{*}{$a^{*}$} & \multirow[b]{2}{*}{$b^{*}$} & \multirow[b]{2}{*}{$\mathrm{L}^{*}$} \\
\hline & CSF & CCSF & $\begin{array}{l}\text { Adjusted } \\
\text { CCSF }\end{array}$ & & & \\
\hline \multicolumn{7}{|l|}{ 321-U1337D- } \\
\hline $1 \mathrm{H}-1,2.6$ & 0.03 & 0.03 & 0.03 & 3.5 & 2.8 & 24.3 \\
\hline $1 \mathrm{H}-1,5.2$ & 0.05 & 0.05 & 0.05 & 6.2 & 11.1 & 33.0 \\
\hline $1 \mathrm{H}-1,7.7$ & 0.08 & 0.08 & 0.07 & 6.1 & 11.1 & 34.6 \\
\hline $1 \mathrm{H}-1,10.2$ & 0.10 & 0.10 & 0.09 & 6.2 & 11.9 & 35.4 \\
\hline $1 \mathrm{H}-1,12.8$ & 0.13 & 0.13 & 0.12 & 4.8 & 10.4 & 39.1 \\
\hline $1 \mathrm{H}-1,15.3$ & 0.15 & 0.15 & 0.14 & 4.7 & 11.2 & 39.1 \\
\hline $1 \mathrm{H}-1,17.9$ & 0.18 & 0.18 & 0.16 & 4.8 & 12.0 & 40.9 \\
\hline $1 \mathrm{H}-1,20.4$ & 0.20 & 0.20 & 0.18 & 6.0 & 14.2 & 40.5 \\
\hline $1 \mathrm{H}-1,22.9$ & 0.23 & 0.23 & 0.21 & 5.9 & 15.0 & 43.7 \\
\hline $1 \mathrm{H}-1,25.5$ & 0.26 & 0.26 & 0.24 & 6.5 & 17.2 & 46.5 \\
\hline $1 \mathrm{H}-1,28$ & 0.28 & 0.28 & 0.26 & 5.5 & 15.4 & 44.4 \\
\hline $1 \mathrm{H}-1,30.6$ & 0.31 & 0.31 & 0.28 & 5.2 & 14.9 & 48.1 \\
\hline $1 \mathrm{H}-1,33.1$ & 0.33 & 0.33 & 0.30 & 7.2 & 18.6 & 48.8 \\
\hline $1 \mathrm{H}-1,35.6$ & 0.36 & 0.36 & 0.33 & 6.7 & 20.0 & 55.5 \\
\hline $1 \mathrm{H}-1,38.2$ & 0.38 & 0.38 & 0.35 & 6.2 & 18.5 & 53.9 \\
\hline $1 \mathrm{H}-1,40.7$ & 0.41 & 0.41 & 0.37 & 5.9 & 17.7 & 50.0 \\
\hline $1 \mathrm{H}-1,43.3$ & 0.43 & 0.43 & 0.39 & 6.0 & 18.7 & 55.0 \\
\hline $1 \mathrm{H}-1,45.8$ & 0.46 & 0.46 & 0.42 & 5.5 & 18.8 & 57.4 \\
\hline $1 \mathrm{H}-1,48.4$ & 0.48 & 0.48 & 0.44 & 4.5 & 15.0 & 59.9 \\
\hline $1 \mathrm{H}-1,50.9$ & 0.51 & 0.51 & 0.47 & 5.0 & 19.6 & 63.0 \\
\hline $1 \mathrm{H}-1,53.4$ & 0.53 & 0.53 & 0.48 & 5.5 & 19.2 & 60.9 \\
\hline $1 \mathrm{H}-1,56$ & 0.56 & 0.56 & 0.51 & 4.2 & 15.6 & 53.9 \\
\hline $1 \mathrm{H}-1,58.5$ & 0.59 & 0.59 & 0.54 & 3.9 & 15.2 & 51.1 \\
\hline $1 \mathrm{H}-1,61.1$ & 0.61 & 0.61 & 0.56 & 5.2 & 17.3 & 57.6 \\
\hline $1 \mathrm{H}-1,63.6$ & 0.64 & 0.64 & 0.58 & 4.9 & 17.1 & 58.4 \\
\hline $1 \mathrm{H}-1,66.1$ & 0.66 & 0.66 & 0.60 & 4.6 & 15.1 & 56.2 \\
\hline $1 \mathrm{H}-1,68.7$ & 0.69 & 0.69 & 0.63 & 4.1 & 15.1 & 53.3 \\
\hline $1 \mathrm{H}-1,71.2$ & 0.71 & 0.71 & 0.65 & 2.2 & 11.7 & 54.1 \\
\hline $1 \mathrm{H}-1,73.8$ & 0.74 & 0.74 & 0.67 & 3.8 & 14.5 & 50.2 \\
\hline $1 \mathrm{H}-1,76.3$ & 0.76 & 0.76 & 0.69 & 4.4 & 14.8 & 54.4 \\
\hline $1 \mathrm{H}-1,78.9$ & 0.79 & 0.79 & 0.72 & 4.8 & 16.5 & 57.3 \\
\hline $1 \mathrm{H}-1,81.4$ & 0.81 & 0.81 & 0.74 & 4.5 & 16.2 & 56.8 \\
\hline $1 \mathrm{H}-1,83.9$ & 0.84 & 0.84 & 0.77 & 4.2 & 15.8 & 53.7 \\
\hline $1 \mathrm{H}-1,86.5$ & 0.87 & 0.87 & 0.79 & 3.9 & 15.6 & 54.0 \\
\hline $1 \mathrm{H}-1,89$ & 0.89 & 0.89 & 0.81 & 3.8 & 15.2 & 54.8 \\
\hline $1 \mathrm{H}-1,91.6$ & 0.92 & 0.92 & 0.84 & 3.9 & 15.3 & 48.0 \\
\hline $1 \mathrm{H}-1,94.1$ & 0.94 & 0.94 & 0.86 & 3.3 & 12.8 & 44.4 \\
\hline $1 \mathrm{H}-1,96.7$ & 0.97 & 0.97 & 0.88 & 3.7 & 10.7 & 36.6 \\
\hline $1 \mathrm{H}-1,99.2$ & 0.99 & 0.99 & 0.90 & 2.4 & 9.4 & 42.3 \\
\hline $1 \mathrm{H}-1,101.7$ & 1.02 & 1.02 & 0.93 & 2.9 & 7.7 & 31.0 \\
\hline $1 \mathrm{H}-1,104.3$ & 1.04 & 1.04 & 0.95 & 3.9 & 9.0 & 36.3 \\
\hline $1 \mathrm{H}-1,106.8$ & 1.07 & 1.07 & 0.97 & 3.0 & 7.9 & 42.0 \\
\hline $1 \mathrm{H}-1,109.4$ & 1.09 & 1.09 & 0.99 & 4.7 & 12.1 & 39.5 \\
\hline $1 \mathrm{H}-1,111.9$ & 1.12 & 1.12 & 1.02 & 6.7 & 17.1 & 39.2 \\
\hline $1 \mathrm{H}-1,114.4$ & 1.14 & 1.14 & 1.04 & 6.1 & 14.3 & 39.7 \\
\hline $1 \mathrm{H}-1,117$ & 1.17 & 1.17 & 1.06 & 5.9 & 15.1 & 41.5 \\
\hline $1 \mathrm{H}-1,119.5$ & 1.20 & 1.20 & 1.09 & 6.6 & 15.7 & 37.9 \\
\hline $1 \mathrm{H}-1,122.1$ & 1.22 & 1.22 & 1.11 & 6.3 & 14.2 & 39.5 \\
\hline $1 \mathrm{H}-1,124.6$ & 1.25 & 1.25 & 1.14 & 6.6 & 14.8 & 39.5 \\
\hline $1 \mathrm{H}-1,127.2$ & 1.27 & 1.27 & 1.16 & 7.1 & 16.4 & 40.4 \\
\hline $1 \mathrm{H}-1,129.7$ & 1.30 & 1.30 & 1.18 & 7.3 & 16.9 & 38.7 \\
\hline $1 \mathrm{H}-1,132.2$ & 1.32 & 1.32 & 1.20 & 7.5 & 16.9 & 37.8 \\
\hline $1 \mathrm{H}-1,134.8$ & 1.35 & 1.35 & 1.23 & 6.9 & 12.8 & 35.4 \\
\hline $1 \mathrm{H}-1,137.3$ & 1.37 & 1.37 & 1.25 & 6.0 & 12.5 & 36.0 \\
\hline $1 \mathrm{H}-1,139.9$ & 1.40 & 1.40 & 1.27 & 4.4 & 10.2 & 37.5 \\
\hline $1 \mathrm{H}-1,142.4$ & 1.42 & 1.42 & 1.29 & 4.6 & 10.2 & 35.4 \\
\hline $1 \mathrm{H}-1,145$ & 1.45 & 1.45 & 1.32 & 2.5 & 5.4 & 39.7 \\
\hline $1 \mathrm{H}-1,147.5$ & 1.48 & 1.48 & 1.35 & 3.5 & 5.1 & 28.0 \\
\hline $1 \mathrm{H}-2,5.2$ & 1.55 & 1.55 & 1.41 & 2.0 & 4.7 & 39.6 \\
\hline $1 \mathrm{H}-2,7.7$ & 1.58 & 1.58 & 1.44 & 3.6 & 6.4 & 41.4 \\
\hline
\end{tabular}

Only a portion of this table appears here. The complete table is available in ASCII. 
Table AT38. Core adjusted data, Hole U1337D. (Continued on next page.)

\begin{tabular}{|c|c|c|c|}
\hline \multirow[b]{2}{*}{$\begin{array}{l}\text { Core, section, } \\
\text { interval }(\mathrm{cm})\end{array}$} & \multicolumn{3}{|c|}{ Depth $(\mathrm{m})$} \\
\hline & $\begin{array}{l}\text { Adjusted } \\
\text { CCSF }\end{array}$ & CCSF & CSF-A \\
\hline \multicolumn{4}{|l|}{ 321-U1337D- } \\
\hline $1 \mathrm{H}-1,0$ & 0.00 & 0.00 & 0.00 \\
\hline $1 \mathrm{H}-1,78$ & 0.71 & 0.78 & 0.78 \\
\hline $1 \mathrm{H}-2,48$ & 1.80 & 1.99 & 1.98 \\
\hline $1 \mathrm{H}-3,90$ & 3.70 & 3.90 & 3.90 \\
\hline $1 \mathrm{H}-5,38$ & 5.99 & 6.38 & 6.38 \\
\hline $1 \mathrm{H}-6,77$ & 7.37 & 7.77 & 7.77 \\
\hline $2 \mathrm{H}-1,0$ & 8.59 & 8.59 & 8.00 \\
\hline $2 \mathrm{H}-1,84$ & 9.56 & 9.43 & 8.84 \\
\hline $2 \mathrm{H}-1,134$ & 9.98 & 9.94 & 9.34 \\
\hline $2 \mathrm{H}-3,72$ & 12.35 & 12.31 & 11.72 \\
\hline $2 \mathrm{H}-5,59$ & 15.19 & 15.18 & 14.59 \\
\hline $2 \mathrm{H}-5,124$ & 15.77 & 15.84 & 15.24 \\
\hline $2 \mathrm{H}-6,39$ & 16.43 & 16.48 & 15.89 \\
\hline $2 \mathrm{H}-6,110$ & 17.19 & 17.19 & 16.60 \\
\hline $3 \mathrm{H}-1,1$ & 19.36 & 18.95 & 17.51 \\
\hline $3 \mathrm{H}-1,128$ & 20.35 & 20.22 & 18.78 \\
\hline $3 \mathrm{H}-2,120$ & 21.71 & 21.64 & 20.20 \\
\hline $3 \mathrm{H}-3,26$ & 22.26 & 22.20 & 20.76 \\
\hline $3 \mathrm{H}-3,118$ & 23.14 & 23.12 & 21.68 \\
\hline $3 \mathrm{H}-4,102$ & 24.43 & 24.46 & 23.02 \\
\hline $3 \mathrm{H}-5,55$ & 25.52 & 25.49 & 24.05 \\
\hline $3 \mathrm{H}-6,9$ & 26.57 & 26.54 & 25.09 \\
\hline $3 \mathrm{H}-7,0$ & 27.39 & 27.44 & 26.00 \\
\hline $3 \mathrm{H}-7,95$ & 28.45 & 28.39 & 26.95 \\
\hline $4 \mathrm{H}-1,1$ & 29.14 & 29.44 & 27.01 \\
\hline $4 \mathrm{H}-1,17$ & 29.24 & 29.60 & 27.17 \\
\hline $4 \mathrm{H}-1,86$ & 30.06 & 30.29 & 27.86 \\
\hline $4 \mathrm{H}-1,150$ & 30.68 & 30.93 & 28.50 \\
\hline $4 \mathrm{H}-2,80$ & 31.60 & 31.73 & 29.30 \\
\hline $4 \mathrm{H}-2,112$ & 31.82 & 32.05 & 29.62 \\
\hline $4 \mathrm{H}-3,44$ & 32.75 & 32.87 & 30.44 \\
\hline $4 \mathrm{H}-4,139$ & 35.21 & 35.32 & 32.89 \\
\hline $4 \mathrm{H}-5,89$ & 36.22 & 36.32 & 33.89 \\
\hline $4 \mathrm{H}-6,50$ & 37.31 & 37.43 & 35.00 \\
\hline $4 \mathrm{H}-6,114$ & 37.86 & 38.07 & 35.64 \\
\hline $5 \mathrm{H}-1,0$ & 39.92 & 40.05 & 36.50 \\
\hline $5 \mathrm{H}-1,103$ & 40.95 & 41.08 & 37.53 \\
\hline $5 \mathrm{H}-2,11$ & 41.49 & 41.66 & 38.11 \\
\hline $5 \mathrm{H}-2,71$ & 42.16 & 42.26 & 38.71 \\
\hline $5 \mathrm{H}-3,41$ & 43.47 & 43.47 & 39.91 \\
\hline $5 \mathrm{H}-4,89$ & 45.47 & 45.44 & 41.89 \\
\hline $5 \mathrm{H}-5,1$ & 46.21 & 46.06 & 42.51 \\
\hline $5 \mathrm{H}-5,90$ & 47.07 & 46.95 & 43.40 \\
\hline $5 \mathrm{H}-5,106$ & 47.36 & 47.11 & 43.56 \\
\hline $5 \mathrm{H}-5,138$ & 47.76 & 47.44 & 43.88 \\
\hline $5 \mathrm{H}-7,5$ & 49.11 & 48.80 & 45.25 \\
\hline $5 \mathrm{H}-7,106$ & 50.08 & 49.81 & 46.26 \\
\hline $6 \mathrm{H}-1,0$ & 50.96 & 51.25 & 46.00 \\
\hline $6 \mathrm{H}-1,44$ & 51.38 & 51.70 & 46.44 \\
\hline $6 \mathrm{H}-2,1$ & 52.84 & 52.76 & 47.51 \\
\hline $6 \mathrm{H}-2,124$ & 53.70 & 53.99 & 48.74 \\
\hline $6 \mathrm{H}-3,43$ & 54.45 & 54.68 & 49.43 \\
\hline $6 \mathrm{H}-4,12$ & 55.64 & 55.87 & 50.62 \\
\hline $6 \mathrm{H}-5,82$ & 58.02 & 58.07 & 52.82 \\
\hline $6 \mathrm{H}-5,105$ & 58.28 & 58.30 & 53.05 \\
\hline $6 \mathrm{H}-8,9$ & 59.87 & 60.11 & 54.86 \\
\hline $6 \mathrm{H}-8,48$ & 60.21 & 60.50 & 55.25 \\
\hline $7 \mathrm{H}-1,0$ & 62.25 & 61.89 & 55.50 \\
\hline $7 \mathrm{H}-1,136$ & 63.47 & 63.25 & 56.86 \\
\hline $7 \mathrm{H}-2,135$ & 64.83 & 64.75 & 58.35 \\
\hline $7 \mathrm{H}-5,58$ & 68.46 & 68.47 & 62.08 \\
\hline $7 \mathrm{H}-6,71$ & 70.12 & 70.10 & 63.71 \\
\hline $7 \mathrm{H}-7,83$ & 71.24 & 71.32 & 64.93 \\
\hline $8 \mathrm{H}-1,0$ & 71.94 & 71.93 & 65.00 \\
\hline $8 \mathrm{H}-1,124$ & 73.24 & 73.17 & 66.24 \\
\hline $8 \mathrm{H}-2,79$ & 74.23 & 74.22 & 67.29 \\
\hline $8 \mathrm{H}-3,40$ & 75.29 & 75.33 & 68.40 \\
\hline
\end{tabular}

\begin{tabular}{|c|c|c|c|}
\hline \multirow[b]{2}{*}{$\begin{array}{l}\text { Core, section, } \\
\text { interval }(\mathrm{cm})\end{array}$} & \multicolumn{3}{|c|}{ Depth (m) } \\
\hline & $\begin{array}{l}\text { Adjusted } \\
\text { CCSF }\end{array}$ & CCSF & CSF-A \\
\hline $8 \mathrm{H}-4,88$ & 77.28 & 77.31 & 70.38 \\
\hline $8 \mathrm{H}-5,102$ & 78.92 & 78.95 & 72.02 \\
\hline $8 \mathrm{H}-6,30$ & 79.74 & 79.74 & 72.80 \\
\hline $8 \mathrm{H}-6,103$ & 80.37 & 80.46 & 73.53 \\
\hline $9 \mathrm{H}-1,1$ & 82.71 & 82.70 & 74.51 \\
\hline $9 \mathrm{H}-2,10$ & 84.23 & 84.30 & 76.10 \\
\hline $9 \mathrm{H}-3,1$ & 85.73 & 85.71 & 77.51 \\
\hline $9 \mathrm{H}-4,81$ & 88.01 & 88.00 & 79.81 \\
\hline $9 \mathrm{H}-5,108$ & 89.85 & 89.77 & 81.58 \\
\hline $9 \mathrm{H}-5,149$ & 90.28 & 90.18 & 81.99 \\
\hline $9 \mathrm{H}-6,37$ & 90.77 & 90.56 & 82.37 \\
\hline $9 \mathrm{H}-6,110$ & 91.44 & 91.29 & 83.10 \\
\hline $9 \mathrm{H}-7,47$ & 92.05 & 91.77 & 83.58 \\
\hline $9 \mathrm{H}-7,68$ & 92.18 & 91.99 & 83.79 \\
\hline $10 \mathrm{H}-1,0$ & 94.07 & 93.79 & 84.00 \\
\hline $10 \mathrm{H}-2,14$ & 95.59 & 95.43 & 85.64 \\
\hline $10 \mathrm{H}-2,42$ & 95.84 & 95.71 & 85.92 \\
\hline $10 \mathrm{H}-2,67$ & 96.15 & 95.96 & 86.17 \\
\hline $10 \mathrm{H}-2,131$ & 96.67 & 96.60 & 86.81 \\
\hline $10 \mathrm{H}-4,74$ & 98.93 & 99.04 & 89.24 \\
\hline $10 \mathrm{H}-5,29$ & 99.97 & 100.08 & 90.29 \\
\hline $10 \mathrm{H}-5,130$ & 100.95 & 101.09 & 91.30 \\
\hline $10 \mathrm{H}-6,32$ & 101.43 & 101.61 & 91.82 \\
\hline $10 \mathrm{H}-7,101$ & 103.55 & 103.50 & 93.71 \\
\hline $11 \mathrm{H}-1,0$ & 105.18 & 105.16 & 93.50 \\
\hline $11 \mathrm{H}-1,88$ & 106.14 & 106.04 & 94.38 \\
\hline $11 \mathrm{H}-3,39$ & 108.64 & 108.55 & 96.89 \\
\hline $11 \mathrm{H}-3,114$ & 109.35 & 109.30 & 97.64 \\
\hline $11 \mathrm{H}-5,0$ & 111.25 & 111.16 & 99.50 \\
\hline $11 \mathrm{H}-5,71$ & 111.99 & 111.87 & 100.21 \\
\hline $11 \mathrm{H}-5,149$ & 112.73 & 112.65 & 100.99 \\
\hline $11 \mathrm{H}-7,42$ & 114.61 & 114.58 & 102.92 \\
\hline $11 \mathrm{H}-7,77$ & 114.94 & 114.94 & 103.27 \\
\hline $12 \mathrm{H}-1,34$ & 115.82 & 115.79 & 103.34 \\
\hline $12 \mathrm{H}-2,131$ & 118.27 & 118.26 & 105.81 \\
\hline $12 \mathrm{H}-3,84$ & 119.30 & 119.30 & 106.84 \\
\hline $12 \mathrm{H}-4,86$ & 120.76 & 120.81 & 108.36 \\
\hline $12 \mathrm{H}-4,137$ & 121.24 & 121.32 & 108.87 \\
\hline $12 \mathrm{H}-5,138$ & 122.82 & 122.83 & 110.38 \\
\hline $12 \mathrm{H}-6,36$ & 123.22 & 123.31 & 110.86 \\
\hline $12 \mathrm{H}-6,109$ & 124.10 & 124.04 & 111.59 \\
\hline $12 \mathrm{H}-7,39$ & 124.62 & 124.44 & 111.99 \\
\hline $12 \mathrm{H}-7,69$ & 124.75 & 124.74 & 112.29 \\
\hline $13 \mathrm{H}-1,0$ & 126.85 & 126.81 & 112.50 \\
\hline $13 \mathrm{H}-1,43$ & 127.23 & 127.24 & 112.93 \\
\hline $13 \mathrm{H}-1,133$ & 128.11 & 128.14 & 113.83 \\
\hline $13 \mathrm{H}-2,105$ & 129.38 & 129.36 & 115.05 \\
\hline $13 \mathrm{H}-3,67$ & 130.50 & 130.48 & 116.17 \\
\hline $13 \mathrm{H}-5,24$ & 133.05 & 133.05 & 118.74 \\
\hline $13 \mathrm{H}-5,119$ & 133.97 & 134.00 & 119.69 \\
\hline $13 \mathrm{H}-5,135$ & 134.16 & 134.16 & 119.85 \\
\hline $13 \mathrm{H}-6,42$ & 134.67 & 134.73 & 120.42 \\
\hline $13 \mathrm{H}-6,120$ & 135.53 & 135.51 & 121.20 \\
\hline $13 \mathrm{H}-7,54$ & 136.32 & 136.35 & 122.04 \\
\hline $13 \mathrm{H}-7,75$ & 136.54 & 136.56 & 122.25 \\
\hline $14 \mathrm{H}-1,1$ & 137.26 & 137.30 & 122.01 \\
\hline $14 \mathrm{H}-1,105$ & 138.34 & 138.34 & 123.05 \\
\hline $14 \mathrm{H}-4,99$ & 142.78 & 142.78 & 127.49 \\
\hline $14 \mathrm{H}-5,116$ & 144.58 & 144.45 & 129.16 \\
\hline $14 \mathrm{H}-6,55$ & 145.49 & 145.34 & 130.05 \\
\hline $14 \mathrm{H}-7,20$ & 146.23 & 145.99 & 130.70 \\
\hline $14 \mathrm{H}-7,63$ & 146.62 & 146.42 & 131.13 \\
\hline $15 \mathrm{H}-1,0$ & 147.95 & 147.95 & 131.50 \\
\hline $15 \mathrm{H}-2,35$ & 149.81 & 149.80 & 133.35 \\
\hline $15 \mathrm{H}-3,5$ & 150.88 & 151.00 & 134.55 \\
\hline $15 \mathrm{H}-3,69$ & 151.53 & 151.64 & 135.19 \\
\hline $15 \mathrm{H}-3,140$ & 152.18 & 152.35 & 135.90 \\
\hline $15 \mathrm{H}-4,63$ & 153.12 & 153.08 & 136.63 \\
\hline
\end{tabular}

\begin{tabular}{|c|c|c|c|}
\hline \multirow[b]{2}{*}{$\begin{array}{l}\text { Core, section, } \\
\text { interval }(\mathrm{cm})\end{array}$} & \multicolumn{3}{|c|}{ Depth $(m)$} \\
\hline & $\begin{array}{l}\text { Adjusted } \\
\text { CCSF }\end{array}$ & CCSF & CSF-A \\
\hline $15 \mathrm{H}-5,81$ & 154.96 & 154.76 & 138.31 \\
\hline $15 \mathrm{H}-5,111$ & 155.32 & 155.06 & 138.61 \\
\hline $15 \mathrm{H}-6,32$ & 156.09 & 155.77 & 139.32 \\
\hline $15 \mathrm{H}-7,26$ & 157.53 & 157.21 & 140.76 \\
\hline $15 \mathrm{H}-7,81$ & 158.08 & 157.76 & 141.31 \\
\hline $16 \mathrm{H}-1,1$ & 159.09 & 159.16 & 141.01 \\
\hline $16 \mathrm{H}-1,46$ & 159.47 & 159.62 & 141.46 \\
\hline $16 \mathrm{H}-1,89$ & 159.87 & 160.04 & 141.89 \\
\hline $16 \mathrm{H}-1,138$ & 160.38 & 160.53 & 142.38 \\
\hline $16 \mathrm{H}-2,54$ & 161.03 & 161.19 & 143.04 \\
\hline $16 \mathrm{H}-3,79$ & 162.84 & 162.95 & 144.79 \\
\hline $16 \mathrm{H}-4,93$ & 164.51 & 164.58 & 146.43 \\
\hline $16 \mathrm{H}-5,55$ & 165.80 & 165.70 & 147.55 \\
\hline $16 \mathrm{H}-6,20$ & 167.05 & 166.85 & 148.70 \\
\hline $16 \mathrm{H}-6,106$ & 167.89 & 167.71 & 149.56 \\
\hline $16 \mathrm{H}-6,154$ & 168.33 & 168.19 & 150.04 \\
\hline $17 \mathrm{H}-1,1$ & 169.72 & 169.64 & 150.51 \\
\hline $17 \mathrm{H}-1,34$ & 170.06 & 169.97 & 150.84 \\
\hline $17 \mathrm{H}-2,124$ & 172.47 & 172.37 & 153.24 \\
\hline $17 \mathrm{H}-3,53$ & 173.27 & 173.16 & 154.03 \\
\hline $17 \mathrm{H}-3,115$ & 173.83 & 173.78 & 154.65 \\
\hline $17 \mathrm{H}-4,91$ & 175.02 & 175.04 & 155.91 \\
\hline $17 \mathrm{H}-5,136$ & 177.05 & 176.99 & 157.86 \\
\hline $17 \mathrm{H}-7,38$ & 178.79 & 178.71 & 159.58 \\
\hline $17 \mathrm{H}-7,75$ & 179.13 & 179.08 & 159.95 \\
\hline $17 \mathrm{H}-7,110$ & 179.52 & 179.43 & 160.30 \\
\hline $18 \mathrm{H}-1,1$ & 179.88 & 179.95 & 160.01 \\
\hline $18 \mathrm{H}-1,39$ & 180.36 & 180.33 & 160.39 \\
\hline $18 \mathrm{H}-1,94$ & 180.87 & 180.88 & 160.94 \\
\hline $18 \mathrm{H}-2,19$ & 181.66 & 181.63 & 161.69 \\
\hline $18 \mathrm{H}-2,118$ & 182.61 & 182.62 & 162.68 \\
\hline $18 \mathrm{H}-4,31$ & 184.67 & 184.75 & 164.81 \\
\hline $18 \mathrm{H}-4,81$ & 185.28 & 185.25 & 165.31 \\
\hline $18 \mathrm{H}-5,11$ & 186.04 & 186.05 & 166.11 \\
\hline $18 \mathrm{H}-5,52$ & 186.53 & 186.46 & 166.52 \\
\hline $18 \mathrm{H}-5,100$ & 186.99 & 186.94 & 167.00 \\
\hline $18 \mathrm{H}-6,24$ & 187.66 & 187.68 & 167.74 \\
\hline $18 \mathrm{H}-7,45$ & 189.09 & 189.09 & 169.15 \\
\hline $18 \mathrm{H}-7,103$ & 189.74 & 189.67 & 169.73 \\
\hline $19 \mathrm{H}-1,0$ & 190.00 & 190.03 & 169.50 \\
\hline $19 \mathrm{H}-1,33$ & 190.30 & 190.36 & 169.83 \\
\hline $19 \mathrm{H}-2,2$ & 191.48 & 191.55 & 171.02 \\
\hline $19 \mathrm{H}-2,148$ & 193.06 & 193.01 & 172.48 \\
\hline $19 \mathrm{H}-3,90$ & 193.88 & 193.93 & 173.40 \\
\hline $19 \mathrm{H}-4,14$ & 194.68 & 194.67 & 174.14 \\
\hline $19 \mathrm{H}-5,78$ & 196.87 & 196.81 & 176.28 \\
\hline $19 \mathrm{H}-6,124$ & 198.98 & 198.78 & 178.24 \\
\hline $19 \mathrm{H}-7,58$ & 199.66 & 199.41 & 178.88 \\
\hline $19 \mathrm{H}-7,92$ & 199.96 & 199.75 & 179.22 \\
\hline $20 \mathrm{H}-1,0$ & 200.32 & 199.87 & 179.00 \\
\hline $20 \mathrm{H}-1,45$ & 200.73 & 200.31 & 179.45 \\
\hline $20 \mathrm{H}-1,84$ & 201.11 & 200.71 & 179.84 \\
\hline $20 \mathrm{H}-2,15$ & 201.81 & 201.51 & 180.65 \\
\hline $20 \mathrm{H}-2,51$ & 202.17 & 201.87 & 181.01 \\
\hline $20 \mathrm{H}-2,132$ & 202.93 & 202.68 & 181.82 \\
\hline $20 \mathrm{H}-4,4$ & 204.43 & 204.40 & 183.54 \\
\hline $20 \mathrm{H}-4,89$ & 205.25 & 205.25 & 184.39 \\
\hline $20 \mathrm{H}-6,18$ & 207.57 & 207.54 & 186.68 \\
\hline $20 \mathrm{H}-6,90$ & 208.26 & 208.27 & 187.40 \\
\hline $20 \mathrm{H}-7,23$ & 209.18 & 209.10 & 188.23 \\
\hline $20 \mathrm{H}-7,48$ & 209.54 & 209.34 & 188.48 \\
\hline $20 \mathrm{H}-7,79$ & 209.78 & 209.65 & 188.79 \\
\hline $21 \mathrm{H}-1,104$ & 210.35 & 210.18 & 189.54 \\
\hline $21 \mathrm{H}-2,108$ & 211.63 & 211.72 & 191.08 \\
\hline $21 \mathrm{H}-3,32$ & 212.33 & 212.46 & 191.82 \\
\hline $21 \mathrm{H}-3,150$ & 213.38 & 213.64 & 193.00 \\
\hline $21 \mathrm{H}-5,0$ & 214.66 & 214.94 & 194.30 \\
\hline $21 \mathrm{H}-5,71$ & 215.31 & 215.65 & 195.01 \\
\hline
\end{tabular}


Table AT38 (continued).

\begin{tabular}{|c|c|c|c|}
\hline \multirow[b]{2}{*}{$\begin{array}{l}\text { Core, section, } \\
\text { interval }(\mathrm{cm})\end{array}$} & \multicolumn{3}{|c|}{ Depth $(m)$} \\
\hline & $\begin{array}{c}\text { Adjusted } \\
\text { CCSF }\end{array}$ & CCSF & CSF-A \\
\hline $22 \mathrm{H}-1,24$ & 216.35 & 216.35 & 193.74 \\
\hline $22 \mathrm{H}-1,49$ & 216.60 & 216.60 & 193.99 \\
\hline $22 \mathrm{H}-2,59$ & 218.21 & 218.21 & 195.59 \\
\hline $22 \mathrm{H}-2,124$ & 218.76 & 218.85 & 196.24 \\
\hline $22 \mathrm{H}-4,6$ & 220.67 & 220.67 & 198.06 \\
\hline $23 \mathrm{H}-1,17$ & 224.10 & 224.21 & 199.87 \\
\hline $23 \mathrm{H}-1,64$ & 224.62 & 224.68 & 200.34 \\
\hline $23 \mathrm{H}-2,18$ & 225.69 & 225.72 & 201.38 \\
\hline $23 \mathrm{H}-3,95$ & 228.23 & 227.99 & 203.65 \\
\hline $23 \mathrm{H}-5,64$ & 230.89 & 230.68 & 206.34 \\
\hline $23 \mathrm{H}-6,68$ & 232.50 & 232.22 & 207.88 \\
\hline $23 \mathrm{H}-6,132$ & 233.07 & 232.86 & 208.52 \\
\hline $23 \mathrm{H}-7,61$ & 233.77 & 233.65 & 209.31 \\
\hline $24 \mathrm{H}-1,1$ & 235.45 & 235.57 & 209.21 \\
\hline $24 \mathrm{H}-1,65$ & 236.10 & 236.21 & 209.85 \\
\hline $24 \mathrm{H}-1,125$ & 236.73 & 236.81 & 210.45 \\
\hline $24 \mathrm{H}-2,97$ & 237.98 & 238.01 & 211.65 \\
\hline $24 \mathrm{H}-3,78$ & 239.33 & 239.32 & 212.96 \\
\hline $24 \mathrm{H}-9,5$ & 245.00 & 245.01 & 218.65 \\
\hline $24 \mathrm{H}-9,27$ & 245.20 & 245.23 & 218.87 \\
\hline $25 \mathrm{H}-2,32$ & 246.35 & 246.35 & 220.52 \\
\hline $25 \mathrm{H}-3,2$ & 247.27 & 247.54 & 221.72 \\
\hline $25 \mathrm{H}-3,135$ & 248.56 & 248.87 & 223.05 \\
\hline $25 \mathrm{H}-4,65$ & 249.27 & 249.67 & 223.85 \\
\hline $25 \mathrm{H}-5,48$ & 250.58 & 251.00 & 225.18 \\
\hline $25 \mathrm{H}-5,88$ & 250.86 & 251.40 & 225.58 \\
\hline $25 \mathrm{H}-5,122$ & 251.21 & 251.74 & 225.92 \\
\hline $25 \mathrm{H}-7,18$ & 252.37 & 253.21 & 227.39 \\
\hline $25 \mathrm{H}-7,79$ & 252.98 & 253.82 & 228.00 \\
\hline $26 \mathrm{H}-1,144$ & 254.93 & 256.26 & 229.64 \\
\hline $26 \mathrm{H}-3,1$ & 257.02 & 257.83 & 231.21 \\
\hline $26 \mathrm{H}-3,50$ & 257.75 & 258.33 & 231.70 \\
\hline $26 \mathrm{H}-5,27$ & 261.00 & 261.09 & 234.47 \\
\hline $26 \mathrm{H}-5,55$ & 261.47 & 261.37 & 234.75 \\
\hline $26 \mathrm{H}-6,43$ & 263.08 & 262.76 & 236.13 \\
\hline $26 \mathrm{H}-7,11$ & 263.71 & 263.43 & 236.81 \\
\hline $26 \mathrm{H}-7,53$ & 264.00 & 263.85 & 237.23 \\
\hline $27 X-1,0$ & 265.02 & 264.92 & 237.70 \\
\hline $27 X-1,33$ & 265.34 & 265.25 & 238.03 \\
\hline $27 X-1,102$ & 265.86 & 265.94 & 238.72 \\
\hline $27 X-2,44$ & 266.45 & 266.61 & 239.39 \\
\hline $27 X-2,81$ & 266.82 & 266.98 & 239.76 \\
\hline $28 \mathrm{H}-1,133$ & 269.35 & 269.35 & 242.73 \\
\hline $28 \mathrm{H}-4,72$ & 273.24 & 273.24 & 246.62 \\
\hline $28 \mathrm{H}-4,138$ & 273.89 & 273.90 & 247.28 \\
\hline $28 \mathrm{H}-5,80$ & 274.94 & 274.82 & 248.20 \\
\hline $28 \mathrm{H}-6,48$ & 276.17 & 276.00 & 249.38 \\
\hline $28 \mathrm{H}-6,107$ & 276.76 & 276.59 & 249.97 \\
\hline $29 \mathrm{H}-1,43$ & 277.41 & 277.30 & 248.43 \\
\hline $29 \mathrm{H}-2,2$ & 278.78 & 278.39 & 249.52 \\
\hline $29 \mathrm{H}-2,31$ & 278.79 & 278.68 & 249.81 \\
\hline $29 \mathrm{H}-2,79$ & 279.23 & 279.17 & 250.29 \\
\hline $29 \mathrm{H}-2,114$ & 279.51 & 279.51 & 250.64 \\
\hline $29 \mathrm{H}-5,48$ & 283.35 & 283.35 & 254.48 \\
\hline $29 \mathrm{H}-6,55$ & 284.92 & 284.92 & 256.05 \\
\hline $29 \mathrm{H}-6,121$ & 285.66 & 285.58 & 256.71 \\
\hline $29 \mathrm{H}-7,89$ & 286.78 & 286.76 & 257.89 \\
\hline
\end{tabular}

\begin{tabular}{|c|c|c|c|}
\hline \multirow[b]{2}{*}{$\begin{array}{l}\text { Core, section, } \\
\text { interval }(\mathrm{cm})\end{array}$} & \multicolumn{3}{|c|}{ Depth $(\mathrm{m})$} \\
\hline & $\begin{array}{l}\text { Adjusted } \\
\text { CCSF }\end{array}$ & CCSF & CSF-A \\
\hline $30 \mathrm{H}-1,0$ & 287.29 & 287.44 & 257.50 \\
\hline $30 \mathrm{H}-1,76$ & 288.06 & 288.21 & 258.26 \\
\hline $30 \mathrm{H}-1,135$ & 288.76 & 288.79 & 258.85 \\
\hline $30 \mathrm{H}-3,1$ & 290.45 & 290.45 & 260.51 \\
\hline $30 \mathrm{H}-6,105$ & 295.99 & 295.99 & 266.05 \\
\hline $31 X-1,0$ & 301.65 & 301.69 & 267.00 \\
\hline $31 X-1,53$ & 302.18 & 302.22 & 267.53 \\
\hline $31 X-1,107$ & 302.96 & 302.76 & 268.07 \\
\hline $31 X-2,22$ & 303.41 & 303.41 & 268.72 \\
\hline $31 X-4,134$ & 307.53 & 307.53 & 272.84 \\
\hline $31 X-5,49$ & 308.23 & 308.18 & 273.49 \\
\hline $31 X-6,1$ & 308.92 & 308.70 & 274.01 \\
\hline $31 X-6,71$ & 309.62 & 309.40 & 274.71 \\
\hline $32 X-1,0$ & 310.36 & 310.51 & 276.20 \\
\hline $32 X-1,53$ & 311.04 & 311.04 & 276.73 \\
\hline $32 X-4,84$ & 315.85 & 315.85 & 281.54 \\
\hline $32 X-4,101$ & 316.11 & 316.03 & 281.71 \\
\hline $32 X-4,150$ & 316.48 & 316.51 & 282.20 \\
\hline $33 X-2,32$ & 320.05 & 321.54 & 287.62 \\
\hline $33 X-2,108$ & 320.81 & 322.30 & 288.38 \\
\hline $33 X-3,73$ & 322.39 & 323.45 & 289.53 \\
\hline $33 X-4,63$ & 324.09 & 324.85 & 290.93 \\
\hline $33 X-5,60$ & 326.21 & 326.32 & 292.40 \\
\hline $33 X-6,119$ & 327.96 & 328.41 & 294.49 \\
\hline $33 X-7,54$ & 328.50 & 328.97 & 295.04 \\
\hline $34 \mathrm{X}-1,79$ & 329.31 & 329.33 & 296.09 \\
\hline $34 X-1,144$ & 329.88 & 329.98 & 296.74 \\
\hline $34 \mathrm{X}-2,22$ & 330.34 & 330.26 & 297.02 \\
\hline $34 X-3,10$ & 330.90 & 331.64 & 298.40 \\
\hline $34 X-4,49$ & 333.35 & 333.53 & 300.29 \\
\hline $34 X-5,64$ & 334.60 & 335.18 & 301.94 \\
\hline $34 X-6,95$ & 337.31 & 336.99 & 303.75 \\
\hline $34 X-7,86$ & 338.02 & 338.00 & 304.76 \\
\hline $35 X-1,13$ & 338.97 & 338.94 & 305.03 \\
\hline $35 X-2,1$ & 340.64 & 340.32 & 306.41 \\
\hline $35 X-3,33$ & 341.76 & 342.14 & 308.23 \\
\hline $35 X-4,40$ & 342.94 & 343.71 & 309.80 \\
\hline $35 X-6,1$ & 345.04 & 345.82 & 311.91 \\
\hline $35 X-6,91$ & 345.93 & 346.72 & 312.81 \\
\hline $36 X-1,1$ & 348.34 & 348.50 & 314.51 \\
\hline $36 X-1,83$ & 348.76 & 349.32 & 315.33 \\
\hline $36 X-1,139$ & 349.64 & 349.88 & 315.89 \\
\hline $36 \mathrm{X}-2,39$ & 350.12 & 350.38 & 316.39 \\
\hline $36 \mathrm{X}-2,93$ & 350.92 & 350.92 & 316.93 \\
\hline $36 X-5,136$ & 355.85 & 355.85 & 321.86 \\
\hline $36 X-6,22$ & 356.61 & 356.21 & 322.22 \\
\hline $36 X-7,1$ & 357.50 & 356.95 & 322.96 \\
\hline $37 X-1,2$ & 358.35 & 358.31 & 324.12 \\
\hline $37 X-1,96$ & 359.23 & 359.25 & 325.06 \\
\hline $37 X-3,144$ & 362.89 & 362.73 & 328.54 \\
\hline $37 X-5,76$ & 365.31 & 365.05 & 330.86 \\
\hline $37 X-6,84$ & 366.48 & 366.63 & 332.44 \\
\hline $37 X-7,80$ & 367.65 & 367.70 & 333.50 \\
\hline $38 X-1,10$ & 368.41 & 368.27 & 333.70 \\
\hline $38 \mathrm{X}-1,79$ & 369.17 & 368.95 & 334.39 \\
\hline $38 \mathrm{X}-2,98$ & 370.62 & 370.64 & 336.08 \\
\hline $38 X-3,2$ & & 71.18 & 36 \\
\hline
\end{tabular}

\begin{tabular}{|c|c|c|c|}
\hline \multirow[b]{2}{*}{$\begin{array}{l}\text { Core, section, } \\
\text { interval }(\mathrm{cm})\end{array}$} & \multicolumn{3}{|c|}{ Depth $(\mathrm{m})$} \\
\hline & $\begin{array}{l}\text { Adjusted } \\
\text { CCSF }\end{array}$ & CCSF & CSF-A \\
\hline $38 X-3,80$ & 372.04 & 371.96 & 337.40 \\
\hline $38 X-3,131$ & 372.57 & 372.47 & 337.91 \\
\hline $38 X-4,138$ & 374.45 & 374.04 & 339.48 \\
\hline $38 X-5,91$ & 375.81 & 375.07 & 340.51 \\
\hline $38 X-6,94$ & 377.24 & 376.60 & 342.04 \\
\hline $38 X-7,69$ & 378.06 & 377.35 & 342.79 \\
\hline $39 X-1,5$ & 379.31 & 379.33 & 343.25 \\
\hline $39 X-1,52$ & 379.82 & 379.80 & 343.72 \\
\hline $39 X-1,99$ & 380.27 & 380.27 & 344.19 \\
\hline $39 X-5,105$ & 386.33 & 386.33 & 350.25 \\
\hline $39 \times-6,96$ & 387.38 & 387.74 & 351.66 \\
\hline 39X-7, 61 & 387.89 & 388.49 & 352.41 \\
\hline $40 X-1,1$ & 389.82 & 389.57 & 352.81 \\
\hline $40 X-1,107$ & 390.88 & 390.63 & 353.87 \\
\hline $40 \times-2,35$ & 391.41 & 391.41 & 354.65 \\
\hline $40 X-6,19$ & 397.25 & 397.25 & 360.49 \\
\hline $40 X-6,51$ & 397.78 & 397.57 & 360.81 \\
\hline $40 X-7,16$ & 398.64 & 398.42 & 361.66 \\
\hline $40 X-7,72$ & 399.11 & 398.98 & 362.22 \\
\hline $41 X-1,7$ & 400.80 & 400.75 & 362.47 \\
\hline $41 X-3,146$ & 405.74 & 405.14 & 366.86 \\
\hline $41 X-6,34$ & 408.36 & 408.52 & 370.24 \\
\hline $41 X-7,81$ & 409.96 & 410.00 & 371.71 \\
\hline $42 X-1,27$ & 413.00 & 413.21 & 372.27 \\
\hline $42 X-2,64$ & 414.57 & 415.07 & 374.14 \\
\hline $42 X-4,127$ & 418.60 & 418.70 & 377.77 \\
\hline $42 X-5,45$ & 419.46 & 419.38 & 378.45 \\
\hline $42 X-7,58$ & 423.30 & 422.12 & 381.18 \\
\hline $43 X-1,3$ & 424.23 & 424.11 & 381.63 \\
\hline $43 X-1,145$ & 425.52 & 425.52 & 383.05 \\
\hline $43 X-6,35$ & 431.92 & 431.92 & 389.45 \\
\hline $43 X-6,101$ & 432.77 & 432.58 & 390.11 \\
\hline $43 X-7,79$ & 433.63 & 433.46 & 390.99 \\
\hline $44 X-1,59$ & 447.34 & 447.22 & 391.79 \\
\hline $44 X-2,7$ & 448.82 & 448.20 & 392.77 \\
\hline $44 X-2,116$ & 449.23 & 449.29 & 393.86 \\
\hline $44 X-3,105$ & 450.68 & 450.68 & 395.25 \\
\hline $44 X-4,85$ & 451.93 & 451.98 & 396.55 \\
\hline $44 X-5,107$ & 453.65 & 453.70 & 398.27 \\
\hline $44 X-6,134$ & 455.43 & 455.47 & 400.04 \\
\hline $45 X-1,0$ & 455.90 & 455.90 & 400.50 \\
\hline $45 X-1,30$ & 456.20 & 456.20 & 400.80 \\
\hline $45 X-6,1$ & 463.41 & 463.41 & 408.01 \\
\hline $45 X-7,70$ & 464.62 & 465.20 & 409.80 \\
\hline $46 X-1,29$ & 467.66 & 467.66 & 410.39 \\
\hline $46 X-2,32$ & 469.19 & 469.19 & 411.92 \\
\hline $46 \mathrm{X}-4,44$ & 472.31 & 472.31 & 415.04 \\
\hline $46 X-5,14$ & 474.17 & 473.51 & 416.24 \\
\hline $46 X-5,85$ & 474.81 & 474.22 & 416.95 \\
\hline $47 X-1,0$ & 476.76 & 476.76 & 419.60 \\
\hline $47 X-3,111$ & 480.87 & 480.87 & 423.71 \\
\hline $48 X-1,0$ & 485.83 & 485.83 & 429.10 \\
\hline $48 X-5,63$ & 492.06 & 492.06 & 435.33 \\
\hline $49 X-1,0$ & 495.53 & 495.53 & 438.80 \\
\hline $49 X-3,32$ & 498.37 & 498.37 & 441.64 \\
\hline
\end{tabular}

This table is also available in ASCII. 
Table AT39. Gamma ray attenuation (GRA) density data, Site U1338.

\begin{tabular}{|c|c|c|c|}
\hline \multirow{2}{*}{$\begin{array}{l}\text { Hole, core, section, } \\
\text { interval }(\mathrm{cm})\end{array}$} & \multicolumn{2}{|c|}{ Depth $(\mathrm{m})$} & \multirow{2}{*}{$\begin{array}{c}\text { GRA } \\
\left(\mathrm{g} / \mathrm{cm}^{3}\right)\end{array}$} \\
\hline & CSF & CCSF & \\
\hline \multicolumn{4}{|l|}{$321-$} \\
\hline U1338A-1H-1, 2.5 & 0.03 & 0.07 & 1.36 \\
\hline U1338A-1H-1, 5 & 0.05 & 0.09 & 1.33 \\
\hline U1338A-1H-1, 7.5 & 0.08 & 0.12 & 1.35 \\
\hline U1338A-1H-1, 12.5 & 0.13 & 0.17 & 1.38 \\
\hline U1338A-1H-1, 15 & 0.15 & 0.19 & 1.38 \\
\hline U1338A-1H-1, 17.5 & 0.18 & 0.22 & 1.37 \\
\hline U1338A-1H-1, 20 & 0.20 & 0.24 & 1.37 \\
\hline U1338A-1H-1, 22.5 & 0.23 & 0.27 & 1.32 \\
\hline U1338A-1H-1, 25 & 0.25 & 0.29 & 1.36 \\
\hline U1338A-1H-1, 27.6 & 0.28 & 0.32 & 1.35 \\
\hline U1338A-1H-1, 30.1 & 0.30 & 0.34 & 1.38 \\
\hline U1338A-1H-1, 32.6 & 0.33 & 0.37 & 1.38 \\
\hline U1338A-1H-1, 35.1 & 0.35 & 0.39 & 1.36 \\
\hline U1338A-1H-1, 37.6 & 0.38 & 0.42 & 1.36 \\
\hline U1338A-1H-1, 40.1 & 0.40 & 0.44 & 1.34 \\
\hline U1338A-1H-1, 42.6 & 0.43 & 0.47 & 1.35 \\
\hline U1338A-1H-1, 45.1 & 0.45 & 0.49 & 1.39 \\
\hline U1338A-1H-1, 47.6 & 0.48 & 0.52 & 1.38 \\
\hline U1338A-1H-1, 50.1 & 0.50 & 0.54 & 1.35 \\
\hline U1338A-1H-1, 52.6 & 0.53 & 0.57 & 1.39 \\
\hline U1338A-1H-1, 55.1 & 0.55 & 0.59 & 1.30 \\
\hline U1338A-1H-1, 57.6 & 0.58 & 0.62 & 1.33 \\
\hline U1338A-1H-1, 60.1 & 0.60 & 0.64 & 1.39 \\
\hline U1338A-1H-1, 62.6 & 0.63 & 0.67 & 1.40 \\
\hline U1338A-1H-1, 65.1 & 0.65 & 0.69 & 1.37 \\
\hline U1338A-1H-1, 67.6 & 0.68 & 0.72 & 1.42 \\
\hline U1338A-1H-1, 70.1 & 0.70 & 0.74 & 1.39 \\
\hline U1338A-1H-1, 72.6 & 0.73 & 0.77 & 1.40 \\
\hline U1338A-1H-1, 75.1 & 0.75 & 0.79 & 1.42 \\
\hline U1338A-1H-1, 77.6 & 0.78 & 0.82 & 1.41 \\
\hline U1338A-1H-1, 80.1 & 0.80 & 0.84 & 1.38 \\
\hline U1338A-1H-1, 82.6 & 0.83 & 0.87 & 1.39 \\
\hline U1338A-1H-1, 85.1 & 0.85 & 0.89 & 1.40 \\
\hline U1338A-1H-1, 87.7 & 0.88 & 0.92 & 1.40 \\
\hline U1338A-1H-1, 90.2 & 0.90 & 0.94 & 1.39 \\
\hline U1338A-1H-1, 92.7 & 0.93 & 0.97 & 1.36 \\
\hline U1338A-1H-1, 95.2 & 0.95 & 0.99 & 1.39 \\
\hline U1338A-1H-1, 97.7 & 0.98 & 1.02 & 1.40 \\
\hline U1338A-1H-1, 100.2 & 1.00 & 1.04 & 1.41 \\
\hline U1338A-1H-1, 102.7 & 1.03 & 1.07 & 1.35 \\
\hline U1338A-1H-1, 105.2 & 1.05 & 1.09 & 1.40 \\
\hline U1338A-1H-1, 107.7 & 1.08 & 1.12 & 1.36 \\
\hline U1338A-1H-1, 110.2 & 1.10 & 1.14 & 1.38 \\
\hline U1338A-1H-1, 112.7 & 1.13 & 1.17 & 1.37 \\
\hline U1338A-1H-1, 115.2 & 1.15 & 1.19 & 1.33 \\
\hline U1338A-1H-1, 117.7 & 1.18 & 1.22 & 1.34 \\
\hline U1338A-1H-1, 120.2 & 1.20 & 1.24 & 1.34 \\
\hline U1338A-1H-1, 122.7 & 1.23 & 1.27 & 1.30 \\
\hline U1338A-1H-1, 125.2 & 1.25 & 1.29 & 1.28 \\
\hline U1338A-1H-1, 127.7 & 1.28 & 1.32 & 1.28 \\
\hline U1338A-1H-1, 130.2 & 1.30 & 1.34 & 1.24 \\
\hline U1338A-1H-1, 132.7 & 1.33 & 1.37 & 1.23 \\
\hline U1338A-1H-1, 135.2 & 1.35 & 1.39 & 1.25 \\
\hline U1338A-1H-1, 137.7 & 1.38 & 1.42 & 1.24 \\
\hline U1338A-1H-1, 140.3 & 1.40 & 1.44 & 1.27 \\
\hline U1338A-1H-1, 142.8 & 1.43 & 1.47 & 1.26 \\
\hline U1338A-1H-2, 5.4 & 1.55 & 1.59 & 1.24 \\
\hline U1338A-1 H-2, 7.9 & 1.58 & 1.62 & 1.25 \\
\hline U1338A-1H-2, 10.4 & 1.60 & 1.64 & 1.26 \\
\hline U1338A-1H-2, 12.9 & 1.63 & 1.67 & 1.25 \\
\hline U1338A-1H-2, 15.4 & 1.65 & 1.69 & 1.23 \\
\hline U1338A-1H-2, 17.9 & 1.68 & 1.72 & 1.24 \\
\hline
\end{tabular}

Only a portion of this table appears here. The complete table is available in ASCII. 
Table AT40. Magnetic susceptibility data, Site U1338.

\begin{tabular}{|c|c|c|c|}
\hline \multirow{2}{*}{$\begin{array}{l}\text { Core, section, } \\
\text { interval }(\mathrm{cm})\end{array}$} & \multicolumn{2}{|c|}{ Depth (m) } & \multirow{2}{*}{$\begin{array}{l}\text { Magnetic } \\
\text { susceptibility } \\
\text { (IU) }\end{array}$} \\
\hline & CSF & CCSF & \\
\hline \multicolumn{4}{|l|}{ 321-U1338A- } \\
\hline $1 \mathrm{H}-1,0.1$ & 0.00 & 0.04 & 0.0 \\
\hline $1 \mathrm{H}-1,2.6$ & 0.03 & 0.07 & 1.4 \\
\hline $1 \mathrm{H}-1,5.1$ & 0.05 & 0.09 & 2.2 \\
\hline $1 \mathrm{H}-1,7.6$ & 0.08 & 0.12 & 2.2 \\
\hline $1 \mathrm{H}-1,10.1$ & 0.10 & 0.14 & 1.9 \\
\hline $1 \mathrm{H}-1,12.6$ & 0.13 & 0.17 & 1.4 \\
\hline $1 \mathrm{H}-1,15.1$ & 0.15 & 0.19 & 1.1 \\
\hline $1 \mathrm{H}-1,17.6$ & 0.18 & 0.22 & 1.4 \\
\hline $1 \mathrm{H}-1,20.1$ & 0.20 & 0.24 & 1.4 \\
\hline $1 \mathrm{H}-1,22.6$ & 0.23 & 0.27 & 2.5 \\
\hline $1 \mathrm{H}-1,25.1$ & 0.25 & 0.29 & 2.2 \\
\hline $1 \mathrm{H}-1,27.6$ & 0.28 & 0.32 & 1.6 \\
\hline $1 \mathrm{H}-1,30.1$ & 0.30 & 0.34 & 1.9 \\
\hline $1 \mathrm{H}-1,32.6$ & 0.33 & 0.37 & 1.1 \\
\hline $1 \mathrm{H}-1,35.1$ & 0.35 & 0.39 & 1.4 \\
\hline $1 \mathrm{H}-1,37.6$ & 0.38 & 0.42 & 0.3 \\
\hline $1 \mathrm{H}-1,40.1$ & 0.40 & 0.44 & 0.0 \\
\hline $1 \mathrm{H}-1,42.6$ & 0.43 & 0.47 & 0.3 \\
\hline $1 \mathrm{H}-1,45.1$ & 0.45 & 0.49 & 0.3 \\
\hline $1 \mathrm{H}-1,47.6$ & 0.48 & 0.52 & 0.3 \\
\hline $1 \mathrm{H}-1,50.1$ & 0.50 & 0.54 & 0.8 \\
\hline $1 \mathrm{H}-1,52.7$ & 0.53 & 0.57 & 0.0 \\
\hline $1 \mathrm{H}-1,55.2$ & 0.55 & 0.59 & 0.0 \\
\hline $1 \mathrm{H}-1,57.7$ & 0.58 & 0.62 & -0.3 \\
\hline $1 \mathrm{H}-1,60.2$ & 0.60 & 0.64 & -0.3 \\
\hline $1 \mathrm{H}-1,62.7$ & 0.63 & 0.67 & -0.5 \\
\hline $1 \mathrm{H}-1,65.2$ & 0.65 & 0.69 & -0.3 \\
\hline $1 \mathrm{H}-1,67.7$ & 0.68 & 0.72 & -0.3 \\
\hline $1 \mathrm{H}-1,70.2$ & 0.70 & 0.74 & -0.3 \\
\hline $1 \mathrm{H}-1,72.7$ & 0.73 & 0.77 & 0.0 \\
\hline $1 \mathrm{H}-1,75.2$ & 0.75 & 0.79 & 0.0 \\
\hline $1 \mathrm{H}-1,77.7$ & 0.78 & 0.82 & -0.3 \\
\hline $1 \mathrm{H}-1,80.2$ & 0.80 & 0.84 & -0.8 \\
\hline $1 \mathrm{H}-1,82.7$ & 0.83 & 0.87 & 0.0 \\
\hline $1 \mathrm{H}-1,85.2$ & 0.85 & 0.89 & 0.0 \\
\hline $1 \mathrm{H}-1,87.7$ & 0.88 & 0.92 & -0.3 \\
\hline $1 \mathrm{H}-1,90.2$ & 0.90 & 0.94 & -0.3 \\
\hline $1 \mathrm{H}-1,92.7$ & 0.93 & 0.97 & -0.3 \\
\hline $1 \mathrm{H}-1,95.2$ & 0.95 & 0.99 & 0.0 \\
\hline $1 \mathrm{H}-1,97.7$ & 0.98 & 1.02 & -0.3 \\
\hline $1 \mathrm{H}-1,100.2$ & 1.00 & 1.04 & 0.0 \\
\hline $1 \mathrm{H}-1,102.7$ & 1.03 & 1.07 & -0.3 \\
\hline $1 \mathrm{H}-1,105.2$ & 1.05 & 1.09 & 0.0 \\
\hline $1 \mathrm{H}-1,107.8$ & 1.08 & 1.12 & 0.8 \\
\hline $1 \mathrm{H}-1,110.2$ & 1.10 & 1.14 & 0.3 \\
\hline $1 \mathrm{H}-1,112.8$ & 1.13 & 1.17 & 0.8 \\
\hline $1 \mathrm{H}-1,115.3$ & 1.15 & 1.19 & 0.8 \\
\hline $1 \mathrm{H}-1,117.8$ & 1.18 & 1.22 & 0.8 \\
\hline $1 \mathrm{H}-1,120.3$ & 1.20 & 1.24 & 1.6 \\
\hline $1 \mathrm{H}-1,122.8$ & 1.23 & 1.27 & 2.5 \\
\hline $1 \mathrm{H}-1,125.3$ & 1.25 & 1.29 & 3.0 \\
\hline $1 \mathrm{H}-1,127.8$ & 1.28 & 1.32 & 2.7 \\
\hline $1 \mathrm{H}-1,130.3$ & 1.30 & 1.34 & 3.3 \\
\hline $1 \mathrm{H}-1,132.8$ & 1.33 & 1.37 & 3.6 \\
\hline $1 \mathrm{H}-1,135.3$ & 1.35 & 1.39 & 3.6 \\
\hline $1 \mathrm{H}-1,137.8$ & 1.38 & 1.42 & 3.8 \\
\hline $1 \mathrm{H}-1,140.3$ & 1.40 & 1.44 & 4.1 \\
\hline $1 \mathrm{H}-1,142.8$ & 1.43 & 1.47 & 3.8 \\
\hline $1 \mathrm{H}-2,0.5$ & 1.50 & 1.54 & 2.5 \\
\hline
\end{tabular}

Only a portion of this table appears here. The complete table is available in ASCII. 
Table AT41. Natural gamma ray (NGR) data, Site U1338.

\begin{tabular}{|c|c|c|c|}
\hline \multirow{2}{*}{$\begin{array}{l}\text { Hole, core, section, } \\
\text { interval }(\mathrm{cm})\end{array}$} & \multicolumn{2}{|c|}{ Depth (m) } & \multirow{2}{*}{$\begin{array}{l}\text { NGR } \\
(\mathrm{cps})\end{array}$} \\
\hline & CSF & CCSF & \\
\hline \multicolumn{4}{|l|}{$321-$} \\
\hline U1338A-1H-1, 0 & 0.0 & 0.04 & 38.6 \\
\hline U1338A-1H-1, 10 & 0.1 & 0.14 & 40.3 \\
\hline U1338A-1H-1, 20 & 0.2 & 0.24 & 44.3 \\
\hline U1338A-1H-1, 30 & 0.3 & 0.34 & 41.3 \\
\hline U1338A-1H-1, 40 & 0.4 & 0.44 & 33.3 \\
\hline U1338A-1H-1, 50 & 0.5 & 0.54 & 28.7 \\
\hline U1338A-1H-1, 60 & 0.6 & 0.64 & 25.7 \\
\hline U1338A-1H-1, 70 & 0.7 & 0.74 & 28.8 \\
\hline U1338A-1H-1, 80 & 0.8 & 0.84 & 27.6 \\
\hline U1338A-1H-1, 90 & 0.9 & 0.94 & 21.7 \\
\hline U1338A-1H-1, 100 & 1.0 & 1.04 & 21.9 \\
\hline U1338A-1H-1, 110 & 1.1 & 1.14 & 29.9 \\
\hline U1338A-1H-1, 120 & 1.2 & 1.24 & 30.4 \\
\hline U1338A-1H-1, 130 & 1.3 & 1.34 & 26.7 \\
\hline U1338A-1H-1, 140 & 1.4 & 1.44 & 24.7 \\
\hline U1338A-1H-2, 0 & 1.5 & 1.54 & 37.4 \\
\hline U1338A-1H-2, 10 & 1.6 & 1.64 & 42.8 \\
\hline U1338A-1H-2, 20 & 1.7 & 1.74 & 39.1 \\
\hline U1338A-1H-2, 30 & 1.8 & 1.84 & 31.3 \\
\hline U1338A-1H-2, 40 & 1.9 & 1.94 & 30.9 \\
\hline U1338A-1H-2, 50 & 2.0 & 2.04 & 26.8 \\
\hline U1338B-1H-2, 50 & 2.0 & 2.00 & 23.3 \\
\hline U1338B-1H-2, 60 & 2.1 & 2.10 & 20.6 \\
\hline U1338B-1H-2, 70 & 2.2 & 2.20 & 14.1 \\
\hline U1338B-1H-2, 80 & 2.3 & 2.30 & 10.5 \\
\hline U1338B-1H-2, 90 & 2.4 & 2.40 & 10.9 \\
\hline U1338B-1H-2, 100 & 2.5 & 2.50 & 11.6 \\
\hline U1338B-1H-2, 110 & 2.6 & 2.60 & 12.5 \\
\hline U1338B-1H-2, 120 & 2.7 & 2.70 & 12.3 \\
\hline U1338B-1H-2, 130 & 2.8 & 2.80 & 12.5 \\
\hline U1338B-1H-2, 140 & 2.9 & 2.90 & 11.6 \\
\hline U1338B-1H-3, 0 & 3.0 & 3.00 & 12.6 \\
\hline U1338B-1H-2, 150 & 3.0 & 3.00 & 11.9 \\
\hline U1338B-1H-3, 10 & 3.1 & 3.10 & 17.8 \\
\hline U1338B-1H-3, 20 & 3.2 & 3.20 & 17.6 \\
\hline U1338B-1H-3, 30 & 3.3 & 3.30 & 11.7 \\
\hline U1338B-1H-3, 40 & 3.4 & 3.40 & 10.7 \\
\hline U1338B-1H-3, 50 & 3.5 & 3.50 & 8.7 \\
\hline U1338B-1H-3, 60 & 3.6 & 3.60 & 8.0 \\
\hline U1338B-1H-3, 70 & 3.7 & 3.70 & 8.7 \\
\hline U1338B-1H-3, 80 & 3.8 & 3.80 & 7.4 \\
\hline U1338B-1H-3, 90 & 3.9 & 3.90 & 8.3 \\
\hline U1338B-1H-3, 100 & 4.0 & 4.00 & 7.1 \\
\hline U1338B-1H-3, 110 & 4.1 & 4.10 & 6.1 \\
\hline U1338B-1H-3, 120 & 4.2 & 4.20 & 6.4 \\
\hline U1338B-1H-3, 130 & 4.3 & 4.30 & 6.6 \\
\hline U1338B-1H-3, 140 & 4.4 & 4.40 & 4.8 \\
\hline U1338B-1H-3, 150 & 4.5 & 4.50 & 4.9 \\
\hline U1338B-1H-4, 0 & 4.5 & 4.50 & 6.1 \\
\hline U1338B-1H-4, 10 & 4.6 & 4.60 & 6.2 \\
\hline U1338B-1H-4, 20 & 4.7 & 4.70 & 5.2 \\
\hline U1338B-1H-4, 30 & 4.8 & 4.80 & 5.0 \\
\hline U1338B-1H-4, 40 & 4.9 & 4.90 & 5.4 \\
\hline U1338B-1H-4, 50 & 5.0 & 5.00 & 5.5 \\
\hline U1338A-2H-1, 80 & 3.5 & 4.98 & 5.2 \\
\hline U1338A-2H-1, 90 & 3.6 & 5.08 & 5.4 \\
\hline U1338A-2H-1, 100 & 3.7 & 5.18 & 4.0 \\
\hline U1338A-2H-1, 110 & 3.8 & 5.28 & 3.4 \\
\hline U1338A-2H-1, 120 & 3.9 & 5.38 & 3.1 \\
\hline U1338A-2H-1, 130 & 4.0 & 5.48 & 2.7 \\
\hline
\end{tabular}

Only a portion of this table appears here. The complete table is available in ASCII. 
Table AT42. Color reflection data, Site U1338.

\begin{tabular}{|c|c|c|c|c|c|}
\hline \multirow{2}{*}{$\begin{array}{l}\text { Core, section, } \\
\text { interval }(\mathrm{cm})\end{array}$} & \multicolumn{2}{|c|}{ Depth (m) } & \multirow[b]{2}{*}{$a^{*}$} & \multirow[b]{2}{*}{$b^{*}$} & \multirow[b]{2}{*}{$L^{*}$} \\
\hline & CSF & CCSF & & & \\
\hline \multicolumn{6}{|l|}{ 321-U1338A- } \\
\hline $1 \mathrm{H}-1,5.2$ & 0.05 & 0.09 & 5.9 & 13.7 & 43.6 \\
\hline $1 \mathrm{H}-1,7.7$ & 0.08 & 0.12 & 5.7 & 13.1 & 43.5 \\
\hline $1 \mathrm{H}-1,10.2$ & 0.10 & 0.14 & 5.3 & 13.2 & 44.6 \\
\hline $1 \mathrm{H}-1,12.8$ & 0.13 & 0.17 & 4.8 & 11.2 & 42.2 \\
\hline $1 \mathrm{H}-1,15.3$ & 0.15 & 0.19 & 5.3 & 12.5 & 43.2 \\
\hline $1 \mathrm{H}-1,17.9$ & 0.18 & 0.22 & 5.4 & 13.5 & 45.0 \\
\hline $1 \mathrm{H}-1,20.4$ & 0.20 & 0.24 & 4.2 & 10.7 & 44.6 \\
\hline $1 \mathrm{H}-1,23$ & 0.23 & 0.27 & 5.3 & 15.3 & 47.7 \\
\hline $1 \mathrm{H}-1,25.5$ & 0.26 & 0.30 & 4.6 & 13.7 & 45.6 \\
\hline $1 \mathrm{H}-1,28$ & 0.28 & 0.32 & 4.2 & 14.6 & 47.8 \\
\hline $1 \mathrm{H}-1,30.6$ & 0.31 & 0.35 & 4.8 & 16.0 & 50.1 \\
\hline $1 \mathrm{H}-1,33.1$ & 0.33 & 0.37 & 5.2 & 18.1 & 52.8 \\
\hline $1 \mathrm{H}-1,35.7$ & 0.36 & 0.40 & 4.9 & 17.7 & 53.7 \\
\hline $1 \mathrm{H}-1,38.2$ & 0.38 & 0.42 & 4.7 & 18.3 & 54.8 \\
\hline $1 \mathrm{H}-1,40.7$ & 0.41 & 0.45 & 4.0 & 17.4 & 54.9 \\
\hline $1 \mathrm{H}-1,43.3$ & 0.43 & 0.47 & 4.4 & 14.8 & 53.7 \\
\hline $1 \mathrm{H}-1,45.8$ & 0.46 & 0.50 & 4.8 & 17.6 & 56.0 \\
\hline $1 \mathrm{H}-1,48.4$ & 0.48 & 0.52 & 4.5 & 16.0 & 55.7 \\
\hline $1 \mathrm{H}-1,50.9$ & 0.51 & 0.55 & 5.0 & 18.1 & 55.8 \\
\hline $1 \mathrm{H}-1,53.4$ & 0.53 & 0.57 & 4.7 & 18.2 & 61.4 \\
\hline $1 \mathrm{H}-1,56$ & 0.56 & 0.60 & 3.8 & 17.9 & 60.2 \\
\hline $1 \mathrm{H}-1,58.5$ & 0.59 & 0.63 & 4.1 & 18.2 & 59.6 \\
\hline $1 \mathrm{H}-1,61.1$ & 0.61 & 0.65 & 4.2 & 17.6 & 60.1 \\
\hline $1 \mathrm{H}-1,63.6$ & 0.64 & 0.68 & 3.6 & 13.9 & 59.6 \\
\hline $1 \mathrm{H}-1,66.2$ & 0.66 & 0.70 & 2.1 & 17.0 & 62.2 \\
\hline $1 \mathrm{H}-1,68.7$ & 0.69 & 0.73 & 4.0 & 15.7 & 56.6 \\
\hline $1 \mathrm{H}-1,71.2$ & 0.71 & 0.75 & 1.4 & 16.3 & 66.7 \\
\hline $1 \mathrm{H}-1,73.8$ & 0.74 & 0.78 & 3.4 & 14.0 & 55.0 \\
\hline $1 \mathrm{H}-1,76.3$ & 0.76 & 0.80 & 3.7 & 17.6 & 62.5 \\
\hline $1 \mathrm{H}-1,78.9$ & 0.79 & 0.83 & 3.5 & 17.8 & 64.6 \\
\hline $1 \mathrm{H}-1,81.4$ & 0.81 & 0.85 & 3.5 & 17.2 & 62.8 \\
\hline $1 \mathrm{H}-1,83.9$ & 0.84 & 0.88 & 3.3 & 14.0 & 53.2 \\
\hline $1 \mathrm{H}-1,86.5$ & 0.87 & 0.91 & 3.2 & 18.2 & 64.3 \\
\hline $1 \mathrm{H}-1,89$ & 0.89 & 0.93 & 3.8 & 18.0 & 64.3 \\
\hline $1 \mathrm{H}-1,91.6$ & 0.92 & 0.96 & 4.5 & 17.9 & 62.1 \\
\hline $1 \mathrm{H}-1,94.1$ & 0.94 & 0.98 & 2.1 & 18.1 & 67.0 \\
\hline $1 \mathrm{H}-1,96.7$ & 0.97 & 1.01 & 2.3 & 17.8 & 64.9 \\
\hline $1 \mathrm{H}-1,99.2$ & 0.99 & 1.03 & 2.5 & 17.9 & 64.0 \\
\hline $1 \mathrm{H}-1,101.7$ & 1.02 & 1.06 & 4.2 & 14.5 & 67.5 \\
\hline $1 \mathrm{H}-1,104.3$ & 1.04 & 1.08 & 3.7 & 16.0 & 59.1 \\
\hline $1 \mathrm{H}-1,106.8$ & 1.07 & 1.11 & 3.1 & 12.3 & 49.5 \\
\hline $1 \mathrm{H}-1,109.4$ & 1.09 & 1.13 & 3.2 & 16.7 & 63.6 \\
\hline $1 \mathrm{H}-1,111.9$ & 1.12 & 1.16 & 3.4 & 14.3 & 55.7 \\
\hline $1 \mathrm{H}-1,114.4$ & 1.14 & 1.18 & 3.3 & 19.0 & 69.7 \\
\hline $1 \mathrm{H}-1,117$ & 1.17 & 1.21 & 5.1 & 17.8 & 55.7 \\
\hline $1 \mathrm{H}-1,119.5$ & 1.20 & 1.24 & 3.8 & 19.0 & 60.5 \\
\hline $1 \mathrm{H}-1,122.1$ & 1.22 & 1.26 & 5.0 & 18.4 & 54.5 \\
\hline $1 \mathrm{H}-1,124.6$ & 1.25 & 1.29 & 5.5 & 17.7 & 52.1 \\
\hline $1 \mathrm{H}-1,127.2$ & 1.27 & 1.31 & 2.3 & 18.8 & 61.9 \\
\hline $1 \mathrm{H}-1,129.7$ & 1.30 & 1.34 & 6.0 & 18.0 & 49.3 \\
\hline $1 \mathrm{H}-1,132.2$ & 1.32 & 1.36 & 5.5 & 16.0 & 46.9 \\
\hline $1 \mathrm{H}-1,134.8$ & 1.35 & 1.39 & 5.1 & 16.2 & 51.8 \\
\hline $1 \mathrm{H}-1,137.3$ & 1.37 & 1.41 & 5.9 & 17.1 & 48.1 \\
\hline $1 \mathrm{H}-1,139.9$ & 1.40 & 1.44 & 6.2 & 16.4 & 53.3 \\
\hline $2 \mathrm{H}-1,3.4$ & 1.53 & 1.57 & 3.7 & 9.0 & 42.1 \\
\hline $2 \mathrm{H}-1,5.9$ & 1.56 & 1.60 & 5.0 & 19.7 & 54.4 \\
\hline $2 \mathrm{H}-1,8.5$ & 1.59 & 1.63 & 5.0 & 11.3 & 35.3 \\
\hline $2 \mathrm{H}-1,11$ & 1.61 & 1.65 & 4.1 & 7.9 & 34.7 \\
\hline $2 \mathrm{H}-1,13.6$ & 1.64 & 1.68 & 4.5 & 14.2 & 41.1 \\
\hline
\end{tabular}

Only a portion of this table appears here. The complete table is available in ASCII. 
Table AT43. Gamma ray attenuation (GRA) density data, Hole U1338A.

\begin{tabular}{|c|c|c|c|c|}
\hline \multirow[b]{2}{*}{$\begin{array}{l}\text { Core, section, } \\
\text { interval }(\mathrm{cm})\end{array}$} & \multicolumn{3}{|c|}{ Depth (m) } & \multirow[b]{2}{*}{$\begin{array}{c}\text { GRA } \\
\left(\mathrm{g} / \mathrm{cm}^{3}\right)\end{array}$} \\
\hline & CSF & CCSF & $\begin{array}{l}\text { Adjusted } \\
\text { CCSF }\end{array}$ & \\
\hline \multicolumn{5}{|l|}{ 321-U1338A- } \\
\hline $1 \mathrm{H}-1,2.5$ & 0.03 & 0.07 & 0.07 & 1.36 \\
\hline $1 \mathrm{H}-1,5$ & 0.05 & 0.09 & 0.09 & 1.33 \\
\hline $1 \mathrm{H}-1,7.5$ & 0.08 & 0.12 & 0.12 & 1.35 \\
\hline $1 \mathrm{H}-1,12.5$ & 0.13 & 0.17 & 0.17 & 1.38 \\
\hline $1 \mathrm{H}-1,15$ & 0.15 & 0.19 & 0.19 & 1.38 \\
\hline $1 \mathrm{H}-1,17.5$ & 0.18 & 0.22 & 0.22 & 1.37 \\
\hline $1 \mathrm{H}-1,20$ & 0.20 & 0.24 & 0.24 & 1.37 \\
\hline $1 \mathrm{H}-1,22.5$ & 0.23 & 0.27 & 0.27 & 1.32 \\
\hline $1 \mathrm{H}-1,25$ & 0.25 & 0.29 & 0.29 & 1.36 \\
\hline $1 \mathrm{H}-1,27.6$ & 0.28 & 0.32 & 0.32 & 1.35 \\
\hline $1 \mathrm{H}-1,30.1$ & 0.30 & 0.34 & 0.34 & 1.38 \\
\hline $1 \mathrm{H}-1,32.6$ & 0.33 & 0.37 & 0.37 & 1.38 \\
\hline $1 \mathrm{H}-1,35.1$ & 0.35 & 0.39 & 0.39 & 1.36 \\
\hline $1 \mathrm{H}-1,37.6$ & 0.38 & 0.42 & 0.42 & 1.36 \\
\hline $1 \mathrm{H}-1,40.1$ & 0.40 & 0.44 & 0.44 & 1.34 \\
\hline $1 \mathrm{H}-1,42.6$ & 0.43 & 0.47 & 0.47 & 1.35 \\
\hline $1 \mathrm{H}-1,45.1$ & 0.45 & 0.49 & 0.49 & 1.39 \\
\hline $1 \mathrm{H}-1,47.6$ & 0.48 & 0.52 & 0.52 & 1.38 \\
\hline $1 \mathrm{H}-1,50.1$ & 0.50 & 0.54 & 0.54 & 1.35 \\
\hline $1 \mathrm{H}-1,52.6$ & 0.53 & 0.57 & 0.57 & 1.39 \\
\hline $1 \mathrm{H}-1,55.1$ & 0.55 & 0.59 & 0.59 & 1.30 \\
\hline $1 \mathrm{H}-1,57.6$ & 0.58 & 0.62 & 0.62 & 1.33 \\
\hline $1 \mathrm{H}-1,60.1$ & 0.60 & 0.64 & 0.64 & 1.39 \\
\hline $1 \mathrm{H}-1,62.6$ & 0.63 & 0.67 & 0.67 & 1.40 \\
\hline $1 \mathrm{H}-1,65.1$ & 0.65 & 0.69 & 0.69 & 1.37 \\
\hline $1 \mathrm{H}-1,67.6$ & 0.68 & 0.72 & 0.72 & 1.42 \\
\hline $1 \mathrm{H}-1,70.1$ & 0.70 & 0.74 & 0.74 & 1.39 \\
\hline $1 \mathrm{H}-1,72.6$ & 0.73 & 0.77 & 0.77 & 1.40 \\
\hline $1 \mathrm{H}-1,75.1$ & 0.75 & 0.79 & 0.79 & 1.42 \\
\hline $1 \mathrm{H}-1,77.6$ & 0.78 & 0.82 & 0.82 & 1.41 \\
\hline $1 \mathrm{H}-1,80.1$ & 0.80 & 0.84 & 0.84 & 1.38 \\
\hline $1 \mathrm{H}-1,82.6$ & 0.83 & 0.87 & 0.87 & 1.39 \\
\hline $1 \mathrm{H}-1,85.1$ & 0.85 & 0.89 & 0.89 & 1.40 \\
\hline $1 \mathrm{H}-1,87.7$ & 0.88 & 0.92 & 0.92 & 1.40 \\
\hline $1 \mathrm{H}-1,90.2$ & 0.90 & 0.94 & 0.94 & 1.39 \\
\hline $1 \mathrm{H}-1,92.7$ & 0.93 & 0.97 & 0.97 & 1.36 \\
\hline $1 \mathrm{H}-1,95.2$ & 0.95 & 0.99 & 0.99 & 1.39 \\
\hline $1 \mathrm{H}-1,97.7$ & 0.98 & 1.02 & 1.02 & 1.40 \\
\hline $1 \mathrm{H}-1,100.2$ & 1.00 & 1.04 & 1.04 & 1.41 \\
\hline $1 \mathrm{H}-1,102.7$ & 1.03 & 1.07 & 1.07 & 1.35 \\
\hline $1 \mathrm{H}-1,105.2$ & 1.05 & 1.09 & 1.09 & 1.40 \\
\hline $1 \mathrm{H}-1,107.7$ & 1.08 & 1.12 & 1.12 & 1.36 \\
\hline $1 \mathrm{H}-1,110.2$ & 1.10 & 1.14 & 1.14 & 1.38 \\
\hline $1 \mathrm{H}-1,112.7$ & 1.13 & 1.17 & 1.17 & 1.37 \\
\hline $1 \mathrm{H}-1,115.2$ & 1.15 & 1.19 & 1.19 & 1.33 \\
\hline $1 \mathrm{H}-1,117.7$ & 1.18 & 1.22 & 1.22 & 1.34 \\
\hline $1 \mathrm{H}-1,120.2$ & 1.20 & 1.24 & 1.24 & 1.34 \\
\hline $1 \mathrm{H}-1,122.7$ & 1.23 & 1.27 & 1.27 & 1.30 \\
\hline $1 \mathrm{H}-1,125.2$ & 1.25 & 1.29 & 1.29 & 1.28 \\
\hline $1 \mathrm{H}-1,127.7$ & 1.28 & 1.32 & 1.32 & 1.28 \\
\hline $1 \mathrm{H}-1,130.2$ & 1.30 & 1.34 & 1.34 & 1.24 \\
\hline $1 \mathrm{H}-1,132.7$ & 1.33 & 1.37 & 1.37 & 1.23 \\
\hline $1 \mathrm{H}-1,135.2$ & 1.35 & 1.39 & 1.39 & 1.25 \\
\hline $1 \mathrm{H}-1,137.7$ & 1.38 & 1.42 & 1.42 & 1.24 \\
\hline $1 \mathrm{H}-1,140.3$ & 1.40 & 1.44 & 1.44 & 1.27 \\
\hline $1 \mathrm{H}-1,142.8$ & 1.43 & 1.47 & 1.47 & 1.26 \\
\hline $1 \mathrm{H}-2,5.4$ & 1.55 & 1.59 & 1.59 & 1.24 \\
\hline $1 \mathrm{H}-2,7.9$ & 1.58 & 1.62 & 1.62 & 1.25 \\
\hline $1 \mathrm{H}-2,10.4$ & 1.60 & 1.64 & 1.64 & 1.26 \\
\hline $1 \mathrm{H}-2,12.9$ & 1.63 & 1.67 & 1.67 & 1.25 \\
\hline
\end{tabular}

Only a portion of this table appears here. The complete table is available in ASCII. 
Table AT44. Magnetic susceptibility data, Hole U1338A.

\begin{tabular}{|c|c|c|c|c|}
\hline \multirow[b]{2}{*}{$\begin{array}{l}\text { Core, section, } \\
\text { interval }(\mathrm{cm})\end{array}$} & \multicolumn{3}{|c|}{ Depth $(\mathrm{m})$} & \multirow{2}{*}{$\begin{array}{c}\text { Magnetic } \\
\text { susceptibility } \\
\text { (IU) }\end{array}$} \\
\hline & CSF & CCSF & $\begin{array}{l}\text { Adjusted } \\
\text { CCSF }\end{array}$ & \\
\hline \multicolumn{5}{|l|}{ 321-U1338A- } \\
\hline $1 \mathrm{H}-1,0.1$ & 0.00 & 0.04 & 0.04 & 0.0 \\
\hline $1 \mathrm{H}-1,2.6$ & 0.03 & 0.07 & 0.07 & 1.4 \\
\hline $1 \mathrm{H}-1,5.1$ & 0.05 & 0.09 & 0.09 & 2.2 \\
\hline $1 \mathrm{H}-1,7.6$ & 0.08 & 0.12 & 0.12 & 2.2 \\
\hline $1 \mathrm{H}-1,10.1$ & 0.10 & 0.14 & 0.14 & 1.9 \\
\hline $1 \mathrm{H}-1,12.6$ & 0.13 & 0.17 & 0.17 & 1.4 \\
\hline $1 \mathrm{H}-1,15.1$ & 0.15 & 0.19 & 0.19 & 1.1 \\
\hline $1 \mathrm{H}-1,17.6$ & 0.18 & 0.22 & 0.22 & 1.4 \\
\hline $1 \mathrm{H}-1,20.1$ & 0.20 & 0.24 & 0.24 & 1.4 \\
\hline $1 \mathrm{H}-1,22.6$ & 0.23 & 0.27 & 0.27 & 2.5 \\
\hline $1 \mathrm{H}-1,25.1$ & 0.25 & 0.29 & 0.29 & 2.2 \\
\hline $1 \mathrm{H}-1,27.6$ & 0.28 & 0.32 & 0.32 & 1.6 \\
\hline $1 \mathrm{H}-1,30.1$ & 0.30 & 0.34 & 0.34 & 1.9 \\
\hline $1 \mathrm{H}-1,32.6$ & 0.33 & 0.37 & 0.37 & 1.1 \\
\hline $1 \mathrm{H}-1,35.1$ & 0.35 & 0.39 & 0.39 & 1.4 \\
\hline $1 \mathrm{H}-1,37.6$ & 0.38 & 0.42 & 0.42 & 0.3 \\
\hline $1 \mathrm{H}-1,40.1$ & 0.40 & 0.44 & 0.44 & 0.0 \\
\hline $1 \mathrm{H}-1,42.6$ & 0.43 & 0.47 & 0.47 & 0.3 \\
\hline $1 \mathrm{H}-1,45.1$ & 0.45 & 0.49 & 0.49 & 0.3 \\
\hline $1 \mathrm{H}-1,47.6$ & 0.48 & 0.52 & 0.52 & 0.3 \\
\hline $1 \mathrm{H}-1,50.1$ & 0.50 & 0.54 & 0.54 & 0.8 \\
\hline $1 \mathrm{H}-1,52.7$ & 0.53 & 0.57 & 0.57 & 0.0 \\
\hline $1 \mathrm{H}-1,55.2$ & 0.55 & 0.59 & 0.59 & 0.0 \\
\hline $1 \mathrm{H}-1,57.7$ & 0.58 & 0.62 & 0.62 & -0.3 \\
\hline $1 \mathrm{H}-1,60.2$ & 0.60 & 0.64 & 0.64 & -0.3 \\
\hline $1 \mathrm{H}-1,62.7$ & 0.63 & 0.67 & 0.67 & -0.5 \\
\hline $1 \mathrm{H}-1,65.2$ & 0.65 & 0.69 & 0.69 & -0.3 \\
\hline $1 \mathrm{H}-1,67.7$ & 0.68 & 0.72 & 0.72 & -0.3 \\
\hline $1 \mathrm{H}-1,70.2$ & 0.70 & 0.74 & 0.74 & -0.3 \\
\hline $1 \mathrm{H}-1,72.7$ & 0.73 & 0.77 & 0.77 & 0.0 \\
\hline $1 \mathrm{H}-1,75.2$ & 0.75 & 0.79 & 0.79 & 0.0 \\
\hline $1 \mathrm{H}-1,77.7$ & 0.78 & 0.82 & 0.82 & -0.3 \\
\hline $1 \mathrm{H}-1,80.2$ & 0.80 & 0.84 & 0.84 & -0.8 \\
\hline $1 \mathrm{H}-1,82.7$ & 0.83 & 0.87 & 0.87 & 0.0 \\
\hline $1 \mathrm{H}-1,85.2$ & 0.85 & 0.89 & 0.89 & 0.0 \\
\hline $1 \mathrm{H}-1,87.7$ & 0.88 & 0.92 & 0.92 & -0.3 \\
\hline $1 \mathrm{H}-1,90.2$ & 0.90 & 0.94 & 0.94 & -0.3 \\
\hline $1 \mathrm{H}-1,92.7$ & 0.93 & 0.97 & 0.97 & -0.3 \\
\hline $1 \mathrm{H}-1,95.2$ & 0.95 & 0.99 & 0.99 & 0.0 \\
\hline $1 \mathrm{H}-1,97.7$ & 0.98 & 1.02 & 1.02 & -0.3 \\
\hline $1 \mathrm{H}-1,100.2$ & 1.00 & 1.04 & 1.04 & 0.0 \\
\hline $1 \mathrm{H}-1,102.7$ & 1.03 & 1.07 & 1.07 & -0.3 \\
\hline $1 \mathrm{H}-1,105.2$ & 1.05 & 1.09 & 1.09 & 0.0 \\
\hline $1 \mathrm{H}-1,107.8$ & 1.08 & 1.12 & 1.12 & 0.8 \\
\hline $1 \mathrm{H}-1,110.2$ & 1.10 & 1.14 & 1.14 & 0.3 \\
\hline $1 \mathrm{H}-1,112.8$ & 1.13 & 1.17 & 1.17 & 0.8 \\
\hline $1 \mathrm{H}-1,115.3$ & 1.15 & 1.19 & 1.19 & 0.8 \\
\hline $1 \mathrm{H}-1,117.8$ & 1.18 & 1.22 & 1.22 & 0.8 \\
\hline $1 \mathrm{H}-1,120.3$ & 1.20 & 1.24 & 1.24 & 1.6 \\
\hline $1 \mathrm{H}-1,122.8$ & 1.23 & 1.27 & 1.27 & 2.5 \\
\hline $1 \mathrm{H}-1,125.3$ & 1.25 & 1.29 & 1.29 & 3.0 \\
\hline $1 \mathrm{H}-1,127.8$ & 1.28 & 1.32 & 1.32 & 2.7 \\
\hline $1 \mathrm{H}-1,130.3$ & 1.30 & 1.34 & 1.34 & 3.3 \\
\hline $1 \mathrm{H}-1,132.8$ & 1.33 & 1.37 & 1.37 & 3.6 \\
\hline $1 \mathrm{H}-1,135.3$ & 1.35 & 1.39 & 1.39 & 3.6 \\
\hline $1 \mathrm{H}-1,137.8$ & 1.38 & 1.42 & 1.42 & 3.8 \\
\hline $1 \mathrm{H}-1,140.3$ & 1.40 & 1.44 & 1.44 & 4.1 \\
\hline $1 \mathrm{H}-1,142.8$ & 1.43 & 1.47 & 1.47 & 3.8 \\
\hline $1 \mathrm{H}-2,0.5$ & 1.50 & 1.54 & 1.54 & 2.5 \\
\hline
\end{tabular}

Only a portion of this table appears here. The complete table is available in ASCII. 
Table AT45. Natural gamma ray (NGR) data, Hole U1338A.

\begin{tabular}{|c|c|c|c|c|}
\hline \multirow[b]{2}{*}{$\begin{array}{l}\text { Core, section, } \\
\text { interval }(\mathrm{cm})\end{array}$} & \multicolumn{3}{|c|}{ Depth (m) } & \multirow[b]{2}{*}{$\begin{array}{l}\text { NGR } \\
\text { (cps) }\end{array}$} \\
\hline & CSF & CCSF & $\begin{array}{l}\text { Adjusted } \\
\text { CCSF }\end{array}$ & \\
\hline \multicolumn{5}{|l|}{ 321-U1338A- } \\
\hline $1 \mathrm{H}-1,0$ & 0.0 & 0.04 & 0.04 & 38.6 \\
\hline $1 \mathrm{H}-1,10$ & 0.1 & 0.14 & 0.14 & 40.3 \\
\hline $1 \mathrm{H}-1,20$ & 0.2 & 0.24 & 0.24 & 44.3 \\
\hline $1 \mathrm{H}-1,30$ & 0.3 & 0.34 & 0.34 & 41.3 \\
\hline $1 \mathrm{H}-1,40$ & 0.4 & 0.44 & 0.44 & 33.3 \\
\hline $1 \mathrm{H}-1,50$ & 0.5 & 0.54 & 0.54 & 28.7 \\
\hline $1 \mathrm{H}-1,60$ & 0.6 & 0.64 & 0.64 & 25.7 \\
\hline $1 \mathrm{H}-1,70$ & 0.7 & 0.74 & 0.74 & 28.8 \\
\hline $1 \mathrm{H}-1,80$ & 0.8 & 0.84 & 0.84 & 27.6 \\
\hline $1 \mathrm{H}-1,90$ & 0.9 & 0.94 & 0.94 & 21.7 \\
\hline $1 \mathrm{H}-1,100$ & 1.0 & 1.04 & 1.04 & 21.9 \\
\hline $1 \mathrm{H}-1,110$ & 1.1 & 1.14 & 1.14 & 29.9 \\
\hline $1 \mathrm{H}-1,120$ & 1.2 & 1.24 & 1.24 & 30.4 \\
\hline $1 \mathrm{H}-1,130$ & 1.3 & 1.34 & 1.34 & 26.7 \\
\hline $1 \mathrm{H}-1,140$ & 1.4 & 1.44 & 1.44 & 24.7 \\
\hline $1 \mathrm{H}-2,0$ & 1.5 & 1.54 & 1.54 & 37.4 \\
\hline $1 \mathrm{H}-2,10$ & 1.6 & 1.64 & 1.64 & 42.8 \\
\hline $1 \mathrm{H}-2,20$ & 1.7 & 1.74 & 1.74 & 39.1 \\
\hline $1 \mathrm{H}-2,30$ & 1.8 & 1.84 & 1.84 & 31.3 \\
\hline $1 \mathrm{H}-2,40$ & 1.9 & 1.94 & 1.94 & 30.9 \\
\hline $1 \mathrm{H}-2,50$ & 2.0 & 2.04 & 2.03 & 26.8 \\
\hline $1 \mathrm{H}-2,60$ & 2.1 & 2.14 & 2.10 & 22.9 \\
\hline $1 \mathrm{H}-2,70$ & 2.2 & 2.24 & 2.18 & 16.5 \\
\hline $1 \mathrm{H}-2,80$ & 2.3 & 2.34 & 2.26 & 10.6 \\
\hline $1 \mathrm{H}-2,90$ & 2.4 & 2.44 & 2.34 & 10.5 \\
\hline $1 \mathrm{H}-2,100$ & 2.5 & 2.54 & 2.42 & 10.7 \\
\hline $2 \mathrm{H}-1,0$ & 2.7 & 4.18 & 4.03 & 11.3 \\
\hline $2 \mathrm{H}-1,10$ & 2.8 & 4.28 & 4.13 & 16.6 \\
\hline $2 \mathrm{H}-1,20$ & 2.9 & 4.38 & 4.25 & 16.3 \\
\hline $2 \mathrm{H}-1,30$ & 3.0 & 4.48 & 4.36 & 8.7 \\
\hline $2 \mathrm{H}-1,40$ & 3.1 & 4.58 & 4.48 & 5.7 \\
\hline $2 \mathrm{H}-1,50$ & 3.2 & 4.68 & 4.59 & 5.1 \\
\hline $2 \mathrm{H}-1,60$ & 3.3 & 4.78 & 4.72 & 4.8 \\
\hline $2 \mathrm{H}-1,70$ & 3.4 & 4.88 & 4.84 & 5.0 \\
\hline $2 \mathrm{H}-1,80$ & 3.5 & 4.98 & 4.97 & 5.2 \\
\hline $2 \mathrm{H}-1,90$ & 3.6 & 5.08 & 5.08 & 5.4 \\
\hline $2 \mathrm{H}-1,100$ & 3.7 & 5.18 & 5.18 & 4.0 \\
\hline $2 \mathrm{H}-1,110$ & 3.8 & 5.28 & 5.28 & 3.4 \\
\hline $2 \mathrm{H}-1,120$ & 3.9 & 5.38 & 5.38 & 3.1 \\
\hline $2 \mathrm{H}-1,130$ & 4.0 & 5.48 & 5.48 & 2.7 \\
\hline $2 \mathrm{H}-1,140$ & 4.1 & 5.58 & 5.58 & 2.3 \\
\hline $2 \mathrm{H}-1,150$ & 4.2 & 5.68 & 5.68 & 2.5 \\
\hline $2 \mathrm{H}-2,0$ & 4.2 & 5.68 & 5.68 & 3.2 \\
\hline $2 \mathrm{H}-2,10$ & 4.3 & 5.78 & 5.78 & 2.9 \\
\hline $2 \mathrm{H}-2,20$ & 4.4 & 5.88 & 5.88 & 2.9 \\
\hline $2 \mathrm{H}-2,30$ & 4.5 & 5.98 & 5.98 & 3.5 \\
\hline $2 \mathrm{H}-2,40$ & 4.6 & 6.08 & 6.08 & 3.7 \\
\hline $2 \mathrm{H}-2,50$ & 4.7 & 6.18 & 6.18 & 3.4 \\
\hline $2 \mathrm{H}-2,60$ & 4.8 & 6.28 & 6.28 & 3.0 \\
\hline $2 \mathrm{H}-2,70$ & 4.9 & 6.38 & 6.38 & 2.2 \\
\hline $2 \mathrm{H}-2,80$ & 5.0 & 6.48 & 6.48 & 2.3 \\
\hline $2 \mathrm{H}-2,90$ & 5.1 & 6.58 & 6.58 & 2.3 \\
\hline $2 \mathrm{H}-2,100$ & 5.2 & 6.68 & 6.68 & 2.4 \\
\hline $2 \mathrm{H}-2,110$ & 5.3 & 6.78 & 6.78 & 2.3 \\
\hline $2 \mathrm{H}-2,120$ & 5.4 & 6.88 & 6.88 & 2.8 \\
\hline $2 \mathrm{H}-2,130$ & 5.5 & 6.98 & 6.98 & 2.7 \\
\hline $2 \mathrm{H}-2,140$ & 5.6 & 7.08 & 7.08 & 2.1 \\
\hline $2 \mathrm{H}-2,150$ & 5.7 & 7.18 & 7.18 & 1.5 \\
\hline $2 \mathrm{H}-3,0$ & 5.7 & 7.18 & 7.18 & 2.2 \\
\hline $2 \mathrm{H}-3,10$ & 5.8 & 7.28 & 7.28 & 2.4 \\
\hline
\end{tabular}

Only a portion of this table appears here. The complete table is available in ASCII. 
Table AT46. Color reflection data, Hole U1338A.

\begin{tabular}{|c|c|c|c|c|c|c|}
\hline \multirow[b]{2}{*}{$\begin{array}{l}\text { Core, section, } \\
\text { interval }(\mathrm{cm})\end{array}$} & \multicolumn{3}{|c|}{ Depth (m) } & \multirow[b]{2}{*}{$a^{*}$} & \multirow[b]{2}{*}{$b^{*}$} & \multirow[b]{2}{*}{$L^{*}$} \\
\hline & CSF & CCSF & $\begin{array}{l}\text { Adjusted } \\
\text { CCSF }\end{array}$ & & & \\
\hline \multicolumn{7}{|l|}{ 321-U1338A- } \\
\hline $1 \mathrm{H}-1,5.2$ & 0.05 & 0.09 & 0.09 & 5.9 & 13.7 & 43.6 \\
\hline $1 \mathrm{H}-1,7.7$ & 0.08 & 0.12 & 0.12 & 5.7 & 13.1 & 43.5 \\
\hline $1 \mathrm{H}-1,10.2$ & 0.10 & 0.14 & 0.14 & 5.3 & 13.2 & 44.6 \\
\hline $1 \mathrm{H}-1,12.8$ & 0.13 & 0.17 & 0.17 & 4.8 & 11.2 & 42.2 \\
\hline $1 \mathrm{H}-1,15.3$ & 0.15 & 0.19 & 0.19 & 5.3 & 12.5 & 43.2 \\
\hline $1 \mathrm{H}-1,17.9$ & 0.18 & 0.22 & 0.22 & 5.4 & 13.5 & 45.0 \\
\hline $1 \mathrm{H}-1,20.4$ & 0.20 & 0.24 & 0.24 & 4.2 & 10.7 & 44.6 \\
\hline $1 \mathrm{H}-1,23$ & 0.23 & 0.27 & 0.27 & 5.3 & 15.3 & 47.7 \\
\hline $1 \mathrm{H}-1,25.5$ & 0.26 & 0.30 & 0.30 & 4.6 & 13.7 & 45.6 \\
\hline $1 \mathrm{H}-1,28$ & 0.28 & 0.32 & 0.32 & 4.2 & 14.6 & 47.8 \\
\hline $1 \mathrm{H}-1,30.6$ & 0.31 & 0.35 & 0.35 & 4.8 & 16.0 & 50.1 \\
\hline $1 \mathrm{H}-1,33.1$ & 0.33 & 0.37 & 0.37 & 5.2 & 18.1 & 52.8 \\
\hline $1 \mathrm{H}-1,35.7$ & 0.36 & 0.40 & 0.40 & 4.9 & 17.7 & 53.7 \\
\hline $1 \mathrm{H}-1,38.2$ & 0.38 & 0.42 & 0.42 & 4.7 & 18.3 & 54.8 \\
\hline $1 \mathrm{H}-1,40.7$ & 0.41 & 0.45 & 0.45 & 4.0 & 17.4 & 54.9 \\
\hline $1 \mathrm{H}-1,43.3$ & 0.43 & 0.47 & 0.47 & 4.4 & 14.8 & 53.7 \\
\hline $1 \mathrm{H}-1,45.8$ & 0.46 & 0.50 & 0.50 & 4.8 & 17.6 & 56.0 \\
\hline $1 \mathrm{H}-1,48.4$ & 0.48 & 0.52 & 0.52 & 4.5 & 16.0 & 55.7 \\
\hline $1 \mathrm{H}-1,50.9$ & 0.51 & 0.55 & 0.55 & 5.0 & 18.1 & 55.8 \\
\hline $1 \mathrm{H}-1,53.4$ & 0.53 & 0.57 & 0.57 & 4.7 & 18.2 & 61.4 \\
\hline $1 \mathrm{H}-1,56$ & 0.56 & 0.60 & 0.60 & 3.8 & 17.9 & 60.2 \\
\hline $1 \mathrm{H}-1,58.5$ & 0.59 & 0.63 & 0.63 & 4.1 & 18.2 & 59.6 \\
\hline $1 \mathrm{H}-1,61.1$ & 0.61 & 0.65 & 0.65 & 4.2 & 17.6 & 60.1 \\
\hline $1 \mathrm{H}-1,63.6$ & 0.64 & 0.68 & 0.68 & 3.6 & 13.9 & 59.6 \\
\hline $1 \mathrm{H}-1,66.2$ & 0.66 & 0.70 & 0.70 & 2.1 & 17.0 & 62.2 \\
\hline $1 \mathrm{H}-1,68.7$ & 0.69 & 0.73 & 0.73 & 4.0 & 15.7 & 56.6 \\
\hline $1 \mathrm{H}-1,71.2$ & 0.71 & 0.75 & 0.75 & 1.4 & 16.3 & 66.7 \\
\hline $1 \mathrm{H}-1,73.8$ & 0.74 & 0.78 & 0.78 & 3.4 & 14.0 & 55.0 \\
\hline $1 \mathrm{H}-1,76.3$ & 0.76 & 0.80 & 0.80 & 3.7 & 17.6 & 62.5 \\
\hline $1 \mathrm{H}-1,78.9$ & 0.79 & 0.83 & 0.83 & 3.5 & 17.8 & 64.6 \\
\hline $1 \mathrm{H}-1,81.4$ & 0.81 & 0.85 & 0.85 & 3.5 & 17.2 & 62.8 \\
\hline $1 \mathrm{H}-1,83.9$ & 0.84 & 0.88 & 0.88 & 3.3 & 14.0 & 53.2 \\
\hline $1 \mathrm{H}-1,86.5$ & 0.87 & 0.91 & 0.91 & 3.2 & 18.2 & 64.3 \\
\hline $1 \mathrm{H}-1,89$ & 0.89 & 0.93 & 0.93 & 3.8 & 18.0 & 64.3 \\
\hline $1 \mathrm{H}-1,91.6$ & 0.92 & 0.96 & 0.96 & 4.5 & 17.9 & 62.1 \\
\hline $1 \mathrm{H}-1,94.1$ & 0.94 & 0.98 & 0.98 & 2.1 & 18.1 & 67.0 \\
\hline $1 \mathrm{H}-1,96.7$ & 0.97 & 1.01 & 1.01 & 2.3 & 17.8 & 64.9 \\
\hline $1 \mathrm{H}-1,99.2$ & 0.99 & 1.03 & 1.03 & 2.5 & 17.9 & 64.0 \\
\hline $1 \mathrm{H}-1,101.7$ & 1.02 & 1.06 & 1.06 & 4.2 & 14.5 & 67.5 \\
\hline $1 \mathrm{H}-1,104.3$ & 1.04 & 1.08 & 1.08 & 3.7 & 16.0 & 59.1 \\
\hline $1 \mathrm{H}-1,106.8$ & 1.07 & 1.11 & 1.11 & 3.1 & 12.3 & 49.5 \\
\hline $1 \mathrm{H}-1,109.4$ & 1.09 & 1.13 & 1.13 & 3.2 & 16.7 & 63.6 \\
\hline $1 \mathrm{H}-1,111.9$ & 1.12 & 1.16 & 1.16 & 3.4 & 14.3 & 55.7 \\
\hline $1 \mathrm{H}-1,114.4$ & 1.14 & 1.18 & 1.18 & 3.3 & 19.0 & 69.7 \\
\hline $1 \mathrm{H}-1,117$ & 1.17 & 1.21 & 1.21 & 5.1 & 17.8 & 55.7 \\
\hline $1 \mathrm{H}-1,119.5$ & 1.20 & 1.24 & 1.24 & 3.8 & 19.0 & 60.5 \\
\hline $1 \mathrm{H}-1,122.1$ & 1.22 & 1.26 & 1.26 & 5.0 & 18.4 & 54.5 \\
\hline $1 \mathrm{H}-1,124.6$ & 1.25 & 1.29 & 1.29 & 5.5 & 17.7 & 52.1 \\
\hline $1 \mathrm{H}-1,127.2$ & 1.27 & 1.31 & 1.31 & 2.3 & 18.8 & 61.9 \\
\hline $1 \mathrm{H}-1,129.7$ & 1.30 & 1.34 & 1.34 & 6.0 & 18.0 & 49.3 \\
\hline $1 \mathrm{H}-1,132.2$ & 1.32 & 1.36 & 1.36 & 5.5 & 16.0 & 46.9 \\
\hline $1 \mathrm{H}-1,134.8$ & 1.35 & 1.39 & 1.39 & 5.1 & 16.2 & 51.8 \\
\hline $1 \mathrm{H}-1,137.3$ & 1.37 & 1.41 & 1.41 & 5.9 & 17.1 & 48.1 \\
\hline $1 \mathrm{H}-1,139.9$ & 1.40 & 1.44 & 1.44 & 6.2 & 16.4 & 53.3 \\
\hline $1 \mathrm{H}-2,3.4$ & 1.53 & 1.57 & 1.57 & 3.7 & 9.0 & 42.1 \\
\hline $1 \mathrm{H}-2,5.9$ & 1.56 & 1.60 & 1.60 & 5.0 & 19.7 & 54.4 \\
\hline $1 \mathrm{H}-2,8.5$ & 1.59 & 1.63 & 1.63 & 5.0 & 11.3 & 35.3 \\
\hline $1 \mathrm{H}-2,11$ & 1.61 & 1.65 & 1.65 & 4.1 & 7.9 & 34.7 \\
\hline $1 \mathrm{H}-2,13.6$ & 1.64 & 1.68 & 1.68 & 4.5 & 14.2 & 41.1 \\
\hline $1 \mathrm{H}-2,16.1$ & 1.66 & 1.70 & 1.70 & 5.0 & 9.9 & 33.5 \\
\hline
\end{tabular}

Only a portion of this table appears here. The complete table is available in ASCII. 
Table AT47. Core adjusted data, Hole U1338A. (Continued on next page.)

\begin{tabular}{|c|c|c|c|}
\hline \multirow[b]{2}{*}{$\begin{array}{l}\text { Core, section, } \\
\text { interval }(\mathrm{cm})\end{array}$} & \multicolumn{3}{|c|}{ Depth $(m)$} \\
\hline & $\begin{array}{l}\text { Adjusted } \\
\text { CCSF }\end{array}$ & CCSF & CSF-A \\
\hline \multicolumn{4}{|l|}{ 321-U1338A- } \\
\hline $1 \mathrm{H}-1,0$ & 0.04 & 0.04 & 0.00 \\
\hline $1 \mathrm{H}-2,47$ & 2.01 & 2.01 & 1.97 \\
\hline $1 \mathrm{H}-2,67$ & 2.16 & 2.22 & 2.17 \\
\hline $1 \mathrm{H}-2,104$ & 2.46 & 2.59 & 2.54 \\
\hline $2 \mathrm{H}-1,0$ & 4.03 & 4.18 & 2.70 \\
\hline $2 \mathrm{H}-1,11$ & 4.15 & 4.29 & 2.81 \\
\hline $2 \mathrm{H}-1,45$ & 4.53 & 4.63 & 3.15 \\
\hline $2 \mathrm{H}-1,84$ & 5.02 & 5.02 & 3.54 \\
\hline $2 \mathrm{H}-6,146$ & 13.14 & 13.14 & 11.66 \\
\hline $2 \mathrm{H}-7,52$ & 13.79 & 13.70 & 12.22 \\
\hline $2 \mathrm{H}-7,75$ & 14.02 & 13.93 & 12.45 \\
\hline $3 \mathrm{H}-1,0$ & 14.71 & 14.43 & 12.20 \\
\hline $3 \mathrm{H}-1,21$ & 14.92 & 14.64 & 12.41 \\
\hline $3 \mathrm{H}-1,44$ & 15.18 & 14.87 & 12.64 \\
\hline $3 \mathrm{H}-1,86$ & 15.60 & 15.29 & 13.06 \\
\hline $3 \mathrm{H}-2,16$ & 16.25 & 16.09 & 13.86 \\
\hline $3 \mathrm{H}-2,120$ & 17.15 & 17.13 & 14.90 \\
\hline $3 \mathrm{H}-3,56$ & 17.99 & 17.99 & 15.76 \\
\hline $3 \mathrm{H}-5,58$ & 21.01 & 21.01 & 18.78 \\
\hline $3 \mathrm{H}-5,121$ & 21.60 & 21.64 & 19.41 \\
\hline $3 \mathrm{H}-6,30$ & 22.21 & 22.23 & 20.00 \\
\hline $3 \mathrm{H}-6,119$ & 23.10 & 23.13 & 20.89 \\
\hline $3 \mathrm{H}-7,3$ & 23.51 & 23.46 & 21.23 \\
\hline $3 \mathrm{H}-7,78$ & 24.21 & 24.21 & 21.98 \\
\hline $4 \mathrm{H}-1,0$ & 25.36 & 25.32 & 21.70 \\
\hline $4 \mathrm{H}-1,18$ & 25.54 & 25.50 & 21.88 \\
\hline $4 \mathrm{H}-1,116$ & 26.62 & 26.48 & 22.86 \\
\hline $4 \mathrm{H}-2,31$ & 27.22 & 27.13 & 23.51 \\
\hline $4 \mathrm{H}-2,125$ & 28.10 & 28.07 & 24.45 \\
\hline $4 \mathrm{H}-3,35$ & 28.67 & 28.67 & 25.05 \\
\hline $4 \mathrm{H}-6,122$ & 34.04 & 34.04 & 30.42 \\
\hline $4 \mathrm{H}-7,19$ & 34.46 & 34.51 & 30.89 \\
\hline $4 \mathrm{H}-7,75$ & 35.02 & 35.07 & 31.45 \\
\hline $5 \mathrm{H}-1,0$ & 36.62 & 36.60 & 31.20 \\
\hline $5 \mathrm{H}-1,52$ & 37.14 & 37.12 & 31.72 \\
\hline $5 \mathrm{H}-1,117$ & 37.76 & 37.78 & 32.37 \\
\hline $5 \mathrm{H}-2,54$ & 38.57 & 38.64 & 33.24 \\
\hline $5 \mathrm{H}-3,89$ & 40.55 & 40.49 & 35.09 \\
\hline $5 \mathrm{H}-4,94$ & 42.31 & 42.04 & 36.64 \\
\hline $5 \mathrm{H}-5,107$ & 44.02 & 43.67 & 38.27 \\
\hline $5 \mathrm{H}-6,59$ & 45.04 & 44.69 & 39.29 \\
\hline $5 \mathrm{H}-6,120$ & 45.66 & 45.30 & 39.90 \\
\hline $5 \mathrm{H}-7,66$ & 46.11 & 46.26 & 40.86 \\
\hline $5 \mathrm{H}-7,28$ & 46.21 & 45.88 & 40.48 \\
\hline $5 \mathrm{H}-7,80$ & 46.73 & 46.40 & 41.00 \\
\hline $6 \mathrm{H}-1,106$ & 47.36 & 47.33 & 41.76 \\
\hline $6 \mathrm{H}-2,53$ & 48.32 & 48.29 & 42.73 \\
\hline $6 \mathrm{H}-3,55$ & 49.81 & 49.81 & 44.25 \\
\hline $6 \mathrm{H}-4,129$ & 52.05 & 52.05 & 46.49 \\
\hline $6 \mathrm{H}-5,147$ & 53.68 & 53.73 & 48.17 \\
\hline $6 \mathrm{H}-6,39$ & 54.07 & 54.16 & 48.59 \\
\hline $6 \mathrm{H}-7,25$ & 55.54 & 55.51 & 49.95 \\
\hline $6 \mathrm{H}-7,82$ & 56.12 & 56.08 & 50.52 \\
\hline $7 \mathrm{H}-1,67$ & 56.45 & 57.08 & 50.87 \\
\hline 7H-1, 143 & 57.84 & 57.84 & 51.63 \\
\hline 7H-4, 111 & 62.03 & 62.03 & 55.81 \\
\hline $7 \mathrm{H}-7,75$ & 66.16 & 66.17 & 59.95 \\
\hline $8 \mathrm{H}-1,0$ & 67.74 & 67.78 & 59.70 \\
\hline $8 \mathrm{H}-1,70$ & 68.44 & 68.48 & 60.40 \\
\hline $8 \mathrm{H}-1,126$ & 69.04 & 69.04 & 60.96 \\
\hline $8 \mathrm{H}-6,79$ & 76.07 & 76.07 & 67.99 \\
\hline $8 \mathrm{H}-7,3$ & 76.84 & 76.81 & 68.73 \\
\hline $8 \mathrm{H}-7,73$ & 77.53 & 77.51 & 69.43 \\
\hline $9 \mathrm{H}-1,0$ & 78.77 & 78.65 & 69.20 \\
\hline $9 \mathrm{H}-1,20$ & 78.97 & 78.85 & 69.40 \\
\hline $9 \mathrm{H}-1,134$ & 79.99 & 79.99 & 70.54 \\
\hline $9 \mathrm{H}-6,79$ & 86.95 & 86.94 & 77.49 \\
\hline
\end{tabular}

\begin{tabular}{|c|c|c|c|}
\hline \multirow[b]{2}{*}{$\begin{array}{l}\text { Core, section, } \\
\text { interval }(\mathrm{cm})\end{array}$} & \multicolumn{3}{|c|}{ Depth (m) } \\
\hline & $\begin{array}{l}\text { Adjusted } \\
\text { CCSF }\end{array}$ & CCSF & CSF-A \\
\hline $9 \mathrm{H}-7,5$ & 87.63 & 87.70 & 78.25 \\
\hline $9 \mathrm{H}-7,45$ & 88.02 & 88.10 & 78.65 \\
\hline $10 \mathrm{H}-1,0$ & 88.90 & 88.93 & 78.70 \\
\hline $10 \mathrm{H}-1,106$ & 89.99 & 89.99 & 79.76 \\
\hline $10 \mathrm{H}-6,54$ & 96.97 & 96.97 & 86.74 \\
\hline $10 \mathrm{H}-6,149$ & 97.93 & 97.92 & 87.69 \\
\hline $10 \mathrm{H}-7,85$ & 98.79 & 98.78 & 88.55 \\
\hline $11 \mathrm{H}-1,0$ & 99.48 & 99.48 & 88.20 \\
\hline $11 \mathrm{H}-1,56$ & 100.04 & 100.04 & 88.76 \\
\hline $11 \mathrm{H}-4,29$ & 104.27 & 104.27 & 92.99 \\
\hline $11 \mathrm{H}-5,51$ & 106.03 & 105.99 & 94.71 \\
\hline $11 \mathrm{H}-5,117$ & 106.68 & 106.65 & 95.37 \\
\hline $11 \mathrm{H}-6,42$ & 107.37 & 107.27 & 95.99 \\
\hline $11 \mathrm{H}-6,84$ & 107.69 & 107.69 & 96.41 \\
\hline $11 \mathrm{H}-6,107$ & 108.00 & 107.92 & 96.64 \\
\hline $12 \mathrm{H}-1,16$ & 110.49 & 110.61 & 97.86 \\
\hline $12 \mathrm{H}-1,100$ & 111.51 & 111.45 & 98.70 \\
\hline $12 \mathrm{H}-2,28$ & 112.23 & 112.23 & 99.48 \\
\hline $12 \mathrm{H}-3,110$ & 114.55 & 114.55 & 101.80 \\
\hline $12 \mathrm{H}-4,85$ & 115.84 & 115.80 & 103.05 \\
\hline $12 \mathrm{H}-5,115$ & 117.58 & 117.60 & 104.85 \\
\hline $12 \mathrm{H}-6,71$ & 118.64 & 118.66 & 105.91 \\
\hline $12 \mathrm{H}-7,32$ & 119.62 & 119.77 & 107.02 \\
\hline $12 \mathrm{H}-7,77$ & 120.07 & 120.22 & 107.47 \\
\hline $13 \mathrm{H}-1,0$ & 121.42 & 121.47 & 107.20 \\
\hline $13 \mathrm{H}-1,29$ & 121.71 & 121.76 & 107.49 \\
\hline $13 \mathrm{H}-1,110$ & 122.57 & 122.57 & 108.30 \\
\hline $13 \mathrm{H}-3,106$ & 125.53 & 125.53 & 111.26 \\
\hline $13 \mathrm{H}-5,1$ & 127.37 & 127.48 & 113.21 \\
\hline $13 \mathrm{H}-6,90$ & 129.79 & 129.87 & 115.60 \\
\hline $13 \mathrm{H}-7,36$ & 130.73 & 130.83 & 116.56 \\
\hline $13 \mathrm{H}-7,70$ & 131.07 & 131.17 & 116.90 \\
\hline $14 \mathrm{H}-1,0$ & 131.67 & 131.48 & 116.70 \\
\hline $14 \mathrm{H}-1,24$ & 131.92 & 131.72 & 116.94 \\
\hline $14 \mathrm{H}-1,50$ & 132.33 & 131.98 & 117.20 \\
\hline $14 \mathrm{H}-2,24$ & 132.83 & 133.22 & 118.44 \\
\hline $14 \mathrm{H}-2,91$ & 133.61 & 133.89 & 119.11 \\
\hline $14 \mathrm{H}-3,100$ & 135.31 & 135.48 & 120.70 \\
\hline $14 \mathrm{H}-4,59$ & 136.57 & 136.57 & 121.79 \\
\hline $14 \mathrm{H}-4,133$ & 137.55 & 137.31 & 122.53 \\
\hline $14 \mathrm{H}-5,136$ & 138.83 & 138.84 & 124.06 \\
\hline $14 \mathrm{H}-7,87$ & 141.34 & 141.35 & 126.57 \\
\hline $15 \mathrm{H}-1,0$ & 141.90 & 142.05 & 126.20 \\
\hline $15 \mathrm{H}-1,95$ & 142.85 & 143.00 & 127.15 \\
\hline $15 \mathrm{H}-2,31$ & 143.86 & 143.86 & 128.01 \\
\hline $15 \mathrm{H}-4,140$ & 147.96 & 147.96 & 132.10 \\
\hline $15 \mathrm{H}-5,85$ & 148.80 & 148.90 & 133.05 \\
\hline $15 \mathrm{H}-6,110$ & 150.82 & 150.65 & 134.80 \\
\hline $15 \mathrm{H}-7,73$ & 151.49 & 151.38 & 135.53 \\
\hline $16 \mathrm{H}-1,0$ & 152.65 & 152.61 & 135.70 \\
\hline $16 \mathrm{H}-1,47$ & 153.12 & 153.09 & 136.17 \\
\hline $16 \mathrm{H}-1,133$ & 153.94 & 153.94 & 137.03 \\
\hline $16 \mathrm{H}-5,129$ & 159.90 & 159.90 & 142.99 \\
\hline $16 \mathrm{H}-6,103$ & 161.26 & 161.15 & 144.23 \\
\hline $16 \mathrm{H}-7,40$ & 162.06 & 162.01 & 145.10 \\
\hline $16 \mathrm{H}-7,75$ & 162.41 & 162.36 & 145.45 \\
\hline $17 \mathrm{H}-1,25$ & 163.88 & 163.99 & 145.45 \\
\hline $17 \mathrm{H}-1,77$ & 164.39 & 164.50 & 145.97 \\
\hline $17 \mathrm{H}-2,27$ & 165.50 & 165.50 & 146.97 \\
\hline $17 \mathrm{H}-4,132$ & 169.55 & 169.55 & 151.02 \\
\hline 17H-5, 109 & 170.38 & 170.82 & 152.29 \\
\hline $17 \mathrm{H}-6,49$ & 171.06 & 171.72 & 153.19 \\
\hline $17 \mathrm{H}-6,145$ & 172.19 & 172.68 & 154.15 \\
\hline $17 \mathrm{H}-7,76$ & 172.68 & 173.50 & 154.96 \\
\hline $18 \mathrm{H}-1,0$ & 174.42 & 174.58 & 154.70 \\
\hline $18 \mathrm{H}-1,23$ & 174.66 & 174.81 & 154.93 \\
\hline $18 \mathrm{H}-2,51$ & 176.59 & 176.59 & 156.71 \\
\hline $18 \mathrm{H}-6,55$ & 182.63 & 182.63 & 162.75 \\
\hline
\end{tabular}

\begin{tabular}{|c|c|c|c|}
\hline \multirow[b]{2}{*}{$\begin{array}{l}\text { Core, section, } \\
\text { interval }(\mathrm{cm})\end{array}$} & \multicolumn{3}{|c|}{ Depth $(m)$} \\
\hline & $\begin{array}{l}\text { Adjusted } \\
\text { CCSF }\end{array}$ & CCSF & CSF-A \\
\hline $18 \mathrm{H}-7,6$ & 183.63 & 183.64 & 163.76 \\
\hline $18 \mathrm{H}-7,75$ & 184.32 & 184.33 & 164.45 \\
\hline $19 \mathrm{H}-1,24$ & 185.06 & 185.09 & 164.44 \\
\hline $19 \mathrm{H}-1,51$ & 185.48 & 185.36 & 164.71 \\
\hline $19 \mathrm{H}-1,144$ & 186.29 & 186.29 & 165.64 \\
\hline $19 \mathrm{H}-6,4$ & 192.40 & 192.39 & 171.74 \\
\hline 19H-6, 137 & 193.69 & 193.72 & 173.07 \\
\hline $19 \mathrm{H}-7,60$ & 194.31 & 194.35 & 173.70 \\
\hline $20 \mathrm{H}-1,0$ & 194.94 & 194.99 & 173.70 \\
\hline $20 \mathrm{H}-1,113$ & 196.07 & 196.12 & 174.83 \\
\hline $20 \mathrm{H}-1,141$ & 196.40 & 196.40 & 175.11 \\
\hline $20 \mathrm{H}-5,63$ & 201.62 & 201.62 & 180.33 \\
\hline $20 \mathrm{H}-6,108$ & 203.71 & 203.58 & 182.28 \\
\hline $20 \mathrm{H}-7,54$ & 204.66 & 204.53 & 183.24 \\
\hline $20 \mathrm{H}-7,77$ & 204.89 & 204.76 & 183.47 \\
\hline $21 \mathrm{H}-1,0$ & 205.69 & 205.69 & 183.20 \\
\hline $21 \mathrm{H}-1,55$ & 206.09 & 206.25 & 183.75 \\
\hline $21 \mathrm{H}-1,103$ & 206.62 & 206.72 & 184.23 \\
\hline $21 \mathrm{H}-2,20$ & 207.39 & 207.39 & 184.90 \\
\hline $21 \mathrm{H}-6,26$ & 213.45 & 213.46 & 190.96 \\
\hline $21 \mathrm{H}-7,15$ & 214.45 & 214.54 & 192.05 \\
\hline $21 \mathrm{H}-7,88$ & 215.19 & 215.27 & 192.78 \\
\hline $22 \mathrm{H}-1,0$ & 216.61 & 216.60 & 192.70 \\
\hline $22 \mathrm{H}-1,28$ & 216.89 & 216.88 & 192.98 \\
\hline $22 \mathrm{H}-1,96$ & 217.56 & 217.56 & 193.66 \\
\hline $22 \mathrm{H}-3,100$ & 220.61 & 220.61 & 196.70 \\
\hline $22 \mathrm{H}-4,99$ & 222.23 & 222.09 & 198.19 \\
\hline $22 \mathrm{H}-5,108$ & 223.87 & 223.69 & 199.78 \\
\hline $22 \mathrm{H}-7,93$ & 226.41 & 226.24 & 202.34 \\
\hline $23 \mathrm{H}-1,0$ & 227.39 & 227.39 & 202.20 \\
\hline $23 \mathrm{H}-1,55$ & 227.94 & 227.94 & 202.75 \\
\hline $23 \mathrm{H}-3,118$ & 231.57 & 231.57 & 206.38 \\
\hline $23 \mathrm{H}-5,1$ & 233.22 & 233.41 & 208.21 \\
\hline $23 \mathrm{H}-6,39$ & 234.99 & 235.28 & 210.09 \\
\hline $23 \mathrm{H}-7,11$ & 236.12 & 236.50 & 211.31 \\
\hline $23 \mathrm{H}-7,70$ & 236.71 & 237.09 & 211.90 \\
\hline $24 \mathrm{H}-1,14$ & 238.60 & 238.67 & 211.84 \\
\hline $24 \mathrm{H}-1,84$ & 239.37 & 239.38 & 212.54 \\
\hline $24 \mathrm{H}-4,61$ & 243.64 & 243.64 & 216.81 \\
\hline $24 \mathrm{H}-4,108$ & 244.11 & 244.11 & 217.28 \\
\hline $24 \mathrm{H}-5,35$ & 244.55 & 244.88 & 218.05 \\
\hline $24 \mathrm{H}-6,2$ & 245.55 & 246.05 & 219.22 \\
\hline $24 \mathrm{H}-6,96$ & 247.16 & 246.99 & 220.16 \\
\hline $24 \mathrm{H}-7,45$ & 247.97 & 247.99 & 221.15 \\
\hline $24 \mathrm{H}-7,71$ & 248.23 & 248.24 & 221.41 \\
\hline $25 \mathrm{H}-1,0$ & 248.98 & 248.98 & 221.20 \\
\hline $25 \mathrm{H}-2,5$ & 250.54 & 250.54 & 222.76 \\
\hline $25 \mathrm{H}-6,87$ & 257.39 & 257.39 & 229.61 \\
\hline $25 \mathrm{H}-7,46$ & 258.50 & 258.29 & 230.51 \\
\hline $25 \mathrm{H}-7,87$ & 258.70 & 258.70 & 230.92 \\
\hline $26 \mathrm{H}-1,0$ & 258.83 & 258.98 & 230.70 \\
\hline $26 \mathrm{H}-1,35$ & 259.29 & 259.33 & 231.05 \\
\hline $26 \mathrm{H}-1,76$ & 260.08 & 259.74 & 231.46 \\
\hline $26 \mathrm{H}-2,122$ & 261.70 & 261.70 & 233.42 \\
\hline $26 \mathrm{H}-6,90$ & 267.38 & 267.38 & 239.10 \\
\hline $26 \mathrm{H}-7,34$ & 268.32 & 268.32 & 240.04 \\
\hline $26 \mathrm{H}-7,69$ & 268.67 & 268.67 & 240.39 \\
\hline $27 X-1,52$ & 268.83 & 268.85 & 240.72 \\
\hline $27 X-1,142$ & 269.48 & 269.75 & 241.62 \\
\hline $27 X-2,37$ & 270.56 & 270.20 & 242.07 \\
\hline $27 X-3,85$ & 272.27 & 272.18 & 244.05 \\
\hline $27 X-5,59$ & 274.67 & 274.92 & 246.79 \\
\hline $27 X-5,134$ & 275.41 & 275.67 & 247.54 \\
\hline $29 X-1,0$ & 289.36 & 288.91 & 259.40 \\
\hline $29 X-1,30$ & 289.66 & 289.21 & 259.70 \\
\hline $29 X-1,92$ & 290.11 & 289.83 & 260.32 \\
\hline $29 X-2,20$ & 290.81 & 290.61 & 261.10 \\
\hline $29 X-3,149$ & 293.16 & 293.40 & 263.89 \\
\hline
\end{tabular}


Table AT47 (continued).

\begin{tabular}{|c|c|c|c|}
\hline \multirow[b]{2}{*}{$\begin{array}{l}\text { Core, section, } \\
\text { interval }(\mathrm{cm})\end{array}$} & \multicolumn{3}{|c|}{ Depth $(\mathrm{m})$} \\
\hline & $\begin{array}{l}\text { Adjusted } \\
\text { CCSF }\end{array}$ & CCSF & CSF-A \\
\hline $29 X-4,65$ & 293.99 & 294.06 & 264.55 \\
\hline 29X-4, 101 & 294.42 & 294.42 & 264.91 \\
\hline $29 X-5,136$ & 296.28 & 296.27 & 266.76 \\
\hline $29 X-6,104$ & 297.76 & 297.46 & 267.94 \\
\hline $29 X-6,124$ & 297.97 & 297.65 & 268.14 \\
\hline $32 X-1,38$ & 321.66 & 321.55 & 288.48 \\
\hline $32 X-2,79$ & 323.62 & 323.46 & 290.39 \\
\hline $32 X-3,47$ & 324.63 & 324.64 & 291.57 \\
\hline $32 X-6,16$ & 328.19 & 328.83 & 295.76 \\
\hline $32 X-6,57$ & 328.78 & 329.24 & 296.17 \\
\hline $33 X-1,21$ & 332.23 & 332.23 & 297.91 \\
\hline $33 X-2,150$ & 335.02 & 335.02 & 300.70 \\
\hline $33 X-4,13$ & 336.51 & 336.65 & 302.33 \\
\hline $33 X-5,4$ & 338.03 & 338.06 & 303.74 \\
\hline $33 X-5,75$ & 338.84 & 338.77 & 304.45 \\
\hline $33 X-5,124$ & 339.39 & 339.26 & 304.94 \\
\hline $33 x-6,37$ & 340.35 & 339.89 & 305.57 \\
\hline $33 X-6,99$ & 341.06 & 340.51 & 306.19 \\
\hline $34 X-1,19$ & 343.44 & 343.32 & 307.49 \\
\hline $34 X-1,62$ & 343.91 & 343.75 & 307.92 \\
\hline $34 X-2,29$ & 344.86 & 344.92 & 309.09 \\
\hline $34 X-2,88$ & 345.45 & 345.51 & 309.68 \\
\hline $34 X-3,10$ & 346.20 & 346.23 & 310.40 \\
\hline $34 X-4,90$ & 348.64 & 348.53 & 312.70 \\
\hline $34 X-5,1$ & 349.45 & 349.14 & 313.31 \\
\hline $34 X-5,100$ & 350.71 & 350.13 & 314.30 \\
\hline $34 X-6,56$ & 351.99 & 351.19 & 315.36 \\
\hline $34 X-6,111$ & 352.53 & 351.74 & 315.91 \\
\hline $35 X-1,84$ & 359.09 & 359.37 & 317.74 \\
\hline $35 X-2,28$ & 360.04 & 360.31 & 318.68 \\
\hline $35 X-2,92$ & 360.98 & 360.95 & 319.32 \\
\hline $35 X-2,120$ & 361.25 & 361.23 & 319.60 \\
\hline $36 X-1,0$ & 365.12 & 365.12 & 326.50 \\
\hline $36 X-1,106$ & 366.18 & 366.18 & 327.56 \\
\hline $37 X-1,0$ & 367.58 & 367.58 & 336.00 \\
\hline $37 X-1,111$ & 368.69 & 368.69 & 337.11 \\
\hline $38 X-1,0$ & 376.53 & 376.53 & 345.50 \\
\hline $38 X-2,33$ & 377.35 & 378.36 & 347.33 \\
\hline $38 X-2,99$ & 377.96 & 379.02 & 347.99 \\
\hline $38 X-2,149$ & 380.02 & 379.52 & 348.49 \\
\hline $38 X-5,25$ & 382.84 & 382.78 & 351.75 \\
\hline $38 X-5,91$ & 383.57 & 383.44 & 352.41 \\
\hline $38 X-6,150$ & 385.53 & 385.53 & 354.50 \\
\hline $39 X-1,0$ & 385.72 & 386.41 & 354.80 \\
\hline $39 X-1,105$ & 386.68 & 387.46 & 355.85 \\
\hline $39 X-6,131$ & 394.44 & 395.22 & 363.61 \\
\hline $40 X-1,0$ & 403.11 & 403.11 & 364.40 \\
\hline $40 X-3,78$ & 406.38 & 406.38 & 367.67 \\
\hline $41 X-1,0$ & 420.97 & 421.52 & 374.00 \\
\hline $41 X-1,39$ & 421.37 & 421.92 & 374.39 \\
\hline $41 X-1,143$ & 422.90 & 422.95 & 375.43 \\
\hline $41 X-3,22$ & 424.79 & 424.74 & 377.22 \\
\hline $41 X-3,74$ & 425.29 & 425.26 & 377.74 \\
\hline $41 X-3,118$ & 425.62 & 425.70 & 378.18 \\
\hline $41 X-4,13$ & 426.09 & 426.15 & 378.63 \\
\hline $41 X-5,42$ & 427.83 & 427.94 & 380.42 \\
\hline $41 X-5,117$ & 428.53 & 428.69 & 381.17 \\
\hline $41 X-6,97$ & 429.83 & 429.99 & 382.47 \\
\hline $42 X-1,0$ & 430.72 & 430.72 & 383.60 \\
\hline $42 X-5,110$ & 437.82 & 437.82 & 390.70 \\
\hline $43 X-1,42$ & 438.13 & 439.89 & 393.62 \\
\hline $43 X-1,64$ & 438.65 & 440.11 & 393.84 \\
\hline $43 X-2,64$ & 441.10 & 441.61 & 395.34 \\
\hline $43 X-2,131$ & 441.41 & 442.28 & 396.01 \\
\hline $44 X-1,0$ & 448.66 & 448.66 & 402.80 \\
\hline $44 X-4,67$ & 453.83 & 453.83 & 407.97 \\
\hline
\end{tabular}

This table is also available in ASCII. 
Table AT48. Gamma ray attenuation (GRA) density data, Hole U1338B.

\begin{tabular}{|c|c|c|c|c|}
\hline \multirow[b]{2}{*}{$\begin{array}{l}\text { Core, section, } \\
\text { interval }(\mathrm{cm})\end{array}$} & \multicolumn{3}{|c|}{ Depth $(\mathrm{m})$} & \multirow[b]{2}{*}{$\begin{array}{c}\text { GRA } \\
\left(\mathrm{g} / \mathrm{cm}^{3}\right)\end{array}$} \\
\hline & CSF & CCSF & $\begin{array}{c}\text { Adjusted } \\
\text { CCSF }\end{array}$ & \\
\hline \multicolumn{5}{|l|}{ 321-U1338B- } \\
\hline $1 \mathrm{H}-1,7.5$ & 0.08 & 0.08 & 0.09 & 1.31 \\
\hline $1 \mathrm{H}-1,10$ & 0.10 & 0.10 & 0.11 & 1.36 \\
\hline $1 \mathrm{H}-1,12.5$ & 0.13 & 0.13 & 0.15 & 1.34 \\
\hline $1 \mathrm{H}-1,15$ & 0.15 & 0.15 & 0.17 & 1.39 \\
\hline $1 \mathrm{H}-1,17.5$ & 0.18 & 0.18 & 0.21 & 1.40 \\
\hline $1 \mathrm{H}-1,20$ & 0.20 & 0.20 & 0.23 & 1.39 \\
\hline $1 \mathrm{H}-1,22.5$ & 0.23 & 0.23 & 0.27 & 1.40 \\
\hline $1 \mathrm{H}-1,25$ & 0.25 & 0.25 & 0.30 & 1.41 \\
\hline $1 \mathrm{H}-1,27.6$ & 0.28 & 0.28 & 0.33 & 1.40 \\
\hline $1 \mathrm{H}-1,30.1$ & 0.30 & 0.30 & 0.36 & 1.37 \\
\hline $1 \mathrm{H}-1,32.6$ & 0.33 & 0.33 & 0.39 & 1.36 \\
\hline $1 \mathrm{H}-1,35.1$ & 0.35 & 0.35 & 0.42 & 1.36 \\
\hline $1 \mathrm{H}-1,37.6$ & 0.38 & 0.38 & 0.45 & 1.38 \\
\hline $1 \mathrm{H}-1,40.1$ & 0.40 & 0.40 & 0.48 & 1.40 \\
\hline $1 \mathrm{H}-1,42.6$ & 0.43 & 0.43 & 0.51 & 1.37 \\
\hline $1 \mathrm{H}-1,45.1$ & 0.45 & 0.45 & 0.54 & 1.41 \\
\hline $1 \mathrm{H}-1,47.6$ & 0.48 & 0.48 & 0.58 & 1.41 \\
\hline $1 \mathrm{H}-1,50.1$ & 0.50 & 0.50 & 0.60 & 1.36 \\
\hline $1 \mathrm{H}-1,52.6$ & 0.53 & 0.53 & 0.64 & 1.35 \\
\hline $1 \mathrm{H}-1,55.1$ & 0.55 & 0.55 & 0.66 & 1.41 \\
\hline $1 \mathrm{H}-1,57.6$ & 0.58 & 0.58 & 0.70 & 1.40 \\
\hline $1 \mathrm{H}-1,60.1$ & 0.60 & 0.60 & 0.72 & 1.34 \\
\hline $1 \mathrm{H}-1,62.6$ & 0.63 & 0.63 & 0.76 & 1.39 \\
\hline $1 \mathrm{H}-1,65.1$ & 0.65 & 0.65 & 0.78 & 1.38 \\
\hline $1 \mathrm{H}-1,67.6$ & 0.68 & 0.68 & 0.82 & 1.38 \\
\hline $1 \mathrm{H}-1,70.1$ & 0.70 & 0.70 & 0.84 & 1.34 \\
\hline $1 \mathrm{H}-1,72.6$ & 0.73 & 0.73 & 0.88 & 1.42 \\
\hline $1 \mathrm{H}-1,75.1$ & 0.75 & 0.75 & 0.90 & 1.38 \\
\hline $1 \mathrm{H}-1,77.6$ & 0.78 & 0.78 & 0.94 & 1.38 \\
\hline $1 \mathrm{H}-1,80.1$ & 0.80 & 0.80 & 0.97 & 1.41 \\
\hline $1 \mathrm{H}-1,82.6$ & 0.83 & 0.83 & 1.00 & 1.37 \\
\hline $1 \mathrm{H}-1,85.1$ & 0.85 & 0.85 & 1.03 & 1.37 \\
\hline $1 \mathrm{H}-1,87.6$ & 0.88 & 0.88 & 1.06 & 1.41 \\
\hline $1 \mathrm{H}-1,90.2$ & 0.90 & 0.90 & 1.09 & 1.40 \\
\hline $1 \mathrm{H}-1,92.7$ & 0.93 & 0.93 & 1.12 & 1.39 \\
\hline $1 \mathrm{H}-1,95.2$ & 0.95 & 0.95 & 1.14 & 1.40 \\
\hline $1 \mathrm{H}-1,97.7$ & 0.98 & 0.98 & 1.16 & 1.39 \\
\hline $1 \mathrm{H}-1,100.2$ & 1.00 & 1.00 & 1.18 & 1.36 \\
\hline $1 \mathrm{H}-1,102.7$ & 1.03 & 1.03 & 1.21 & 1.37 \\
\hline $1 \mathrm{H}-1,105.2$ & 1.05 & 1.05 & 1.22 & 1.37 \\
\hline $1 \mathrm{H}-1,107.7$ & 1.08 & 1.08 & 1.25 & 1.34 \\
\hline $1 \mathrm{H}-1,110.2$ & 1.10 & 1.10 & 1.26 & 1.29 \\
\hline $1 \mathrm{H}-1,112.7$ & 1.13 & 1.13 & 1.29 & 1.31 \\
\hline $1 \mathrm{H}-1,115.2$ & 1.15 & 1.15 & 1.31 & 1.31 \\
\hline $1 \mathrm{H}-1,117.7$ & 1.18 & 1.18 & 1.33 & 1.32 \\
\hline $1 \mathrm{H}-1,120.2$ & 1.20 & 1.20 & 1.35 & 1.26 \\
\hline $1 \mathrm{H}-1,122.7$ & 1.23 & 1.23 & 1.37 & 1.27 \\
\hline $1 \mathrm{H}-1,125.2$ & 1.25 & 1.25 & 1.39 & 1.27 \\
\hline $1 \mathrm{H}-1,127.7$ & 1.28 & 1.28 & 1.41 & 1.24 \\
\hline $1 \mathrm{H}-1,130.2$ & 1.30 & 1.30 & 1.43 & 1.26 \\
\hline $1 \mathrm{H}-1,132.7$ & 1.33 & 1.33 & 1.45 & 1.28 \\
\hline $1 \mathrm{H}-1,135.2$ & 1.35 & 1.35 & 1.47 & 1.27 \\
\hline $1 \mathrm{H}-1,137.7$ & 1.38 & 1.38 & 1.50 & 1.26 \\
\hline $1 \mathrm{H}-1,140.2$ & 1.40 & 1.40 & 1.51 & 1.28 \\
\hline $1 \mathrm{H}-2,3.3$ & 1.53 & 1.53 & 1.62 & 1.30 \\
\hline $1 \mathrm{H}-2,5.8$ & 1.56 & 1.56 & 1.65 & 1.28 \\
\hline $1 \mathrm{H}-2,8.3$ & 1.58 & 1.58 & 1.66 & 1.26 \\
\hline $1 \mathrm{H}-2,10.8$ & 1.61 & 1.61 & 1.69 & 1.27 \\
\hline $1 \mathrm{H}-2,13.3$ & 1.63 & 1.63 & 1.70 & 1.25 \\
\hline $1 \mathrm{H}-2,15.8$ & 1.66 & 1.66 & 1.73 & 1.25 \\
\hline $1 \mathrm{H}-2,18.3$ & 1.68 & 1.68 & 1.74 & 1.24 \\
\hline
\end{tabular}

Only a portion of this table appears here. The complete table is available in ASCII. 
Table AT49. Magnetic susceptibility data, Hole U1338B.

\begin{tabular}{|c|c|c|c|c|}
\hline \multirow[b]{2}{*}{$\begin{array}{l}\text { Core, section, } \\
\text { interval }(\mathrm{cm})\end{array}$} & \multicolumn{3}{|c|}{ Depth $(m)$} & \multirow{2}{*}{$\begin{array}{c}\text { Magnetic } \\
\text { susceptibility } \\
\text { (IU) }\end{array}$} \\
\hline & CSF & CCSF & $\begin{array}{l}\text { Adjusted } \\
\text { CCSF }\end{array}$ & \\
\hline \multicolumn{5}{|l|}{ 321-U1338B- } \\
\hline $1 \mathrm{H}-1,0.1$ & 0.00 & 0.00 & -0.01 & 2.7 \\
\hline $1 \mathrm{H}-1,2.6$ & 0.03 & 0.03 & 0.03 & 3.3 \\
\hline $1 \mathrm{H}-1,5.1$ & 0.05 & 0.05 & 0.05 & 4.4 \\
\hline $1 \mathrm{H}-1,7.6$ & 0.08 & 0.08 & 0.09 & 4.4 \\
\hline $1 \mathrm{H}-1,10.1$ & 0.10 & 0.10 & 0.11 & 4.4 \\
\hline $1 \mathrm{H}-1,12.6$ & 0.13 & 0.13 & 0.15 & 4.4 \\
\hline $1 \mathrm{H}-1,15.1$ & 0.15 & 0.15 & 0.17 & 4.4 \\
\hline $1 \mathrm{H}-1,17.6$ & 0.18 & 0.18 & 0.21 & 4.4 \\
\hline $1 \mathrm{H}-1,20.1$ & 0.20 & 0.20 & 0.23 & 5.5 \\
\hline $1 \mathrm{H}-1,22.6$ & 0.23 & 0.23 & 0.27 & 5.5 \\
\hline $1 \mathrm{H}-1,25.1$ & 0.25 & 0.25 & 0.30 & 4.9 \\
\hline $1 \mathrm{H}-1,27.6$ & 0.28 & 0.28 & 0.33 & 5.5 \\
\hline $1 \mathrm{H}-1,30.1$ & 0.30 & 0.30 & 0.36 & 4.1 \\
\hline $1 \mathrm{H}-1,32.6$ & 0.33 & 0.33 & 0.39 & 3.3 \\
\hline $1 \mathrm{H}-1,35.1$ & 0.35 & 0.35 & 0.42 & 3.6 \\
\hline $1 \mathrm{H}-1,37.6$ & 0.38 & 0.38 & 0.45 & 3.0 \\
\hline $1 \mathrm{H}-1,40.1$ & 0.40 & 0.40 & 0.48 & 3.3 \\
\hline $1 \mathrm{H}-1,42.6$ & 0.43 & 0.43 & 0.51 & 3.3 \\
\hline $1 \mathrm{H}-1,45.1$ & 0.45 & 0.45 & 0.54 & 3.0 \\
\hline $1 \mathrm{H}-1,47.6$ & 0.48 & 0.48 & 0.58 & 3.3 \\
\hline $1 \mathrm{H}-1,50.1$ & 0.50 & 0.50 & 0.60 & 2.5 \\
\hline $1 \mathrm{H}-1,52.6$ & 0.53 & 0.53 & 0.64 & 2.2 \\
\hline $1 \mathrm{H}-1,55.1$ & 0.55 & 0.55 & 0.66 & 2.2 \\
\hline $1 \mathrm{H}-1,57.7$ & 0.58 & 0.58 & 0.70 & 2.2 \\
\hline $1 \mathrm{H}-1,60.2$ & 0.60 & 0.60 & 0.72 & 2.2 \\
\hline $1 \mathrm{H}-1,62.7$ & 0.63 & 0.63 & 0.76 & 1.9 \\
\hline $1 \mathrm{H}-1,65.2$ & 0.65 & 0.65 & 0.78 & 2.5 \\
\hline $1 \mathrm{H}-1,67.7$ & 0.68 & 0.68 & 0.82 & 2.5 \\
\hline $1 \mathrm{H}-1,70.2$ & 0.70 & 0.70 & 0.84 & 2.5 \\
\hline $1 \mathrm{H}-1,72.7$ & 0.73 & 0.73 & 0.88 & 2.2 \\
\hline $1 \mathrm{H}-1,75.2$ & 0.75 & 0.75 & 0.90 & 1.9 \\
\hline $1 \mathrm{H}-1,77.7$ & 0.78 & 0.78 & 0.94 & 2.7 \\
\hline $1 \mathrm{H}-1,80.2$ & 0.80 & 0.80 & 0.97 & 2.5 \\
\hline $1 \mathrm{H}-1,82.7$ & 0.83 & 0.83 & 1.00 & 2.5 \\
\hline $1 \mathrm{H}-1,85.2$ & 0.85 & 0.85 & 1.03 & 2.5 \\
\hline $1 \mathrm{H}-1,87.7$ & 0.88 & 0.88 & 1.06 & 2.2 \\
\hline $1 \mathrm{H}-1,90.2$ & 0.90 & 0.90 & 1.09 & 2.2 \\
\hline $1 \mathrm{H}-1,92.7$ & 0.93 & 0.93 & 1.12 & 2.2 \\
\hline $1 \mathrm{H}-1,95.2$ & 0.95 & 0.95 & 1.14 & 2.5 \\
\hline $1 \mathrm{H}-1,97.7$ & 0.98 & 0.98 & 1.16 & 2.5 \\
\hline $1 \mathrm{H}-1,100.2$ & 1.00 & 1.00 & 1.18 & 2.7 \\
\hline $1 \mathrm{H}-1,102.7$ & 1.03 & 1.03 & 1.21 & 3.0 \\
\hline $1 \mathrm{H}-1,105.2$ & 1.05 & 1.05 & 1.22 & 3.0 \\
\hline $1 \mathrm{H}-1,107.7$ & 1.08 & 1.08 & 1.25 & 3.6 \\
\hline $1 \mathrm{H}-1,110.2$ & 1.10 & 1.10 & 1.26 & 3.6 \\
\hline $1 \mathrm{H}-1,112.7$ & 1.13 & 1.13 & 1.29 & 3.6 \\
\hline $1 \mathrm{H}-1,115.2$ & 1.15 & 1.15 & 1.31 & 3.6 \\
\hline $1 \mathrm{H}-1,117.8$ & 1.18 & 1.18 & 1.33 & 4.1 \\
\hline $1 \mathrm{H}-1,120.2$ & 1.20 & 1.20 & 1.35 & 4.9 \\
\hline $1 \mathrm{H}-1,122.8$ & 1.23 & 1.23 & 1.37 & 5.5 \\
\hline $1 \mathrm{H}-1,125.3$ & 1.25 & 1.25 & 1.39 & 6.0 \\
\hline $1 \mathrm{H}-1,127.8$ & 1.28 & 1.28 & 1.41 & 6.9 \\
\hline $1 \mathrm{H}-1,130.3$ & 1.30 & 1.30 & 1.43 & 6.6 \\
\hline $1 \mathrm{H}-1,132.8$ & 1.33 & 1.33 & 1.45 & 6.3 \\
\hline $1 \mathrm{H}-1,135.3$ & 1.35 & 1.35 & 1.47 & 6.3 \\
\hline $1 \mathrm{H}-1,137.8$ & 1.38 & 1.38 & 1.50 & 6.3 \\
\hline $1 \mathrm{H}-1,140.3$ & 1.40 & 1.40 & 1.51 & 6.3 \\
\hline $1 \mathrm{H}-1,142.8$ & 1.43 & 1.43 & 1.54 & 5.8 \\
\hline
\end{tabular}

Only a portion of this table appears here. The complete table is available in ASCII. 
Table AT50. Natural gamma ray (NGR) data, Hole U1338B.

\begin{tabular}{|c|c|c|c|c|}
\hline \multirow[b]{2}{*}{$\begin{array}{l}\text { Core, section, } \\
\text { interval }(\mathrm{cm})\end{array}$} & \multicolumn{3}{|c|}{ Depth $(\mathrm{m})$} & \multirow[b]{2}{*}{$\begin{array}{l}\text { NGR } \\
\text { (cps) }\end{array}$} \\
\hline & CSF & CCSF & $\begin{array}{l}\text { Adjusted } \\
\text { CCSF }\end{array}$ & \\
\hline \multicolumn{5}{|l|}{ 321-U1338B- } \\
\hline $1 \mathrm{H}-1,0$ & 0.0 & 0.0 & -0.01 & 38.6 \\
\hline $1 \mathrm{H}-1,10$ & 0.1 & 0.1 & 0.11 & 39.1 \\
\hline $1 \mathrm{H}-1,20$ & 0.2 & 0.2 & 0.23 & 42.0 \\
\hline $1 \mathrm{H}-1,30$ & 0.3 & 0.3 & 0.36 & 35.0 \\
\hline $1 \mathrm{H}-1,40$ & 0.4 & 0.4 & 0.48 & 28.1 \\
\hline $1 \mathrm{H}-1,50$ & 0.5 & 0.5 & 0.60 & 25.5 \\
\hline $1 \mathrm{H}-1,60$ & 0.6 & 0.6 & 0.72 & 26.2 \\
\hline $1 \mathrm{H}-1,70$ & 0.7 & 0.7 & 0.84 & 23.8 \\
\hline $1 \mathrm{H}-1,80$ & 0.8 & 0.8 & 0.97 & 22.6 \\
\hline $1 \mathrm{H}-1,90$ & 0.9 & 0.9 & 1.09 & 20.2 \\
\hline $1 \mathrm{H}-1,100$ & 1.0 & 1.0 & 1.18 & 24.6 \\
\hline $1 \mathrm{H}-1,110$ & 1.1 & 1.1 & 1.26 & 29.0 \\
\hline $1 \mathrm{H}-1,120$ & 1.2 & 1.2 & 1.35 & 29.4 \\
\hline $1 \mathrm{H}-1,130$ & 1.3 & 1.3 & 1.43 & 33.5 \\
\hline $1 \mathrm{H}-1,140$ & 1.4 & 1.4 & 1.51 & 43.7 \\
\hline $1 \mathrm{H}-2,0$ & 1.5 & 1.5 & 1.60 & 41.5 \\
\hline $1 \mathrm{H}-2,10$ & 1.6 & 1.6 & 1.68 & 42.2 \\
\hline $1 \mathrm{H}-2,20$ & 1.7 & 1.7 & 1.76 & 33.1 \\
\hline $1 \mathrm{H}-2,30$ & 1.8 & 1.8 & 1.84 & 28.9 \\
\hline $1 \mathrm{H}-2,40$ & 1.9 & 1.9 & 1.93 & 28.7 \\
\hline $1 \mathrm{H}-2,50$ & 2.0 & 2.0 & 2.01 & 23.3 \\
\hline $1 \mathrm{H}-2,60$ & 2.1 & 2.1 & 2.10 & 20.6 \\
\hline $1 \mathrm{H}-2,70$ & 2.2 & 2.2 & 2.20 & 14.1 \\
\hline $1 \mathrm{H}-2,80$ & 2.3 & 2.3 & 2.30 & 10.5 \\
\hline $1 \mathrm{H}-2,90$ & 2.4 & 2.4 & 2.40 & 10.9 \\
\hline $1 \mathrm{H}-2,100$ & 2.5 & 2.5 & 2.50 & 11.6 \\
\hline $1 \mathrm{H}-2,110$ & 2.6 & 2.6 & 2.60 & 12.5 \\
\hline $1 \mathrm{H}-2,120$ & 2.7 & 2.7 & 2.70 & 12.3 \\
\hline $1 \mathrm{H}-2,130$ & 2.8 & 2.8 & 2.80 & 12.5 \\
\hline $1 \mathrm{H}-2,140$ & 2.9 & 2.9 & 2.90 & 11.6 \\
\hline $1 \mathrm{H}-2,150$ & 3.0 & 3.0 & 3.00 & 11.9 \\
\hline $1 \mathrm{H}-3,0$ & 3.0 & 3.0 & 3.00 & 12.6 \\
\hline $1 \mathrm{H}-3,10$ & 3.1 & 3.1 & 3.10 & 17.8 \\
\hline $1 \mathrm{H}-3,20$ & 3.2 & 3.2 & 3.20 & 17.6 \\
\hline $1 \mathrm{H}-3,30$ & 3.3 & 3.3 & 3.30 & 11.7 \\
\hline $1 \mathrm{H}-3,40$ & 3.4 & 3.4 & 3.40 & 10.7 \\
\hline $1 \mathrm{H}-3,50$ & 3.5 & 3.5 & 3.50 & 8.7 \\
\hline $1 \mathrm{H}-3,60$ & 3.6 & 3.6 & 3.60 & 8.0 \\
\hline $1 \mathrm{H}-3,70$ & 3.7 & 3.7 & 3.70 & 8.7 \\
\hline $1 \mathrm{H}-3,80$ & 3.8 & 3.8 & 3.80 & 7.4 \\
\hline $1 \mathrm{H}-3,90$ & 3.9 & 3.9 & 3.90 & 8.3 \\
\hline $1 \mathrm{H}-3,100$ & 4.0 & 4.0 & 4.00 & 7.1 \\
\hline $1 \mathrm{H}-3,110$ & 4.1 & 4.1 & 4.10 & 6.1 \\
\hline $1 \mathrm{H}-3,120$ & 4.2 & 4.2 & 4.20 & 6.4 \\
\hline $1 \mathrm{H}-3,130$ & 4.3 & 4.3 & 4.30 & 6.6 \\
\hline $1 \mathrm{H}-3,140$ & 4.4 & 4.4 & 4.40 & 4.8 \\
\hline $1 \mathrm{H}-3,150$ & 4.5 & 4.5 & 4.50 & 4.9 \\
\hline $1 \mathrm{H}-4,0$ & 4.5 & 4.5 & 4.50 & 6.1 \\
\hline $1 \mathrm{H}-4,10$ & 4.6 & 4.6 & 4.60 & 6.2 \\
\hline $1 \mathrm{H}-4,20$ & 4.7 & 4.7 & 4.70 & 5.2 \\
\hline $1 \mathrm{H}-4,30$ & 4.8 & 4.8 & 4.80 & 5.0 \\
\hline $1 \mathrm{H}-4,40$ & 4.9 & 4.9 & 4.90 & 5.4 \\
\hline $1 \mathrm{H}-4,50$ & 5.0 & 5.0 & 5.00 & 5.5 \\
\hline $1 \mathrm{H}-4,60$ & 5.1 & 5.1 & 5.10 & 4.3 \\
\hline $1 \mathrm{H}-4,70$ & 5.2 & 5.2 & 5.20 & 3.6 \\
\hline $1 \mathrm{H}-4,80$ & 5.3 & 5.3 & 5.30 & 3.0 \\
\hline $1 \mathrm{H}-4,90$ & 5.4 & 5.4 & 5.40 & 2.7 \\
\hline $1 \mathrm{H}-4,100$ & 5.5 & 5.5 & 5.49 & 2.4 \\
\hline $1 \mathrm{H}-4,110$ & 5.6 & 5.6 & 5.59 & 2.6 \\
\hline $1 \mathrm{H}-4,120$ & 5.7 & 5.7 & 5.69 & 3.2 \\
\hline $1 \mathrm{H}-4,130$ & 5.8 & 5.8 & 5.79 & 2.7 \\
\hline
\end{tabular}

Only a portion of this table appears here. The complete table is available in ASCII. 
Table AT51. Color reflection data, Hole U1338B.

\begin{tabular}{|c|c|c|c|c|c|c|}
\hline \multirow[b]{2}{*}{$\begin{array}{l}\text { Core, section, } \\
\text { interval }(\mathrm{cm})\end{array}$} & \multicolumn{3}{|c|}{ Depth (m) } & \multirow[b]{2}{*}{$a^{*}$} & \multirow[b]{2}{*}{$b^{*}$} & \multirow[b]{2}{*}{$\mathrm{L}^{*}$} \\
\hline & CSF & CCSF & $\begin{array}{l}\text { Adjusted } \\
\text { CCSF }\end{array}$ & & & \\
\hline \multicolumn{7}{|l|}{ 321-U1338B- } \\
\hline $1 \mathrm{H}-1,2.6$ & 0.03 & 0.03 & 0.03 & 6.1 & 12.8 & 45.7 \\
\hline $1 \mathrm{H}-1,5.1$ & 0.05 & 0.05 & 0.05 & 5.5 & 13.1 & 50.6 \\
\hline $1 \mathrm{H}-1,7.7$ & 0.08 & 0.08 & 0.09 & 6.1 & 14.3 & 49.9 \\
\hline $1 \mathrm{H}-1,10.2$ & 0.10 & 0.10 & 0.11 & 4.4 & 11.4 & 51.2 \\
\hline $1 \mathrm{H}-1,12.8$ & 0.13 & 0.13 & 0.15 & 5.0 & 11.6 & 44.2 \\
\hline $1 \mathrm{H}-1,15.3$ & 0.15 & 0.15 & 0.17 & 5.5 & 12.4 & 45.2 \\
\hline $1 \mathrm{H}-1,17.9$ & 0.18 & 0.18 & 0.21 & 5.6 & 14.9 & 49.5 \\
\hline $1 \mathrm{H}-1,20.4$ & 0.20 & 0.20 & 0.23 & 4.9 & 14.0 & 47.3 \\
\hline $1 \mathrm{H}-1,22.9$ & 0.23 & 0.23 & 0.27 & 4.6 & 15.6 & 52.6 \\
\hline $1 \mathrm{H}-1,25.5$ & 0.26 & 0.26 & 0.31 & 4.8 & 16.4 & 55.3 \\
\hline $1 \mathrm{H}-1,28$ & 0.28 & 0.28 & 0.33 & 2.8 & 20.8 & 72.2 \\
\hline $1 \mathrm{H}-1,30.6$ & 0.31 & 0.31 & 0.37 & 2.8 & 21.2 & 71.3 \\
\hline $1 \mathrm{H}-1,33.1$ & 0.33 & 0.33 & 0.39 & 1.4 & 18.4 & 65.6 \\
\hline $1 \mathrm{H}-1,35.6$ & 0.36 & 0.36 & 0.43 & 4.2 & 19.1 & 59.7 \\
\hline $1 \mathrm{H}-1,38.2$ & 0.38 & 0.38 & 0.45 & 4.5 & 19.0 & 62.3 \\
\hline $1 \mathrm{H}-1,40.7$ & 0.41 & 0.41 & 0.49 & 3.7 & 19.2 & 63.7 \\
\hline $1 \mathrm{H}-1,43.3$ & 0.43 & 0.43 & 0.51 & 4.7 & 18.6 & 63.0 \\
\hline $1 \mathrm{H}-1,45.8$ & 0.46 & 0.46 & 0.55 & 3.9 & 20.1 & 64.7 \\
\hline $1 \mathrm{H}-1,48.3$ & 0.48 & 0.48 & 0.58 & 3.7 & 17.0 & 64.4 \\
\hline $1 \mathrm{H}-1,50.9$ & 0.51 & 0.51 & 0.61 & 4.2 & 19.0 & 67.1 \\
\hline $1 \mathrm{H}-1,53.4$ & 0.53 & 0.53 & 0.64 & 3.3 & 15.6 & 60.4 \\
\hline $1 \mathrm{H}-1,56$ & 0.56 & 0.56 & 0.67 & 4.0 & 19.5 & 69.6 \\
\hline $1 \mathrm{H}-1,58.5$ & 0.59 & 0.59 & 0.71 & 3.4 & 14.7 & 55.2 \\
\hline $1 \mathrm{H}-1,61.1$ & 0.61 & 0.61 & 0.73 & 3.8 & 17.1 & 63.3 \\
\hline $1 \mathrm{H}-1,63.6$ & 0.64 & 0.64 & 0.77 & 3.8 & 18.9 & 67.8 \\
\hline $1 \mathrm{H}-1,66.1$ & 0.66 & 0.66 & 0.79 & 3.5 & 15.7 & 61.3 \\
\hline $1 \mathrm{H}-1,68.7$ & 0.69 & 0.69 & 0.83 & 3.7 & 17.8 & 68.1 \\
\hline $1 \mathrm{H}-1,71.2$ & 0.71 & 0.71 & 0.86 & 2.8 & 18.4 & 70.0 \\
\hline $1 \mathrm{H}-1,73.8$ & 0.74 & 0.74 & 0.89 & 3.7 & 18.5 & 69.4 \\
\hline $1 \mathrm{H}-1,76.3$ & 0.76 & 0.76 & 0.92 & 4.1 & 17.5 & 69.4 \\
\hline $1 \mathrm{H}-1,78.8$ & 0.79 & 0.79 & 0.95 & 4.1 & 18.1 & 65.6 \\
\hline $1 \mathrm{H}-1,81.4$ & 0.81 & 0.81 & 0.98 & 4.5 & 17.5 & 66.1 \\
\hline $1 \mathrm{H}-1,83.9$ & 0.84 & 0.84 & 1.01 & 3.1 & 18.5 & 75.3 \\
\hline $1 \mathrm{H}-1,86.5$ & 0.87 & 0.87 & 1.05 & 2.6 & 18.4 & 73.7 \\
\hline $1 \mathrm{H}-1,89$ & 0.89 & 0.89 & 1.07 & 0.1 & 16.3 & 79.1 \\
\hline $1 \mathrm{H}-1,91.6$ & 0.92 & 0.92 & 1.11 & 2.9 & 18.9 & 76.5 \\
\hline $1 \mathrm{H}-1,94.1$ & 0.94 & 0.94 & 1.13 & 3.9 & 18.7 & 72.5 \\
\hline $1 \mathrm{H}-1,96.6$ & 0.97 & 0.97 & 1.16 & 4.1 & 18.1 & 67.3 \\
\hline $1 \mathrm{H}-1,99.2$ & 0.99 & 0.99 & 1.17 & 3.2 & 15.7 & 61.8 \\
\hline $1 \mathrm{H}-1,101.7$ & 1.02 & 1.02 & 1.20 & 3.2 & 14.9 & 57.5 \\
\hline $1 \mathrm{H}-1,104.3$ & 1.04 & 1.04 & 1.21 & 3.7 & 14.8 & 62.9 \\
\hline $1 \mathrm{H}-1,106.8$ & 1.07 & 1.07 & 1.24 & 3.9 & 19.1 & 66.1 \\
\hline $1 \mathrm{H}-1,109.4$ & 1.09 & 1.09 & 1.26 & 5.0 & 19.8 & 62.2 \\
\hline $1 \mathrm{H}-1,111.9$ & 1.12 & 1.12 & 1.28 & 5.1 & 18.9 & 60.5 \\
\hline $1 \mathrm{H}-1,114.4$ & 1.14 & 1.14 & 1.30 & 4.7 & 17.4 & 55.2 \\
\hline $1 \mathrm{H}-1,117$ & 1.17 & 1.17 & 1.32 & 5.7 & 20.6 & 56.5 \\
\hline $1 \mathrm{H}-1,119.5$ & 1.20 & 1.20 & 1.35 & 5.8 & 20.9 & 57.3 \\
\hline $1 \mathrm{H}-1,122.1$ & 1.22 & 1.22 & 1.36 & 5.9 & 20.9 & 56.8 \\
\hline $1 \mathrm{H}-1,124.6$ & 1.25 & 1.25 & 1.39 & 4.9 & 19.7 & 58.3 \\
\hline $1 \mathrm{H}-1,127.2$ & 1.27 & 1.27 & 1.41 & 5.4 & 18.0 & 57.3 \\
\hline $1 \mathrm{H}-1,129.7$ & 1.30 & 1.30 & 1.43 & 5.4 & 17.4 & 52.1 \\
\hline $1 \mathrm{H}-1,132.2$ & 1.32 & 1.32 & 1.45 & 5.7 & 17.8 & 51.5 \\
\hline $1 \mathrm{H}-1,134.8$ & 1.35 & 1.35 & 1.47 & 5.3 & 16.1 & 49.1 \\
\hline $1 \mathrm{H}-1,137.3$ & 1.37 & 1.37 & 1.49 & 5.7 & 19.7 & 56.1 \\
\hline $1 \mathrm{H}-1,139.9$ & 1.40 & 1.40 & 1.51 & 4.0 & 11.3 & 52.5 \\
\hline $1 \mathrm{H}-2,5.1$ & 1.55 & 1.55 & 1.64 & 3.7 & 11.8 & 45.3 \\
\hline $1 \mathrm{H}-2,7.7$ & 1.58 & 1.58 & 1.66 & 4.2 & 12.1 & 42.8 \\
\hline $1 \mathrm{H}-2,10.2$ & 1.60 & 1.60 & 1.68 & 4.0 & 10.9 & 40.0 \\
\hline $1 \mathrm{H}-2,12.8$ & 1.63 & 1.63 & 1.70 & 4.4 & 10.9 & 39.4 \\
\hline $1 \mathrm{H}-2,15.3$ & 1.65 & 1.65 & 1.72 & 3.9 & 10.9 & 46.4 \\
\hline
\end{tabular}

Only a portion of this table appears here. The complete table is available in ASCII. 
Table AT52. Core adjusted data, Hole U1338B. (Continued on next page.)

\begin{tabular}{|c|c|c|c|c|c|c|c|c|c|c|c|}
\hline \multirow[b]{2}{*}{$\begin{array}{l}\text { Core, section, } \\
\text { interval }(\mathrm{cm})\end{array}$} & \multicolumn{3}{|c|}{ Depth $(\mathrm{m})$} & \multirow[b]{2}{*}{$\begin{array}{l}\text { Core, section, } \\
\text { interval }(\mathrm{cm})\end{array}$} & \multicolumn{3}{|c|}{ Depth (m) } & \multirow[b]{2}{*}{$\begin{array}{l}\text { Core, section, } \\
\text { interval }(\mathrm{cm})\end{array}$} & \multicolumn{3}{|c|}{ Depth (m) } \\
\hline & $\begin{array}{l}\text { Adjusted } \\
\text { CCSF }\end{array}$ & CCSF & CSF-A & & $\begin{array}{l}\text { Adjusted } \\
\text { CCSF }\end{array}$ & CCSF & CSF-A & & $\begin{array}{l}\text { Adjusted } \\
\text { CCSF }\end{array}$ & CCSF & CSF-A \\
\hline 321-U1338B- & & & & $11 \mathrm{H}-1,104$ & 104.26 & 104.26 & 94.14 & $21 \mathrm{H}-7,73$ & 218.58 & 218.58 & 197.83 \\
\hline $1 \mathrm{H}-1,4$ & 0.04 & 0.04 & 0.04 & $11 \mathrm{H}-7,23$ & 112.25 & 112.25 & 102.13 & $22 \mathrm{H}-1,0$ & 219.16 & 219.22 & 197.60 \\
\hline $1 \mathrm{H}-1,93$ & 1.12 & 0.93 & 0.93 & $11 \mathrm{H}-7,82$ & 112.84 & 112.84 & 102.72 & $22 \mathrm{H}-1,39$ & 219.56 & 219.61 & 197.99 \\
\hline $1 \mathrm{H}-2,56$ & 2.06 & 2.06 & 2.06 & $12 \mathrm{H}-1,0$ & 114.19 & 114.19 & 102.60 & $22 \mathrm{H}-1,123$ & 220.36 & 220.45 & 198.83 \\
\hline $1 \mathrm{H}-4,52$ & 5.02 & 5.02 & 5.02 & $12 \mathrm{H}-1,37$ & 114.56 & 114.56 & 102.97 & $22 \mathrm{H}-1,140$ & 220.62 & 220.62 & 199.00 \\
\hline $1 \mathrm{H}-5,12$ & 6.11 & 6.13 & 6.12 & $12 \mathrm{H}-6,92$ & 122.61 & 122.61 & 111.02 & $22 \mathrm{H}-6,117$ & 227.90 & 227.90 & 206.27 \\
\hline $1 \mathrm{H}-5,106$ & 7.05 & 7.06 & 7.06 & $12 \mathrm{H}-7,100$ & 123.99 & 123.99 & 112.40 & $22 \mathrm{H}-7,41$ & 228.83 & 228.63 & 207.01 \\
\hline $2 \mathrm{H}-1,1$ & 8.74 & 8.90 & 7.61 & $13 \mathrm{H}-1,0$ & 124.95 & 125.07 & 112.10 & $22 \mathrm{H}-7,75$ & 229.18 & 228.97 & 207.35 \\
\hline $2 \mathrm{H}-1,67$ & 9.57 & 9.56 & 8.27 & $13 \mathrm{H}-1,26$ & 125.20 & 125.33 & 112.36 & $23 \mathrm{H}-1,0$ & 230.49 & 230.49 & 207.10 \\
\hline $2 \mathrm{H}-2,61$ & 10.96 & 11.00 & 9.71 & $13 \mathrm{H}-1,48$ & 125.55 & 125.55 & 112.58 & $23 \mathrm{H}-1,111$ & 231.60 & 231.60 & 208.21 \\
\hline $2 \mathrm{H}-3,91$ & 12.73 & 12.80 & 11.51 & $13 \mathrm{H}-6,1$ & 132.58 & 132.58 & 119.61 & $23 \mathrm{H}-6,137$ & 239.36 & 239.36 & 215.97 \\
\hline $2 \mathrm{H}-3,126$ & 13.15 & 13.15 & 11.86 & $13 \mathrm{H}-6,98$ & 133.61 & 133.55 & 120.58 & $23 \mathrm{H}-7,31$ & 239.85 & 239.80 & 216.41 \\
\hline $2 \mathrm{H}-7,7$ & 17.96 & 17.96 & 16.67 & $13 \mathrm{H}-6,118$ & 133.81 & 133.75 & 120.78 & $23 \mathrm{H}-7,70$ & 240.25 & 240.19 & 216.80 \\
\hline $2 \mathrm{H}-7,51$ & 18.36 & 18.40 & 17.11 & $14 \mathrm{H}-1,0$ & 135.39 & 135.50 & 121.60 & $24 \mathrm{H}-1,0$ & 241.63 & 241.81 & 216.60 \\
\hline $2 \mathrm{H}-7,71$ & 18.60 & 18.60 & 17.31 & $14 \mathrm{H}-1,60$ & 135.99 & 136.10 & 122.20 & $24 \mathrm{H}-1,56$ & 242.19 & 242.37 & 217.16 \\
\hline $3 \mathrm{H}-1,0$ & 19.34 & 19.17 & 17.10 & $14 \mathrm{H}-1,72$ & 136.26 & 136.22 & 122.32 & $24 \mathrm{H}-1,93$ & 242.65 & 242.74 & 217.53 \\
\hline $3 \mathrm{H}-1,65$ & 19.98 & 19.82 & 17.75 & $14 \mathrm{H}-1,140$ & 136.90 & 136.90 & 123.00 & $24 \mathrm{H}-2,34$ & 243.65 & 243.65 & 218.44 \\
\hline $3 \mathrm{H}-1,127$ & 20.51 & 20.44 & 18.37 & $14 \mathrm{H}-6,82$ & 143.82 & 143.82 & 129.92 & $24 \mathrm{H}-6,117$ & 250.49 & 250.49 & 225.27 \\
\hline $3 \mathrm{H}-2,35$ & 21.02 & 21.02 & 18.95 & $14 \mathrm{H}-7,69$ & 145.01 & 144.99 & 131.09 & $24 \mathrm{H}-7,79$ & 251.60 & 251.60 & 226.39 \\
\hline $3 \mathrm{H}-7,50$ & 28.67 & 28.67 & 26.60 & $14 \mathrm{H}-7,98$ & 145.31 & 145.28 & 131.38 & $25 \mathrm{H}-1,0$ & 252.44 & 252.81 & 226.10 \\
\hline $3 \mathrm{H}-7,73$ & 28.90 & 28.90 & 26.83 & $15 \mathrm{H}-1,0$ & 146.30 & 146.30 & 131.10 & $25 \mathrm{H}-1,45$ & 252.89 & 253.27 & 226.55 \\
\hline $4 \mathrm{H}-1,1$ & 29.27 & 29.27 & 26.61 & $15 \mathrm{H}-1,43$ & 146.73 & 146.73 & 131.53 & $25 \mathrm{H}-1,149$ & 254.02 & 254.30 & 227.59 \\
\hline $4 \mathrm{H}-1,136$ & 30.63 & 30.62 & 27.96 & $15 \mathrm{H}-1,73$ & 147.16 & 147.04 & 131.83 & $25 \mathrm{H}-3,18$ & 255.70 & 255.99 & 229.28 \\
\hline $4 \mathrm{H}-4,28$ & 34.04 & 34.04 & 31.38 & $15 \mathrm{H}-2,12$ & 147.92 & 147.92 & 132.72 & $25 \mathrm{H}-3,141$ & 256.89 & 257.22 & 230.51 \\
\hline $4 \mathrm{H}-6,126$ & 38.02 & 38.02 & 35.36 & $15 \mathrm{H}-6,11$ & 153.91 & 153.91 & 138.71 & $25 \mathrm{H}-4,9$ & 257.40 & 257.40 & 230.69 \\
\hline $4 \mathrm{H}-7,77$ & 39.03 & 39.03 & 36.37 & $15 \mathrm{H}-7,2$ & 155.03 & 155.13 & 139.92 & $25 \mathrm{H}-6,137$ & 261.69 & 261.69 & 234.97 \\
\hline $5 \mathrm{H}-1,0$ & 40.18 & 40.40 & 36.10 & $15 \mathrm{H}-7,91$ & 155.91 & 156.01 & 140.81 & $25 \mathrm{H}-7,56$ & 262.04 & 262.38 & 235.66 \\
\hline $5 \mathrm{H}-1,38$ & 40.56 & 40.78 & 36.48 & $16 \mathrm{H}-1,0$ & 157.13 & 157.13 & 140.60 & $25 \mathrm{H}-7,78$ & 262.26 & 262.59 & 235.88 \\
\hline $5 \mathrm{H}-1,122$ & 41.64 & 41.63 & 37.32 & $16 \mathrm{H}-1,123$ & 158.24 & 158.36 & 141.83 & $26 \mathrm{H}-1,0$ & 265.58 & 265.63 & 238.10 \\
\hline $5 \mathrm{H}-3,61$ & 44.01 & 44.01 & 39.71 & $16 \mathrm{H}-2,128$ & 159.92 & 159.92 & 143.38 & $26 \mathrm{H}-1,11$ & 265.69 & 265.74 & 238.21 \\
\hline $5 \mathrm{H}-7,38$ & 49.78 & 49.78 & 45.48 & $16 \mathrm{H}-6,91$ & 165.54 & 165.54 & 149.01 & $26 \mathrm{H}-2,54$ & 267.59 & 267.67 & 240.14 \\
\hline $5 \mathrm{H}-7,56$ & 50.07 & 49.96 & 45.66 & $16 \mathrm{H}-7,1$ & 165.81 & 165.84 & 149.31 & $26 \mathrm{H}-2,120$ & 268.33 & 268.33 & 240.80 \\
\hline $5 \mathrm{H}-7,82$ & 50.33 & 50.22 & 45.92 & $16 \mathrm{H}-7,51$ & 166.46 & 166.34 & 149.81 & $26 \mathrm{H}-3,86$ & 269.49 & 269.49 & 241.96 \\
\hline $6 \mathrm{H}-1,0$ & 50.67 & 50.74 & 45.60 & $16 \mathrm{H}-7,100$ & 166.95 & 166.83 & 150.30 & $26 \mathrm{H}-6,148$ & 274.62 & 274.62 & 247.08 \\
\hline $6 \mathrm{H}-1,13$ & 50.80 & 50.87 & 45.73 & $17 \mathrm{H}-1,0$ & 167.61 & 167.89 & 150.10 & $26 \mathrm{H}-7,77$ & 275.40 & 275.40 & 247.87 \\
\hline $6 \mathrm{H}-1,134$ & 52.08 & 52.08 & 46.94 & $17 \mathrm{H}-1,16$ & 167.92 & 168.06 & 150.26 & $27 \mathrm{H}-1,0$ & 275.93 & 275.96 & 247.60 \\
\hline $6 \mathrm{H}-5,110$ & 57.84 & 57.84 & 52.70 & $17 \mathrm{H}-1,63$ & 168.37 & 168.52 & 150.73 & $27 \mathrm{H}-1,56$ & 276.49 & 276.52 & 248.16 \\
\hline $6 \mathrm{H}-6,44$ & 58.67 & 58.68 & 53.54 & $17 \mathrm{H}-1,103$ & 168.92 & 168.92 & 151.13 & $27 \mathrm{H}-1,96$ & 276.77 & 276.92 & 248.56 \\
\hline $6 \mathrm{H}-7,24$ & 60.10 & 59.98 & 54.84 & $17 \mathrm{H}-2,17$ & 169.56 & 169.56 & 151.77 & $27 \mathrm{H}-3,4$ & 278.75 & 279.00 & 250.64 \\
\hline $6 \mathrm{H}-7,83$ & 60.69 & 60.57 & 55.43 & $17 \mathrm{H}-6,119$ & 176.59 & 176.59 & 158.79 & $27 \mathrm{H}-6,75$ & 283.96 & 284.21 & 255.85 \\
\hline $7 \mathrm{H}-1,0$ & 61.01 & 60.96 & 55.10 & $17 \mathrm{H}-7,74$ & 177.37 & 177.43 & 159.64 & $28 \mathrm{H}-1,5$ & 285.13 & 285.20 & 257.15 \\
\hline $7 \mathrm{H}-1,36$ & 61.38 & 61.32 & 55.46 & $17 \mathrm{H}-7,104$ & 177.67 & 177.73 & 159.94 & $28 \mathrm{H}-1,51$ & 285.66 & 285.66 & 257.61 \\
\hline $7 \mathrm{H}-1,108$ & 62.04 & 62.04 & 56.18 & $18 \mathrm{H}-1,0$ & 178.32 & 178.16 & 159.60 & $28 \mathrm{H}-7,44$ & 294.40 & 294.39 & 266.34 \\
\hline $7 \mathrm{H}-6,57$ & 69.03 & 69.03 & 63.17 & $18 \mathrm{H}-1,50$ & 178.83 & 178.66 & 160.10 & $28 \mathrm{H}-7,82$ & 294.85 & 294.77 & 266.72 \\
\hline $7 \mathrm{H}-7,46$ & 70.28 & 70.22 & 64.36 & $18 \mathrm{H}-2,10$ & 179.85 & 179.76 & 161.20 & $28 \mathrm{H}-7,105$ & 294.97 & 295.00 & 266.95 \\
\hline 7H-7, 89 & 70.70 & 70.65 & 64.79 & $18 \mathrm{H}-3,26$ & 181.36 & 181.42 & 162.86 & $29 \mathrm{H}-1,0$ & 295.37 & 295.20 & 266.60 \\
\hline $8 \mathrm{H}-1,0$ & 71.12 & 71.18 & 64.60 & $18 \mathrm{H}-3,137$ & 182.49 & 182.53 & 163.97 & $29 \mathrm{H}-1,40$ & 295.77 & 295.60 & 267.00 \\
\hline $8 \mathrm{H}-1,20$ & 71.32 & 71.38 & 64.80 & $18 \mathrm{H}-3,146$ & 182.62 & 182.62 & 164.06 & $29 \mathrm{H}-1,108$ & 296.28 & 296.28 & 267.68 \\
\hline $8 \mathrm{H}-1,105$ & 72.15 & 72.23 & 65.65 & $18 \mathrm{H}-6,64$ & 186.30 & 186.30 & 167.74 & $29 \mathrm{H}-7,76$ & 304.86 & 304.86 & 276.26 \\
\hline $8 \mathrm{H}-4,42$ & 76.10 & 76.10 & 69.52 & $18 \mathrm{H}-7,84$ & 187.63 & 187.70 & 169.14 & $29 \mathrm{H}-7,90$ & 305.00 & 305.00 & 276.40 \\
\hline $8 \mathrm{H}-6,132$ & 80.00 & 80.00 & 73.42 & $18 \mathrm{H}-7,98$ & 187.74 & 187.84 & 169.28 & $30 \mathrm{H}-1,1$ & 305.59 & 305.59 & 276.11 \\
\hline $8 \mathrm{H}-7,84$ & 81.02 & 81.02 & 74.44 & $19 \mathrm{H}-1,44$ & 188.07 & 188.14 & 169.54 & $30 \mathrm{H}-1,1$ & 305.59 & 305.59 & 276.11 \\
\hline $9 \mathrm{H}-1,0$ & 81.85 & 82.13 & 74.10 & $19 \mathrm{H}-2,33$ & 189.68 & 189.53 & 170.93 & $30 \mathrm{H}-1,147$ & 307.04 & 307.05 & 277.57 \\
\hline $9 \mathrm{H}-1,105$ & 82.91 & 83.18 & 75.15 & $19 \mathrm{H}-4,20$ & 192.40 & 192.40 & 173.80 & $30 \mathrm{H}-2,133$ & 307.81 & 308.41 & 278.93 \\
\hline $9 \mathrm{H}-2,92$ & 84.44 & 84.55 & 76.52 & $19 \mathrm{H}-6,121$ & 196.41 & 196.41 & 177.81 & $30 \mathrm{H}-4,130$ & 311.38 & 311.38 & 281.90 \\
\hline $9 \mathrm{H}-4,30$ & 86.93 & 86.93 & 78.90 & $19 \mathrm{H}-7,75$ & 197.50 & 197.46 & 178.85 & $30 \mathrm{H}-4,141$ & 311.49 & 311.49 & 282.01 \\
\hline $9 \mathrm{H}-6,34$ & 89.98 & 89.98 & 81.94 & $20 \mathrm{H}-1,0$ & 198.70 & 198.63 & 178.60 & $31 \mathrm{H}-1,64$ & 312.02 & 312.02 & 282.74 \\
\hline $9 \mathrm{H}-7,8$ & 90.68 & 90.71 & 82.68 & $20 \mathrm{H}-1,18$ & 198.88 & 198.81 & 178.78 & $32 \mathrm{H}-1,0$ & 313.52 & 313.52 & 282.90 \\
\hline $9 \mathrm{H}-7,60$ & 91.20 & 91.23 & 83.20 & $20 \mathrm{H}-1,123$ & 199.92 & 199.86 & 179.83 & $32 \mathrm{H}-1,57$ & 314.09 & 314.09 & 283.47 \\
\hline $10 \mathrm{H}-1,0$ & 92.19 & 92.60 & 83.60 & $20 \mathrm{H}-2,100$ & 201.10 & 201.13 & 181.10 & $32 \mathrm{H}-7,22$ & 322.54 & 322.54 & 291.92 \\
\hline $10 \mathrm{H}-1,59$ & 92.78 & 93.19 & 84.19 & $20 \mathrm{H}-3,2$ & 201.65 & 201.65 & 181.62 & $32 \mathrm{H}-7,96$ & 323.28 & 323.28 & 292.66 \\
\hline $10 \mathrm{H}-1,101$ & 93.28 & 93.61 & 84.61 & $20 \mathrm{H}-6,128$ & 207.41 & 207.41 & 187.38 & $33 \mathrm{H}-1,0$ & 323.66 & 323.55 & 292.40 \\
\hline $10 \mathrm{H}-3,0$ & 95.43 & 95.60 & 86.60 & $20 \mathrm{H}-7,39$ & 208.09 & 208.02 & 187.99 & $33 \mathrm{H}-1,17$ & 323.83 & 323.72 & 292.57 \\
\hline $10 \mathrm{H}-3,98$ & 96.39 & 96.58 & 87.58 & $20 \mathrm{H}-7,71$ & 208.41 & 208.34 & 188.31 & $33 \mathrm{H}-1,112$ & 324.67 & 324.67 & 293.52 \\
\hline $10 \mathrm{H}-3,138$ & 96.98 & 96.98 & 87.98 & $21 \mathrm{H}-1,0$ & 208.84 & 208.85 & 188.10 & $33 \mathrm{H}-6,117$ & 332.22 & 332.22 & 301.07 \\
\hline $10 \mathrm{H}-5,140$ & 100.00 & 100.00 & 91.00 & $21 \mathrm{H}-1,104$ & 209.87 & 209.89 & 189.14 & $33 \mathrm{H}-7,80$ & 332.82 & 333.05 & 301.90 \\
\hline $10 \mathrm{H}-7,82$ & 102.19 & 102.12 & 93.12 & $21 \mathrm{H}-4,9$ & 213.44 & 213.44 & 192.69 & $34 \mathrm{H}-1,0$ & 334.33 & 334.33 & 301.90 \\
\hline $11 \mathrm{H}-1,0$ & 103.22 & 103.22 & 93.10 & $21 \mathrm{H}-6,120$ & 217.55 & 217.55 & 196.80 & $34 \mathrm{H}-1,68$ & 335.02 & 335.02 & 302.58 \\
\hline
\end{tabular}


Table AT52 (continued).

\begin{tabular}{|c|c|c|c|}
\hline \multirow[b]{2}{*}{$\begin{array}{l}\text { Core, section, } \\
\text { interval }(\mathrm{cm})\end{array}$} & \multicolumn{3}{|c|}{ Depth $(\mathrm{m})$} \\
\hline & $\begin{array}{l}\text { Adjusted } \\
\text { CCSF }\end{array}$ & CCSF & CSF-A \\
\hline $34 \mathrm{H}-4,129$ & 340.14 & 340.14 & 307.71 \\
\hline $34 \mathrm{H}-6,50$ & 342.44 & 342.36 & 309.93 \\
\hline $34 \mathrm{H}-7,84$ & 343.78 & 343.70 & 311.27 \\
\hline $35 \mathrm{H}-1,0$ & 345.17 & 345.18 & 311.40 \\
\hline $35 \mathrm{H}-1,27$ & 345.44 & 345.45 & 311.67 \\
\hline $35 \mathrm{H}-1,100$ & 346.18 & 346.18 & 312.40 \\
\hline $35 \mathrm{H}-2,49$ & 347.17 & 347.17 & 313.39 \\
\hline $35 \mathrm{H}-5,84$ & 352.03 & 352.02 & 318.24 \\
\hline $35 \mathrm{H}-6,119$ & 353.98 & 353.87 & 320.09 \\
\hline $35 \mathrm{H}-7,10$ & 354.42 & 354.29 & 320.50 \\
\hline $36 \mathrm{H}-1,0$ & 354.96 & 355.04 & 320.90 \\
\hline $35 \mathrm{H}-7,72$ & 355.04 & 354.90 & 321.12 \\
\hline $36 \mathrm{H}-1,110$ & 356.15 & 356.15 & 322.00 \\
\hline $36 \mathrm{H}-6,37$ & 362.91 & 362.91 & 328.77 \\
\hline $36 \mathrm{H}-6,138$ & 363.92 & 363.92 & 329.78 \\
\hline $36 \mathrm{H}-7,75$ & 364.79 & 364.79 & 330.65 \\
\hline $37 \mathrm{H}-1,0$ & 365.48 & 365.58 & 330.40 \\
\hline $37 \mathrm{H}-1,129$ & 366.77 & 366.87 & 331.69 \\
\hline $37 \mathrm{H}-2,104$ & 368.12 & 368.12 & 332.94 \\
\hline 37Н-6, 31 & 373.39 & 373.39 & 338.21 \\
\hline $37 \mathrm{H}-7,42$ & 375.07 & 375.00 & 339.82 \\
\hline $37 \mathrm{H}-7,73$ & 375.38 & 375.31 & 340.13 \\
\hline $38 \mathrm{H}-1,0$ & 376.94 & 376.98 & 339.90 \\
\hline $38 \mathrm{H}-1,23$ & 377.26 & 377.21 & 340.13 \\
\hline $38 \mathrm{H}-2,3$ & 378.49 & 378.51 & 341.43 \\
\hline $38 \mathrm{H}-2,66$ & 379.14 & 379.14 & 342.06 \\
\hline $38 \mathrm{H}-5,68$ & 383.66 & 383.66 & 346.58 \\
\hline $38 \mathrm{H}-6,73$ & 385.21 & 385.21 & 348.13 \\
\hline $38 \mathrm{H}-7,69$ & 386.86 & 386.67 & 349.59 \\
\hline $39 \mathrm{H}-1,1$ & 392.12 & 391.64 & 349.41 \\
\hline $39 \mathrm{H}-2,86$ & 393.75 & 393.99 & 351.76 \\
\hline $39 \mathrm{H}-3,124$ & 395.76 & 395.87 & 353.64 \\
\hline $39 \mathrm{H}-5,142$ & 398.92 & 399.05 & 356.82 \\
\hline $39 \mathrm{H}-6,68$ & 399.70 & 399.81 & 357.58 \\
\hline $39 \mathrm{H}-7,72$ & 401.35 & 401.35 & 359.12 \\
\hline $40 \mathrm{H}-2,56$ & 403.36 & 403.36 & 360.96 \\
\hline $40 \mathrm{H}-4,45$ & 406.15 & 406.26 & 363.85 \\
\hline $40 \mathrm{H}-6,144$ & 409.95 & 410.24 & 367.84 \\
\hline $40 \mathrm{H}-7,72$ & 410.73 & 411.02 & 368.62 \\
\hline $41 \mathrm{H}-1,9$ & 412.16 & 412.16 & 368.49 \\
\hline $41 \mathrm{H}-6,74$ & 420.31 & 420.31 & 376.64 \\
\hline $41 \mathrm{H}-7,8$ & 421.11 & 420.87 & 377.20 \\
\hline $41 \mathrm{H}-7,18$ & 421.27 & 420.97 & 377.30 \\
\hline $41 \mathrm{H}-7,55$ & 421.59 & 421.34 & 377.67 \\
\hline $41 \mathrm{H}-7,80$ & 421.84 & 421.59 & 377.92 \\
\hline $42 \mathrm{H}-1,0$ & 422.01 & 421.94 & 377.90 \\
\hline $42 \mathrm{H}-1,85$ & 422.86 & 422.80 & 378.75 \\
\hline $42 \mathrm{H}-2,38$ & 423.82 & 423.82 & 379.78 \\
\hline $42 \mathrm{H}-5,103$ & 429.00 & 429.00 & 384.96 \\
\hline $42 \mathrm{H}-7,91$ & 431.73 & 431.73 & 387.69 \\
\hline $43 X-1,134$ & 433.21 & 433.15 & 388.74 \\
\hline $43 X-2,117$ & 434.70 & 434.48 & 390.07 \\
\hline $43 X-3,40$ & 435.79 & 435.21 & 390.80 \\
\hline $43 X-4,86$ & 437.26 & 436.68 & 392.27 \\
\hline $44 X-1,0$ & 442.33 & 442.33 & 397.00 \\
\hline $44 X-2,88$ & 444.71 & 444.71 & 399.38 \\
\hline $45 X-1,0$ & 451.93 & 451.93 & 406.60 \\
\hline $45 X-2,42$ & 453.91 & 453.76 & 408.43 \\
\hline $45 X-3,13$ & 455.29 & 454.97 & 409.64 \\
\hline
\end{tabular}

This table is also available in ASCII. 
Table AT53. Gamma ray attenuation (GRA) density data, Hole U1338C.

\begin{tabular}{|c|c|c|c|c|}
\hline \multirow[b]{2}{*}{$\begin{array}{l}\text { Core, section, } \\
\text { interval }(\mathrm{cm})\end{array}$} & \multicolumn{3}{|c|}{ Depth (m) } & \multirow[b]{2}{*}{$\begin{array}{c}\text { GRA } \\
\left(\mathrm{g} / \mathrm{cm}^{3}\right)\end{array}$} \\
\hline & CSF & CCSF & $\begin{array}{l}\text { Adjusted } \\
\text { CCSF }\end{array}$ & \\
\hline \multicolumn{5}{|l|}{ 321-U1338C- } \\
\hline $1 \mathrm{H}-1,2.5$ & 0.03 & 0.03 & 0.03 & 1.31 \\
\hline $1 \mathrm{H}-1,5$ & 0.05 & 0.05 & 0.05 & 1.30 \\
\hline $1 \mathrm{H}-1,7.5$ & 0.08 & 0.08 & 0.08 & 1.31 \\
\hline $1 \mathrm{H}-1,10$ & 0.10 & 0.10 & 0.10 & 1.35 \\
\hline $1 \mathrm{H}-1,12.5$ & 0.13 & 0.13 & 0.13 & 1.35 \\
\hline $1 \mathrm{H}-1,15$ & 0.15 & 0.15 & 0.15 & 1.32 \\
\hline $1 \mathrm{H}-1,17.5$ & 0.18 & 0.18 & 0.18 & 1.34 \\
\hline $1 \mathrm{H}-1,20$ & 0.20 & 0.20 & 0.19 & 1.35 \\
\hline $1 \mathrm{H}-1,22.6$ & 0.23 & 0.23 & 0.22 & 1.44 \\
\hline $1 \mathrm{H}-1,25.1$ & 0.25 & 0.25 & 0.24 & 1.40 \\
\hline $1 \mathrm{H}-1,27.6$ & 0.28 & 0.28 & 0.27 & 1.37 \\
\hline $1 \mathrm{H}-1,30.1$ & 0.30 & 0.30 & 0.29 & 1.41 \\
\hline $1 \mathrm{H}-1,32.6$ & 0.33 & 0.33 & 0.32 & 1.40 \\
\hline $1 \mathrm{H}-1,35.1$ & 0.35 & 0.35 & 0.34 & 1.36 \\
\hline $1 \mathrm{H}-1,37.6$ & 0.38 & 0.38 & 0.37 & 1.38 \\
\hline $1 \mathrm{H}-1,40.1$ & 0.40 & 0.40 & 0.39 & 1.39 \\
\hline $1 \mathrm{H}-1,42.6$ & 0.43 & 0.43 & 0.42 & 1.39 \\
\hline $1 \mathrm{H}-1,45.1$ & 0.45 & 0.45 & 0.44 & 1.39 \\
\hline $1 \mathrm{H}-1,47.6$ & 0.48 & 0.48 & 0.46 & 1.36 \\
\hline $1 \mathrm{H}-1,50.1$ & 0.50 & 0.50 & 0.48 & 1.34 \\
\hline $1 \mathrm{H}-1,52.6$ & 0.53 & 0.53 & 0.51 & 1.36 \\
\hline $1 \mathrm{H}-1,55.1$ & 0.55 & 0.55 & 0.53 & 1.34 \\
\hline $1 \mathrm{H}-1,57.6$ & 0.58 & 0.58 & 0.56 & 1.43 \\
\hline $1 \mathrm{H}-1,60.1$ & 0.60 & 0.60 & 0.58 & 1.40 \\
\hline $1 \mathrm{H}-1,62.6$ & 0.63 & 0.63 & 0.61 & 1.35 \\
\hline $1 \mathrm{H}-1,67.6$ & 0.68 & 0.68 & 0.66 & 1.37 \\
\hline $1 \mathrm{H}-1,70.1$ & 0.70 & 0.70 & 0.68 & 1.39 \\
\hline $1 \mathrm{H}-1,72.6$ & 0.73 & 0.73 & 0.71 & 1.41 \\
\hline $1 \mathrm{H}-1,75.1$ & 0.75 & 0.75 & 0.72 & 1.41 \\
\hline $1 \mathrm{H}-1,77.6$ & 0.78 & 0.78 & 0.75 & 1.40 \\
\hline $1 \mathrm{H}-1,80.2$ & 0.80 & 0.80 & 0.77 & 1.40 \\
\hline $1 \mathrm{H}-1,82.7$ & 0.83 & 0.83 & 0.80 & 1.43 \\
\hline $1 \mathrm{H}-1,85.2$ & 0.85 & 0.85 & 0.82 & 1.37 \\
\hline $1 \mathrm{H}-1,87.7$ & 0.88 & 0.88 & 0.85 & 1.38 \\
\hline $1 \mathrm{H}-1,90.2$ & 0.90 & 0.90 & 0.87 & 1.41 \\
\hline $1 \mathrm{H}-1,92.7$ & 0.93 & 0.93 & 0.90 & 1.38 \\
\hline $1 \mathrm{H}-1,95.2$ & 0.95 & 0.95 & 0.92 & 1.41 \\
\hline $1 \mathrm{H}-1,100.2$ & 1.00 & 1.00 & 0.97 & 1.39 \\
\hline $1 \mathrm{H}-1,102.7$ & 1.03 & 1.03 & 0.99 & 1.40 \\
\hline $1 \mathrm{H}-1,105.2$ & 1.05 & 1.05 & 1.01 & 1.40 \\
\hline $1 \mathrm{H}-1,107.7$ & 1.08 & 1.08 & 1.04 & 1.39 \\
\hline $1 \mathrm{H}-1,110.2$ & 1.10 & 1.10 & 1.06 & 1.39 \\
\hline $1 \mathrm{H}-1,112.7$ & 1.13 & 1.13 & 1.09 & 1.37 \\
\hline $1 \mathrm{H}-1,115.2$ & 1.15 & 1.15 & 1.11 & 1.36 \\
\hline $1 \mathrm{H}-1,117.7$ & 1.18 & 1.18 & 1.14 & 1.35 \\
\hline $1 \mathrm{H}-1,120.2$ & 1.20 & 1.20 & 1.16 & 1.35 \\
\hline $1 \mathrm{H}-1,122.7$ & 1.23 & 1.23 & 1.19 & 1.36 \\
\hline $1 \mathrm{H}-1,125.2$ & 1.25 & 1.25 & 1.21 & 1.31 \\
\hline $1 \mathrm{H}-1,127.7$ & 1.28 & 1.28 & 1.24 & 1.29 \\
\hline $1 \mathrm{H}-1,130.2$ & 1.30 & 1.30 & 1.26 & 1.30 \\
\hline $1 \mathrm{H}-1,132.7$ & 1.33 & 1.33 & 1.28 & 1.29 \\
\hline $1 \mathrm{H}-1,135.3$ & 1.35 & 1.35 & 1.30 & 1.30 \\
\hline $1 \mathrm{H}-1,137.8$ & 1.38 & 1.38 & 1.33 & 1.28 \\
\hline $1 \mathrm{H}-1,140.3$ & 1.40 & 1.40 & 1.35 & 1.26 \\
\hline $1 \mathrm{H}-1,142.8$ & 1.43 & 1.43 & 1.38 & 1.26 \\
\hline $1 \mathrm{H}-1,145.3$ & 1.45 & 1.45 & 1.40 & 1.27 \\
\hline $1 \mathrm{H}-1,147.8$ & 1.48 & 1.48 & 1.43 & 1.33 \\
\hline $1 \mathrm{H}-1,150.3$ & 1.50 & 1.50 & 1.45 & 1.32 \\
\hline $1 \mathrm{H}-2,4.3$ & 1.54 & 1.54 & 1.49 & 1.33 \\
\hline
\end{tabular}

Only a portion of this table appears here. The complete table is available in ASCII. 
Table AT54. Magnetic susceptibility data, Hole U1338C.

\begin{tabular}{|c|c|c|c|c|}
\hline \multirow[b]{2}{*}{$\begin{array}{l}\text { Core, section, } \\
\text { interval }(\mathrm{cm})\end{array}$} & \multicolumn{3}{|c|}{ Depth $(m)$} & \multirow{2}{*}{$\begin{array}{l}\text { Magnetic } \\
\text { susceptibility } \\
\text { (IU) }\end{array}$} \\
\hline & CSF & CCSF & $\begin{array}{l}\text { Adjusted } \\
\text { CCSF }\end{array}$ & \\
\hline \multicolumn{5}{|l|}{ 321-U1338C- } \\
\hline $1 \mathrm{H}-1,0.1$ & 0.00 & 0.00 & 0.00 & 1.4 \\
\hline $1 \mathrm{H}-1,2.6$ & 0.03 & 0.03 & 0.03 & 2.7 \\
\hline $1 \mathrm{H}-1,5.1$ & 0.05 & 0.05 & 0.05 & 3.0 \\
\hline $1 \mathrm{H}-1,7.6$ & 0.08 & 0.08 & 0.08 & 3.0 \\
\hline $1 \mathrm{H}-1,10.1$ & 0.10 & 0.10 & 0.10 & 3.3 \\
\hline $1 \mathrm{H}-1,12.6$ & 0.13 & 0.13 & 0.13 & 3.6 \\
\hline $1 \mathrm{H}-1,15.1$ & 0.15 & 0.15 & 0.15 & 3.0 \\
\hline $1 \mathrm{H}-1,17.6$ & 0.18 & 0.18 & 0.18 & 3.0 \\
\hline $1 \mathrm{H}-1,20.1$ & 0.20 & 0.20 & 0.19 & 3.3 \\
\hline $1 \mathrm{H}-1,22.6$ & 0.23 & 0.23 & 0.22 & 4.1 \\
\hline $1 \mathrm{H}-1,25.1$ & 0.25 & 0.25 & 0.24 & 4.1 \\
\hline $1 \mathrm{H}-1,27.6$ & 0.28 & 0.28 & 0.27 & 4.1 \\
\hline $1 \mathrm{H}-1,30.1$ & 0.30 & 0.30 & 0.29 & 3.3 \\
\hline $1 \mathrm{H}-1,32.6$ & 0.33 & 0.33 & 0.32 & 2.7 \\
\hline $1 \mathrm{H}-1,35.1$ & 0.35 & 0.35 & 0.34 & 1.9 \\
\hline $1 \mathrm{H}-1,37.6$ & 0.38 & 0.38 & 0.37 & 2.2 \\
\hline $1 \mathrm{H}-1,40.1$ & 0.40 & 0.40 & 0.39 & 1.4 \\
\hline $1 \mathrm{H}-1,42.6$ & 0.43 & 0.43 & 0.42 & 1.9 \\
\hline $1 \mathrm{H}-1,45.1$ & 0.45 & 0.45 & 0.44 & 1.9 \\
\hline $1 \mathrm{H}-1,47.7$ & 0.48 & 0.48 & 0.46 & 1.9 \\
\hline $1 \mathrm{H}-1,50.2$ & 0.50 & 0.50 & 0.48 & 1.1 \\
\hline $1 \mathrm{H}-1,52.7$ & 0.53 & 0.53 & 0.51 & 0.8 \\
\hline $1 \mathrm{H}-1,55.2$ & 0.55 & 0.55 & 0.53 & 0.8 \\
\hline $1 \mathrm{H}-1,57.7$ & 0.58 & 0.58 & 0.56 & 1.4 \\
\hline $1 \mathrm{H}-1,60.2$ & 0.60 & 0.60 & 0.58 & 0.8 \\
\hline $1 \mathrm{H}-1,62.7$ & 0.63 & 0.63 & 0.61 & 1.1 \\
\hline $1 \mathrm{H}-1,65.2$ & 0.65 & 0.65 & 0.63 & 0.3 \\
\hline $1 \mathrm{H}-1,67.7$ & 0.68 & 0.68 & 0.66 & 0.5 \\
\hline $1 \mathrm{H}-1,70.2$ & 0.70 & 0.70 & 0.68 & 0.3 \\
\hline $1 \mathrm{H}-1,72.7$ & 0.73 & 0.73 & 0.71 & 0.0 \\
\hline $1 \mathrm{H}-1,75.2$ & 0.75 & 0.75 & 0.72 & 0.8 \\
\hline $1 \mathrm{H}-1,77.7$ & 0.78 & 0.78 & 0.75 & 0.5 \\
\hline $1 \mathrm{H}-1,80.2$ & 0.80 & 0.80 & 0.77 & 1.1 \\
\hline $1 \mathrm{H}-1,82.7$ & 0.83 & 0.83 & 0.80 & 0.3 \\
\hline $1 \mathrm{H}-1,85.2$ & 0.85 & 0.85 & 0.82 & 1.1 \\
\hline $1 \mathrm{H}-1,87.7$ & 0.88 & 0.88 & 0.85 & 0.5 \\
\hline $1 \mathrm{H}-1,90.2$ & 0.90 & 0.90 & 0.87 & 0.0 \\
\hline $1 \mathrm{H}-1,92.7$ & 0.93 & 0.93 & 0.90 & 0.3 \\
\hline $1 \mathrm{H}-1,95.2$ & 0.95 & 0.95 & 0.92 & 0.5 \\
\hline $1 \mathrm{H}-1,97.7$ & 0.98 & 0.98 & 0.95 & 0.5 \\
\hline $1 \mathrm{H}-1,100.2$ & 1.00 & 1.00 & 0.97 & 0.8 \\
\hline $1 \mathrm{H}-1,102.8$ & 1.03 & 1.03 & 0.99 & 0.5 \\
\hline $1 \mathrm{H}-1,105.2$ & 1.05 & 1.05 & 1.01 & 0.5 \\
\hline $1 \mathrm{H}-1,107.8$ & 1.08 & 1.08 & 1.04 & 0.3 \\
\hline $1 \mathrm{H}-1,110.3$ & 1.10 & 1.10 & 1.06 & 1.4 \\
\hline $1 \mathrm{H}-1,112.8$ & 1.13 & 1.13 & 1.09 & 1.4 \\
\hline $1 \mathrm{H}-1,115.3$ & 1.15 & 1.15 & 1.11 & 1.4 \\
\hline $1 \mathrm{H}-1,117.8$ & 1.18 & 1.18 & 1.14 & 1.1 \\
\hline $1 \mathrm{H}-1,120.3$ & 1.20 & 1.20 & 1.16 & 1.6 \\
\hline $1 \mathrm{H}-1,122.8$ & 1.23 & 1.23 & 1.19 & 1.9 \\
\hline $1 \mathrm{H}-1,125.3$ & 1.25 & 1.25 & 1.21 & 3.0 \\
\hline $1 \mathrm{H}-1,127.8$ & 1.28 & 1.28 & 1.24 & 3.8 \\
\hline $1 \mathrm{H}-1,130.3$ & 1.30 & 1.30 & 1.26 & 3.3 \\
\hline $1 \mathrm{H}-1,132.8$ & 1.33 & 1.33 & 1.28 & 4.1 \\
\hline $1 \mathrm{H}-1,135.3$ & 1.35 & 1.35 & 1.30 & 4.4 \\
\hline $1 \mathrm{H}-1,137.8$ & 1.38 & 1.38 & 1.33 & 4.4 \\
\hline $1 \mathrm{H}-1,140.3$ & 1.40 & 1.40 & 1.35 & 4.4 \\
\hline $1 \mathrm{H}-1,142.8$ & 1.43 & 1.43 & 1.38 & 4.4 \\
\hline $1 \mathrm{H}-1,145.3$ & 1.45 & 1.45 & 1.40 & 4.7 \\
\hline
\end{tabular}

Only a portion of this table appears here. The complete table is available in ASCII. 
Table AT55. Natural gamma ray (NGR) data, Hole U1338C.

\begin{tabular}{|c|c|c|c|c|}
\hline \multirow[b]{2}{*}{$\begin{array}{l}\text { Core, section, } \\
\text { interval }(\mathrm{cm})\end{array}$} & \multicolumn{3}{|c|}{ Depth (m) } & \multirow[b]{2}{*}{$\begin{array}{l}\text { NGR } \\
\text { (cps) }\end{array}$} \\
\hline & CSF & CCSF & $\begin{array}{l}\text { Adjusted } \\
\text { CCSF }\end{array}$ & \\
\hline \multicolumn{5}{|l|}{ 321-U1338C- } \\
\hline $1 \mathrm{H}-1,0$ & 0.0 & 0.00 & 0.00 & 40.1 \\
\hline $1 \mathrm{H}-1,10$ & 0.1 & 0.10 & 0.10 & 42.2 \\
\hline $1 \mathrm{H}-1,20$ & 0.2 & 0.20 & 0.19 & 47.0 \\
\hline $1 \mathrm{H}-1,30$ & 0.3 & 0.30 & 0.29 & 41.0 \\
\hline $1 \mathrm{H}-1,40$ & 0.4 & 0.40 & 0.39 & 30.9 \\
\hline $1 \mathrm{H}-1,50$ & 0.5 & 0.50 & 0.48 & 25.8 \\
\hline $1 \mathrm{H}-1,60$ & 0.6 & 0.60 & 0.58 & 26.9 \\
\hline $1 \mathrm{H}-1,70$ & 0.7 & 0.70 & 0.68 & 27.3 \\
\hline $1 \mathrm{H}-1,80$ & 0.8 & 0.80 & 0.77 & 23.4 \\
\hline $1 \mathrm{H}-1,90$ & 0.9 & 0.90 & 0.87 & 20.4 \\
\hline $1 \mathrm{H}-1,100$ & 1.0 & 1.00 & 0.97 & 20.9 \\
\hline $1 \mathrm{H}-1,110$ & 1.1 & 1.10 & 1.06 & 27.4 \\
\hline $1 \mathrm{H}-1,120$ & 1.2 & 1.20 & 1.16 & 33.9 \\
\hline $1 \mathrm{H}-1,130$ & 1.3 & 1.30 & 1.26 & 33.1 \\
\hline $1 \mathrm{H}-1,140$ & 1.4 & 1.40 & 1.35 & 26.1 \\
\hline $1 \mathrm{H}-1,150$ & 1.5 & 1.50 & 1.45 & 25.1 \\
\hline $1 \mathrm{H}-2,0$ & 1.5 & 1.50 & 1.45 & 29.2 \\
\hline $1 \mathrm{H}-2,10$ & 1.6 & 1.60 & 1.54 & 39.6 \\
\hline $1 \mathrm{H}-2,20$ & 1.7 & 1.70 & 1.64 & 45.5 \\
\hline $1 \mathrm{H}-2,30$ & 1.8 & 1.80 & 1.74 & 32.0 \\
\hline $1 \mathrm{H}-2,40$ & 1.9 & 1.90 & 1.83 & 30.6 \\
\hline $1 \mathrm{H}-2,50$ & 2.0 & 2.00 & 1.93 & 29.7 \\
\hline $1 \mathrm{H}-2,60$ & 2.1 & 2.10 & 2.02 & 25.1 \\
\hline $1 \mathrm{H}-2,70$ & 2.2 & 2.20 & 2.11 & 22.9 \\
\hline $1 \mathrm{H}-2,80$ & 2.3 & 2.30 & 2.20 & 15.6 \\
\hline $1 \mathrm{H}-2,90$ & 2.4 & 2.40 & 2.29 & 11.0 \\
\hline $1 \mathrm{H}-2,100$ & 2.5 & 2.50 & 2.38 & 11.2 \\
\hline $1 \mathrm{H}-2,110$ & 2.6 & 2.60 & 2.46 & 11.9 \\
\hline $1 \mathrm{H}-2,120$ & 2.7 & 2.70 & 2.55 & 11.3 \\
\hline $1 \mathrm{H}-3,0$ & 2.7 & 2.70 & 2.55 & 12.2 \\
\hline $1 \mathrm{H}-3,10$ & 2.8 & 2.80 & 2.65 & 13.3 \\
\hline $1 \mathrm{H}-3,20$ & 2.9 & 2.90 & 2.76 & 14.1 \\
\hline $1 \mathrm{H}-3,30$ & 3.0 & 3.00 & 2.87 & 13.5 \\
\hline $1 \mathrm{H}-3,40$ & 3.1 & 3.10 & 2.98 & 13.3 \\
\hline $1 \mathrm{H}-3,50$ & 3.2 & 3.20 & 3.07 & 14.1 \\
\hline $1 \mathrm{H}-3,60$ & 3.3 & 3.30 & 3.15 & 19.8 \\
\hline $1 \mathrm{H}-3,70$ & 3.4 & 3.40 & 3.23 & 16.8 \\
\hline $1 \mathrm{H}-3,80$ & 3.5 & 3.50 & 3.32 & 10.2 \\
\hline $1 \mathrm{H}-3,90$ & 3.6 & 3.60 & 3.40 & 8.6 \\
\hline $2 \mathrm{H}-1,0$ & 3.8 & 4.35 & 4.20 & 8.6 \\
\hline $2 \mathrm{H}-1,10$ & 3.9 & 4.45 & 4.31 & 12.4 \\
\hline $2 \mathrm{H}-1,20$ & 4.0 & 4.55 & 4.42 & 11.9 \\
\hline $2 \mathrm{H}-1,30$ & 4.1 & 4.65 & 4.53 & 8.0 \\
\hline $2 \mathrm{H}-1,40$ & 4.2 & 4.75 & 4.64 & 7.4 \\
\hline $2 \mathrm{H}-1,50$ & 4.3 & 4.85 & 4.75 & 7.8 \\
\hline $2 \mathrm{H}-1,60$ & 4.4 & 4.95 & 4.86 & 7.6 \\
\hline $2 \mathrm{H}-1,70$ & 4.5 & 5.05 & 4.96 & 7.3 \\
\hline $2 \mathrm{H}-1,80$ & 4.6 & 5.15 & 5.07 & 6.3 \\
\hline $2 \mathrm{H}-1,90$ & 4.7 & 5.25 & 5.18 & 7.1 \\
\hline $2 \mathrm{H}-1,100$ & 4.8 & 5.35 & 5.29 & 9.7 \\
\hline $2 \mathrm{H}-1,110$ & 4.9 & 5.45 & 5.40 & 12.5 \\
\hline $2 \mathrm{H}-1,120$ & 5.0 & 5.55 & 5.51 & 12.3 \\
\hline $2 \mathrm{H}-1,130$ & 5.1 & 5.65 & 5.62 & 11.4 \\
\hline $2 \mathrm{H}-1,140$ & 5.2 & 5.75 & 5.72 & 10.9 \\
\hline $2 \mathrm{H}-1,150$ & 5.3 & 5.85 & 5.83 & 12.6 \\
\hline $2 \mathrm{H}-2,0$ & 5.3 & 5.85 & 5.83 & 11.8 \\
\hline $2 \mathrm{H}-2,10$ & 5.4 & 5.95 & 5.94 & 11.1 \\
\hline $2 \mathrm{H}-2,20$ & 5.5 & 6.05 & 6.05 & 10.7 \\
\hline
\end{tabular}

Only a portion of this table appears here. The complete table is available in ASCII. 
Table AT56. Color reflection data, Hole U1338C.

\begin{tabular}{|c|c|c|c|c|c|c|}
\hline \multirow[b]{2}{*}{$\begin{array}{l}\text { Core, section, } \\
\text { interval }(\mathrm{cm})\end{array}$} & \multicolumn{3}{|c|}{ Depth (m) } & \multirow[b]{2}{*}{$a^{*}$} & \multirow[b]{2}{*}{$b^{*}$} & \multirow[b]{2}{*}{$L^{*}$} \\
\hline & CSF & CCSF & $\begin{array}{l}\text { Adjusted } \\
\text { CCSF }\end{array}$ & & & \\
\hline \multicolumn{7}{|l|}{ 321-U1338C- } \\
\hline $1 \mathrm{H}-1,2.6$ & 0.03 & 0.03 & 0.03 & 3.7 & 5.1 & 33.6 \\
\hline $1 \mathrm{H}-1,5.2$ & 0.05 & 0.05 & 0.05 & 5.6 & 12.4 & 45.0 \\
\hline $1 \mathrm{H}-1,7.7$ & 0.08 & 0.08 & 0.08 & 5.8 & 12.5 & 45.7 \\
\hline $1 \mathrm{H}-1,10.2$ & 0.10 & 0.10 & 0.10 & 5.4 & 12.2 & 46.1 \\
\hline $1 \mathrm{H}-1,12.8$ & 0.13 & 0.13 & 0.13 & 5.1 & 11.8 & 46.0 \\
\hline $1 \mathrm{H}-1,15.3$ & 0.15 & 0.15 & 0.15 & 5.2 & 12.0 & 47.2 \\
\hline $1 \mathrm{H}-1,17.9$ & 0.18 & 0.18 & 0.18 & 4.7 & 10.3 & 42.2 \\
\hline $1 \mathrm{H}-1,20.4$ & 0.20 & 0.20 & 0.19 & 5.0 & 11.4 & 43.6 \\
\hline $1 \mathrm{H}-1,22.9$ & 0.23 & 0.23 & 0.22 & 4.7 & 12.4 & 46.4 \\
\hline $1 \mathrm{H}-1,25.5$ & 0.26 & 0.26 & 0.25 & 4.7 & 15.4 & 54.8 \\
\hline $1 \mathrm{H}-1,28$ & 0.28 & 0.28 & 0.27 & 1.1 & 16.1 & 63.7 \\
\hline $1 \mathrm{H}-1,30.6$ & 0.31 & 0.31 & 0.30 & 4.5 & 18.7 & 59.9 \\
\hline $1 \mathrm{H}-1,33.1$ & 0.33 & 0.33 & 0.32 & 5.1 & 18.5 & 59.2 \\
\hline $1 \mathrm{H}-1,35.6$ & 0.36 & 0.36 & 0.35 & 4.2 & 18.6 & 58.1 \\
\hline $1 \mathrm{H}-1,38.2$ & 0.38 & 0.38 & 0.37 & 4.8 & 17.7 & 59.0 \\
\hline $1 \mathrm{H}-1,40.7$ & 0.41 & 0.41 & 0.40 & 5.1 & 17.9 & 60.7 \\
\hline $1 \mathrm{H}-1,43.3$ & 0.43 & 0.43 & 0.42 & 3.0 & 17.8 & 64.3 \\
\hline $1 \mathrm{H}-1,45.8$ & 0.46 & 0.46 & 0.45 & 4.4 & 17.4 & 63.9 \\
\hline $1 \mathrm{H}-1,48.4$ & 0.48 & 0.48 & 0.46 & 4.6 & 18.3 & 64.8 \\
\hline $1 \mathrm{H}-1,50.9$ & 0.51 & 0.51 & 0.49 & 2.9 & 17.8 & 67.3 \\
\hline $1 \mathrm{H}-1,53.4$ & 0.53 & 0.53 & 0.51 & -0.1 & 15.9 & 72.0 \\
\hline $1 \mathrm{H}-1,56$ & 0.56 & 0.56 & 0.54 & 3.5 & 19.4 & 72.8 \\
\hline $1 \mathrm{H}-1,58.5$ & 0.59 & 0.59 & 0.57 & 4.3 & 15.7 & 66.0 \\
\hline $1 \mathrm{H}-1,61.1$ & 0.61 & 0.61 & 0.59 & 3.3 & 17.1 & 68.1 \\
\hline $1 \mathrm{H}-1,63.6$ & 0.64 & 0.64 & 0.62 & 3.0 & 10.5 & 55.3 \\
\hline $1 \mathrm{H}-1,66.1$ & 0.66 & 0.66 & 0.64 & 4.2 & 15.5 & 66.0 \\
\hline $1 \mathrm{H}-1,68.7$ & 0.69 & 0.69 & 0.67 & 3.0 & 16.8 & 72.1 \\
\hline $1 \mathrm{H}-1,71.2$ & 0.71 & 0.71 & 0.69 & 2.2 & 16.5 & 73.4 \\
\hline $1 \mathrm{H}-1,73.8$ & 0.74 & 0.74 & 0.72 & 3.7 & 12.4 & 61.9 \\
\hline $1 \mathrm{H}-1,76.3$ & 0.76 & 0.76 & 0.73 & 4.3 & 16.7 & 69.5 \\
\hline $1 \mathrm{H}-1,78.9$ & 0.79 & 0.79 & 0.76 & 3.2 & 17.3 & 69.9 \\
\hline $1 \mathrm{H}-1,81.4$ & 0.81 & 0.81 & 0.78 & 2.3 & 15.8 & 69.9 \\
\hline $1 \mathrm{H}-1,83.9$ & 0.84 & 0.84 & 0.81 & 3.2 & 17.1 & 71.6 \\
\hline $1 \mathrm{H}-1,86.5$ & 0.87 & 0.87 & 0.84 & 4.2 & 17.5 & 71.4 \\
\hline $1 \mathrm{H}-1,89$ & 0.89 & 0.89 & 0.86 & 3.7 & 17.3 & 71.3 \\
\hline $1 \mathrm{H}-1,91.6$ & 0.92 & 0.92 & 0.89 & 4.2 & 16.2 & 66.8 \\
\hline $1 \mathrm{H}-1,94.1$ & 0.94 & 0.94 & 0.91 & 4.1 & 15.0 & 60.4 \\
\hline $1 \mathrm{H}-1,96.7$ & 0.97 & 0.97 & 0.94 & 2.3 & 16.6 & 70.0 \\
\hline $1 \mathrm{H}-1,99.2$ & 0.99 & 0.99 & 0.96 & 3.7 & 17.4 & 73.1 \\
\hline $1 \mathrm{H}-1,101.7$ & 1.02 & 1.02 & 0.99 & 3.7 & 15.6 & 70.1 \\
\hline $1 \mathrm{H}-1,104.3$ & 1.04 & 1.04 & 1.00 & 2.2 & 16.2 & 74.9 \\
\hline $1 \mathrm{H}-1,106.8$ & 1.07 & 1.07 & 1.03 & 5.0 & 16.6 & 65.7 \\
\hline $1 \mathrm{H}-1,109.4$ & 1.09 & 1.09 & 1.05 & 3.3 & 16.9 & 69.4 \\
\hline $1 \mathrm{H}-1,111.9$ & 1.12 & 1.12 & 1.08 & 3.0 & 11.4 & 55.4 \\
\hline $1 \mathrm{H}-1,114.4$ & 1.14 & 1.14 & 1.10 & 2.9 & 10.4 & 55.8 \\
\hline $1 \mathrm{H}-1,117$ & 1.17 & 1.17 & 1.13 & 4.5 & 16.7 & 63.6 \\
\hline $1 \mathrm{H}-1,119.5$ & 1.20 & 1.20 & 1.16 & 2.0 & 17.3 & 71.6 \\
\hline $1 \mathrm{H}-1,122.1$ & 1.22 & 1.22 & 1.18 & 4.7 & 13.9 & 55.2 \\
\hline $1 \mathrm{H}-1,124.6$ & 1.25 & 1.25 & 1.21 & 4.0 & 13.5 & 50.0 \\
\hline $1 \mathrm{H}-1,127.2$ & 1.27 & 1.27 & 1.23 & 5.0 & 15.8 & 52.2 \\
\hline $1 \mathrm{H}-1,129.7$ & 1.30 & 1.30 & 1.26 & 4.8 & 18.9 & 63.3 \\
\hline $1 \mathrm{H}-1,132.2$ & 1.32 & 1.32 & 1.27 & 5.7 & 17.6 & 56.3 \\
\hline $1 \mathrm{H}-1,134.8$ & 1.35 & 1.35 & 1.30 & 4.2 & 16.0 & 61.5 \\
\hline $1 \mathrm{H}-1,137.3$ & 1.37 & 1.37 & 1.32 & 6.0 & 18.8 & 54.8 \\
\hline $1 \mathrm{H}-1,139.9$ & 1.40 & 1.40 & 1.35 & 6.2 & 19.1 & 55.6 \\
\hline $1 \mathrm{H}-1,142.4$ & 1.42 & 1.42 & 1.37 & 5.7 & 19.4 & 56.0 \\
\hline $1 \mathrm{H}-1,145$ & 1.45 & 1.45 & 1.40 & 5.3 & 18.8 & 56.1 \\
\hline $1 \mathrm{H}-1,147.5$ & 1.48 & 1.48 & 1.43 & 2.8 & 10.5 & 39.9 \\
\hline
\end{tabular}

Only a portion of this table appears here. The complete table is available in ASCII. 
Table AT57. Core adjusted data, Hole U1338C. (Continued on next page.)

\begin{tabular}{|c|c|c|c|c|c|c|c|c|c|c|c|}
\hline \multirow[b]{2}{*}{$\begin{array}{l}\text { Core, section, } \\
\text { interval }(\mathrm{cm})\end{array}$} & \multicolumn{3}{|c|}{ Depth $(m)$} & \multirow[b]{2}{*}{$\begin{array}{l}\text { Core, section, } \\
\text { interval }(\mathrm{cm})\end{array}$} & \multicolumn{3}{|c|}{ Depth (m) } & \multirow[b]{2}{*}{$\begin{array}{l}\text { Core, section, } \\
\text { interval }(\mathrm{cm})\end{array}$} & \multicolumn{3}{|c|}{ Depth (m) } \\
\hline & $\begin{array}{l}\text { Adjusted } \\
\text { CCSF }\end{array}$ & CCSF & CSF-A & & $\begin{array}{l}\text { Adjusted } \\
\text { CCSF }\end{array}$ & CCSF & CSF-A & & $\begin{array}{l}\text { Adjusted } \\
\text { CCSF }\end{array}$ & CCSF & CSF-A \\
\hline 321-U1338C- & & & & $9 \mathrm{H}-7,77$ & 89.14 & 89.44 & 80.07 & $18 \mathrm{H}-5,91$ & 179.28 & 179.37 & 162.71 \\
\hline $1 \mathrm{H}-1,4$ & 0.04 & 0.04 & 0.04 & $10 \mathrm{H}-1,0$ & 89.95 & 90.07 & 79.80 & $18 \mathrm{H}-5,113$ & 179.49 & 179.59 & 162.93 \\
\hline $1 \mathrm{H}-2,58$ & 2.00 & 2.08 & 2.08 & $10 \mathrm{H}-1,50$ & 90.22 & 90.57 & 80.30 & $19 \mathrm{H}-1,78$ & 182.05 & 181.74 & 162.08 \\
\hline $1 \mathrm{H}-3,6$ & 2.61 & 2.76 & 2.76 & $10 \mathrm{H}-1,94$ & 90.69 & 91.01 & 80.74 & $19 \mathrm{H}-1,121$ & 182.47 & 182.16 & 162.51 \\
\hline $1 \mathrm{H}-3,41$ & 2.99 & 3.11 & 3.11 & $10 \mathrm{H}-3,9$ & 92.99 & 93.16 & 82.89 & $19 \mathrm{H}-2,99$ & 183.63 & 183.44 & 163.79 \\
\hline $1 \mathrm{H}-3,95$ & 3.45 & 3.65 & 3.65 & $10 \mathrm{H}-4,80$ & 95.23 & 95.37 & 85.10 & $19 \mathrm{H}-3,7$ & 184.20 & 184.02 & 164.37 \\
\hline $2 \mathrm{H}-2,49$ & 6.36 & 6.34 & 5.79 & $10 \mathrm{H}-5,40$ & 96.41 & 96.47 & 86.20 & $19 \mathrm{H}-4,99$ & 186.42 & 186.44 & 166.79 \\
\hline $2 \mathrm{H}-2,126$ & 7.16 & 7.11 & 6.56 & $10 \mathrm{H}-5,112$ & 97.13 & 97.19 & 86.92 & $19 \mathrm{H}-5,60$ & 187.52 & 187.55 & 167.90 \\
\hline $2 \mathrm{H}-3,43$ & 7.94 & 7.79 & 7.23 & $10 \mathrm{H}-6,20$ & 97.74 & 97.77 & 87.50 & $19 \mathrm{H}-6,108$ & 189.37 & 189.53 & 169.88 \\
\hline $2 \mathrm{H}-3,83$ & 8.33 & 8.18 & 7.63 & $10 \mathrm{H}-6,128$ & 98.80 & 98.85 & 88.58 & $19 \mathrm{H}-7,1$ & 189.66 & 189.78 & 170.13 \\
\hline $2 \mathrm{H}-4,110$ & 10.06 & 9.95 & 9.40 & $10 \mathrm{H}-7,90$ & 99.72 & 99.77 & 89.50 & $19 \mathrm{H}-7,98$ & 190.63 & 190.75 & 171.10 \\
\hline $2 \mathrm{H}-5,67$ & 11.07 & 11.02 & 10.47 & $11 \mathrm{H}-1,32$ & 100.27 & 99.87 & 89.62 & $20 \mathrm{H}-1,91$ & 191.79 & 191.83 & 171.71 \\
\hline $2 \mathrm{H}-7,31$ & 13.60 & 13.66 & 13.11 & $11 \mathrm{H}-1,68$ & 100.63 & 100.23 & 89.98 & $20 \mathrm{H}-3,25$ & 194.11 & 194.17 & 174.05 \\
\hline $2 \mathrm{H}-7,46$ & 13.78 & 13.81 & 13.26 & $11 \mathrm{H}-1,143$ & 101.40 & 100.98 & 90.73 & $20 \mathrm{H}-3,95$ & 194.84 & 194.87 & 174.75 \\
\hline $2 \mathrm{H}-7,78$ & 13.99 & 14.13 & 13.58 & $11 \mathrm{H}-3,8$ & 102.90 & 102.63 & 92.38 & $20 \mathrm{H}-5,32$ & 197.18 & 197.24 & 177.12 \\
\hline $3 \mathrm{H}-1,72$ & 16.48 & 16.33 & 14.02 & $11 \mathrm{H}-4,122$ & 105.18 & 105.28 & 95.02 & $20 \mathrm{H}-6,41$ & 198.84 & 198.83 & 178.71 \\
\hline $3 \mathrm{H}-1,148$ & 17.12 & 17.09 & 14.78 & $11 \mathrm{H}-5,124$ & 106.67 & 106.79 & 96.54 & $20 \mathrm{H}-7,42$ & 200.41 & 200.34 & 180.22 \\
\hline $3 \mathrm{H}-2,37$ & 17.59 & 17.48 & 15.17 & $11 \mathrm{H}-6,102$ & 107.90 & 108.07 & 97.82 & $20 \mathrm{H}-7,69$ & 200.68 & 200.61 & 180.49 \\
\hline $3 \mathrm{H}-2,123$ & 18.35 & 18.34 & 16.03 & $11 \mathrm{H}-7,78$ & 109.18 & 109.33 & 99.08 & $21 \mathrm{H}-1,56$ & 202.64 & 202.57 & 180.86 \\
\hline $3 \mathrm{H}-5,0$ & 21.59 & 21.61 & 19.30 & $12 \mathrm{H}-1,1$ & 109.94 & 110.10 & 98.81 & $21 \mathrm{H}-1,129$ & 203.38 & 203.31 & 181.59 \\
\hline $3 \mathrm{H}-6,23$ & 23.40 & 23.34 & 21.03 & $12 \mathrm{H}-1,39$ & 110.45 & 110.48 & 99.19 & $21 \mathrm{H}-2,16$ & 203.70 & 203.67 & 181.96 \\
\hline $3 \mathrm{H}-6,125$ & 24.50 & 24.36 & 22.05 & $12 \mathrm{H}-1,122$ & 111.46 & 111.31 & 100.02 & $21 \mathrm{H}-2,112$ & 204.65 & 204.63 & 182.92 \\
\hline $3 \mathrm{H}-7,77$ & 25.35 & 25.38 & 23.07 & $12 \mathrm{H}-3,55$ & 113.64 & 113.64 & 102.35 & $21 \mathrm{H}-3,50$ & 205.46 & 205.51 & 183.80 \\
\hline $4 \mathrm{H}-1,0$ & 26.38 & 25.66 & 22.80 & $12 \mathrm{H}-4,105$ & 115.69 & 115.64 & 104.35 & $21 \mathrm{H}-4,31$ & 206.82 & 206.82 & 185.11 \\
\hline $4 \mathrm{H}-1,61$ & 26.99 & 26.27 & 23.41 & $12 \mathrm{H}-5,59$ & 116.65 & 116.68 & 105.39 & $21 \mathrm{H}-5,121$ & 209.34 & 209.22 & 187.51 \\
\hline $4 \mathrm{H}-1,85$ & 27.34 & 26.51 & 23.65 & $12 \mathrm{H}-6,125$ & 118.65 & 118.84 & 107.55 & $21 \mathrm{H}-6,21$ & 209.84 & 209.72 & 188.01 \\
\hline $4 \mathrm{H}-1,133$ & 28.34 & 26.99 & 24.13 & $12 \mathrm{H}-7,78$ & 119.60 & 119.67 & 108.38 & $21 \mathrm{H}-7,7$ & 211.24 & 211.08 & 189.37 \\
\hline $4 \mathrm{H}-5,15$ & 31.41 & 31.81 & 28.95 & $13 \mathrm{H}-4,15$ & 124.65 & 124.70 & 112.95 & $22 \mathrm{H}-1,0$ & 212.04 & 211.93 & 189.80 \\
\hline $4 \mathrm{H}-5,82$ & 32.07 & 32.48 & 29.62 & $13 \mathrm{H}-4,89$ & 125.49 & 125.44 & 113.69 & $21 \mathrm{H}-7,77$ & 212.07 & 211.78 & 190.07 \\
\hline $4 \mathrm{H}-6,27$ & 33.48 & 33.43 & 30.57 & $13 \mathrm{H}-5,66$ & 126.49 & 126.71 & 114.96 & $22 \mathrm{H}-1,71$ & 212.75 & 212.64 & 190.51 \\
\hline $4 \mathrm{H}-6,46$ & 33.88 & 33.63 & 30.76 & $13 \mathrm{H}-6,22$ & 127.36 & 127.77 & 116.02 & $22 \mathrm{H}-2,127$ & 214.64 & 214.70 & 192.57 \\
\hline $4 \mathrm{H}-6,133$ & 34.74 & 34.49 & 31.63 & $13 \mathrm{H}-7,39$ & 128.76 & 129.15 & 117.40 & $22 \mathrm{H}-4,86$ & 217.15 & 217.29 & 195.16 \\
\hline $5 \mathrm{H}-1,0$ & 37.56 & 37.56 & 32.30 & $13 \mathrm{H}-7,71$ & 129.27 & 129.47 & 117.72 & $22 \mathrm{H}-6,1$ & 219.19 & 219.44 & 197.31 \\
\hline $5 \mathrm{H}-1,48$ & 38.04 & 38.04 & 32.78 & $13 \mathrm{H}-7,90$ & 129.47 & 129.66 & 117.91 & $22 \mathrm{H}-6,130$ & 220.36 & 220.73 & 198.60 \\
\hline $5 \mathrm{H}-5,46$ & 44.02 & 44.02 & 38.76 & $14 \mathrm{H}-1,0$ & 131.38 & 131.38 & 117.80 & $22 \mathrm{H}-7,59$ & 221.02 & 221.32 & 199.19 \\
\hline $5 \mathrm{H}-6,85$ & 45.72 & 45.91 & 40.65 & $14 \mathrm{H}-1,119$ & 132.57 & 132.57 & 118.99 & $22 \mathrm{H}-7,55$ & 221.15 & 221.28 & 199.15 \\
\hline $5 \mathrm{H}-7,50$ & 46.73 & 47.06 & 41.80 & $14 \mathrm{H}-4,101$ & 136.89 & 136.89 & 123.31 & $22 \mathrm{H}-7,85$ & 221.28 & 221.58 & 199.45 \\
\hline $5 \mathrm{H}-7,77$ & 47.01 & 47.33 & 42.07 & $14 \mathrm{H}-5,130$ & 138.85 & 138.68 & 125.10 & $23 \mathrm{H}-1,145$ & 223.00 & 222.73 & 200.75 \\
\hline $6 \mathrm{H}-1,0$ & 49.41 & 49.46 & 41.80 & $14 \mathrm{H}-6,90$ & 139.80 & 139.78 & 126.20 & $23 \mathrm{H}-2,78$ & 223.79 & 223.56 & 201.58 \\
\hline $6 \mathrm{H}-1,44$ & 49.86 & 49.91 & 42.24 & $14 \mathrm{H}-7,98$ & 141.18 & 141.17 & 127.59 & $23 \mathrm{H}-2,146$ & 224.36 & 224.25 & 202.26 \\
\hline $6 \mathrm{H}-1,71$ & 50.09 & 50.17 & 42.51 & $15 \mathrm{H}-1,0$ & 142.14 & 141.88 & 127.30 & $23 \mathrm{H}-4,55$ & 226.38 & 226.33 & 204.35 \\
\hline $6 \mathrm{H}-1,134$ & 50.76 & 50.80 & 43.14 & $15 \mathrm{H}-1,73$ & 142.88 & 142.61 & 128.03 & $23 \mathrm{H}-5,9$ & 227.45 & 227.38 & 205.39 \\
\hline $6 \mathrm{H}-3,89$ & 53.32 & 53.35 & 45.69 & $15 \mathrm{H}-2,133$ & 144.96 & 144.71 & 130.13 & $23 \mathrm{H}-5,124$ & 228.81 & 228.52 & 206.54 \\
\hline $6 \mathrm{H}-5,86$ & 56.41 & 56.32 & 48.66 & $15 \mathrm{H}-4,77$ & 147.15 & 147.15 & 132.57 & $23 \mathrm{H}-6,51$ & 229.68 & 229.29 & 207.31 \\
\hline $6 \mathrm{H}-6,89$ & 57.91 & 57.85 & 50.19 & $15 \mathrm{H}-5,83$ & 148.64 & 148.71 & 134.13 & $23 \mathrm{H}-7,78$ & 231.45 & 231.06 & 209.08 \\
\hline $6 \mathrm{H}-7,1$ & 58.55 & 58.47 & 50.81 & $15 \mathrm{H}-6,57$ & 149.72 & 149.95 & 135.37 & $24 \mathrm{H}-1,0$ & 232.49 & 232.45 & 208.80 \\
\hline $6 \mathrm{H}-7,80$ & 59.34 & 59.26 & 51.60 & $15 \mathrm{H}-6,104$ & 150.16 & 150.42 & 135.84 & $24 \mathrm{H}-1,74$ & 233.23 & 233.19 & 209.54 \\
\hline $7 \mathrm{H}-2,33$ & 60.09 & 59.93 & 53.13 & $15 \mathrm{H}-7,71$ & 151.34 & 151.59 & 137.01 & $24 \mathrm{H}-2,98$ & 234.99 & 234.93 & 211.28 \\
\hline $7 \mathrm{H}-3,60$ & 61.78 & 61.70 & 54.90 & $16 \mathrm{H}-1,36$ & 154.05 & 154.00 & 137.16 & $24 \mathrm{H}-3,150$ & 236.97 & 236.95 & 213.30 \\
\hline $7 \mathrm{H}-4,77$ & 63.36 & 63.37 & 56.57 & $16 \mathrm{H}-1,71$ & 154.40 & 154.35 & 137.51 & $24 \mathrm{H}-5,28$ & 238.68 & 238.73 & 215.08 \\
\hline $7 \mathrm{H}-6,59$ & 66.13 & 66.19 & 59.39 & $16 \mathrm{H}-2,1$ & 155.12 & 155.15 & 138.31 & $24 \mathrm{H}-6,114$ & 241.04 & 241.09 & 217.44 \\
\hline $7 \mathrm{H}-6,90$ & 66.44 & 66.50 & 59.70 & $16 \mathrm{H}-5,100$ & 160.18 & 160.64 & 143.80 & $24 \mathrm{H}-7,71$ & 242.11 & 242.16 & 218.51 \\
\hline $7 \mathrm{H}-7,79$ & 67.76 & 67.89 & 61.09 & $16 \mathrm{H}-6,58$ & 161.29 & 161.72 & 144.88 & $25 \mathrm{H}-1,7$ & 243.40 & 243.40 & 218.37 \\
\hline $8 \mathrm{H}-2,100$ & 71.96 & 72.28 & 63.30 & $16 \mathrm{H}-6,123$ & 162.00 & 162.37 & 145.53 & $25 \mathrm{H}-1,83$ & 244.09 & 244.16 & 219.13 \\
\hline $8 \mathrm{H}-3,3$ & 72.26 & 72.81 & 63.83 & $16 \mathrm{H}-7,73$ & 162.73 & 163.17 & 146.33 & $25 \mathrm{H}-2,67$ & 245.51 & 245.50 & 220.47 \\
\hline $8 \mathrm{H}-3,56$ & 72.91 & 73.34 & 64.36 & $16 \mathrm{H}-7,85$ & 162.86 & 163.29 & 146.45 & $25 \mathrm{H}-3,21$ & 246.57 & 246.55 & 221.51 \\
\hline $8 \mathrm{H}-3,72$ & 73.35 & 73.50 & 64.52 & $17 \mathrm{H}-1,71$ & 164.54 & 164.54 & 147.01 & $25 \mathrm{H}-5,44$ & 249.84 & 249.77 & 224.74 \\
\hline $8 \mathrm{H}-4,6$ & 74.52 & 74.34 & 65.36 & $17 \mathrm{H}-2,123$ & 166.59 & 166.56 & 149.03 & $25 \mathrm{H}-6,83$ & 251.82 & 251.66 & 226.63 \\
\hline $8 \mathrm{H}-4,84$ & 75.39 & 75.12 & 66.14 & $17 \mathrm{H}-3,71$ & 167.54 & 167.54 & 150.01 & $25 \mathrm{H}-7,76$ & 253.25 & 253.09 & 228.06 \\
\hline $8 \mathrm{H}-5,133$ & 77.09 & 77.11 & 68.13 & $17 \mathrm{H}-3,108$ & 167.83 & 167.91 & 150.38 & $26 \mathrm{H}-1,0$ & 254.09 & 254.04 & 227.80 \\
\hline $8 \mathrm{H}-6,117$ & 78.06 & 78.45 & 69.47 & $17 \mathrm{H}-4,53$ & 168.92 & 168.86 & 151.33 & $26 \mathrm{H}-1,108$ & 255.17 & 255.12 & 228.88 \\
\hline $8 \mathrm{H}-6,148$ & 78.37 & 78.76 & 69.78 & $17 \mathrm{H}-6,52$ & 171.81 & 171.85 & 154.32 & $26 \mathrm{H}-2,113$ & 256.66 & 256.67 & 230.43 \\
\hline $9 \mathrm{H}-1,87$ & 80.46 & 80.54 & 71.17 & $17 \mathrm{H}-6,125$ & 172.63 & 172.59 & 155.05 & $26 \mathrm{H}-2,137$ & 256.89 & 256.91 & 230.67 \\
\hline $9 \mathrm{H}-1,131$ & 81.12 & 80.98 & 71.61 & $17 \mathrm{H}-7,79$ & 173.65 & 173.62 & 156.09 & $26 \mathrm{H}-3,23$ & 257.56 & 257.27 & 231.03 \\
\hline $9 \mathrm{H}-4,30$ & 84.45 & 84.47 & 75.10 & $18 \mathrm{H}-2,113$ & 175.25 & 175.09 & 158.43 & $26 \mathrm{H}-4,111$ & 259.79 & 259.65 & 233.41 \\
\hline $9 \mathrm{H}-5,1$ & 85.59 & 85.69 & 76.31 & $18 \mathrm{H}-2,148$ & 175.61 & 175.44 & 158.78 & $26 \mathrm{H}-6,15$ & 261.67 & 261.69 & 235.45 \\
\hline $9 \mathrm{H}-6,7$ & 87.09 & 87.24 & 77.87 & $18 \mathrm{H}-3,54$ & 176.16 & 176.00 & 159.34 & $26 \mathrm{H}-6,148$ & 262.49 & 263.02 & 236.78 \\
\hline $9 \mathrm{H}-7,22$ & 88.59 & 88.89 & 79.52 & $18 \mathrm{H}-3,104$ & 176.68 & 176.50 & 159.84 & $26 \mathrm{H}-7,50$ & 263.16 & 263.54 & 237.30 \\
\hline
\end{tabular}


Table AT57 (continued).

\begin{tabular}{lccc}
\hline & \multicolumn{3}{c}{ Depth (m) } \\
\cline { 2 - 4 } Core, section, & Adjusted & & \\
interval (cm) & CCSF & CCSF & CSF-A \\
\hline $26 \mathrm{H}-7,76$ & 263.43 & 263.80 & 237.56 \\
$27 \mathrm{H}-1,6$ & 264.70 & 264.62 & 237.36 \\
$27 \mathrm{H}-1,42$ & 265.05 & 264.98 & 237.72 \\
$27 \mathrm{H}-1,82$ & 265.47 & 265.38 & 238.12 \\
$27 \mathrm{H}-2,134$ & 267.40 & 267.40 & 240.14 \\
$27 \mathrm{H}-4,41$ & 269.47 & 269.47 & 242.21 \\
$27 \mathrm{H}-5,129$ & 271.75 & 271.86 & 244.59 \\
$27 \mathrm{H}-6,77$ & 272.69 & 272.83 & 245.57 \\
$27 \mathrm{H}-6,147$ & 273.39 & 273.53 & 246.27 \\
$28 \mathrm{H}-1,7$ & 274.33 & 274.33 & 246.87 \\
$28 \mathrm{H}-1,38$ & 274.64 & 274.64 & 247.18 \\
$28 \mathrm{H}-7,54$ & 283.80 & 283.80 & 256.34 \\
$29 \mathrm{H}-1,35$ & 284.11 & 284.11 & 256.65 \\
$29 \mathrm{H}-2,41$ & 285.67 & 285.67 & 258.21 \\
$29 \mathrm{H}-3,73$ & 287.50 & 287.49 & 260.03 \\
$29 \mathrm{H}-4,60$ & 288.62 & 288.86 & 261.40 \\
$29 \mathrm{H}-4,128$ & 289.45 & 289.54 & 262.08 \\
$29 \mathrm{H}-5,46$ & 290.14 & 290.23 & 262.76 \\
$29 \mathrm{H}-5,125$ & 290.80 & 291.01 & 263.55 \\
$29 \mathrm{H}-6,101$ & 292.02 & 292.27 & 264.81 \\
$29 \mathrm{H}-7,68$ & 293.19 & 293.44 & 265.98 \\
$30 \mathrm{H}-1,14$ & 294.64 & 294.38 & 265.94 \\
$30 \mathrm{H}-1,95$ & 295.47 & 295.20 & 266.75 \\
$30 \mathrm{H}-2,2$ & 296.02 & 295.76 & 267.32 \\
$30 \mathrm{H}-2,97$ & 296.74 & 296.71 & 268.27 \\
$30 \mathrm{H}-3,104$ & 298.27 & 298.28 & 269.84 \\
$30 \mathrm{H}-5,124$ & 301.47 & 301.48 & 273.04 \\
$30 \mathrm{H}-6,95$ & 302.67 & 302.69 & 274.25 \\
$30 \mathrm{H}-7,83$ & 303.87 & 303.88 & 275.44 \\
$31 \mathrm{H}-1,13$ & 304.85 & 304.86 & 275.43 \\
$31 \mathrm{H}-5,142$ & 312.14 & 312.14 & 282.72 \\
$32 \mathrm{H}-1,55$ & 312.87 & 312.87 & 283.45 \\
$32 \mathrm{H}-2,27$ & 314.09 & 314.09 & 284.67 \\
$32 \mathrm{H}-3,24$ & 315.63 & 315.56 & 286.14 \\
$32 \mathrm{H}-4,31$ & 316.79 & 316.64 & 287.22 \\
$32 \mathrm{H}-4,75$ & 317.23 & 317.08 & 287.66 \\
$33 \mathrm{H}-1,9$ & 322.53 & 322.53 & 292.49 \\
$33 \mathrm{H}-2,71$ & 324.65 & 324.65 & 294.61 \\
$33 \mathrm{H}-5,3$ & 328.60 & 328.47 & 298.43 \\
$33 \mathrm{H}-5,116$ & 329.53 & 329.60 & 299.56 \\
$33 \mathrm{H}-5,144$ & 329.98 & 329.88 & 299.84 \\
$33 \mathrm{H}-6,49$ & 330.59 & 330.43 & 300.39 \\
$33 \mathrm{H}-6,108$ & 331.24 & 331.02 & 300.98 \\
$33 \mathrm{H}-7,53$ & 332.13 & 331.78 & 301.74 \\
$33 \mathrm{H}-7,91$ & 332.38 & 332.16 & 302.12 \\
$34 \mathrm{H}-2,78$ & 332.73 & 332.97 & 304.18 \\
$34 \mathrm{H}-3,51$ & 333.96 & 334.20 & 305.41 \\
$34 \mathrm{H}-3,149$ & 335.12 & 335.18 & 306.39 \\
$34 \mathrm{H}-4,76$ & 335.83 & 335.95 & 307.16 \\
$34 \mathrm{H}-6,111$ & 339.38 & 339.30 & 310.51 \\
$35 \mathrm{H}-1,10$ & 340.13 & 340.13 & 307.00 \\
$35 \mathrm{H}-5,109$ & 347.15 & 347.15 & 314.02 \\
$35 \mathrm{H}-6,115$ & 348.61 & 348.71 & 315.58 \\
$35 \mathrm{H}-7,70$ & 349.44 & 349.58 & 316.44 \\
$35 \mathrm{H}-7,90$ & 349.64 & 349.77 & 316.64 \\
$36 \mathrm{H}-1,0$ & 351.01 & 350.97 & 316.40
\end{tabular}

\begin{tabular}{lccc}
\hline & \multicolumn{3}{c}{ Depth (m) } \\
\cline { 2 - 4 } Core, section, & Adjusted & & \\
interval (cm) & CCSF & CCSF & CSF-A \\
\hline $36 \mathrm{H}-1,30$ & 351.31 & 351.27 & 316.70 \\
$36 \mathrm{H}-1,73$ & 351.54 & 351.70 & 317.13 \\
$36 \mathrm{H}-1,103$ & 352.01 & 352.01 & 317.43 \\
$36 \mathrm{H}-4,68$ & 356.15 & 356.15 & 321.58 \\
$36 \mathrm{H}-5,45$ & 357.42 & 357.42 & 322.85 \\
$36 \mathrm{H}-6,18$ & 358.56 & 358.65 & 324.08 \\
$36 \mathrm{H}-7,33$ & 360.04 & 360.30 & 325.73 \\
$36 \mathrm{H}-7,70$ & 360.41 & 360.67 & 326.10 \\
$37 \mathrm{H}-1,0$ & 362.07 & 362.07 & 325.90 \\
$37 \mathrm{H}-1,86$ & 362.93 & 362.93 & 326.76 \\
$37 \mathrm{H}-5,5$ & 368.13 & 368.13 & 331.96 \\
$37 \mathrm{H}-5,89$ & 369.06 & 368.97 & 332.80 \\
$37 \mathrm{H}-5,145$ & 369.53 & 369.53 & 333.36 \\
$37 \mathrm{H}-7,40$ & 371.56 & 371.48 & 335.31 \\
$37 \mathrm{H}-7,67$ & 371.83 & 371.75 & 335.58 \\
$38 \mathrm{H}-1,4$ & 372.22 & 372.24 & 335.44 \\
$38 \mathrm{H}-1,48$ & 372.66 & 372.67 & 335.88 \\
$38 \mathrm{H}-1,119$ & 373.38 & 373.38 & 336.59 \\
$38 \mathrm{H}-5,96$ & 379.15 & 379.15 & 342.36 \\
$38 \mathrm{H}-5,120$ & 379.66 & 379.39 & 342.60 \\
$38 \mathrm{H}-6,98$ & 381.02 & 380.67 & 343.88 \\
$38 \mathrm{H}-7,63$ & 381.99 & 381.82 & 345.03 \\
$39 \mathrm{H}-1,0$ & 382.10 & 382.20 & 344.90 \\
$39 \mathrm{H}-1,25$ & 382.35 & 382.45 & 345.15 \\
$39 \mathrm{H}-1,76$ & 383.00 & 382.96 & 345.66 \\
$39 \mathrm{H}-1,148$ & 383.69 & 383.69 & 346.38 \\
$39 \mathrm{H}-7,74$ & 391.94 & 391.94 & 354.64 \\
$40 \mathrm{H}-1,0$ & 392.08 & 392.08 & 354.40 \\
$40 \mathrm{H}-7,48$ & 401.16 & 401.16 & 363.48 \\
$41 \mathrm{H}-1,11$ & 402.26 & 402.30 & 364.01 \\
$41 \mathrm{H}-1,116$ & 403.35 & 403.35 & 365.06 \\
$41 \mathrm{H}-7,71$ & 411.92 & 411.92 & 373.63 \\
$42 \mathrm{H}-2,6$ & 413.69 & 413.87 & 374.96 \\
$42 \mathrm{H}-2,110$ & 415.06 & 414.91 & 376.00 \\
$42 \mathrm{H}-3,25$ & 415.55 & 415.56 & 376.65 \\
$42 \mathrm{H}-3,63$ & 415.81 & 415.94 & 377.03 \\
$42 \mathrm{H}-4,123$ & 418.06 & 418.04 & 379.13 \\
$42 \mathrm{H}-5,67$ & 418.73 & 418.73 & 379.82 \\
$42 \mathrm{H}-5,80$ & 418.88 & 418.86 & 379.95 \\
$43 \mathrm{H}-2,46$ & 420.31 & 420.32 & 379.86 \\
$43 \mathrm{H}-4,96$ & 423.83 & 423.83 & 383.38 \\
$43 \mathrm{H}-5,85$ & 425.28 & 425.23 & 384.78 \\
$43 \mathrm{H}-5,109$ & 425.59 & 425.47 & 385.02 \\
$43 \mathrm{H}-7,35,85$ & 427.70 & 427.77 & 387.32 \\
$43 \mathrm{H}-7,93$ & 428.19 & 428.35 & 387.90 \\
$44 \mathrm{H}-1,0$ & 428.61 & 428.61 & 387.40 \\
$44 \mathrm{H}-1,40$ & 429.01 & 429.01 & 387.80 \\
$44 \mathrm{H}-6,78$ & 436.68 & 436.68 & 395.47 \\
$45 \mathrm{H}-1,0$ & 438.11 & 438.11 & 396.90 \\
46 & 445.03 & 445.03 & 403.82 \\
& 445.21 & 445.21 & 404.00 \\
& 451.44 & 451.44 & 410.23 \\
465.27 & 451.85 & 410.64 \\
& & & 414.06 \\
\hline
\end{tabular}

This table is also available in ASCII. 